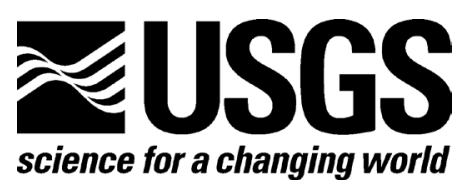

\title{
HAWAIIAN VOLCANO OBSERVATORY 1975 Annual Administrative Report
}

\author{
INTRODUCTORY NOTE BY THOMAS L. WRIGHT AND JENNIFER S. NAKATA
}

COMPILED BY JENNIFER S. NAKATA

\author{
SUMMARY 75 \\ JANUARY TO DECEMBER 1975 \\ By Robert Y. Koyanagi, Karen Meagher, \\ Fred W. KLEIN, AND ARnOld T. OKAMura \\ CHRONOLOGICAL SUMMARY \\ BY DONALD W. PETERSON
}

OPEN-FILE REPORT 2007-1335

U.S. DEPARTMENT OF THE INTERIOR

U.S. GEOLOGICAL SURVEY 


\title{
U.S. Department of the Interior DIRK KEMPTHORNE, Secretary \\ U.S. Geological Survey Mark D. Myers, Director
}

\section{U.S. Geological Survey, Reston, Virginia 2007}

\author{
For product and ordering information: \\ World Wide Web: http://www.usgs.gov/pubprod \\ Telephone: 1-888-ASK-USGSFor more information on the USGS-the Federal source for \\ science about the Earth, \\ its natural and living resources, natural hazards, and the environment: \\ World Wide Web: http://www.usgs.gov \\ Telephone: 1-888-ASK-USGS
}

Any use of trade, product, or firm names is for descriptive purposes only and does not imply endorsement by the U.S. Government.

Although this report is in the public domain, permission must be secured from the individual copyright owners to reproduce any copyrighted material contained within this report. 


\section{INTRODUCTORY NOTE}

The Hawaiian Volcano Observatory Summaries have been published in the current format since 1956. The Quarterly Summaries (1956 through 1973) and the Annual Summaries (1974 through 1985) were originally published as Administrative Reports. These reports have been compiled and published as U.S. Geological Survey Open-File Reports. The quarterly reports have been combined and published as one annual summary. All the summaries from 1956 to the present are now available as .pdf files at http://www.usgs.gov/pubprod.

The earthquake summary data are presented as a listing of origin time, depth, magnitude, and other location parameters. Network instrumentation, field station sites, and location algorithms are described. Tilt and other deformation data are included until Summary 77, January to December 1977. From 1978, the seismic and deformation data are published separately, due to differing schedules of data reduction.

There are eight quarters - from the fourth quarter of 1959 to the third quarter of 1961 — that were never published. Two of these ( $4^{\text {th }}$ quarter $1959,1^{\text {st }}$ quarter 1960) have now been published, using handwritten notes of Jerry Eaton (HVO seismologist at the time) and his colleagues. The seismic records for the remaining six summaries went back to California in 1961 with Jerry Eaton. Other responsibilities intervened, and the seismic summaries were never prepared.

\section{Chronology}

The following Kìlauea eruption chronology covers the two recent reports and the six missing quarters:

\begin{tabular}{|l|l|l|l|}
\hline Location & Beginning Date & Ending Date & Comment \\
\hline Kìlauea Iki crater (Kîlauea's summit) & $11 / 14 / 1959$ & $12 / 20 / 1959$ & 19 eruptive episodes \\
\hline Kapoho (lower east rift zone) & $1 / 13 / 1960$ & $2 / 18 / 1960$ & 4 eruption stages \\
\hline Halemaumau (Kīlauea's summit) & $2 / 24 / 1961$ & $2 / 24 / 1961$ & $\begin{array}{l}\text { Intermittent activity during } \\
\text { uninterrupted inflation fol- } \\
\text { lowing the 1960 eruption }\end{array}$ \\
\hline Halemaumau (Kīlauea's summit) & $3 / 22 / 1961$ & $3 / 25 / 1961$ & Same as above. \\
\hline Halemaumau (Kīlauea's summit) & $7 / 10 / 1961$ & $7 / 17 / 1961$ & Same as above. \\
\hline Heiheiahulu (middle east rift zone) & $9 / 22 / 1961$ & $9 / 25 / 1961$ & $\begin{array}{l}\text { First historical east rift erup- } \\
\text { tion at this location }\end{array}$ \\
\hline
\end{tabular}

The 1959-1960 eruptions were among two of the most spectacular Kilauea eruptions. The HVO staff was kept busy with acquisition of unusually high quantities of instrumental data and observations of the two sequences, which were separated by less than one month. Even with a year's interval before the beginning of the summit-east rift sequence in 1961, the staff never caught up, and the seismic records were set aside for later study.

A total of 1,672 earthquakes-1,106 for 1960 and 566 for 1961-are part of HVO's cataloged database. The annual listings have been appended to the $1^{\text {st }}$ Quarter Report of 1960 and to the $4^{\text {th }}$ Quarter Report for 1961 . The number of earthquakes is probably low, biased toward the larger magnitudes. The entire HVO catalog, including 1960 and 1961, is accessible from the ANSS CATALOG SEARCH site at http://www.ncedc.org/anss/catalog-search. 
UNITED STATES

DEPARTMENT OF THE INTERIOR

GEOLOGICAL SURVEY

\section{HAWAIIAN VOLCANO OBSERVATORY}

\section{SUMMARY 75}

JANUARY TO DECEMBER 1975

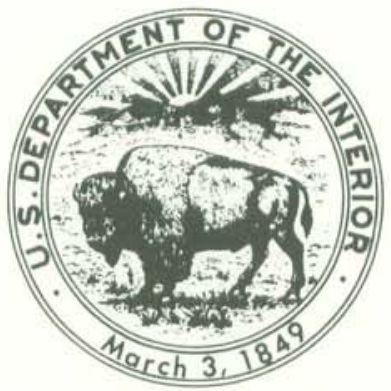

This report is preliminary and has not been edited or reviewed for conformity with Geological Survey standards and nomenclature

Menlo Park, California 


\author{
UNITED STATES \\ DEPARTMENT OF THE INTERIOR \\ GEOLOGICAL SURVEY \\ HAWAIIAN VOLCANO OBSERVATORY \\ SUMMARY 75 \\ JANUARY TO DECEMBER 1975
}

BY

ROBERT Y. KOYANAGI, KAREN MEAGHER*, FRED W. KLEIN*, AND ARNOLD T. OKAMURA

CHRONOLOGICAL SUMMARY

BY

DONALD W. PETERSON

OBSERVATORY STAFF

GEOLOGY

GEOCHEMISTRY

ROBIN T. HOLCOMB

REGINALD T. OKAMURA

PETER W. LIPMAN

JOHN P. LOCKWOOD

DONALD W. PETERSON (SCIENTIST-IN-CHARGE)

ROBERT I. TILLING (SCIENTIST-IN-CHARGE)

GEOPHYSICS

LENNART A. ANDERSON

JENNIFER S. EBISU

ELLIOT T. ENDO

KENNETH T. HONMA

GEORGE KOJIMA

ROBERT Y. KOYANAGI

ERWIN MCPHERSON, JR.

ARNOLD T. OKAMURA

GARY S. PUN IWAI

KENNETH M. YAMASHITA

CHARLES J. ZABLOCKI $\underline{\text { SUPPORT }}$

JOHN C. FORBES

WILLIAM H. FRANCIS

MARIE S. ONOUYE

MAURICE K. SAKO

AKIRA YAMAMOTO

*OFFICE OF EARTHQUAKE STUDIES, MENLO PARK, CALIFORNIA 
Introduction. . . . . . . . .

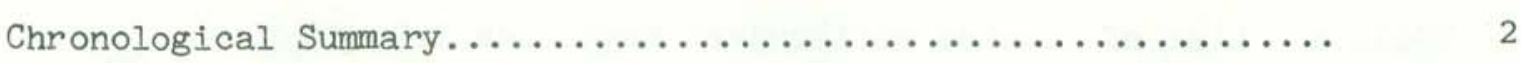

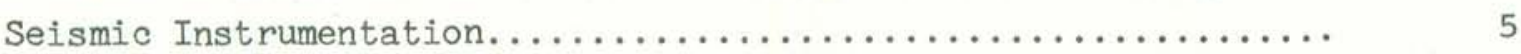

Figure 1 Map of Hawaii showing geographic and geologic

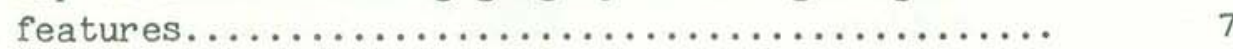

Figure 2 Map of Hawaii showing active seismic stations..... 8

Table 1 Seismic stations on Hawaii operated by the USGS... 9

Table 2 Seismic instrumentation types in use by HVO...... 10

Figure 3 System response curve of the four basic seismograph types in use by HVO............... 11

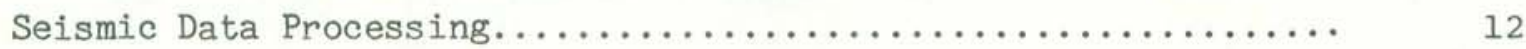

Figure 4 Relationship between duration time and local

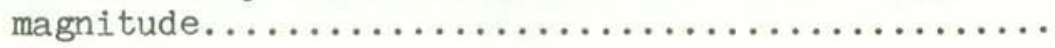

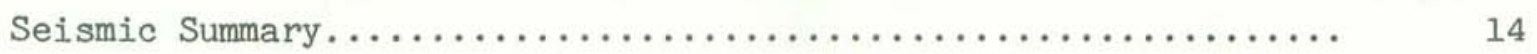

Table 3 Number of earthquakes and minutes of tremor recorded on seismographs around Kilauea..........

Figure 5 Epicenter map of Hawaii showing only magnitudes

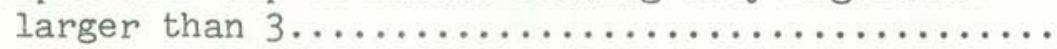

Figure 6 Epicenter map of Hawail showing all earthquakes...

Figure 7 Epicenter map of the Kilauea/Mauna Loa region showing only magnitudes larger than $3 \ldots \ldots \ldots \ldots$.

Figure $४$ Epicenter map of the Kilauea/Mauna Loa region

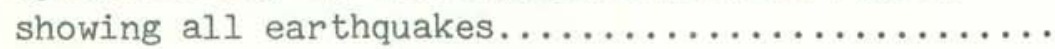

Figure 9 Map of Hawaii showing geographic codes used for classifying earthquakes by region..............

Table 4 Coordinates of named regions used for classifying

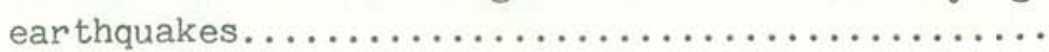

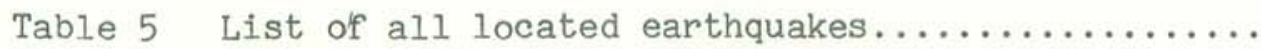


CONTENTS (continued)

$\underline{\text { Page }}$

Table 6 List of located earthquakes magnitude 3.5 and larger................................ 99

Table 7 List of felt earthquakes, January-September, 1975. 105

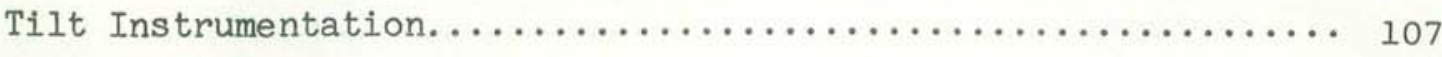

Tilting of the ground around Kilauea Caldera............... 108

Table 8 Weekly tilt coordinates at Uwekahuna........... 108

Table 9 Water-tube tilt stations in Hawaii ............ 110

Table 10 Tilt coordinates and changes, January-April, 1975. 111

Figure 10 Tilt vectors around Kilauea, January-April, 1975.. 112

Table 11 Tilt coordinates and changes, April-September,

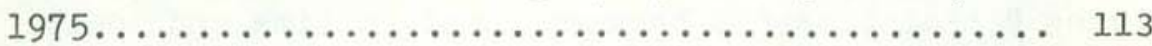

Figure 11 Tilt vectors around Kilauea, April-September,

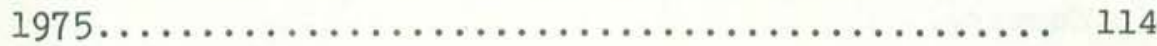

Table 12 Tilt coordinates and changes, September-December,

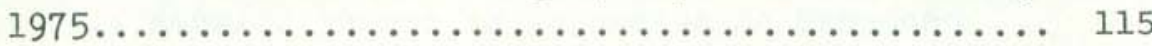

Figure 12 Tilt vectors around Kilauea, September-December,

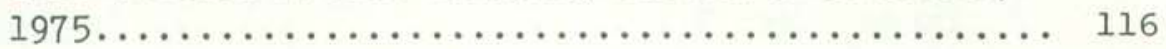

References cited.................................. 117 


\section{INTRODUCTION}

The Hawaiian Volcano Observatory (HVO) summaries present data gathered during the year together with a chronological narrative intended to describe in geologic terms the volcanic activity associated with the seismic events and tilt data included. The seismic, tilt, and chronological summaries are offered without interpretation as a source of preliminary data. The seismic summary is complete in the sense that all data routinely gathered by the observatory are included. The emphasis in collection of tilt and deformation data has recently shifted from quarterly measurements at a few water-tube tilt stations ("wet" tilt) to a larger number of continuously recording borehole tiltmeters and repeated measurements at numerous spirit-level tilt stations ("dry" tilt). To maintain continuity with past summaries, we will continue to publish weekly data from the Uwekahuna vault tiltmeter (Kilauea summit) and from water-tube tilt stations as they are reoccupied. A comprehensive summary of the numerous and varied tilt and deformation data now gathered is beyond the scope of this publication.

The HVO summaries have been published in various formats since 1956. Summaries prior to 1974 were issued quarterly, but cost, convenience of preparation and distribution, and the large quantities of data dictated an annual format beginning with summary 74 (Koyanagi, et al.) for the year 1974. Summary 74 includes an extensive description of the seismic instrumentation, calibration and processing used in recent years. The present summary includes enough background information on the seismic network and processing to use the data and understand the essentials of how it was gathered.

Publication of the summary represents a group effort by the staffs of the Hawaiian Volcano Observatory and the National Center for Earthquake Research in Menlo Park, California. 


\section{CHRONOLOGICAL SUMMARY}

\section{Kilauea}

The year 1975 opened with Kilauea adjusting to the effect of the 6-hour-long eruption of December 31, 1974. This eruption occurred in the $\mathrm{Ka}^{\prime} \mathrm{u}$ Desert, between the southwest rift zone and the Koae fault system. About $15 \times 10^{6} \mathrm{~m}^{3}$ of lava was erupted from a set of en echelon fissures about $4 \mathrm{~km}$ long. The eruption was preceded, accompanied, and followed by a strong earthquake swarm, and the resultant ground deformation was severe. Deflation at Kilavea's summit continued until about January 5, and later the magma reservoir beneath Kilauea's summit began to reinflate.

Inflation continued at moderate rates for many months, interrupted occasionally by brief episodes of rapidly fluctuating deflation and inflation. The pattern of ground deformation and seismicity on Kilauea was not affected in any perceptible way by an eruption of Mauna Loa on July 5-6.

By early November, inflation had reached a level sufficient for the observatory staff to speculate about renewed activity on Kilauea. This high level of inflation coincided with an appreciable increase in felt earthquakes, which were centered on the south flank and provided additional credence for these speculations. However, the increase in seismicity was later to be interpreted as foreshocks of a much more momentous event, the greatest earthquake in Hawaii in more than a century.

On November 29 at 03:36 a 5.7-magnitude earthquake (Richter Scale) proved to be the largest foreshock. The main shock, of magnitude 7.2, struck at 04:48. It was centered beneath the southeastern coast of Hawaii (1at $19^{\circ} 20.1^{\prime}$ N., long $155^{\circ} 01.4^{\prime}$ W.) at a depth of about $5 \mathrm{~km}$, and it was accompanied or followed by massive ground movements, hundreds of aftershocks, a tsunami, and a brief summit eruption of Kilauea. Although the epicenter of the quake was located near Kalapana, most of the aftershocks as well as the major ground deformation occurred throughout a region extending $40 \mathrm{~km}$ to the west of the epicenter. Ground subsidence along the south coast of the island reached a maximum of $3.5 \mathrm{~m}$ in the area due south of Kilauea caldera, and subsidence of more than $0.3 \mathrm{~m}$ occurred throughout a segment of the south coast at least $70 \mathrm{~km}$ in length. Extensive landslides, rockfalls, and ground cracks were induced by the quake throughout the summit and south flank regions of Kilauea. Structural damage was estimated to be about $\$ 2.7$ million; the most severe damage was in Hilo and Hawaii Volcanoes National Park.

A tsunami was generated by sudden ground motion associated with the main earthquake. It produced one of the largest waves recorded in Hawaiian history, which had a maximum runup height of 
$14.6 \mathrm{~m}$ above the postsubmergence shoreline. The greatest runups were recorded along the sparsely populated south coast of the island, but waves in both the Hilo and the Kona districts reached heights as much as $2.4 \mathrm{~m}$. The tsunami claimed two lives and caused damage estimated at $\$ 1.4$ million.

About a half hour after the main quake, harmonic tremor began to be recorded near Kilauea's summit, and at 05:32 lava began to fountain from a 500-m long, east-northeast-trending fissure on the caldera floor just northeast of Halemaumau. Fountains initially were about $50 \mathrm{~m} \mathrm{high}$, but in about 15 minutes they declined to 5-10 $\mathrm{m}$ high, and they finally ended about 07:00. The resultant lava flow covered about $0.25 \mathrm{~km}^{2}$ and had a total volume of about $0.25 \mathrm{x}$ $10^{6} \mathrm{~m}^{3}$. Noisy, jetlike degassing from the vents continued for some time.

At about $08: 30$ a vent opened on Halemaumau's northeast wal1, $21 \mathrm{~m}$ above the crater floor. It emitted low fountains whose lava built a small spatter cone and formed a sluggish flow that pooled at the base of the wall. At 09:53 another vent opened nearby on Halemaumau's northeast wall. Weak emission of lava from both vents was intermittent throughout the day and was interspersed with vigorous degassing episodes. Eruption of lava ended at 22:00; degassing continued until 02:00 on November 30. Lava from the 08:30 vent had a volume of $3.2 \times 10^{3} \mathrm{~m}^{3}$, and from the 09:53 vent about $300 \mathrm{~m}^{3}$.

A more complete summary of the events of November 29 has been prepared by Tilling and others (1976).

\section{Mauna Loa}

Following a strong earthquake swarm in December 1974, Mauna Loa seismicity dropped to moderate levels through the first half of 1975, with occasional swarms of microearthquakes during March and April. The huge volcano apparently was continuing to stir from its quarter-century of slumber. The heaviest cover of snow and ice in many years thwarted efforts by observatory personnel to expand deformation-monitoring networks in the summit region, although several geodimeter and tilt stations were installed on the lower and middle slopes. Aerial surveillance indicated anomalously high rates of snow melting in Lua Hou pit crater and along some zones on the floor of Mokuaweoweo. In early June, when weather finally permitted the summit geodimeter network to be reoccupied, measurements revealed that tumescence was continuing unabated. All these signs suggested a buildup toward an eruption, but they did not convey the imminence of the anticipated outbreak.

On July 5, harmonic tremor began to be recorded at 22:51 on Mauna Loa summit seismometers. The intensity of the tremor quickly increased and soon was recorded across the entire seismic network; 
swarms of microearthquakes accompanied the tremor. Observatory personnel had just enough time to issue warnings to National Park and Civil Defense authorities before lava broke out from the floor of Mokuaweoweo at 23:42. The eruption, as seen from the observatory $30 \mathrm{~km}$ to the east and $2,750 \mathrm{~m}$ lower, began with a small glow above the southwest end of the caldera, but within minutes the glow extended across the entire summit area. By the time aerial surveillance was established, at about 01:45 on July 6, a northeasttrending line of fountains extended across the caldera floor and about $1 \mathrm{~km}$ down the southwest rift zone. Fountains were $20-50 \mathrm{~m}$ high; lava was flooding much of the caldera floor and was pouring into the pit craters south of the caldera; narrow flows were advancing southward and westward away from the rift zone.

Thereafter the line of active fountains extended northeastward across North Pit and into the upper part of the northeast rift zone. At 03:15 the fountains within the caldera and on the southwest rift zone began to wane, but others continued to propagate into the northeast rift zone. Caldera and southwest rift zone fountains ended about 06:00, while those on the northeast rift zone continued unabated and fed copious flows, most of which traveled northward. The main eruptive activity became localized along short fissures near Pohaku Hanalei at the 3,700-m level. These vents remained vigorous until about 08:00, after which activity gradually declined throughout the day. The longest flow reached about $5 \mathrm{~km}$ north of the rift zone, while other flows extended 2-3 km eastward and northeastward. Eruptive activity ceased between 16:30 and 19:30; the duration of the eruption was 17 to 20 hours. Approximately $30 \times 10^{6} \mathrm{~m}^{3}$ of lava covered $13.5 \mathrm{~km}^{2}$ of land in the summit area and upper flanks of the volcano.

When extrusion of lava ceased, strong harmonic tremor accompanied by abundant earthquakes continued. Earthquake epicenters migrated down the northeast rift zone to about the 2,900-m leve1 near Puu Ulaula (Red Hill). Frequent reoccupation of the nearby geodimeter network revealed significant dilation for a few days, and further eruptive outbreaks seemed possible. However, about July 10, the tremor diminished, and geodimeter measurements indicated detumescence. By July 12 the volcano had stabilized and no further immediate activity seemed likely. Throughout the remainder of 1975, however, geodetic measurements indicated that tumescence had resumed and seismometers showed a relatively high level of microseismicity, suggesting that magma was building toward Mauna Loa's next eruption. Further details on Mauna Loa's eruption and subsequent status are provided by Lockwood and others (1976). 


\section{SEISMIC INSTRUMENTATION}

The network. The Hawailan Volcano Observatory has installed and maintains an extensive telemetering seismometer network on the island of Hawaii. In January 1975 the seismometer network consisted of thirty-six vertical high gain short period (1 sec.) stations spread over an area with a diameter of 125 kilometres (Figs. 1 and 2). The coverage is most complete on and around the main center of seismic and volcanic activity, Kilauea Volcano. Other stations in the network are part of a larger net located on other volcanoes of the island of Hawaii. With the exception of HIL, all seismometer signals from the short period network are telemetered to the observatory for recording.

The network expanded during 1975. Two new stations were added on the middle portion of the East Rift Zone (LUA, TAN), and two more were added on the northwest flank of Mauna Loa (HSS, KII). Twelve horizontal seismometers were added to nine existing vertical stations to make a total of twenty-nine vertical only, seven two-component and four three-component stations on the island.

Figure $I$ is a map of selected geographic and geologic features, and Figure 2 shows the seismic stations which were operated or added during the year. Table 1 lists all seismic stations operated by the U.S. Geological Survey in Hawaii during 1975. Listed are station name, three letter code, coordinates in degrees and minutes, elevation in meters, and other data described below.

Instrumentation and recording. Each telemetering station has a voltage controlled oscillator (VCO) for FM multiplex transmission to HVO via either hardwire or VHF radio. The VCO frequencies are listed in Table 1 . These telemetering stations are now all of Type 1, the NCER standard system used in USGS seismic networks (see Table 2 for details). After discrimination, the analog signals from thirty-two stations are recorded on two Develocorders using $16 \mathrm{~mm}$ microfilm. Beginning in late 1975, FM signals from the telemetering network were recorded directly on one inch magnetic tape. Selected larger events are copied onto condensed library tapes which are currently archived in Menlo Park.

Develocorder records are read on a film viewer with $20 x$ magnification. Arrivals are read to the nearest 0.05 second. The recorded arrival times, amplitudes (where readable), and other key data are routinely sent to N.C.E.R. in Menlo Park for computer processing.

In addition to the standard stations, optical seismographs are maintained at Uwekahuna (HVO), Hilo, Maui, and on Oahu (Kipapa station operated by Honolulu Observatory). The less sensitive short period records are used primarily for $\mathrm{S}$ data and amplitude measurements for magnitude calculations to supplement readings from $16 \mathrm{~mm}$ film. Optical seismographs listed in Table 1 are of four types. Types numbered three and four are electro-mechanical systems of high and low gain respectively. Hilo and Haleakala are each equipped with two low-gain Wood-Anderson torsion seismographs. Long period Press-Ewing seismographs record in three components in the Uwekahuna vault. The paper (optical) records as well as the $16 \mathrm{~mm}$ develocorder microfilm are archived at HVO. 
Seismograph response and calibration. Displacement response curves for the four short-period seismograph types in use are given in Figure 3. Types three and four are electro-mechanical systems recorded on paper records. The Type 1 curve gives the displacement magnification of the standard NCER system from ground motion at the seismometer to the seismic trace as seen on a $20 \mathrm{x}$ Develocorder film viewer. The curves plot the unit response. which should be multiplied by the factors CAL listed in Table 1 to get the response for an individual station. Individual CAL factors for Type 1 seismographs are equal to the peak-to-peak amplitude measured in $\mathrm{mm}$ on the 20x Develocorder viewer of a 10 microvolt $5 \mathrm{~Hz}$ signal introduced to the preamp/VCO in place of the geophone. Calibration is normally done each time a station is visited, and major changes in attenuation or recalibrations are listed in Table 1 along with the dates they took place. Minor changes in CAL factors may not be listed if they are less than $20 \%$. 


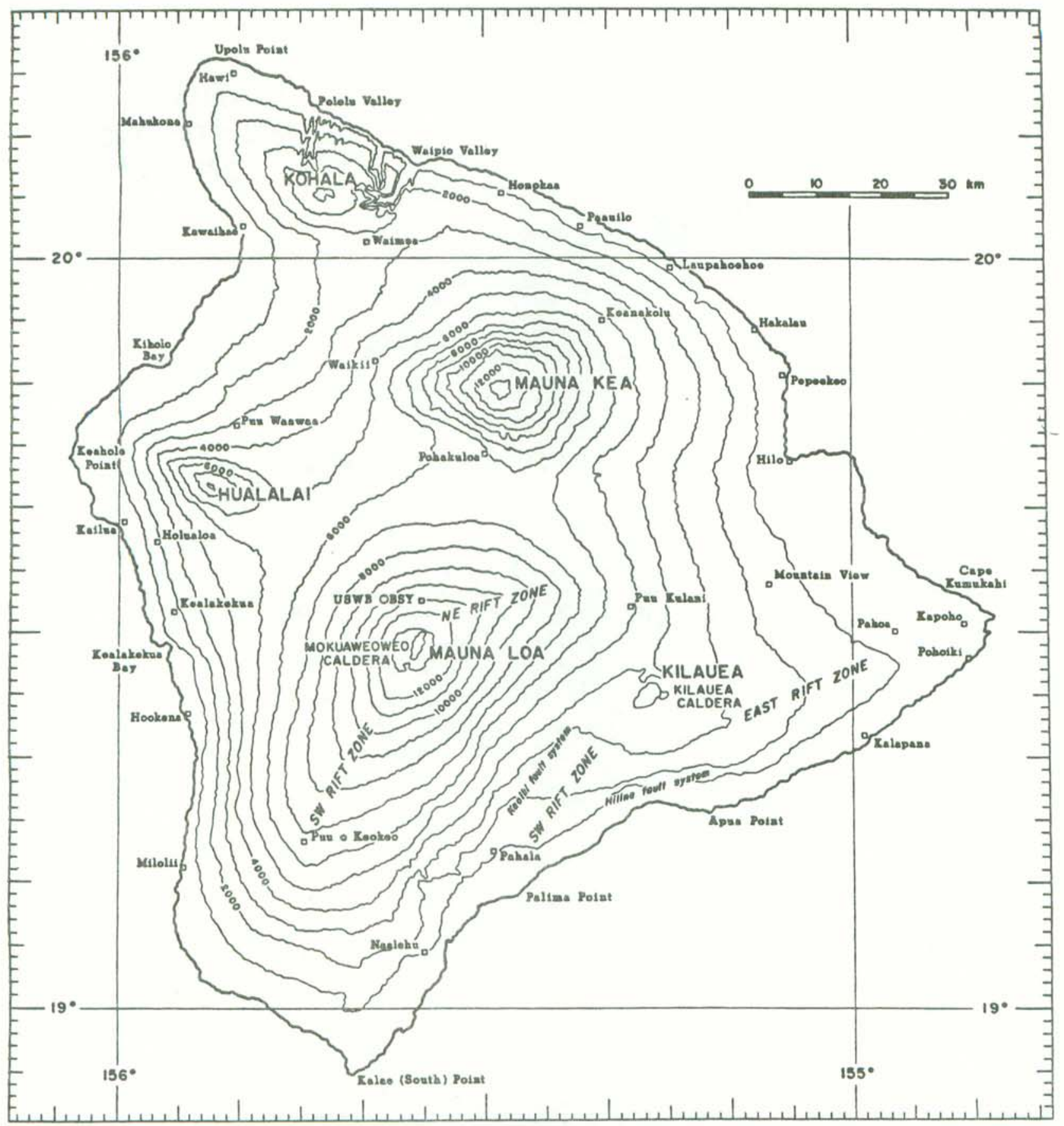

Figure 1 Map of the island of Hawaii showing principal settlements and selected geographic and geologic features. 


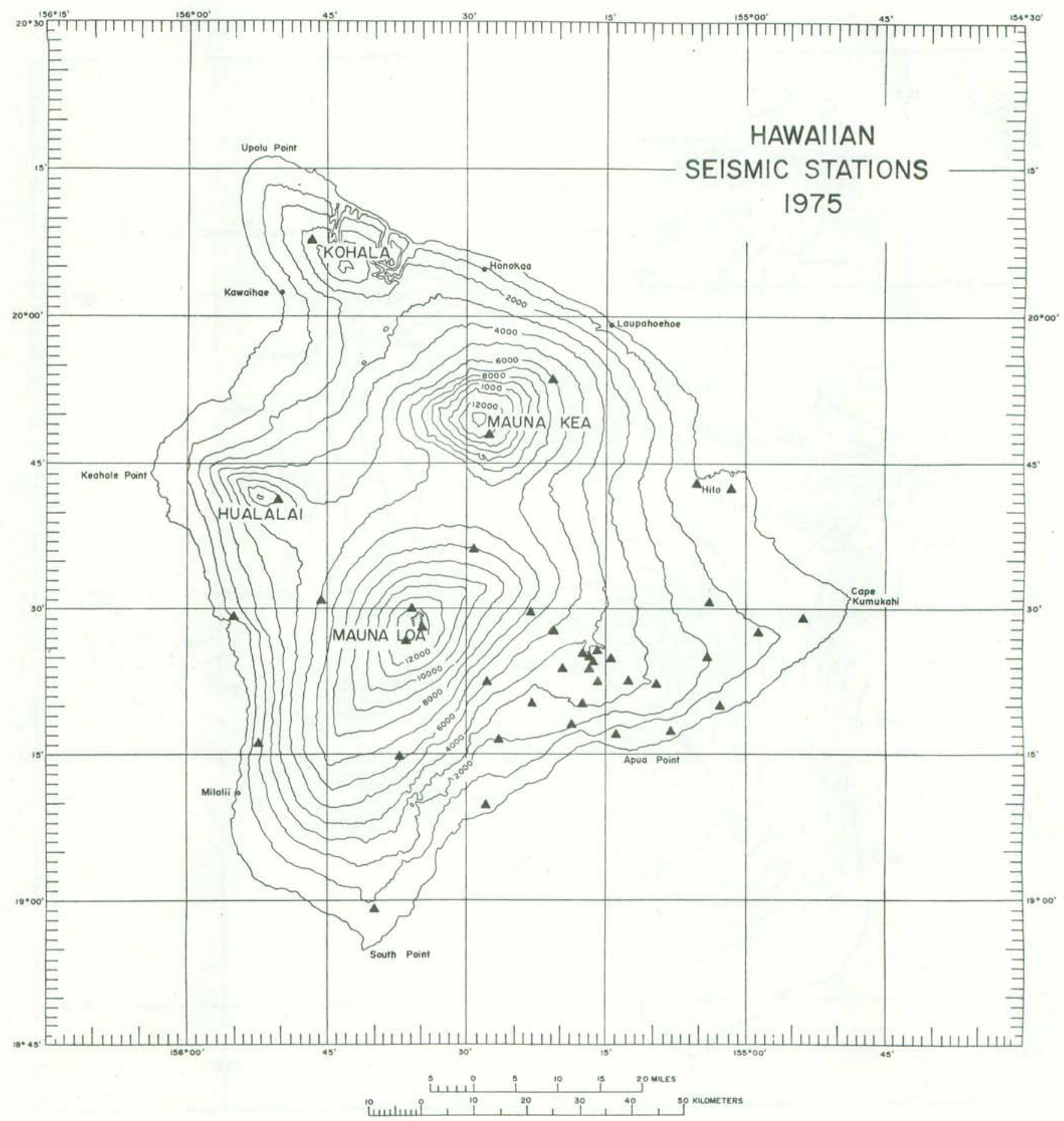

Figure 2 Map of the island of Hawaii showing seismic stations operated by the U.S. Geological Survey. 
TABLE 1. Seismometer stations in Hawaii operated by the U. S. Geological Survey, 1975

\begin{tabular}{|c|c|c|c|c|c|c|c|c|c|c|c|c|}
\hline Station Name & Code & \multicolumn{2}{|c|}{ LAT-N } & \multicolumn{2}{|c|}{ LON-W } & Delay & ELEV & VCO & $\begin{array}{c}\text { 0ld } \\
\text { Type/Cal }\end{array}$ & $\begin{array}{l}\text { Date of } \\
\text { Change }\end{array}$ & \multicolumn{2}{|c|}{$\begin{array}{c}\text { New } \\
\text { Type/Cal }\end{array}$} \\
\hline AHUA & AHU & 19 & 22.40 & 155 & 15.90 & .06 & 1070 & 2380 & 13.8 & & & \\
\hline \multirow[t]{3}{*}{ AINAPO } & AIN & 19 & 22.50 & 155 & 27.62 & .33 & 1524 & 1020 & 18.5 & & & \\
\hline & AINE & & & & & & & 2380 & none & $75 / 11 / 14$ & 1 & \\
\hline & AINN & & & & & & & 2720 & none & $75 / 11 / 14$ & 1 & \\
\hline CAPTAIN COOK & $\mathrm{CAC}$ & 19 & 29.29 & 155 & 55.09 & .15 & 323 & 1360 & 13.3 & & & \\
\hline \multirow[t]{2}{*}{ CONE PEAK } & CPK & 19 & 23.70 & 155 & 19.70 & -.04 & 1038 & 1700 & 13.5 & $75 / 12 / 30$ & 1 & 4.0 \\
\hline & $\mathrm{CPKH}$ & & & & & & & 1020 & none & $75 / 12 / 22$ & 1 & \\
\hline DESERT & DES & 19 & 20.20 & 155 & 23.30 & -.10 & 815 & 680 & $\begin{array}{ll}3 & 1.34\end{array}$ & $75 / 04 / 30$ & 1 & 4.8 \\
\hline ESCAPE ROAD & ESR & 19 & 24.68 & 155 & 14.33 & .01 & 1177 & 1360 & 11.7 & & & \\
\hline HALE POHAKU & HPU & 19 & 46.85 & 155 & 27.50 & .42 & 3396 & 2720 & 1 & $75 / 11 / 18$ & 1 & 4.5 \\
\hline HILINA PALI & HLP & 19 & 17.96 & 155 & 18.63 & .18 & 707 & 2040 & 12.5 & $75 / 05 / 21$ & 1 & 5.0 \\
\hline HUALALAI & HUA & 19 & 41.25 & 155 & 50.32 & .58 & 2189 & 1700 & 15.3 & $75 / 02 / 04$ & 1 & 2.2 \\
\hline \multirow[t]{3}{*}{ HUMUULA } & HSS & 19 & 36.31 & 155 & 29.13 & .35 & 2445 & 1700 & none & $75 / 07 / 07$ & 1 & 6.2 \\
\hline & HSSE & & & & & & & 680 & none & $75 / 10 / 29$ & 1 & \\
\hline & HSSN & & & & & & & 2720 & none & $75 / 10 / 29$ & 1 & \\
\hline KAAPUNA & KAA & 19 & 15.98 & 155 & 52.28 & .00 & 524 & 1020 & 16.0 & $75 / 03 / 28$ & 1 & 4.9 \\
\hline KAENA & $\mathrm{KAE}$ & 19 & 17.35 & 155 & 7.95 & .15 & 37 & 2380 & 12.2 & & & \\
\hline KAHUKU & $\mathrm{KHU}$ & 19 & 14.90 & 155 & 37.10 & .08 & 1939 & 1700 & 15.7 & $75 / 02 / 18$ & 1 & 3.7 \\
\hline KALALUA & LUA & 19 & 24.55 & 155 & 04.25 & -.02 & 622 & 1020 & none & $75 / 12 / 09$ & 1 & \\
\hline \multirow[t]{3}{*}{ KANEKII } & KII & 19 & 30.56 & 155 & 45.90 & .18 & 1841 & 1700 & none & $75 / 11 / 13$ & 1 & 7.1 \\
\hline & KIIE & & & & & & & 1020 & none & $75 / 11 / 13$ & 1 & \\
\hline & KIIN & & & & & & & 1360 & none & $75 / 11 / 13$ & 1 & \\
\hline KAPAPALA RANCH & $\mathrm{KPR}$ & 19 & 16.40 & 155 & 26.70 & .05 & 610 & 1700 & 14.33 & & & \\
\hline KEANAKOLU & KKU & 19 & 53.39 & 155 & 20.58 & .86 & 1863 & 2380 & 12.3 & & & \\
\hline KIPUKA NENE & KPN & 19 & 20.10 & 155 & 17.40 & .07 & 924 & 1360 & 15.0 & & & \\
\hline KOHALA & $\mathrm{KOH}$ & 20 & 7.69 & 155 & 46.77 & .21 & 1166 & 2380 & 13.2 & $75 / 12$ & 1 & 2.4 \\
\hline \multirow[t]{2}{*}{ MAUNA LOA } & MLO & 19 & 29.80 & 155 & 23.30 & .24 & 2010 & 1360 & 111.8 & $75 / 05 / 09$ & 1 & 10.5 \\
\hline & MLOH & & & & & & & 2040 & none & $75 / 09 / 25$ & 1 & \\
\hline \multirow[t]{2}{*}{ MAUNA LOA X } & $\operatorname{MLX}$ & 19 & 27.60 & 155 & 20.70 & .27 & 1475 & 1360 & 1 & $75 / 04 / 24$ & 1 & 2.1 \\
\hline & MLXH & & & & & & & 2720 & none & $75 / 11 / 19$ & 1 & \\
\hline MAKAOPUHI & MPR & 19 & 22.07 & 155 & 9.85 & -.01 & 881 & 2720 & 15.7 & $75 / 11 / 07$ & 1 & 2.1 \\
\hline MOKUAWEOWEO & MOK & 19 & 29.28 & 155 & 35.98 & .28 & 4104 & 2040 & 17.5 & & & \\
\hline MOUNTAIN VIEW & MTV & 19 & 30.25 & 155 & 3.75 & .17 & 409 & 680 & 16.2 & $75 / 05 / 13$ & 1 & 3.2 \\
\hline NATIONAL GUARD & NAG & 19 & 42.12 & 155 & 1.72 & .63 & 18 & 1360 & 18.5 & & & \\
\hline NORTH PIT & NPT & 19 & 24.90 & 155 & 17.00 & -.06 & 1115 & 680 & 19.0 & & & \\
\hline \multirow[t]{2}{*}{ OUTLET } & OTL & 19 & 23.40 & 155 & 16.80 & .02 & 1084 & 1360 & 14.15 & & & \\
\hline & OTLH & & & & & & & 2040 & none & $75 / 09 / 15$ & 1 & \\
\hline PAU & PAU & 19 & 22.62 & 155 & 13.10 & -.06 & 994 & 2040 & 13.8 & & & \\
\hline & PAUH & & & & & & & 1020 & none & $75 / 01 / 19$ & 1 & \\
\hline POLIOKEAWE PALI & POL & 19 & 17.02 & 155 & 13.47 & .10 & 169 & 2720 & 13.0 & $75 / 12 / 10$ & 1 & 4.8 \\
\hline PUU HONUALA & PHO & 19 & 28.90 & 154 & 53.40 & .03 & 215 & 2720 & 14.8 & & & \\
\hline PUU PILI & PPL & 19 & 9.50 & 155 & 27.87 & .24 & 35 & 1360 & 12.2 & & & \\
\hline RIM & RIM & 19 & 23.90 & 155 & 16.60 & .12 & 1128 & 1020 & 11.1 & $75 / 05 / 7$ & 1 & 7.3 \\
\hline & RIMH & & & & & & & 2040 & & & 1 & \\
\hline SOUTH POINT & SPT & 18 & 58.91 & 155 & 39.92 & -.07 & 244 & 2040 & 14.8 & $75 / 06 / 10$ & 1 & 3.6 \\
\hline SOUTHWEST RIFT & SWR & 19 & 27.26 & 155 & 36.30 & .14 & 4048 & 1020 & 111.1 & $75 / 10 / 15$ & 1 & 2.4 \\
\hline SUMMIT CABIN & SCA & 19 & 28.20 & 155 & 35.08 & .30 & 4048 & 1700 & 111.5 & & & \\
\hline TANGERINE & TAN & 19 & 27.79 & 154 & 58.51 & .02 & 351 & 1020 & none & $75 / 12 / 02$ & 1 & \\
\hline WAHAULA & WHA & 19 & 19.90 & 155 & 2.92 & .06 & 29 & 680 & 13.66 & & & \\
\hline WALDRON LEDGE & WLG & 19 & 25.49 & 155 & 15.69 & -.02 & 1067 & 2380 & 12.2 & & & \\
\hline
\end{tabular}


Optical Seismographs

\begin{tabular}{|c|c|c|c|c|c|c|c|c|c|}
\hline HALEAKALA $\mathrm{Z}$ & HAL & 20 & 46.00 & 156 & 15.00 & & 2090 & 3 & 0.71 \\
\hline HALEAKALA EW & HAE & 20 & 46.00 & 156 & 15.00 & & 2090 & WA & 1.0 \\
\hline HALEAKALA NS & HAN & 20 & 46.00 & 156 & 15.00 & & 2090 & WA & 1.0 \\
\hline HILO Z & HIL & 19 & 43.20 & 155 & 5.30 & .64 & 20 & 3 & 1.0 \\
\hline HILO EW & HIE & 19 & 43.20 & 155 & 5.30 & .64 & 20 & WA & 1.0 \\
\hline HILO NS & HIN & 19 & 43.20 & 155 & 5.30 & .64 & 20 & WA & 1.0 \\
\hline KIPAPA & KIP & 21 & 25.40 & 158 & .90 & & 76 & 3 & 0.56 \\
\hline UWEKAHUNA Z & UWE & 19 & 25.40 & 155 & 17.60 & .06 & 1240 & 3 & 0.7 \\
\hline UWEKAHUNA Z & USZ & 19 & 25.40 & 155 & 17.60 & .06 & 1240 & 4 & 1.0 \\
\hline UWEKAHUNA EW & USE & 19 & 25.40 & 155 & 17.60 & .06 & 1240 & 4 & 1.0 \\
\hline UWEKAHUNA & PEZ & 19 & 25.40 & 155 & 17.60 & & 1240 & PE & \\
\hline UWEKAHUNA & PEE & 19 & 25.40 & 155 & 17.60 & & 1240 & $\mathrm{PE}$ & \\
\hline UWEKAHUNA & PEN & 19 & 25.40 & 155 & 17.60 & & 1240 & $\mathrm{PE}$ & \\
\hline
\end{tabular}

Table 2. -- Seismic Instrumentation Types

Type 1. Consists of:

a) EV-17 - Electrotech EV-17 1.0 sec. period moving magnet vertical component seismometer or horizontal component adjusted for an output of 0.5 volts $/ \mathrm{cm} / \mathrm{sec}$. and 0.8 critically damped.

b) Preamp/VCO Develco Model 6202 voltage controlled oscillator or a USGS/NCER Model JE202. 3 db points for bandpass filter at $0.1 \mathrm{~Hz}$ and $30 \mathrm{~Hz}$. Signals are transmitted on audio FM carrier over cable or FM radio link to HVO. USGS Model J302 was introducted in 1974.

Type 3. Consists of:

a) $\mathrm{EV}-17$ Electrotech EV-17 (as described above), Hall-Sears
HS-10 $0.5 \mathrm{sec}$. period moving coil seismometer or
Observatory-built 0.3 sec. period moving coil
seismometer with HVO-built solid state seismic
preamplifier (voltage gain, 2000x), or
Observatory-built electronmagnetic seismometer
approximately 40,000 at $4 \mathrm{~Hz}$.

Type 4. Consists of:

Sprengnether short period vertical and horizontal seismometers (E-W) with $1.5 \mathrm{sec}$ galvanometers, coupling factor $=0.25,2 \mathrm{X}$ critically damped. Peak magnitication approximately $1500 \mathrm{X}$ at $2 \mathrm{~Hz}$.

Experimental type amplifier systems are not given type numbers. Type 2 instruments have been discontinued. 


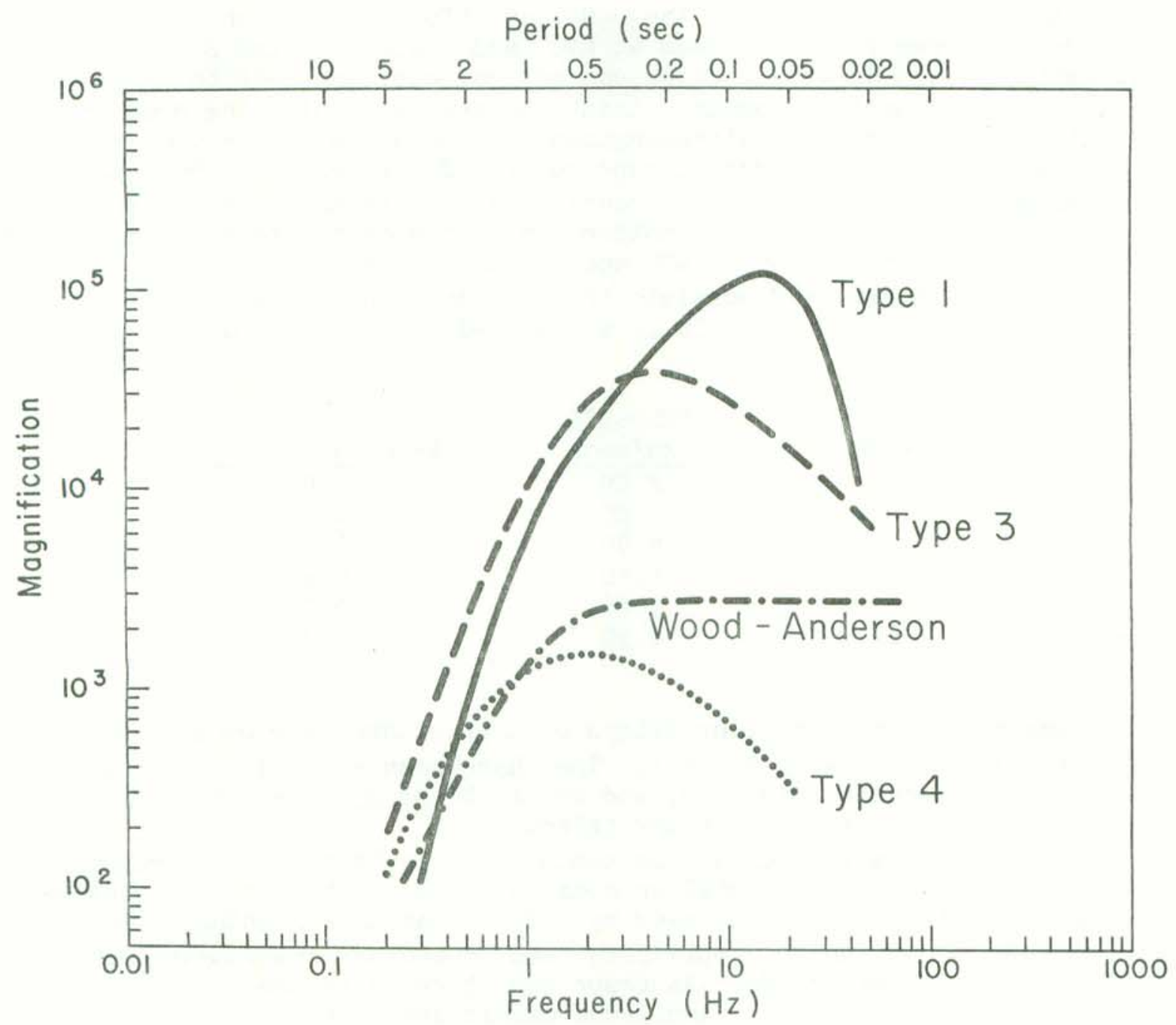

Figure 3. System response curves for the Wood-Anderson torsion seismograph and for the 3 different types of seismometers in use by the Hawaiian Volcano Observatory. Types 3 and 4 are electro-mechanical seismographs recorded optically on photographic paper. Type 1 is the standard NCER seismometer recorded on Develocorder film. The curve for Type 1 includes response of the geophone, all electronics including telemetry, Develocorder galvanometer, and projection of film by a $20 \mathrm{x}$ viewer. The curves plot the unit response which should be multiplied by the factors CAL listed in Table 1 to get the response for an individual station. 


\section{SEISMIC DATA PROCESSING}

Earthquakes are located by the combined efforts of HVO and NCER in Menlo Park. Develocorder films are read at HVO, and lists of $\mathrm{P}$ and $\mathrm{S}$ arrival times, event amplitude and duration, clock correction, etc. are sent to Menlo Park. Data are then keypunched, computer locations are made using the program HYPOELLIPSE (Lahr, et al., in preparation), and problem events are reread at HVO and rerun. Card and magnetic tape copies of all arrival time (phase) and output summary data (one card per event), are kept in Menlo Park. All computer output (including first motion plots) are on microfiche, and copies are available for inspection at HVO and in Menlo Park.

The crustal model used consists of flat, homogeneous layers and contains a embedded low velocity zone. It is a modified version of Crosson's (1976) model and is:

$\begin{array}{ccc}\text { LAYER } & \begin{array}{c}\text { VELOCITY } \\ (\mathrm{km} / \mathrm{sec})\end{array} & \text { DEPTH TO TOP }(\mathrm{km}) \\ 1 & 2.00 & 0.0 \\ 2 & 3.25 & 0.8 \\ 3 & 6.00 & 2.0 \\ 4 & 6.40 & 5.5 \\ 5 & 5.30 & 9.5 \\ 6 & 8.30 & 13.0\end{array}$

An empirical set of station delays or corrections were used in the locations, and are given in Table 1. They have been adjusted so the mean delay of Kilauea stations is zero, and are most appropriate for locating earthquakes on the south side of the island.

Magnitudes for most events were computed using both recorded amplitudes on calibrated stations and signal or coda duration on short-period vertical stations. Amplitude magnitudes used by HVO are based on readings from Wood-Anderson seismographs. Amplitudes read from other instruments are corrected to an equivalent Wood-Anderson amplitude using the curves of Figure 3 and CAL factors of Table 1. Amplitude magnitudes larger than 2.5 are generally based on the Wood-Anderson instruments in Hilo or Type 4 seismographs at Uwekahuna. Smaller events may occasionally include amplitude readings from stations AHU, KAA, OTL, or PPL.

Duration magnitudes are determined from the length of signal in seconds read from the Develocorder viewer. This time, also called the "F-P time" is measured from the first $P$ arrival to the point where the earthquake signal has decayed to about twice the noise level, or to about $1 \mathrm{~cm}$ peak-to-peak on the Develocorder viewer. A plot of log (F-P time) versus local (amplitude) magnitude appears in Figure 4. The bilinear relation shown in the figure is an appropriate fit to the data sample and is used to compute all duration magnitudes. Duration times are only read from Type 1 seismographs. Because duration magnitudes are relatively insensitive to station response and can be determined using the high-gain short-period stations, it is felt that duration magnitudes are more accurate and complete at the lower magnitudes (below 2). 


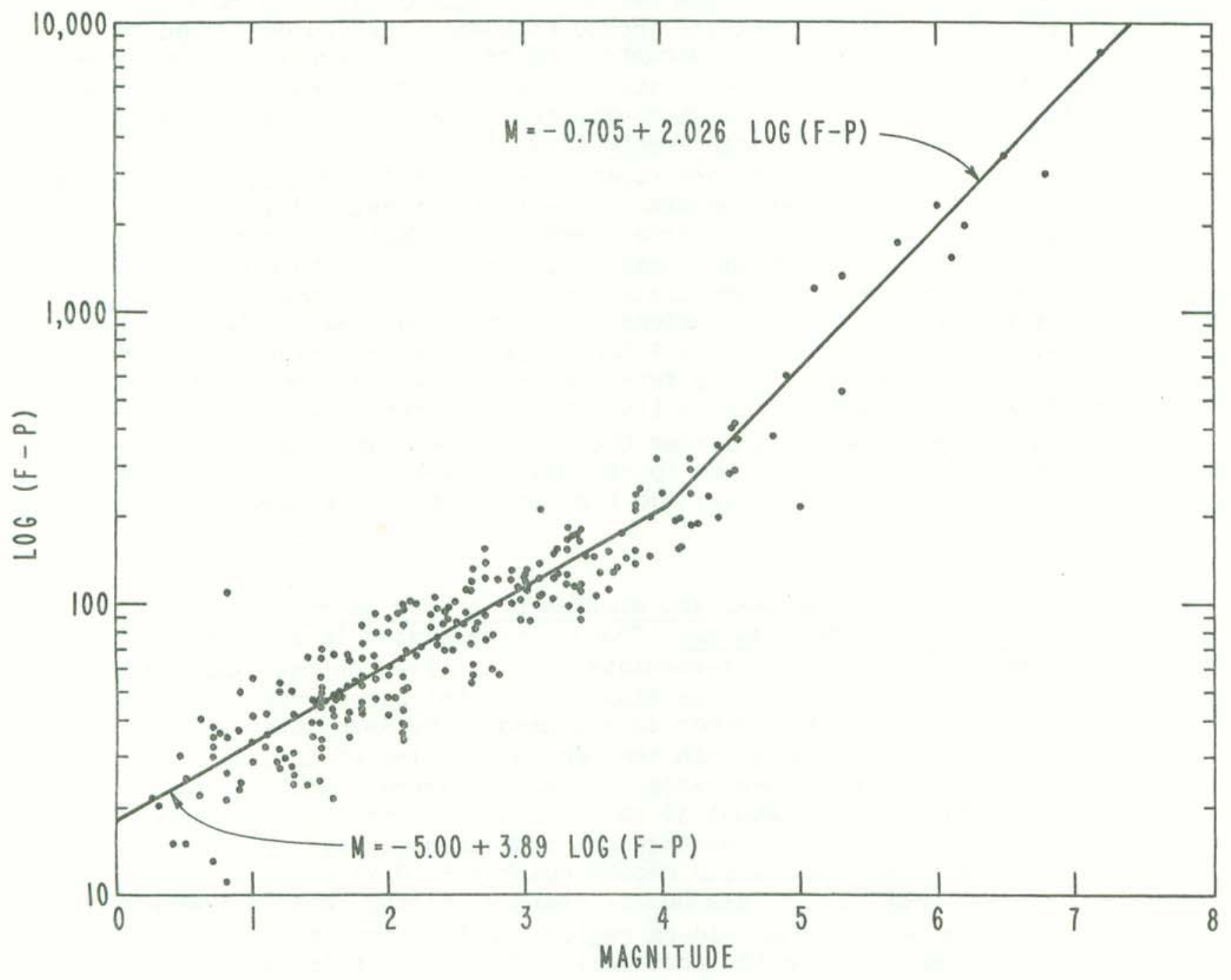

Figure 4. Relationship between signal duration (F-P time) and local magnitude for a large number of earthquakes which occurred during 1975 and 1976. Local magnitude is determined from amplitudes read on Wood-Anderson and other calibrated seismographs. The dual linear relationship between magnitude and $\log (\mathrm{F}-\mathrm{P})$ appears to hold over a magnitude range of 7 units. 
The emphasis in both station coverage and detailed data analysis is on the highly active south flank of the island of Hawaii. Hundreds of earthquakes too small to locate are counted daily, and the set of located earthquakes in the Kilauea region is nearly complete above magnitude about 2.0 to 2.5. Many smaller events are located also. Substantial effort is made to locate earthquakes elsewhere on the island and within about $150 \mathrm{~km}$ of the island. Such coverage cannot be as complte as on the south flank, but nearly all events above magnitude 3.0 to 3.5 are located.

Data presented in the seismic summary is in four parts. Table 3 gives duration of harmonic tremor and numbers of earthquakes (most too small to locate) from several source regions around Kilauea. The source region is determined visually from signal character and pattern of arrival times at key stations. Maps showing computer located epicenters of all depths are given in Figures 5, 6, 7 and 8 . The epicenter maps are on two scales, and show both all located earthquakes and large events only.

The list of computer locations constitutes the bulk of this summary, and is given in Table 5. Each earthquake in the list is assigned a three-letter code based on its location and in some cases on its depth. Figure 9 is a map of the regions used to assign the location codes. The latitude and longitude limits of rectangular regions are listed in Table 4 . When the listed coordinates imply an overlap, precedence is given according to Figure 9 . Table 6 relists the events in Table 5 for which either duration or amplitude magnitude is 3.5 or larger. It is felt that this list is a more objective measure of large earthquakes than a list of felt earthquakes.

Earthquakes reported felt during the first three quarters of 1975 are listed in Table 7 . A list for the fourth quarter would be long and dominated by the $M=7.2$ Kalapana earthquake and its many aftershocks, and is omitted here.

Table 3. Number of earthquakes and minutes of tremor recorded on seismographs around Kilauea. Tremor is separated into three categories: Deep, Intermediate, and Shallow, on the basis of relative amplitude on seismographs in the summit region. Unless otherwise stated, tremor is presumed to be associated with movement of magma within the central complex of Kilauea Volcano. Earthquake categories are: Kilauea Summit $30 \mathrm{~km}$, earthquakes from about $30 \mathrm{~km}$ beneath the summit region; Kilauea Summit long-period, earthquakes characterized by low-frequency waves from intermediate depths roughly $5-10 \mathrm{~km}$ beneath the summit region: Kilauea Summit Shallow, earthquakes from within a few $\mathrm{km}$ beneath the caldera region; SW Rift and Kaoiki, earthquakes along the southwest rift zone of Kilauea and the adjacent portions of the Kaoiki fault system; Upper East Rift, earthquakes from the upper east rift zone of Kilauea; Koae, earthquake from along the northeast-trending Koae fault system south of the caldera; Lower East Rift, earthquakes from the lower east rift zone of Kilauea; South Flank, faults on the south flank of Kilauea; Mauna Loa L-P, earthquakes characterized by low-frequency waves from Mauna Loa volcano; Mauna Loa S-P earthquakes from within a few kilometers beneath the summit of Mauna Loa; Offshore PPL, earthquakes from mostly of fshore areas south of Puu Pili station. 


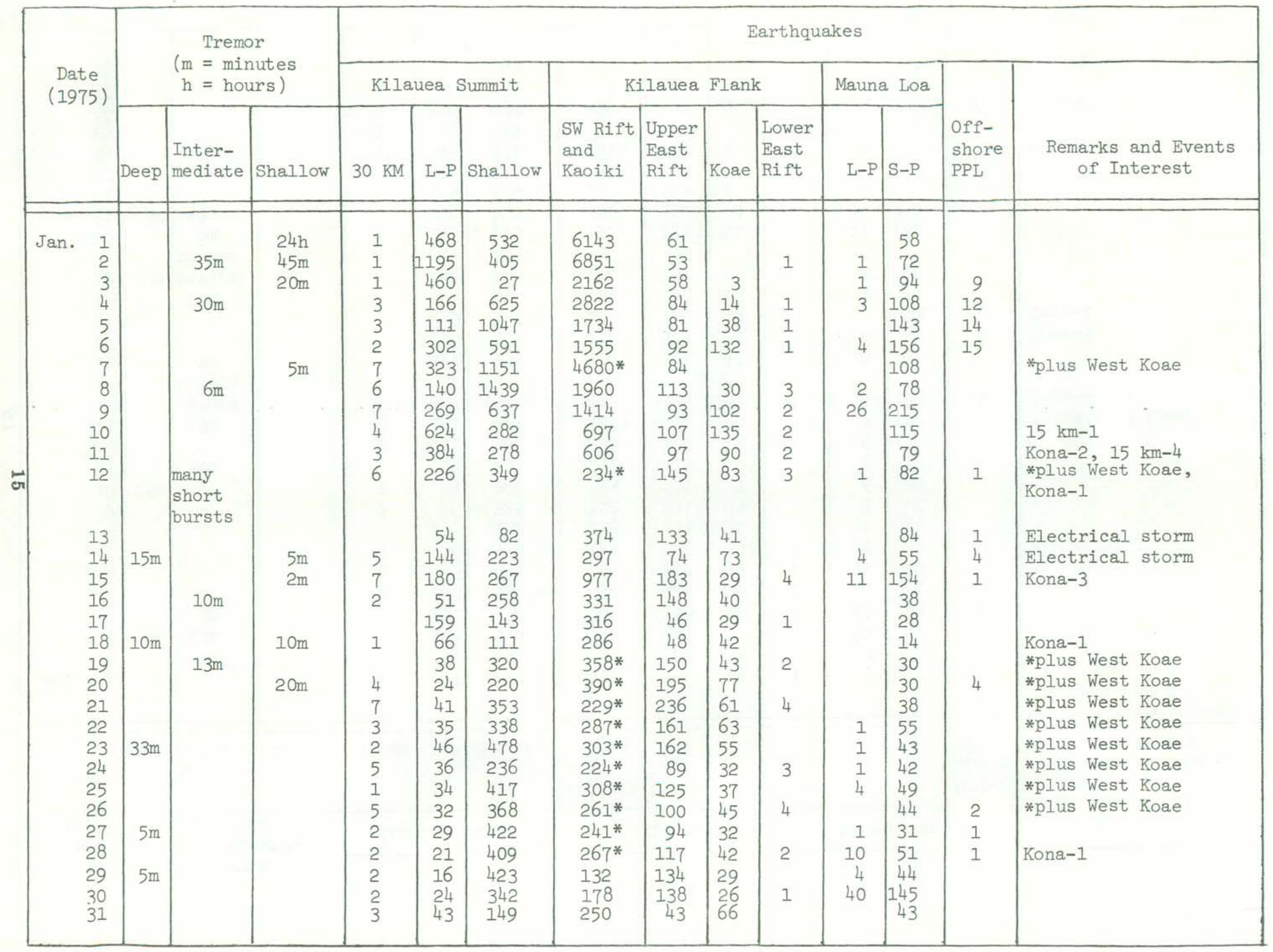




\begin{tabular}{|c|c|c|c|c|c|c|c|c|c|c|c|c|c|c|}
\hline \multirow{3}{*}{$\begin{array}{c}\text { Date } \\
(1975)\end{array}$} & \multirow{2}{*}{\multicolumn{3}{|c|}{$\begin{array}{c}\text { Tremor } \\
(\mathrm{m}=\text { minutes } \\
\mathrm{h}=\text { hours) }\end{array}$}} & \multicolumn{10}{|c|}{ Earthquakes } & \multirow[b]{3}{*}{$\begin{array}{c}\text { Remarks and Events } \\
\text { of Interest }\end{array}$} \\
\hline & & & & \multicolumn{3}{|c|}{ Kilauea Summit } & \multicolumn{4}{|c|}{ Kilauea Flank } & \multicolumn{2}{|c|}{ Mauna Loa } & \multirow[b]{2}{*}{$\begin{array}{l}\text { Off- } \\
\text { shore } \\
\text { PPL }\end{array}$} & \\
\hline & Deep & $\begin{array}{l}\text { Inter- } \\
\text { mediate }\end{array}$ & Shallow & $30 \mathrm{KM}$ & L-P & Shallow & $\begin{array}{l}\text { SW Rift } \\
\text { and } \\
\text { Kaoiki }\end{array}$ & $\begin{array}{l}\text { Upper } \\
\text { East } \\
\text { Rift }\end{array}$ & Koae & \begin{tabular}{|l|} 
Lower \\
East \\
Rift
\end{tabular} & $\mathrm{L}-\mathrm{P}$ & S-P & & \\
\hline $\begin{array}{r}\text { Feb. } \\
2 \\
3 \\
4 \\
5 \\
6 \\
7 \\
8 \\
9 \\
10 \\
11 \\
12 \\
13 \\
14 \\
\\
15 \\
16 \\
17 \\
18 \\
19 \\
\\
20 \\
21 \\
22 \\
23 \\
24 \\
25 \\
26 \\
27 \\
28\end{array}$ & $25 \mathrm{~m}$ & $\begin{array}{c}4 \mathrm{~m} \\
13 \mathrm{~m} \\
3 \mathrm{~m} \\
\\
\\
\\
10 \mathrm{~m} \\
15 \mathrm{~m} \\
\text { several } \\
\text { bursts } \\
5 \mathrm{~m} \\
\\
20 \mathrm{~m} \\
\text { several } \\
\text { bursts } \\
8 \mathrm{~m} \\
13 \mathrm{~m} \\
10 \mathrm{~m} \\
5 \mathrm{~m}\end{array}$ & & $\begin{array}{l}4 \\
3 \\
\\
4 \\
3 \\
9 \\
3 \\
2 \\
2 \\
2 \\
2 \\
1\end{array}$ & $\begin{array}{r}43 \\
59 \\
318 \\
101 \\
37 \\
35 \\
38 \\
115 \\
6 \\
44 \\
56 \\
515 \\
191 \\
907 \\
197 \\
1238 \\
45 \\
344 \\
160 \\
\\
169 \\
116 \\
49 \\
65 \\
45 \\
35 \\
399 \\
88 \\
117\end{array}$ & $\begin{array}{l}210 \\
491 \\
546 \\
352 \\
598 \\
425 \\
139 \\
132 \\
525 \\
298 \\
223 \\
361 \\
452 \\
440 \\
\\
499 \\
317 \\
427 \\
970 \\
603 \\
\\
576 \\
415 \\
559 \\
408 \\
526 \\
345 \\
238 \\
485 \\
199\end{array}$ & $\begin{array}{r}355 \\
87 \\
110 \\
82 \\
92 \\
86 \\
144 \\
173 \\
103 \\
83 \\
111 \\
88 \\
128 * \\
293 \\
\\
110 \\
132 \\
108 \\
64 \\
72 \\
95 \\
90 \\
103 \\
52 \\
64 \\
71 \\
806 \\
75 \\
126\end{array}$ & $\begin{array}{r}56 \\
104 \\
117 \\
118 \\
125 \\
179 \\
30 \\
31 \\
221 \\
167 \\
116 \\
146 \\
114 \\
124 \\
\\
44 \\
305 \\
72 \\
102 \\
131 \\
\\
155 \\
110 \\
112 \\
110 \\
125 \\
122 \\
125 \\
85 \\
37\end{array}$ & $\begin{array}{r}93 \\
23 \\
18 \\
20 \\
19 \\
38 \\
36 \\
23 \\
15 \\
9 \\
3 \\
28 \\
61 \\
\\
17 \\
62 \\
19 \\
13 \\
21 \\
\\
11 \\
8 \\
15 \\
6 \\
21 \\
11 \\
101 \\
20 \\
37\end{array}$ & $\begin{array}{r}8 \\
5 \\
10\end{array}$ & $\begin{array}{r}2 \\
9 \\
69 \\
65 \\
41 \\
21 \\
7 \\
7 \\
8 \\
6 \\
7 \\
6 \\
5 \\
3 \\
\\
10 \\
6 \\
3 \\
3 \\
6 \\
\\
11 \\
14 \\
4 \\
\\
1 \\
2 \\
14 \\
\\
1\end{array}$ & $\begin{array}{r}17 \\
76 \\
52 \\
43 \\
41 \\
36 \\
24 \\
34 \\
45 \\
82 \\
72 \\
110 \\
172 \\
122 \\
\\
67 \\
95 \\
53 \\
74 \\
93 \\
162 \\
330 \\
124 \\
73 \\
73 \\
53 \\
369 \\
45 \\
68\end{array}$ & $\begin{array}{c}16 \\
1\end{array}$ & $\begin{array}{l}\text { *plus West Koae } \\
\text { Kona-4 }\end{array}$ \\
\hline
\end{tabular}




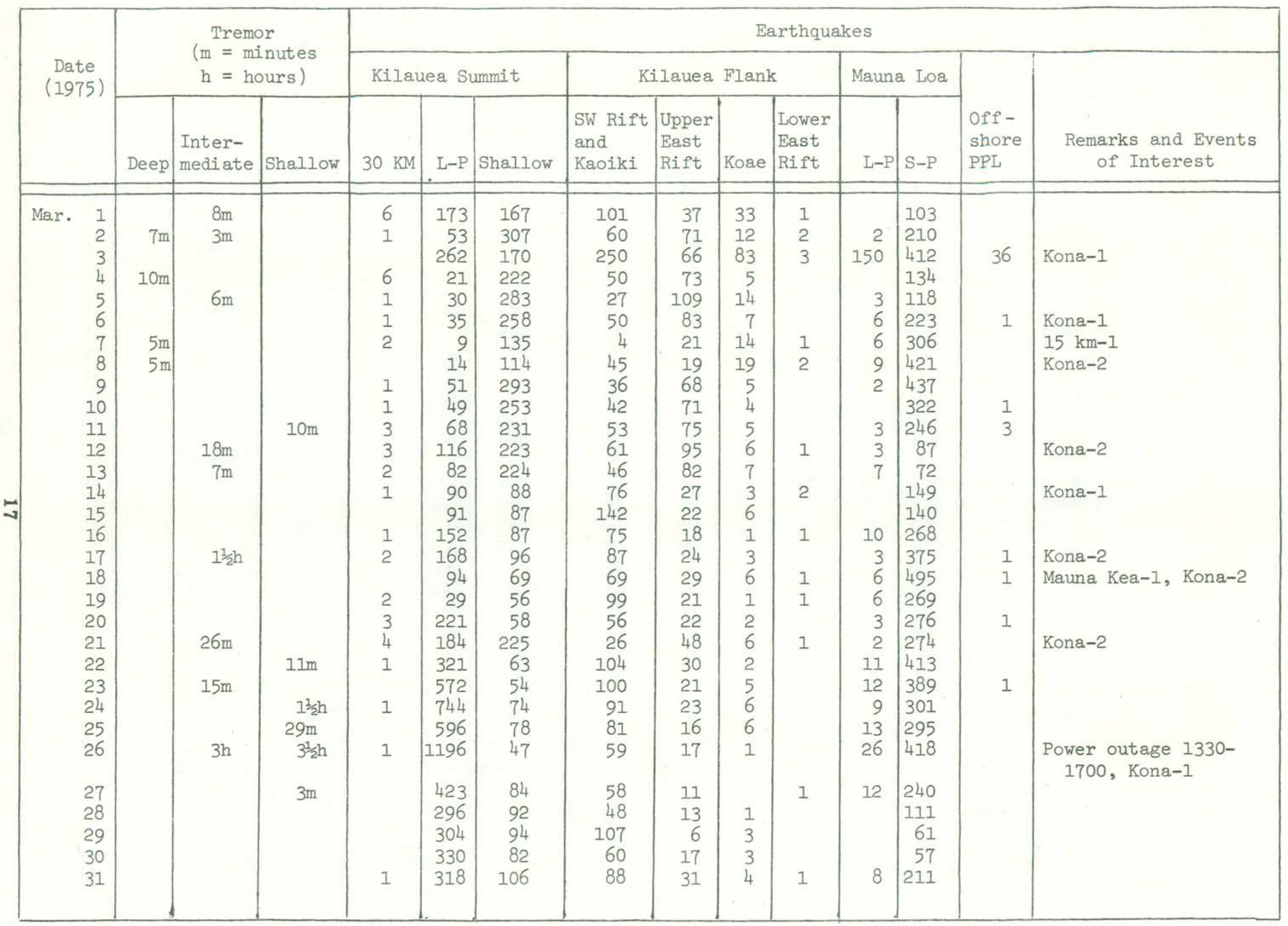




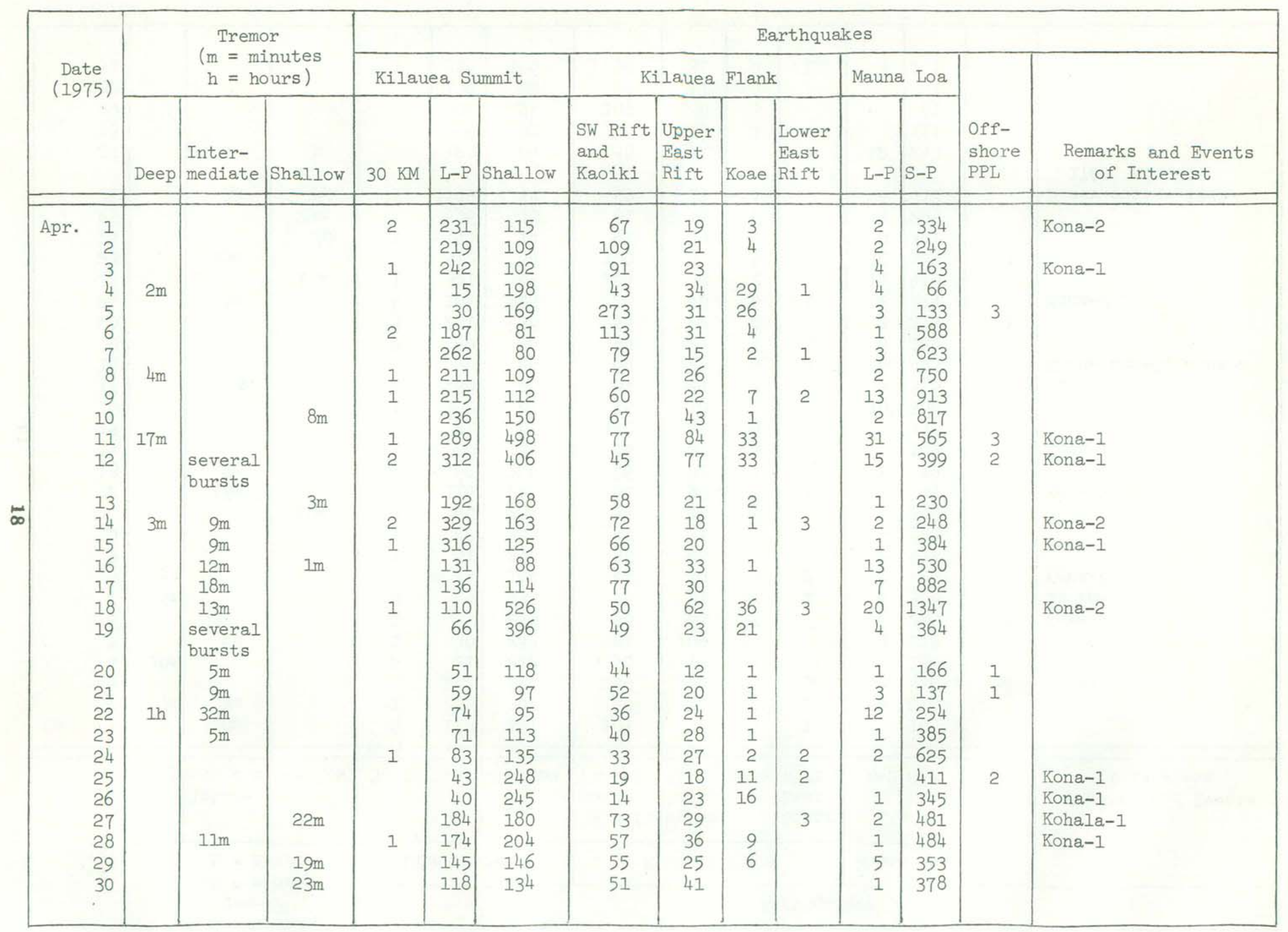




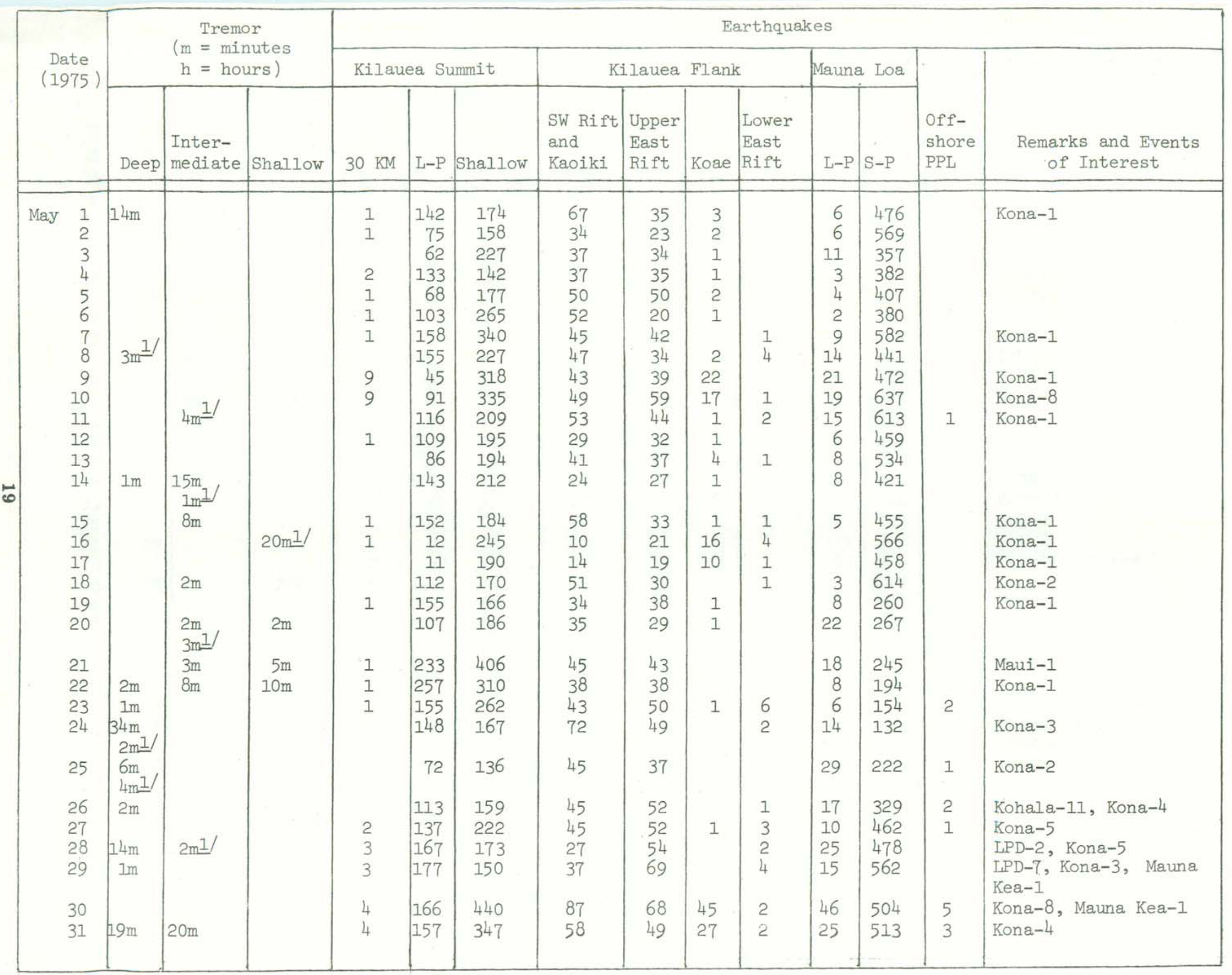




\begin{tabular}{|c|c|c|c|c|c|c|c|c|c|c|c|c|c|c|}
\hline \multirow{3}{*}{$\begin{array}{l}\text { Date } \\
\text { (1975) }\end{array}$} & \multirow{2}{*}{\multicolumn{3}{|c|}{$\begin{array}{c}\text { Tremor } \\
(\mathrm{m}=\text { minutes } \\
\mathrm{h}=\text { hours })\end{array}$}} & \multicolumn{11}{|c|}{ Earthquakes } \\
\hline & & & & \multicolumn{3}{|c|}{ Kilauea Summit } & \multicolumn{4}{|c|}{ Kilauea Flank } & \multicolumn{2}{|c|}{ Mauna Loa } & \multirow[b]{2}{*}{$\begin{array}{l}\text { Off- } \\
\text { shore } \\
\text { PPL }\end{array}$} & \multirow[b]{2}{*}{$\begin{array}{c}\text { Remarks and Events } \\
\text { of Interest }\end{array}$} \\
\hline & Deep & $\begin{array}{l}\text { Inter- } \\
\text { mediate }\end{array}$ & Shallow & $30 \mathrm{KM}$ & L-P & Shallow & $\begin{array}{l}\text { SW Rift } \\
\text { and } \\
\text { Kaoiki }\end{array}$ & $\begin{array}{l}\text { Upper } \\
\text { East } \\
\text { Rift }\end{array}$ & Koae & $\begin{array}{l}\text { Lower } \\
\text { East } \\
\text { Rift }\end{array}$ & $\mathrm{L}-\mathrm{P}$ & $S-P$ & & \\
\hline $\begin{array}{r}\text { Jun. } \\
2 \\
3 \\
4 \\
5 \\
6 \\
7 \\
8 \\
9 \\
10 \\
11 \\
12 \\
13 \\
14 \\
15 \\
16 \\
17 \\
18 \\
19 \\
20 \\
21 \\
22 \\
23 \\
24 \\
25 \\
26 \\
27 \\
28 \\
29 \\
30\end{array}$ & $\begin{array}{l}5 \mathrm{~m} \\
30 \mathrm{~m} \\
20 \mathrm{~m} \\
1 \mathrm{~h} \\
18 \mathrm{~m}\end{array}$ & $\begin{array}{r}10 \mathrm{~m} \\
20 \mathrm{~m} \\
7 \mathrm{~m} \\
40 \mathrm{~m}\end{array}$ & $10 \mathrm{~m}$ & $\begin{array}{l}7 \\
4 \\
1 \\
4 \\
2 \\
2 \\
1 \\
2 \\
2 \\
1 \\
2 \\
3 \\
3 \\
3\end{array}$ & $\begin{array}{r}105 \\
153 \\
202 \\
\\
93 \\
131 \\
4 \\
3 \\
134 \\
27 \\
94 \\
85 \\
96 \\
142 \\
105 \\
52 \\
151 \\
97 \\
97 \\
233 \\
267 \\
81 \\
97 \\
41 \\
48 \\
33 \\
38 \\
3 \\
10 \\
180 \\
46\end{array}$ & $\begin{array}{r}134 \\
130 \\
82 \\
\\
108 \\
175 \\
381 \\
383 \\
204 \\
337 \\
117 \\
167 \\
149 \\
214 \\
183 \\
133 \\
184 \\
365 \\
343 \\
187 \\
374 \\
262 \\
127 \\
121 \\
97 \\
188 \\
211 \\
393 \\
487 \\
210 \\
173\end{array}$ & $\begin{array}{l}43 \\
38 \\
42 \\
61 \\
45 \\
29 \\
20 \\
47 \\
23 \\
43 \\
83 \\
54 \\
42 \\
52 \\
66 \\
43 \\
19 \\
32 \\
40 \\
55 \\
56 \\
41 \\
35 \\
24 \\
23 \\
33 \\
11 \\
22 \\
22 \\
38\end{array}$ & $\begin{array}{r}40 \\
32 \\
47 \\
\\
34 \\
28 \\
26 \\
28 \\
61 \\
79 \\
72 \\
54 \\
67 \\
61 \\
35 \\
54 \\
46 \\
80 \\
110 \\
63 \\
51 \\
50 \\
45 \\
19 \\
18 \\
39 \\
28 \\
17 \\
14 \\
42 \\
47\end{array}$ & $\begin{array}{r}1 \\
12 \\
17 \\
1 \\
1 \\
1 \\
1 \\
5 \\
4 \\
3\end{array}$ & $\begin{array}{l}2 \\
1 \\
1 \\
3 \\
2 \\
3 \\
1 \\
1 \\
1 \\
2 \\
1\end{array}$ & $\begin{array}{r}13 \\
8 \\
10 \\
12 \\
24 \\
8 \\
7 \\
20 \\
21 \\
16 \\
2 \\
2 \\
4 \\
1 \\
9\end{array}$ & $\begin{array}{l}509 \\
436 \\
528 \\
\\
230 \\
193 \\
117 \\
176 \\
181 \\
212 \\
286 \\
195 \\
227 \\
410 \\
461 \\
331 \\
227 \\
325 \\
246 \\
504 \\
506 \\
597 \\
403 \\
110 \\
283 \\
514 \\
428 \\
276 \\
145 \\
120 \\
117\end{array}$ & $\begin{array}{l}1 \\
1 \\
1 \\
7\end{array}$ & $\begin{array}{l}\text { Kona-7 } \\
\text { Kona-4 } \\
\text { LPD-1, Offshore-1, } \\
\text { Kona-1 } \\
\text { LPD-1, Kona-3, Kohala-2 } \\
\text { LPD-2, Kona-2 } \\
\text { Kona-6 } \\
\text { LPD-5, Kona-2 } \\
\text { LPD-11, Kona-4 } \\
\text { LPD-3 } \\
\text { LPD-5 } \\
\text { Kona-3 } \\
\text { Kona-1 } \\
\text { LPD-2 } \\
\text { Kona-2, Hilo-1 } \\
\text { Kona-2 } \\
\text { Kona-1 } \\
\text { Kona-12 } \\
\text { Kona-2 } \\
\text { Kona-2 } \\
\text { LPD-1 } \\
\text { LPD-11 } \\
\text { Kona-1, LPD-3, Kohala-1 } \\
\text { Kona-2 } \\
\text { 15-KM-1, Kona-1 } \\
\text { LPD-1, Kona-3 }\end{array}$ \\
\hline
\end{tabular}




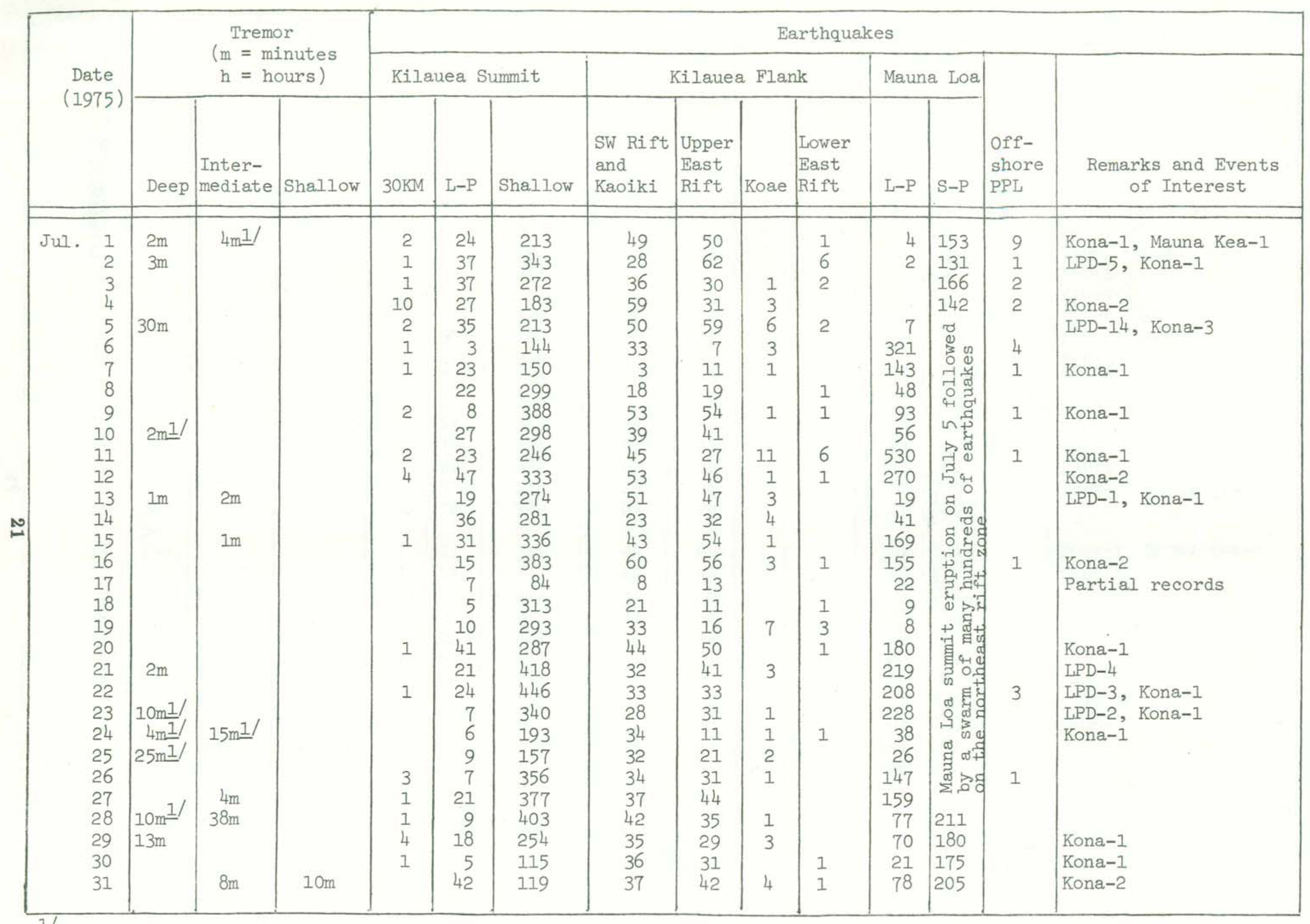

1/MOK-tremor beneath Mauna Loa Volcano. 


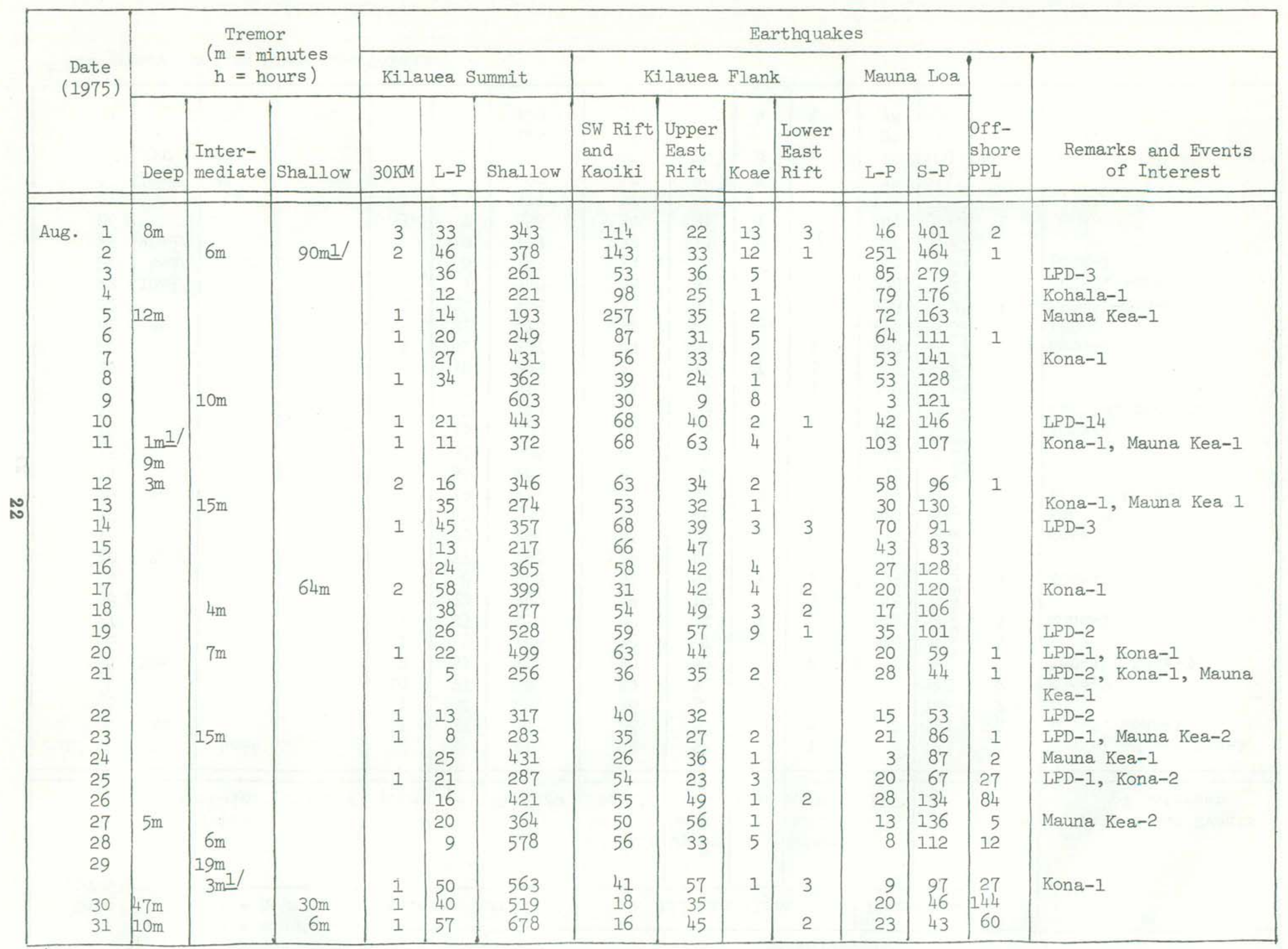

1/MOK-tremor beneath Mauna Loa Volcano 


\begin{tabular}{|c|c|c|c|c|c|c|c|c|c|c|c|c|c|c|}
\hline \multirow{3}{*}{$\begin{array}{c}\text { Date } \\
\text { (1975) }\end{array}$} & \multirow{2}{*}{\multicolumn{3}{|c|}{$\begin{array}{c}\text { Tremor } \\
(\mathrm{m}=\text { minutes } \\
\mathrm{h}=\text { hours })\end{array}$}} & \multicolumn{11}{|c|}{ Earthquakes } \\
\hline & & & & \multicolumn{3}{|c|}{ Kilauea Summit } & \multicolumn{4}{|c|}{ Kilauea Flank } & \multicolumn{2}{|c|}{ Mauna Loa } & \multirow[b]{2}{*}{$\begin{array}{l}\text { off- } \\
\text { shore } \\
\text { PPL }\end{array}$} & \multirow[b]{2}{*}{$\begin{array}{c}\text { Remarks and Events } \\
\text { of Interest }\end{array}$} \\
\hline & Deep & $\begin{array}{l}\text { Inter- } \\
\text { mediate }\end{array}$ & Shallow & $30 \mathrm{KM}$ & $\mathrm{L}-\mathrm{P}$ & Shallow & $\begin{array}{l}\text { SW Rift } \\
\text { and } \\
\text { Kaoiki }\end{array}$ & $\begin{array}{l}\text { Upper } \\
\text { East } \\
\text { Rift }\end{array}$ & Koae & $\begin{array}{l}\text { Lower } \\
\text { East } \\
\text { Rift }\end{array}$ & L-P & $S-P$ & & \\
\hline $\begin{array}{r}\text { Sep. } \\
2 \\
2 \\
3 \\
4 \\
5 \\
6 \\
7 \\
8 \\
9 \\
10 \\
11 \\
12 \\
13 \\
14 \\
15 \\
16 \\
17 \\
18 \\
19 \\
20 \\
21 \\
22 \\
23 \\
24 \\
25 \\
26 \\
27 \\
28 \\
29 \\
30\end{array}$ & $\begin{array}{c}3 \mathrm{~m} \\
3 \mathrm{~m} 1 / \\
29 \mathrm{~m} \\
17 \mathrm{~m} \\
32 \mathrm{~m} \\
\\
4 \mathrm{~m} \\
15 \mathrm{~m} \\
\\
1 \mathrm{~m} 1 / \\
70 \mathrm{~m} \\
20 \mathrm{~m} \\
30 \mathrm{~m}\end{array}$ & $\begin{array}{l}15 \mathrm{~m} \\
3 \mathrm{~m} \\
\\
5 \mathrm{~m} \\
5 \mathrm{~m}\end{array}$ & $\begin{array}{l}20 \mathrm{~m} \\
85 \mathrm{~m} \\
10 \mathrm{~m} \\
20 \mathrm{~m}\end{array}$ & $\begin{array}{l}1 \\
2 \\
2 \\
3 \\
1 \\
2 \\
2 \\
1 \\
2 \\
1\end{array}$ & $\begin{array}{l}54 \\
94 \\
67 \\
61 \\
89 \\
23 \\
52 \\
40 \\
13 \\
87 \\
42 \\
47 \\
43 \\
30 \\
26 \\
30 \\
21 \\
36 \\
40 \\
39 \\
35 \\
10 \\
38 \\
23 \\
10 \\
23 \\
26 \\
19 \\
40 \\
21\end{array}$ & $\begin{array}{r}686 \\
\\
543 \\
583 \\
456 \\
367 \\
259 \\
356 \\
378 \\
478 \\
900 \\
932 \\
700 \\
598 \\
492 \\
511 \\
558 \\
530 \\
309 \\
331 \\
1223 \\
928 \\
673 \\
549 \\
615 \\
305 \\
466 \\
647 \\
577 \\
501 \\
289\end{array}$ & $\begin{array}{r}78 \\
47 \\
48 \\
36 \\
49 \\
41 \\
39 \\
58 \\
43 \\
46 \\
26 \\
37 \\
31 \\
28 \\
49 \\
59 \\
54 \\
68 \\
16 \\
16 \\
76 \\
48 \\
53 \\
70 \\
38 \\
59 \\
103 \\
73 \\
44 \\
58\end{array}$ & $\begin{array}{l}47 \\
24 \\
48 \\
36 \\
64 \\
57 \\
46 \\
58 \\
42 \\
61 \\
53 \\
34 \\
29 \\
33 \\
38 \\
31 \\
41 \\
46 \\
12 \\
9 \\
44 \\
47 \\
33 \\
41 \\
38 \\
54 \\
41 \\
42 \\
33 \\
44\end{array}$ & $\begin{array}{l}1 \\
5 \\
4 \\
2 \\
9 \\
1 \\
1 \\
1 \\
3 \\
2 \\
8 \\
1 \\
1 \\
2 \\
3 \\
6 \\
3 \\
6 \\
4 \\
3 \\
4 \\
5 \\
3 \\
3 \\
5\end{array}$ & $\begin{array}{l}1 \\
1 \\
1 \\
3 \\
2 \\
3 \\
2 \\
3 \\
2\end{array}$ & $\begin{array}{r}8 \\
17 \\
14 \\
7 \\
9 \\
58 \\
54 \\
57 \\
36 \\
77 \\
19 \\
12 \\
20 \\
17 \\
23 \\
21 \\
42 \\
47 \\
\\
7 \\
92 \\
44 \\
78 \\
62 \\
74 \\
33 \\
78 \\
46 \\
17 \\
20\end{array}$ & \begin{tabular}{|r}
152 \\
107 \\
102 \\
126 \\
81 \\
85 \\
65 \\
63 \\
87 \\
62 \\
51 \\
62 \\
58 \\
101 \\
45 \\
83 \\
77 \\
62 \\
60 \\
71 \\
51 \\
58 \\
58 \\
47 \\
25 \\
56 \\
19 \\
18 \\
82 \\
89
\end{tabular} & $\begin{array}{r}61 \\
80 \\
110 \\
23 \\
19 \\
118 \\
66 \\
31 \\
36 \\
9 \\
101 \\
10 \\
114 \\
41 \\
17 \\
19 \\
6 \\
32 \\
8 \\
\\
10 \\
9 \\
12 \\
17 \\
37 \\
41 \\
27 \\
34 \\
57 \\
30\end{array}$ & $\begin{array}{l}\text { Kona-2 } \\
\text { Kona-1 } \\
\text { Kona-1 } \\
\text { LPD-1, Kona-2 } \\
\text { Kona-1 } \\
\text { Kona-1, LPD-1 } \\
\text { Kona-2 } \\
\text { Kona-1 } \\
\text { Kona-1, Kohala-1 } \\
\text { Kona-1 } \\
\text { LPD-1, Kona-1 } \\
\text { LPD-1, Kona-2 }\end{array}$ \\
\hline
\end{tabular}

I/MOK-tremor beneath Mauna Loa Volcano 


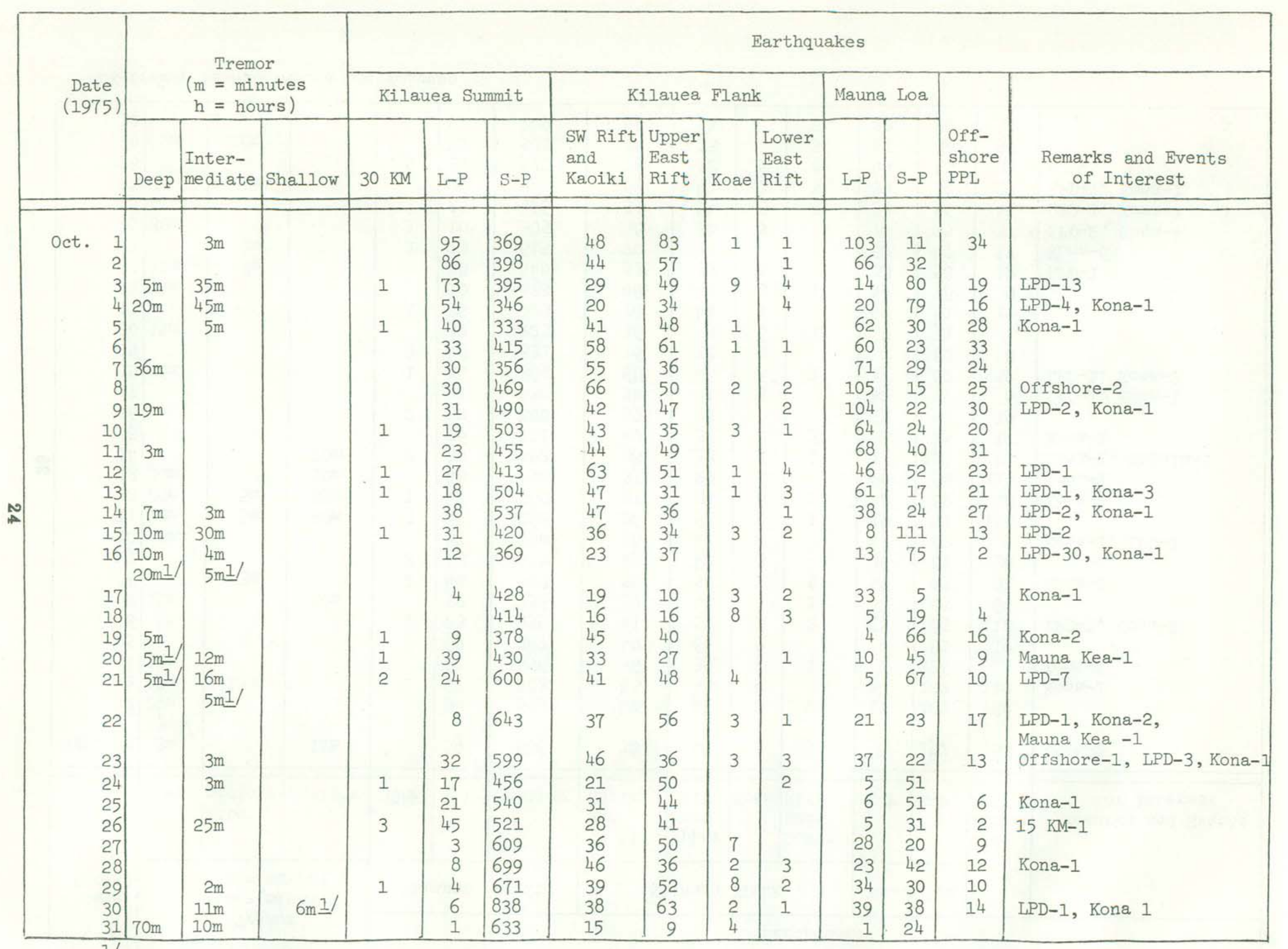




\begin{tabular}{|c|c|c|c|c|c|c|c|c|c|c|c|c|c|c|}
\hline \multirow{3}{*}{$\begin{array}{l}\text { Date } \\
\text { (1975) }\end{array}$} & \multirow{2}{*}{\multicolumn{3}{|c|}{$\begin{array}{c}\text { Tremor } \\
(\mathrm{m}=\text { minutes } \\
\mathrm{h}=\text { hours })\end{array}$}} & \multicolumn{11}{|c|}{ Earthquakes } \\
\hline & & & & \multicolumn{3}{|c|}{ Kilauea Summit } & \multicolumn{4}{|c|}{ Kilauea Flank } & \multicolumn{2}{|c|}{ Mauna Loa } & \multirow[b]{2}{*}{$\begin{array}{l}\text { Off- } \\
\text { shore } \\
\text { PPL }\end{array}$} & \multirow[b]{2}{*}{$\begin{array}{c}\text { Remarks and Events } \\
\text { of Interest }\end{array}$} \\
\hline & Deep & $\begin{array}{l}\text { Inter- } \\
\text { mediate }\end{array}$ & Shallow & $30 \mathrm{KM}$ & L-P & S-P & $\begin{array}{l}\text { SW Rift } \\
\text { and } \\
\text { Kaoiki }\end{array}$ & $\begin{array}{l}\text { Upper } \\
\text { East } \\
\text { Rift }\end{array}$ & Koae & $\begin{array}{l}\text { Lower } \\
\text { East } \\
\text { Rift }\end{array}$ & L-P & S-P & & \\
\hline Nov. $\begin{array}{r}1 \\
2 \\
3 \\
4 \\
5 \\
6 \\
7 \\
8 \\
\\
9 \\
10 \\
11 \\
12 \\
13 \\
14 \\
15 \\
16 \\
17 \\
18 \\
19 \\
20 \\
21 \\
22 \\
23 \\
24 \\
25 \\
26 \\
27 \\
28 \\
29 \\
30\end{array}$ & $\begin{array}{l}7 \mathrm{~m} \\
4 \mathrm{~m} \\
30 \mathrm{~m} \\
4 \mathrm{~m} \\
17 \mathrm{~m} \\
6 \mathrm{~m}\end{array}$ & $\begin{array}{r}2 \mathrm{~m} \\
5 \mathrm{~m} \\
5 \mathrm{~m} \\
\\
2 \mathrm{~m} \\
\\
2 \mathrm{~m} \\
139 \mathrm{~m} \\
30 \mathrm{~m} \\
5 \mathrm{~m} \\
\\
2 \mathrm{~m}\end{array}$ & $\begin{array}{c}2 \mathrm{~m} \\
52 \mathrm{~m} 1 /\end{array}$ & $\begin{array}{l}2 \\
3 \\
1 \\
1 \\
1 \\
2 \\
1 \\
1 \\
2 \\
1 \\
1 \\
1 \\
3\end{array}$ & $\begin{array}{r}10 \\
16 \\
12 \\
8 \\
20 \\
26 \\
20 \\
39 \\
\\
12 \\
9 \\
6 \\
15 \\
36 \\
72 \\
39 \\
24 \\
45 \\
19 \\
26 \\
21 \\
7 \\
1 \\
4 \\
4 \\
10 \\
21 \\
24 \\
32 \\
32 \\
9\end{array}$ & $\begin{array}{r}1068 \\
300 \\
787 \\
553 \\
784 \\
894 \\
743 \\
672 \\
\\
872 \\
823 \\
1055 \\
962 \\
928 \\
601 \\
484 \\
610 \\
552 \\
585 \\
526 \\
453 \\
379 \\
498 \\
429 \\
415 \\
397 \\
415 \\
643 \\
225 \\
66\end{array}$ & $\begin{array}{r}7 \\
38 \\
43 \\
45 \\
74 \\
55 \\
34 \\
28 \\
39 \\
50 \\
49 \\
42 \\
38 \\
18 \\
15 \\
43 \\
36 \\
38 \\
42 \\
42 \\
59 \\
8 \\
33 \\
29 \\
41 \\
46 \\
32 \\
30 \\
1458\end{array}$ & $\begin{array}{r}16 \\
15 \\
56 \\
73 \\
47 \\
67 \\
74 \\
66 \\
\\
79 \\
84 \\
74 \\
104 \\
74 \\
59 \\
183 \\
99 \\
110 \\
102 \\
102 \\
78 \\
19 \\
28 \\
57 \\
66 \\
80 \\
72 \\
83 \\
233 \\
1161\end{array}$ & $\begin{array}{l}1 \\
7 \\
4 \\
2 \\
1 \\
2\end{array}$ & $\begin{array}{l}3 \\
1 \\
3 \\
1 \\
4 \\
3 \\
2 \\
\\
1 \\
2 \\
1 \\
2 \\
6 \\
\\
1 \\
3 \\
4 \\
1 \\
3 \\
3 \\
9 \\
1 \\
3 \\
\\
1 \\
2 \\
1 \\
3 \\
3 \\
489\end{array}$ & $\begin{array}{r}1 \\
31 \\
36 \\
41 \\
152 \\
45 \\
19 \\
2 \\
5 \\
9 \\
30 \\
28 \\
38 \\
3 \\
2 \\
45 \\
55 \\
39 \\
25 \\
19 \\
4 \\
1 \\
6 \\
3 \\
5 \\
12 \\
25 \\
13\end{array}$ & $\begin{array}{r}30 \\
21 \\
42 \\
47 \\
47 \\
36 \\
14 \\
31 \\
\\
19 \\
5 \\
56 \\
26 \\
28 \\
33 \\
32 \\
17 \\
36 \\
24 \\
22 \\
19 \\
26 \\
23 \\
18 \\
5\end{array}$ & $\begin{array}{r}2 \\
11 \\
9 \\
8 \\
7 \\
9 \\
2 \\
2 \\
\\
1 \\
1 \\
54 \\
1 \\
1\end{array}$ & $\begin{array}{l}15 \mathrm{KM}-1 \\
\text { Kona-1, LPD-1 } \\
\text { LPD-2, 15 KM-1 } \\
\text { Kona-2, Mauna Kea-1 } \\
\text { Kona-3 } \\
\text { LPD-6, Kona-1 } \\
\text { LPD-6, Kona-2, Mauna } \\
\text { Kea-2 } \\
\text { LPD-2, Kona-2 } \\
\text { LPD-4, Kona-2 } \\
\text { Kona-1 } \\
\text { LPD-20, Kona-1 } \\
\text { LPD-16 } \\
\\
\text { LPD-11, } \\
\text { LPD-1, Kona-2 } \\
\text { LPD-1 } \\
\text { LPD-8, Kona-1 } \\
\text { LPD-8 } \\
\text { LPD-5, Kona-1 } \\
\text { LPD-2 } \\
\text { LPD-3 } \\
\text { LPD-15, Kona-3 } \\
\text { LPD-3, Kona-3, Mauna } \\
\text { Kea-1 } \\
\text { LPD-5, Kilauea eruption } \\
\text { @0552 } \\
\text { Counts incomplete-- } \\
\text { totals are estimated } \\
\text { Quakes not counted }\end{array}$ \\
\hline
\end{tabular}




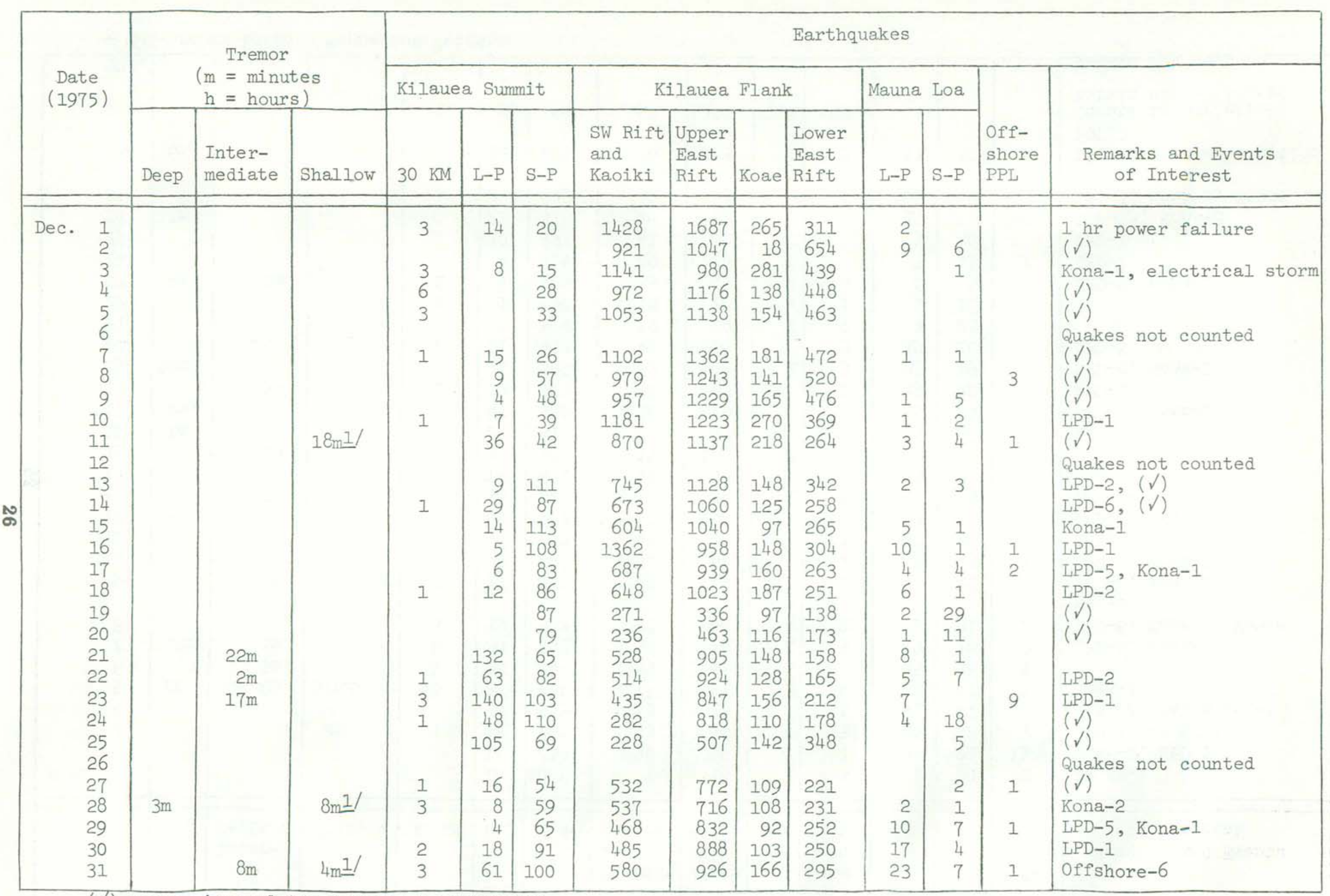

$(\checkmark)$ counts incomplete; totals are estimated

If MOK-tremor beneath Mauna Loa Volcano 


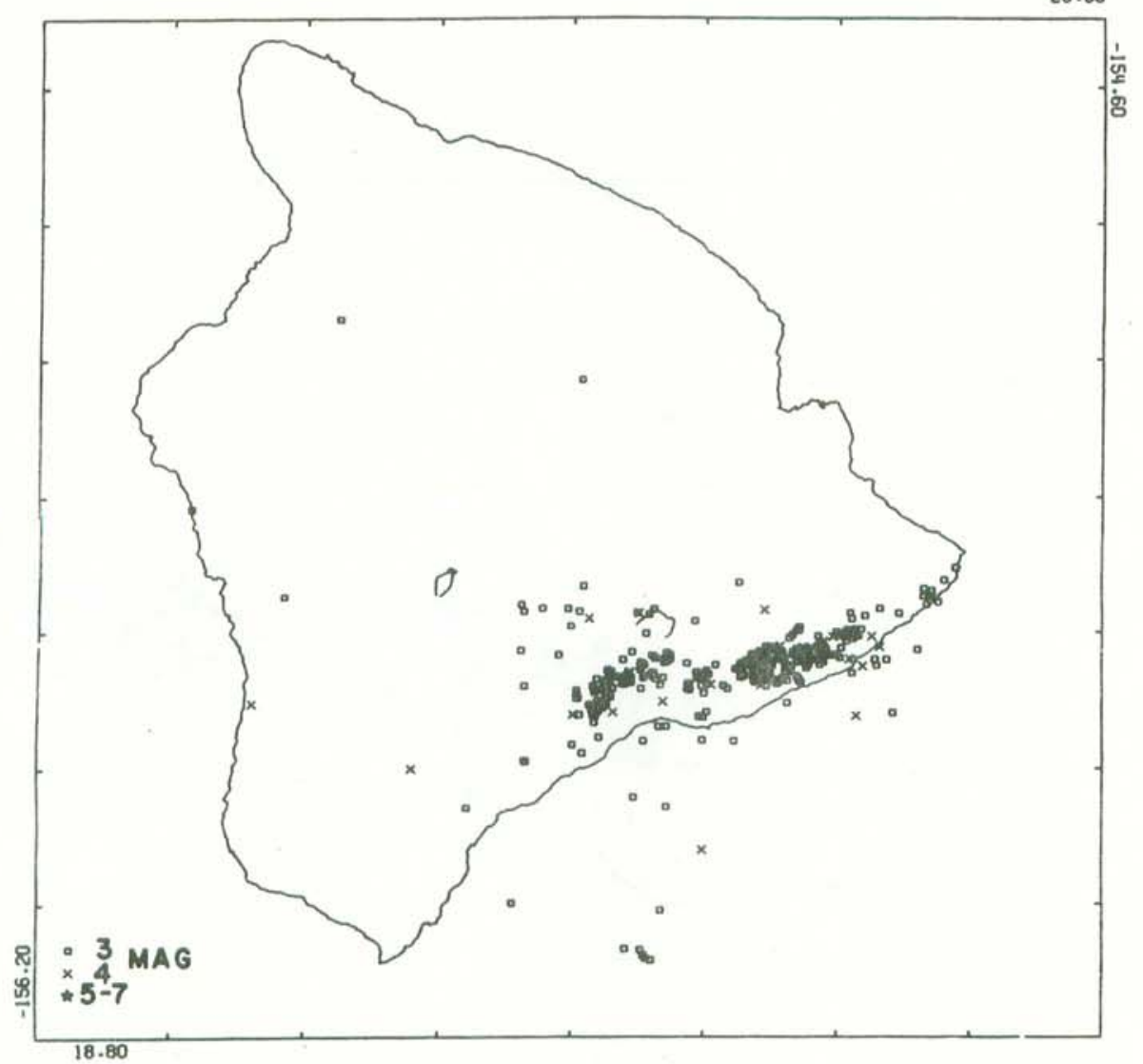

18.80

Figure 5 Epicenter plot of magnitude 3 and above earthquakes for the year 1975.

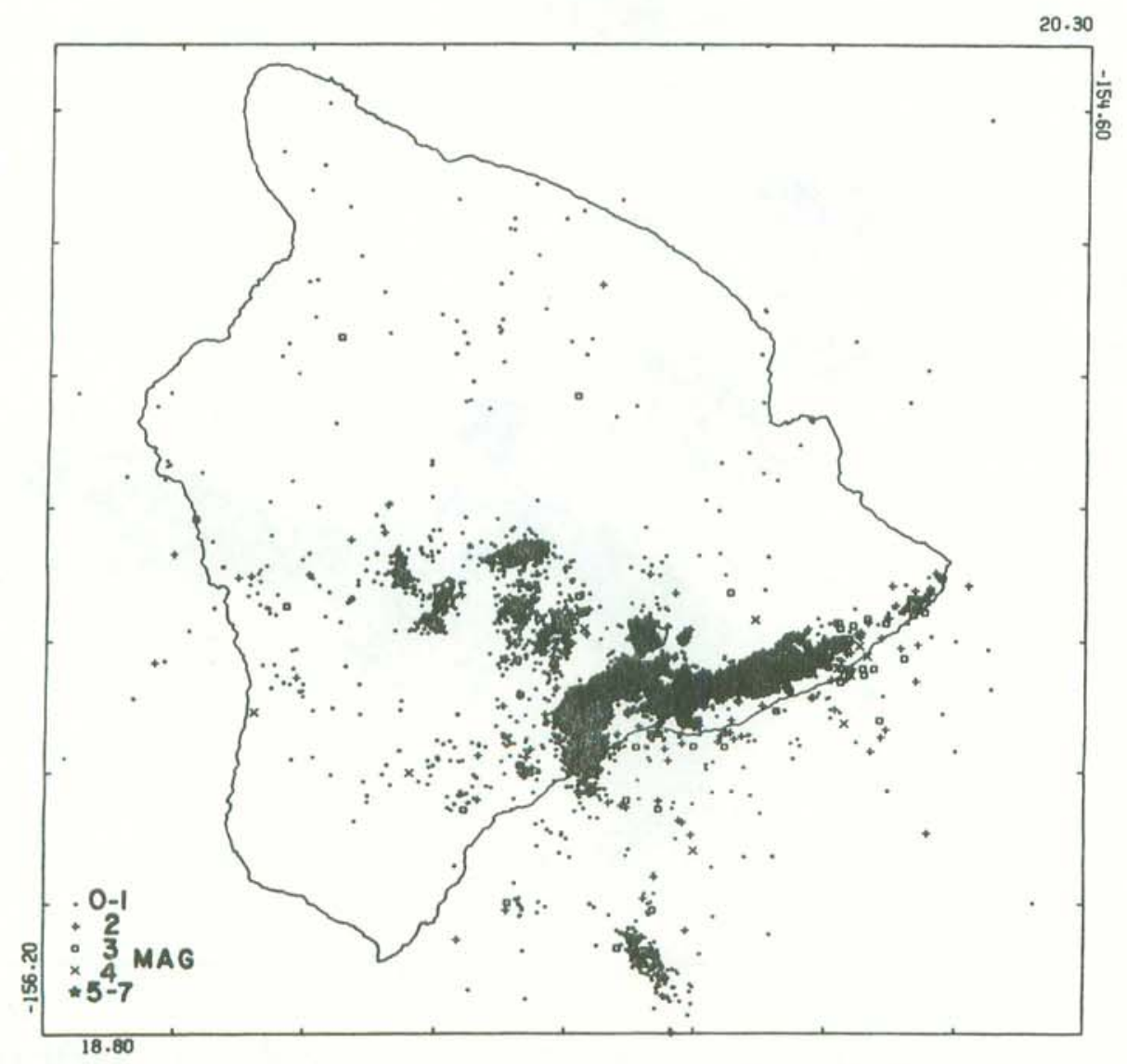

Figure 6. Epicenter plot of all events located for the year 1975. 


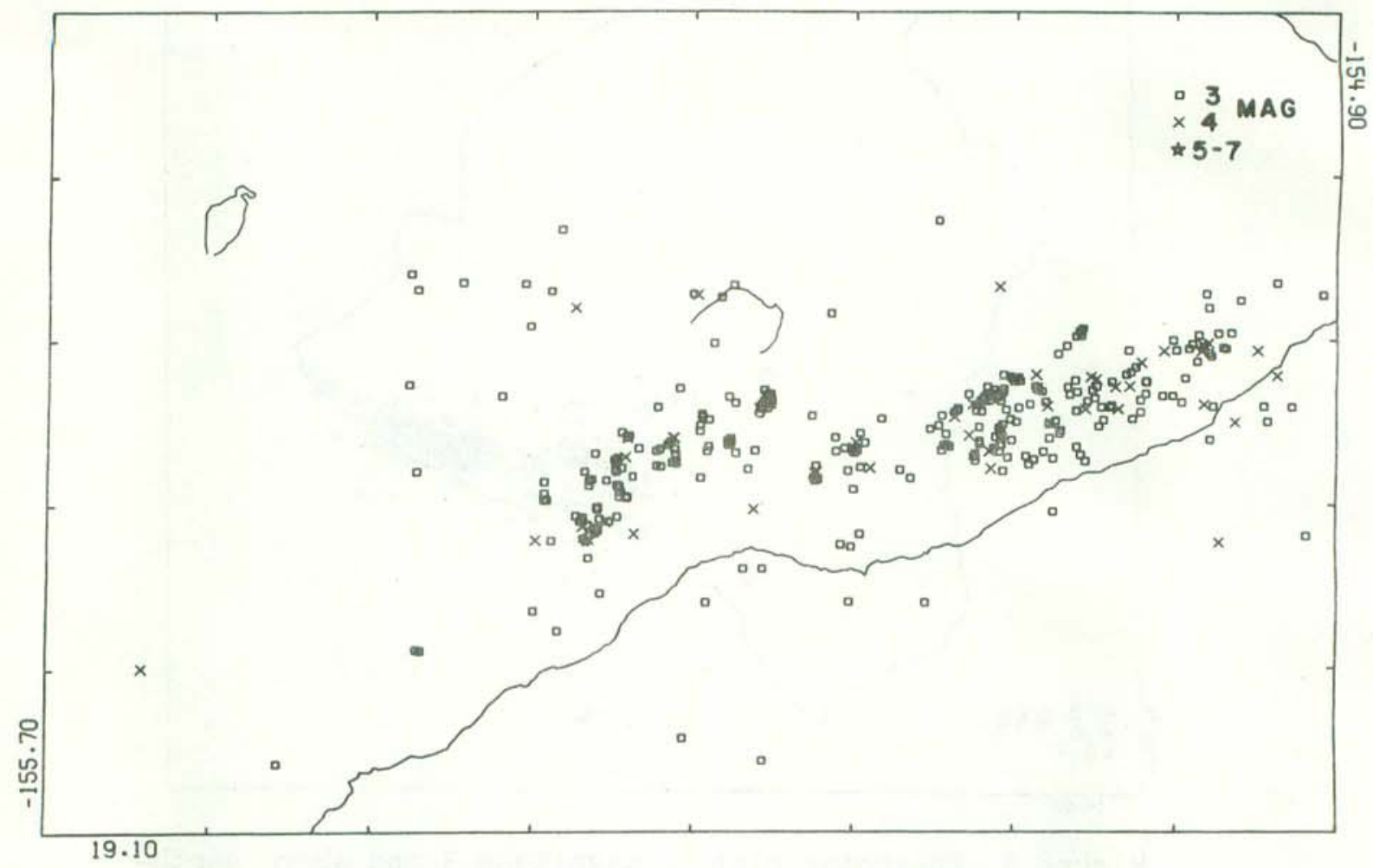

Figure 7. Epicenter plot of Mauna Loa and Kilauea earthquakes magnitude 3 and above for the year 1975.

19.60

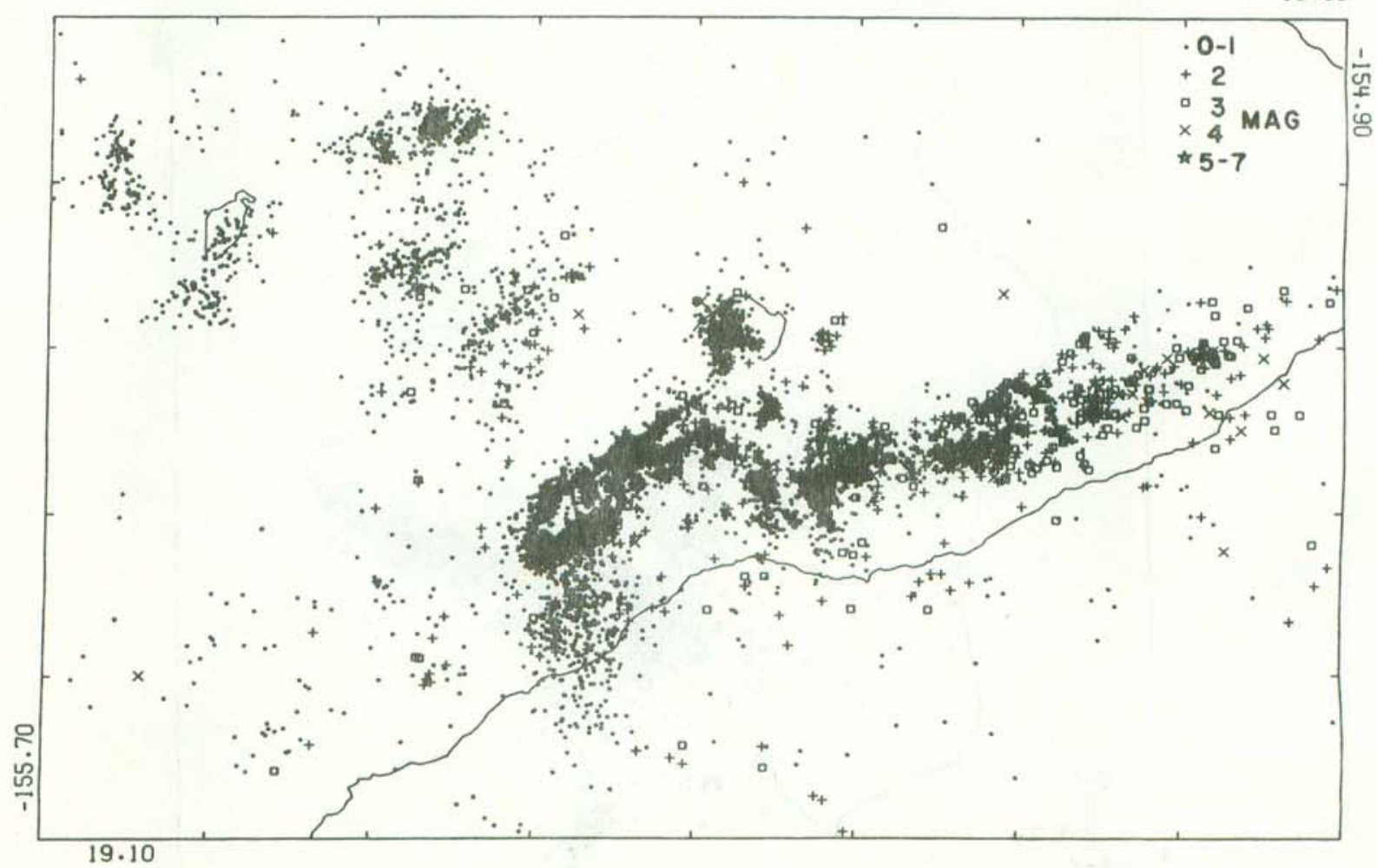

Figure 8. Epicenter plot of all Mauna Loa and Kilauea earthquakes located for the year 1975. 


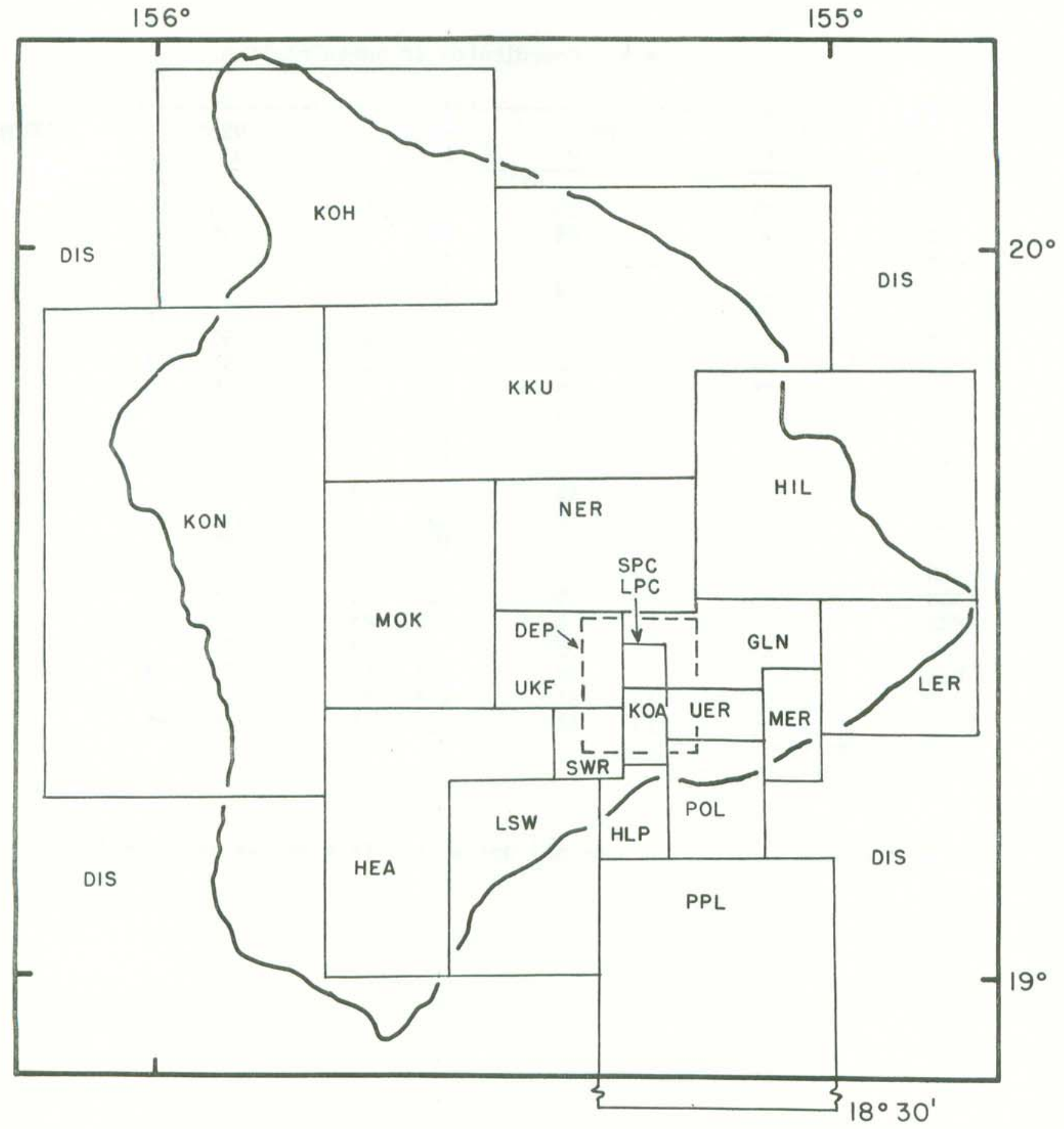

Figure 9. Map indicating limits of areas for specific mnemonic codes used in the remarks column of the earthquake summary. DEP is a code applied to earthquakes with depths greater than 13 kilometres in the Kilauea region. SPC is used for depths between 0 and $6 \mathrm{~km}$, and LPC is used between 6 and 13 $\mathrm{km}$. 
Table 4. Coordinates of named regions.

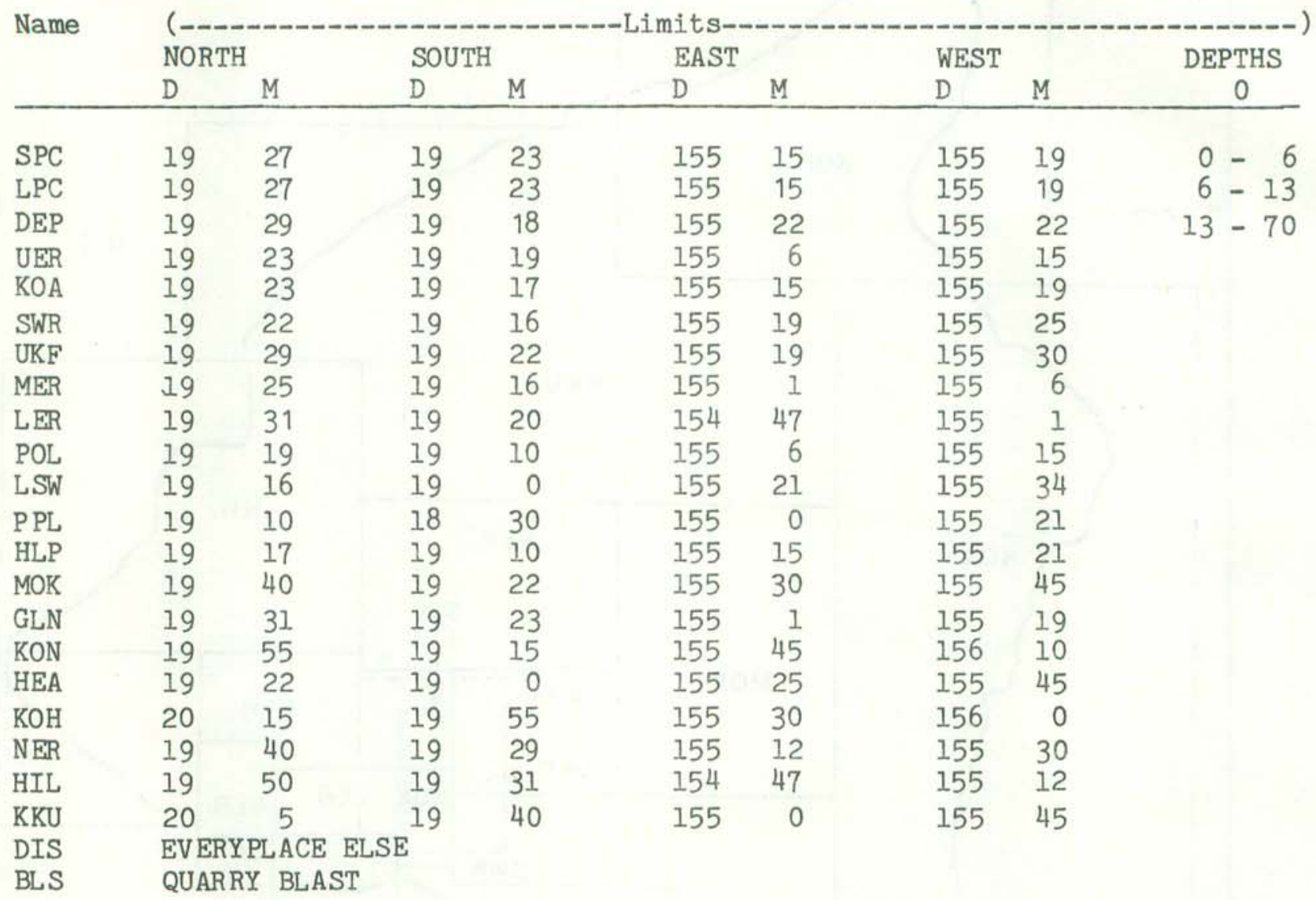

When coordinates imply an overlap, precedence is given as shown in Figure 9. 
Table 5 is a chronological listing of successfully located earthquakes. For each event the following data are presented:

Origin time in Hawaiian Standard Time: date, hour (HR), minute (MN), and second (SEC).

Epicenter in degrees and minutes of North latitude (LAT N) and west longitude (LON W).

DEPTH - Depth of focus in $\mathrm{km}$.

AMP MAG - Amplitude magnitude, if determined.

DUR MAG - Duration magnitude, if determined.

NR - Number of arrivals ( $P$ or $S$ ) used for solution.

NS - Number of $\mathrm{S}$ arrivals used for solution.

GAP DEG - Largest azimuthal separation in degrees between stations.

RMS SEC - Root mean square error of time residuals in sec.

$$
\mathrm{RMS}=\left(\sum \mathrm{R}_{i}^{2} / \mathrm{NR}\right)^{1 / 2}
$$

MIN DIS - Epicentral distance in $\mathrm{km}$ to the third nearest station.

ERH $\mathrm{km}$ - Standard error of the epicenter in $\mathrm{km}$.

ERZ $\mathrm{km}$ - Standrad error of depth of focus in $\mathrm{km}$.

REMK - Remarks, three letter code for geographic location of event. See Figure 9 for location of mnemonic code.

Table 5 lists all events located during 1975. Table 6 lists only events of magnitude 3.5 or larger. 


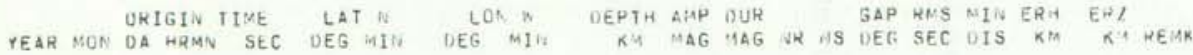

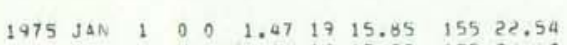
0.534 .071915 .85915522 .54

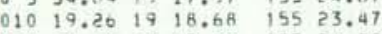
$\begin{array}{rrrrrr}010 & 57.17 & 19 & 17.86 & 155 & 24.20 \\ 014 & 4,87 & 19 & 17.62 & 155 & 24.22\end{array}$

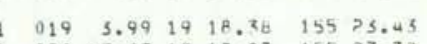

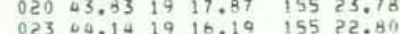
$\begin{array}{llllll}024 & 30.67 & 19 & 16.56 & 155 & 22.47 \\ 026 & 33.15 & 19 & 17.42 & 155 & 24.22\end{array}$

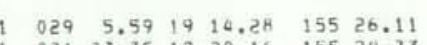

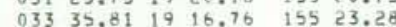

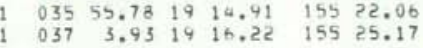

$\begin{array}{lllllll}1 & 040 & 17.32 & 19 & 19.19 & 155 & 22.29\end{array}$ $\begin{array}{llllll}046 & 18.03 & 14 & 16.10 & 155 & 24.47\end{array}$

$049 \quad 46.0919 \quad 9.41 \quad 155 \quad 15.25$

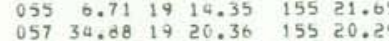

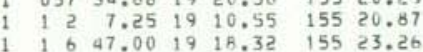

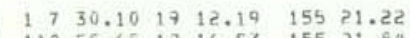
$\begin{array}{llllll}110 & 55.65 & 17 & 16.53 & 155 & 21.64 \\ 123 & 40.98 & 19 & 16.33 & 155 & 22.70\end{array}$

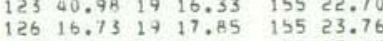

$\begin{array}{llllll}137 & 33.72 & 19 & 13.39 & 155 & 23.56\end{array}$ $\begin{array}{llllll}147 & 24.44 & 19 & 13.22 & 155 & 23.22 \\ 153 & 52.76 & 19 & 16.56 & 155 & 24.20\end{array}$ $154 \quad 49.811917 .74 \quad 15524.00$ $\begin{array}{llllll}210 & 52.52 & 17 & 18.21 & 155 & 23.91\end{array}$

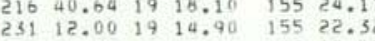
$\begin{array}{llllll}233 & 6.10 & 19 & 15.44 & 155 & 22.7\end{array}$

$\begin{array}{llllll}240 & 25.46 & 19 & 15.4 n & 155 & 23.44 \\ 241 & 10.96 & 19 & 12.43 & 155 & 21.30\end{array}$ $\begin{array}{lllll}241 & 10.36 & 19 & 12.43 & 155 \\ 256 & 52.19 & 19 & 17.84 & 155 \\ 24.11\end{array}$

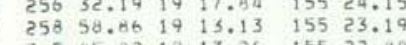

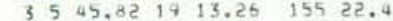

$\begin{array}{lllllll}1 & 311 & 2.75 & 17 & 15.45 & 155 & 23.10\end{array}$ $\begin{array}{llllll}313 & 11.39 & 14 & 17.17 & 155 & 21.74 \\ 335 & 38.03 & 19 & 16.17 & 15522.31\end{array}$ $\begin{array}{llllllllll}5.51 & 2.9 & 14 & 0 & 154 & .14 & 8 & 1.6 & 2.9 & \text { LSA }\end{array}$

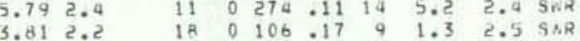
$\begin{array}{rllllllll}.092 .1 & 13 & 0 & 122 & .19 & 10 & 1.5 & 7.5 & \text { SnR } \\ 1.551 .8 & 14 & 0 & 117 & .11 & 10 & .0 & 58.1 & \text { SNR }\end{array}$

$1.13 \begin{array}{lllllllll}2.4 & 17 & 0 & 124 & .20 & 11 & 1.6 & 52.8 & \text { SAR }\end{array}$ .082 .5 $\begin{array}{ll}5.28 & 1.8 \\ 3.64 & 2.3\end{array}$

$5.16 \quad 3.5$ 3.160 .040 $\begin{array}{ll}7.42 & 2.2 \\ 2.39 & 1.9\end{array}$ 5.15 2. 2 2.77 . 1.172 .6 2.362 . $4.25 \quad 2.1$ 4.53 1.9 $7.66 \quad 4.1$ 9. $47 \quad 2.9$ 0.882 .3 $\begin{array}{ll}6.08 & 2.7 \\ 4.57 & 2.1\end{array}$ $4,533,3$

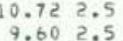
2.442 .3 6.003. $\begin{array}{ll}4.35 & 2.7 \\ 2.32 & 2.5\end{array}$ $\begin{array}{lll}6.74 & 2.2 \\ 5.29 & 2.5\end{array}$

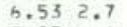
$\begin{array}{ll}0.97 \\ 3.97 & 0.6\end{array}$ 2.152 .6 9.303 .5 0.192 .5 0.352 .9
4.35

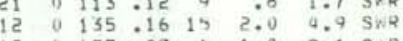
$\begin{array}{rrrrrrrr}17 & 0 & 133 & .23 & 8 & 1.8 & 2.1 & \text { SNR } \\ 16 & 0 & 119 & .09 & 10 & .7 & 3.2 & \text { SWR }\end{array}$ यद 0119.18 is $1.4 \quad 1.6$ LSW 500156.15 a $1.5 \quad 4.0$ Snk $\begin{array}{lllllllll}22 & 0 & 151 & 11 & 10 & .0 & 1.3 & \text { LSF } \\ 12 & 0 & 121 & 117 & 12 & 1.2 & 4.3 & \text { HEA }\end{array}$ $\begin{array}{lllllllllll}14 & 0 & 100 & -11 & 9 & .9 & 1.0 & \text { SWR }\end{array}$ $\begin{array}{llllllll}15 & 0 & 127 & 21 & 13 & 1.2 & 1.7 & \text { LSN }\end{array}$ $\begin{array}{rrrrrrrr}13 & 0 & 170 & .17 & 13 & 2.3 & 65.5 & \text { SWR } \\ 21 & 0 & 118 & .14 & 10 & 1.0 & 2.5 & \text { SWR }\end{array}$ $\begin{array}{llllllll}8 & 0 & 229 & .13 & 20 & 3.4 & 4.2 & P P L\end{array}$ $\begin{array}{llllllll}15 & 0 & 156 & .14 & 11 & 1.3 & 1.5 & \text { LSN } \\ 15 & 0 & 120 & .05 & 5 & .6 & 2.3 & 5 W R\end{array}$

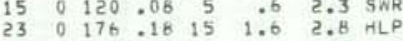
0.15 \& 99 1.3 SNR $\begin{array}{llllllll}25 & 0 & 165 & .16 & 15 & 1.3 & 1.0 & \text { LSW } \\ 22 & 0 & 133 & : 13 & 9 & 1.0 & 1.7 & \text { SWR } \\ 16 & 0 & 130 & .15 & 9 & 1.3 & 2.3 & \text { SW }\end{array}$ 160130.15 a 1.3 2.3 SWR

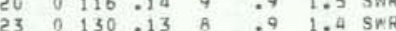
$\begin{array}{llllllll}23 & 0 & 151 & .13 & 12 & 1.3 & .6 & \text { LSN }\end{array}$ $\begin{array}{llllllll}22 & 0 & 157 & .15 & 12 & 1.3 & .9 & \text { LSW }\end{array}$

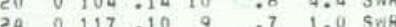
$20 \quad 0 \quad 125 \quad 20 \quad 12 \quad 1.5 \quad 3.5-5 \times 8$ $21001100.16 \quad 9 \quad 1.0 \quad 1.7$ SNR 51 0161.09 lo 1.0 4.0 Snस

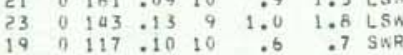

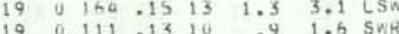
200 15月 .11 l2 99 3.4 LS $200 \begin{array}{lllll}164 & .16 & 12 & 1.7 & 2.1 \\ 20 & \text { LSN }\end{array}$ 25 0 134.54 9 $1.7 \quad 3.4$ LS*

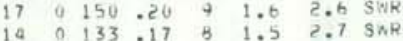

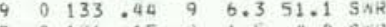
$\begin{array}{llllllllll}13 & 0 & 131 & .20 & 9 & 1.7 & 3.7 & \text { LSw }\end{array}$

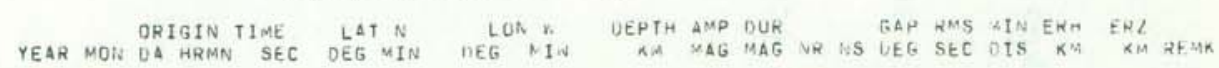

1975 JAN $1344 \quad 16.53 \quad 19 \quad 14.81 \quad 15324.93$

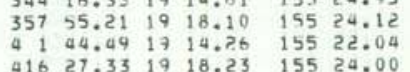
$\begin{array}{llllll}416 & 27.33 & 19 & 18.23 & 155 & 24.00 \\ 431 & 35.56 & 19 & 16.55 & 155 & 24.09\end{array}$

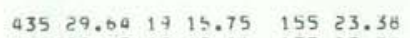

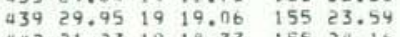

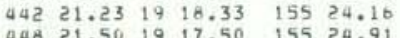
$\begin{array}{llllll}448 & 21.50 & 19 & 17.50 & 155 & 24.91 \\ 53 & 41.35 & 19 & 18.91 & 155 & 23.74\end{array}$

$\begin{array}{llllll}511 & 25.29 & 19 & 18.37 & 155 & 23.50\end{array}$ $\begin{array}{llllll}515 & 15.03 & 19 & 17.87 & 155 & 24.70\end{array}$ $\begin{array}{llllll}518 & 13.01 & 19 & 10.46 & 155 & 23.50\end{array}$

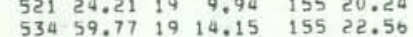

$540 \quad 37.43 \quad 19 \quad 18.75 \quad 15522.93$

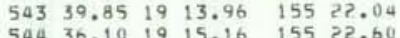
$\begin{array}{cccccc}544 & 36.10 & 19 & 15.16 & 155 & 22.60 \\ 548 & 7.47 & 19 & 11.98 & 155 & 21.92\end{array}$

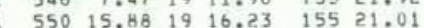
$\begin{array}{llllll}552 & 16.24 & 19 & 13.49 & 155 & 21.21 \\ 553 & 29.79 & 19 & 13.63 & 155 & 21.08\end{array}$ $6310.661917 .80 \quad 15524.60$

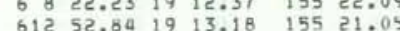

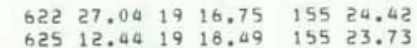

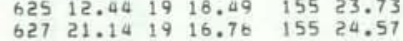

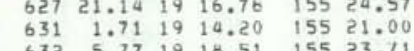

$\begin{array}{lllll}633 & 24.66 \quad 19 & 20.07 \quad 155 \quad 21.20\end{array}$ $63438.551918 .52 \quad 15521.78$

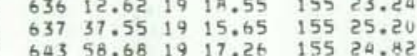
$\begin{array}{llllll}646 & 42.83 & 19 & 15.03 & 155 & 22.53\end{array}$

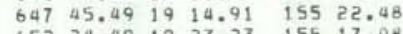

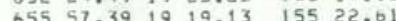

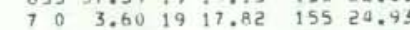
$\begin{array}{llllll}1 & 2 & 55.14 & 19 & 14.55 \quad 155 \quad 23.29\end{array}$

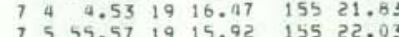

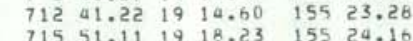
$\begin{array}{llllll}717 & 7.57 & 19 & 14.52 & 155 & 22.35\end{array}$ $731 \quad 3.37 \quad 19 \quad 18.35 \quad 155 \quad 25.36$
8.243 .3

3.061 .9

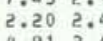

4.912 .4

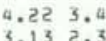

$\begin{array}{lll}5.85 & 5.0\end{array}$

1.743 .1

$\begin{array}{rr}2.80 & 2.1 \\ 2.00 & 5.5\end{array}$ $\begin{array}{rl}.00 & 0.25 \\ 6.61 & 3.4\end{array}$

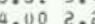
5.012 .5

$\begin{array}{ll}2.67 & 1.5 \\ 7.69 & 2.0\end{array}$

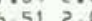
0.06 ?. 6.071 .8

$\begin{array}{ll}7.59 & 3.1 \\ 7.01 & 3.3 \\ 3.57 & 3.3\end{array}$ $\begin{array}{ll}.57 & 3.2 \\ 3.56 & 2.6\end{array}$ 6.36 ट.,

6.152 .5 6.302 .4 1.992 .0

4.752 .0 $\begin{array}{ll}2.38 & 1.9 \\ 4.03 & 0.3\end{array}$ 4.842 .6 $4,4<2.0$ 5.43
6.833 .0 4.6320 .692 .1 $\begin{array}{lll}1.35 & 2.7 \\ 7.74 & 2.7\end{array}$ 1.832 .5 .27
1.47
1.97

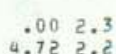
4.72
4.492 .2
4.0

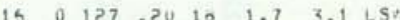
$\begin{array}{llllllll}19 & 0 & 111 & 0.14 & 10 & 1.0 & 1.2 & \text { Sivk } \\ 19 & 4 & 155 & 013 & 13 & 1.3 & 2.7 & \text { LS }\end{array}$

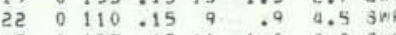
2000139,13 is $1.0 \quad 1.7$ LS

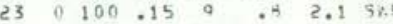

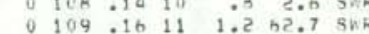

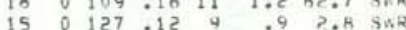

$\begin{array}{llllllll}19 & 0 & 145 & .15 & 9 & 1.0 & 3.0 & 5 \text { mid }\end{array}$

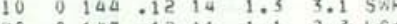

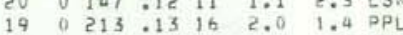
$1902154.10+110.95$

$\begin{array}{llllllll}16 & 0 & 118 & 00 & 0 & .5 & 1.7 & \text { SWR }\end{array}$

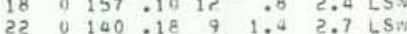

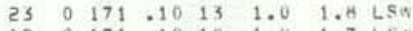

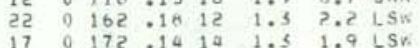

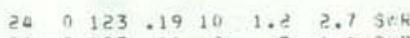

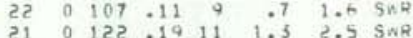
$\begin{array}{llllllll}17 & 0 & 158 & -10 & 12 & .4 & 29.5 & \text { LSR } \\ 17 & 0 & 137 & .12 & 9 & 99 & 59.4 & 5 N R\end{array}$ $\begin{array}{lllllllll}17 & 0 & 89 & .09 & 7 & .7 & .9 & 5 W R\end{array}$

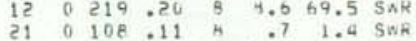

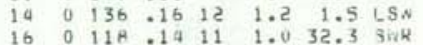
$\begin{array}{lllllllll}12 & 0 & 194 & .14 & 13 & 2.7 & 1.7 & \text { LSW }\end{array}$

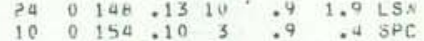

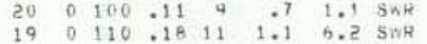
$\begin{array}{rrrrrrrrr}20 & 0 & 158 & .13 & 10 & .9 & 31.7 & \text { L SW }\end{array}$ $\begin{array}{llllllll}22 & 0 & 137 & 14 & 8 & 1.0 & 36.0 & \text { LSW }\end{array}$ $\begin{array}{llllllll}19 & 0 & 168 & .13 & 10 & 00 & 44.7 & \text { LSi } \\ 21 & 0 & 104 & .16 & 10 & 06 & 50.6 & 5 \text { W. }\end{array}$

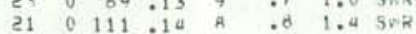
1500176 .1a 12 1.6 3.3 Ls 1901716.10141 .01 .7 LSW

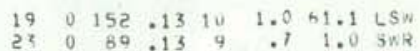


HVO EARTHQUAKE SUMMARY LIST

PAge 3

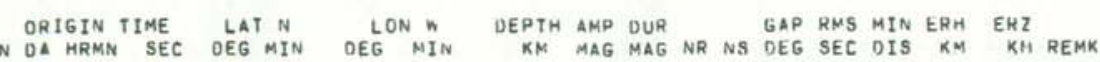
$\begin{array}{llllllll}1975 \text { JAN } 1 & 723 & 5.23 & 19 & 19.00 & 155 & 22.53\end{array}$ 1
1 $724 \quad 17.34 \quad 19 \quad 14.97 \quad 155 \quad 22.65$

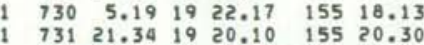

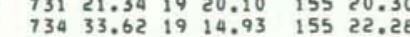

$735 \quad 10.98 \quad 19 \quad 18.97 \quad 155 \quad 23.77$
1 $\begin{array}{llllll}742 & 40.29 & 19 & 24.56 & 155 & 16.78\end{array}$

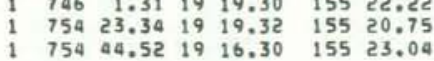

$758 \quad 51.0019$
$12.19 \quad 155 \quad 23.18$

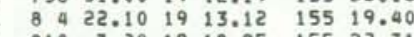
$\begin{array}{lllll}810 & 7.20 & 19 & 18.95 & 155 \\ 8122.71\end{array}$

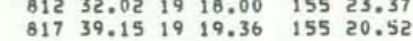

$\begin{array}{llllll}851 & 42.87 \quad 19 & 19.19 & 155 & 22.94\end{array}$

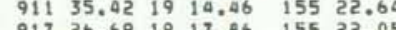

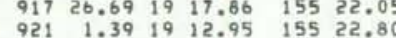

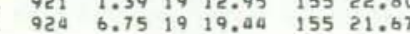

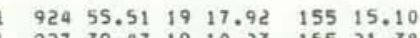

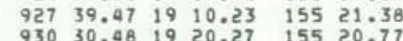
$900 \quad 11.5719 .0 .14 \quad 15525.13$ $94217.441912 .41 \quad 15521.10$

$1946 \quad 45.66 \quad 19 \quad 14.40 \quad 155 \quad 24.31$

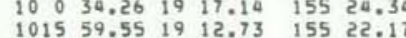
$101716.541919 .25 \quad 15523.13$

$\begin{array}{lllllll}1 & 1020 \quad 994 & 19 & 18.49 & 155 & 23.57\end{array}$

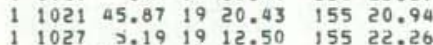
$1033 \quad 47.741919 .12 \quad 15513.90$

$1103933.71 \quad 19 \quad 10.90 \quad 155 \quad 21.97$ $\begin{array}{lllllll}1 & 1042 & 15.53 & 19 & 17.77 & 155 & 23.46\end{array}$ 104649.3919 16.61 15523.49

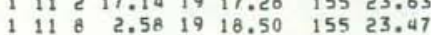

$\begin{array}{lllllll}1 & 1114 & 31.45 & 19 & 16.77 & 155 & 25.02 \\ 1 & 1119 & 45.13 & 19 & 16.08 & 155 & 23.70\end{array}$ $1112854.5619 \quad 10.95 \quad 15521.80$

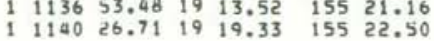
$\begin{array}{lllllll}1 & 1143 & 52.44 & 19 & 19.08 & 155 & 22.18\end{array}$

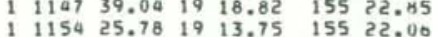
$\begin{array}{lllllllllll}1.91 & 2.2 & 17 & 0 & 114 & .13 & 9 & .8 & 59.9 & \text { SWR }\end{array}$ $1.171 .5 \quad 7 \quad 0154.07 \quad 450.5 \%$

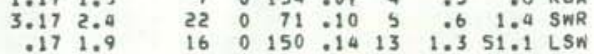
$\begin{array}{llllllllll}4.56 & 2.5 & 16 & 0 & 126 & .13 & 9 & 1.1 & 1.5 & \text { SWR }\end{array}$

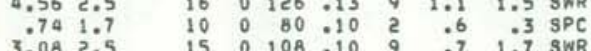
$\begin{array}{llllllllll}3.08 & 2.5 & 15 & 0 & 108 & 110 & 9 & .7 & 1.7 & \text { SWR } \\ 8.00 & 2.3 & 18 & 0 & 98 & 014 & 6 & 1.0 & 1.3 & \text { SWR }\end{array}$

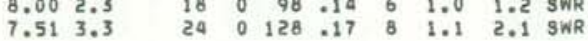

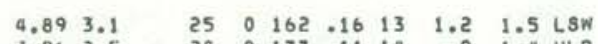

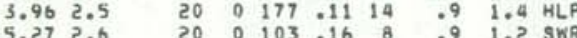

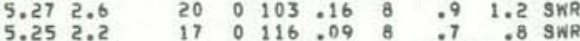

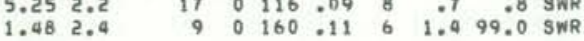
$\begin{array}{llllllllll}5.06 & 2.2 & 21 & 0 & 99 & 11 & 8 & .7 & .9 & \text { SWR }\end{array}$

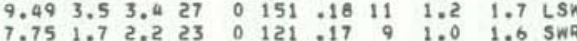

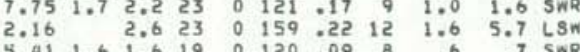

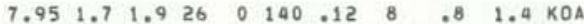

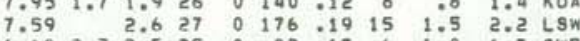

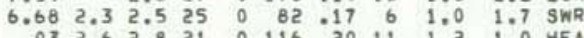

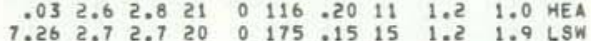
$\begin{array}{llllllllllll}\text { S. } 81 & 4.3 & 4.1 & 22 & 0 & 140 & .15 & 11 & 1.2 & 2.6 & \text { LSW } \\ 4.82 & 2.9 & 3.0 & 27 & 0 & 114 & .18 & 10 & 1.0 & 1.3 & \text { SWRR }\end{array}$

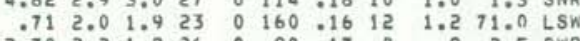

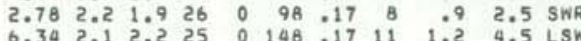

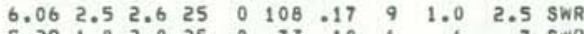

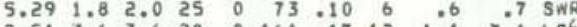

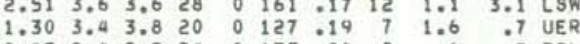
$\begin{array}{lllllllllll}7.72 & 2.9 & 3.0 & 23 & 0 & 171 & .18 & 14 & 1.6 & 3.3 & \text { LSN }\end{array}$

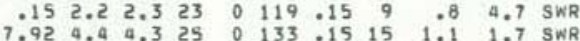
.952 .32 .2 22 $0137: 19$ 14 1.444 .1 SWR

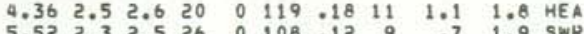

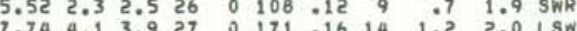

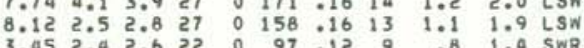

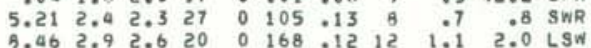

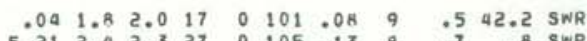

HVO EARTHQUAKE SUMMARY LIST

PAGE

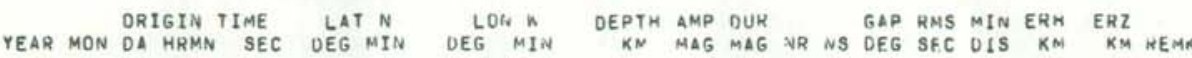

1975 JAN $11155 \quad 40.8419 \quad 19.42 \quad 155 \quad 22.21$ $\begin{array}{lllllll}1210 & 31.66 & 19 & 11.06 & 155 & 21.25 \\ 1 & 1213 & 34.51 & 19 & 17.89 & 155 & 13.92\end{array}$

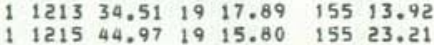

$\begin{array}{lllllll}1 & 1235 & 7.87 & 19 & 17.37 & 155 & 24.75\end{array}$ $\begin{array}{lllllll}1 & 1237 & 43.82 & 19 & 12.19 & 15521.09\end{array}$ $1 \quad 1240 \quad 37.00 \quad 19 \quad 18.23 \quad 15523.27$

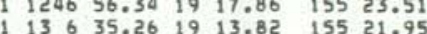

$\begin{array}{llllllll}1 & 13 & 8 & 16.33 & 19 & 13.12 & 155 & 21.96\end{array}$ $1 \quad 13933.01 \quad 1914.12 \quad 15522.34$

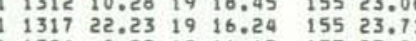
$\begin{array}{llllll}1324 & 8.22 & 19 & 16.67 & 155 & 23.22\end{array}$

$1 \quad 1331 \quad 16.99 \quad 1911.69 \quad 15521.40$
13333 1333
13 $1.92 \quad 19 \quad 18.24 \quad 155 \quad 13.13$

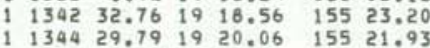
$134731.0819 \quad 15.47 \quad 15524.69$

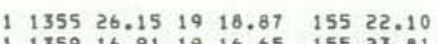

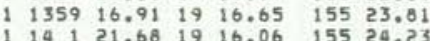

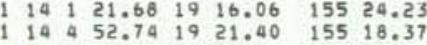
$11472.961918 .61 \quad 15523.47$

$\begin{array}{llllllll}1 & 14 & 7 & 39.77 & 19 & 18.41 & 155 & 22.55 \\ 1 & 14 & 23.52 & 19 & 20.70 & 155 & 20.21\end{array}$ $1410 \quad 58.84 \quad 19 \quad 19.78 \quad 15521.33$ $141555.171918 .97 \quad 15522.00$ $\begin{array}{llllll}1426 & 37.78 & 19 & 18.31 & 155 & 23.37\end{array}$

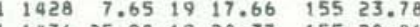
$143625.98 \quad 1920.37 \quad 15520.86$

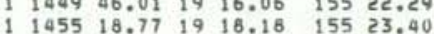

$\begin{array}{lllllll}1 & 1456 & 49.08 & 19 & 24.94 & 155 & 16.55 \\ 1 & 1457 & 45.00 & 19 & 15.81 & 155 & 20.98\end{array}$

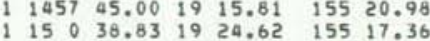
$15434.441920 .12 \quad 15521.15$

$\begin{array}{lllllll}1 & 1521 & 5.70 & 19 & 12.49 & 155 & 23.39\end{array}$

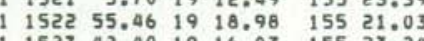
$152742.401916 .03 \quad 15523.24$

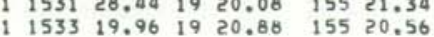

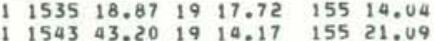

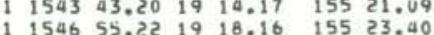

$\begin{array}{lllllllllllll}5.33 & 2.1 & 2.5 & 27 & 0 & 95 & .12 & 9 & .6 & .8 & \text { SWR } \\ 6.33 & 2.0 & 2.3 & 24 & 0 & 139 & : 17 & 9 & 1.2 & 3.0 & \text { LSW }\end{array}$

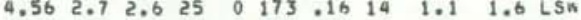
$\begin{array}{lllllllllll}9.38 & 2.2 & 2.5 & 27 & 0 & 137 & .09 & 9 & .6 & .0 & \text { POL } \\ 4.47 & 2.8 & 3.0 & 24 & 0 & 150 & .20 & 9 & 1.3 & 1.9 & \text { LS }\end{array}$

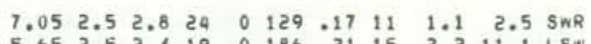

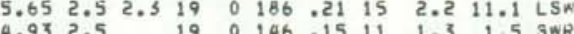

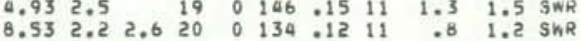

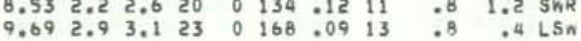
$\begin{array}{lllllllllllll}7.53 & 2.3 & 2.2 & 19 & 0 & 178 & .11 & 15 & 1.1 & 1.7 & \text { LSW }\end{array}$ $5.562 .4200133: 10$ id 0.5 SkR

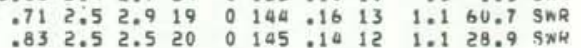
$\begin{array}{lllllllllll}5.75 & 2.6 & 2.5 & 20 & 0 & 177 & .12 & 16 & 1.3 & 3.4 & \text { LSA }\end{array}$

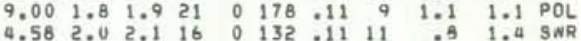

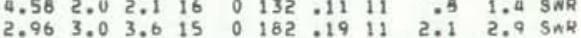

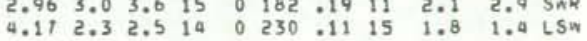

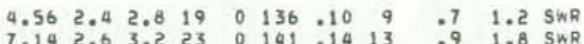

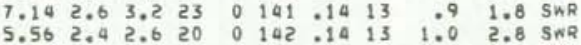

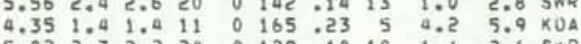
$\begin{array}{lllllllllll}5.46 & 2.2 & 2.1 & 15 & 0 & 170 & .16 & 10 & 1.7 & 1.3 & \text { SWR }\end{array}$

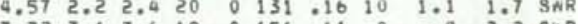

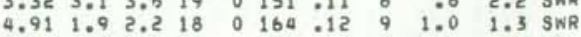
$5.212^{2.2} 2.6 \quad 18$ o $174: 10$ o 1.0 . $\begin{array}{lllllllllllll}5.46 & 2.3 & 2.4 & 25 & 0 & 117 & .12 & 6 & .6 & .6 & \text { SNR }\end{array}$

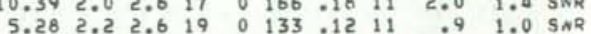
$\begin{array}{rrrrrrrrrrrr}13.53 & 2.1 & 2.4 & 22 & 0 & 95 & .10 & 2 & .8 & .8 & \text { DEP } \\ 2.56 & 1.8 & 1.6 & 20 & 0 & 163 & 111 & 10 & .8 & 2.4 & \text { HLP }\end{array}$ $\begin{array}{lllllllllll}.05 & 1.6 & 2.1 & 14 & 0 & 67 & 116 & 2 & .6 & .7 & \text { SPC }\end{array}$

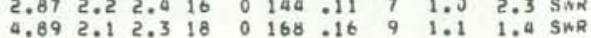

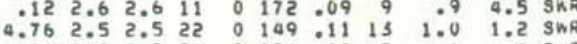

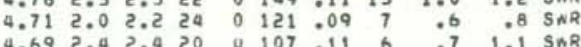
$\begin{array}{rrrrrrrrrrrr}10.29 & 3.7 & 3.6 & 25 & 0 & 172 & .08 & 9 & . .3 & .3 & \text { POL } \\ 8.59 & 2.8 & 2.8 & 25 & 0 & 169 & .13 & 13 & 1.1 & 2.0 & \text { LS }\end{array}$

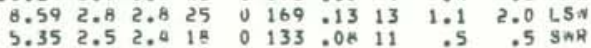
$\begin{array}{lllllllllll}5.70 & 1.7 & 2.2 & 15 & 0 & 165 & .11 & 11 & 1.0 & 1.9 & \text { SWR } \\ 5.67 & 2.7 & 2.9 & 25 & 0 & 134 & 1.9 & 11 & .0 & 1.3 & \text { SNR }\end{array}$ $\begin{array}{lllllllllll}7.12 & 2.2 & 2.5 & 20 & 0 & 182 & .12 & 19 & 1.2 & 2.5 & \mathrm{LSW}\end{array}$ 
HVO EARTHGUAKE SUMMAKY LIST

HaGE 5

ORIGIN TTME LAT W LOR W DEPTA AMP DUK LAP RMS MIN ERA ENZ

व.37

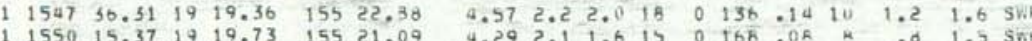

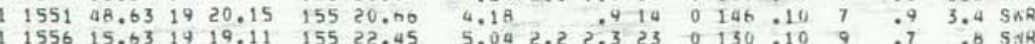

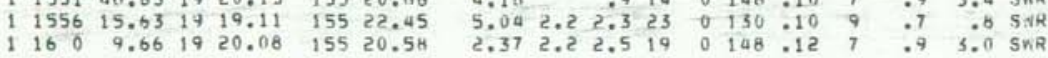

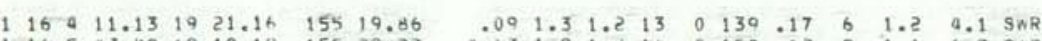

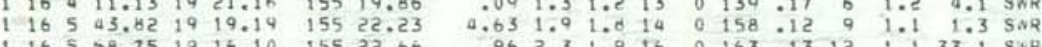

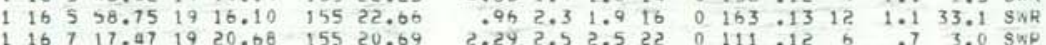

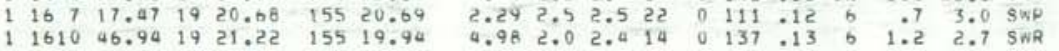
$\begin{array}{llllllllllllllllll}1 & 1611 & 55.94 & 19 & 18.89 & 155 & 22.01 & 3.74 & 2.0 & 2.0 & 16 & 0 & 159 & .07 & 10 & .6 & 1.0 & \text { SNR }\end{array}$

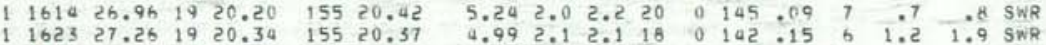

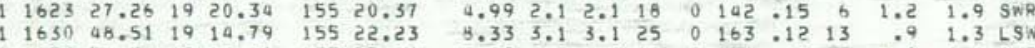

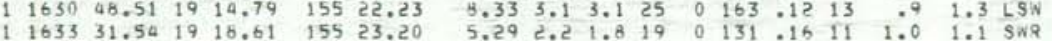

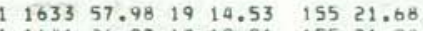

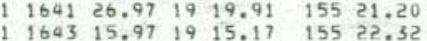

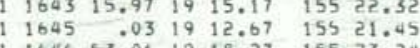

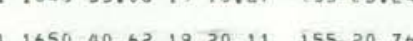

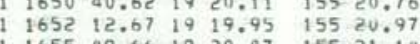

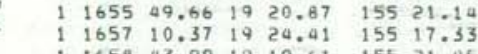

$\begin{array}{lllll}1659 & 21.30 & 19 & 14.08 & 15520.89\end{array}$ 1170 टा.7त 191919. त4 15520.63

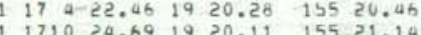

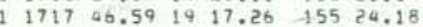

$\begin{array}{lllll}1719 & 29.10 \quad 19 & 20.55 \quad 155 & 20.53\end{array}$ $172252.46 \quad 19$
1723 172532.18 19 $13.11 \quad 15522.46$ $11726 \quad 29.35 \quad 19 \quad 19.43 \quad 15521.82$

$11730 \quad 34.49 \quad 19 \quad 16.21 \quad 1.5521 .47$
1733

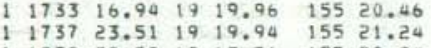

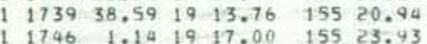
$\begin{array}{llllll}1 & 1755 & 51.20 \quad 19 & 20.04 & 155 & 21.30\end{array}$ 1 A11 $6.0519 \quad 18.15$ 155 23.46

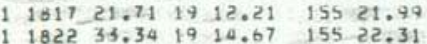

$11823 \quad 39.34 \quad 19 \quad 15.34 \quad 155 \quad 22.67$

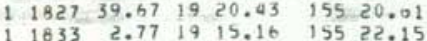

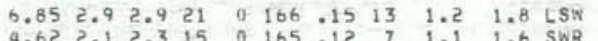

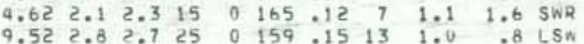

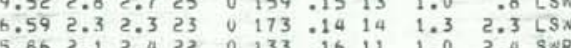

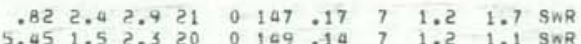

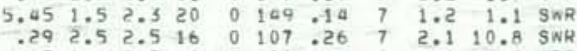

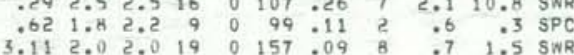
$\begin{array}{rrrrrrrrrrr}5.45 & 2.1 & 1.9 & 21 & 0 & 177 & .12 & 13 & 1.0 & 1.5 & \mathrm{HLP} \\ 2.92 & 2.1 & 2.1 & 14 & 0 & 167 & .18 & 7 & 1.8 & 13.4 & \text { SWR }\end{array}$

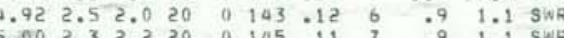
$6.992 .21 .923 \quad 0 \quad 130.14 \quad 11 \quad 99 \quad 1.4$ SWR

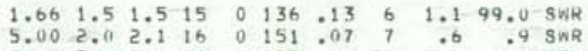

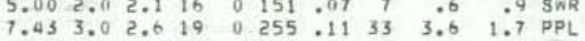

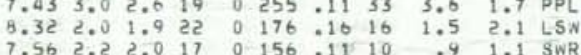
$\begin{array}{lllllllllll}6.48 & 2.2 & 1.8 & 15 & 0 & 163 & .08 & 16 & 1.1 & 4.2 & \text { SWR }\end{array}$

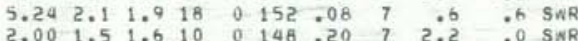

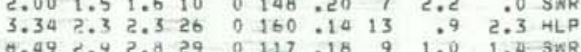
$\begin{array}{llllllllllll}3.61 & 2.0 & 2.1 & 23 & 0 & 91 & .12 & 7 & .6 & 1.6 & \text { SWR }\end{array}$

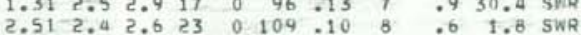

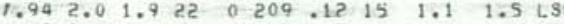
$9.492 .5 ? .425 \quad 0 \quad 147.13 \quad 10 \quad 94 \quad 1.5$ LSW

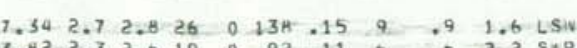

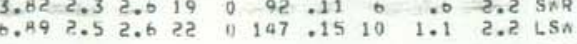

aVO EARTHIDUAKE. SUMMARY LIST

PaGE G

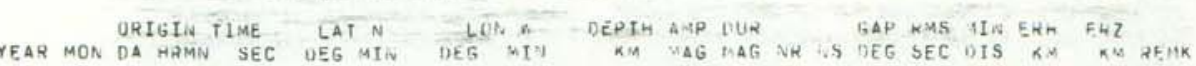

1975 JAN $1183456.76 \quad 1918.90 \quad 15523.50$ $185054.02 \quad 1920.65 \quad 15520.2$

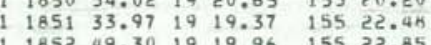

$1855 \quad 32.791920 .41 \quad 15520.70$

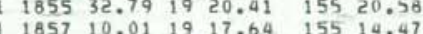
$1912 \quad 44.76 \quad 19 \quad 18.71 \quad 15522.91$

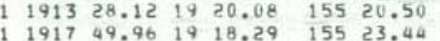

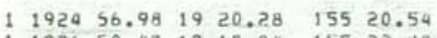
$1192650.471918 .84 \quad 15522.80$ $\begin{array}{lllllll}1 & 1931 & 17.39 & 19 & 19.32 & 155 & 22.58 \\ 1 & 1932 & 39.70 & 19 & 20.54 & 155 & 20.14\end{array}$

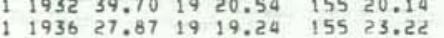

$1937 \quad 26.31 \quad 1922.86 \quad 155 \quad 18.82$
1

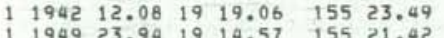
$\begin{array}{lllllll}1 & 1949 & 23.94 & 19 & 14.57 & 155 & 21.42 \\ 1 & 1950 & 41.93 & 19 & 16.57 & 155 & 23.19\end{array}$ $11952 \quad 47.33 \quad 1917.04 \quad 155 \quad 23.64$

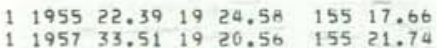
$\begin{array}{llllllll}1 & 20 & 0 & 1.36 & 19 & 20.56 & 155 & 20.40 \\ 1 & 20 & 3 & 2.03 & 19 & 13.13 & 155 & 23.12\end{array}$ $\begin{array}{lllll}20 & 320.11 & 19 & 19.90 & 15521.92\end{array}$ $\begin{array}{llllllll}1 & 20 & 7 & 17.14 & 19 & 20.25 & 155 & 20.44 \\ 1 & 20 & 8 & 22.04 & 19 & 18.57 & 155 & 23.39\end{array}$

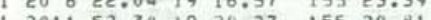

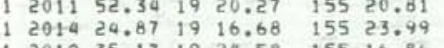
$\begin{array}{lllll}1.2023 \quad 7.43 \quad 19 & 20.60 \quad 155 \quad 20.49\end{array}$

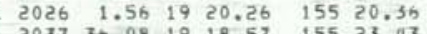

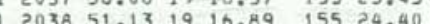
$12040 \quad 5.70 \quad 19 \quad 19.38 \quad 155 \quad 22.99$

$\begin{array}{lllllll}12006 & 10.47 & 19 & 19.93 & 155 & 20.25\end{array}$

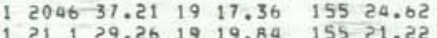

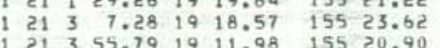
$\begin{array}{lllllll}1 & 2117 & 52.68 & 19 & 17.43 & 155 & 24.44\end{array}$

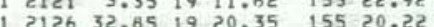
$\begin{array}{lllll}12126 & 55.02 \quad 19 & 17.78 & 155 & 20.32\end{array}$ $\begin{array}{llllll}12128 & 2.05 & 19 & 19.19 & 155-23.37\end{array}$ $\begin{array}{llllll}12120 & 24.06 \quad 19 & 18.51 & 155 & 23.4\end{array}$

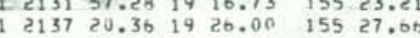

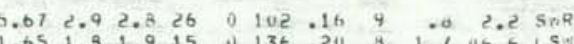

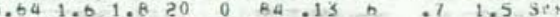

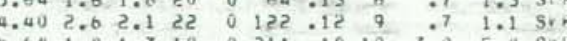

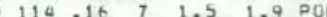

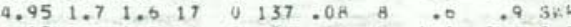

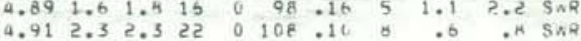

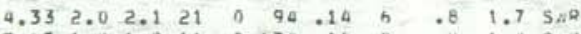

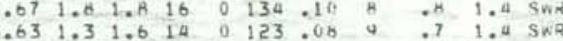
1.812 .41 .615 o 85.15 b .989.0 Sn

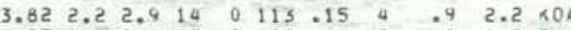

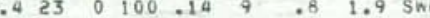

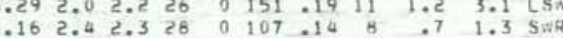

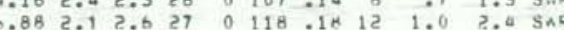

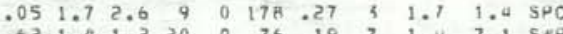

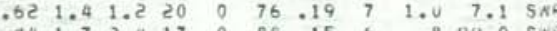

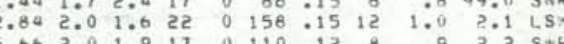

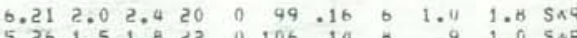

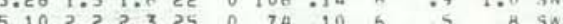

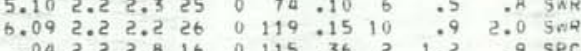

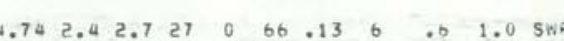

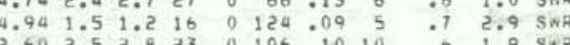

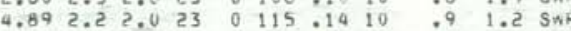

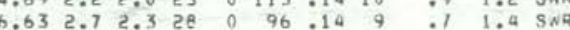

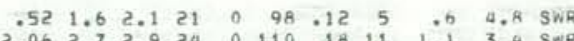

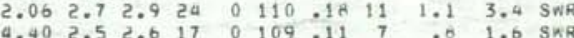

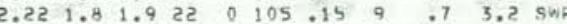

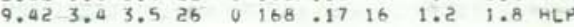
$\begin{array}{lllllllllll}1.78 & 3.1 & 3.9 & 23 & 0 & 110 & .16 & 10 & .928 .2 & 5 N R\end{array}$

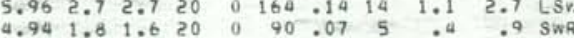

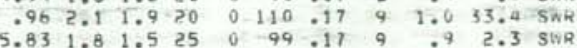
$\begin{array}{lllllllllll}5.44 & 2.6 & 2.3 & 20 & 0 & 106 & .15 & 8 & 1.0 & \text { A A } & \text { SwR }\end{array}$

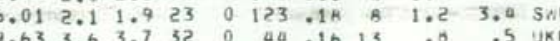


hVO EARTHISUAKE SUMMARY LIST

PAGE

ORIGIN TIME LAT N LOF N OEPTH AMP DUR GAP RMS MIN ERH ERZ

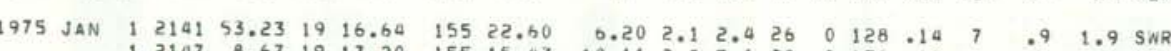

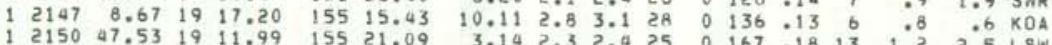

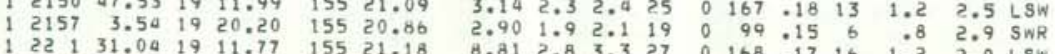

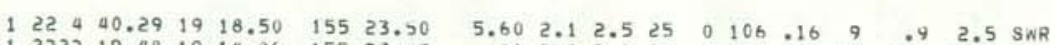

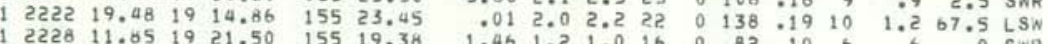

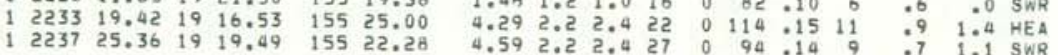

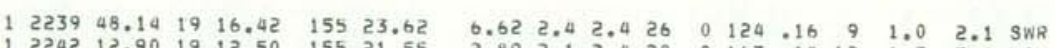

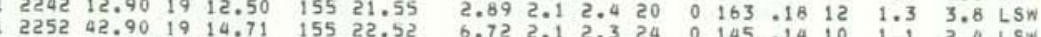

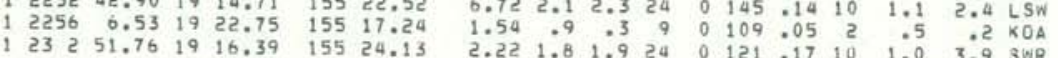

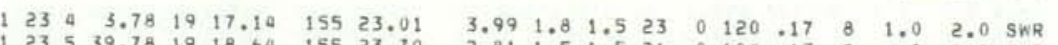

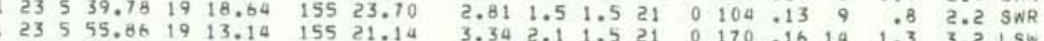

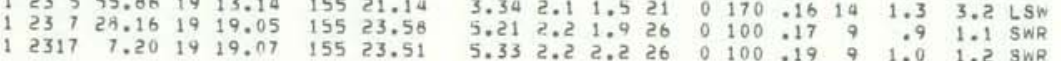

$\begin{array}{lllllll}1 & 2322 & 10.63 & 19 & 15.23 & 155 & 24.18 \\ 1 & 2325 & 1.76 & 19 & 14.59 & 155 & 22.73 \\ 1 & 2326 & 58 & 07 & 19 & 14.01 & 155 \\ 1 & 23.28\end{array}$

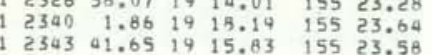

$\begin{array}{lllll}2348 & 6.77 \quad 19 & 12.13 & 155 & 22.34\end{array}$

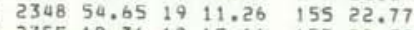
$1235519.761915 .11 \quad 15522.59$ $0170.3419,13.33$ 155 16.07

$\begin{array}{lllllll}\text { द } & 022 & 10.13 & 19 & 19.46 & 155 & 22.35\end{array}$ $\begin{array}{rrrrrr}029 & 2.24 & 19 & 14.85 & 155 & 21.81 \\ 039 & 44.27 & 19 & 19.27 & 155 & 22.92 \\ 0 & 0.92\end{array}$

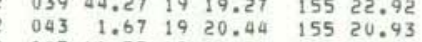

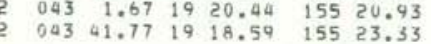

$\begin{array}{lllllll}2 & 047 & 18.05 & 19 & 18.91 & 155 & 22.57 \\ 2 & 351 & 54.56 & 19 & 20.96 & 155 & 19.36\end{array}$ $05435.201920 .13 \quad 15520.25$
10 $11114,13 \quad 17 \quad 14,12 \quad 155,23.45$ $\begin{array}{llllll}114 & 12.62 & 19 & 13.16 & 155 & 23.2\end{array}$ 5 127 47.21 1919.28 155 18.55 $145 \quad 8.42 \quad 19 \quad 17.16 \quad 15522.44$ $\begin{array}{llllll}147 & 33.14 & 19 & 12.22 \quad 155 & 23.35\end{array}$

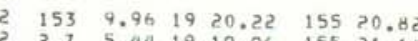

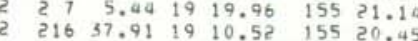

$\begin{array}{llllllllllllll}7.05 & 2.3 & 2.4 & 22 & 0 & 131 & .17 & 11 & 1.2 & 1.9 & \text { LSw } \\ 5.63 & 2.2 & 2.0 & 25 & 0 & 149 & 15 & 10 & 1.2 & 2.9 & \text { LSw }\end{array}$

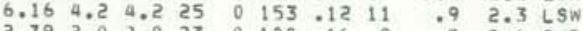

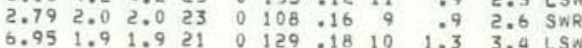
$\begin{array}{lllllllllllllll}2.02 & 2.5 & 2.0 & 24 & 0 & 163 & .19 & 13 & 1.4 & 5.8 & \text { LS* }\end{array}$

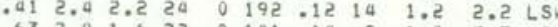

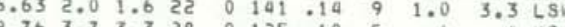

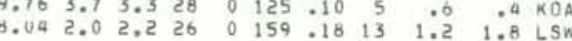
$\begin{array}{lllllllllll}4.98 & 1.6 & 1.6 & 20 & 0 & 120 & .14 & 9 & 1.0 & 1.3 & \text { SWP }\end{array}$

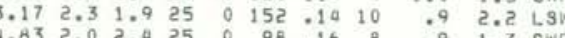

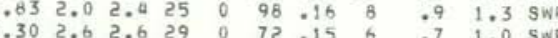

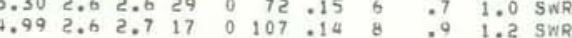

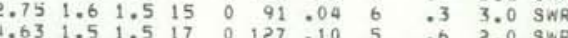

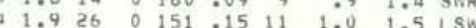
$\begin{array}{lllllllllll}3.51 & 2.0 & 1.4 & 21 & 0 & 156 & -15 & 12 & 1.1 & 2.4 & \text { LSW }\end{array}$

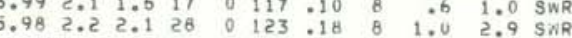
$152.01 .621 \quad 0 \quad 169.20 \quad 13 \quad 1.8 \quad 99.0$ LS

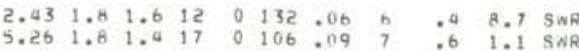

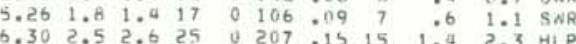

$\begin{array}{lllllllllll}3.29 & 1.6 & 1.2 & 17 & 0 & 133 & .11 & 9 & .8 & 3.2 & \text { SWR }\end{array}$

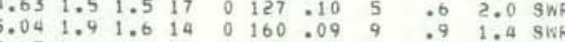

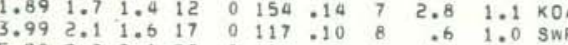

HVO EARTHQUAKE SUMMARY LIST

PAGE A

ORIGIN TIME LAT" LON N DEPTH AMP DUR YEAR MON DA HRM SEC DEG MIN DEG MIN KM MAG MAG NR NS DEG SEC DIS KM KM REMK

1975 JAN 2 2 $219 \begin{array}{llllll}8.43 & 19 & 20.36 & 155 & 20.74\end{array}$

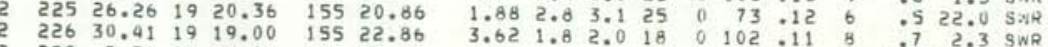
\begin{tabular}{l}
$229 \quad 7.76 \quad 1925.16 \quad 155 \quad 18.00$ \\
\hline
\end{tabular}

$\begin{array}{lllllll}2 & 229 & 27.48 & 19 & 22.51 & 155 & 13.90 \\ 2 & 233 & 11.20 & 19 & 12.57 & 155 & 20.98\end{array}$

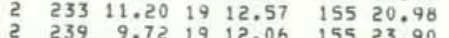

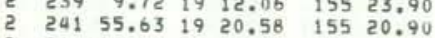
$\begin{array}{lllllll}2 & 241 & 55.63 & 19 & 20.58 & 155 & 20.90 \\ 2 & 246 & 22.48 & 19 & 17.34 & 155 & 24.07\end{array}$

$\begin{array}{lllllll}2 & 247 & 42.46 & 19 & 16.62 & 155 & 23.61 \\ 2 & 255 & 59.36 & 19 & 19.57 & 155 & 21.49\end{array}$ $\begin{array}{lllllll}2 & 255 & 59.36 & 19 & 19.57 & 155 & 23.61 \\ 2 & 3 & 2 & 32.23 & 19 & 19.57 & 155 \\ 2 & 3 & 32.23 & 19 & 17.59 & 155 & 24.03\end{array}$ $\begin{array}{llllllll}2 & 3 & 2 & 32.23 & 19 & 17.59 & 155 & 24.03 \\ 2 & 3 & 3 & 33.27 & 19 & 20.32 & 155 & 20.34\end{array}$ $\begin{array}{llllllll}3 & 6 & 7.61 & 19 & 19.55 & 155 & 21.06\end{array}$

$\begin{array}{llllllll}2 & 3 & 7 & 23.38 & 19 & 19.58 & 155 & 22.44 \\ 2 & 310 & 39.95 & 19 & 19.91 & 155 & 21.35\end{array}$ \begin{tabular}{lllllll} 
e & 310 & 39.95 & 19 & 19.91 & 155 & 22.45 \\
2 & 311 & 15.39 & 19 & 19.49 & 155 & 21.35 \\
\hline & 311 & 155 & 21.69
\end{tabular}

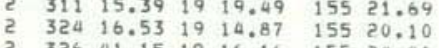

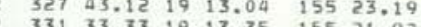

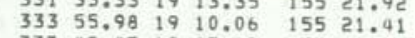

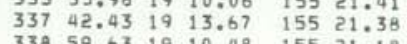

$\begin{array}{lllll}339 & 53.43 \quad 19 & 11.25 & 155 & 22.45\end{array}$

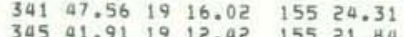
$\begin{array}{lrrrrr}349 & 7.85 & 19 & 18.65 & 155 & 23.56 \\ 352 & 40.11 & 19 & 20.47 & 155 & 20.43\end{array}$

$\begin{array}{lllllll}2 & 354 & 52.41 & 19 & 16.88 & 155 & 23.89\end{array}$ $\begin{array}{llllllll}2 & 4 & 4 & 14.18 & 19 & 13.66 & 155 & 20.49 \\ 2 & 4 & 4 & 45.49 & 19 & 20.47 & 155 & 20\end{array}$

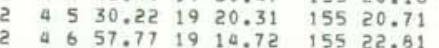
$\begin{array}{llllllll}2 & 4 & 8 & 23.05 & 19 & 16.63 & 155 & 24.58\end{array}$

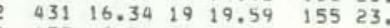

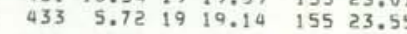

$\begin{array}{llllll}436 & 13.31 & 19 & 20.49 & 155 & 20.79\end{array}$

$441 \quad 16.96 \quad 1920.26 \quad 155 \quad 20.06$
499

$449 \quad 57.8019 \quad 17.82 \quad 155 \quad 23.85$
45558.36

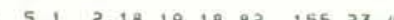
$\begin{array}{llllll}510 & 53.19 & 19 & 14.85 & 155 & 23.73 \\ 527 & 11.34 & 19 & 18.15 & 155 & 13.09\end{array}$

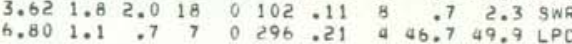
$\begin{array}{lllllllllllll}9.33 & 2.1 & 1.5 & 8 & 0 & 143 & -10 & 5 & 10.6 & 24.7 & \text { DEP }\end{array}$ $\begin{array}{llllllllll}8.78 & 2.5 & 2.2 & 30 & 0 & 164 & .16 & 15 & 1.0 & 1.4\end{array}$

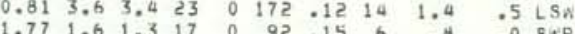

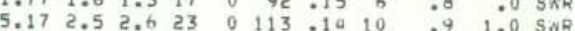
$\begin{array}{lllllllllll}6.81 & 2.4 & 2.5 & 29 & 0 & 122 & .18 & 9 & 1.1 & 1.8 & \text { SUR } \\ 5.55 & 3.0 & 1.6 & 17 & 0 & 1.7 & 09 & 9 & .7 & 1.5 & \text { SWR }\end{array}$

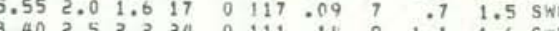

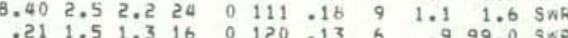
.331 .91 .916 o 115.11 6 0.799 .0 5NA $\begin{array}{lllllllllll}4.38 & 2.0 & 1.9 & 23 & 0 & 93 & .13 & 9 & .8 & 1.4 & \text { SMR }\end{array}$

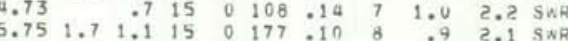

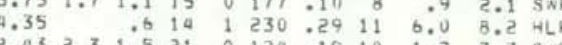
$\begin{array}{lllllllllll}6.15 & 5.0 & 4.7 & 24 & 0 & 158 & .19 & 12 & 1.5 & 3.8 & \text { LS }\end{array}$

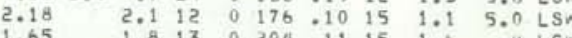
$3.722 .31 .422 \quad 0 \quad 160.14$ 14 1.00 2.0 LS $6.762 .82 .925 \quad 0 \quad 200.14$ is $1.5 \quad 2.04$ Ls $\begin{array}{lllllllllll}9.68 & 3.6 & 3.5 & 18 & 0 & 194 & .08 & 17 & 1.2 & .4 & \text { LSA }\end{array}$

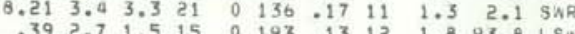

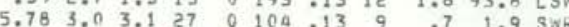

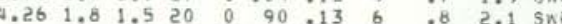
$\begin{array}{lllllllllll}5.30 & 2.3 & 2.0 & 24 & 0 & 118 & .15 & 9 & .9 & 3.1 & \text { SWR }\end{array}$

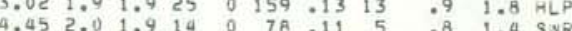

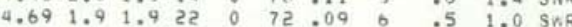
$6.622 .62 .326 \quad 0 \quad 143.14 \quad 10 \quad .923 .458$ $\begin{array}{llllllllll}8.44 & 3.4 & 3.5 & 26 & 0 & 132 & .14 & 12 & .9 & 1.5\end{array}$ SWR

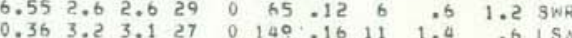

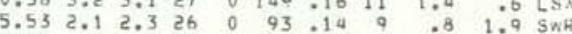

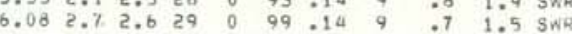
$\begin{array}{lllllllllll}4.47 & 1.7 & 1.6 & 19 & 0 & 93 & .12 & 6 & .6 & 1.4 & \text { SWF }\end{array}$

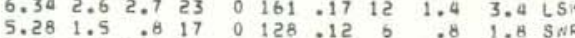

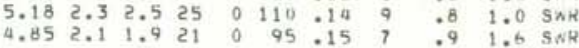
$\begin{array}{lllllllllllll}5.54 & 2.3 & 2.5 & 26 & 0 & 103 & .15 & 9 & .8 & 2.2 & 5 W 4\end{array}$

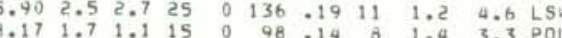


HVO EARTMQHAKE SURMARY LIST

NaGE 9

ORIGIN THAE LATH LOR A DEPTH AMP OUR GIP KRS MIN LRIA ERZ

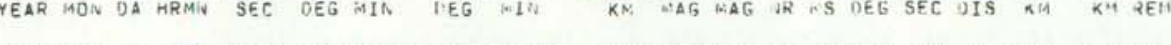

1975 JAN $253549.111916 .48 \quad 15523.24$

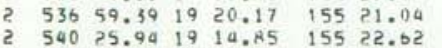

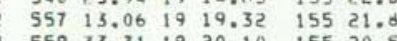

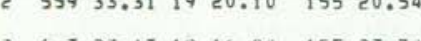

$\begin{array}{llllllll}2 & 6 & 3 & 23.17 & 19 & 16.8 B & 155 & 23.70\end{array}$ 2
2 $10 \quad 39.8019 \quad 19.76 \quad 155 \quad 22.5$

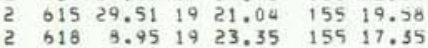
$\begin{array}{lllllll}2 & 619 & 7.90 & 19 & 13.35 & 155 & 19.88 \\ 2 & 620 & 51.72 & 19 & 20.79 & 155 & 19.44\end{array}$

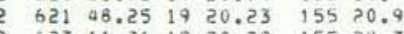
$\begin{array}{lllllll}2 & 623 & 11.26 & 19 & 20.29 & 155 & 20.39 \\ 2 & 626 & 7.72 & 19 & 18.98 & 155 & 22.9\end{array}$

$\begin{array}{lllllll}2 & 628 & 54.50 & 19 & 15.31 & 155 & 22.81 \\ ? & 629 & 28.89 & 19 & 18.35 & 155 & 23.29\end{array}$

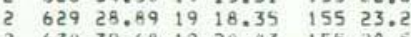

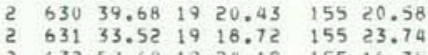

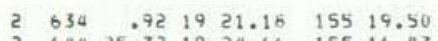
$\begin{array}{lllllll}2 & 644 & 25.32 & 19 & 24.66 & 155 & 16.83 \\ 2 & 647 & 33.92 & 19 & 24.67 & 155 & 16.77\end{array}$

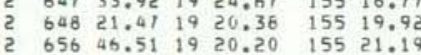
$\begin{array}{lllllll}2 & 658 & 25.48 & 19 & 19.33 & 155 & 22.24\end{array}$

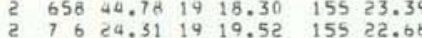
$\begin{array}{lllllll}2 & 710 & 19.23 & 19 & 19.40 & 155 & 20.45 \\ 2 & 711 & 17.45 & 19 & 17.06 & 155 & 23.38\end{array}$ $\begin{array}{lllllll}2 & 714 & 7.61 & 19 & 18.84 & 155 & 23.04\end{array}$

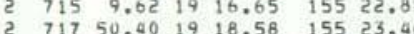

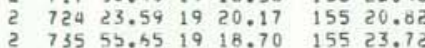

$\begin{array}{lllllll}2 & 73724.70 & 1920.33 & 15520.40\end{array}$

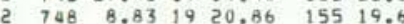

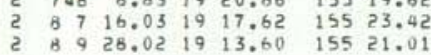

$\begin{array}{lllllll}2 & 810 & 19.79 & 19 & 17.58 \quad 155 & 23.96\end{array}$ $\begin{array}{lllllll}2 & 811 & 35.12 & 19 & 12.11 & 155 & 23.85 \\ 2 & 825 & 20.20 & 19 & 20.43 & 155 & 20.28\end{array}$

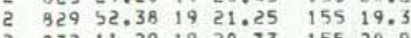

$30054.431919 .20 \quad 15522.17$ 80316.76 19 19.20155 . $2846 \quad 7.5919 \quad 18.81 \quad 15523.52$

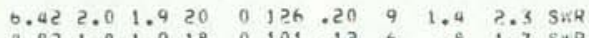

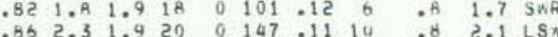

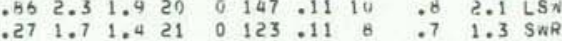

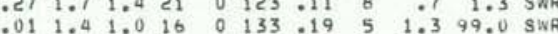

$\begin{array}{llllllllllll}.52 & 2.2 & 1.9 & 20 & 0 & 119 & .16 & 9 & 1.1 & 83.3 & \text { SWR }\end{array}$

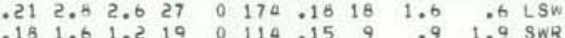

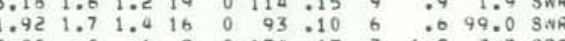
$\begin{array}{lllllllllll}7.40 & 2.5 & 2.3 & 23 & 0 & 174 & .10 & 13 & .9 & 1.7 & \text { HLP }\end{array}$ $\begin{array}{lllllllllll}.93 & 1.7 & 1.0 & 14 & 0 & 97 & .17 & 5 & 1.7 & 1.6 & \text { SWR }\end{array}$

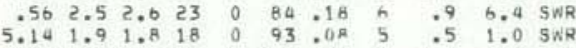

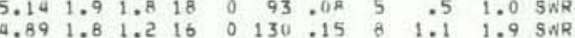
$\begin{array}{rrrrrrrrrrr}1.47 & 2.3 & 2.1 & 21 & 0 & 77 & .14 & 6 & .7 & 37.8 & \text { SWR } \\ 2.63 & 1.5 & 1.4 & 21 & 0 & 103 & .14 & 9 & .8 & 2.4 & \text { SWR }\end{array}$

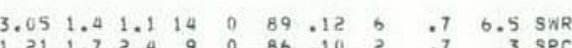

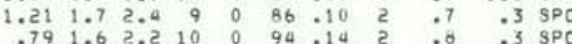

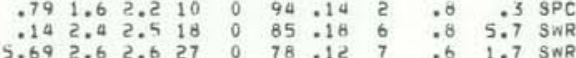
$\begin{array}{llllllllllll}5.14 & 1.6 & 1.3 & 15 & 0 & 123 & .11 & 9 & .7 & 1.3 & \text { SWR }\end{array}$

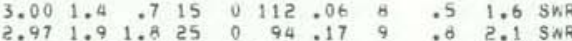

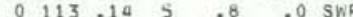

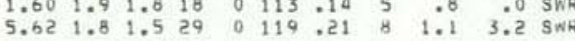
$\begin{array}{lllllllllllll}3.43 & 2.0 & 2.3 & 20 & 0 & 133 & .11 & 8 & .7 & 1.5 & \text { SWR }\end{array}$

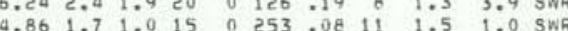

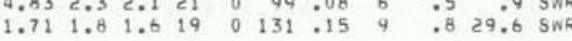
$\begin{array}{lllllllllll}5.79 & 2.0 & 1.9 & 20 & 0 & 97 & .19 & 6 & 1.1 & 3.1 & \text { SWR }\end{array}$

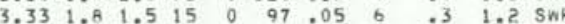

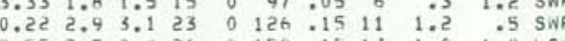

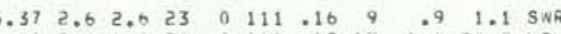

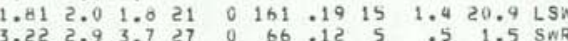

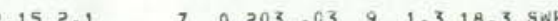

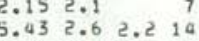

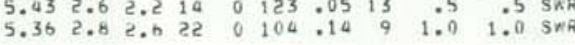

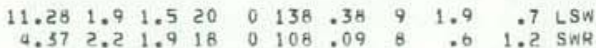

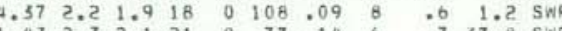

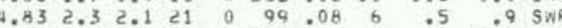

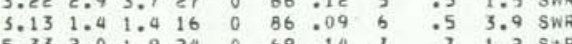

HVO EAKTHQUAKE SUMMARY LIST

PAIFE 11

DRIGIN TIME LAT D LON K TEAR MON DA HRMN SEC JEG MIN DEG MIN

$\begin{array}{lllllllll}1975 & \text { JAN } 2 & 848 & 57.69 & 19 & 13.04 & 155 & 22.54 \\ & 2 & 852 & 12.57 & 19 & 20.03 & 155 & 20.54\end{array}$

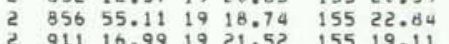
$c$
$z$ $91133.851919 .04 \quad 15523.05$

$\begin{array}{llllllll}2 & 914 & 55.39 & 19 & 20.29 & 155 & 20.18 \\ 2 & 916 & 28.26 & 19 & 21.63 & 155 & 18.81\end{array}$ $919 \quad 36.591919 .20 \quad 15523.1$

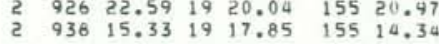

$\begin{array}{lllllll}2 & 940 & 24.96 & 19 & 19.37 & 155 & 22.3\end{array}$ $\begin{array}{lllllll}2 & 941 & 37.92 & 19 & 16.53 & 155 & 23.78 \\ 5 & 946 & 39.24 & 19 & 24.69 & 155 & 16.79\end{array}$

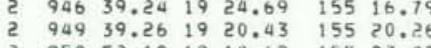
$2958 \quad 52.1919 \quad 18.62 \quad 155$ $\begin{array}{llllllll}2 & 10 & 0 & 45.25 & 19 & 18.36 & 155 & 23.75 \\ 2 & 10 & 2 & 10.79 & 19 & 16.52 & 155 & 23.5\end{array}$ ट $10210.791916 .52 \quad 15523.52$

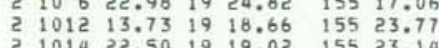
$\begin{array}{lllllll}2 & 1017 & 8.09 & 19 & 18.63 & 155 & 23.4\end{array}$

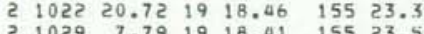

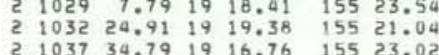
$\begin{array}{lllllll}2 & 1042 & 18.97 & 19 & 17.80 & 155 & 23.62\end{array}$ $\begin{array}{lllllll}2 & 1048 & 9.76 & 19 & 13.67 & 155 & 20.62\end{array}$

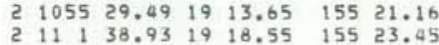

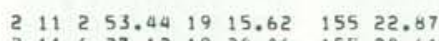
2 11 8 52.47 19 $20.06 \quad 155520.64$

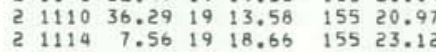
$\begin{array}{lllllll}2 & 1116 & 47.51 & 19 & 20.14 & 155 & 20.86\end{array}$

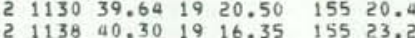

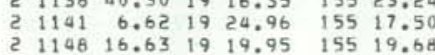

$\begin{array}{lllllll}2 & 1150 & 19.57 & 19 & 24.50 & 155 & 17.15\end{array}$

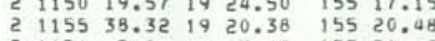
$\begin{array}{lllllll}2 & 1156 & 5.84 & 19 & 13.44 & 155 & 21.33\end{array}$

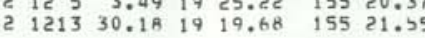

$\begin{array}{lllllll}2 & 1214 & 58.16 \quad 14 & 11.65 \quad 155 & 22.17\end{array}$

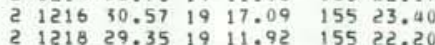

DEPTH AMP DUR GAP KUS $11 \%$ ERH ERT $4.92 \quad 1.414$ o 136.11 \& .9 l.t Sw

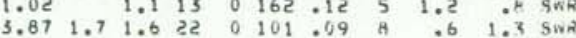

$2.09 \quad 1.3 \quad 12 \quad 0 \quad 900.0 R \quad 5 \quad$. $557 . A$ SNR

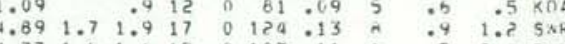
$\begin{array}{llllllllllll}5.37 & 1.3 & 1.6 & 15 & 0 & 103 & 11 & 6 & .7 & 2.4 & 5.4 \\ 7.01 & 1.7 & 1.8 & 20 & 0 & 102 & .09 & 6 & .6 & 1.3 & \text { POL }\end{array}$

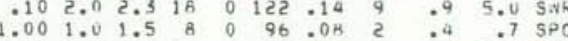

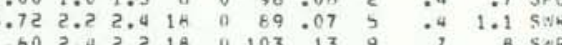
$\begin{array}{llllllllllllll}1.41 & 2.2 & 2.2 & 17 & 0 & 106 & .13 & 9 & .6 & 1.0 & 5 \% 4\end{array}$

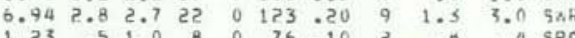

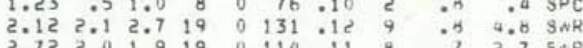
$\begin{array}{lllllllllll}2.59 & 2.4 & 2.7 & \text { 21 } & 0 & 105 & -11 & 9 & .7 & 1.6 & 5 \mathrm{sih}\end{array}$

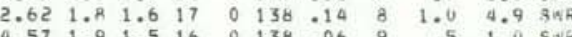

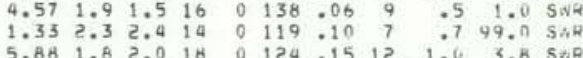
$\begin{array}{llllllllllll}5.30 & 2.4 & 2.5 & 25 & 0 & 111 & .13 & 9 & .7 & .4 & \text { SWR }\end{array}$

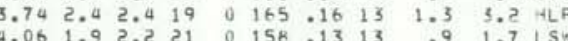
$\begin{array}{llllllllllll}7.75 & 2.9 & 2.6 & 27 & 0 & 157 & 16 & 13 & 1.0 & 2.0 & 15 & 16\end{array}$

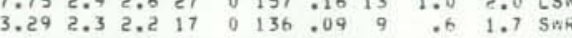

$\begin{array}{lllllllllll}5.81 & 2.3 & 2.3 & 26 & 0 & 135 & .17 & 13 & 1.1 & 3.4 & \text { LSW }\end{array}$

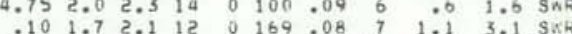

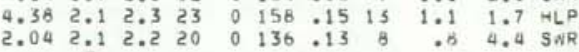
$\begin{array}{lllllllllll}5.34 & 2.1 & 2.2 & 22 & 0 & 55 & .09 & 0 & .5 & .7 & \text { SMR }\end{array}$

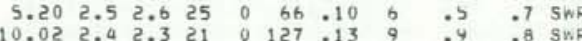

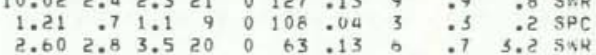
$\begin{array}{llllllllllll}1.11 & .7 & 1.2 & 7 & 0 & 172 & .13 & 2 & 1.2 & . \text { B SPC }\end{array}$

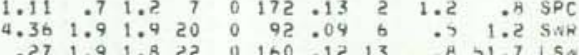

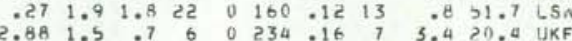
$\begin{array}{cccccccccccc}2.88 & 1.5 & .7 & 6 & 0 & 234 & .16 & 7 & 3.4 & 20.4 & \text { UKF } \\ 3.68 & 1.5 & 1.1 & 13 & 0 & 114 & .04 & 7 & .7 & 2.9 & \text { SWR }\end{array}$

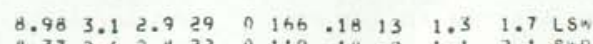

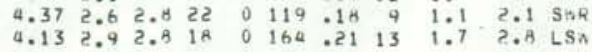
$\begin{array}{llllllllllll}.94 & 2.0 & 1.5 & 20 & 0 & 156 & -16 & 11 & 1.1 & 2.3 & \text { LS } \\ .50 & 2.5 & 2.4 & 27 & 0 & 67 & 13 & 6 & .6 & 1 . ? & 544\end{array}$

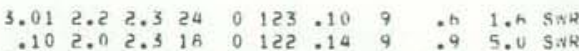




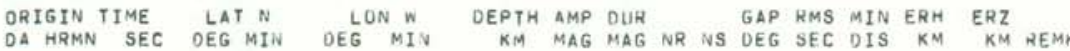

1975 JAN $2 \quad 1230 \quad 34.32 \quad 19 \quad 18.41 \quad 155 \quad 23.64$ $\begin{array}{lllllll}2 & 1239 & 4.28 & 19 & 19.14 & 155 & 22.46 \\ 2 & 1238 & 6.77 & 19 & 17.61 & 155 & 22.15\end{array}$

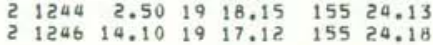

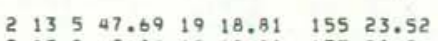
9.881919 .9115520 .76

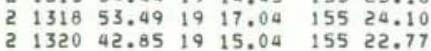

$\begin{array}{lllllll}2 & 1321 & 37.40 & 19 & 14.45 & 155 & 22.74\end{array}$

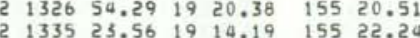

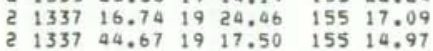

2 $1340 \quad 15.03 \quad 1921.06 \quad 155 \quad 19.56$ $\begin{array}{lllllll}2 & 1353 & 3.06 & 19 & 21.05 & 155 & 19.46\end{array}$

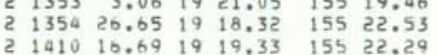

$\begin{array}{lllllll}2 & 1415 & 11.41 & 19 & 21.04 & 155 & 19.49 \\ 2 & 1420 & 24.55 & 19 & 18.98 & 155 & 22.96\end{array}$ $\begin{array}{lllllll}2 & 1429 & 19.32 & 19 & 11.67 & 155 & 23.76 \\ 2 & 1433 & 33.22 & 19 & 20.31 & 155 & 20.43\end{array}$

$\begin{array}{lllllll}2 & 1440 & 29.04 & 19 & 12.81 & 155 & 25.02\end{array}$

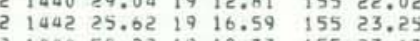
$\begin{array}{lllll}1444 & 58.22 & 19 & 18.73 & 155 \\ 23.17\end{array}$ $2144632.291919 .77 \quad 15522.75$

$\begin{array}{llllll}1457 & 36.66 & 19 & 24.82 & 155 & 17.04\end{array}$

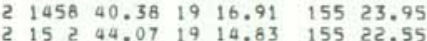

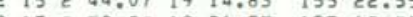
15
15

$\begin{array}{llllllll}2 & 15 & 7 & 38.49 & 19 & 20.97 & 155 & 19.39 \\ 2 & 15 & 8 & 38.09 & 19 & 20.28 & 155 & 20.76\end{array}$

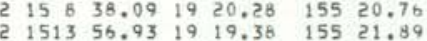

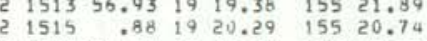

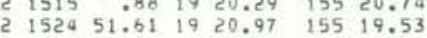

$\begin{array}{lllllll}2 & 1526 & 29.40 & 19 & 16.67 & 155 & 23.48\end{array}$ 2 153319.511919 .76 155 21.26

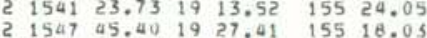
$21548 \quad 36.811911 .20 \quad 155 \quad 22.19$

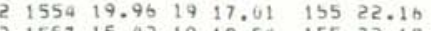

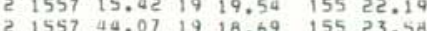

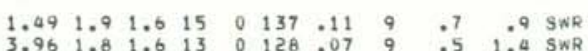

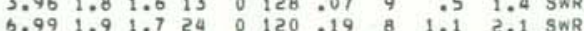
$\begin{array}{lllllllllllll}5.19 & 3.0 & 3.1 & 29 & 0 & 106 & 14 & 10 & 0 & 1.7 & 1.0 & \text { SNR } \\ .30 & 2.3 & 2.4 & 22 & 0 & 114 & .13 & 10 & .0 & 3.9 & \text { SWR }\end{array}$ $\begin{array}{lllllllllll}5.52 & 3.1 & 3.1 & 28 & 0 & 103 & .16 & 9 & .9 & 2.3 & \text { SWR }\end{array}$ $\begin{array}{lllllllllll}.23 & 1.9 & 1.9 & 20 & 0 & 148 & .15 & 11 & 1.9 & 71.7 & \text { LSN }\end{array}$

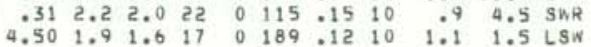
$\begin{array}{lllllllllll}5.77 & 2.6 & 3.1 & 26 & 0 & 151 & .16 & 11 & 1.0 & 2.9 & \text { LSW }\end{array}$ $2.752 .52 .622 \quad 0 \quad 155,1211 \quad 082.645 x$

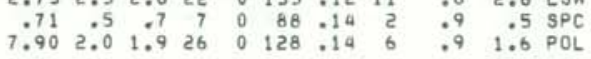

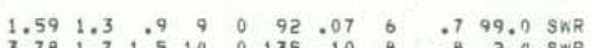

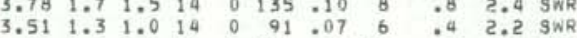

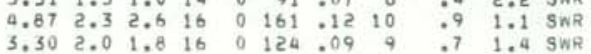
$\begin{array}{llllllllllll}1.51 & 1.7 & 1.6 & 13 & 0 & 172 & .08 & 6 & .8 & .0 & \text { SWR }\end{array}$ $\begin{array}{lllllllllll}4.84 & 2.3 & 1.9 & 21 & 0 & 130 & .12 & 8 & .8 & 1.1 & \text { SWR } \\ 3.75 & .0 & 0.2 & 25 & 0 & 161 & .40 & 16 & .7 & 8.7 & \text { SW }\end{array}$

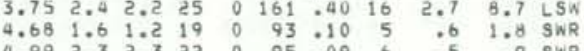
$\begin{array}{lllllllllll}8.89 & 2.2 & 2.0 & 19 & 0 & 160 & .16 & 14 & 1.4 & 2.7 & \text { LSW }\end{array}$

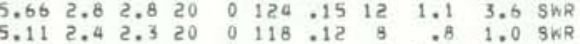

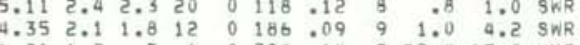
$\begin{array}{llllllllllll}1.12 & .7 & 1.0 & 8 & 0 & 75 & .13 & 2 & 1.0 & .6 & 9 P C\end{array}$

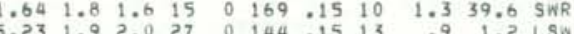

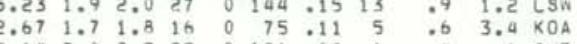

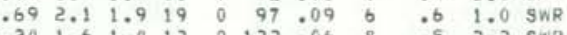

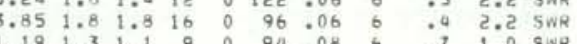

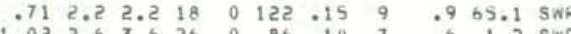

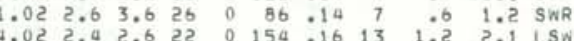
$1.581 .31 .0 \quad 8 \quad 0214.23555 .799 .0$ GLN

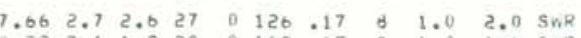

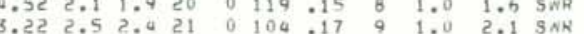

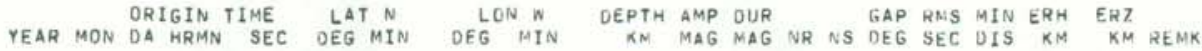

1975 JAN $216 \quad 0 \quad 26.43 \quad 19 \quad 17.10 \quad 155 \quad 22.16$

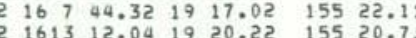

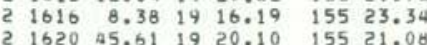

2 \begin{tabular}{l}
$162152.6019 \quad 18.75 \quad 15523.28$ \\
\hline
\end{tabular}

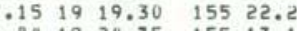

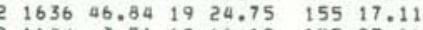
$\begin{array}{lllllll}2 & 1644 & 3.51 & 19 & 16.19 & 155 & 23.66 \\ 2 & 1646 & 35.19 & 19 & 20.51 & 155 & 20.31\end{array}$ $\begin{array}{lllllll}2 & 1647 & 49.78 & 19 & 16.41 & 155 & 23.78\end{array}$ $21648 \quad 54.691919 .82 \quad 155 \quad 21,73$

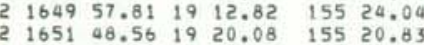

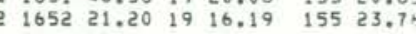

$\begin{array}{lllllll}2 & 1654 & 6.45 & 19 & 16.14 & 155 & 23.69\end{array}$ $\begin{array}{llllll}1656 & 9.79 & 19 & 19.94 & 155 & 23.09\end{array}$ $\begin{array}{llllllll}2 & 17 & 2 & 54.09 & 19 & 11.76 & 155 & 22.13 \\ 2 & 17 & 6 & 47.95 & 19 & 20.24 & 155 & 20.78\end{array}$

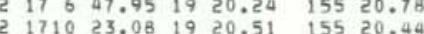

$\begin{array}{lllllll}2 & 1711 & 35.58 & 19 & 12.50 & 155 & 22.03\end{array}$ $\begin{array}{lllllll}1713 & 41.47 & 19 & 20.51 & 155 & 20.29\end{array}$ 172150.57 19 $18.29 \quad 155523.59$

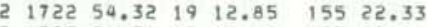

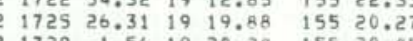
$1733 \quad 30.17$ 19 20.20 15520.45 $173359.94 \quad 1920.30 \quad 15520.71$

$\begin{array}{llllll}1736 & 11.67 & 19 & 18.48 & 155 & 15.80\end{array}$

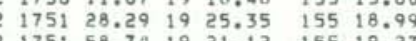

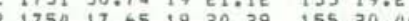
175833.58 19 $19.21 \quad 15523.15$

$\begin{array}{llllllll}2 & 18 & 2 & 1.67 & 19 & 20.17 & 155 & 20.73\end{array}$

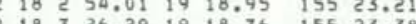
$\begin{array}{lllllll}18 & 3 & 26.29 & 19 & 18.76 & 155 & 23.36 \\ 18 & 5 & 49.35 & 19 & 19.74 & 155 & 23.39\end{array}$ $\begin{array}{lllllllll}2 & 18 & 6 & 36.16 & 19 & 12.74 & 155 & 22.38\end{array}$

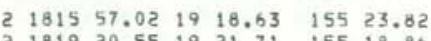

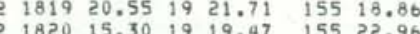
$182427.36 \quad 1921.10 \quad 15519.43$ $\begin{array}{lllll}1824 & 42.15 \quad 19 & 18.54 & 155 & 21.60\end{array}$ $\begin{array}{lllllll}1828 & 16.84 & 19 & 19.10 & 155 & 22.96\end{array}$ 1834 51.9219 19.32 155

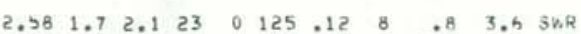

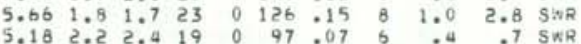

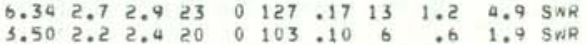
$\begin{array}{llllllllllllll}4.27 & 1.7 & 1.6 & 14 & 0 & 134 & .05 & 8 & .4 & 1.1 & \text { Swir }\end{array}$

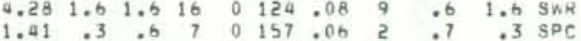

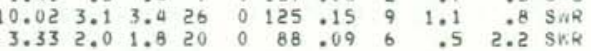
$\begin{array}{lllllllllll}5.62 & 1.9 & 1.9 & 25 & 0 & 123 & .16 & 9 & 1.0 & 3.1 & \text { SwR }\end{array}$ $3.552 .93 .126 \quad 0 \quad 157 \quad .18013 \quad 1.9$ $\begin{array}{llllllllllll}2.01 & 1.6 & 1.6 & 10 & 0 & 101 & .12 & 6 & .4 & .0 & \text { SWR }\end{array}$

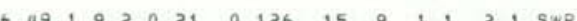
. 11 0107.15 97 t. 5 99.0 SWR

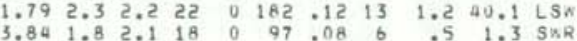

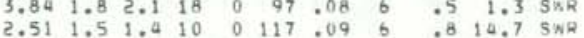

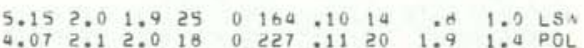
$\begin{array}{rrrrrrrrrrr}1.06 & 1.4 & 1.3 & 12 & 0 & 88 & .14 & 6 & .9 & .0 & \text { SWA } \\ 2.63 & 1.6 & 1.4 & 12 & 0 & 126 & .06 & 9 & .5 & 2.7 & \text { SNR }\end{array}$

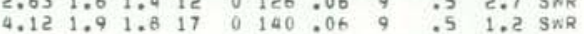

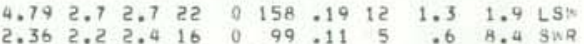

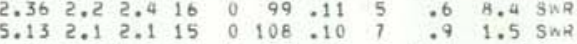

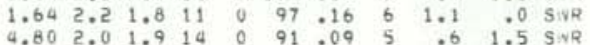
$\begin{array}{llllllllllll}9.00 & 2.2 & 2.1 & 26 & 0 & 108 & .09 & 5 & .6 & .9 & K O A\end{array}$

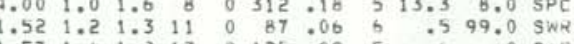
$\begin{array}{lllllllllll}1.57 & 1.4 & 1.2 & 13 & 0 & 125 & .09 & 5 & .6 & .0 & \text { SwR } \\ 5.25 & 2.2 & 2.1 & 22 & 0 & 124 & .14 & 9 & .9 & 1.0 & \text { SwR }\end{array}$

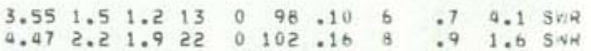

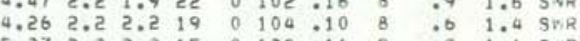

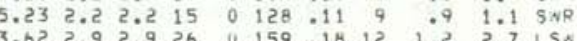
$1.931 .71 .619 \quad 0 \quad 131.15$ q 1.043 .3 SWR

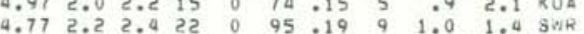

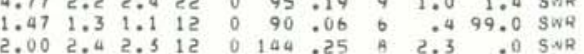
$\begin{array}{lllllllllll}5.06 & 1.9 & 1.8 & 23 & 0 & 127 & .13 & 8 & .8 & 1.1 & \text { SNR }\end{array}$

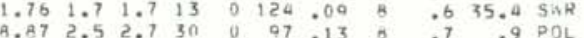


EPTH AMP OIIR GAP KMS MIN ERM ERZ KM MAG MAG VR AS IEEF SEC OIS KM KA REIMK

$\begin{array}{llllllll}1975 \text { JAN } 2 & 1848 & 10.34 & 19 & 18.95 & 155 & 23.32 \\ 2 & 1850 & 3.27 & 19 & 20.27 & 155 & 20.40\end{array}$

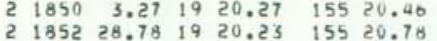

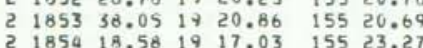
द $1859 \quad 18.72 \quad 19 \quad 20.31 \quad 155 \quad 20.99$

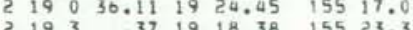

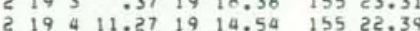
$\begin{array}{lllll}19 & 8 & 22.99 \quad 19 \quad 16.76 \quad 155 \quad 23.82\end{array}$ $\begin{array}{lllllll}2 & 1916 & 46.04 & 19 & 20.07 \quad 155 & 21.12\end{array}$ \begin{tabular}{llllll}
1917 & 3.83 & 19 & 17.32 & 155 & 23.7 \\
\hline & 1918 & 19 & &
\end{tabular}

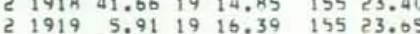

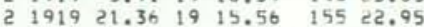
2 $192459.191924 .62 \quad 15516.80$

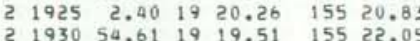
2 $1932 \quad 17.30 \quad 1924.81 \quad 15516.45$ $\begin{array}{lllllll}1938 & 52.60 & 19 & 17.71 & 155 & 23.87\end{array}$

$319419.85 \quad 1924.68 \quad 15516.92$ $1941 \quad 56.94 \quad 19 \quad 17.10 \quad 155 \quad 24.65$

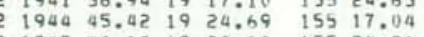

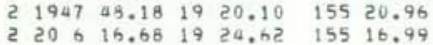
द $20 \quad 9 \quad 18.9919 \quad 19 .+3 \quad 155 \quad 21.52$

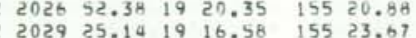
5 203022.091914 .3115522 .37

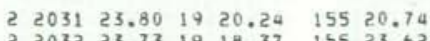

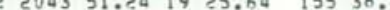

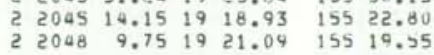
$\begin{array}{lllllll}2 & 2052 & 16.03 & 19 & 18.91 & 155 & 23.76\end{array}$ $\begin{array}{llrllll}2 & 2121 & 3.63 & 19 & 19.17 & 155 & 22.21 \\ 2 & 2122 & 56.79 & 19 & 21.20 & 155 & 19.29\end{array}$

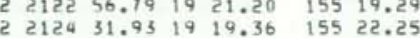
2 $2127 \quad 40.32 \quad 19 \quad 19.64 \quad 15521.49$

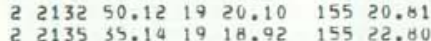

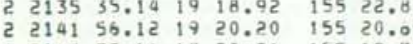
1.0519 20.04 15550.29

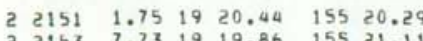

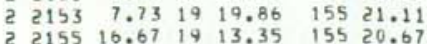

$\begin{array}{lllllllllll}4.61 & 2.1 & 2.1 & 20 & 0 & 102 & .12 & 8 & .7 & 1.1 & \text { SWR }\end{array}$

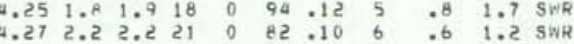
0.482 .62 .628 0 $65.16 \quad 6 \quad .8 \quad 1.6$ SWH $\begin{array}{lllllllllll}2.41 & 1.4 & .7 & 8 & 0 & 131 & .13 & 6 & 1.4 & 26.0 & \text { SWR }\end{array}$

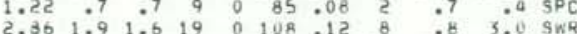

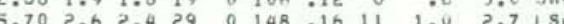
$\begin{array}{llllllllll}4.10 & 1.5 & 1.6 & 15 & 0 & 130.08 & 7 & .6 & 1.6 & 5 W\end{array}$

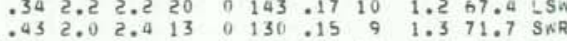
$\begin{array}{llllllllllllll}7.16 & 2.3 & 2.2 & 17 & 0 & 153 & .08 & 15 & .8 & 1.7 & \text { LS }\end{array}$ $\begin{array}{lllllllllll}1.02 & .5 & 1.4 & 10 & 0 & 85 & .18 & 2 & .9 & .7 & \text { SP }\end{array}$

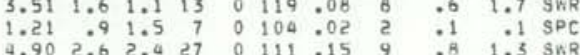
$\begin{array}{lllllllllllll}. \text { AO } & 1.0 & 1.2 & 9 & 0 & 110 & .13 & 2 & .9 & .6 & \text { SPC }\end{array}$

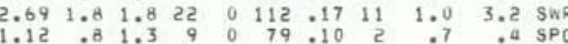

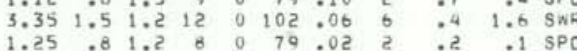
$\begin{array}{lllllllllll}4.26 & 1.9 & 1.7 & 13 & 0 & 115 & .14 & 7 & 1.0 & 2.2 & \text { SWR }\end{array}$

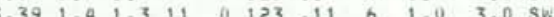
2.401 .91 .618 o 150.1611 1.2 5.2 LSW $\begin{array}{lllllllllll}4.23 & 1.5 & 1.4 & 16 & 0 & 123 & .05 & 6 & .3 & .9 & \text { SWR }\end{array}$ $\begin{array}{llllllllll}1.03 & 3.0 & 2.8 & 16 & 0 & 151 & .19 & 19 & 1.4 & 1.7 \\ \text { MOK }\end{array}$

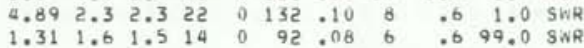
$\begin{array}{lllllllllll}8.31 & 3.2 & 3.3 & 29 & 0 & 102 & .16 & 9 & .9 & 1.2 & \text { SNR }\end{array}$

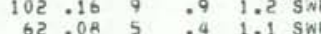
$\begin{array}{llllllllllll}4.38 & 1.9 & 1.6 & 17 & 0 & 127 & .09 & 9 & .6 & 1.1 & \text { SWR } \\ 3.23 & & 1.2 & 12 & 0 & 86 & .05 & 6 & .4 & 12.2 & \text { SWR }\end{array}$

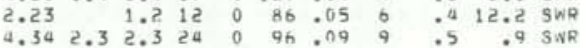

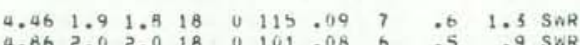

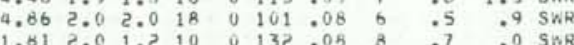
$\begin{array}{rrrrrrrrrrr}1.61 & 2.0 & 1.2 & 10 & 0 & 132 & .05 & 8 & .7 & .0 & \text { SNR } \\ 4.58 & 2.4 & 2.6 & 25 & 0 & 73 & .11 & 6 & .5 & 1.1 & \text { SNR }\end{array}$

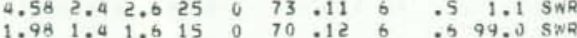
$\begin{array}{lllllllllll}4.57 & 1.9 & 2.1 & 23 & 0 & \text { AQ } & .09 & 5 & .5 & 1.0 & \text { SNR }\end{array}$

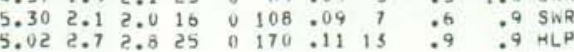

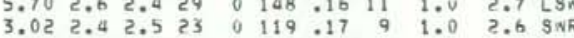

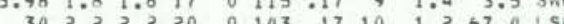

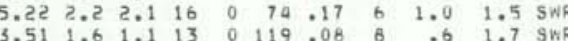

HVO EARTMOUARE SLHARAKY LIST

patic 14

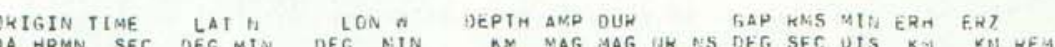

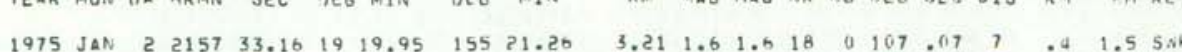
52
5 $57.891920 .75 \quad 15520.16$

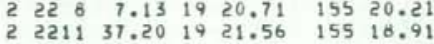

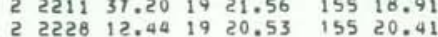

$\begin{array}{lllllll}2 & 2238 & 4.25 & 19 & 18.74 & 155 & 22.90 \\ 2 & 2241 & 5.80 & 19 & 18.51 & 155 & 23.07\end{array}$

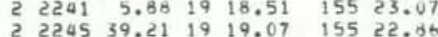

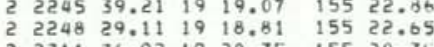

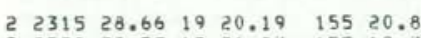

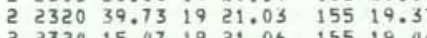

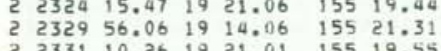
$\begin{array}{lllll}2 & 2332 & 40.51 & 19 & 18.55 \quad 155 \quad 23.35\end{array}$ $\begin{array}{lllllll}2 & 2332 & 40.51 & 19 & 18.55 & 155 & 23.35 \\ 2 & 2333 & 19.10 & 19 & 13.57 & 155 & 20.9\end{array}$ $\begin{array}{lllllll}2 & 2333 & 19.10 & 19 & 13.57 & 155 & 20.35 \\ 2 & 2337 & 55.36 & 19 & 19.70 & 155 & 20.9 \\ 2 & 2346 & 8.24 & 19 & 20.39 & 155 & 20.9\end{array}$

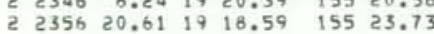
$\begin{array}{lllllll}2 & 2357 & 25.66 & 19 & 12.35 & 155 & 22.59\end{array}$ $\begin{array}{llllllll}3 & 0 & 1 & 18.91 & 19 & 16.41 & 155 & 23.6\end{array}$

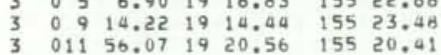

$\begin{array}{lllllll}3 & 023 & 55.96 & 19 & 20.17 & 155 & 19.19\end{array}$ $302959.9919 \quad 16.76 \quad 15520.75$ $\begin{array}{lllll}045 & 27.15 & 19 & 16.58 & 15523.86\end{array}$ $3057 \quad 10.98 \quad 19 \quad 20.16 \quad 155 \quad 21.09$ $\begin{array}{lllllll}3 & 058 & 29.89 & 19 & 19.38 & 155 & 22.27\end{array}$ $\begin{array}{llllllll}3 & 1 & 8 & 27.80 & 19 & 20.36 & 155 & 20.30\end{array}$

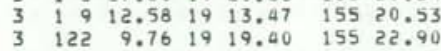

$\begin{array}{lllllll}3 & 126 & 55.05 & 19 & 9.97 & 155 & 22.89\end{array}$ $\begin{array}{lllllll}3 & 126 & 55.05 & 19 & 9.97 & 155 & 22.89 \\ 3 & 132 & 9.27 & 19 & 21.09 & 155 & 19.58\end{array}$

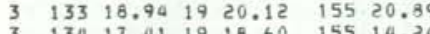
$\begin{array}{lllllll}3 & 134 & 17.41 & 19 & 18.60 & 155 & 14.24 \\ 3 & 138 & 35.09 & 19 & 21.24 & 155 & 19.06\end{array}$

$\begin{array}{lllllll}3 & 142 & 36.11 & 19 & 18.59 & 155 & 14.18\end{array}$

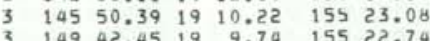
$\begin{array}{rrrrrr}149 & 42.45 & 19 & 9.74 & 155 & 22.74\end{array}$ $\begin{array}{lrrrrrr}3 & 151 & 23.13 & 19 & 16.84 & 155 & 23.41 \\ 3 & 158 & 5.85 & 19 & 18.81 & 155 & 14.11\end{array}$

$\begin{array}{lllllllll}3 & 2 & 2 & 2.07 & 19 & 20.86 & 155 & 19.70\end{array}$ $\begin{array}{rrrrrrrr}3 & 2 & 2 & 30.27 & 19 & 11.53 & 155 & 22.11 \\ 3 & 2 & 5 & 3.00 & 19 & 20.32 & 155 & 20.04\end{array}$ $\begin{array}{rrrrrrrrrrr}3.21 & 1.6 & 1.6 & 18 & 0 & 107 & .07 & 7 & .4 & 1.5 & 5 \times 4 \\ 3.51 & 2.4 & 2.5 & 24 & 0 & 83 & .14 & 0 & : 7 & 1.5 & 5 \times 2\end{array}$ $1.091 .31 .412 \quad 0109.0750 .594 .0$ SWA $4.581 .41,2$ 16 0117 .06 $50.50 .6 \mathrm{kma}$ $\begin{array}{llllllllllll}1.57 & 1.4 & 1.4 & 17 & 0 & 136.06 & \text { a } & .6 & .6 & \text { SNE }\end{array}$

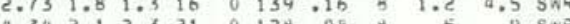
\begin{tabular}{lllllllllll}
4.34 & 2.1 & 2.3 & 21 & 0 & 128 & .06 & 8 & .5 & .4 & SNR \\
3.79 & 2.1 & 2.4 & 19 & 0 & 136 & 12 & 9 & $: 9$ & 1.4 & SNR \\
\hline
\end{tabular} $\begin{array}{lllllllllll}42 & 1.4 & 1.8 & 18 & 0 & 91.07 & 5 & .4 & 1.1 & 5 \times 4\end{array}$ $\begin{array}{lllllllllllll}5.08 & 1.6 & 1.8 & 21 & 0 & 99.08 & 6 & .5 & .8 & 5 \times R\end{array}$

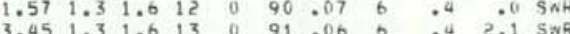
$5.262 .32 .426 \quad 0154.1812 \quad 1.121 .545$

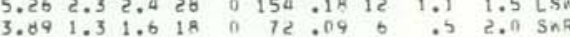

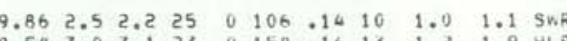

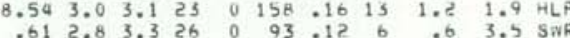

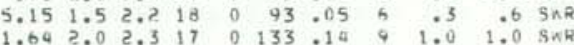
$\begin{array}{lllllllllll}3.19 & 2.0 & 2.1 & 25 & 0 & 161 & .15 & 12 & 1.0 & 2.8 & \text { LSW }\end{array}$

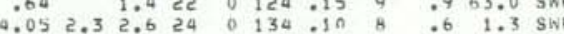

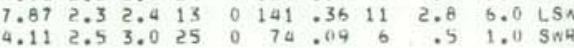
$\begin{array}{llllllllllllll}2.00 & 1.0 & 2.0 & 15 & 0 & 76 & .15 & 7 & .0 & .0 & \text { SWR }\end{array}$ $\begin{array}{lllllllllllll}5.53 & 2.2 & 2.4 & 20 & 0 & 121 & .14 & 10 & .9 & 2.8 & \text { SWR }\end{array}$ 1.

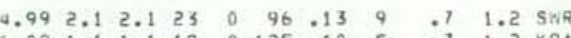
$\begin{array}{lllllllllll}.89 & 1.1 & 1.1 & 19 & 0 & 125 & .10 & 5 & .7 & 1.2 & k 0\end{array}$

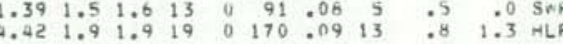

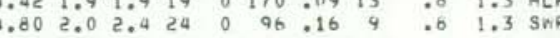

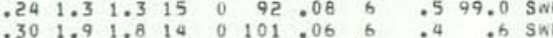

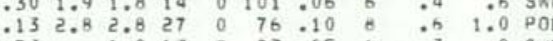

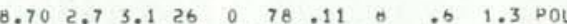

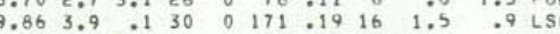

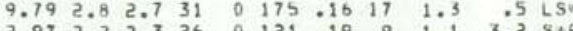

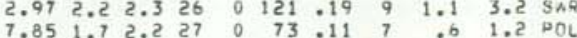

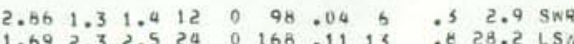

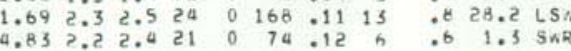

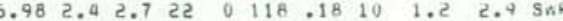
$\begin{array}{lllllllllllll}0.09 & 2.6 & 2.9 & 25 & 0 & 173 & .15 & 17 & 1.1 & 5.4 & \text { LSN }\end{array}$ 
ORIGIN TIME LATN LON W DEPTH AMP OUR GAP RMS MIN ERH ERZ YEAR MON DA HRMN SEC DEG MIN DEg MIN KM MAG MAG NR NS OEG SEC UIS KM KM REMK 1975 JAN 3 2 $553.851918 .57 \quad 155 \quad 14.20$

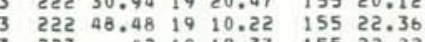
$\begin{array}{lllllll}3 & 227 & 042 & 19 & 19.37 & 155 & 22.22 \\ 3 & 230 & 41.41 & 19 & 21.37 & 155 & 19.15\end{array}$

$\begin{array}{lllllll}3 & 231 & 15.94 & 19 & 9.25 & 155 & 13.97 \\ 3 & 232 & 53.81 & 19 & 18.57 & 155 & 23.53\end{array}$

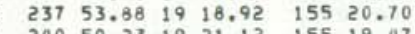

(3)

$\begin{array}{lllllll}3 & 242 & 41.56 & 19 & 18.47 & 155 & 23.33\end{array}$ $\begin{array}{lllllll}3 & 244 & 34.97 & 19 & 19.19 & 155 & 16.30\end{array}$

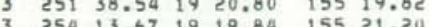
$33017.82 \quad 1920.43 \quad 15520.25$

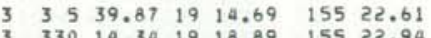

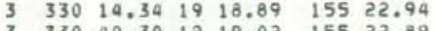

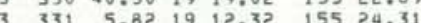
$333 \quad 39.83 \quad 19 \quad 13.14 \quad 15524.42$

$\begin{array}{llllll}341 & 56.47 & 19 & 10.71 & 155 & 23.49\end{array}$ $\begin{array}{llllll}3 & 355 & 16.83 & 19 & 17.91 & 155 \\ 3 & 357 & 23.03 & 155 & 23.53\end{array}$ $\begin{array}{llllll}357 & 16.28 & 19 & 20.15 & 155 & 20.77 \\ 359 & 39.47 & 19 & 20.57 & 155 & 20.19\end{array}$ $\begin{array}{lllllll}3 & 413 & 32.72 & 19 & 20.29 & 155 & 20.26\end{array}$

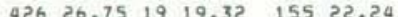
$\begin{array}{llllll}427 & 27.55 & 19 & 24.47 & 155 & 17.18 \\ 435 & 14.27 & 19 & 20.23 & 155 & 20.84\end{array}$ $\begin{array}{lllllll}4 & 445 & 29.51 & 19 & 20.46 & 155 & 20.91\end{array}$ $\begin{array}{lllll}454 & 55.12 \quad 19 & 20.51 & 155 & 20.27\end{array}$ $\begin{array}{llllll}458 & 58.18 & 19 & 24.60 & 155 & 17.07 \\ 459 & 25.37 & 19 & 20.21 & 155 & 21.10\end{array}$

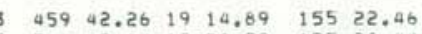

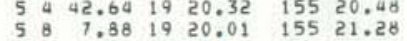
$\begin{array}{lllllll}3 & 516 & 6.74 & 19 & 10.34 & 155 & 22.88 \\ 3 & 522 & 37.12 & 19 & 20.73 & 155 & 19.48\end{array}$

$\begin{array}{lllllll}3 & 524 & 1.34 & 19 & 18.55 & 155 & 23.22\end{array}$ $\begin{array}{lllllll}3 & 526 & 24.53 & 19 & 10.13 & 155 & 22.29 \\ 3 & 533 & .45 & 19 & 14.93 & 155 & 21.70\end{array}$ $\begin{array}{lllllll}3 & 541 & 1.92 & 19 & 11.94 & 155 & 23.18\end{array}$

$\begin{array}{llllllll}3 & 6 & 0 & 35.36 & 19 & 20.35 & 155 & 21.12\end{array}$

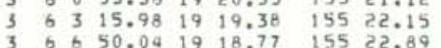

$\begin{array}{lllllllllll}8.73 & 2.0 & 2.2 & 27 & 0 & 75 & .14 & 8 & .8 & 1.2 & \text { POL } \\ 3.4 & .5 & .8 & 16 & 0 & 114 & .09 & 6 & .5 & 4.7 & \text { SWR }\end{array}$

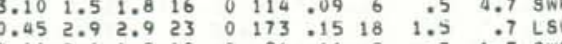

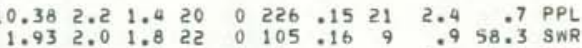
$.21 \quad 1.71 .612 \quad 00181.12$ 6 $2.150 .0 \mathrm{sw}$

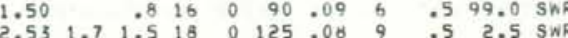
$\begin{array}{lllllllllll}4.51 & 2.1 & 2.3 & 22 & 0 & 107 & .12 & 8 & .7 & 1.3 & \text { SWR }\end{array}$

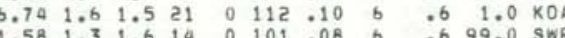

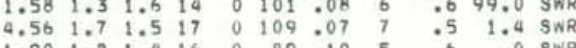
$1.901 .81 .4 \quad 16 \quad 0 \quad 89$

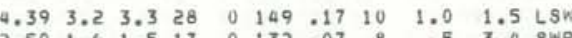

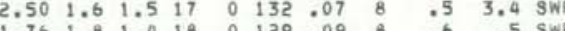

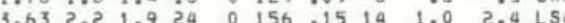
$\begin{array}{lllllllllll}5.00 & 2.5 & 2.5 & 19 & 0 & 188 & .16 & 16 & 1.8 & 2.6 & \text { LSW }\end{array}$

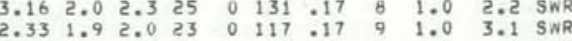
$\begin{array}{lllllllllll}5.34 & 1.8 & 1.8 & 16 & 0 & 99 & .07 & 6 & .5 & .8 & \text { SNR } \\ 3.03 & 1.4 & 1.5 & 15 & 0 & 85 & .07 & 6 & .4 & 2.6 & \text { SNR }\end{array}$ $\begin{array}{lllllllllll}4.80 & 2.1 & 2.3 & 19 & 0 & 91 & .08 & 5 & .5 & 1.2 & \text { SNR }\end{array}$

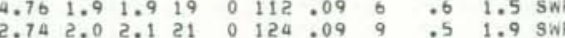

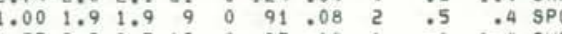
$\begin{array}{lllllllllll}6.26 & 2.5 & 2.8 & 28 & 0 & 72 & .15 & 6 & .7 & 1.5 & \text { SWR }\end{array}$

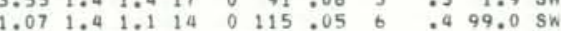

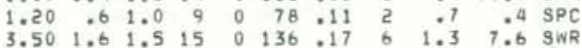
$\begin{array}{lllllllllllllll}9.06 & 2.6 & 2.7 & 23 & 0 & 144 & .15 & 10 & 1.0 & 1.3 & \text { LSN }\end{array}$

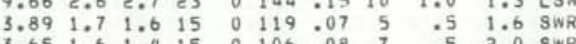
$\begin{array}{llllllllllll}3.65 & 1.6 & 1.4 & 15 & 0 & 106 & 08 & 7 & .5 & 2.0 & \text { SWR }\end{array}$

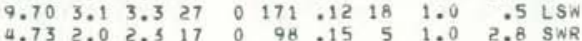
$\begin{array}{lllllllllllll}4.24 & 2.1 & 2.2 & 20 & 0 & 137 & .09 & 8 & .7 & 1.4 & \text { SNR }\end{array}$

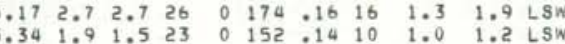

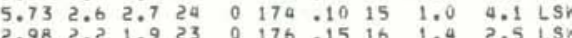
$\begin{array}{lllllllllll}4.97 & 2.5 & 2.8 & 23 & 0 & 75 & .12 & 7 & .7 & 1.3 & \text { SW }\end{array}$

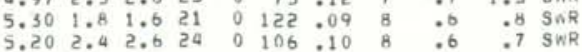
$\begin{array}{lllllllllll}.11 & 2.1 & 1.9 & 18 & 0 & 96 & 11 & 9 & .7 & 1.5 & \text { SWR }\end{array}$

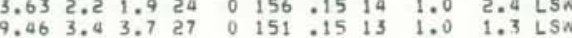

HVO EARTHQUAKE SURMAKY LIST

PAGE 16

ORIGIN TIME LAT N LON W YEAR MON DA MRMN SEC DES MIN UEO MII

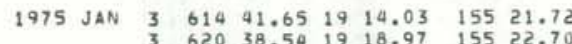
$\begin{array}{lllllll}3 & 620 & 38.54 & 19 & 18.97 & 155 & 22.7 \\ 3 & 621 & 28.77 & 19 & 18.92 & 155 & 22.80\end{array}$

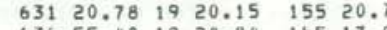

$\begin{array}{llllll}640 & 50.45 & 19 & 13.42 \quad 155 & 20.55\end{array}$ $3 \quad 64739.5219 \quad 15.35 \quad 15523.12$

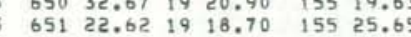

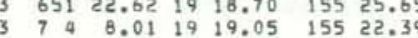

$\begin{array}{llllllll}3 & 7 & 4 & 57.87 & 19 & 20.85 & 155 & 19.79 \\ 3 & 7 & 9 & 24.00 & 19 & 16.69 & 155 & 22.50\end{array}$ $\begin{array}{llllllll}3 & 7 & 9 & 24.00 & 19 & 16.69 & 155 & 22.50\end{array}$

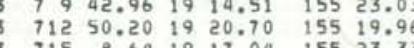

$\begin{array}{lllllll}3 & 717 & 31.30 & 19 & 11.68 & 155 & 21.69\end{array}$ $\begin{array}{lrrrrrr}3 & 718 & 23.21 & 19 & 16.40 & 155 & 23.37 \\ 3 & 722 & 7.32 & 19 & 16.44 & 155 & 24.48\end{array}$

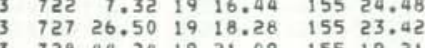

$\begin{array}{llllll}730 & 35.13 & 19 & 14.67 & 155 & 21.75\end{array}$

$\begin{array}{lllllll}3 & 730 & 35.13 & 19 & 14.67 & 155 & 21.75 \\ 3 & 732 & 26.55 & 19 & 17.82 & 155 & 14.38\end{array}$

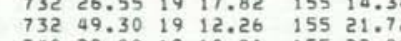
74028.80
743

$\begin{array}{lllllll}3 & 743 & 43.17 & 19 & 14.26 & 155 & 23.56\end{array}$

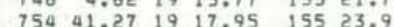

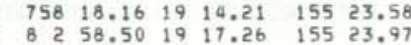

$\begin{array}{llllll}811 & 42.43 \quad 19 & 14.63 & 155 & 23.43\end{array}$ $\begin{array}{llllll}811 & 42.43 & 19 & 14.63 & 155 & 23.43 \\ 814 & 46.08 & 19 & 13.01 & 155 & 23.00\end{array}$

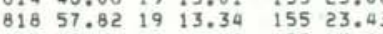
$\begin{array}{rrrrrr}823 & 32.15 & 19 & 10.59 & 155 & 23.04 \\ 831 & 9.58 & 19 & 12.72 & 155 & 22.31\end{array}$

$\begin{array}{lllllll}3 & 832 & 18.86 & 19 & 18.35 & 155 & 21.85\end{array}$ $\begin{array}{llllll}832 & 18.86 & 19 & 18.35 & 155 & 21.85 \\ 835 & 2.58 & 19 & 20.68 & 155 & 20.34\end{array}$ $\begin{array}{llllll}837 & 33.71 & 19 & 20.50 & 155 & 20.2\end{array}$ $\begin{array}{lllllll}3 & 847 & 6.58 & 19 & 15.42 & 155 & 22.75 \\ 3 & 848 & 4.39 & 19 & 15.52 & 155 & 22.46\end{array}$

$\begin{array}{llllll}8566 & 37.09 & 19 & 18.74 & 155 & 23.06\end{array}$ $\begin{array}{llllll}857 & 56.75 & 19 & 18.74 & 155 & 22.87\end{array}$

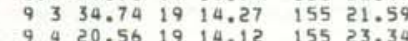

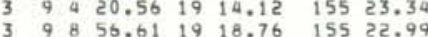

$\begin{array}{llllllll}3 & 9 & 9 & 35.27 & 19 & 16.90 & 155 & 22.49\end{array}$ $\begin{array}{lllllll}3 & 911 & 49.47 & 19 & 16.28 & 155 & 22.35 \\ 3 & 914 & 28.49 & 19 & 16.86 & 155 & 22.40\end{array}$
DEPTH AMP OUR GAP RMS MIN ERH ERZ $\begin{array}{llllllllllll}4.01 & 1.9 & 1.8 & 19 & 0 & 153 & .15 & 12 & 1.1 & 2.1 & \text { LSN }\end{array}$ .841 .91 .921 0 99.08 6 50.51 .1 SWR

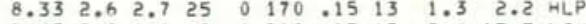

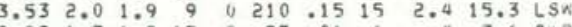

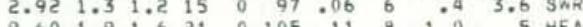
$\begin{array}{lllllllllllll}1.28 & 1.3 & 1.1 & 11 & 0 & 100 & 08 & 6 & .6 & 99.0 & \text { SwR }\end{array}$ 5.991 .81 .921 o 128.15 t 1.133 .1 Swm

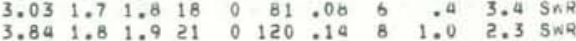
$\begin{array}{rrrrrrrrrrr}2.99 & 2.7 & 2.6 & 26 & 0 & 167 & .17 & 13 & 1.2 & 3.0 & \text { LSW }\end{array}$

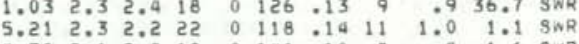

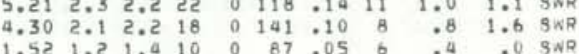
$\begin{array}{lllllllllll}5.82 & 2.2 & .5 & 55 & 0 & 154 & .12 & 11 & .8 & 3.6 & \text { LSN }\end{array}$

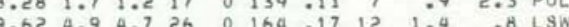

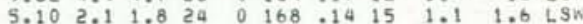
$\begin{array}{llllllllll}10.54 & 2.8 & 2.8 & 19 & 0 & 166 & .16 & 16 & 2.1 & .8 \text { LSN }\end{array}$

\begin{tabular}{llllllllll}
3.41 & 3.2 & 3.0 & 14 & 0 & 142 & .15 & 11 & 1.1 & 3.7 \\
\hline
\end{tabular}

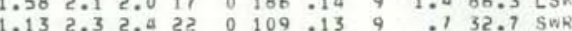
$\begin{array}{lllllllllll}7.30 & 2.4 & 2.4 & 22 & 0 & 147 & .13 & 11 & 1.0 & 2.6 & \text { LSW } \\ 3.18 & 2.5 & 2.7 & 26 & 0 & 114 & .10 & 9 & 1.0 & 2.9 & \text { SWR }\end{array}$

\begin{tabular}{llllllllll}
8.89 & 3.4 & 3.8 & 26 & 0 & 144 & .16 & 10 & 1.1 & 1.6 \\
\hline
\end{tabular}

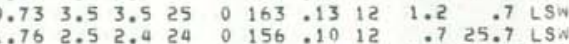

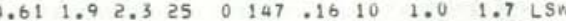
$8.842 .01 .621 \quad 0 \quad 162.1414 \quad 1.2$ 2.0 Ls" $\begin{array}{llllllllll}0.47 & 2.0 & 2.3 & 21 & 0 & 113 & .16 & 8 & 1.1 & 1.0\end{array}$

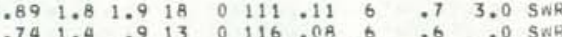

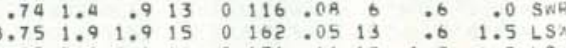

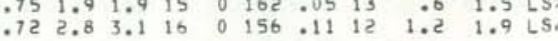
$\begin{array}{lllllllllll}5.11 & 1.7 & 1.6 & 19 & 0 & 135 & .13 & \text { B } & 1.0 & 1.2 & \text { SNR }\end{array}$

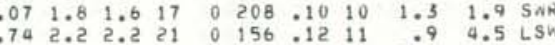

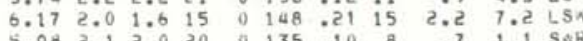

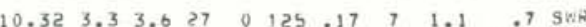
$\begin{array}{llllllllll}.93 & 1.0 & 14 & 0 & 153 & .12 & 8 & 1.3 & 3.1 & \text { SWR } \\ 9.95 & 1.6 & 25 & 0 & 126 & .14 & 8 & .9 & 2.7 & \text { SWA }\end{array}$

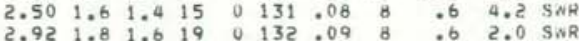

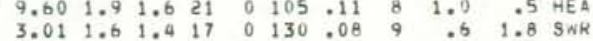
392.82 .818000 14. 


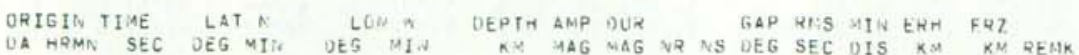

$\begin{array}{lllllll}1975 \text { JAN } 3 & 917 & 3.12 & 19 & 13.42 & 155 & 22.35\end{array}$ $\begin{array}{llllll}918 & 5.23 & 19 & 16.70 & 155 & 22.03 \\ 919 & 1.11 & 19 & 16.90 & 155 & 22.10\end{array}$ $920 \quad 53.72 \quad 19 \quad 16.66 \quad 15522.25$

$\begin{array}{lllllll}9 & 924 & 48.24 & 19 & 16.78 & 155 & 22.43\end{array}$

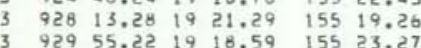
$\begin{array}{lllllll}3 & 929 & 55.22 & 19 & 18.59 & 155 & 23.27 \\ 3 & 934 & 23.38 & 19 & 14.06 & 155 & 23.27\end{array}$ $936 \quad 4.11 \quad 19 \quad 16.14 \quad 15523.46$

$\begin{array}{lllllll}3 & 941 & 6.79 & 19 & 18.04 & 155 & 23.40\end{array}$

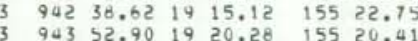

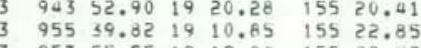
$95755.55 \quad 1919.040455$

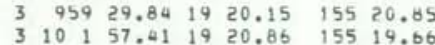

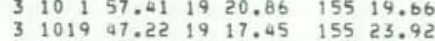
$\begin{array}{lllllll}3 & 1019 & 47.22 & 19 & 17.45 & 155 & 23.92 \\ 3 & 1021 & 38.79 & 19 & 19.07 & 155 & 22.77 \\ 3 & 1022 & 34.06 & 19 & 17.22 & 155 & 23.61\end{array}$

$\begin{array}{lllllll}3 & 1023 & 7.01 & 19 & 16.19 & 155 & 20.63\end{array}$ $\begin{array}{rrrrrr}3 & 1027 & 9.55 & 19 & 14.45 & 155 \\ 3 & 1031 & 56.60 & 19.68 \\ 3 & 1033 & 33.80 & 19.83 & 155 & 16.88\end{array}$ $\begin{array}{llllll}3 & 1033 & 33.10 & 19 & 14.20 & 155 \\ 3 & 1038 & 16.04\end{array}$

$\begin{array}{lllllll}3 & 1041 & 6.84 & 19 & 9.38 & 155 & 19.67\end{array}$ $\begin{array}{lllllll}3 & 1042 & 17.84 & 19 & 14.52 & 155 & 21.93 \\ 3 & 1049 & 2.45 & 19 & 19.26 & 155 & 22.91\end{array}$

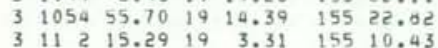

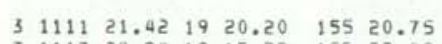

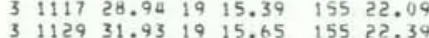

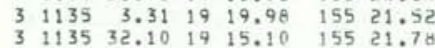

$\begin{array}{lllllll}3 & 1149 & 51.68 & 19 & 15.48 & 155 & 22.27\end{array}$ $3115310.1019 \quad 19.28 \quad 155231.29$

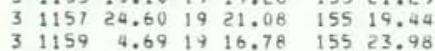

$\begin{array}{llllllll}3 & 12 & 3 & 23.71 & 19 & 18.50 & 155 & 23.51\end{array}$

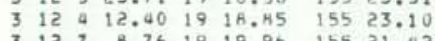
$\begin{array}{lllllll}3 & 12 & 8.76 & 19 & 19.96 & 155 & 21.42 \\ 3 & 1210 & 11.18 & 19 & 14.77 & 155 & 22.52\end{array}$

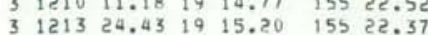

$\begin{array}{lllllll}3 & 1228 & 20.01 & 19 & 16.86 & 155 & 22.57\end{array}$ $\begin{array}{lllllll}3 & 1236 & 3.61 & 19 & 14.37 & 195 & 22.19 \\ 3 & 1247 & 8.91 & 19 & 14.56 & 155 & 22.35\end{array}$ $\begin{array}{lllllllllll}6.93 & 2.5 & 2.6 & 26 & 0 & 15 H & .13 & 12 & .9 & 3.1 & \text { LSA }\end{array}$

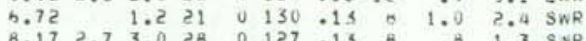

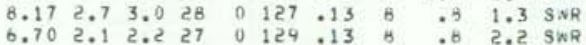

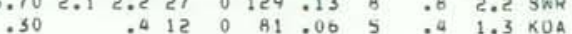
$\begin{array}{lllllllllll}6.09 & 1.8 & 1.8 & 22 & 0 & 127 & .13 & 8 & .9 & 5.6 & \text { SWR }\end{array}$

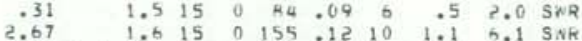

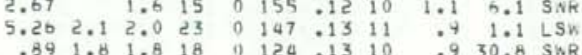

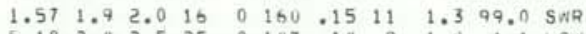

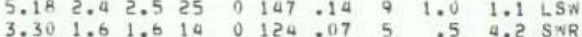

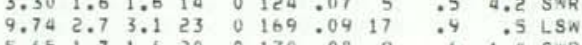

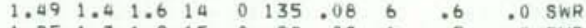

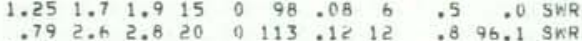

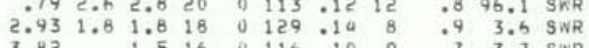

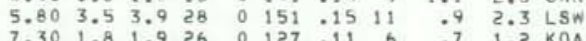

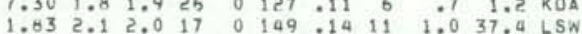
$\begin{array}{llllllllllll}7.65 & 2.7 & 2.6 & 27 & 0 & 159 & .16 & 12 & 1.1 & 2.1 & 45 W\end{array}$ $\begin{array}{llllllllllllll}12.09 & 2.7 & 2.6 & 58 & 0 & 185 & .13 & 18 & 1.7 & .4 & \text { PPL }\end{array}$

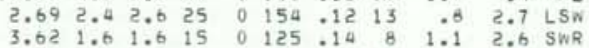

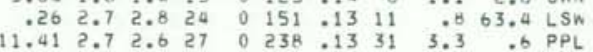
$\begin{array}{lllllllllll}4.70 & 2.3 & 2.2 & 25 & 0 & 74 & .11 & 6 & .6 & 1.1 & \text { SWR }\end{array}$

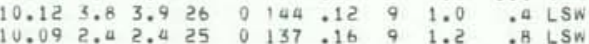

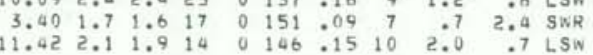
$\begin{array}{llllllllllll}9.54 & 5.1 & 3.3 & 24 & 0 & 140 & .14 & 9 & .9 & .8 & .85 w\end{array}$

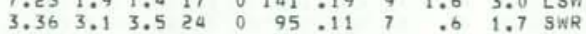

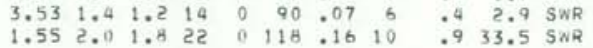
$\begin{array}{llllllllllll}3.38 & 1.8 & 1.6 & 15 & 0 & 137 & .10 & 8 & .8 & 2.4 & \text { SWR }\end{array}$

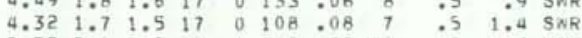

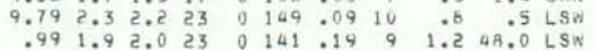
$\begin{array}{lllllllllllll}.54 & 1.8 & 1.9 & 20 & 0 & 125 & .15 & 7 & .9 & \text { A7.7 SWR } & \end{array}$

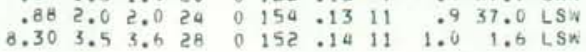
$\begin{array}{llllllllllll}6.95 & 1.8 & 1.6 & 16 & 0 & 141 & .14 & 9 & 1.1 & 2.8 & \text { SWR }\end{array}$

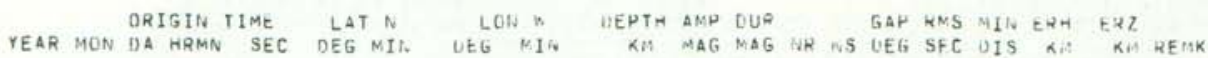

1975 JAN $31257 \quad 11.4919 \quad 9.19 \quad 15522.66 \quad 5.20 \quad 1.016 \quad 0 \quad 225 \quad .1518 \quad 2.5$ 2.A LS $\begin{array}{lllllll}3 & 13 & 36.65 & 19 & 18.06 & 155 & 23.66\end{array}$ 3
3 $133318.401914 .11 \quad 15525.00$ $\begin{array}{llllllll}3 & 13 & 3 & 37.06 & 14 & 14.92 & 155 & 22.82 \\ 3 & 13 & 8 & 39.40 & 19 & 13.76 & 155 & 22.33\end{array}$ $\begin{array}{lllllll}3 & 1315 & 28.36 & 19 & 14.52 & 155 & 21.80 \\ 3 & 1317 & 57.87 & 19 & 16.76 & 155 & 22.42\end{array}$

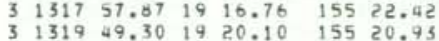
3
3 $32918.871918 .62 \quad 15523.21$ $31349 \quad 14.69 \quad 19 \quad 18.63 \quad 155 \quad 23.42$

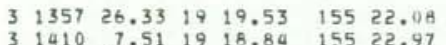
$\begin{array}{lllllll}3 & 1410 & 7.51 & 19 & 18.83 & 155 & 22.97\end{array}$ 3 1411 17.891914 .61 155 2e.03

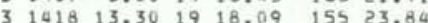

$\begin{array}{lllllll}3 & 1428 & 44.37 & 19 & 14.55 & 155 & 21.93 \\ 3 & 1430 & 55.91 & 19 & 17.71 & 155 & 23.05\end{array}$

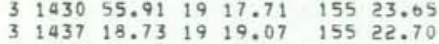
3
3 $143946.191920 .94 \quad 15519.49$ $\begin{array}{lllllll}3 & 1448 & 50.17 & 19 & 20.28 & 155 & 20.58\end{array}$ $\begin{array}{lllllll}3 & 1448 & 50.17 & 19 & 20.28 & 155 & 20.58 \\ 3 & 1454 & 29.37 & 19 & 16.86 & 155 & 23.43 \\ 3 & 152 & 48.17 & 19 & 19.39 & 155 & 22.41\end{array}$

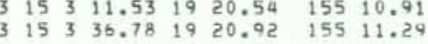

$\begin{array}{lllllll}3 & 1512 & 3.29 & 19 & 19.59 & 155 & 22.80\end{array}$ 35190.71 19 17.05 155523.10 $\begin{array}{lllllll}3 & 1532 & 41.49 & 19 & 20.51 & 155 & 20.13 \\ 3 & 1536 & 41.37 & 19 & 20.82 & 155 & 19.68\end{array}$

$\begin{array}{lllllll}3 & 1539 & 49.47 & 19 & 12.44 & 155 & 22.45\end{array}$

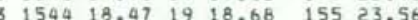
$\begin{array}{lllllll}3 & 1554 & 8.75 & 19 & 19.23 & 155 & 23.15\end{array}$

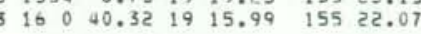
$\begin{array}{llllllll}3 & 16 & 6 & 28.97 & 19 & 19.21 & 155 & 22.25\end{array}$

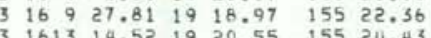

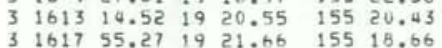

$\begin{array}{lllllll}3 & 1624 & 20.72 & 19 & 20.06 & 155 & 20.78\end{array}$

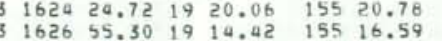
$\begin{array}{lllllll}3 & 1630 & 9.44 & 19 & 17.45 & 155 & 43.06 \\ 3 & 1630 & .21 & 19 & 20.60 & 155 & 19.41\end{array}$ $\begin{array}{rrrrrrr}3 & 1634 & .21 & 19 & 20.60 & 155 & 19.41 \\ 3 & 1635 & 18.97 & 19 & 1.09 & 155 & 28.72\end{array}$

$\begin{array}{lllllll}3 & 1641 & 55.01 & 19 & 20.19 & 155 & 20.56\end{array}$

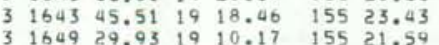

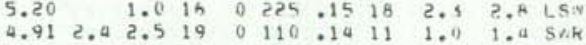

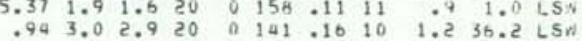

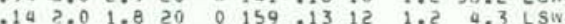

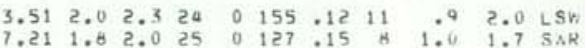

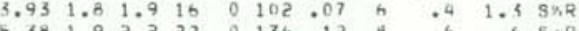

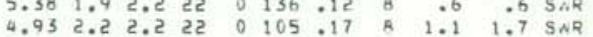
$\begin{array}{llllllllllll}2.89 & 1.7 & 1.6 & 14 & 0 & 147 & .07 & \text { B } & .6 & 1.7 & 5.0 R \\ 2.48 & 1.8 & 1.6 & 16 & 0 & 133 & .07 & \text { H } & .5 & 2.0 & \text { SNR }\end{array}$ 3.581 .91 .816 o 164.11 is 1.1 S.9 LSN

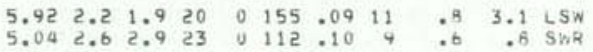

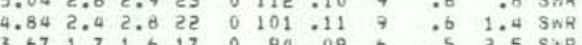

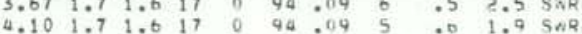

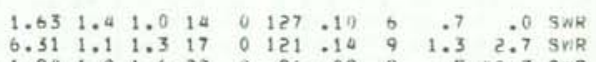

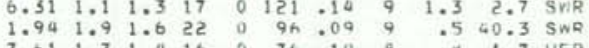

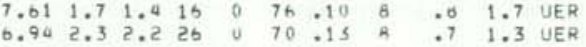
$\begin{array}{lllllllllll}5.07 & 2.2 & 2.5 & 25 & 0 & 93 & 0.16 & 9 & .4 & 1.2 & \text { SwR }\end{array}$

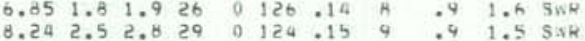

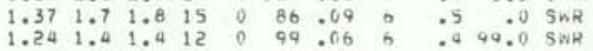
$\begin{array}{lllllllllll}7.19 & 2.0 & 1.5 & 19 & 0 & 179 & .14 & 12 & 1.5 & 2.0 & \text { LSW }\end{array}$

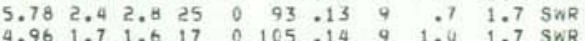
$\begin{array}{rrrrrrrrrrr}4.13 & 2.0 & 2.0 & 19 & 0 & 98 & .10 & 0 & .0 & 1.8 & \text { SWR } \\ 7.73 & 2.2 & 2.3 & 28 & 0 & 136 & .17 & 0 & 1.0 & 1.8 & \text { LSK }\end{array}$

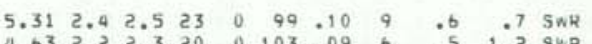

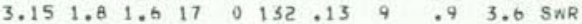

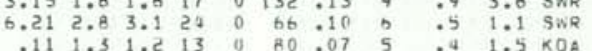

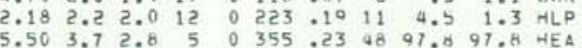

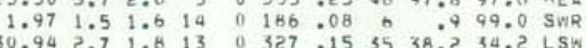
$\begin{array}{lllllllllll}1.90 & 2.2 & 2.1 & 19 & 0 & 138 & 10 & 8 & .7 & 37.9 & \text { SHR } \\ 6.47 & 2.8 & 7.9 & 26 & 0 & 202 & .11 & 15 & 1.2 & 2.9 & \text { LSW }\end{array}$

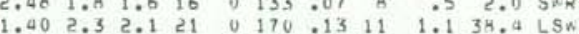
$\begin{array}{llllllllllllll}4.70 & 2.0 & 1.9 & 17 & 0 & 116 & .07 & 0 & .5 & 1.1 & \operatorname{sh} R\end{array}$

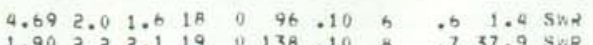




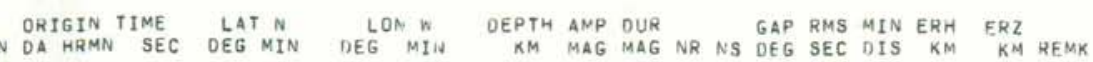

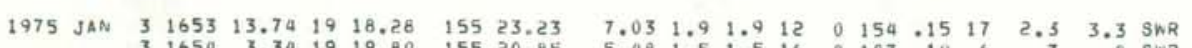

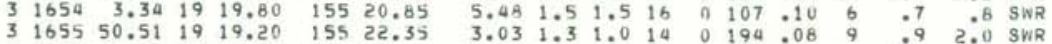

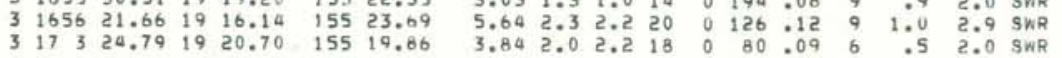
$\begin{array}{lllllll}3 & 1730 & 7.25 & 19 & 21.25 & 155 & 19.28\end{array}$ $\begin{array}{lllllll}3 & 1741 & 7.54 & 19 & 16.76 & 155 & 24.29\end{array}$

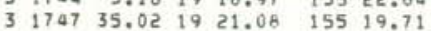
$\begin{array}{lllllll}3 & 1757 & 45.10 & 19 & 20.44 & 155 & 20.18\end{array}$

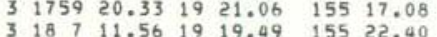

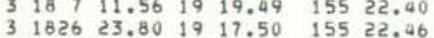

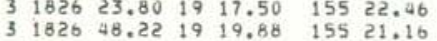

$\begin{array}{lllllll}3 & 1828 & 13.21 & 19 & 20.35 & 155 & 20.30\end{array}$ $\begin{array}{lllllll}3 & 1831 & 6.15 & 19 & 20.07 & 155 & 20.89\end{array}$ 3 3 $1845 \quad 35.79$ 19 20.82 155 23.58

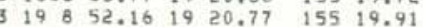

$\begin{array}{lllllll}3 & 1930 & 2.96 & 19 & 20.18 & 155 & 20.99 \\ 3 & 1946 & .72 & 19 & 21.00 & 155 & 19.33\end{array}$ $\begin{array}{lllllll}3 & 1946 & .72 & 19 & 21.00 & 155 & 19.33 \\ 3 & 1947 & 51.65 & 19 & 20.28 & 155 & 20.64\end{array}$

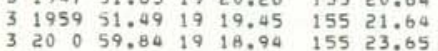
$\begin{array}{lllllll}3200 & 38.39 & 19 & 18.91 & 155 & 22.90\end{array}$

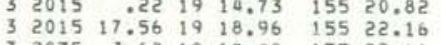

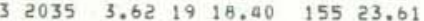
$\begin{array}{lllllll}3 & 2035 & 52.38 & 19 & 20.27 & 155 & 7.65\end{array}$

$\begin{array}{lllllll}3 & 2041 & 30.59 & 19 & 19.95 \quad 155 & 20.78\end{array}$ $\begin{array}{lllllll}3 & 2045 & 2.56 & 19 & 21.66 & 155 & 18.79\end{array}$

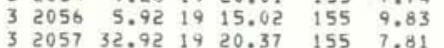
$\begin{array}{lllllll}32059 & 1.49 & 19 & 7.31 & 156 & 13.30\end{array}$

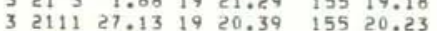

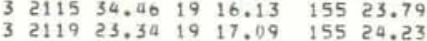

$\begin{array}{lllllll}3 & 2136 & 10.52 & 19 & 18.72 & 155 & 22.79\end{array}$

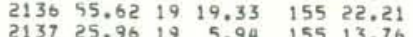
$\begin{array}{llllllll}3 & 2145 & .79 & 19 & 18.37 & 155 & 21.25 \\ 3 & 22 & 2 & 13.34 & 19 & 20.64 & 155 & 11.73\end{array}$

$\begin{array}{llllllll}3 & 22 & 5 & 34.19 & 19 & 20.58 & 155 & 20.17\end{array}$

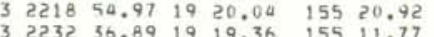

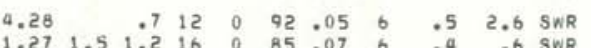

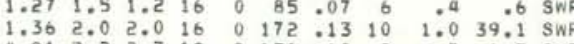

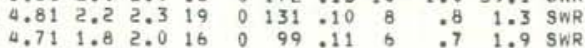
$\begin{array}{llllllllllll}1.73 & 1.5 & 1.6 & 13 & 0 & 112 & .07 & 5 & .5 & 54.7 & \text { SWR }\end{array}$

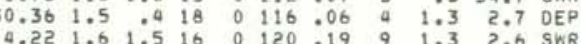

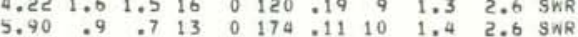
$\begin{array}{lllllllllll}2.89 & 1.9 & 1.8 & 17 & 0 & 108 & .08 & 7 & .5 & 2.4 & \text { SWR }\end{array}$

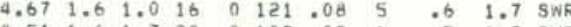
$\begin{array}{rrrrrrrrrrr}4.51 & 1.6 & 1.3 & 20 & 0 & 102 & .09 & 6 & .5 & 1.3 & \text { SWR } \\ 1.60 & 2.2 & 2.3 & 23 & 0 & 163 & .12 & 14 & .9 & 28.6 & \text { LSW }\end{array}$

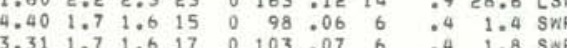

$\begin{array}{llllllllll}3.39 & 1.9 & 1.9 & 19 & 0 & 100 & .09 & 6 & .5 & 2.0\end{array}$ Sw

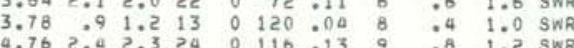
$\begin{array}{lllllllllll}3.38 & 2.0 & 1.9 & 17 & 0 & 132 & .06 & 8 & .4 & 1.3 & \text { SWA }\end{array}$

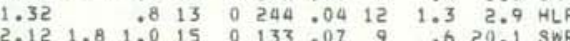

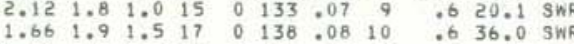
$8.984 .4 \quad 4.2$ 26 0091.10 8 $9.7 \%$ $\begin{array}{lllllllllllll}4.61 & 1.8 & 1.6 & 15 & 0 & 104 & -10 & 6 & .7 & 3.1 & \text { SWR }\end{array}$

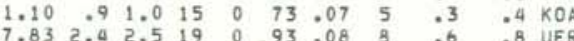

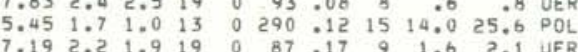
$\begin{array}{lllllllllll}3.93 & 3.1 & 1.9 & 15 & 0 & 350 & .19 & 98 & 76.9 & 76.9 & 01 S\end{array}$

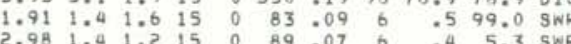
$\begin{array}{llllllllllll}2.17 & 1.8 & 1.6 & 23 & 0 & 125 & .15 & 10 & 1.0 & 3.4 & \text { SWR } \\ 1.89 & 2.8 & 3.2 & 15 & 0 & 124 & .13 & 10 & 1.1 & 99.0 & \text { SWR }\end{array}$ $\begin{array}{llllllllllllll}4.39 & 1.7 & 1.6 & 1 \mathrm{~A} & 0 & 137 & .10 & 10 & .8 & 5.5 & \text { SWR }\end{array}$

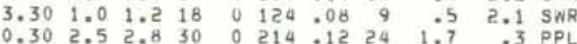

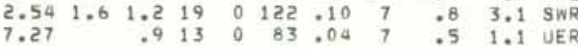
$\begin{array}{lllllllllll}1.12 & 1.5 & 1.3 & 15 & 0 & 112 & .09 & 6 & .5 & 99.0 & \text { SWR }\end{array}$

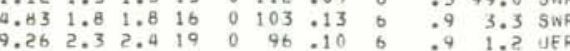

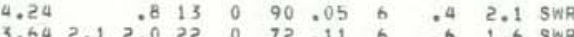

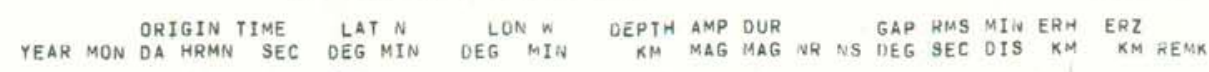

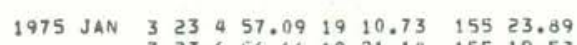

$\begin{array}{llllllll}3 & 23 & 6 & 56.66 & 19 & 21.14 & 155 & 19.53\end{array}$

$\begin{array}{lllllll}3 & 2311 & 19.04 & 19 & 20.02 & 155 & 21.15 \\ 3 & 2331 & 22.81 & 19 & 10.67 & 155 & 23.95\end{array}$

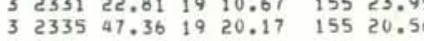

a $016 \quad 52.14 \quad 1920.85 \quad 155 \quad 19.81$

4
4 $126 \quad 45.96 \quad 1920.26 \quad 15520.5$

$4 \quad 127 \quad 38.9919 \quad 11.54 \quad 15522.4$

$\begin{array}{lllll}4 & 154 & 52.18 & 19 & 20.27 \quad 155 \quad 20.8\end{array}$

$4 \quad 158 \quad 36.54 \quad 19 \quad 18.58 \quad 155 \quad 23.26$

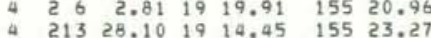

$422129.66 \quad 1912.56 \quad 155 \quad 23.020$

$\begin{array}{lllllll}4 & 224 & 45.10 & 19 & 20.35 & 155 & 20.47\end{array}$

$4 \quad 249 \quad 30.46 \quad 1920.39 \quad 155 \quad 20.31$

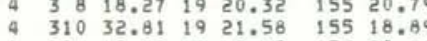

$1920.22 \quad 15520.80$

$\begin{array}{lllllll}4 & 340 & 10.69 & 19 & 14.31 & 155 & 23.28 \\ 4 & 341 & 39.65 & 19 & 14.00 & 155 & 23.07\end{array}$

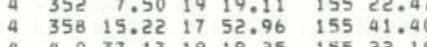

$4 \quad 5 \quad 35.03 \quad 19 \quad 19.08 \quad 155 \quad 22.7$
49.79

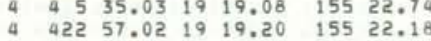

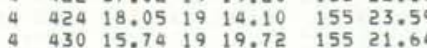

$4 \quad 443 \quad 16,25 \quad 19 \quad 17.75 \quad 155 \quad 23.78$

$\begin{array}{lllllll}4 & 456 & 17.76 & 19 & 24.64 & 155 & 17.77 \\ 4 & 5 & 2 & 23.04 & 19 & 18.98 & 155 \\ 22.87\end{array}$ $\begin{array}{llllllll}4 & 5 & 2 & 23.04 & 19 & 18.98 & 155 & 22.87 \\ 4 & 5 & 3 & 57.42 & 19 & 20.38 & 155 & 20.34\end{array}$ $451026.151924 .40 \quad 15517.33$

$\begin{array}{lllll}511 & 39.55 \quad 19 & 17.81 \quad 155 & 23.68\end{array}$ $51510.46 \quad 1920.69 \quad 155 \quad 19.9$
$528 \quad 4.41 \quad 19 \quad 19.30$

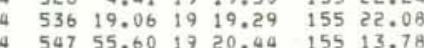

$4550 \quad 12.75 \quad 19 \quad 18.69 \quad 155 \quad 23.45$

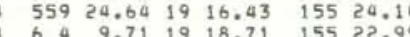

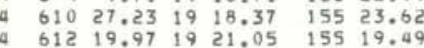

$\begin{array}{llllll}633 & 1.59 & 19 & 18.78 & 155 & 13.1\end{array}$

4
4 \begin{tabular}{llllllllll}
4.50 & 2.3 & 2.2 & 25 & 0 & 166 & .14 & 16 & 1.1 & 1.5 \\
\hline
\end{tabular}

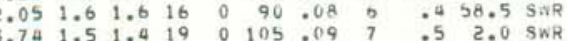

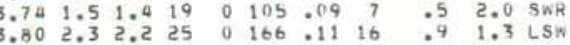

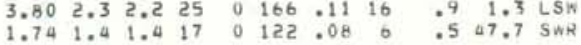
$\begin{array}{llllllllllllll}2.34 & 1.7 & 1.9 & 17 & 0 & 100 & .05 & 0 & .3 & 2.8 & \text { SNR }\end{array}$

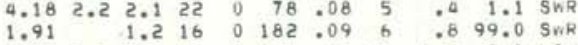

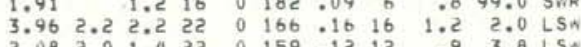

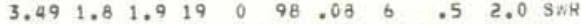

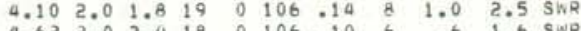

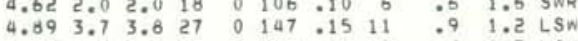

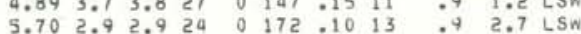

$\begin{array}{lllllllllll}3.84 & 2.1 & 2.2 & 19 & 0 & 92 & .10 & 5 & .6 & 1.8 & S W R\end{array}$

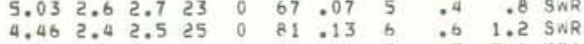

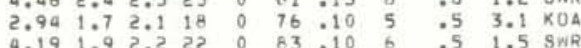
$\begin{array}{lllllllllllll}3.86 & 3.1 & 3.2 & 25 & 0 & 148 & .14 & 11 & 99 & 2.2 & \text { LSN } \\ 2.19 & 2.9 & 3.0 & 26 & 0 & 153 & .13 & 11 & 99 & 2.9 & \text { LSN }\end{array}$

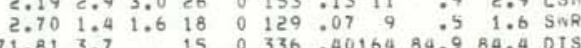

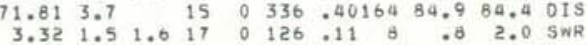

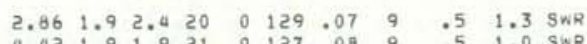

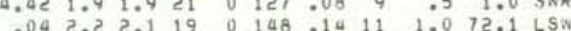
$3.501 .51 .61900114 .07 \quad 7 \quad .4 \quad 1.3$ SWR $\begin{array}{lllllllllll}5.28 & 2.1 & 2.2 & 22 & 0 & 111 & .15 & 9 & .9 & 1.1 & \text { SnR }\end{array}$ $\begin{array}{lllllllllllll}18.46 & 1.2 & 27 & 0 & 38 & .09 & 2 & .7 & 1.0 & \text { OEP }\end{array}$

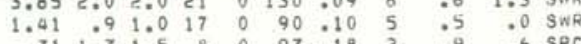

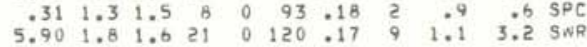
$\begin{array}{lllllllllll}5.69 & 2.8 & 3.0 & 27 & 0 & 111 & .15 & 9 & .8 & 1.9 & \text { SwK }\end{array}$

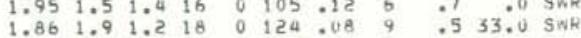

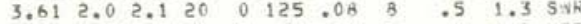
$6.911 .51 .426 \quad 0$ 67 1.14 o .4 1.5 UJEA $\begin{array}{llllllllllllll}5.39 & 2.4 & 2.7 & 25 & 0 & 105 & .14 & 9 & .8 & .9 & S W R\end{array}$

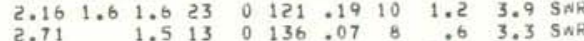

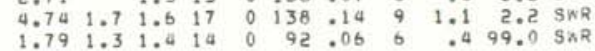
$\begin{array}{lllllllllll}4.21 & .7 & .4 & 16 & 0 & 85 & .13 & 8 & .9 & 1.8 & \text { POL }\end{array}$

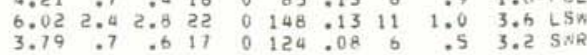


HVO EARTHIJIAKE SURMARY LIST

PAGE 21

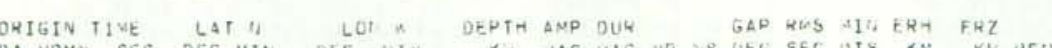
EAR MOH DA HQM TIUE SEO

1975 JAN \& $716 \quad 34.61 \quad 19 \quad 19.16 \quad 155 \quad 22.52$ \begin{tabular}{l}
728 \\
750.80 \\
\hline
\end{tabular}

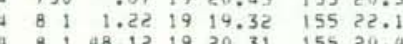

$\begin{array}{lllllll}8 & 2 & 30.59 & 19 & 18.20 & 155 & 13.27\end{array}$

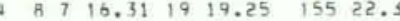

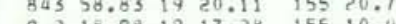
4350.201920 .3515520 .36 $4 \quad 924 \quad 20.91 \quad 19 \quad 13.03 \quad 15521.03$ $\begin{array}{llll}102 & 13.3719 & 21.0 t & 15519 \\ 10.01\end{array}$

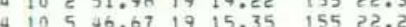

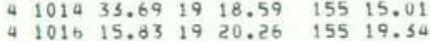
105019.4319 10.76 15514.99

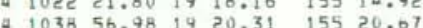

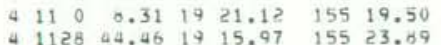
4 1128 44.26 19 15.97 155 23.09

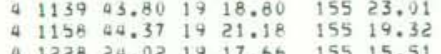

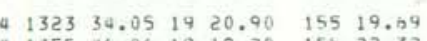

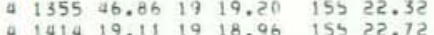

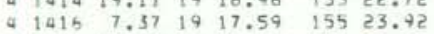

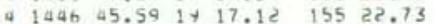

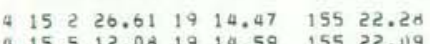

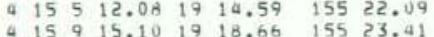

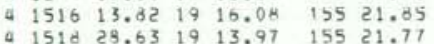
4. $1519 \quad 18.34 \quad 19 \quad 16.31 \quad 155 \quad 22.45$

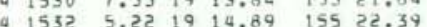
$\begin{array}{lllllll}4 & 1542 & 13.06 & 19 & 16.71 & 155 & 22.78 \\ 4 & 1507 & 26.97 & 19 & 13.60 & 155 & 23.47\end{array}$

$\begin{array}{lllllll}4 & 1550 \quad 0.77 \quad 19 & 16.50 \quad 155 & 22.54\end{array}$

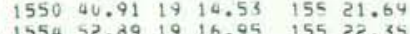
4155032.361912 .45 155 21.65 $4155819.421915 .28 \quad 15521.96$ 4 in $214.44 \quad 1917.12 \quad 15524.10$ 4 it 5 .04 1916.06 155 22.8ट $\begin{array}{lllllllllll}4.9 \times & 2.2 & 2.4 & 25 & 0 & 100 & .13 & 9 & .7 & 1.1 & 3 W R\end{array}$

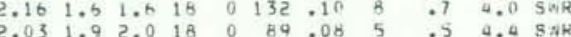

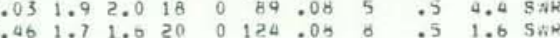

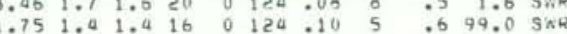

$\begin{array}{lllllllllll}.70 & 2.3 & 2.3 & 26 & 0 & 90 & .11 & 8 & .7 & 1.3 & \text { POL }\end{array}$

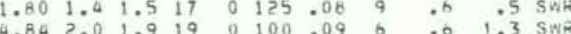

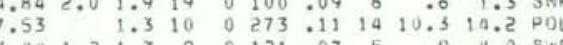

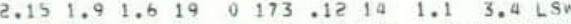

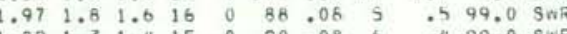
1.891 .31 .4 is o 90.08 o 0499.0 SWR

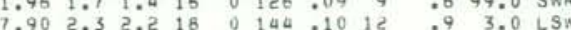

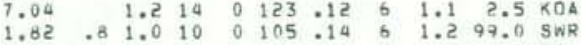
\begin{tabular}{lllllllll}
.08 & 1.6 & 25 & 0 & 93 & .10 & 7 & .0 & 1.3 \\
\hline
\end{tabular}

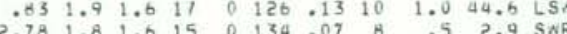

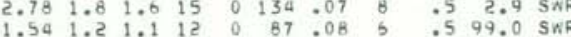

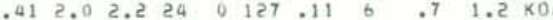

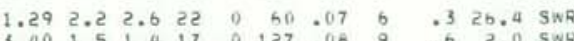

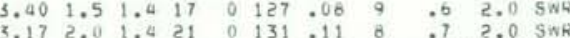

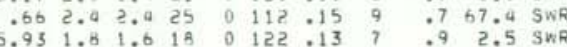
$\begin{array}{llllllllllllll}0.26 & 3.7 & 3.8 & 26 & 0 & 153 & .11 & 11 & .8 & .6 & .6 & \text { LSW }\end{array}$

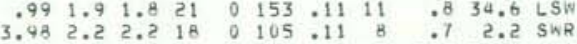

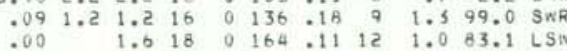
$\begin{array}{lllllllllll}0.12 & 1.8 & 1.6 & 18 & 0 & 132 & .14 & 7 & 1.2 & 3.0 & \text { SWR }\end{array}$ $\begin{array}{lllllllllll}5.49 & 2.3 & 2.8 & 23 & 0 & 165 & .09 & 12 & .8 & 3.1 & \text { LS } \\ 9.70 & 5.0 & 4.8 & 28 & 0 & 144 & 16 & 10 & 1.1 & .7 & \text { LSW }\end{array}$

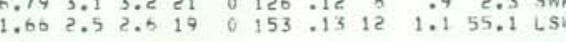
$\begin{array}{lllllllllll}6.79 & 1.4 & 1.5 & 16 & 0 & 133 & .13 & 11 & 1 . ? & 3.5 & \text { SWR }\end{array}$

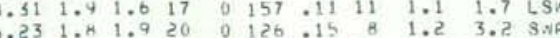

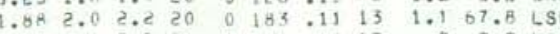

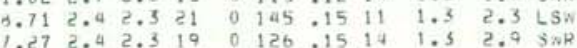

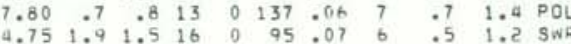
2.25 $1.6 \quad 1.6 \quad 150000.07 \quad 6 \quad .9416 .1$ SNH

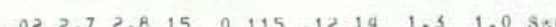

HVO EARTHCUANF SHMMANY LIST

PuGE Z2

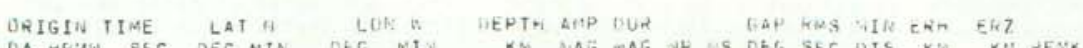

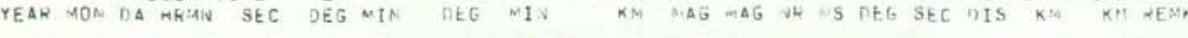

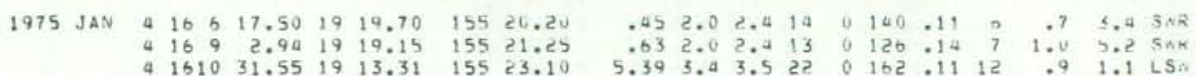

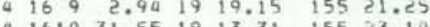
$4161031.5519 \quad 13.31 \quad 15523.10$

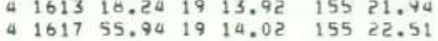

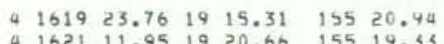
4621
4
4 $1621.951920 .66 \quad 15519.53$

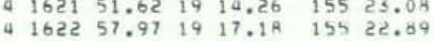

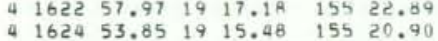

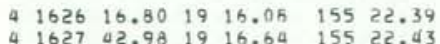

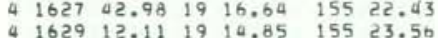

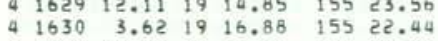
$\begin{array}{llllll}4 & 1630 & 3.62 & 19 & 16.88 & 155 \\ 4 & 1631 & 40.99 & 19 & 16.02 & 155 \\ 42.50\end{array}$ $\begin{array}{lllllll}4 & 1635 & 1.03 & 19 & 13.54 & 155 & 23.70 \\ 4 & 1638 & 8.11 & 19 & 13.64 & 155 & 22.85\end{array}$ $\begin{array}{llllll}4 & 1643 & 39.72 & 19 & 14.63 & 15520.78 \\ 4 & 1649 & 38.87 & 19 & 16.03 & 155 \\ 4 & 23.41\end{array}$

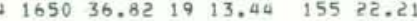

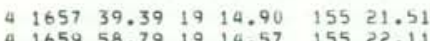
4. 17598.79 19 $14.57 \quad 15522.11$ 17
4
4 $\begin{array}{lllllll}4 & 1715 & 31.27 & 19 & 16.29 & 155 & 23.75\end{array}$

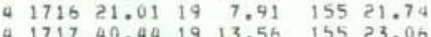

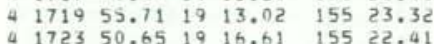

$\begin{array}{lllll}4 & 1725 & 31.95 \quad 19 & 11.48 \quad 155 & 21.82\end{array}$ $4 \begin{aligned} & 1728 \\ & 4\end{aligned} 15.17 \quad 19 \quad 17.05 \quad 155 \quad 22.76$ $\begin{array}{lllllll}4 & 1706 & 7.92 & 19 & 13.11 & 155 & 23.20 \\ 4 & 1748 & 1.71 & 19 & 19.25 & 155 & 22.28\end{array}$ $\begin{array}{lllllll}4 & 1755 & 3.31 & 19 & 20.25 & 155 & 20.41\end{array}$

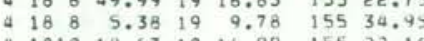
$41819 \quad 10.67 \quad 19 \quad 16.99 \quad 15522.16$

$\begin{array}{lllllll}4 & 1824 & 32.14 & 19 & 15.04 & 155 & 21.34\end{array}$

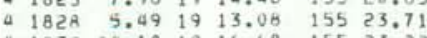
$4 \quad 1832 \quad 24.10 \quad 19 \quad 16.68 \quad 15523.22$ 41836

$\begin{array}{lllllll}4838 & 34.74 & 19 & 16.68 & 155 & 23.12\end{array}$

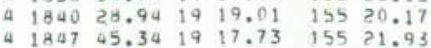

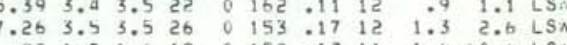

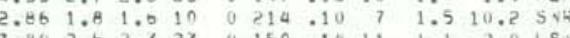
.093 .03 .526 0 121.16 y 1.1 1.5 SNR

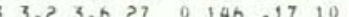

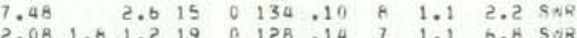

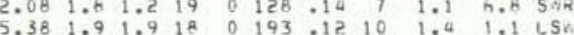

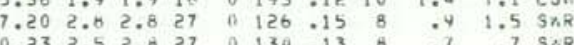
$\begin{array}{rrrrrrrrrrr}.98 & 2.6 & 2.7 & 17 & 0 & 152 & .11 & 12 & .7 & .0 & \text { LSW } \\ 3.01 & 2.3 & 2.4 & 20 & 0 & 160 & .17 & 12 & 1.0 & 3.8 & \text { LSN }\end{array}$

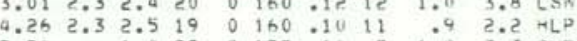

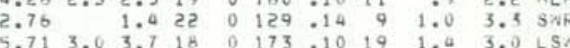

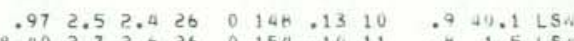

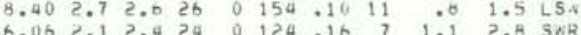

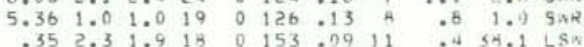

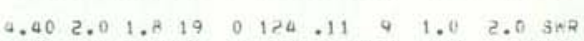

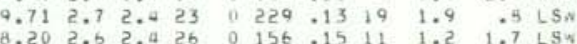

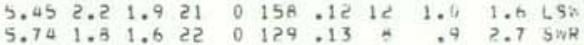
$\begin{array}{lllllllllll}5.83 & 3.0 & 2.9 & 16 & 0 & 202 & .10 & 13 & 2.0 & 3.7 & \text { LSA }\end{array}$

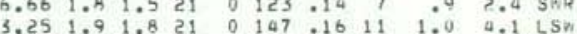
$\begin{array}{ccccccccccc}5.39 & 2.0 & 1.6 & 21 & 0 & 160 & .13 & 12 & 1.3 & 1.4 & \text { LSN } \\ 2.76 & 1.9 & 2.2 & 20 & 0 & 98 & .08 & 9 & .5 & 2.1 & \text { SNR }\end{array}$ $\begin{array}{lllllllllll}2.84 & 2.1 & 2.5 & 23 & 0 & 84 & 11 & 5 & .6 & 2.7 & \text { SWR }\end{array}$

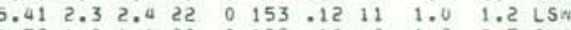

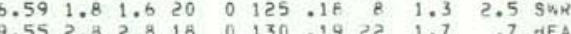

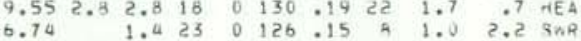
$\begin{array}{lllllllllll}7.09 & 2.0 & 1.8 & 22 & 0 & 153 & .10 & 10 & .4 & 1.7 & \text { LSN }\end{array}$

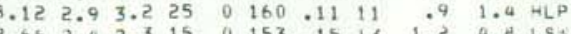

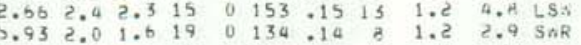

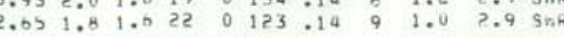

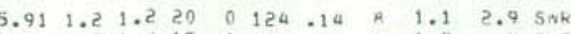

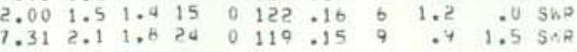


HVO EARTHOUAKE SUMMARY LIST

PAGE 23 ORIGIN TIME LATN LON N OEPTH AMP OUR N GAP RMS MIN ERH ERZ
DA HRMN SEC DEG MIN DEG NIN KM MAG MAG NR NS DEG SEC UIS KM KM REMK 1975 JAN $4 \begin{array}{lllllllllllllllllll}1858 & 2.40 & 19 & 14.78 & 155 & 21.33 & 5.95 & 2.0 & 2.0 & 25 & 0 & 154 & .14 & 11 & 1.0 & 3.2 & \text { LSN }\end{array}$ 1919
4
4 4192713.10
4
4

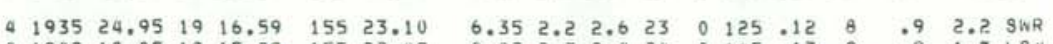

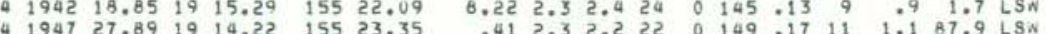
$41948 \quad 7.781920 .61 \quad 15520.39$

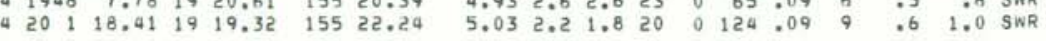

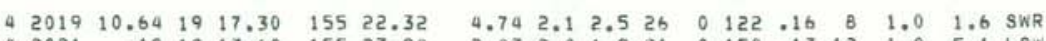

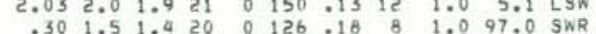

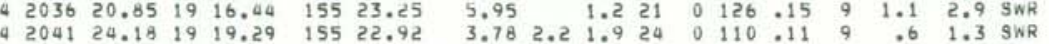

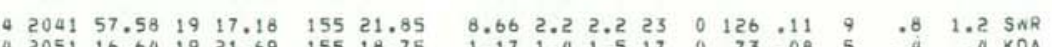

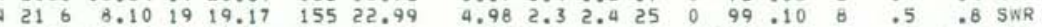

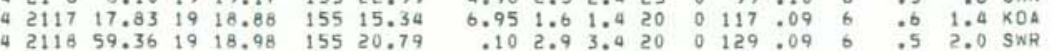

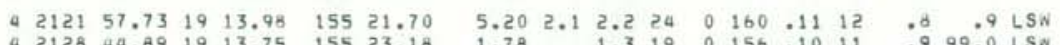
a $2133 \quad 58.5819 \quad 18.65 \quad 15523.28$

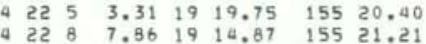

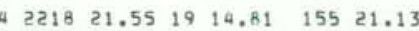
42525

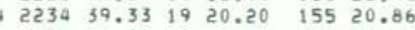
4 . $223756.301916 .42 \quad 15523.10$

$\begin{array}{lllllll}23 & 7 & 5.11 & 19 & 19.11 & 155 & 22.18\end{array}$

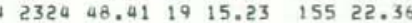

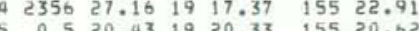
5 01920.431920 .33 155 20.82

$\begin{array}{llllll}5 & 023 \quad 19.6419 & 20.63 \quad 155 \quad 20.68\end{array}$

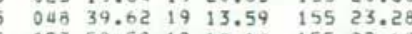
$5 \quad 053 \quad 58.58 \quad 19 \quad 14.14 \quad 155 \quad 22.64$ $5,350.9619,12.96$ 155 23.53 $\begin{array}{lllllll}5 & 117 & 40.25 & 19 & 11.52 & 155 & 21.11 \\ 5 & 140 & 41.60 & 19 & 19.68 & 155 & 21.74\end{array}$ 50.56 19 19.46 155 12.55 $\begin{array}{llllllll}5 & 2 & 8 & 1.84 & 19 & 19.44 & 155 & 22.03\end{array}$

$\begin{array}{lllllll}5 & 214 & 18.01 & 19 & 10.69 & 155 & 21.56 \\ 5 & 215 & 50.02 & 19 & 19.34 & 155 & 21.35\end{array}$

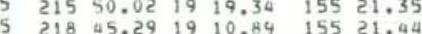

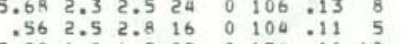

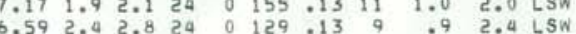
$\begin{array}{lllllllllll}1.54 & 1.8 & 1.6 & 16 & 0 & 99.07 & 6 & .4 & .0 & \text { SWR }\end{array}$ $\begin{array}{rrrrrrrrrrr}5.62 & 1.8 & 1.7 & 21 & 0 & 127 & .15 & 8 & 1.2 & 3.3 & \text { SWR } \\ 1.28 & 2.1 & 2.1 & 20 & 0 & 152 & .14 & 12 & 1.2 & 65.0 & \text { LSW }\end{array}$ $\begin{array}{lllllllllll}3.24 & 2.0 & 1.8 & 20 & 0 & 129 & .07 & 9 & .4 & 1.4 & \text { SWR }\end{array}$

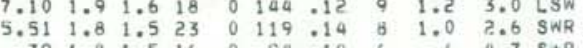
$\begin{array}{rrrrrrrrrrr}.79 & 1.8 & 1.5 & 16 & 0 & 94 & .10 & 6 & .6 & 4.7 & \text { SAR } \\ 1.41 & 2.7 & 2.8 & 23 & 0 & 154 & .13 & 12 & .0 & 33.5 & \text { LSN }\end{array}$ $\begin{array}{llllllllllll}1.97 & 2.2 & 2.6 & 16 & 0 & 117 & .09 & 6 & .7 & 50.2 & \text { SWR }\end{array}$

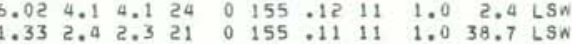
$7.492 .42 .226 \quad 0 \quad 159: 10 \quad 110.7 \quad 9$ HLP $\begin{array}{rrrrrrrrrrr}3.96 & 2.2 & 2.5 & 23 & 0 & 170 & .16 & 13 & 1.4 & 2.2 & \text { LSW } \\ 9.94 & 1.7 & 1.8 & 17 & 0 & 169 & .08 & 8 & .6 & .9 & \text { SWR }\end{array}$

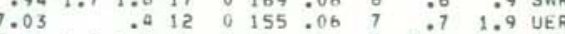

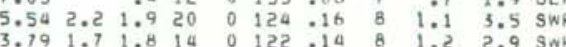

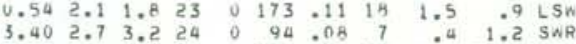

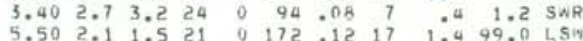

HVO EARTHOUAKE SUMMARY LIST

PAGE 24 ORIGIN TIME LATN LON N DEPTH AMP DUR N GAP RMS MIN EHM ERZ RME

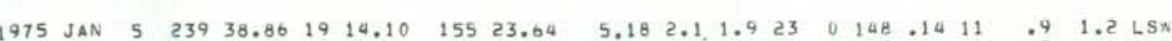

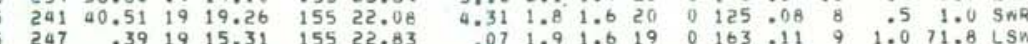

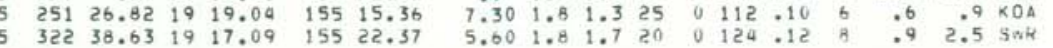

5 $325 \quad 59.16 \quad 19 \quad 15.02 \quad 155 \quad 20.75$ $\begin{array}{lllllll}5 & 334 & 24.31 & 19 & 21.66 & 155 & 18.80\end{array}$ $\begin{array}{lllll}5 & 353 \quad 47.99 & 19 & 20.20 & 155 \quad 20.79\end{array}$

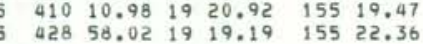

$\begin{array}{lllllll}5 & 430 & 3.34 & 19 & 12.13 & 155 & 21.69\end{array}$

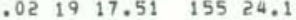
$5 \quad 441 \quad 10.26 \quad 1918.22 \quad 15523.12$

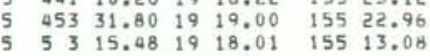

$\begin{array}{lllllll}5 & 511 & 10.82 & 19 & 19.22 & 155 & 22.34\end{array}$ $\begin{array}{llllll}516 & 39.84 & 19 & 18.95 & 155 & 22.71\end{array}$

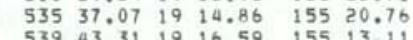

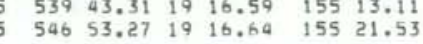
$\begin{array}{lllllll}5 & 6 & 64.29 & 19 & 16.99 & 155 & 23.33\end{array}$ $\begin{array}{llllll}632 & 8.22 & 19 & 16.50 & 155 & 23.29\end{array}$

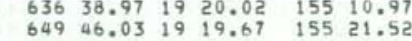

$\begin{array}{lllllll}5 & 715 & 24.80 & 19 & 16.03 & 155 & 22.03\end{array}$ $\begin{array}{lllllll}5 & 733 & 30.77 & 19 & 16.49 & 155 & 21.52 \\ 5 & 755 & 40.34 & 19 & 17.09 & 155 & 23.32\end{array}$

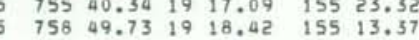

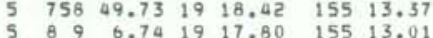

$\begin{array}{lllllll}5 & 812 & 53.75 & 19 & 17.67 & 155 & 13.13 \\ 5 & 823 & 34.58 & 19 & 18.17 & 155 & 13.13\end{array}$

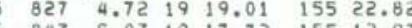
54.79317 .7215512 .93

$\begin{array}{lllllll}5 & 856 & 35.83 & 19 & 18.65 & 155 & 8.32\end{array}$ $\begin{array}{llllllll}5 & 9 & 1 & 54.34 & 19 & 20.06 & 155 & 21.01 \\ 5 & 9 & 4 & 54.54 & 19 & 17.77 & 155 & 14.33\end{array}$

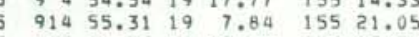
\begin{tabular}{l}
$917 \quad 16.57 \quad 19 \quad 13.94 \quad 155 \quad 23.35$ \\
\hline
\end{tabular}

$5 \quad 947 \quad 42.75 \quad 19 \quad 19.43 \quad 15522.23$

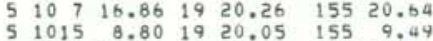
$5 \begin{aligned} & 502453.7719 \\ & 5\end{aligned}$ $503010.82 \quad 1917.94 \quad 155251.75$

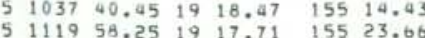
$51125 \quad 40.31$ 19 19.17 155 22.41 $\begin{array}{lllllllllllll}4.17 & .9 & .4 & 19 & 0 & 157 & .09 & 11 & .8 & 1.4 & \text { HLP }\end{array}$

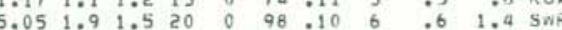

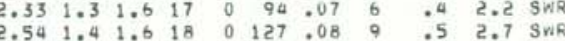
$\begin{array}{lllllllllll}1.36 & 2.0 & 1.9 & 21 & 0 & 175 & .15 & 12 & 1.3 & 50.4 & \text { LSW }\end{array}$

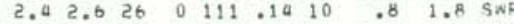

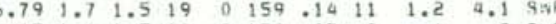

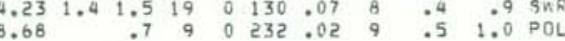
$\begin{array}{llllllllllllll}3.62 & 1.6 & .6 & 18 & 0 & 126 & .08 & 9 & .6 & 2.1 & \text { SWR }\end{array}$

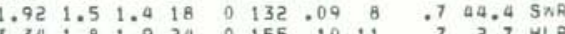

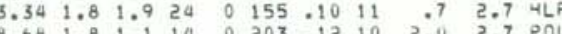

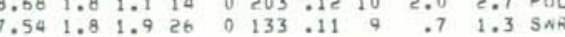
$\begin{array}{lllllllllll}5.55 & 1.8 & 1.4 & 25 & 0 & 120 & .15 & 6 & 1.0 & 2.9 & 5 \mathrm{NNK}\end{array}$

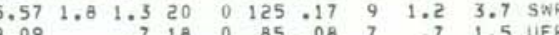

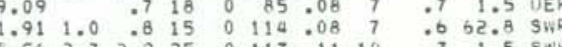
$\begin{array}{lllllllllll}5.86 & 1.8 & 1.5 & 23 & 0 & 136 & .17 & 8 & 1.1 & 3.7 & \text { SWR }\end{array}$

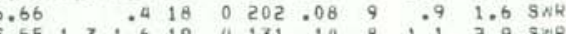

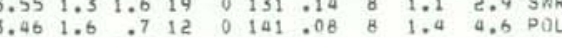

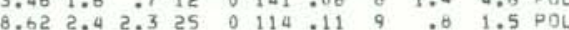
$\begin{array}{llllllllll}6.95 & .8 & 17 & 0 & 113 & .11 & 9 & 1.0 & 2.3 & \text { POL } \\ 3.76 & .5 & 12 & 0 & 96 & .09 & 8 & 1.0 & 3.4 & \text { POL }\end{array}$ $\begin{array}{lllllllllll}3.34 & 1.6 & 1.2 & 18 & 0 & 130 & .09 & 8 & .6 & 5.2 & 5 x\end{array}$

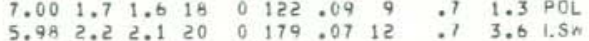

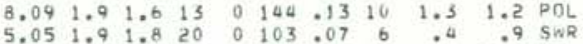
$\begin{array}{llllllllll}6.87 & 1.0 & 18 & 0 & 140 & .10 & 8 & .8 & 1.8 & \text { pol }\end{array}$

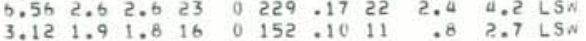

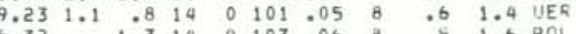

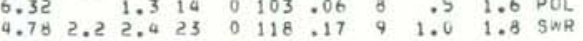

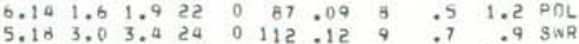
$\begin{array}{lllllllllll}5.10 & 3.0 & 3.4 & 24 & 0 & 112 & -12 & 9 & -7 & .9 & \text { SNR } \\ 3.03 & 1.6 & 1.9 & 19 & 0 & 126 & .10 & 8 & 0 & 1.4 & \text { SNR }\end{array}$

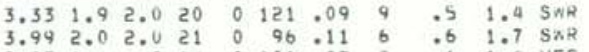




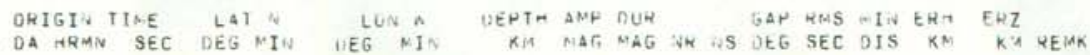

1775 SAN $5112719.031+21.01 \quad 13511.12$ 5
5 $155536.93 \quad 19 \quad 13.75 \quad 155 \quad 23.26$ 5122131.141919 .1315523 .84 $51231 \quad 47.06 \quad 19 \quad 16.90 \quad 15523.15$
0

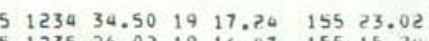
51235
5 $26.02 \quad 19 \quad 16.87 \quad 155 \quad 15.20$ 5 1244 3.65 19 $13.01 \quad 15523.45$ $\begin{array}{llllll}5 & 1318 & 6.97 \quad 19 & 21.26 & 155 & 4.51\end{array}$

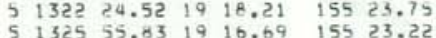

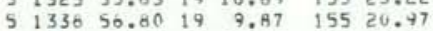

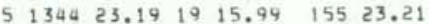
$\begin{array}{lllllll}5 & 1357 & 10.74 & 19 & 17.65 & 155 & 21.49\end{array}$

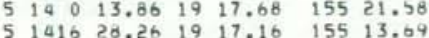

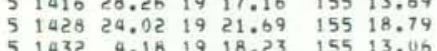

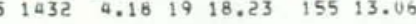

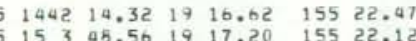
$515836.131917 .00 \quad 15523.21$

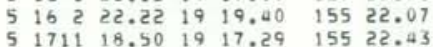
$\begin{array}{lllllll}5 & 1727 & 26.66 & 19 & 17.63 & 155 & 15.56\end{array}$

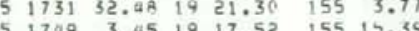

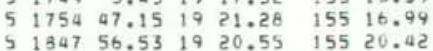
$\begin{array}{lllllll}5 & 1856 & 25.82 & 19 & 18.85 & 155 & 15.08\end{array}$ $55 \quad 6.0319 \quad 19.57 \quad 155 \quad 8.41$

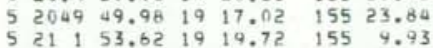

$\begin{array}{lllllll}5 & 2141 & 21.02 \quad 19 & 16.71 \quad 155 & 23.56\end{array}$ $\begin{array}{llllll}2146 & 57.50 & 19 & 19.12 & 155 & 22.21\end{array}$

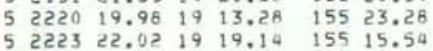

$\begin{array}{lllllll}5 & 2226 & 1.79 & 19 & 19.21 & 155 & 22.39\end{array}$

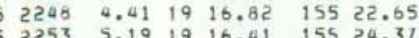

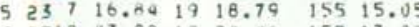

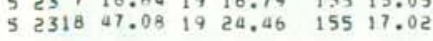
$\begin{array}{lllllll}5 & 2335 & 17.75 & 19 & 20.33 & 155 & 20.57\end{array}$

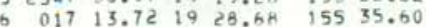

$\begin{array}{lllllllllll}0.75 & 1.7 & 1.4 & 19 & 0 & 160 & .13 & 8 & 1.3 & 1.8 & \text { IJER }\end{array}$

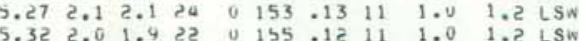

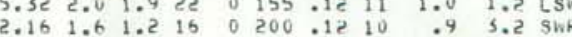

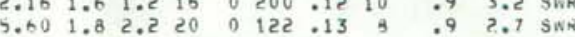
$\begin{array}{lllllllllll}4.84 & 1.8 & 1.6 & 21 & 0 & 119 & .14 & 8 & .9 & 1.7 & \text { SWR }\end{array}$

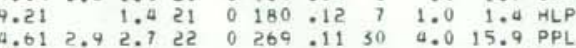

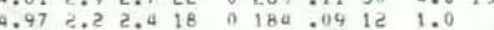

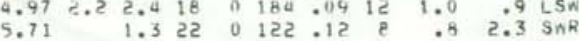
$\begin{array}{lllllllllll}8.97 & 2.7 & 3.1 & 22 & 0 & 212 & .11 & 17 & 1.1 & 1.8 & \text { MER }\end{array}$

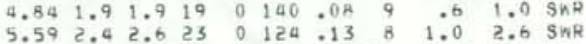

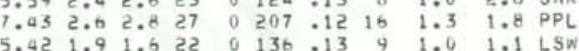
$\begin{array}{llllllllllll}8.67 & 2.8 & 3.0 & 25 & 0 & 122 & .12 & 0 & .9 & 1.3 & \text { SWR }\end{array}$ $\begin{array}{lllllllllll}3.01 & 1.8 & 1.6 & 20 & 0 & 121 & 11 & 9 & .7 & 2.8 & \text { SWR } \\ 7.92 & 1.9 & 2.1 & 25 & 0 & 117 & 011 & 9 & -8 & 1.2 & 901\end{array}$

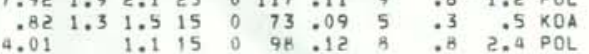

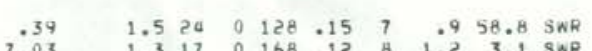

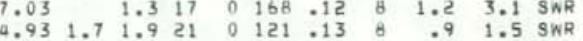

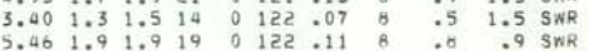
$\begin{array}{llllllllll}6.84 & 1.5 & 20 & 0 & 155 & .11 & 6 & .8 & 1.7 & \text { KOA }\end{array}$

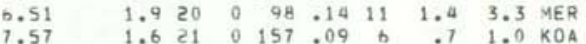

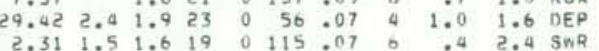
$\begin{array}{lllllllllll}7.18 & 1.2 & 25 & 0 & 120 & 10 & 0 & .6 & .9 & K O A\end{array}$ $6.653 .62 .827 \quad 0 \quad 71.1460 .91 .55$ SWR

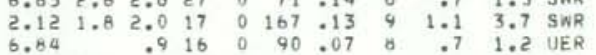
$\begin{array}{lllllllllll}5.36 & 1.6 & 20 & 0 & 133 & .13 & 9 & .0 & 1.0 & \text { SNR }\end{array}$

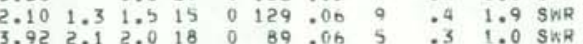

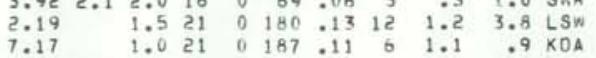
$\begin{array}{llllllllllll}4.28 & 1.8 & 1.6 & 19 & 0 & 126 & .08 & 9 & .6 & .9 & \text { SNR }\end{array}$

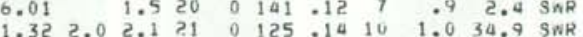

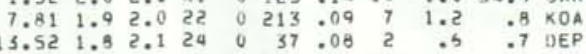
$\begin{array}{llllllllllllll}3.29 & 1.2 & 1.3 & 17 & 0 & 120 & .07 & 6 & .5 & 2.1 & \text { SWR }\end{array}$

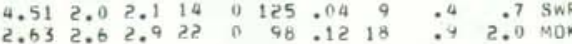

HVO EAKTHQUAKE SUMMARY HIST

Lat 26

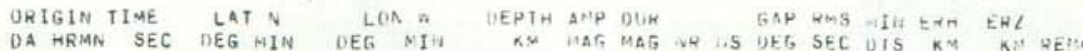

1975 JAN $603125.271921 .52 \quad 15518.75$ 603019.251913 .2515522 .80 6 6 $03424.1019 \quad 13.62 \quad 15523.1$ $6 \quad 116 \quad 2.091915 .18 \quad 15522.10$

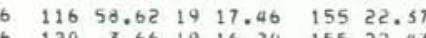

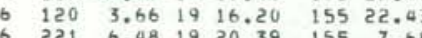
$6253 \quad 4.8019 \quad 11.84 \quad 155$ 23.6 \begin{tabular}{l}
$6 \quad 38$ \\
\hline
\end{tabular} $3.6819 \quad 19.18 \quad 155222.26$

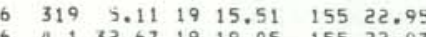
$\begin{array}{llllllll}6 & 4 & 1 & 32.67 & 19 & 19.05 & 155 & 22.05\end{array}$ 6
6 $51331.52 \quad 19 \quad 19.26 \quad 15515.79$

$6 \quad 545 \quad 24.07 \quad 19 \quad 14.63 \quad 155 \quad 20.5$ $6 \quad 552 \quad 13.86 \quad 19 \quad 13.10 \quad 155 \quad 22.5$

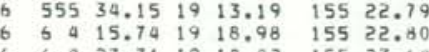
$6.827 .711918 .02 \quad 15523.64$ $6 \quad 633 \quad 21.61 \quad 19 \quad 19.10 \quad 15522.91$ 60 $6 \quad 714 \quad 0.061915 .0515521 .10$ $\begin{array}{ll}12.27 & 19 \\ 16.28 & 155 \\ 22.97\end{array}$

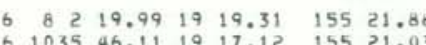
61030.17 19 $19.120 \quad 155 \quad 15.85$ $\begin{array}{llllllll}6 & 12 & 8 & 57.94 & 19 & 18.96 & 155 & 16.13 \\ 6 & 14 & 1 & 10.99 & 19 & 16.17 & 155 & 22.90\end{array}$ $\begin{array}{lllllll}6 & 1938 & 10.99 & 19 & 17.82 & 155 & 15.58\end{array}$ $\begin{array}{lllllll}6 & 1449 & 17.23 & 19 & 18.80 & 155 & 22.75\end{array}$ $6 \quad 1533 \quad 14.07 \quad 1920.29 \quad 15520.67$

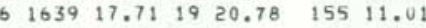

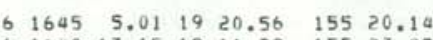
$\begin{array}{lllllll}6 & 1648 & 17.15 & 19 & 16.96 & 155 & 20.19 \\ 6 & 1728 & 18.91 & 19 & 19.45 & 155 & 23.37\end{array}$

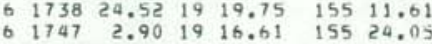

6 $184124.01 \quad 19 \quad 17.86 \quad 155 \quad 14.40$ $\begin{array}{lllllll}6 & 1857 & 42.88 & 19 & 13.81 & 155 & 23.18\end{array}$ $61922 \quad 41.74 \quad 19 \quad 10.15 \quad 155222.50$ 6193945.081910 .89 155 22.20 $\begin{array}{lllllll}6 & 2031 & 1.46 & 19 & 16.98 & 155 & 12.92\end{array}$

$\begin{array}{lllllll}6 & 2040 \quad 53.69 & 19 & 13.75 \quad 155 \quad 23.28\end{array}$ $\begin{array}{r}2048 \quad 16.46 \quad 1920.52 \quad 155 \quad 20.65 \\ \hline\end{array}$ $\begin{array}{rrrrrrrrrrr}2.28 & 1.5 & 1.6 & 14 & 0 & 75 & .06 & 5 & .0 & 10.8 & 004 \\ 2.02 & 1.3 & 20 & 0 & 174 & .13 & 13 & 1.2 & 4.5 & 63\end{array}$

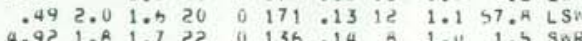
$8.242 .42 .4240165 .11+4.4 \quad 1.345$ $4.411 .71 .022 \quad 0131.10$ o 1.001 .0 Snk

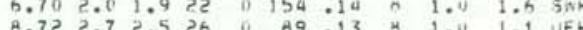

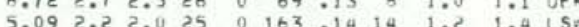
$3.721 .81 .618 \quad 0 \quad 127.06 \quad 9$.5 1.6548 $11.04 \quad 1.5$ 2ट $0165 \cdot 1007.1 .9$ LS

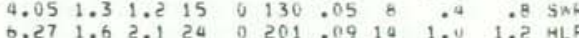
$1.68 \quad .6 \quad .617$ o $181: 10$ 6 1.1 1.A KUA

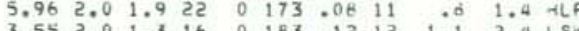

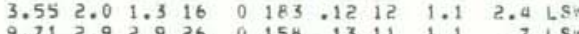

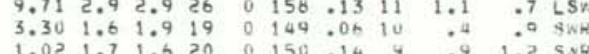

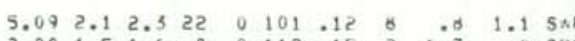

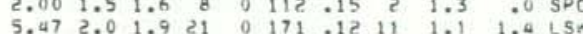

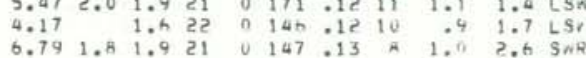
$\begin{array}{llllllllllll}3.27 & 1.2 & 15 & 0 & 150 & 0.06 & 3 & .3 & 1.2 & 3+4\end{array}$

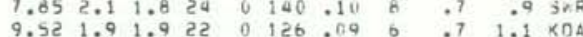

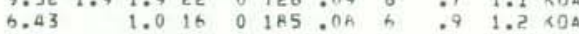
$\begin{array}{lllllllllll}5.67 & 1.1 & 1.5 & 20 & 0 & 131 & .13 & 8 & 1.0 & 5.0 & 5 w h\end{array}$ $\begin{array}{lllllllllll}7.57 & 1.7 & 1.9 & 24 & 0 & 123 & .09 & 5 & .5 & 1.1 & \mathrm{KOA}\end{array}$

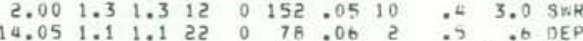

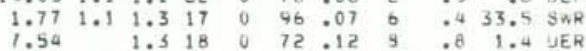
$\begin{array}{lllllllllll}3.23 & 1.4 & 1.6 & 14 & 0 & 45 & .06 & 6 & .4 & 3.4 & \text { SIR }\end{array}$

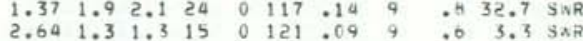
$\begin{array}{llllllllllll}7.91 & & 1.0 & 16 & 0 & 86 & 06 & 6 & 0.5 & 1.3 & \text { JER } \\ 8.68 & 4.5 & 4.3 & 27 & 0 & 119 & 118 & 10 & 1.1 & 1.7 & \text { SNR }\end{array}$ $\begin{array}{llllllllllllll}7.64 & 2.0 & 2.0 & 25 & 0 & 103 & .08 & 7 & .3 & .4 & P O L\end{array}$

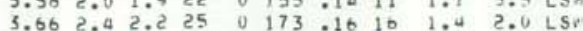
$10.392 .12 .022 \quad 0 \quad 170.1917 \quad 1.4 \quad 1 . ?$ LSA $\begin{array}{llllllllll}7.05 & 1.2 & 16 & 0 & 241 & 10 & 10 & 1.0 & 2.1 & \text { POL }\end{array}$ $\begin{array}{lllllllllll}4.28 & 2.3 & 2.4 & 22 & 0 & 155 & .1 \text { व } & 11 & 1.3 & 2.4 & \text { L9n }\end{array}$

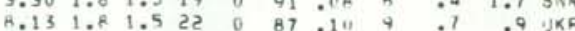


HVO EARTHQUAKE SUMMARY LIST

PAGE 27

ORIGIN TIME LATN LON K OEPTH AMP OUR GAP RMS MIN ERH ERZ RT

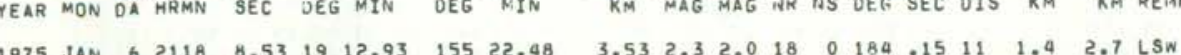
$62147 \quad 35.43 \quad 19 \quad 18.80 \quad 15513.13$

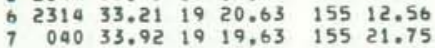
$\begin{array}{lllllll}7 & 040 & 33.92 & 19 & 19,63 & 155 & 21.75 \\ 7 & 051 & 45.12 & 19 & 17.16 & 155 & 24.29\end{array}$ $\begin{array}{llllllll}7 & 1 & 2 & 59.23 & 19 & 21.02 & 155 & 19.59 \\ 7 & 1 & 7 & 14.27 & 19 & 12.80 & 155 & 22.87\end{array}$ $\begin{array}{llllllll}7 & 1 & 7 & 14.27 & 19 & 12.00 & 155 & 22.87 \\ 7 & 1 & 9 & 9.06 & 19 & 24.09 & 155 & 26.36\end{array}$

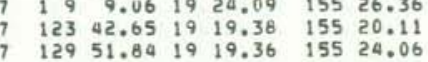

$\begin{array}{lllllll}7 & 238 & 55.62 & 19 & 13.31 & 155 & 23.44 \\ 7 & 254 & 44.59 & 19 & 19.43 & 155 & 22.09\end{array}$

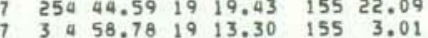

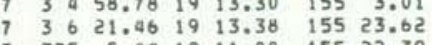

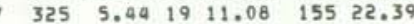

$7 \quad \begin{array}{llllll}335 & 55.19 & 19 & 19.43 & 155 & 22.20\end{array}$ 7 336 $10.14 \quad 1921.25 \quad 155 \quad 19.26$ $734955.981920 .19 \quad 15520.63$ $7 \quad 559 \quad 44.56 \quad 19 \quad 13.82 \quad 155 \quad 23.63$

$\begin{array}{lllllll}7 & 614 & 7.59 & 19 & 20.22 & 155 & 20.81\end{array}$ $\begin{array}{lllllll}7 & 627 & 39.87 & 19 & 19.34 & 155 & 22.10 \\ 7 & 638 & 36.52 & 19 & 13.69 & 155 & 23.12\end{array}$

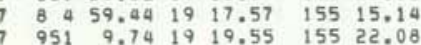
$\begin{array}{llllllll}7 & 11 & 6 & 49.67 & 19 & 17.51 & 155 & 15.03\end{array}$

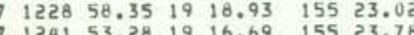

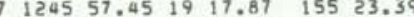

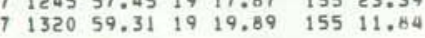

$\begin{array}{llllllll}7 & 1328 & 55.90 & 19 & 19.61 & 155 & 17.78\end{array}$ $1426 \quad 30.071920 .69 \quad 15519.74$

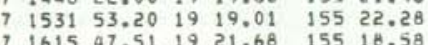

$\begin{array}{lllllll}7 & 1621 & 12.56 & 19 & 16.91 & 155 & 23.10\end{array}$ $7164156.51 \quad 1913.86 \quad 15523.20$

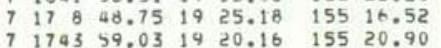

$\begin{array}{lllllll}7 & 1752 & 11.69 & 19 & 19.54 & 155 & 22.18\end{array}$ $\begin{array}{lllllll}7 & 1752 & 11.69 & 19 & 19.54 & 155 & 22.10 \\ 7 & 949 & 14.27 & 19 & 20.51 & 155 & 20.55\end{array}$

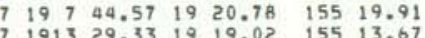

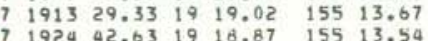

\begin{tabular}{l}
$72156 \quad 42.06 \quad 1921.41 \quad 15518.62$ \\
\hline
\end{tabular}

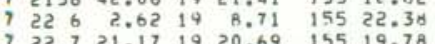

$\begin{array}{rrrrrrrrrrr}3.53 & 2.3 & 2.0 & 18 & 0 & 184 & .15 & 11 & 1.4 & 2.7 & \text { LSW } \\ 8.45 & & .6 & 19 & 0 & 84 & .09 & 8 & .7 & 1.6 & \text { POL }\end{array}$ $\begin{array}{rrrrrrrrrrr}9.37 & 1.7 & 1.6 & 20 & 0 & 67 & .09 & 7 & .6 & 1.4 & \text { UER } \\ 3.57 & 1.6 & 1.4 & 15 & 0 & 127 & .11 & 8 & : 9 & 2.8 & \text { SWR } \\ 4.93 & 3.1 & 3.3 & 24 & 0 & 113 & .14 & 10 & .9 & 1.1 & \text { SWR }\end{array}$ $\begin{array}{rrrrrrrrrrrr}2.33 & 1.3 & 1.5 & 18 & 0 & 93 & 0.08 & 6 & .4 & 3.4 & \text { SWR } \\ 2.68 & & 1.3 & 24 & 0 & 160 & 012 & 12 & 1.0 & 2.5 & \text { LSW }\end{array}$

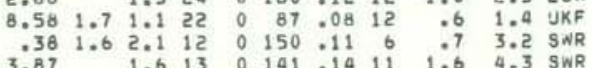
$\begin{array}{lllllllllll}5.03 & 2.0 & 1.8 & 20 & 0 & 159 & .10 & 12 & .8 & 1.0 & \text { LSW }\end{array}$

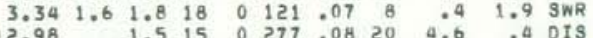

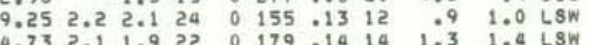
$\begin{array}{lllllllllll}3.49 & 1.2 & 15 & 0 & 121 & .09 & 8 & .7 & 2.4 & \text { SWR }\end{array}$

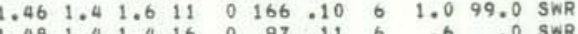

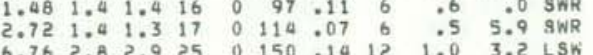

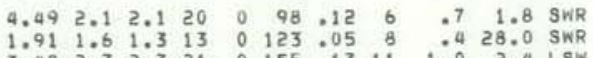
.482 .32 .3210 $\begin{array}{lllllllllll}5.67 & & 1.4 & 21 & 0 & 158 & 09 & 6 & .6 & 1.2 & \text { KOA } \\ 3.19 & 1.6 & 1.6 & 17 & 0 & 118 & .08 & 8 & .6 & 2.6 & \text { SNR }\end{array}$ $\begin{array}{lllllllllll}7.11 & 1.8 & 1.9 & 22 & 0 & 128 & .12 & 6 & .8 & 1.8 & \text { KOA }\end{array}$ $8.602 .52 .627 \quad 00120.16$ 9 1.0 1.4 SKR $\begin{array}{rrrrrrrrrrr}1.20 & 1.6 & 1.8 & 23 & 0 & 112 & .15 & 8 & .7 & 33.1 & \text { SWR } \\ 8.97 & 1.7 & 1.9 & 13 & 0 & 212 & .07 & 8 & 1.5 & 2.4 & \text { UER }\end{array}$ $\begin{array}{llllllllllllll}5.52 & 1.6 & 1.0 & 14 & 1 & 134 & .10 & 6 & 2.0 & 2.0 & \text { DEP }\end{array}$

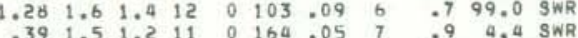

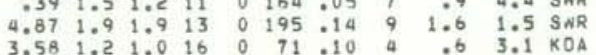
$\begin{array}{lllllllllll}4.02 & 1.7 & 1.2 & 22 & 0 & 122 & .17 & 8 & 1.1 & 2.3 & \text { SWR }\end{array}$

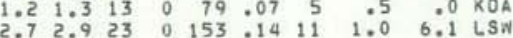

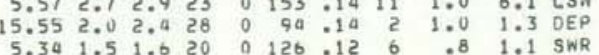
$\begin{array}{llllllllllllll}1.87 & 1.6 & 1.7 & 16 & 0 & 119 & .07 & 8 & .4 & .4 & \text { SWR }\end{array}$

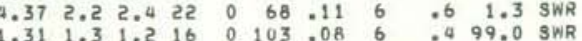

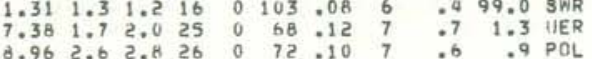

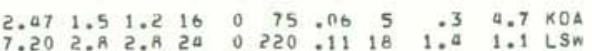

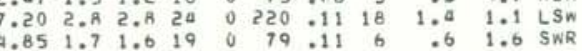

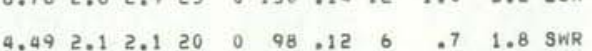

hVO EARTHQUAKE SUMmahY LIST

PAGE 28 ORIGIN TIME LATN LON W UEPTH AMP OUR GAP RMS MIN ERH ERZ REMT $\begin{array}{llllllllllllllllllll}1975 \text { JAN } 7 & 2251 & 16.52 & 19 & 16.75 & 155 & 23.84 & 4.90 & 1.6 & 1.9 & 20 & 0 & 132 & 13 & 9 & 1.1 & 1.2 & \text { SNR } \\ & 7 & 2330 & 27.23 & 19 & 26.97 & 155 & 35.54 & 5.50 & 3.4 & 3.4 & 22 & 0 & 121 & 11 & 22 & .9 & 99.0 & \text { MOK }\end{array}$

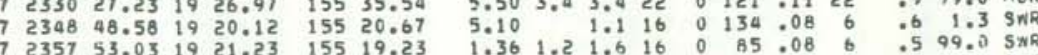
$805 \quad 8.35 \quad 1920.12 \quad 15520.70$

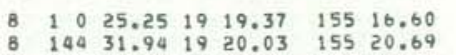

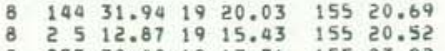
8 . $257 \quad 30.481913 .36 \quad 15523.92$ $\begin{array}{llllll}410 & 1.04 & 19 & 21.43 \quad 155 & 18.74\end{array}$ - 534.191917 .9115515 .34 \begin{tabular}{l}
$542 \quad 21.7019 \quad 19.06 \quad 155 \quad 22.19$ \\
\hline
\end{tabular} \begin{tabular}{l}
$55250.23 \quad 19 \quad 15.32 \quad 155 \quad 20.55$ \\
\hline
\end{tabular}

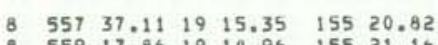
55917.86
6

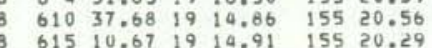

$813 \quad 31.33 \quad 19 \quad 14.62 \quad 155 \quad 20.38$

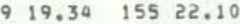
$\begin{array}{llll}21.67 & 1919.44 & 155 & 20.34\end{array}$

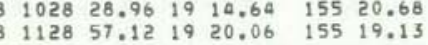
B $1340 \quad 7.53 \quad 19 \quad 15.33 \quad 155 \quad 20.14$ $145018.021920 .01 \quad 15520.00$ 15516.99

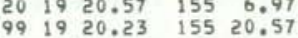

$\begin{array}{llllllll}8 & 21 & 7 & 6.15 & 19 & 16.63 & 155 & 22.82\end{array}$

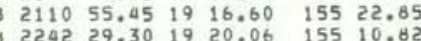

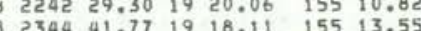

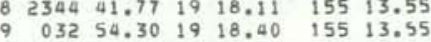

$\begin{array}{lllllll}038 \quad 31.23 & 19 & 17.75 & 155 & 15.72\end{array}$ $1524.26 \quad 1926.80 \quad 15517.13$
1920 $\begin{array}{llllll}237 & .71 & 19 & 14.72 & 155 & 22.42\end{array}$ $\begin{array}{lllllll}9 & 322 & 40.38 & 19 & 13.89 & 155 & 22.11 \\ 9 & 358 & 29.87 & 19 & 17.93 & 155 & 17.95\end{array}$ $\begin{array}{llllllll}9 & 4 & 2 & 4.32 & 19 & 20.53 & 155 & 17.30 \\ 9 & 4 & 5 & 48.58 & 19 & 16.08 & 155 & 24.07\end{array}$ $\begin{array}{llllll}457 & 3.42 & 19 & 16.39 & 155 & 22.68\end{array}$ 52010.631913 .66 155 19.90 $\begin{array}{lllllll}9 & 623 & 13.86 & 19 & 17.33 & 155 & 23.60\end{array}$

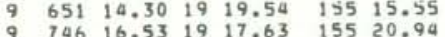

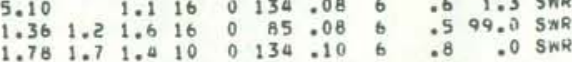

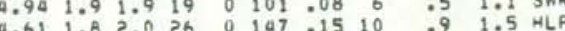
$\begin{array}{lllllllllll}5.14 & 2.3 & 2,3 & 18 & 0 & 154 & .10 & 13 & .9 & 1.0 & \text { LSW } \\ 3.64 & 2.0 & 1.9 & 20 & 0 & 149 & .14 & 12 & 1.1 & 2.7 & \text { LS }\end{array}$ $\begin{array}{lllllllllll}1.88 & 1.2 & 1.2 & 15 & 0 & 76 & .07 & 5 & .4 & .0 & \mathrm{kOA}\end{array}$

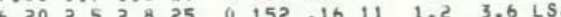

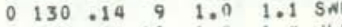

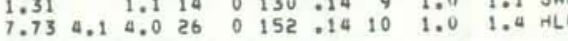

$\begin{array}{llllllllllll}4.68 & 2.4 & 2.6 & 28 & 0 & 147 & .18 & 10 & 1.0 & 1.8 & \text { HLP }\end{array}$

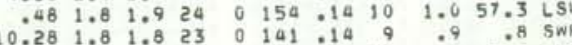

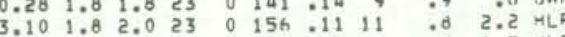

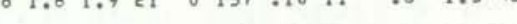

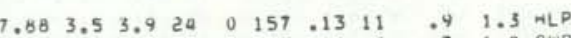
$\begin{array}{rrrrrrrrrrrr}3.27 & 1.6 & 1.6 & 18 & 0 & 123 & -10 & 8 & 07 & 1.8 & \text { SWR } \\ 55 & 2.9 & 3.3 & 12 & 0 & 63 & 0.15 & 5 & 1.0 & 7.6 & \text { SNE }\end{array}$

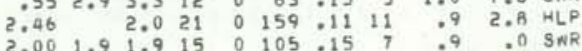
$\begin{array}{lllllllllll}.98 & 1.8 & 1.8 & 24 & 0 & 149 & .10 & 11 & .6 & 33.1 & \text { HLP }\end{array}$

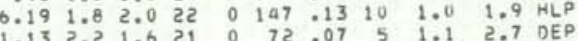
$\begin{array}{lllllllllll}1.13 & 2.2 & 1.6 & 21 & 0 & 72 & .07 & 5 & 1.1 & 2.7 & \text { DEP } \\ 3.51 & 1.9 & 1.4 & 22 & 0 & 96 & .19 & 7 & 1.2 & 3.1 & \text { UER }\end{array}$

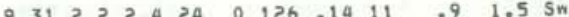

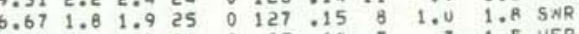
$\begin{array}{lllllllllllll}8.87 & 1.7 & 1.7 & 21 & 0 & 85 & 10 & 10 & 9 & .7 & 1.5 & \text { UER } \\ 7.68 & & .9 & 18 & 0 & 92 & 0.13 & 8 & 1.0 & 2.5 & \text { POL }\end{array}$

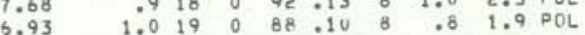

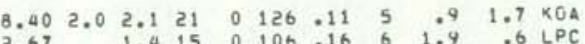

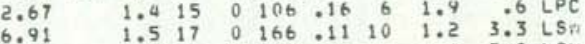

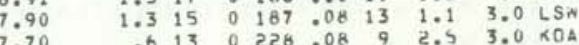

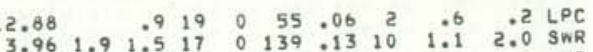

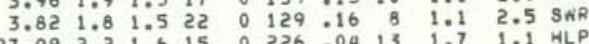

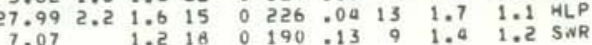
$\begin{array}{lllllllllll}5.48 & 2.1 & 1.9 & 22 & 0 & 125 & .14 & 9 & 1.0 & 1.0 & \text { SWR }\end{array}$

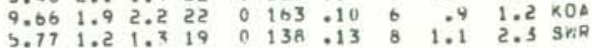
$\begin{array}{lllllllllll}1.77 & 1.6 & .9 & 19 & 0 & 160 & .10 & 6 & 2.0 & 5.0 & \text { DFP }\end{array}$ 


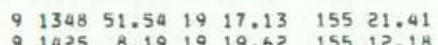

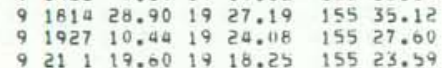

$92226 \quad 56.64 \quad 19 \quad 15.11 \quad 15522.49$ $92330 \quad 8.031919 .80 \quad 15520.72$ $10 \quad 35550.2819 \quad 16.13 \quad 15523.59$

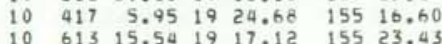

$\begin{array}{lllllll}10 & 713 & 34.66 & 19 & 17.90 & 155 & 13.39\end{array}$ $\begin{array}{lllllll}10 & 815 & 3.83 & 19 & 18.25 & 155 & 23.80 \\ 10 & 827 & 4.86 & 19 & 21.17 & 155 & 19.26\end{array}$ $\begin{array}{lllllll}10 & 827 & 4.86 & 19 & 21.17 & 155 & 19.26 \\ 10 & 832 & 21.07 & 19 & 20.09 & 155 & 20.67\end{array}$ $10,1051,40.77$ 19 $16.32 \quad 155,20.30$

$\begin{array}{llllllllll}2.34 & 1.5 & 1.5 & 14 & 0 & 76.09 & 5 & 0 & 13.5 & \text { KOA }\end{array}$

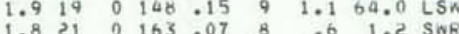

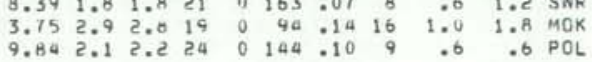
$\begin{array}{lllllllllll}4.26 & 2.0 & 2.1 & 21 & 0 & 138 & .14 & 9 & 1.0 & 1.7 & \text { SWR }\end{array}$

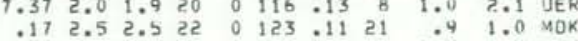

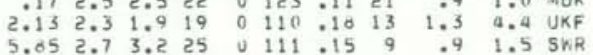
$\begin{array}{lllllllllll}5.70 & 1.9 & 1.9 & 23 & 0 & 147 & .13 & 13 & 1.0 & 2.7 & \text { LSW }\end{array}$

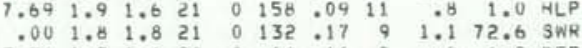

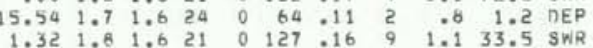
$\begin{array}{llllllllllll}8.72 & 1.8 & 1.4 & 18 & 0 & 175 & 11 & 9 & 1.5 & 1.0 & \text { POL } \\ 1.01 & 1.7 & 1.6 & 19 & 0 & 103 & 015 & 9 & 1.1 & 35.2 & \text { SWR }\end{array}$

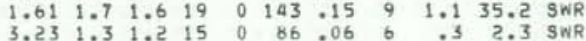

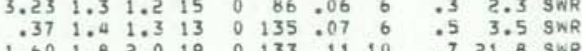

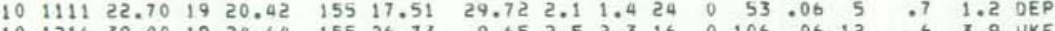

1303 $51.001924 .64 \quad 15526.73$ $10 \quad 1441 \quad 0.771916 .78 \quad 15522.15$

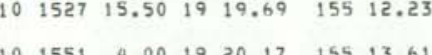
$\begin{array}{rrrrrrr}10 & 1551 & 4.00 & 19 & 20.17 & 155 & 13.61 \\ 10 & 2248 & 48.38 & 19 & 20.01 & 155 & 17.20\end{array}$

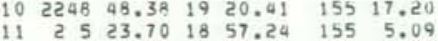
11 422 $6.881921 .22 \quad 155 \quad 5.95$

$11 \quad 549 \quad 40.55 \quad 19 \quad 16.73 \quad 155 \quad 23.3$ $11 \quad 556 \quad 25.55 \quad 19 \quad 13.59 \quad 15523.3$ $11 \quad 616 \quad 12.68 \quad 19 \quad 16.54 \quad 155$ 22.8 $11 \quad 819 \quad 32.19 \quad 19 \quad 17.80 \quad 155 \quad 20.97$

$\begin{array}{lllllll}11 & 840 & 44.07 & 19 & 19.37 & 155 & 15.97\end{array}$

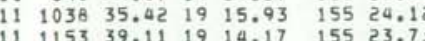

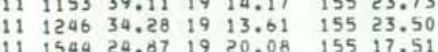
$\begin{array}{lllllll}11 & 1545 & 57.82 & 19 & 20.94 \quad 155 & 17.12\end{array}$ $111162528.561919 .25 \quad 155 \quad 13.07$ $\begin{array}{rrrrrrr}11 & 1853 & 8.27 & 19 & 17.41 & 155 & 24.29 \\ 11 & 1855 & 42.63 & 19 & 17.33 & 155 & 22.45\end{array}$

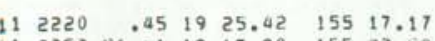
$11.233429 .28 \quad 1913.52 \quad 15522.60$

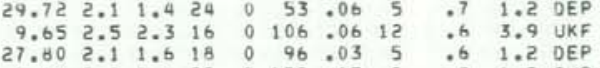

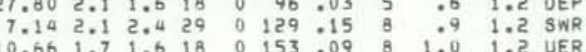
\begin{tabular}{lllllllllll}
8.97 & 1.6 & 1.2 & 19 & 0 & 151 & .14 & 7 & 1.4 & 2.2 & UER \\
\hline
\end{tabular}

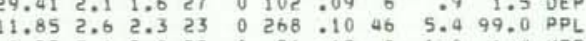

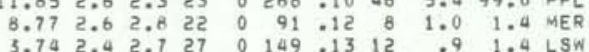
$\begin{array}{llllllllll}5.39 & 1.8 & 1.6 & 24 & 0 & 130 & .19 & 12 & 1.0 & 1.5\end{array}$

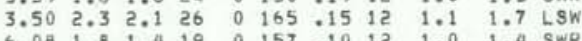

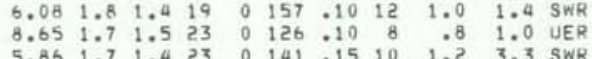
$\begin{array}{llllllllllll}7.35 & 1.6 & 21 & 0 & 153 & .09 & 8 & .6 & .8 & \text { KOA }\end{array}$ $5.312 .32 .12400142 .1911 \quad 1.3 \quad 1.7$ LSW

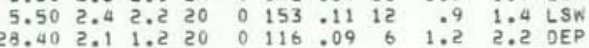
$\begin{array}{lllllllllll}30.12 & 2.1 & 1.2 & 19 & 0 & 85 & .06 & 5 & .9 & 1.7 & \text { DEP }\end{array}$

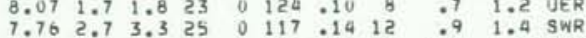

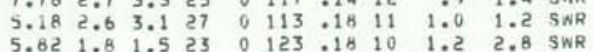
$\begin{array}{llllllllllll}17.03 & 1.7 & 1.5 & 28 & 0 & 48 & .08 & 3 & .5 & .8 & .8 & \text { DEP }\end{array}$

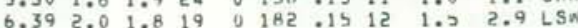

HVO EAKTHDUAKE SLMMARY LIST

patit so

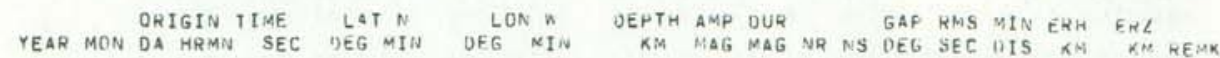

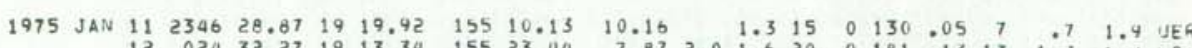

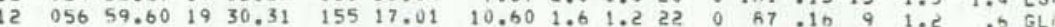

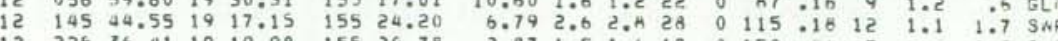
$\begin{array}{lllllllllllllllll}12 & 225 & 36.41 & 19 & 19.98 & 155 & 20.78 & 2.87 & 1.5 & 1.6 & 18 & 0 & 150 & .06 & 7 & .4 & 1.3\end{array}$ $\begin{array}{llllllllllllllllll}12 & 234 & 7.35 & 19 & 20.06 & 155 & 20.75 & 3.56 & 1.5 & 1.5 & 17 & 0 & 1408 & .07 & 7 & .0 & 1.3 & \text { SNE }\end{array}$

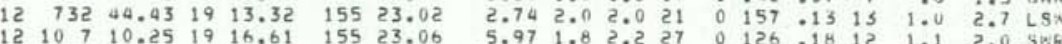

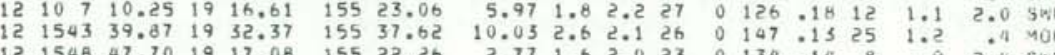

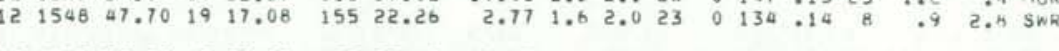

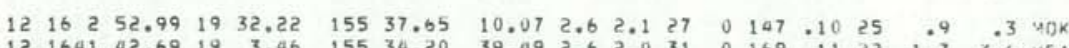

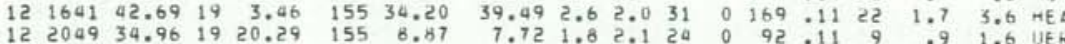

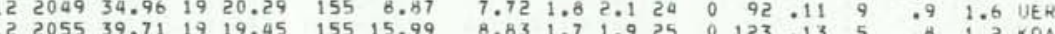

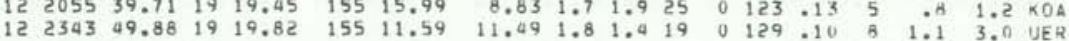

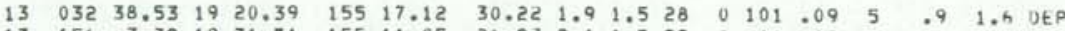

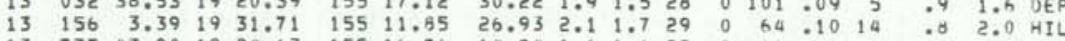

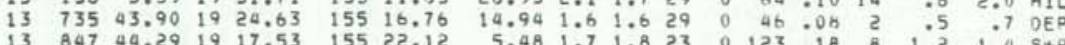

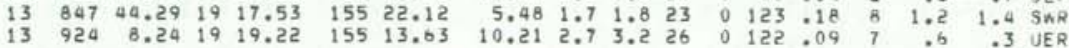

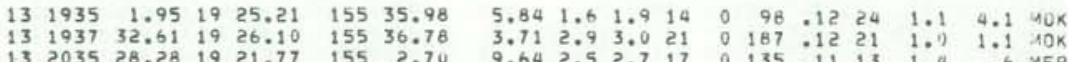

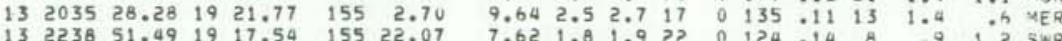

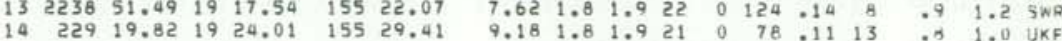

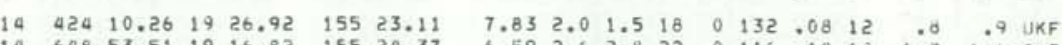

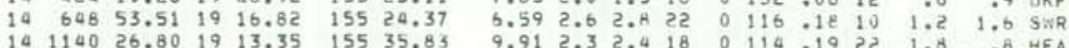

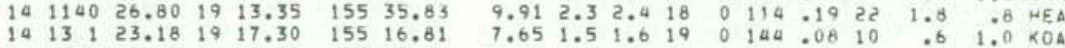

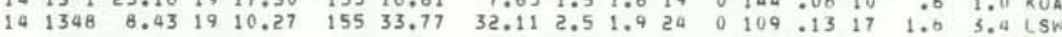

$\begin{array}{llllllllllllllllll}14 & 1513 & 52.31 & 19 & 24.75 & 155 & 16.77 & 13.65 & 2.0 & 2.0 & 21 & 0 & 73 & .06 & 2 & .5 & .4 & \text { DEP }\end{array}$

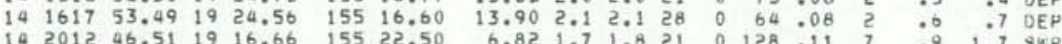

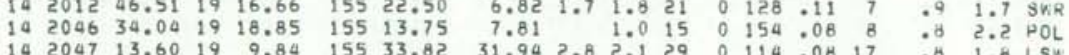

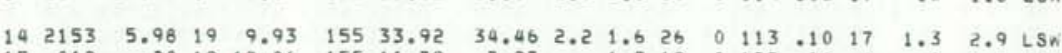

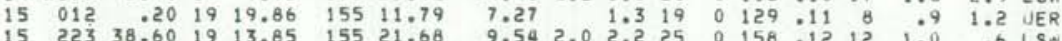

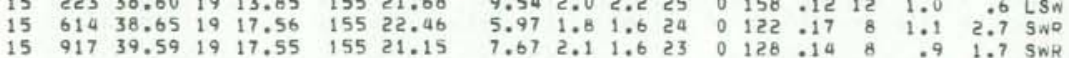
$\begin{array}{llllllllllllllllll}15 & 1024 & 59.49 & 19 & 24.80 & 155 & 16.49 & 14.12 & 2.9 & 2.7 & 19 & 0 & 47 & .08 & 2 & .7 & . \text {. D DEP }\end{array}$

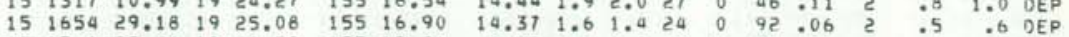

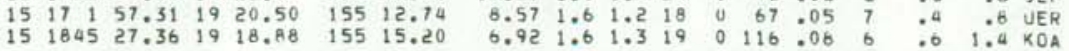

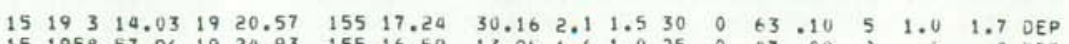

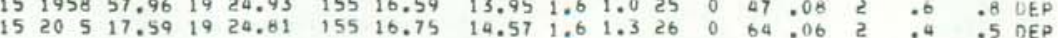




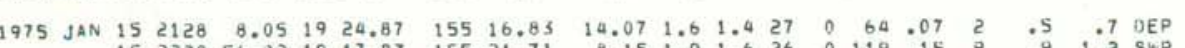

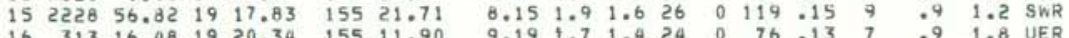

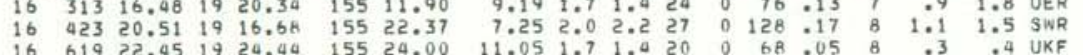
$\begin{array}{lllllllllllllllll}16 & 631 & 21.10 & 19 & 18.61 & 155 & 15.03 & 7.78 & 1.1 & 19 & 0 & 122 & .07 & 6 & .5 & 1.2 & \text { KOA }\end{array}$

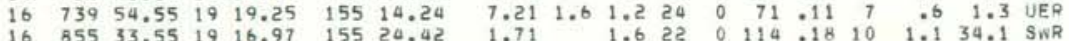

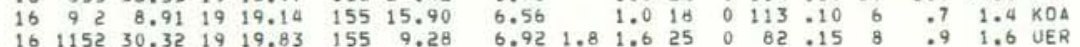

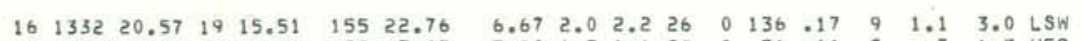

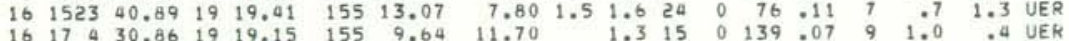

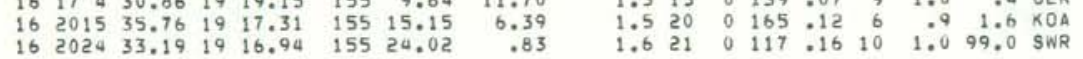

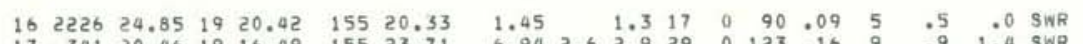

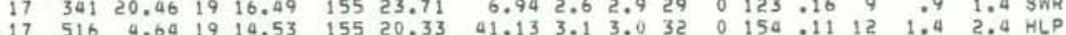

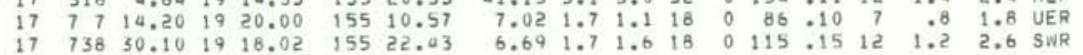

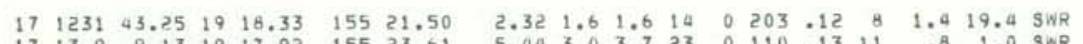

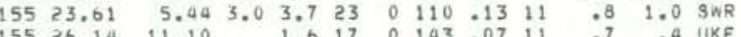

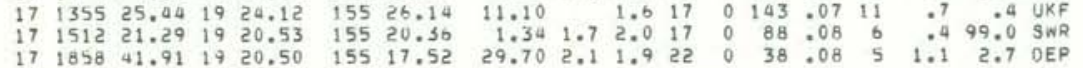

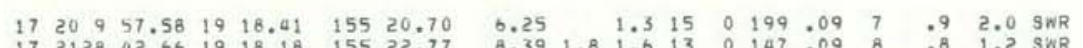

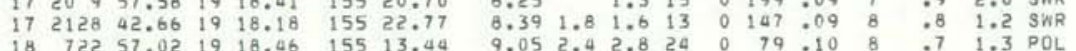

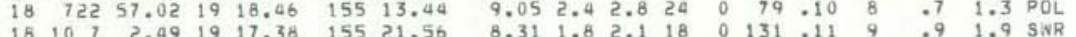

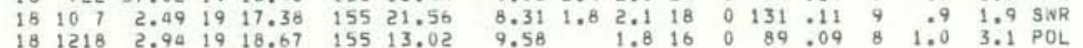
$\begin{array}{lllllllllllllllllll}18 & 1223 & 28.84 & 19 & 25.91 & 155 & 25.01 & 12.02 & 1.7 & 1.8 & 16 & 0 & 105 & .07 & 8 & .8 & .4 & \text { UKF }\end{array}$

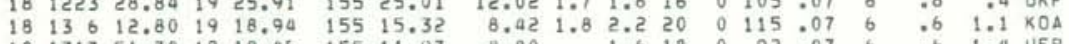

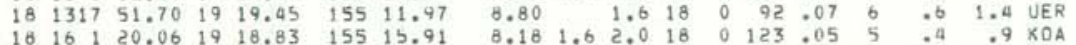

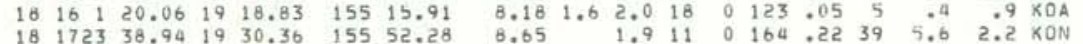

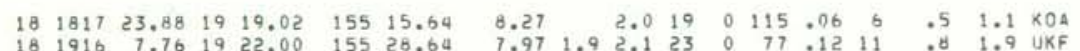

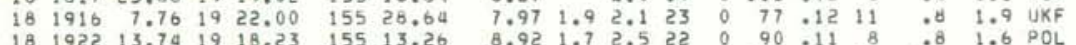

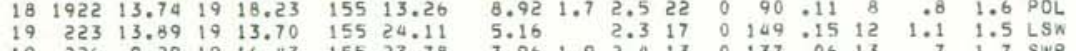

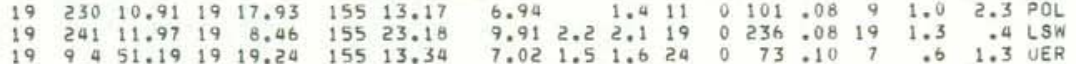

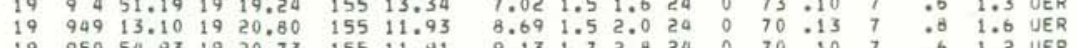

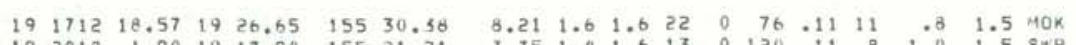

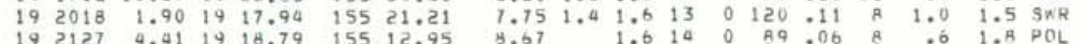

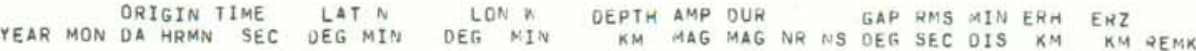

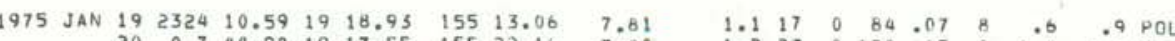

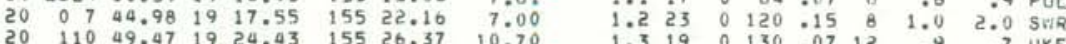

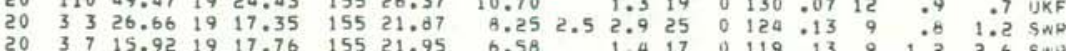
$\begin{array}{llllllllllllllllll}20 & 4 & 0 & 58.38 & 19 & 17.97 & 155 & 21.33 & 6.91 & 2.2 & 2.0 & 24 & 0 & 119 & .12 & 8 & .8 & 1.2\end{array}$

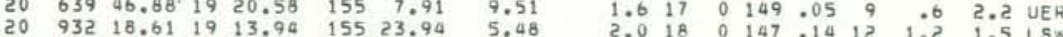

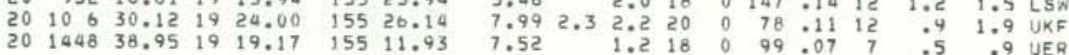

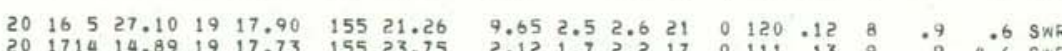

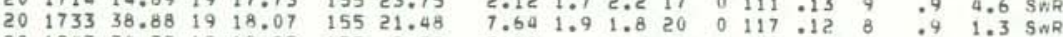

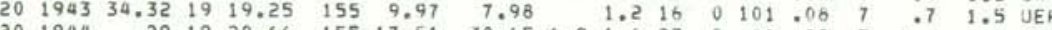

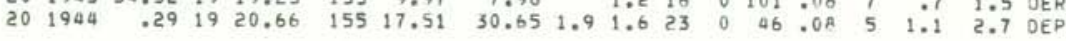

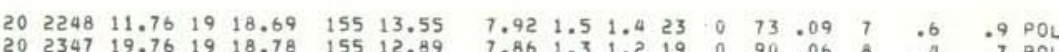

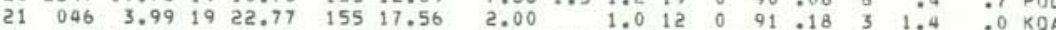

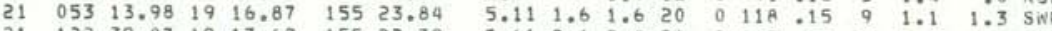
$21 \quad 12239.071917 .62 \quad 15522.79 \quad 7.612 .12 .42400117 .18,1.2 \quad 1.6$ SwR

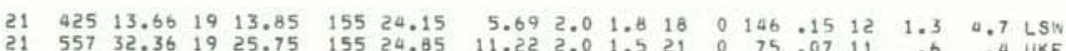

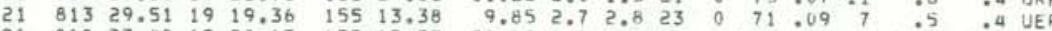

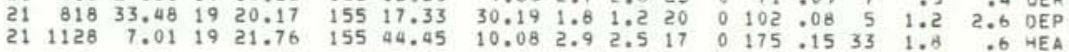

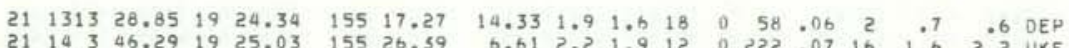

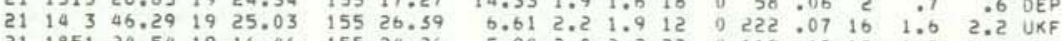

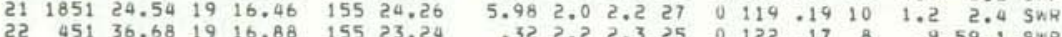

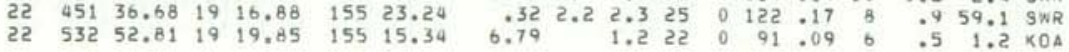

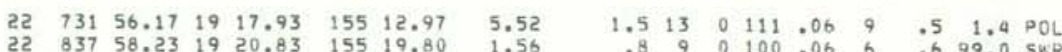

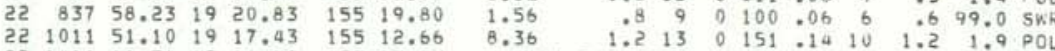

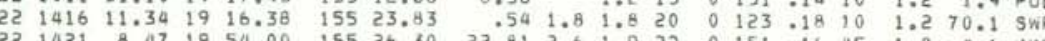

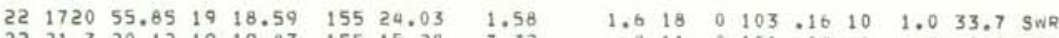

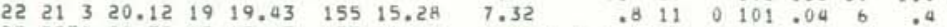

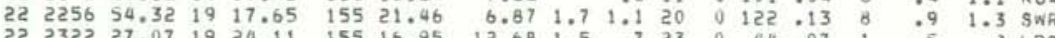

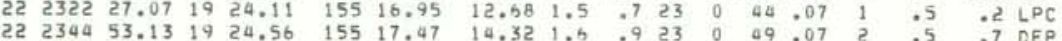

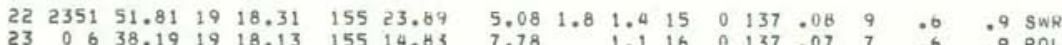

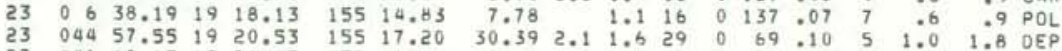

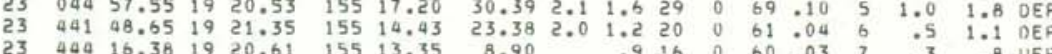

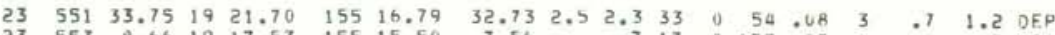

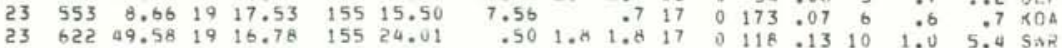


HVO EAKTHIJUARE. SUMAAGY LIST

DAGE 33 ORIGIN TIME LATN LOW A DEPTH AMP DUR GAP KMS MIN ERH FRZ

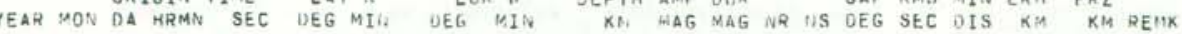
1975 JAN $23 \quad 746 \quad 7.01 \quad 19 \quad 19.35 \quad 15520.43$

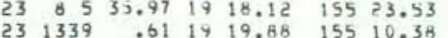
$23 \quad 134520.9819 \quad 18.24 \quad 15513.69$

$23 \quad 16 \quad 5 \quad 58.94 \quad 19 \quad 16.64 \quad 15523.67$ $23 \quad 173 \quad 53.891915 .58$ 155 22.45 $\begin{array}{lllllll}23 & 1716 & 50.07 & 19 & 19.13 & 155 & 15.25 \\ 23 & 1936 & 26.50 & 19 & 33.96 & 155 & 36.52\end{array}$

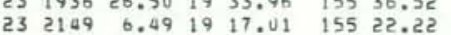

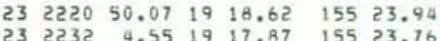

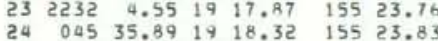
$24 \quad 16 \quad 42.371918 .97 \quad 15513.54$

24 a $951.13 \quad 1932.90 \quad 155 \quad 32.14$ 24 429 41.041914 .86 155 19.08

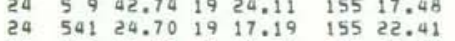

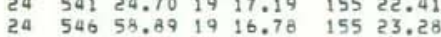

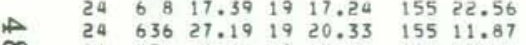
$\begin{array}{lllllll}24 & 734 & 48.92 & 19 & 17.86 & 155 & 13.87 \\ 24 & 95 & 39.21 & 19 & 19.00 & 155 & 13.34\end{array}$ $24922 \quad 36.78 \quad 1924.39 \quad 15517.28$ $24 \quad 1051 \quad 34.09 \quad 19 \quad 17.86 \quad 155 \quad 14.47$ $\begin{array}{lllllll}24 & 1133 & 36.50 & 19 & 17.79 & 155 & 10.79 \\ 24 & 1224 & 12.21 & 19 & 16.70 & 155 & 21.78\end{array}$ $\begin{array}{llllll}24 & 1258 \quad 59.63 & 19 & 17.68 & 155 & 14.30\end{array}$

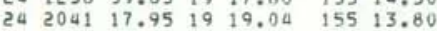

$\begin{array}{lllllll}24 & 2112 & 22.26 & 19 & 19.70 & 155 & 11.84\end{array}$

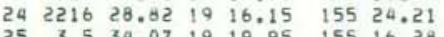
$\begin{array}{lllllll}25 & 336 & 27.54 & 19 & 18.33 & 155 & 13.58\end{array}$

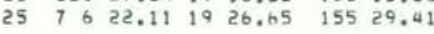
$\begin{array}{lllllll}25 & 748 & 3.20 & 19 & 17.85 & 155 & 14.58\end{array}$

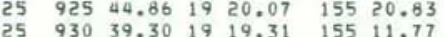
$\begin{array}{lllllll}25 & 1131 & 5.46 & 19 & 17.87 & 155 & 21.93 \\ 25 & 1213 & 45.33 & 19 & 19.02 & 155 & .89\end{array}$

$\begin{array}{lllllll}25 & 1343 & 52.52 & 19 & 17.15 & 155 & 22.56\end{array}$

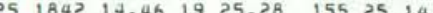
$\begin{array}{llllllll}25 & 19 & 4 & 40.06 & 19 & 22.04 & 155 & 18.17 \\ 25 & 20 & 3 & 54.67 & 19 & 22.00 & 155 & 4.6\end{array}$

$\begin{array}{lllllll}25 & 2236 & 18.77 & 19 & 19.26 & 155 & 16.07\end{array}$

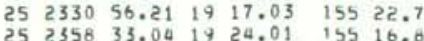

$\begin{array}{llllllllllll}4.68 & 1.5 & 1.6 & 18 & 0 & 135 & .14 & 5 & 1.0 & 1.6 & \text { SNR }\end{array}$

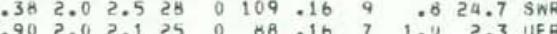

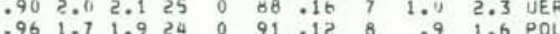

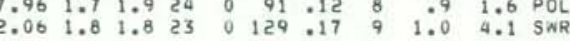

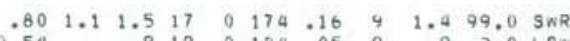
$\begin{array}{lllllllllll}.54 & .9 & 19 & 0 & 184 & 165 & 9 & .9 & 9.0 & \text { LS }\end{array}$

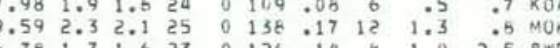
$1.791 .71 .6 \quad 19 \quad 0 \quad 103.14 \quad 9 \quad .434 .8$ SWR

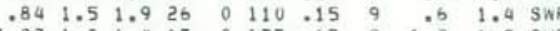

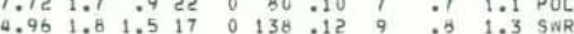
$\begin{array}{llllllllllll}39.82 & 2.5 & 1.6 & 12 & 0 & 276 & .15 & 22 & 16.0 & 24.6 & \text { MOK } \\ 27.20 & & & 20 & 0 & 150 & -13 & 11 & 1.9 & 3.5 & \text { HLP }\end{array}$ $\begin{array}{lllllllllll}14.30 & 1.7 & 1.7 & 28 & 0 & 35 & .08 & 2 & .5 & .7 & 0 E P\end{array}$

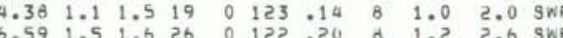
$\begin{array}{rrrrrrrrrrr}4.36 & 1.6 & 1.8 & 22 & 0 & 122 & .18 & 7 & .9 & 1.8 & \text { SWR } \\ 0.29 & 2.8 & 3.2 & 28 & 0 & 76 & .09 & 7 & .6 & .4 & \text { UER }\end{array}$

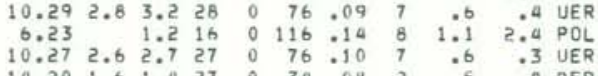
$\begin{array}{llll}.50 & 1.6 & 1.4 & 27\end{array}$ $\begin{array}{rrrrrrrrrrr}9.72 & 2.8 & 2.9 & 26 & 0 & 101 & .11 & 7 & .6 & .9 & \text { POL } \\ 4.22 & 1.3 & 9 & 17 & 0 & 119 & .17 & 4 & 1.1 & 2.0 & \text { SWR }\end{array}$

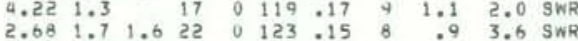

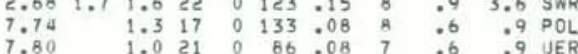
$\begin{array}{llllllllllll}9.90 & 2.2 & 2.2 & 27 & 0 & 87 & .12 & 6 & .7 & 1.2 & \text { UER }\end{array}$ $\begin{array}{llllllllll}5.97 & 1.8 & 2.2 & 26 & 0 & 122 & .16 & 10 & 1.1 & 1.9\end{array}$

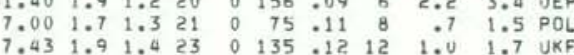

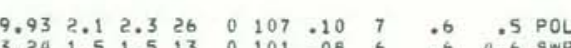
$\begin{array}{lllllllllll}3.24 & 1.5 & 1.5 & 13 & 0 & 101 & 0.08 & 6 & .6 & 4.6 & \text { SWR } \\ 4.94 & 1.4 & .7 & 31 & 0 & 97 & 0.0 & 7 & .6 & .0 & \text { UER }\end{array}$

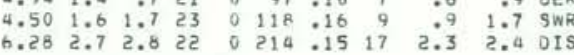

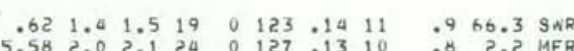

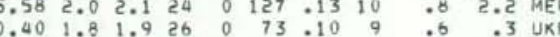

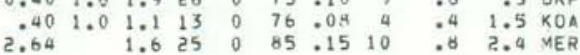
$\begin{array}{llllllllllll}8.09 & 1.4 & 25 & 0 & 110 & .12 & \text { b } & .7 & 1.1 & \mathrm{kOA}\end{array}$

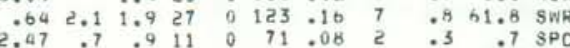

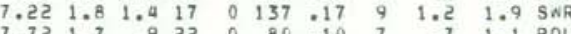

HVO EARTHEULARE SURNAKY LIST

PAGE 34

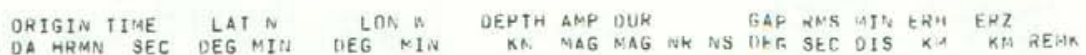

1975 JAN 26 O42 $34.091916 .55 \quad 15520.01$ $\begin{array}{lllllll}26 & 053 & 15.10 & 19 & 16.56 & 155 & 24.13 \\ 26 & 146 & 28.17 & 19 & 19.55 & 155 & 15.80\end{array}$ $\begin{array}{lllllll}26 & 146 & 28.17 & 19 & 19.15 & 155 & 15.80 \\ 26 & 147 & 24.66 & 19 & 16.36 & 155 & 23.75\end{array}$

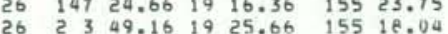

$\begin{array}{lllllll}26 & 322 & 38.67 & 19 & 16.20 & 155 & 23.92\end{array}$ $\begin{array}{lllllll}26 & 348 & 52.71 & 19 & 20.14 & 155 & 11.84\end{array}$

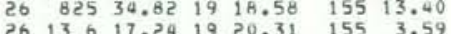
$\begin{array}{llllllll}26 & 13 & 6 & 17.24 & 19 & 20.31 & 155 & 3.59 \\ 26 & 1310 & 1.43 & 19 & 21.41 & 155 & 4.11\end{array}$

$26 \quad 151455.2919 \quad 19.85 \quad 155 \quad 9.41$ $\begin{array}{lllllll}26 & 1822 & 19.53 & 19 & 16.88 & 155 & 23.75\end{array}$

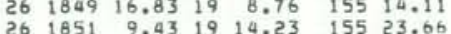
$2619423.561916 .55 \quad 15524.04$

$\begin{array}{lllllll}26 & 1923 & 7.20 & 19 & 18.06 & 155 & 21.80\end{array}$

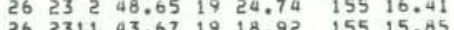

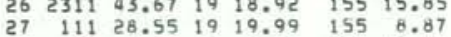

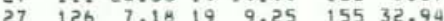

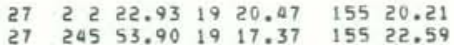

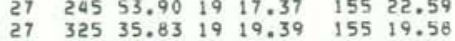
$\begin{array}{lllllll}27 & 325 & 35.83 & 19 & 19.39 & 155 & 19.58 \\ 27 & 532 & 57.08 & 19 & 21.20 & 155 & 19.18\end{array}$

$\begin{array}{lllllll}27 & 714 & 9.86 \quad 19 & 22.95 \quad 155 & 23.95\end{array}$ $\begin{array}{lllllll}27 & 741 & 9.55 & 19 & 15.66 & 155 & 22.79 \\ 27 & 819 & 32.18 & 19 & 15.45 & 155 & 22.50\end{array}$

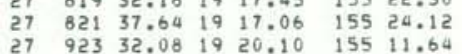
$\begin{array}{lrrrrrr}27 & 930 & 6.83 & 19 & 30.83 & 155 & 16.21\end{array}$

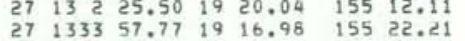
$271414 \quad 30.41 \quad 1917.64 \quad 15521.70$ $\begin{array}{llllllll}27 & 15 & 4 & 39.62 & 19 & 9.88 & 155 & 6.90\end{array}$ $27 \quad 1555 \quad 58.77 \quad 19 \quad 18.43 \quad 155 \quad 15.42$ ट7 161541.791918 .0915514 .62 $\begin{array}{lllllll}27 & 1726 & 57.19 & 19 & 18.98 & 155 & 12.65 \\ 27 & 1939 & 49.31 & 19 & 20.85 & 155 & 17.27\end{array}$

$\begin{array}{lllllll}27 & 2049 & 46.83 & 19 & 17.10 & 155 & 22.39\end{array}$

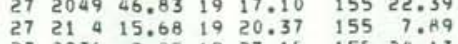

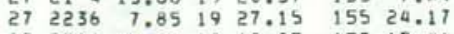

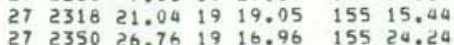
$\begin{array}{lllllll}27 & 2353 & 12.67 & 19 & 16.59 & 155 & 24.08\end{array}$

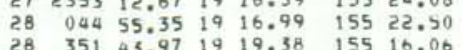

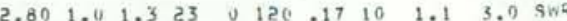

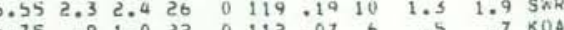

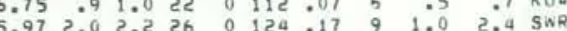

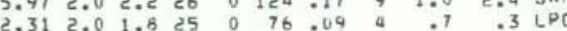
$\begin{array}{llllllllll}2.17 & 1.1 & 1.2 & 23 & 0 & 124 & .16 & 10 & 1.0 & \text { S.3 S SNR }\end{array}$

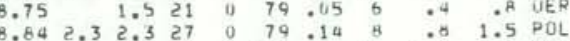

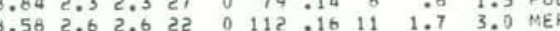

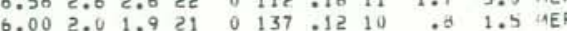
$\begin{array}{rrrrrrrrrrr}8.00 & 2.6 & 2.9 & 27 & 0 & 87 & .11 & 8 & .7 & .9 & \text { UER } \\ .03 & 1.7 & 1.9 & 26 & 0 & 119 & .17 & 9 & .9 & 58.1 & \text { SNR }\end{array}$ $36.06 \quad 1.71 .92600204 .0919 \quad 1.4 \quad 2.7$ PPL $\begin{array}{lllllllllll}5.21 & 2.1 & 1.8 & 27 & 0 & 142 & .16 & 11 & 1.0 & 1.4 & \text { LSW } \\ .97 & 1.0 & 1.4 & 24 & 0 & 120 & .19 & 10 & 1.1 & 34.2 & \text { SWR }\end{array}$ $\begin{array}{lllllllllll}6.83 & 1.7 & 1.6 & 27 & 0 & 116 & .19 & 9 & 1.0 & 1.7 & \text { SNR }\end{array}$

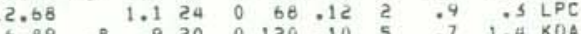
$6.762 .32 .94240074 .13 \quad 9 \quad: 92.11$ UER $\begin{array}{rrrrrrrrrrr}88 & .8 & 1.5 & 14 & 0 & 87 & .09 & 5 & .5 & .0 & 5 m\end{array}$

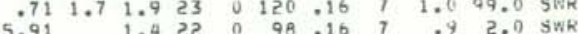

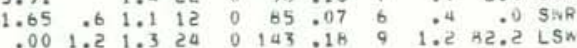
$\begin{array}{lllllllllll}1.86 & 1.3 & 1.4 & 19 & 0 & 69 & .06 & 8 & .4 & .4 & 4 K F\end{array}$

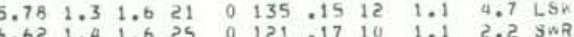

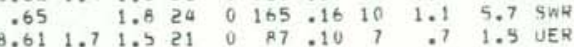

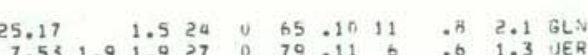
090

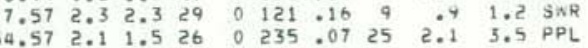
$\begin{array}{llllllllllll}6.83 & 1.4 & 1.4 & 26 & 0 & 106 & .11 & 0 & .6 & .9 & \text { KOA } & 0\end{array}$ $\begin{array}{llllllllllll}7.23 & 1.9 & 2.3 & 29 & 0 & 153 & .13 & 10 & 9 & 9 & 1.3 & \text { LSW }\end{array}$

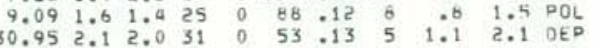
$\begin{array}{lllllllllllll}6.26 & 1.7 & 1.7 & 20 & 0 & 124 & .12 & 6 & .0 & 2.2 & \text { SNR }\end{array}$ 6.921 .91 .6210075 .0910 .5 1.6 UKF

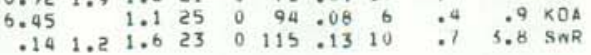

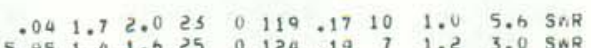

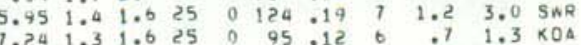


hVO EARTHQUAKE SUMMARY LIST

PAGE 35 ORIGIN TIME LATN LON W DEPTH AMP OUR GAP RMS MIN ERM ERZ YEAR MON DA HRMN SEC DEG MIN DEG NIN KM MAG MAG NR US DEG SEC DIS KM KM REMK

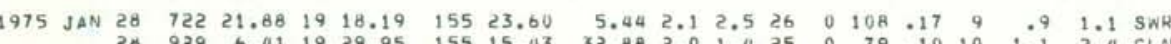

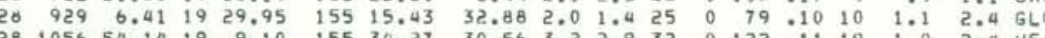

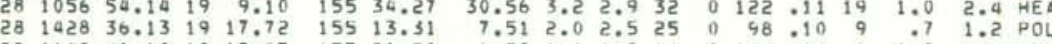

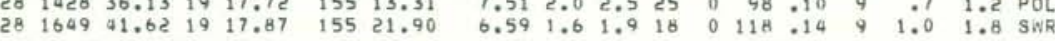
$\begin{array}{llllllllllllllllll}28 & 1824 & 56.34 & 19 & 19.43 & 155 & 13.89 & 8.47 & 1.6 & 1.5 & 24 & 0 & 63 & .11 & 6 & .7 & 1.4 & \text { UER }\end{array}$

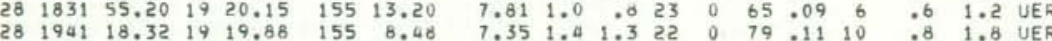

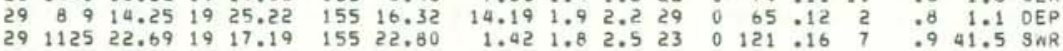

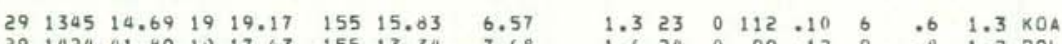

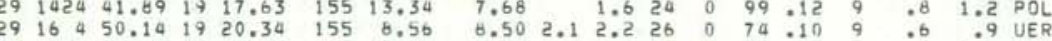

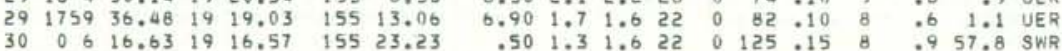
$\begin{array}{lllllllllllllllll}30 & 019 & 47.15 & 19 & 17.86 & 155 & 21.51 & 7.32 & 1.8 & 2.1 & 25 & 0 & 120 & .14 & 8 & .9 & 1.2\end{array}$ SNR $\begin{array}{lllllllllllllllll}30 & 6 & 5 & 1.50 & 19 & 18.66 & 155 & 15.21 & 7.73 & 1.5 & 20 & 0 & 116 & .09 & 6 & .6 & 1.1 \\ 3004\end{array}$

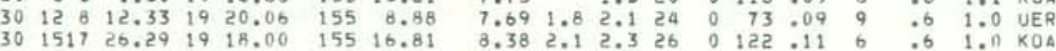

$30.738 \quad 18.8919 \quad 19.72 \quad 155 \quad 8.60$ $30 \quad 1747 \quad 56.3919 \quad 19.800155 \quad 8.72$ $30174924.03 \quad 1932.47 \quad 15533.01$

$\begin{array}{llllll}30 & 1853 & 57.71 & 19 & 19.26 \quad 155 \quad 13.62\end{array}$ $30 \quad 185929.81 \quad 19 \quad 18.45 \quad 155 \quad 13.68$

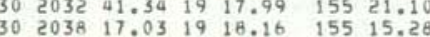
$30210 \quad 4.251919 .49 \quad 15513.24$

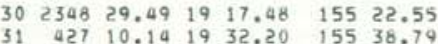
$31 \quad 442 \quad 6.991916 .33 \quad 15523.6$

31 542 45.491924 .08 155 17.47

$31 \quad 1137 \quad 7.951916 .54 \quad 15513.26$

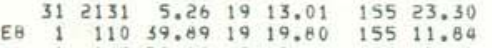

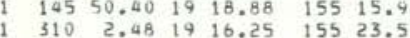

$1 \quad 428 \quad 31.46 \quad 19 \quad 16.63 \quad 15522.92$ 172621.151923 .69 155 29.36

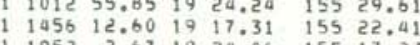

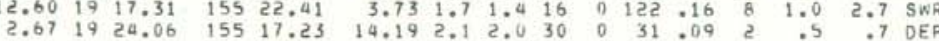

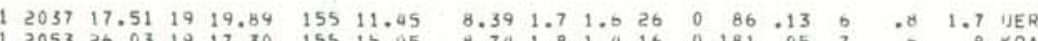

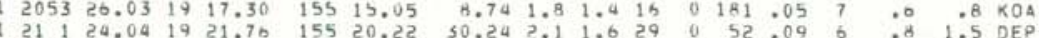

HVO EARTHDUAKE SUMMARY LIST

PAGE 36 ORIGIN TIME LATN LONN DEPTH AMP DUR GAP HMS AIN ERH ERZ

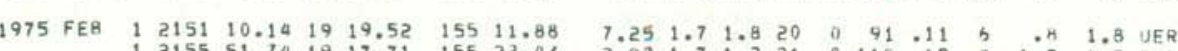

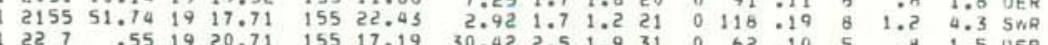

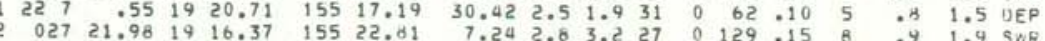

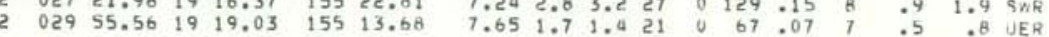

$\begin{array}{lllllllllllllllllll}2 & 042 & 55.48 & 19 & 25.15 & 155 & 29.09 & 8.76 & 1.9 & 1.8 & 15 & 0 & 122 & .09 & 13 & .8 & 2.3 & \text { UKF }\end{array}$

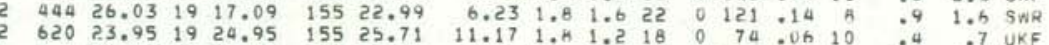

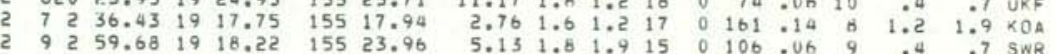

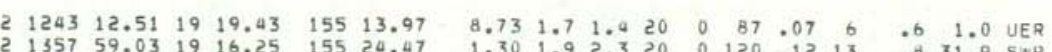

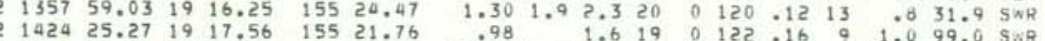

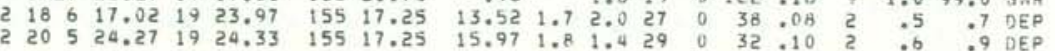
$\begin{array}{llllllllllllllllll}2 & 2344 & 24.82 & 19 & 20.66 & 155 & 11.85 & 9.43 & 1.7 & 1.7 & 21 & 0 & 71 & .10 & 7 & .7 & 1.5 \text { UER }\end{array}$

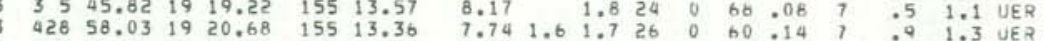

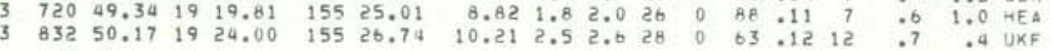

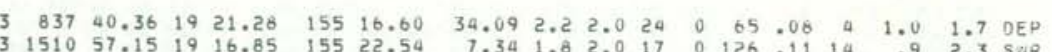

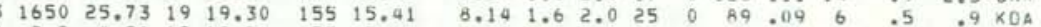

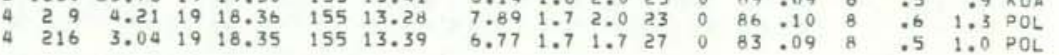
$\begin{array}{llllllllllllllllllll}3 & 3 & 14.46 & 19 & 17.15 & 155 & 22.56 & 6.66 & 1.8 & 2.1 & 22 & 0 & 123 & .13 & 7 & .9 & 2.4 & \text { SWR }\end{array}$ 433
4

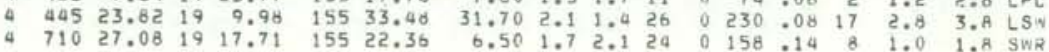

$\begin{array}{lllllllllllllllll}4 & 1210 & 3.56 & 19 & 20.26 & 155 & 17.58 & 29.86 & 2.1 & 1.7 & 25 & 0 & 70 & .08 & 5 & 9 & 1.6\end{array}$

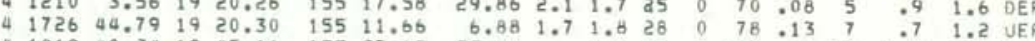

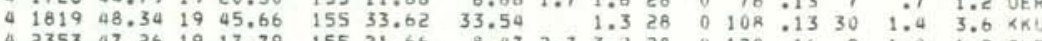

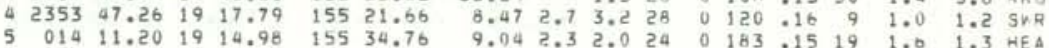

$\begin{array}{llllllllllllllllll}5 & 022 & 36.49 & 19 & 17.59 & 155 & 23.31 & 6.66 & 1.8 & 1.7 & 20 & 0 & 115 & -16 & 11 & 1.2 & 3.2 & \text { SNR }\end{array}$

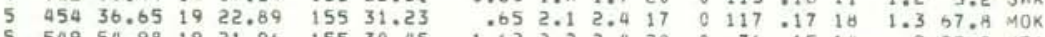

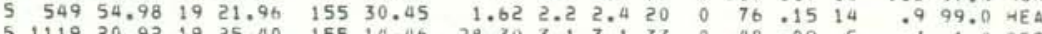

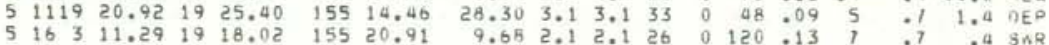

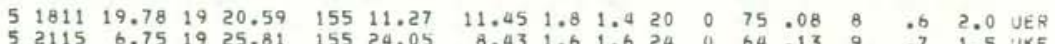

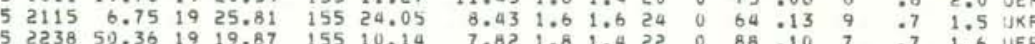

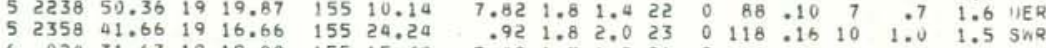

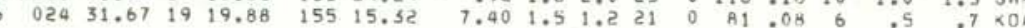

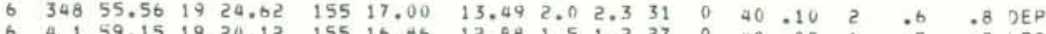

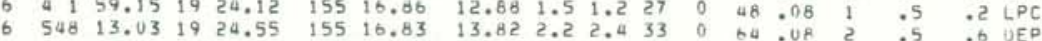




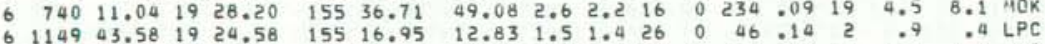

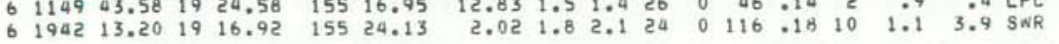
$\begin{array}{lllllllllllllllllll}7 & 1 & 57.82 & 19 & 20.67 & 155 & 17.31 & 50.20 & 2.3 & 2.5 & 33 & 0 & 54 & .16 & 5 & .4 & 1.4 & \text { OEP } & \end{array}$

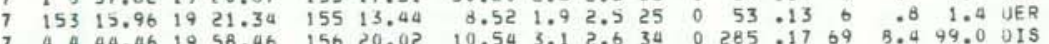

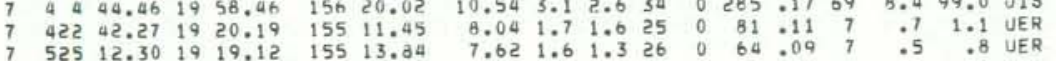

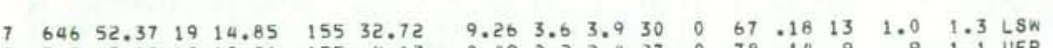

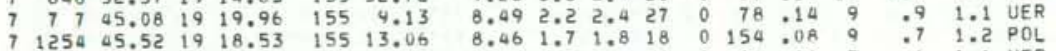

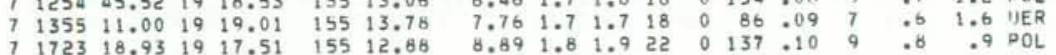

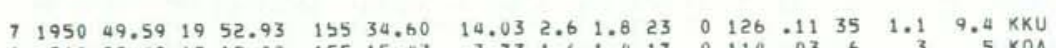

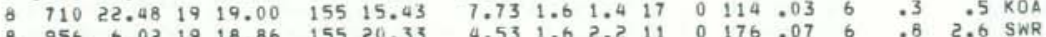

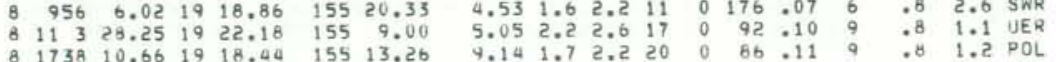

a) $2232 \quad 32.511918 .20 \quad 15515.69$ $913455.351919 .64 \quad 15512.61$

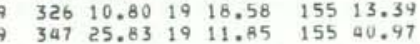

9
$912 \quad 47.92 \quad 19 \quad 17.64 \quad 155 \quad 22.65$ $740250.191919 .69 \quad 15511.9$ $911237.36 \quad 1917.98 \quad 15513.26$ $91117 \quad 3.23 \quad 19 \quad 17.31 \quad 155 \quad 13.25$

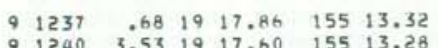

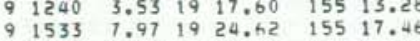

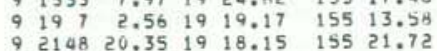

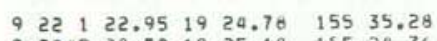
$9 \begin{aligned} & 254528.72 \\ & 9\end{aligned} 2925.18 \quad 15524.36$ 923 4 $34.22 \quad 19 \quad 10.31 \quad 155 \quad 8.64$ $10 \quad 25131.391917 .08 \quad 15522.37$

$\begin{array}{llllllll}10 \quad 6 \quad & 46.45 & 19 & 16.67 & 155 & 22.62\end{array}$ $10124746.431917 .48 \quad 15522.11$

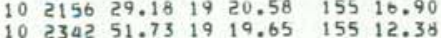
$1100342.921924 .55 \quad 15516.89$

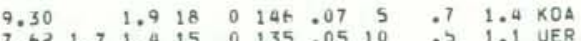

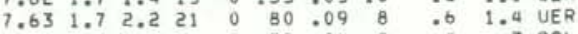

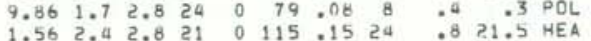
$\begin{array}{lllllllllll}6.66 & 1.8 & 2.3 & 18 & 0 & 158 & .14 & 7 & 1.2 & 1.8 & \text { SWR }\end{array}$

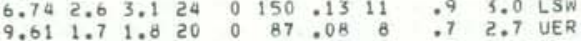

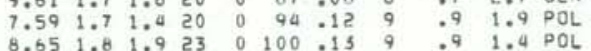
$0.44 \quad 1.8 \quad 1.6 \quad 18 \quad 0 \quad 143 \quad 16 \quad 9 \quad 1.6 \quad 9004$

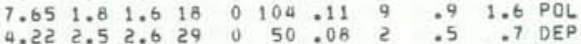

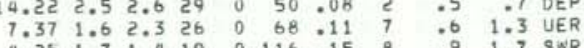
$\begin{array}{lllllllllll}5.42 & 2.7 & 3.0 & 19 & 0 & 143 & .12 & 23 & 1.3 & 10.1 & \text { MOK }\end{array}$

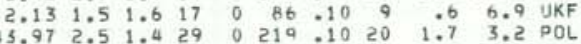

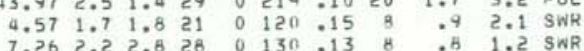

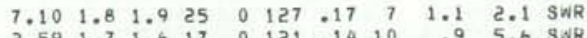

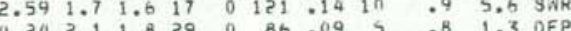

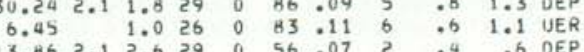

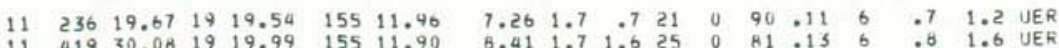

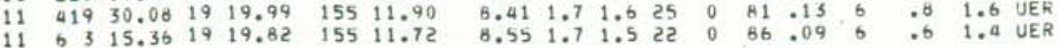

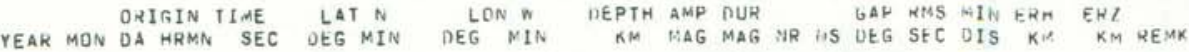

1975 FEB $11 \quad 626 \quad 55.98 \quad 19 \quad 27.75 \quad 155 \quad 36.01$

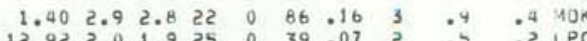

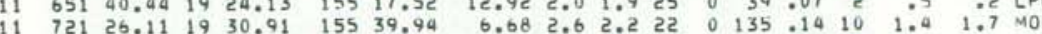

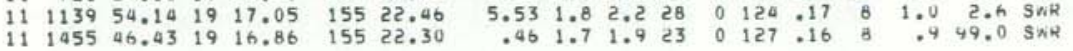

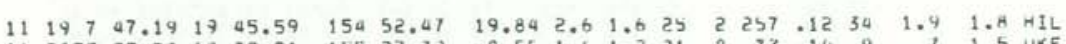

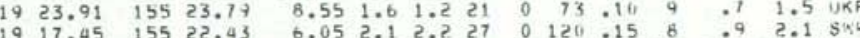

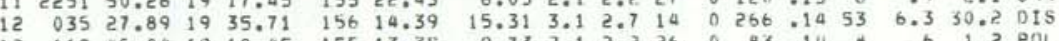
$12 \quad 119 \quad 45.04 \quad 19 \quad 18.45 \quad 155 \quad 13.35$

$12 \quad 338 \quad 14.19 \quad 19 \quad 17.89 \quad 155 \quad 14.65$ $12 \quad 445 \quad 4.96 \quad 19 \quad 27.15 \quad 155 \quad 27.60$ $12 \quad 448 \quad 9.26 \quad 19 \quad 17.95 \quad 15514.46$

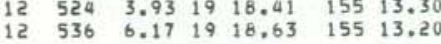

$\begin{array}{lllllll}12 & 544 & 22.45 & 19 & 18.54 & 155 & 13.41\end{array}$

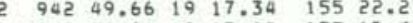

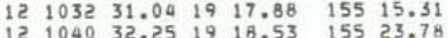

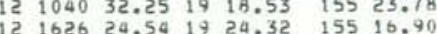

$\begin{array}{lllllll}12 & 1630 & 23.21 & 19 & 20.67 & 155 & 12.46\end{array}$ $12170 \quad 11.57 \quad 19 \quad 9.68 \quad 15532.52$

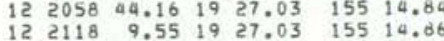
$13 \quad 748 \quad 42.31 \quad 1911.84 \quad 155 \quad 32.54$

$\begin{array}{lllllll}13 & 755 & 46.91 & 19 & 11.54 & 155 & 32.24\end{array}$ $\begin{array}{lllllll}13 & 12 & 34.11 & 19 & 16.53 & 155 & 23.64 \\ 13 & 1243 & 13.92 & 19 & 16.42 & 155 & 23.41\end{array}$

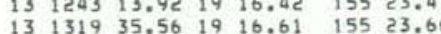
$\begin{array}{lllllll}13 & 1319 & 35.56 & 19 & 16.61 & 155 & 23.66 \\ 13 & 1359 & 18.08 & 19 & 16.81 & 155 & 23.50\end{array}$

$\begin{array}{lllllll}13 & 1522 & 27.73 & 18 & 52.41 & 155 & 12.09\end{array}$ $\begin{array}{lllllll}13 & 1610 & 42.72 & 19 & 18.04 & 155 & 23.63\end{array}$

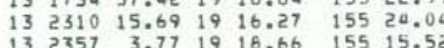
$\begin{array}{lllllll}14 & 028 & 27.66 & 19 & 16.95 & 155 & 23.05\end{array}$

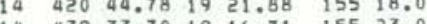
$14 \quad 43933.701916 .71 \quad 15523.03$ $14 \quad 633 \quad 15.891920 .83 \quad 15517.24$

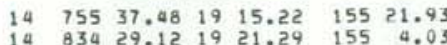
14 834 29.121921 .29 155 4.03 $14.1127 \quad 32.85 \quad 19 \quad 18.14 \quad 15515.26$ $14153924.8919 \quad 30.73 \quad 15539.55$ $141935 \quad 16.07 \quad 19 \quad 19.04 \quad 155 \quad 15.5$ $\begin{array}{lllllll}14 & 1936 & 35.74 & 19 & 16.85 & 155 & 21.91 \\ 14 & 2122 & 12.01 & 19 & 20.32 & 155 & 50.07\end{array}$ $\begin{array}{lllllllllllll}6.71 & 1.7 & 1.9 & 21 & 0 & 134 & .12 & 7 & .8 & 1.8 & \text { POL }\end{array}$ $\begin{array}{lllllllllll}9.25 & 1.8 & 1.6 & 25 & 0 & 75 & .11 & 12 & .6 & 1.1 & \text { UKF }\end{array}$

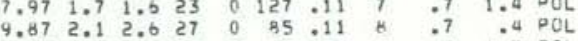

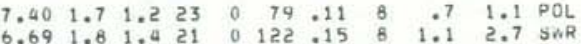

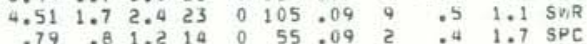

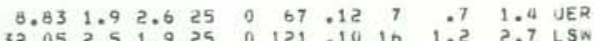

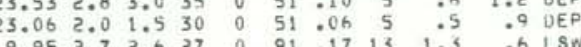

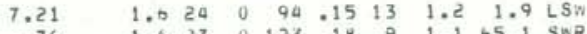

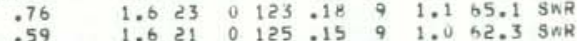
$5.38 \quad 2.226 \quad 0122.18$ 9 $1.0 \quad 1.4$ SWR $\begin{array}{lllllllllll}48.43 & 1.6 & 28 & 2 & 284 & .09 & 47 & 2.0 & 2.3 & \text { PPL }\end{array}$

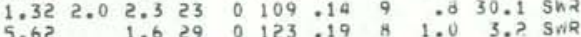

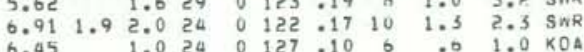
$\begin{array}{llllllllll}.51 & 1.4 & 20 & 0 & 140 & .15 & 8 & 1.2 & 66.1 & \text { SNR }\end{array}$

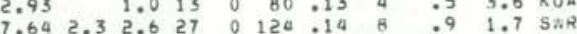

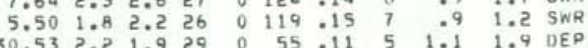
$\begin{array}{llllllllllllllllllll}7.82 & 1.9 & 21 & 0 & 147 & -10 & 10 & .8 & 2.0 & \text { LSW }\end{array}$ 6.481 .81 .918 o 77.1110 9 90 1.8 UER $\begin{array}{rrrrrrrrrrr}7.48 & .9 & .6 & 16 & 0 & 162 & .09 & 8 & .7 & .9 & K 04 \\ 8.37 & 2.8 & 2.5 & 23 & 0 & 79 & .16 & 27 & 1.0 & 1.9 & 40 K\end{array}$ $\begin{array}{llllllllll}8.84 & 1.8 & 2.2 & 25 & 0 & 95 & .14 & 6 & .8 & 1.2 \\ 604\end{array}$ $221.7 \quad 1.423$
0 $8.441 .7 \quad 1.62100119 .16$ 6 1.1 1., 16 KOA

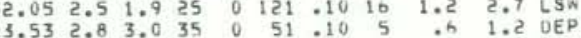

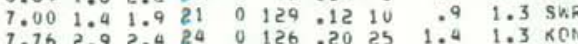


HVO EARTHGUARE SUMMARY LIST

PAGE 39

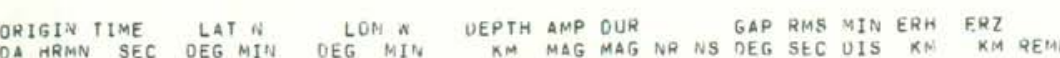

1975 FEB $142249 \quad 1.73 \quad 19 \quad 14.37 \quad 155 \quad 34.31$

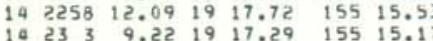
i4 $2356 \quad 40.671925 .70 \quad 15524.7$

$\begin{array}{lllllll}15 & 227 & 51.97 & 19 & 17.35 & 155 & 22.40 \\ 15 & 320 & 36.21 & 19 & 18.31 & 155 & 13.35\end{array}$ $\begin{array}{lllllll}15 & 320 & 36.21 & 19 & 18.31 & 155 & 13.35\end{array}$ $1532123.4419 \quad 18.73 \quad 155 \quad 13.30$ $\begin{array}{lllllll}15 & 348 & 12.99 & 19 & 18.71 & 155 & 13.33\end{array}$

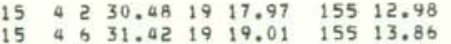
$15 \quad 441 \quad 47.041926 .53 \quad 15525.49$

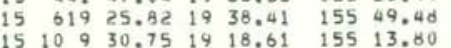
$\begin{array}{lllllll}15 & 1633 & 53.18 & 19 & 18.40 & 155 & 22.00\end{array}$ $15 \quad 214041.58 \quad 1920.57 \quad 155 \quad 13.25$ $16 \quad 62710.41 \quad 1920.11 \quad 15511.36$

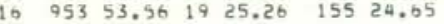

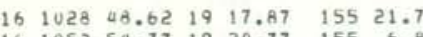

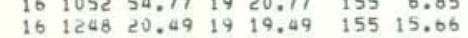
$\begin{array}{rrrrrrr}16 & 1320 & 11.29 & 19 & 16.84 & 155 & 21.93 \\ 16 & 1821 & 6.71 & 19 & 19.75 & 155 & 11.20\end{array}$

$\begin{array}{lllllll}16 & 2046 & 33.79 & 19 & 18.70 & 155 & 15.67\end{array}$

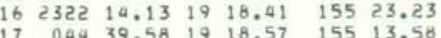

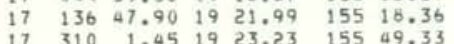
$\begin{array}{lllllll}17 & 635 & .11 & 19 & 18.95 & 155 & 15.59\end{array}$ $\begin{array}{lllllll}17 & 711 & 33.91 & 19 & 15.54 & 155 & 19.29\end{array}$ $\begin{array}{llllll}728 & 19.99 & 19 & 19.98 & 155 & 10.62\end{array}$

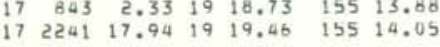
$\begin{array}{llllllll}17 & 23 & 5 & 27.46 & 19 & 19.07 & 155 & 13.09\end{array}$ $\begin{array}{lllllll}18 & 046 & 16.26 & 19 & 20.29 & 155 & 25.00\end{array}$

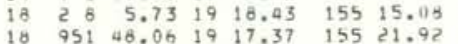
$\begin{array}{lllllll}18 & 1024 & 2.28 & 19 & 20.38 & 155 & 6.68\end{array}$

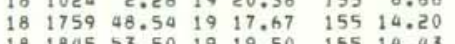
$\begin{array}{lllllll}18 & 1845 & 53.50 & 19 & 19.50 & 155 & 14.43 \\ 18 & 1933 & 38.48 & 19 & 29.49 & 155 & 39.83\end{array}$ $\begin{array}{lllllll}18 & 1933 & 38.48 & 19 & 29.49 & 155 & 39.83 \\ 18 & 2347 & 36.04 & 19 & 24.57 & 155 & 16.57\end{array}$

$\begin{array}{llllllll}19 & 0 & 1 & 46.66 & 19 & 24.57 & 155 & 16.57\end{array}$

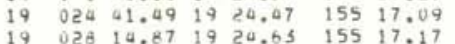
$\begin{array}{rrrrrrrrrrr}8.42 & 2.3 & 1.9 & 23 & 0 & 116 & 0.17 & 22 & 1.3 & 1.9 & \text { HEA } \\ 5.98 & & 1.3 & 14 & 0 & 165 & 06 & 5 & .0 & 1.2 & K D O A\end{array}$

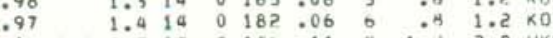

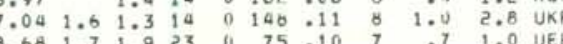
$\begin{array}{lllllllllllll}5.61 & 1.4 & 1.2 & 13 & 0 & 121 & .10 & 10 & .9 & 2.2 & \text { SWR }\end{array}$

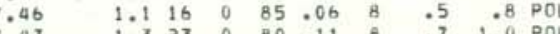
$\begin{array}{rrrrrrrrrr}0.47 & 1.3 & 23 & 0 & 80 & .11 & 6 & .7 & 1.0 & \text { POL } \\ 6.26 & .0 & 12 & 0 & 143 & .07 & 8 & 1.2 & 2.6 & \text { pOL }\end{array}$ $\begin{array}{lllllllllll}7.26 & 1.4 & 1.6 & 19 & 0 & 80 & .08 & 8 & .6 & 1.0 & \text { POL }\end{array}$ $\begin{array}{lllllllllll}8.01 & 1.0 & 10 & 0 & 127 & 10 & 9 & 1.7 & 3.8 & \text { POL }\end{array}$

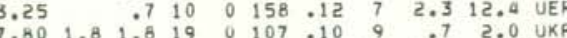

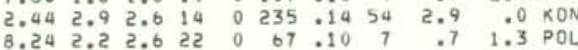
$\begin{array}{llllllllll}1.93 \quad 1.6 & 18 & 0 & 208 & .10 & 9 & 1.2 & 99.0 & \text { SNR }\end{array}$

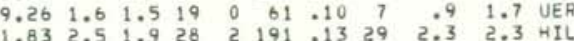

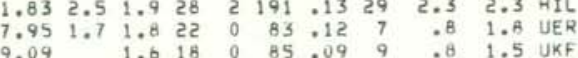
$\begin{array}{llllllllllll}5.80 & 1.7 & 2.0 & 18 & 0 & 119 & .12 & 9 & .4 & 1.7 & \text { SWR }\end{array}$

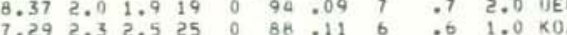

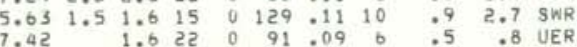
$\begin{array}{lllllllllll}7.04 & 1.6 & 1.6 & 22 & 0 & 127 & .14 & 5 & .9 & 1.1 & \times 04\end{array}$

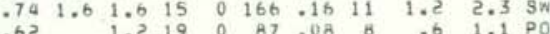

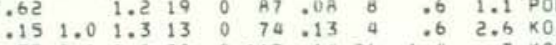

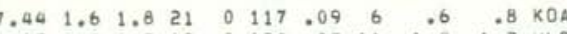
$\begin{array}{lllllllllll}15 & 1.8 & 1.9 & 18 & 0 & 190 & .09 & 11 & 1.0 & 1.2 & \text { HLP }\end{array}$

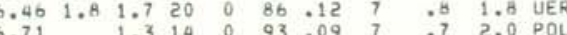
$\begin{array}{lllllllllll}6.71 & & 1.3 & 14 & 0 & 93 & .09 & 7 & 97 & 2.0 & \text { POL } \\ .68 & 1.6 & 2.2 & 21 & 0 & 84 & .14 & 6 & .9 & 1.2 & \text { UER }\end{array}$ $\begin{array}{llllllllllll}7.85 & 1.7 & 2.3 & 25 & 0 & 67 & .11 & 7 & .7 & 1.1 & \text { UEE }\end{array}$

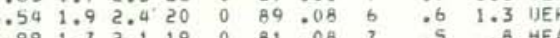

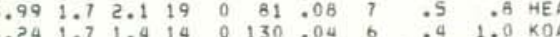

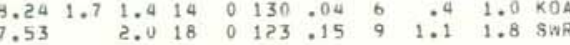
$\begin{array}{llllllllllllll}1.20 & 2.0 & 2.2 & 17 & 0 & 186 & .06 & 12 & .0 & .0 & .4 & \text { UER }\end{array}$ $\begin{array}{llllllllllll}8.48 & 1.5 & 18 & 0 & 141 & .08 & 8 & .6 & 1.0 & \text { POL }\end{array}$ $\begin{array}{rrrrrrrrrrr}.41 & 1.6 & 1.8 & 22 & 0 & 72 & .09 & 6 & .6 & 1.3 & \text { UER } \\ 53 & 2.3 & 2.0 & 13 & 0 & 129 & .16 & 27 & 1.5 & 5.7 & 40 K\end{array}$ $.83 \quad .31 .3$ 9 0 Q 92.09 a 1.5 . $9 \mathrm{SPC}$ $\begin{array}{llllllllllll}1.34 & .4 & .8 & 8 & 0 & 95 & 0.05 & 5 & .4 & .5 & 5 P C\end{array}$

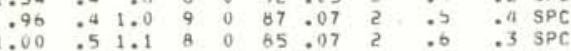

HVO EARTHQUAKE SUMMAHY LIST

PAGE 40

ORIGIN TIME LATN LON W UEPTH AMP DUR GAP RMS MIN ERM ERL

1975 FEB 19 029 $9.66 \quad 1924.59 \quad 155 \quad 17.04$

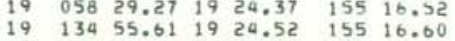
19 137 $15.051924 .52 \quad 15516.60$

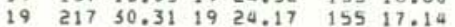
$\begin{array}{lllllll}19 & 220 & 15.96 & 19 & 24.40 & 155 & 17.28\end{array}$ 19.103026 .5019 18.76 15513.59 $191522 \quad 7.841924 .35 \quad 15516.11$ $\begin{array}{llllll}19 & 17 & 43.23 & 19 & 10.09 & 155 \quad 33.17\end{array}$ $\begin{array}{rrrrrrr}19 & 17 & 43.23 & 19 & 10.09 & 155 & 33.17 \\ 20 & 037 & 22.84 & 19 & 26.70 & 155 & 35.23\end{array}$ $20 \quad 915 \quad 45.46 \quad 1926.15 \quad 155 \quad 37.03$ $20 \quad 1016 \quad 23.6119 \quad 16.22 \quad 15523.82$ $\begin{array}{lllllll}20 & 1440 & 44.87 & 19 & 25.57 & 155 & 35.98\end{array}$

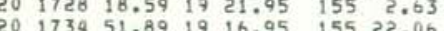
$20 \quad 1814 \quad 19.24 \quad 19 \quad 16.75 \quad 155 \quad 21.99$ $20 \quad 1815 \quad 19.25 \quad 1916.80 \quad 155 \quad 22.13$

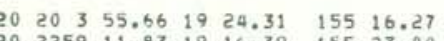
$\begin{array}{lllll}20 & 2338 \quad 3.79 & 19 & 16.37 \quad 155 & 23.88\end{array}$

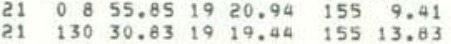

$\begin{array}{llllllll}21 & 2 & 6 & 47.76 & 19 & 16.90 & 155 & 22.05\end{array}$ 55914.18 19 16.76 155 25.73 $\begin{array}{rrrrrrr}21 & 821 & 54.37 & 19 & 30.81 & 155 & 29.72 \\ 21 & 1119 & 40.47 & 19 & 21.51 & 155 & 6.48\end{array}$

$\begin{array}{lllllll}21 & 1127 & 39.97 & 19 & 21.17 & 155 & 6.27\end{array}$ $21 \quad \begin{array}{llllll}1132 & 52.52 & 19 & 16.34 & 155 & 22.83\end{array}$

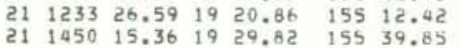

$\begin{array}{lllllll}21 & 1731 & 18.92 & 19 & 18.72 \quad 155 \quad 23.12\end{array}$ $21 \quad 1739 \quad 58.68 \quad 19 \quad 31.20 \quad 155 \quad 39.73$

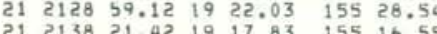
$223444.08 \quad 1929.98 \quad 155 \quad 39.87$ $\begin{array}{lllllll}22 & 1318 & 59.38 & 19 & 38.51 & 155 & 4.04\end{array}$

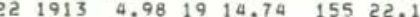
$\begin{array}{lllllll}23 & 121 & 15.04 & 19 & 20.03 & 155 & 7.23 \\ 23 & 3 & 91.45 & 19 & 26.37 & 155 & 35.90\end{array}$ $\begin{array}{lllllll}23 & 1035 & 34.27 & 19 & 20.23 & 155 & 17.18 \\ 23 & 1236 & 31.72 & 19 & 17.92 & 155 & 24.07\end{array}$ $\begin{array}{llllllllllll}1.14 & .2 & .4 & 8 & 0 & 82 & .02 & 2 & .2 & .1 & \mathrm{SPC}\end{array}$ $\begin{array}{rrrrrrrrrrr}.86 & 1.1 & 1.5 & 11 & 0 & 76 & .19 & 2 & .6 & .9 & \mathrm{SPC} \\ .30 & .3 & 1.0 & 9 & 0 & 87 & .06 & 2 & .0 & .5 & \mathrm{SPC}\end{array}$

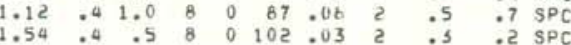

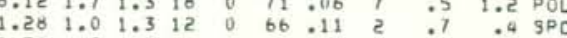

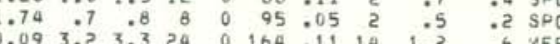
$\begin{array}{lllllllllll}9.13 & 2.8 & 1.8 & 20 & 0 & 140 & .12 & 25 & 3.2 & 11.9 & \text { LSA }\end{array}$ 9.38 .

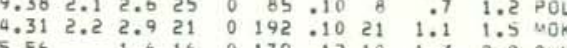
$\begin{array}{llllllllllll}5.56 & 1.6 & 16 & 0 & 179 & .12 & 10 & 1.3 & 2.9 & \text { swe }\end{array}$

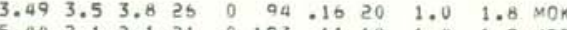

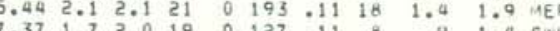

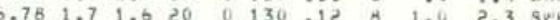

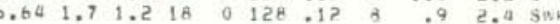

$\begin{array}{lllllllllll}1.68 & .9 & 1.5 & 12 & 0 & 74 & .05 & 2 & .3 & .2 & \text { SPc }\end{array}$

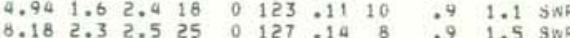

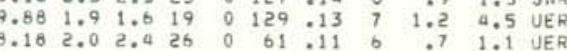
$\begin{array}{lllllllllll}6.39 & 1.3 & 1.5 & 19 & 0 & 127 & .13 & 0 & 1.1 & 2.3 & \text { SNA }\end{array}$ $\begin{array}{llll}0 & .5 & 1.5 \\ \text { UEH }\end{array}$

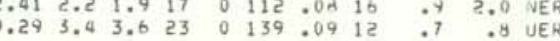
$\begin{array}{llllllllll}.58 & 1.9 & 1.8 & 18 & 0 & 145 & .19 & 12 & 1.6 & 3.6 \\ 0.5 & \text { UER }\end{array}$

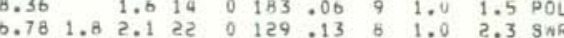

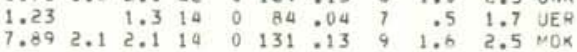
$\begin{array}{llllllllllll}5.81 & 1.7 & 2.0 & 19 & 0 & 135 & .09 & 8 & .7 & 1.6 & \text { SNR }\end{array}$ $\begin{array}{lllllllllll}.22 & 2.4 & 2.3 & 18 & 0 & 81 & .16 & 10 & 1.3 & 3.2 & 10 \mathrm{~K}\end{array}$ $\begin{array}{lllllllllll}9.80 & 2.4 & 2.7 & 29 & 0 & 49 & .13 & 11 & .7 & .4 & \text { UKF }\end{array}$

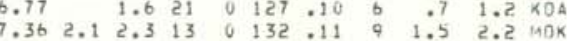
$\begin{array}{lllllllllllll}2.27 & 2.5 & 2.2 & 7 & 0 & 248 & .14 & 35 & 6.8 & 6.1 & \text { BLS }\end{array}$

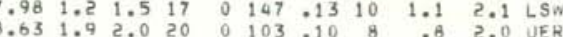

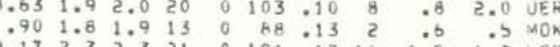

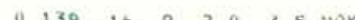

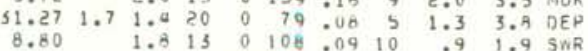

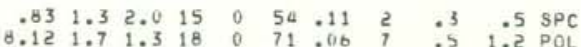




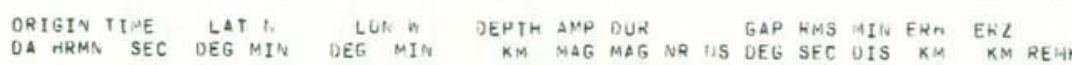
$\begin{array}{llllllllllllllllllll}1975 \text { FEd } 23 & 18 & 3 & 0.17 & 19 & 17.17 & 155 & 22.74 & 4.76 & 1.8 & 2.3 & 18 & 0 & 121 & .13 & 7 & .4 & 1.4 & \text { SWR }\end{array}$

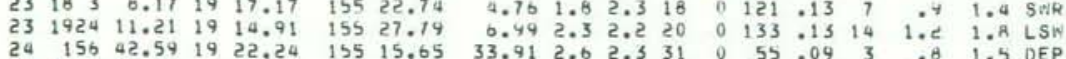

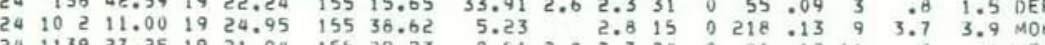
$\begin{array}{llllllllllll}1 & 2.8 & 2.7 & 24 & 0 & 81 & .12 & 11 & .9 & .6 & .6 & \text { HEA }\end{array}$

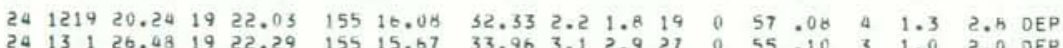

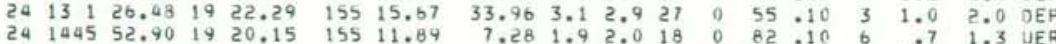

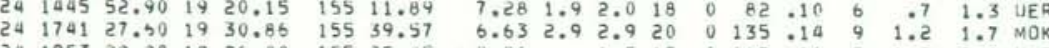
24. $195309.2019326 .40 \quad 15525.45$

$\begin{array}{lllllll}25 & 638 & 9.46 & 19 & 20.16 & 155 & 7.63 \\ 25 & 746 & 42.60 & 19 & 22.24 & 155 & 25.47\end{array}$

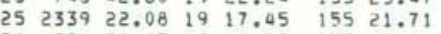
$26 \quad 22150.831919 .05 \quad 15513.86$

$\begin{array}{lllllll}26 & 816 & 56.80 & 19 & 23.41 & 155 & 29.57 \\ 26 & 1114 & 15.33 & 19 & 16.78 & 155 & 23.50\end{array}$ $\begin{array}{lllllll}26 & 1114 & 15.33 & 19 & 16.78 & 155 & 23.50 \\ 26 & 1322 & 39.90 & 19 & 20.80 & 155 & 17.59\end{array}$ $26 \quad 1543 \quad 17.811928 .85$ 155 27.12

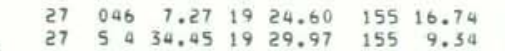

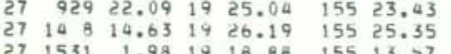

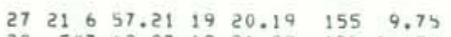

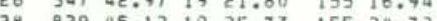
28. $857 \quad 50.88$ 19 18.81 155 13.40 $\begin{array}{lllllll}28 & 914 & 1.11 & 19 & 22.36 & 155 & 15.59\end{array}$

$\begin{array}{rrrrrrr}28 & 949 & 3.65 & 19 & 17.86 & 155 & 15.90 \\ 28 & 959 & 15.79 & 19 & 24.97 & 155 & 16.00\end{array}$ $\begin{array}{rrrrrrr}28 & 959 & 15.79 & 19 & 24.97 & 155 & 16.68 \\ 28 & 1028 & 1.30 & 19 & 22.25 & 155 & 30.04\end{array}$ $\begin{array}{lllllll}28 & 1256 & 32.08 & 19 & 29.18 & 155 & 39.16 \\ 28 & 1737 & 21.31 & 19 & 19.98 & 155 & 12.19\end{array}$

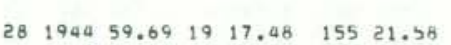

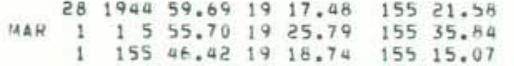

$\begin{array}{lllllll}1 & 416 & 45.26 & 19 & 18.54 & 155 & 15.01 \\ 1 & 758 & 48.53 & 19 & 18.53 & 155 & 15.05\end{array}$

1.938
1 $33.99 \quad 19 \quad 20.03 \quad 155 \quad 12.20$ $114355.43 \quad 19 \quad 19.61 \quad 15517.33$

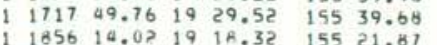

$\begin{array}{lllllll}2 & 020 & 1.49 & 19 & 18.98 & 155 & 13.60\end{array}$

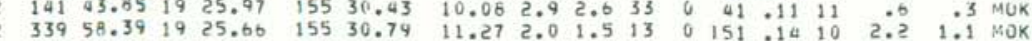

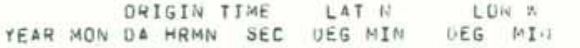

1975 MAR $2 \quad 448 \quad 50.00 \quad 1923.92 \quad 155 \quad 15.43$ 20040.13 19

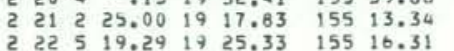

$\begin{array}{lllllll}2 & 2236 & 41.31 & 19 & 30.54 & 155 & 34.54\end{array}$ 3 053 37.84 19 $53.26 \quad 155 \quad 47.49$

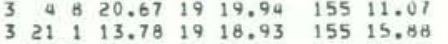
$32131 \quad 5.8819 \quad 16.50 \quad 15523.29$

$\begin{array}{lllllll}3 & 2150 & 9.60 & 19 & 17.18 & 155 & 22.45\end{array}$

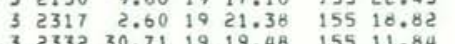

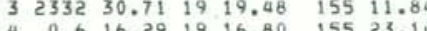
$4.01258 .3019 \quad 18.41$ 155 15.30

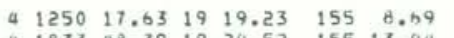
$4 \begin{aligned} & 1833 \\ & 4\end{aligned} 28.391920 .52 \quad 15513.04$ 4.2030
4 $525345.511919 .68 \quad 15512.15$

$\begin{array}{rrrrrrr}5 & 523 & 44.21 & 19 & 20.00 & 155 & 11.67 \\ 5 & 1556 & 18.09 & 19 & 20.39 & 155 & 13.29\end{array}$ $67157.591920 .46 \quad 155 \quad 0.31$

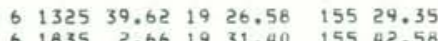

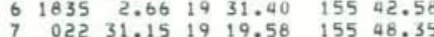

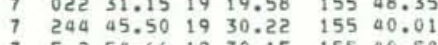
$75254.66 \quad 1930.15 \quad 155 \quad 40.58$

$\begin{array}{llllllll}7 & 5 & 4 & 14.28 & 19 & 26.36 & 155 & 36.80\end{array}$

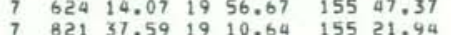

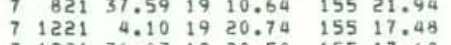
7 1221 $36.031920 .50 \quad 15517.60$ $\begin{array}{rrrrrrr}7 & 1237 & 48.08 & 19 & 20.95 & 155 & 5.95 \\ 8 & 513 & 32.64 & 19 & 24.40 & 155 & 17.48\end{array}$

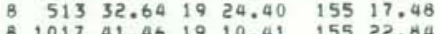

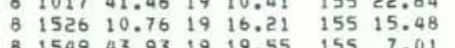
$8 \quad 1549 \quad 43.93 \quad 19$
$19.55 \quad 155 \quad 7.01$

$\begin{array}{lllllll}8 & 1859 & 55.38 & 19 & 19.38 & 155 & 13.30\end{array}$ 9
9 $157 \quad 22.08 \quad 1924.12 \quad 155 \quad 24.49$ $97521.241930 .21 \quad 15538.78$

$9 \quad 956 \quad 48.62 \quad 19 \quad 19.46 \quad 155 \quad 11.57$ $910120.491929 .53 \quad 15536.93$

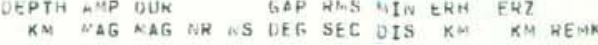
$\begin{array}{llllllllll}28.73 & 2.6 & 5.2 & 31 & 0 & 51.04 & 3 & .7 & 1.3 & \text { DEP }\end{array}$

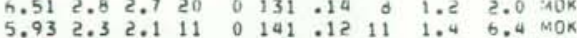

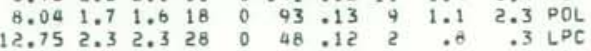

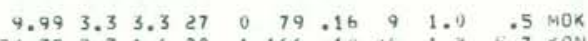

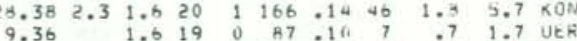

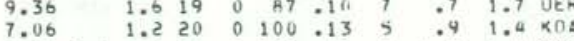
$\begin{array}{llllllllll}0.87 & 2.1 & 2.4 & 24 & 0 & 125 & .17 & 9 & 1.2 & 1.4\end{array}$

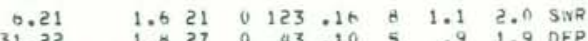

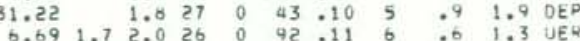

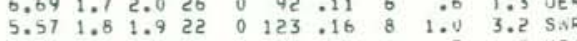
$\begin{array}{lllllll} & \end{array}$

$\begin{array}{lllllllllll}9.64 & 1.6 & 14 & 0 & 136 & .13 & 10 & 1.6 & \text {. A UEN }\end{array}$

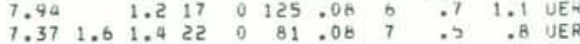

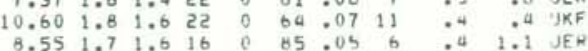

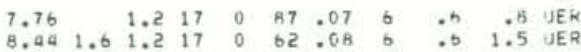
6.871 .5 1.5 18 0 $110.07 \quad 5 \quad: 5$ 1. 0 K04

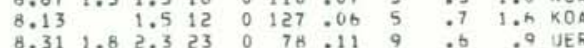
$\begin{array}{lllllllllll}12.08 & 2.0 & 1.8 & 13 & 0 & 128 & \text {. OA } & 12 & 1.2 & .5 & .5 \\ \text { UKF }\end{array}$

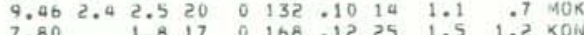

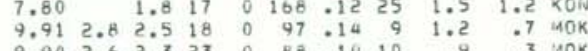

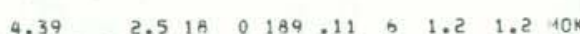

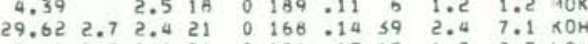

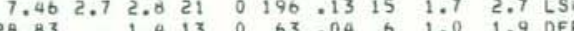
$\begin{array}{llllllllll}28.83 & 1.4 & 13 & 0 & 63 & .04 & 6 & 1.0 & 1.9 & \text { DEP } \\ 29.07 & 1.1 & 13 & 0 & 69 & .06 & 5 & 1.4 & 2.9 & \text { DEP }\end{array}$

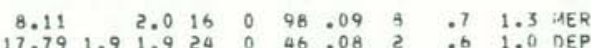

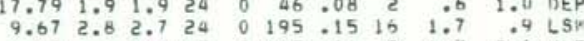
$\begin{array}{rrrrrrrrrr}7.52 & 2.0 & 15 & 0 & 185 & : 11 & 8 & 1.3 & 2.1 & \text { HLP } \\ 11.02 & 1.5 & 16 & 0 & 117 & .06 & 7 & .8 & .3 & \text { UER } \\ 11.02 & .06 & & \end{array}$ $\begin{array}{llllllllllll}.53 & 1.6 & 1.6 & 20 & 0 & 72 & .08 & 7 & .5 & .9 & \text { IJER }\end{array}$

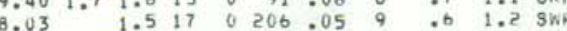

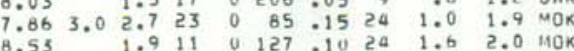
$\begin{array}{lllllllllll}7.04 & 1.7 & 1.6 & 19 & 0 & 95 & .10 & 6 & .7 & 2.0 & \text { UER }\end{array}$

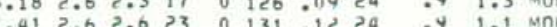


hVo earthquaKe SUMMAKY List

PAGE 43 ORIGIN TIME LAT N LON $N$ DEPTH AMP DUR GAP RMS MIN EKH ERZ TEAR MON ORAGHN TIME SEC LAT MIN

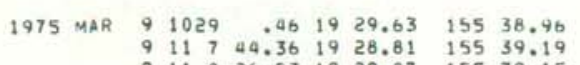

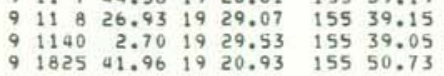

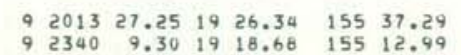

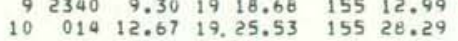
1001938.5619 .25 .5315528 .29 $10 \quad 629 \quad 48.07 \quad 19 \quad 19.84 \quad 15514.03$ $\begin{array}{rrrrrrr}10 & 723 & 31.44 & 19 & 25.76 & 155 & 44.91 \\ 10 & 745 & 7.88 & 19 & 18.06 & 155 & 13.30\end{array}$ $\begin{array}{lllllll}10 & 745 & 7.88 & 19 & 18.06 & 155 & 13.30 \\ 10 & 839 & 17.75 & 19 & 15.58 & 155 & 23.29\end{array}$

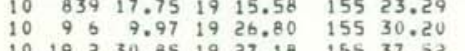
1019 2 $30.851927 .1 \mathrm{~A}$ 155 37.52

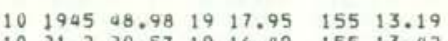

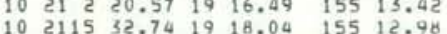
$\begin{array}{lllllll}11 & 036 & 6.20 & 19 & 27.26 & 155 & 52.33 \\ 11 & 045 & 39.99 & 19 & 29.98 & 155 & 40.11\end{array}$

$\begin{array}{lllllll}11 & 333 & .92 & 19 & 16.58 & 155 & 13.52\end{array}$

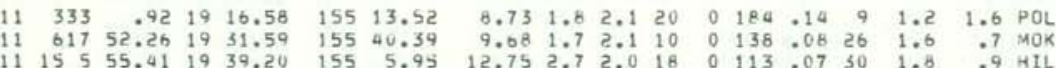

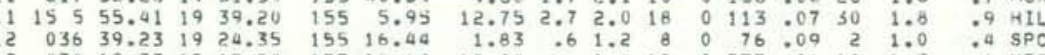
, 31.57 19 15.60

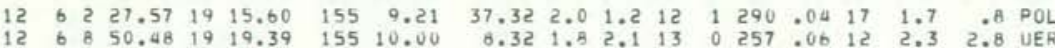
$\begin{array}{llllllllllllllll}12 & 726 & 28.50 & 19 & 19.32 & 155 & 12.37 & 8.74 & 1.3 & 12 & 0 & 216 & .04 & 8 & .6 & 1.4\end{array}$

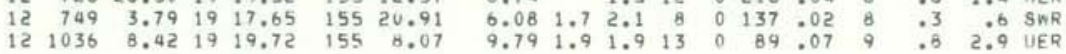
$\begin{array}{llllllllllllllllll}12 & 1254 & 19.97 & 19 & 30.34 & 155 & 42.63 & 9.58 & 2.4 & 2.4 & 21 & 0 & 75 & .14 & 22 & 1.1 & .5 & \text { MOK }\end{array}$

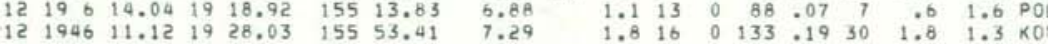

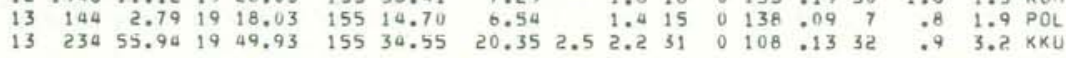

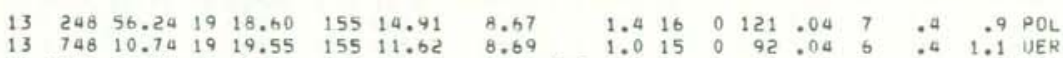

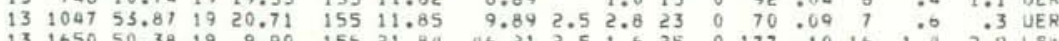

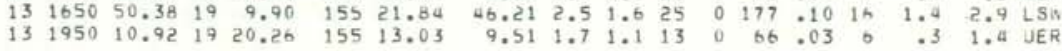

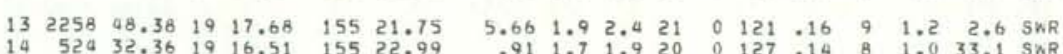

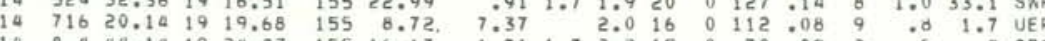

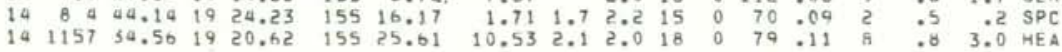

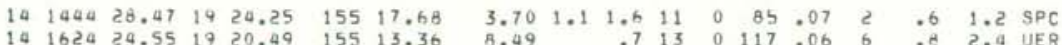

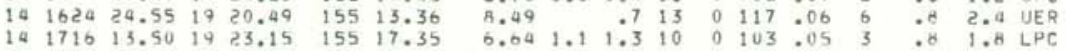

HVO EARTHQUAKE SUMMARY LIST

PAGE 44 ORIGIN TIME LATN LON N DEPTH AMP DUR GAP RMS MIN ERT ERZ $\begin{array}{lllllllllllllllllll}1975 \text { MAR } 14 & 1856 & 46.92 & 19 & 24.93 & 155 & 16.80 & 2.01 & .6 & 1.8 & 13 & 0 & 113 & .12 & 2 & .9 & 1.8 & 5 P C \\ & 14 & 19 & 63.03 & 19 & 24.91 & 155 & 17.12 & 10.14 & 0 & 1.5 & 10 & 0 & 119 & 05 & 3 & .9 & 3.5 & \text { LPC }\end{array}$

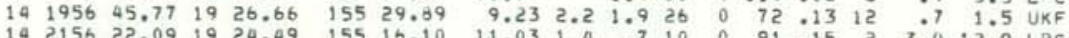

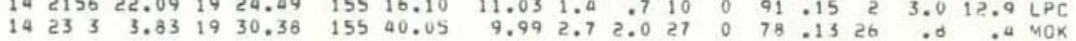

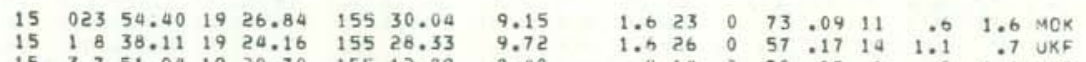

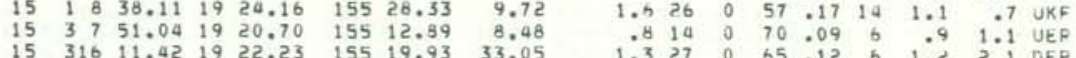

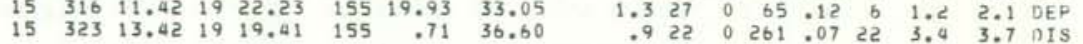
$\begin{array}{lllllllllllllllllll}15 & 432 & 8.64 & 19 & 26.04 & 155 & 36.98 & 3.55 & 2.4 & 2.0 & 19 & 0 & 190 & 11 & 18 & .8 & 1.2 & \text { MOK } \\ 15 & 528 & 25.92 & 19 & 24.05 & 155 & 25.13 & 9.15 & 1.8 & 1.6 & 22 & 0 & 57 & 0.10 & 10 & .7 & 1.11 & \text { UKF }\end{array}$

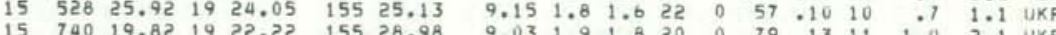

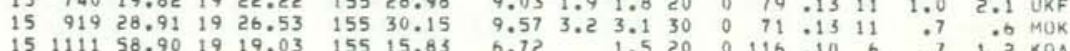

$15.1626 \quad 8.95 .1919 .11015515 .53$ $15 \quad 1758 \quad 58.691926 .26 \quad 155 \quad 36.72$ $15203254.57 \quad 1919.94$

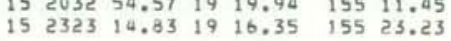
$15 \quad 2355 \quad 48.07 \quad 1926.21 \quad 155 \quad 36.76$ $16 \quad 11657.021919 .02-15515.56$ $\begin{array}{lllllll}16 & 139 & 11.17 & 19 & 18.87 & 155 & 15.68 \\ 16 & 316 & 28.21 & 19 & 25.29 & 155 & 36.67\end{array}$ $\begin{array}{lllllll}16 & 546 & 9.68 & 19 & 19.73 & 155 & 11.20\end{array}$ $\begin{array}{lllllll}16 & 740 & 4.10 & 19 & 11.96 & 155 & 37.58\end{array}$

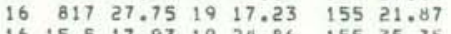

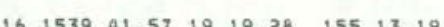
$16 \quad 2053 \quad 7.5519$ 19.03 155513.0

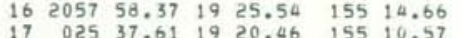

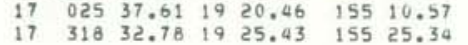

$\begin{array}{lllllll}17 & 1231 & 40.50 & 19 & 25.36 & 155 & 35.45\end{array}$

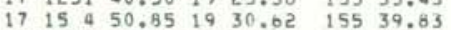
$171518 \quad 34.78 \quad 1930.13 \quad 15540.12$ $17195210.701918 .61 \quad 15515.20$

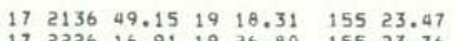

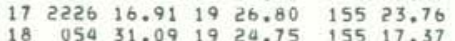

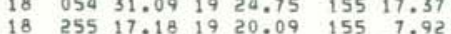
18 $58 \begin{array}{lllll}18.91 & 19 & 13.54 & 154 & 28.27\end{array}$

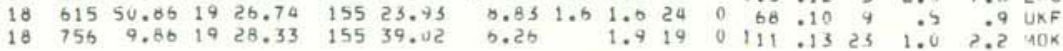




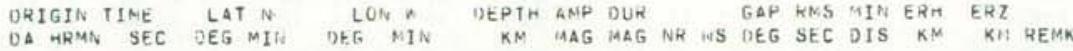

1775 MAR $18 \quad 1230 \quad 57.46 \quad 1929.57 \quad 155 \quad 36.01$

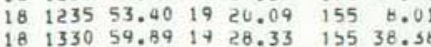

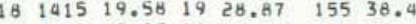
$18 \quad 1416 \quad 45.2519 \quad 15.20 \quad 155 \quad 2.60$

$\begin{array}{lllllll}18 & 1439 & .93 & 20 & 18.78 & 155 & 20.34 \\ 18 & 1449 & 21.46 & 19 & 28.98 & 155 & 38.23\end{array}$ $\begin{array}{llllllll}18 & 17 & 5 & 1.48 & 19 & 29.03 & 155 & 39.78\end{array}$

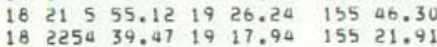

$18 \quad 2314 \quad 58.14 \quad 1926.42 \quad 155 \quad 50.07$ $\begin{array}{lllllll}19 & 037 & 3.36 & 19 & 30.08 & 15539.0 & 30.0\end{array}$ $\begin{array}{lllllll}19 & 043 & .27 & 19 & 19.61 & 155 & 8.59\end{array}$ $19 \quad 3528.57 \quad 19 \quad 18.63 \quad 155 \quad 13.7$ $\begin{array}{llllllll}19 & 335 & 13.51 & 19 & 18.76 & 155 & 15.69\end{array}$

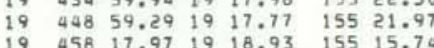

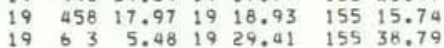

$19 \quad 6 \quad 5 \quad 32.18 \quad 19 \quad 17.45 \quad 155 \quad 13.63$ $19 \quad 646 \quad 36.7519 \quad 17.98 \quad 155 \quad 16.7$ $\begin{array}{lllllll}19 & 7 & 22.17 & 19 & 29.94 & 155 & 39.20 \\ 19 & 725 & 32.30 & 19 & 26.27 & 155 & 28.33\end{array}$

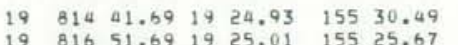

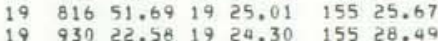

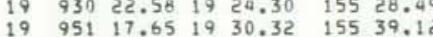
$19 \quad 952 \quad 45.37 \quad 1929.52 \quad 15539.10$

$\begin{array}{lllllll}19 & 15 & 32.17 & 19 & 28.86 & 155 & 36.85 \\ 19 & 1510 & 49.09 & 19 & 29.27 & 155 & 38.93\end{array}$ $19 \quad 16 \quad 6 \quad 26.01 \quad 1924.62 \quad 15525.6$ $190239 \quad .71916 .23 \quad 15513.19$

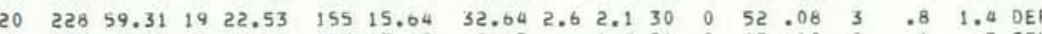

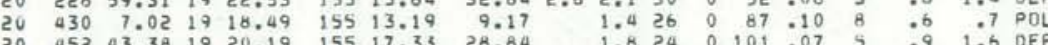

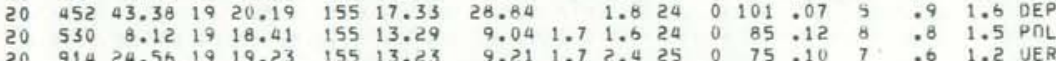

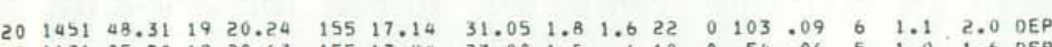

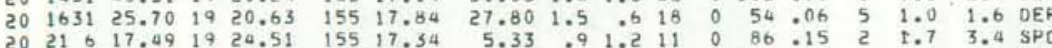

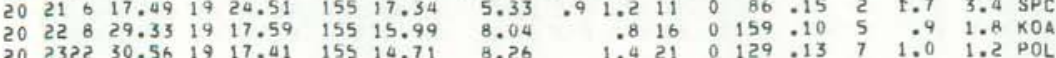

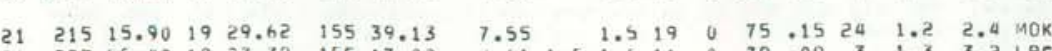

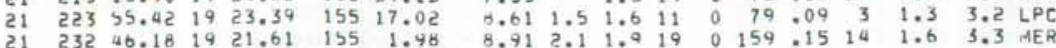

ORIGIN TIME LAT N LON W DEPTH AMP CUR GAP RMS MIV ERH ERZ KR YEAR MON DA HRMN SEC DEG MIN DEG MIN KM MAG MAG WIR WS DEG SEC DIS KM KM REMK 1975 HAR $21 \quad 430 \quad 39.5019 \quad 16.78 \quad 155 \quad 23.00$ $38 \quad 15517$ 21 $812 \quad 41.06 \quad 1924.67 \quad 155 \quad 16.17$ $21 \quad 815 \quad 11.37 \quad 1920.47 \quad 155 \quad 16.71$ $211349 \quad 48.93 \quad 1920.02 \quad 155 \quad 6.02$ $21 \quad 146 \begin{array}{lllll}2.38 & 19 & 24.93 & 155 & 16.64\end{array}$ $211434 \quad 46.43 \quad 1925.33$ 155 17.14 द1 $150 \quad 43.201929 .73 \quad 15539.16$

$212224 \quad 36.11 \quad 19 \quad 19.20 \quad 155 \quad 11.84$ $21 \quad 2225 \quad 3.34$ 19 $19.07 \quad 15625.36$

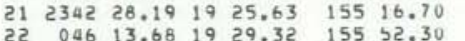
$22046 \quad 13.68 \quad 1929.32 \quad 155 \quad 32.30$

$22 \quad 123 \quad .32 \quad 19 \quad 18.62 \quad 155 \quad 13.05$ $\begin{array}{lllllll}22 & 256 & 24.36 & 19 & 25.12 & 155 & 16.66 \\ 22 & 257 & 44.63 & 19 & 24.85 & 155 & 16.46\end{array}$ $22 \quad 257 \quad 44.63 \quad 1924.85 \quad 15516.46$

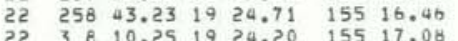
$\begin{array}{llllllll}22 & 5 & 70.71 & 19 & 19.25 & 155 & 14.05 \\ 22 & 536 & 26.10 & 19 & 22.35 & 155 & 17.57\end{array}$ $22 \quad 636 \quad 43.66 \quad 1924.62 \quad 155 \quad 15.98$ $22 \quad 93951.97$ 19 24.79 155 17.11

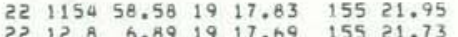
$22128 \quad 6.891917 .69$ 154 21.73 22 $1241 \quad 4.36 \quad 1925.42$ 155 16.39 $\begin{array}{lllllll}22 & 1626 & 49.82 & 19 & 24.37 & 155 & 16.51\end{array}$ $\begin{array}{lllllll}22 & 1752 & 30.94 & 19 & 24.29 & 155 & 13.72\end{array}$

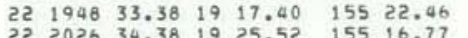
$222135 \quad 11.89 \quad 1924.66 \quad 15517.01$

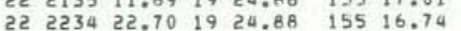
$\begin{array}{lllllll}22 & 2322 & 14.72 & 19 & 25.35 & 155 & 16.75\end{array}$

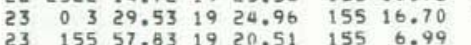

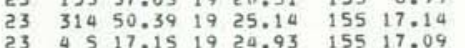

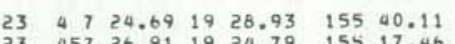
$23 \quad 51256.76$ 19 $18.63 \quad 15515.30$ $\begin{array}{llrllll}23 & 625 & 6.78 & 19 & 25.11 & 155 & 17.04 \\ 23 & 645 & 58.08 & 19 & 24.30 & 155 & 16.95\end{array}$

$\begin{array}{llllllll}23 & 720 & 59.99 & 19 & 23.37 \quad 155 \quad 16.75\end{array}$ $\begin{array}{llll}36.57 \quad 19 & 24.20 & 155 & 26.03\end{array}$ $\begin{array}{lllll}946 & 16.30 \quad 19 & 17.84 & 155 & 31.42\end{array}$ $\begin{array}{rrrrrrrrrrr}6.68 & 1.8 & 2.0 & 20 & 0 & 133 & .14 & 0 & 1.2 & 2.1 & \text { SwP } \\ 5.33 & 1.1 & 1.4 & 11 & 0 & 77 & .09 & 2 & 1.0 & 2.0 & \text { SPC }\end{array}$ \begin{tabular}{lllllllllll}
4.19 & 1.3 & 1.8 & 12 & 0 & 87 & .14 & 3 & 1.3 & 2.7 & $\mathrm{SPC}$ \\
\hline
\end{tabular}

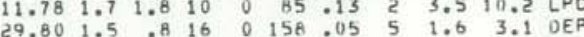

$\begin{array}{llllllllllll}.07 & 1.9 & 1.9 & 19 & 0 & 87 & .08 & 9 & .7 & 1.3 & \text { UER }\end{array}$

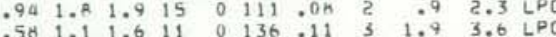

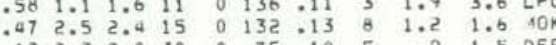

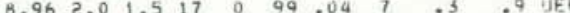

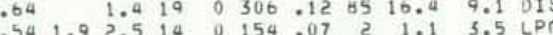

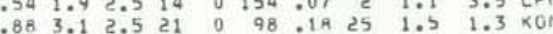

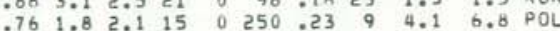

$\begin{array}{llllllllllll}9.53 & 1.7 & 1.5 & 18 & 0 & 89 & 09 & 0 & .0 & 3.3 & P O L\end{array}$

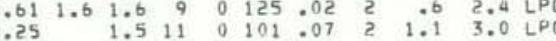

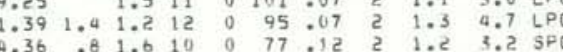

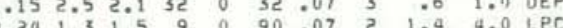

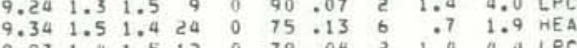
$\begin{array}{llllllllll}.10 & 2.7 & 2.8 & 28 & 0 & 118 & .10 & 4 & .7\end{array}$

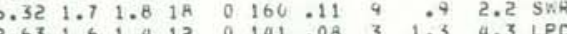

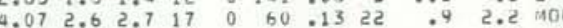

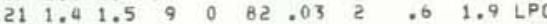
$\begin{array}{lllllllllllll}7.90 & 2.7 & 2.7 & 9 & 0 & 276 & .11 & 5 & 17.0 & 58.1 & \text { DEP }\end{array}$

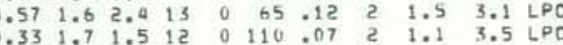
$\begin{array}{lllllllllll}0.25 & 1.5 & 1.6 & 12 & 0 & 137 & .04 & 2 & .0 & 1.9 & \mathrm{LPC}\end{array}$

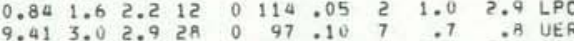

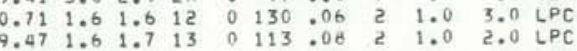
$\begin{array}{llllllllll}5.12 & 1.6 & 20 & 0 & 72 & .21 & 25 & 1.4 & 5.4 & \text { moK }\end{array}$

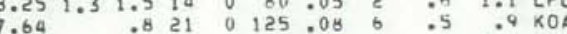
$\begin{array}{rrrrrrrrrr}8.56 & 1.4 & 1.8 & 14 & 0 & 125 & .05 & 2 & .6 & 1.1 \\ 7.77 & 1.3 & 1.5 & 12 & 0 & 71.07 & 2 & .8 & 1 . A & L P C\end{array}$ $\begin{array}{lllllllllll}0.67 & 1.6 & 1.5 & 14 & 0 & 67 & .10 & 2 & 1.0 & 1.4 & \text { LPC }\end{array}$ $5.40 \quad 1.623 \quad 0 \quad 120.13$ B $\begin{array}{lllllllllll}8.37 & .7 & 17 & 0 & 87 & 07 & 6 & .5 & 1.3 & \text { JER }\end{array}$ 
hVO EARTHQUAKE SUMMARY LIST

HAGE 47

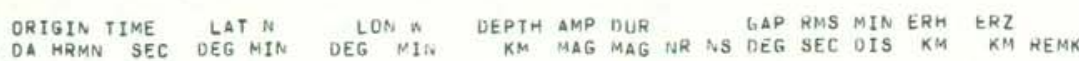

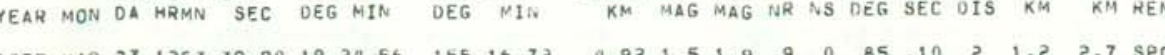

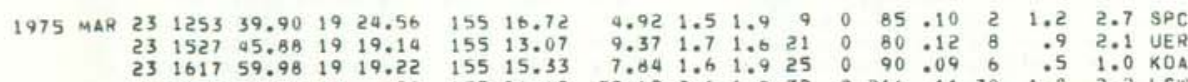

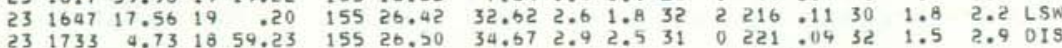
$\begin{array}{llllllllllllllllll}23 & 1840 & 45.21 & 19 & 19.59 & 155 & 15.50 & 6.70 & 1.5 & 2.3 & 27 & 0 & 95 & .12 & 5 & .6 & 1.2 & \text { KOA } \\ 23 & 2248 & 33.85 & 19 & 20.16 & 155 & 11.86 & 9.53 & 1.7 & 1.8 & 23 & 0 & 79 & .08 & 6 & .5 & .7 & \text { UER }\end{array}$

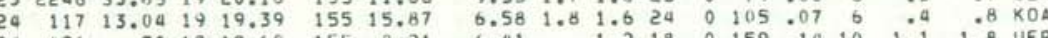

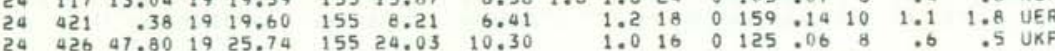

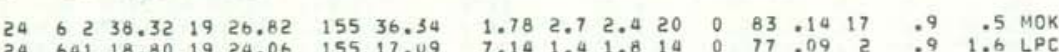

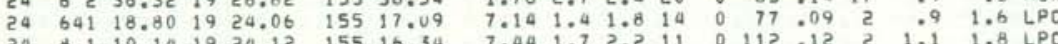

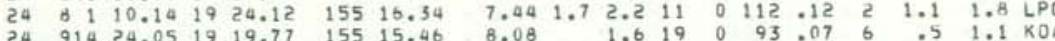

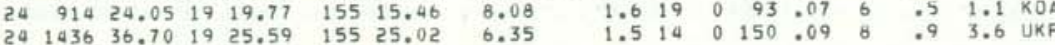

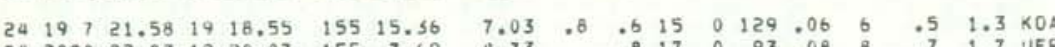

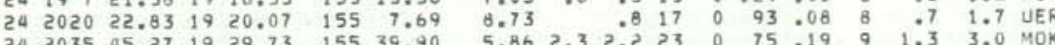

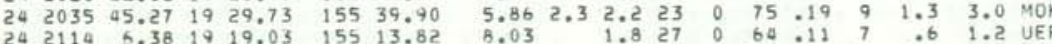

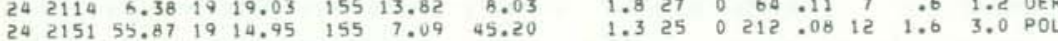

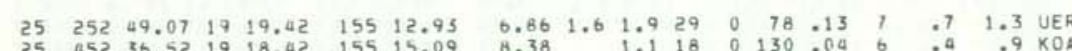

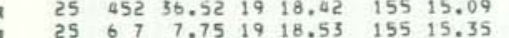
$\begin{array}{lllllllllll}7.53 & .9 & .6 & 18 & 0 & 129 & .07 & 6 & .5 & .9 & 04\end{array}$

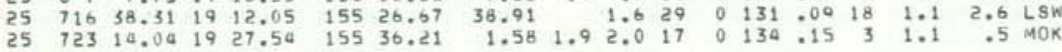
$\begin{array}{llll}25 & 1145 \quad 7.55 & 17 & 19.46 \quad 155 \quad 13.42\end{array}$ $251611 \quad 45.26 \quad 17 \quad 18.62 \quad 15513.02$

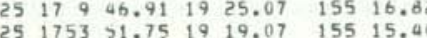

$25 \quad 1917 \quad 20.53 \quad 19 \quad 18.80 \quad 155 \quad 15.50$ $252050 \quad 55.87 \quad 19 \quad 19.11 \quad 155 \quad 13.60$ $25 \quad 2127 \quad 45.89 \quad 19 \quad 23.90$

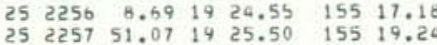

$26 \quad 250 \quad 20.93 \quad 1920.19 \quad 155 \quad 11.66$ $26 \quad 553 \quad 2.43 \quad 1920.71 \quad 15511.51$ $\begin{array}{rrrrrrr}26 & 711 & 17.49 & 19 & 25.85 & 155 & 37.46 \\ 26 & 10 & 7.73 & 19 & 27.23 & 155 & 35.47\end{array}$

$26 \quad 1013 \quad 29.03 \quad 1927.83 \quad 155 \quad 35.81$

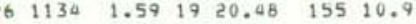

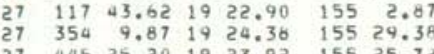
$27 \quad 44525.201923 .92 \quad 15525.7$

$27532 \quad 44.8219 \quad 16.06 \quad 155 \quad 14.01$

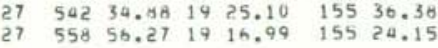

$\begin{array}{lllllllllllll}6.64 & 1.6 & 19 & 0 & 118 & .13 & 7 & 1.0 & 2.2 & \text { UER } \\ 7.74 & .9 & 15 & 0 & 154 & 08 & 8 & .9 & 1.9 & \text { POL }\end{array}$

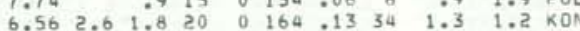

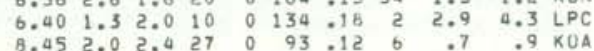
$\begin{array}{lllllllllllll}7.78 & 1.0 & .5 & 15 & 0 & 122 & .00 & 6 & .4 & .6 & \text { KOA }\end{array}$

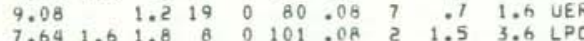

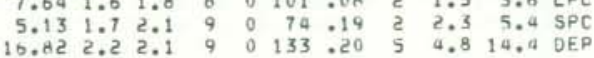
$\begin{array}{llllllllll}8.79 & 2.3 & 2.4 & 27 & 0 & 80 & .12 & 7 & .7 & 1.2\end{array}$ UER

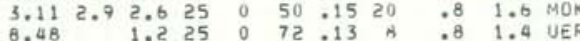

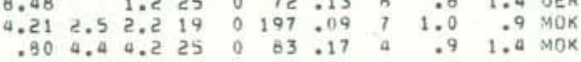
$\begin{array}{lllllllllll}1.16 & 3.3 & 3.4 & 21 & 0 & 79 & 14 & 3 & .8 & .5 & \text { MOK } \\ 7.14 & & 1.0 & 19 & 0 & 77 & .08 & 8 & .6 & 1.2 & \text { UER }\end{array}$

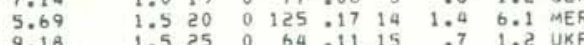
$\begin{array}{llllllllll}9.18 & 1.5 & 25 & 0 & 64 & .11 & 15 & .7 & 1.2 & \text { UKF } \\ 9.44 & 1.2 & 20 & 0 & 83 & .09 & 11 & .6 & 2.2 & \text { UKF }\end{array}$ $\begin{array}{rrrrrrrrrrr}7.83 & 1.9 & .8 & 13 & 0 & 134 & .05 & 7 & .8 & 2.0 & \text { POL } \\ 2.35 & 2.8 & 2.5 & 19 & 0 & 96 & .15 & 19 & .9 & 3.6 & 40 K \\ 5.94 & & 2.0 & 24 & 0 & 130 & .17 & 10 & 1.1 & 2.9 & \text { SNK }\end{array}$
hVO EARTHQUAKE SUMMahY LIST

PAGE 48

DRIGIN TIME LATN LON N DEPTH AMP DUR GAP RMS MIN ERH ERZ

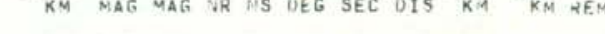
$\begin{array}{rlrrrrrrrrrrrrrrrr}1975 \text { MAR } & 27 & 1645 & 34.07 & 19 & 20.82 & 155 & 7.81 & 8.49 & 1.9 & 1.9 & 26 & 0 & 61 & .17 & 4 & 1.1 & 1.3 \\ 27 & 1919 & 1.57 & 19 & 17.83 & 155 & 3.80 & 47.28 & & 1.1 & 26 & 0 & 221 & .07 & 13 & 1.0 & 2.4 & \text { MER }\end{array}$

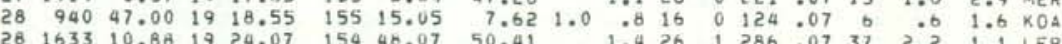

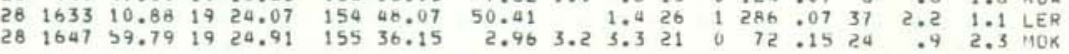

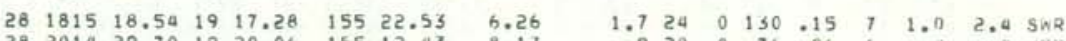

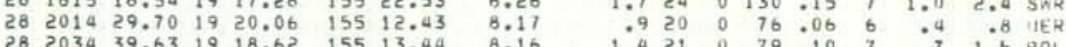

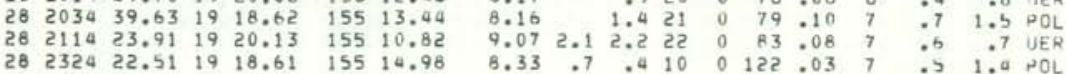

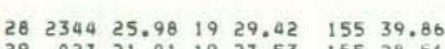
$\begin{array}{lllllll}29 & 027 & 21.01 & 19 & 29.53 & 155 & 28.56\end{array}$

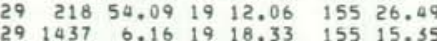
$29 \begin{array}{lllll}1443 & 53.16 & 19 & 20.05 & 155 \quad 20.76\end{array}$ $\begin{array}{llllll}29 & 1448 & 27.51 & 19 & 17.03 \quad 155 & 22.58\end{array}$

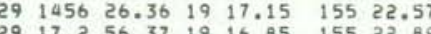
$2920052.42 \quad 19$

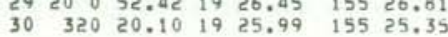

$\begin{array}{lllllll}30 & 327 & 55.11 & 17 & 19.75 \quad 155 \quad 11.93\end{array}$

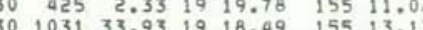

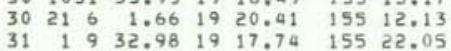

$\begin{array}{lllllll}31 & 637 & 20.14 & 19 & 17.18 & 155 & 21.47\end{array}$

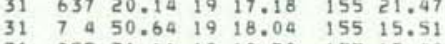
$\begin{array}{lrlllll}31 & 855 & 31.66 & 19 & 18.78 & 155 & 15.40\end{array}$

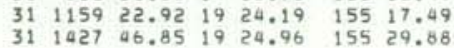

$\begin{array}{lllllll}31 & 1455 & 48.42 & 19 & 19.77 \quad 155 & 10.26\end{array}$

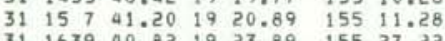
$\begin{array}{lllllll}31 & 1639 & 40.82 & 19 & 23.89 & 155 & 27.22\end{array}$ $\begin{array}{lllllll}31 & 2322 & 4.47 & 19 & 24.49 & 155 & 16.60 \\ 31 & 2336 & 24.07 & 19 & 28.59 & 155 & 35.59\end{array}$

$\begin{array}{llllll}014 & 43.23 \quad 19 & 18.88 & 155 & 13.00\end{array}$ $\begin{array}{llllll}016 & 43.74 & 19 & 19.08 & 155 & 13.25 \\ 049 & 28.27 & 19 & 18.55 & 155 & 13.38\end{array}$

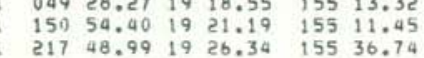

$\begin{array}{llllll}35 & 19.35 & 19 & 19.60 & 155 & 11.97\end{array}$

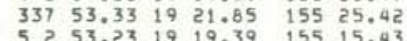

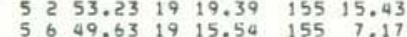

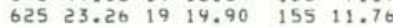

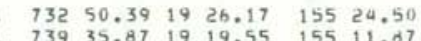

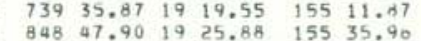

$\begin{array}{llllllllll}6.07 & 1.1 & 16 & 0 & 129 & 0.16 & 27 & 2.1 & 4.0 & \text { MOK }\end{array}$

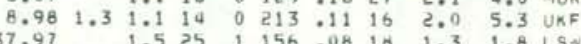

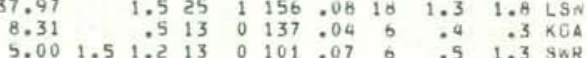
$\begin{array}{lllllllllll}.96 & 1.9 & 2.0 & 20 & 0 & 132 & .16 & 7 & 1.1 & 46.2 & \text { SNR }\end{array}$

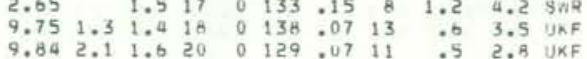
$\begin{array}{lllllllllll}9.46 & 1.9 & 2.3 & 21 & 0 & 86 & .09 & 6 & .7 & 1.4 & \text { UER }\end{array}$

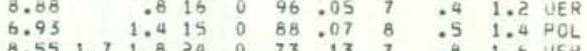

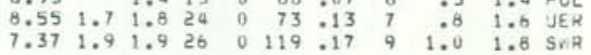
$\begin{array}{llllllllll}5.90 & 1.4 & 22 & 0 & 127 & .16 & 9 & 1.1 & 2.9 & \text { SNR }\end{array}$

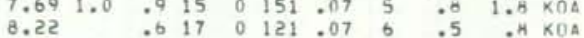
$\begin{array}{rrrrrrrrrrr}14.84 & 1.6 & 1.1 & 21 & 0 & 75 & 06 & 2 & .06 & .7 & \text { DEP } \\ 7.96 & 1.9 & 1.2 & 21 & 0 & 105 & .14 & 14 & 1.0 & 2.0 & \text { UXF }\end{array}$ $\begin{array}{llllllllll}7.67 & 1.2 & 22 & 0 & 90 & .12 & 7 & .8 & 1.9 & \text { UER }\end{array}$ $\begin{array}{lllllllllll}5.47 & 1.8 & 1.3 & 14 & 0 & 108 & .14 & 13 & 1.1 & 1.6 & \text { UKF }\end{array}$ $\begin{array}{rrrrrrrrrrr}14.94 & 1.8 & 1.7 & 30 & 0 & 46 & .07 & 2 & .5 & .6 & .6 \\ 1.40 & 2.3 & 2.4 & 21 & 0 & 125 & .11 & 3 & .7 & .3 & \text { MUK }\end{array}$ $\begin{array}{lllllllllll}9.46 & 1.6 & 1.3 & 21 & 0 & 85 & .09 & 8 & .7 & 1.5 & \text { POL }\end{array}$

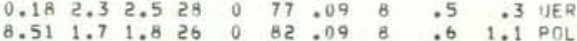

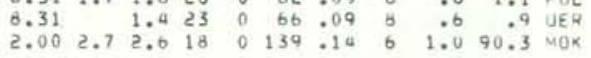
$\begin{array}{llllllllll}6.76 & .9 & 16 & 0 & 88 & .10 & 6 & .8 & 1.7 & \text { NER }\end{array}$

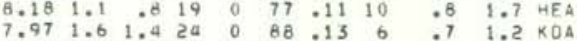

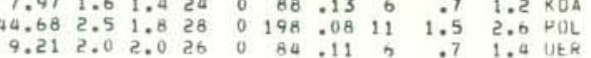
$\begin{array}{llllllllllll}7.87 & 2.2 & 2.1 & 29 & 0 & 52 & 13 & 9 & .7 & 1.1 & \text { UKF } \\ 9.56 & 1.1 & .6 & 19 & 0 & 90 & .15 & 6 & -5 & 3.7 & \text { UE }\end{array}$

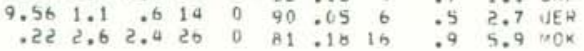

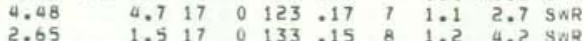


ORIGIN TIME LATN LON A DEPTH AMP IUR W GAP RMS AIN ERH ERZ
UA HRMN SEC DEG MIV DEG NIN KM MAG MAG NR NS DEG SEC UIS KM KM KEMK

1975 APH $1153045.23 \quad 1426.54 \quad 15551.46 \quad 10.052 .92 .422$ 0 120.14 26 1.110 .4 KON 169
17
1

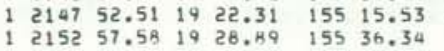
$\begin{array}{llllllll}2 & 2 & 0 & 56.10 & 19 & 19.77 \quad 155 & 13.78\end{array}$ $\begin{array}{lllllll}2 & 8 & 41.16 & 19 & 20.03 & 155 & 13.07\end{array}$ $\begin{array}{lllllll}2 & 456 & 2.52 & 19 & 19.39 & 155 & 13.03 \\ 2 & 529 & 7.67 & 19 & 20.77 & 155 & 25\end{array}$ $253656.2519 \quad 33.34 \quad 15536.87$

$2 \quad 61939.32 \quad 19 \quad 16.19 \quad 15523.73$

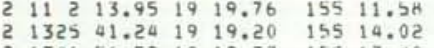

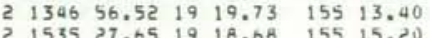

$\begin{array}{llllll}2 & 1743 \quad 39.98 \quad 19 & 29.17 & 155 & 39.86\end{array}$

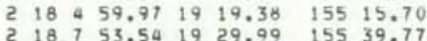

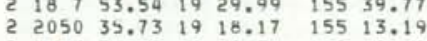

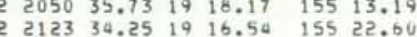

$\begin{array}{lllllll}2 & 2234 & 1.21 & 19 & 16.47 & 155 & 22.70\end{array}$

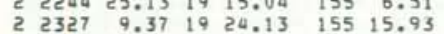

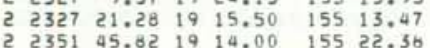

$\begin{array}{lllllll}3 & 345 & 17.26 & 19 & 20.31 & 155 & 11.31\end{array}$

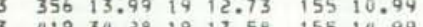
$\begin{array}{lllllll}3 & 419 & 34.28 & 19 & 17.58 & 155 & 14.99 \\ 3 & 724 & 7.84 & 19 & 18.85 & 155 & 13.51\end{array}$

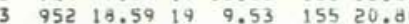

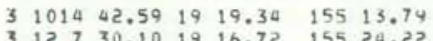
3
3 12730.101919 .3015015524 .22

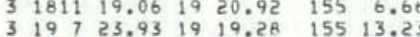

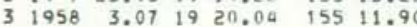

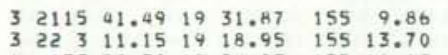

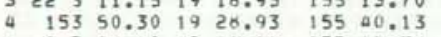

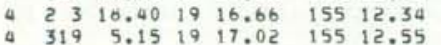

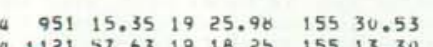
$4.13955 .861919 .24 \quad 155 \quad 13.50$

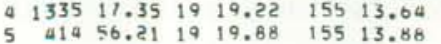

$\begin{array}{lllllllll}5 & 8 & 3 & 3.20 & 19 & 16.70 & 155 & 22.87\end{array}$ $94150.171915 .48-155$ 22.

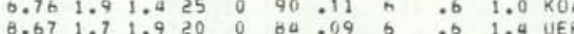

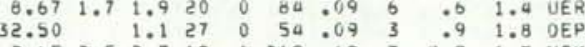

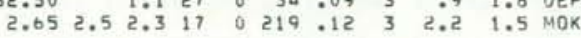
$\begin{array}{llllllllll}0.43 & 1.9 & 2.0 & 23 & 0 & 75 & .10 & 6 & .6 & 1.3 \\ 0 \text { UER }\end{array}$ 8.1001 .61 .527 O 60.10 b 50.5 .9 UER

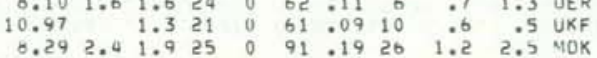

$\begin{array}{llllllllllll}5.55 & 1.9 & 1.6 & 20 & 0 & 125 & .14 & 10 & 1.1 & 3.0 & \text { SWR }\end{array}$

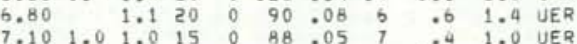

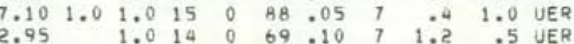

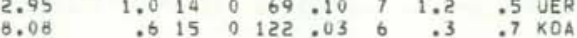

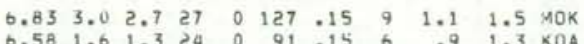

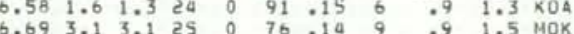

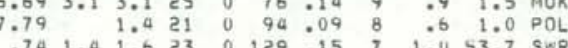

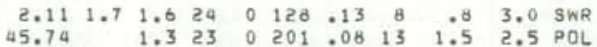
$\begin{array}{lllllllllll}8.36 & 1.4 & 1.0 & 16 & 0 & 226 & .14 & 10 & 2.4 & 1.5 & \text { POL } \\ 5.94 & 2.5 & 2.8 & 27 & 0 & 153 & .15 & 12 & 1.1 & 1.9 & \text { S }\end{array}$ $\begin{array}{rrrrrrrrrrr}7.29 & 1.1 & .7 & 19 & 0 & 95 & .09 & 7 & .7 & 1.5 & \text { UER } \\ 13.54 & 1.5 & .8 & 12 & 0 & 255 & .13 & 18 & 4.4 & 3.7 & \text { POL }\end{array}$

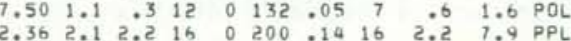
$\begin{array}{rrrrrrrrrrr}7.34 & 1.7 & 1.6 & 20 & 0 & 63 & .10 & 6 & .7 & 1.5 & \text { UER } \\ 4.38 & 1.8 & 1.8 & 22 & 0 & 117 & .14 & 10 & .9 & 1.8 & \text { SWR } \\ 0.66 & .86 & .7 & 25 & 0 & 93 & .14 & 7 & .8 & 1.1 & \text { UER }\end{array}$

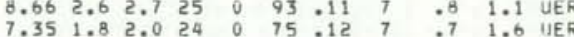
$\begin{array}{rrrrrrrrrrr}4.32 & 2.9 & 2.6 & 30 & 0 & 64 & .09 & 16 & .6 & 2.0 & \text { HIL }\end{array}$

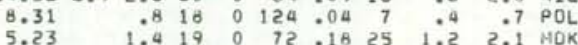

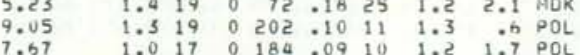
$\begin{array}{llllllllll}8.52 & 1.6 & 13 & 0 & 118 & -11 & 10 & 1.0 & 1.8 & \text { MOK }\end{array}$

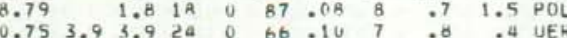

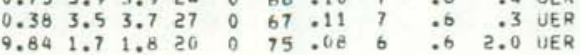
$\begin{array}{lllllllllll}7.49 & 5.6 & 3.6 & 27 & 0 & 125 & .13 & 8 & .0 & 1.4 & \text { SWR }\end{array}$

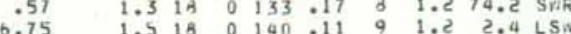

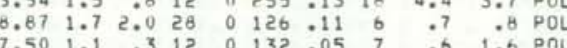

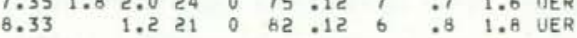

ORIGIN TIME LAT N LON N DEPTH AMF DUK GAP KMS MIIA FRH F.KL YEAR MON DA HRMN SEC DEG MIN DEG MIN KM MAG KAG NR IS DEG SEC DIS KHA KM KEM

1975 APR $5 \quad 942 \quad 42.48 \quad 19 \quad 16.97 \quad 155 \quad 22.48$ $51024 \quad 28 \quad 19 \quad 18.11 \quad 1552323.05$

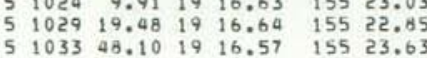

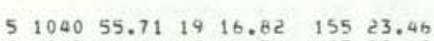

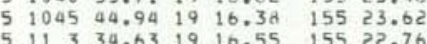

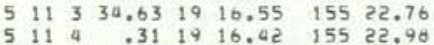
$\begin{array}{llllllll}5 & 11 & 4 & 0.31 & 19 & 16.42 & 155 & 22.98 \\ 5 & 1110 & 56.05 & 19 & 16.02 & 155 & 22.98\end{array}$

$\begin{array}{lllllll}5 & 1143 \quad 39.83 & 19 & 16.61 & 155 & 22.90\end{array}$

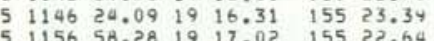

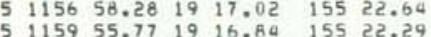
5 12 $142.351916 .18 \quad 15521.74$

$\begin{array}{llllllll}5 & 12 & 3 & 20.73 & 19 & 17.16 & 155 & 21.85\end{array}$ 5129
5 5
5
5 $1223 \quad 39.371917 .17 \quad 15522.33$

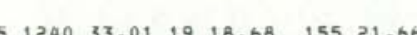
$\begin{array}{lllllll}5 & 1240 & 33.01 & 19 & 18.68 & 155 & 21.66 \\ 5 & 132 & 17.12 & 19 & 17.34 & 155 & 22.85\end{array}$ $\begin{array}{lllllll}5 & 1311 & 10.37 & 19 & 16.45 & 155 & 23.10\end{array}$ $5 \quad 134031.8319 \quad 17.26 \quad 15522.03$ $\begin{array}{lllllll}5 & 1345 & 16.32 & 19 & 17.35 & 155 & 22.01\end{array}$ $514011.211917 .15 \quad 15522.15$ 55172114.0919 16.52 15523.52 $5 \quad 197.42 .86 \quad 19 \quad 16.72 \quad 15523.10$

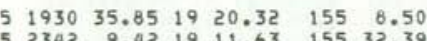

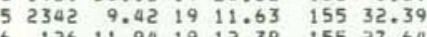

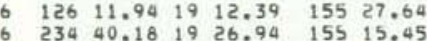

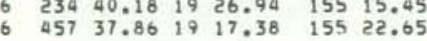

$\begin{array}{lllllll}6 & 458 & 14.92 & 19 & 17.17 & 155 & 22.62\end{array}$ 6
647 $40.78 \quad 1926.21 \quad 15528.18$

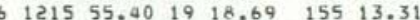

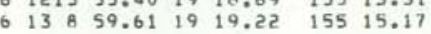
$6 \quad 1444 \quad 39.78 \quad 19 \quad 19.03 \quad 155 \quad 13.80$
0 $6183333.8319 \quad 17.38 \quad 155 \quad 13.09$ $619635.921926 .60 \quad 15530.02$ 61954.061919 .22 155 12.11

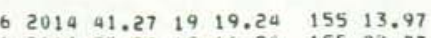

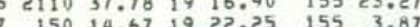

$\begin{array}{lllllllllll}0.78 & 1.8 & 1.8 & 23 & 0 & 122 & .14 & 8 & 1.3 & 2.3 & \text { SWR }\end{array}$

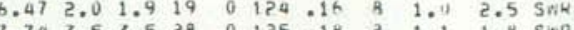

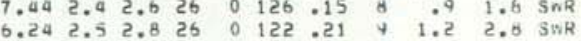
$\begin{array}{lllllllllll}.38 & 1.8 & 2.2 & 50 & 0 & 121 & .211 & 9 & 1.3 & 99.0 & 5\end{array}$

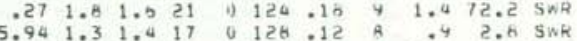

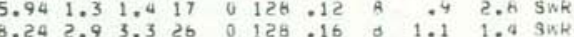

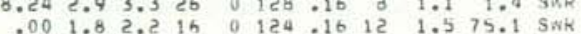
$\begin{array}{llllllllll}5.91 & 1.4 & 17 & 0 & 126 & .14 & \text { A } & 1.2 & 5.2 & \text { Snk }\end{array}$

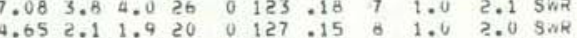
082.230220159 .09 a 8 .8 SWR

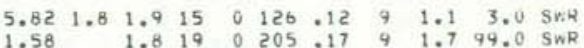

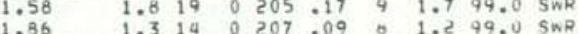

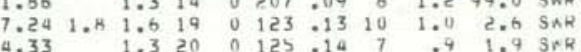
$\begin{array}{lllllllllll}4.03 & & 1.1 & 16 & 0 & 200 & 11 & 8 & 1.1 & 3.0 & \text { SWR } \\ 3.65 & 1.8 & 1.0 & 21 & 0 & 119 & : 19 & 7 & 1.2 & 3.1 & \text { SWRR }\end{array}$ 6.53 1.5 19 0 157.15 a 1.5 c.0. SAR

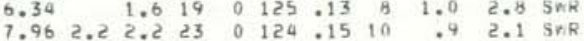

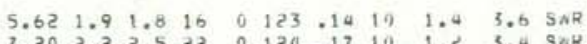

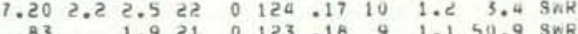

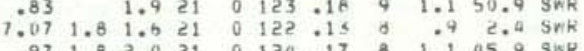

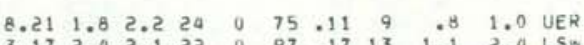

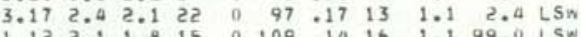
$\begin{array}{llllllllllll}2.74 & 1.7 & 1.8 & 22 & 0 & 131 & .16 & 7 & 1.0 & 3.8 & \text { SWR }\end{array}$

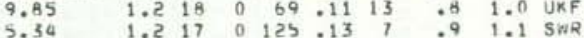

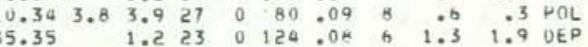
$\begin{array}{lllllllllll}7.74 & 1.7 & 1.6 & 21 & 0 & 86 & .13 & 7 & .9 & 1.7 & \\ 0.3 E R\end{array}$

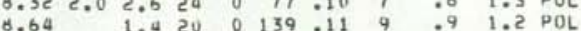

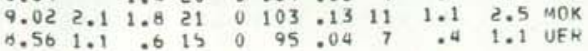
$\begin{array}{llllllllll}8.45 & 5.0 & 5.0 & 23 & 0 & 66 & .12 & 6 & .7 & 1.5 \\ 0 \text { UER }\end{array}$

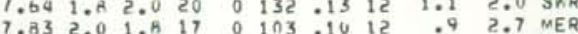

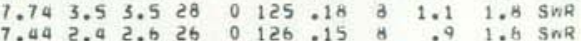

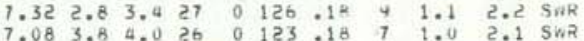

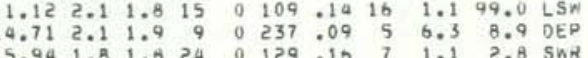


HVO EARTHQHAKE SUMRARY LIST

PAGE 51

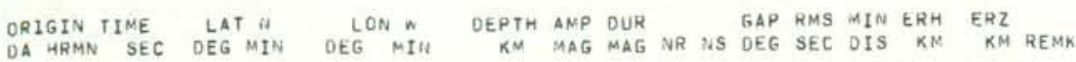

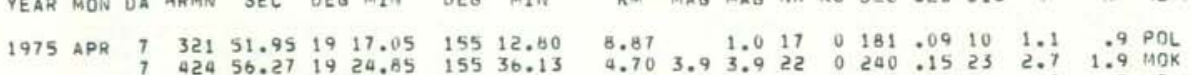

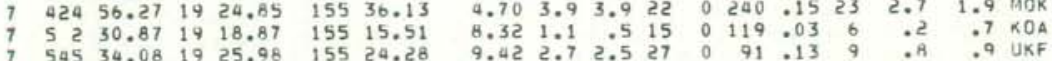

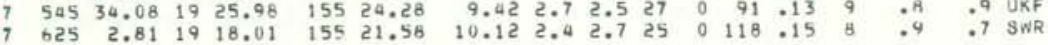
$\begin{array}{llllllllllllllllll}7 & 7 & 0 & 30.87 & 19 & 22.17 & 155 & 3.91 & 8.59 & 2.0 & 1.8 & 18 & 0 & 102 & .11 & 11 & 1.0 & 2.7 \\ 7 & \text { MER }\end{array}$

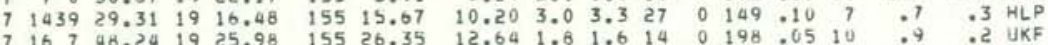

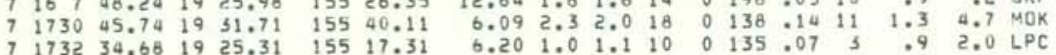
$\begin{array}{lllllllllllllllll}7 & 1750 & 38.68 & 19 & 18.69 & 155 & 15.56 & 6.00 & 1.6 & 1.2 & 20 & 0 & 126 & .12 & 6 & .9 & 1.8 \\ \text { KOA }\end{array}$

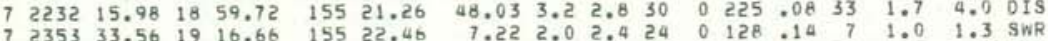

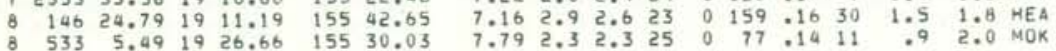

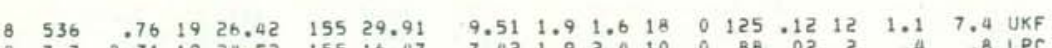

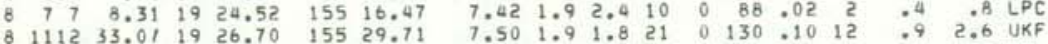

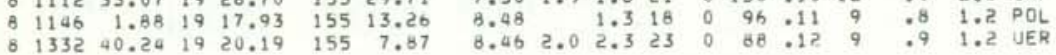

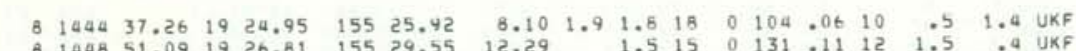

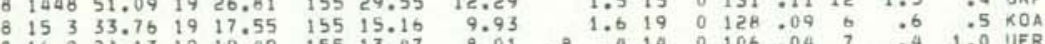

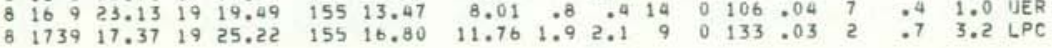

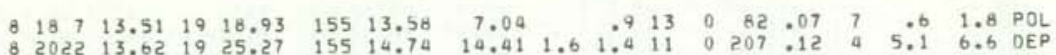

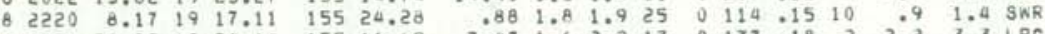

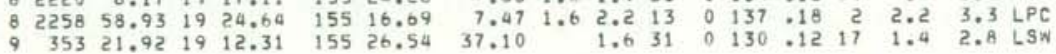
$\begin{array}{rrrrrrrrrrrrrrrr}712 & 14.45 & 19 & 24.08 & 155 & 16.89 & 9.20 & 2.1 & 2.8 & 10 & 0 & 83 & .05 & 2 & 1.0 & 1.8 \text { LPC } \\ 9 & 12 & 31.12 & 19 & 18.71 & 155 & 15.76 & 9.16 & 1.7 & 1.8 & 23 & 0 & 104.10 & 5 & .6 & .8 \text { KOA }\end{array}$

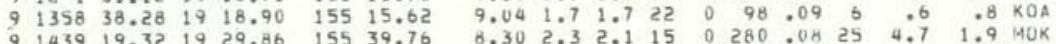

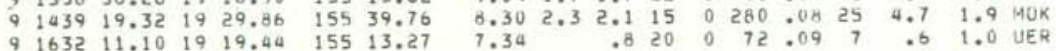

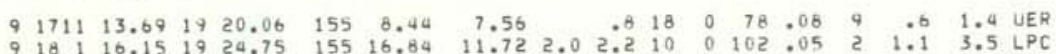

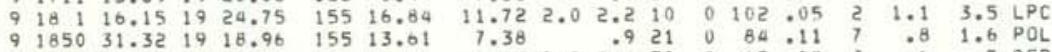

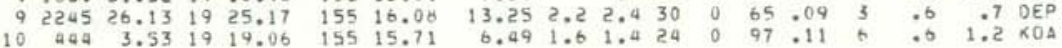
$\begin{array}{rrrrrrrrrrrrrrrrrrrrr}10 & 541 & 2.90 & 19 & 23.99 & 155 & 16.71 & 5.29 & 1.6 & 2.2 & 14 & 0 & 50 & .18 & 2 & 1.4 & 2.9 & 5 P C \\ 10 & 656 & 10.86 & 19 & 26.52 & 155 & 36.41 & 3.51 & 3.0 & 2.8 & 21 & 0 & 133 & .13 & 21 & .7 & 1.2 & 40 \mathrm{~K}\end{array}$

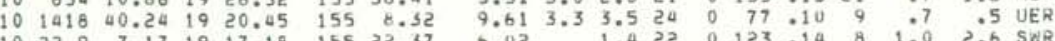

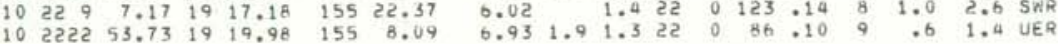
$\begin{array}{llllllllllllllllll}10 & 2243 & 8.70 & 19 & 25.00 & 155 & 16.05 & 10.43 & 1.3 & 1.1 & 11 & 0 & 120 & .03 & 2 & .5 & 1.5 & \mathrm{LPC}\end{array}$

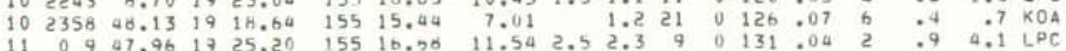

HVO EARTHQUAKE SULOARYY LISI

PAGt 52

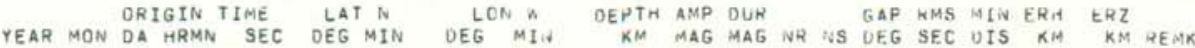

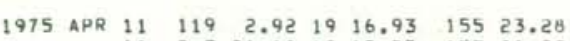

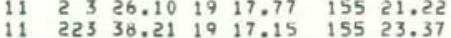

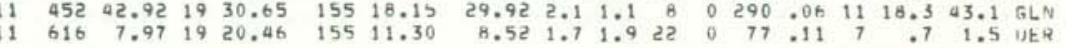

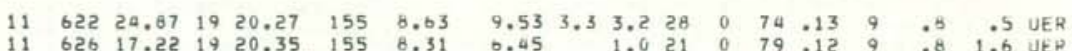

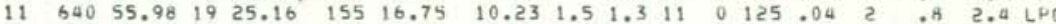

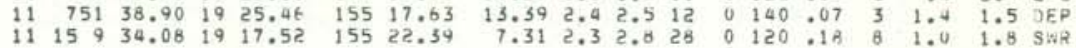
$\begin{array}{lllllll}11 & 1520 & 50.94 & 19 & 9.59 & 155 & 28.81\end{array}$ 11 1715 $17.301925 .56 \quad 15537.06$

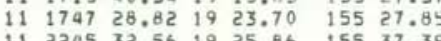
15537.39 $\begin{array}{lllllll}12 & 010 & 24.75 & 19 & 25.09 & 155 & 36.49\end{array}$

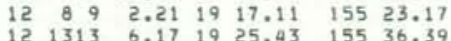

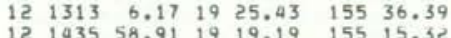
$\begin{array}{rrrrrrrr}12 & 1435 & 58.91 & 19 & 19.19 & 155 & 15.32 \\ 12 & 1458 & 5.39 & 19 & 19.27 & 155 & 10.50\end{array}$

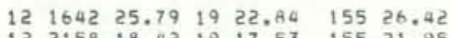

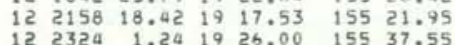

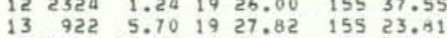

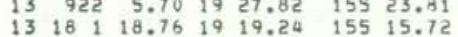

$\begin{array}{rrrrrrr}13 & 2251 & 4.11 & 19 & 26.33 & 155 & 24.95 \\ 13 & 2327 & 24.92 & 19 & 20.55 & 155 & 4.20\end{array}$ $\begin{array}{rrrrrrr}13 & 2327 & 24.92 & 19 & 20.55 & 155 & 4.20 \\ 14 & 253 & 54.55 & 19 & 32.28 & 155 & 39.72\end{array}$ $14 \quad 64855.231918 .24 \quad 15515.46$ $14 \quad 64855.231918 .24 \quad 15515.46$

$\begin{array}{lrrrrrr}14 & 1824 & 2.21 & 19 & 25.10 & 155 & 16.69\end{array}$ 14233935.221918 .9115526 .06 15 15. 18.57 19 25.05 155 16.05 $15944 \quad 26.88 \quad 1924.54 \quad 155 \quad 17.05$

$\begin{array}{lllllll}15 & 1433 & 13.97 & 19 & 24.97 & 155 & 17.46\end{array}$

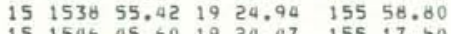

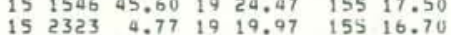
$\begin{array}{llllllll}16 & 0 & 7 & 15.94 & 19 & 20.00 & 155 & 8.77\end{array}$ $\begin{array}{lllllll}16 & 012 & 51.90 & 19 & 28.34 & 155 & 35.10 \\ 16 & 1 & 5 & 12.67 & 19 & 17.23 & 155 \\ 22.54\end{array}$ $\begin{array}{lllllll}16 & 1 & 512.67 & 19 & 17.23 & 155 & 22.54 \\ 16 & 835 & 57.32 & 19 & 20.43 & 155 & 11.22\end{array}$ $\begin{array}{lllllll}16 & 1447 & 9.62 & 19 & 19.17 & 155 & 13.26\end{array}$ $\begin{array}{lllllll}16 & 1550 & 56.77 & 19 & 20.58 & 155 & 10.63\end{array}$ $\begin{array}{lllllll}16 & 1627 & 53.49 & 19 & 19.94 & 155 & 11.92\end{array}$

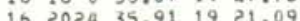

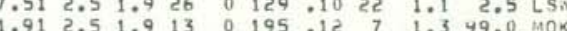

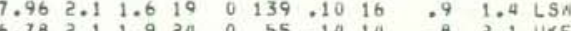

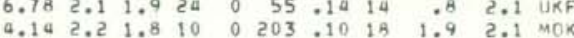
$\begin{array}{lllllllllllll}3.35 & 2.6 & 2.3 & 12 & 0 & 213 & .09 & 19 & 1.2 & 1.5 & 40 \mathrm{~K}\end{array}$

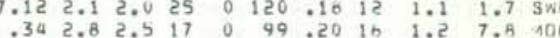

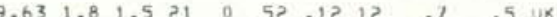

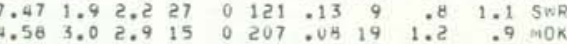
$\begin{array}{llllllllllll}6.34 & 1.7 & 1.6 & 16 & 0 & 102 & .08 & 10 & .5 & 1.7 & \text { UKR } \\ 7.24 & 1.6 & 1.3 & 24 & 0 & 109 & .12 & 6 & .7 & 1.1 & \times 04\end{array}$

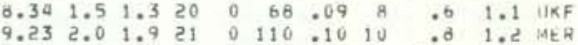

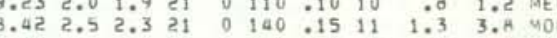
$\begin{array}{lllllllllll}7.25 & 1.7 & 1.6 & 24 & 0 & 138 & .08 & 6 & .5 & .8 & \text { KOA } \\ 8.73 & 1.6 & 1.8 & 11 & 0 & 162 & .09 & 4 & 1.0 & 2.7 & \text { LPC }\end{array}$ $\begin{array}{lllllllllllll}9.08 & 1.4 & 1.5 & 13 & 0 & 121 & .07 & 2 & .9 & 1.8 & \text { LPC }\end{array}$

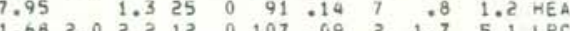

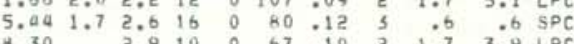
$\begin{array}{lllllllllll}9.39 & 1.4 & 1.6 & 12 & 0 & 122 & .09 & 3 & 1.3 & 2.7 & L P C\end{array}$

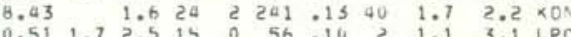

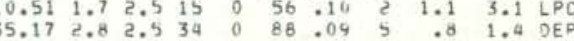

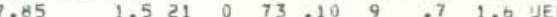
$\begin{array}{lllllllllllll}1.14 & 2.7 & 2.6 & 22 & 0 & 122 & .14 & 3 & .7 & .4 & \times 0 K\end{array}$

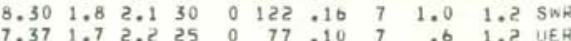
6.90
7.34 $\begin{array}{lllllllllll}7.65 & 1.7 & 1.2 & 23 & 0 & 62 & .10 & 6 & .0 & 1.4 & \text { UEH }\end{array}$

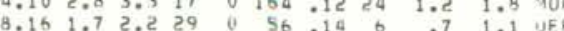
6.291 .61 .6 21 0090.1260 . 9 1.a k0 


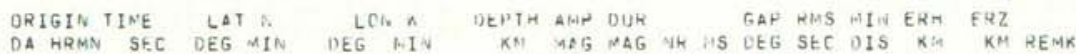
1975 APR $16 \quad 2027 \quad 31.98 \quad 19 \quad 31.63 \quad 15539.01$ $162223 \quad 46.741920 .62 \quad 15513.7$

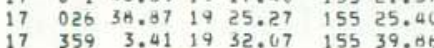
$\begin{array}{lllllll}17 & 451 & 12.53 & 19 & 19.01 \quad 155 \quad 1 \% .13\end{array}$

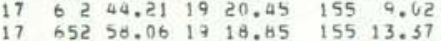

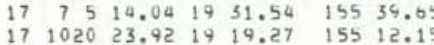
$17 \quad 1134 \quad 58.68 \quad 19 \quad 17.62 \quad 15525.33$ $17.625 \quad 52.461918 .74 \quad 15515.25$ $\begin{array}{lllllll}17 & 1653 & 22.33 & 19 & 31.83 & 155 & 39.71 \\ 17 & 1752 & 35.19 & 19 & 29.29 & 155 & 26.32\end{array}$

$\begin{array}{lllllll}17 & 1821 & 32.48 & 19 & 12.01 & 155 & 28.41\end{array}$

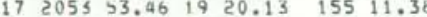

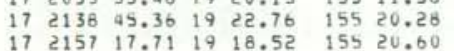

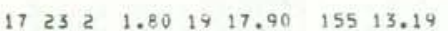

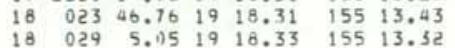

$\begin{array}{lllllll}18 & 057 & 11.60 & 19 & 29.37 & 155 & 41.91\end{array}$ 18 $32228.92 \quad 1929.56 \quad 155 \quad 13.35$ $18 \quad 335 \quad 30.74 \quad 19 \quad 18.11 \quad 155 \quad 13.36$

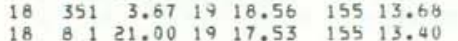

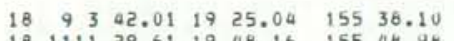

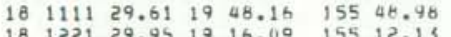

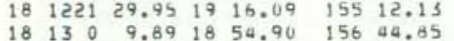
$\begin{array}{lllllllll}18 & 13 & 0 & 9.89 & 18 & 54.90 & 156 & 44.85 \\ 18 & 1415 & 4.06 & 19 & 31.86 & 155 & 39.86\end{array}$ $\begin{array}{lllllll}18 & 1430 & 48.80 & 19 & 19.86 & 155 & 12.04\end{array}$

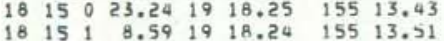

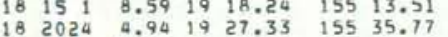
$18205935.89 \quad 1926.17 \quad 15536.71$

$\begin{array}{rrrrrrr}19 & 010 & 28.00 & 19 & 19.42 & 155 & 11.35 \\ 19 & 15 & 8.30 & 19 & 19.84 & 155 & 11.52\end{array}$ $19 \quad 410 \quad 12.94 \quad 19 \quad 16.86 \quad 155 \quad 13.62$ 190407.01 19 $22.48 \quad 15524.05$

$\begin{array}{lllllll}19 & 714 & 21.44 & 19 & 31.35 & 155 & 39.17\end{array}$ $\begin{array}{lllllll}19 & 747 & 4.69 & 19 & 20.57 & 155 & 12.83 \\ 19 & 859 & 7.84 & 19 & 31.46 & 154 & 39.43\end{array}$
$7.742 .3 \quad 1.921 \quad 0 \quad 84.18 \quad 10 \quad 1.1 \quad 1.4$ MOK

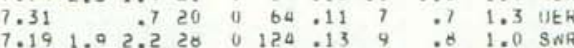

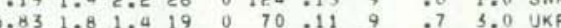

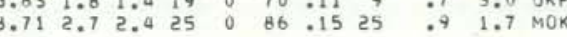
$\begin{array}{lllllllllllll}8.24 & 1.7 & 1.5 & 21 & 0 & 114 & .11 & 7 & .7 & .8 & \mathrm{KOA}\end{array}$

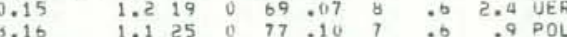

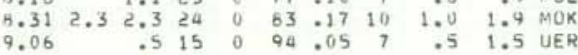
$\begin{array}{lllllllllllll}9.51 & 2.5 & 2.8 & 26 & 0 & 104 & .14 & 10 & .7 & .6 & \text { HEA }\end{array}$ $\begin{array}{lllllllllll}.41 & 1,0 & 14 & 0 & 123 & 0.04 & 5 & .4 & 1.0 & \times 04 & 0\end{array}$ $\begin{array}{lllllllll}7.06 & .7 & 19 & 0 & 121 & .10 & 0\end{array}$ $\begin{array}{lllllllllll}.66 & 3.1 & 2.8 & 25 & 0 & 84 & 1.13 & 11 & .0 & 1.5 & 1.50 \mathrm{~K} \\ 4.87 & 2.0 & 1.5 & 18 & 0 & 93 & .16 & 13 & 1.3 & 4.4 & \text { NER }\end{array}$ $\begin{array}{llllllllll}32.11 & 2.5 & 2.4 & 28 & 0 & 94 & .09 & 18 & 1.0 & 2.3 \\ 12.3 W\end{array}$

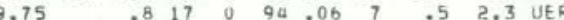

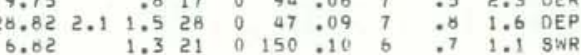
$\begin{array}{lllllllll}8.12 & 1.0 & 16 & 0 & 101 & .11 & 9 & 1.0 & 2.4 \\ 0 O L\end{array}$

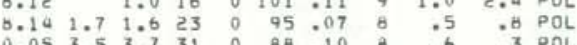

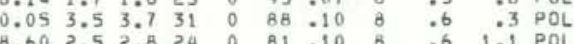

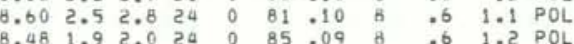

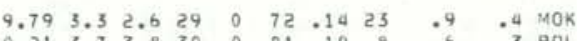
$\begin{array}{lllllllllll}10.21 & 3.7 & 3.8 & 30 & 0 & 81 & .10 & 8 & .6 & .3 & \text { POL }\end{array}$

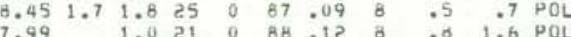

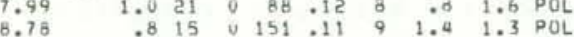
$\begin{array}{lllllllllll}3.90 & 2.5 & 2.2 & 9 & 0 & 211 & .08 & 9 & 1.6 & 3.2 & \text { MOK }\end{array}$

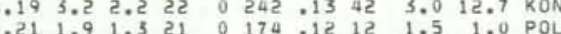

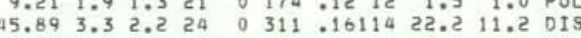

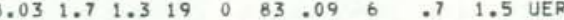

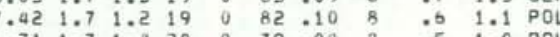
6.311 .71 .4200079 .08 o 51.51 .0 POL $3.783 .03 .02100186: 16$ b $\begin{array}{lllllllllll}7.09 & 1.7 & 1.4 & 26 & 0 & 88 & .13 & 6 & .8 & 1.3 & \text { UER } \\ 6.98 & & 1.1 & 19 & 0 & 91 & .12 & 6 & .9 & 1.5 & \text { UER }\end{array}$

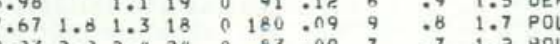

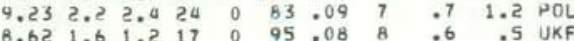
$\begin{array}{llllllllll}7.78 & 2.3 & 2.2 & 16 & 0 & 82 & .15 & 10 & 1.1 & 3.0 \\ 7.90 K\end{array}$

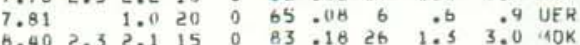

HVO EAKTHIUAKE SIMWARY LIST

PAGE 54 ORIGIN TIME LAT N LON
DA HRMN SEC OEG MIN DEG MIN

1975 APK $19 \quad 1013 \quad 46.83 \quad 19 \quad 31.19 \quad 15534.65$

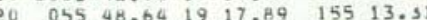
$\begin{array}{lllllll}20 & 138 & 16.63 & 19 & 17.59 & 155 & 21.95 \\ 20 & 98 & 41.75 & 19 & 20.20 & 155 & 8.72\end{array}$

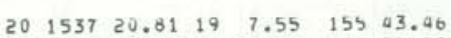

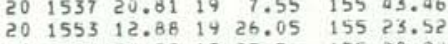
$\begin{array}{lllllll}20 & 1724 & 34.92 & 19 & 25.76 & 155 & 28.9 \mathrm{H}\end{array}$ $\begin{array}{lllllll}20 & 20 & 14.26 & 19 & 16.48 & 155 & 23.71 \\ 20 & 2044 & 50.52 & 19 & 22.87 & 155 & 29.05\end{array}$ $21 \quad 1240 \quad 26.67 \quad 19 \quad 19.29 \quad 155 \quad 15.95$ $211650 \quad 55.41$ 19 $15.04 \quad 155 \quad 35.95$ $212227 \quad 3.25 \quad 1919.84 \quad 155 \quad 11.44$ $\begin{array}{rrrrrrr}22 & 250 & 11.44 & 19 & 17.06 & 155 & 23.54 \\ 22 & 254 & 9.85 & 19 & 18.72 & 155 & 12.85\end{array}$ 22. $513 \quad 28.12 \quad 19 \quad 17.16 \quad 155 \quad 22.10$ $2211952.4919 \quad 17.98 \quad 15522.80$ $\begin{array}{lllllll}22 & 1140 & 22.03 & 19 & 19.53 & 155 & 13.65 \\ 22 & 2040 & 12.20 & 19 & 17.80 & 155 & 22.83\end{array}$ $\begin{array}{rrrrrrr}22 & 2040 & 12.20 & 19 & 17.80 & 155 & 22.83 \\ 23 & 233 & 58.19 & 19 & 25.19 & 155 & 25.30\end{array}$

$\begin{array}{lllllll}23 & 932 & 16.87 & 19 & 24.27 & 155 & 16.17\end{array}$ $\begin{array}{llllllll}23 & 10 & 56.75 & 49 & 30.22 & 155 & 40.23 & \\ 2 & 40.25 & & \end{array}$

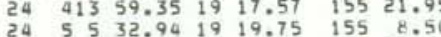

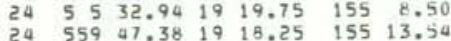

$\begin{array}{lllllll}24 & 647 & 42.12 & 19 & 19.62 & 155 & 11.10\end{array}$

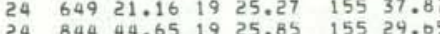

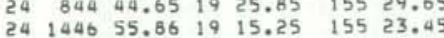
$\begin{array}{lllllll}24 & 1446 & 55.86 & 19 & 15.25 & 155 & 23.45 \\ 24 & 1448 & 23.53 & 19 & 16.17 & 155 & 23.73\end{array}$

$24 \begin{array}{lllllll}16 & 9 & 37.89 & 19 & 18.54 & 155 & 13.50\end{array}$

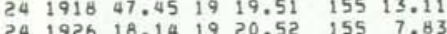
25 2 $429.921920 .02 \quad 155 \quad 8.30$ $25 \quad 243 \quad 52.69 \quad 19 \quad 31.03 \quad 155 \quad 40.36$

$\begin{array}{lllllll}25 & 324 & 21.00 & 19 & 31.08 & 155 & 39.81 \\ 25 & 527 & 29.27 & 19 & 19.04 & 155 & 13.50\end{array}$ $\begin{array}{lllllll}25 & 527 & 29.27 & 19 & 19.04 & 155 & 13.50 \\ 25 & 67 & 18.81 & 19 & 23.22 & 155 & 26.69\end{array}$ $\begin{array}{lllllll}25 & 6 & 18.81 & 19 & 23.22 & 155 & 26.69 \\ 25 & 628 & 47.68 & 19 & 27.27 & 155 & 36.38\end{array}$ $\begin{array}{llllllll}25 & 8 & 0 & 4.39 & 19 & 18.97 & 155 & 15.45\end{array}$

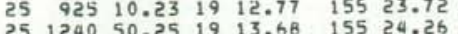

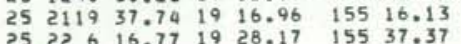
$\begin{array}{ll}26 & 8 \quad 90.47 \quad 19 \quad 18.57 \quad 155 \quad 13.14\end{array}$ $\begin{array}{llllllll}26 & 1 & 8 & 40.47 & 19 & 18.57 & 155 & 13.14 \\ 26 & 4 & 5 & 35.08 & 19 & 27.71 & 155 & 36.06 \\ 26 & 552 & 33.11 & 19 & 31.05 & 155 & 40.36\end{array}$
DEPTH AMP DUR GAP RNS MIN ERH EKI

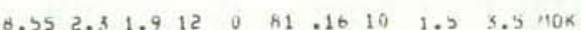

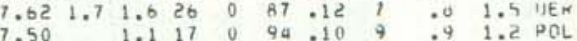
$\begin{array}{llllllllll}7.50 & 1.1 & 17 & 0 & 94 & -10 & 9 & .9 & 1.2 & P O L\end{array}$

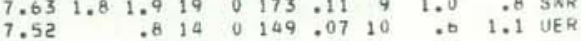
$6.513 .52 .918 \quad 0 \quad 303.18$ 48 $38.4 \quad 13.4$ HEA

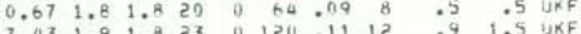

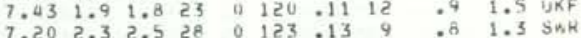

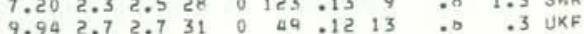

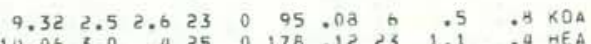

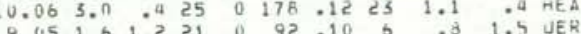

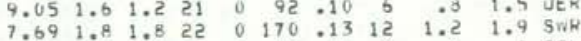

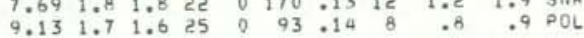

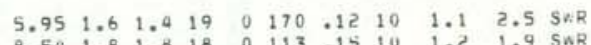

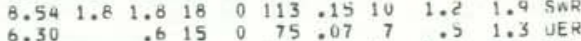

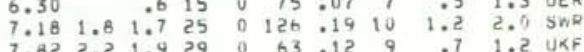

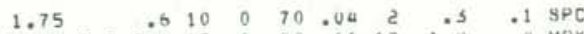

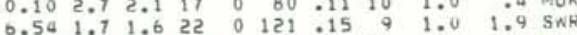

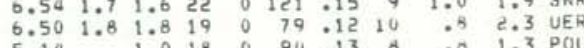
$9.141 .81 .820 \quad 0 \quad 94.0197 \quad .5 \%$.9 UFR

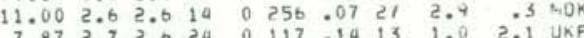

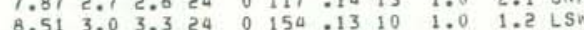

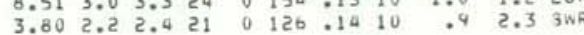

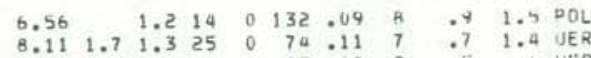

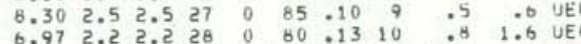
$9.652 .52 .517 \quad 0 \quad 135$.12 26 $1.2 \quad .540 \mathrm{~K}$

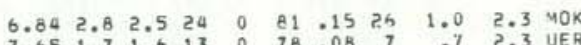

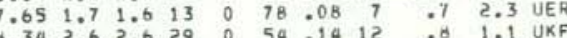

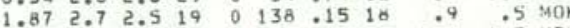
5012.9240135 .1326 1.1 1.5 mox $\begin{array}{lllllllllllll}7.36 & .8 & .6 & 13 & 0 & 116 & .05 & 6 & .5 & .6 & \times 04\end{array}$

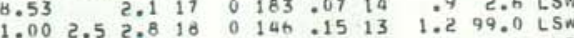
$\begin{array}{lllllllllll}8.34 & 1.8 & 15 & 0 & 176 & .05 & 6 & .6 & 1.0 & \text { HLP } \\ 3.05 & 2.4 & 11 & 0 & 231 & .12 & 20 & 2.7 & 1.6 & \text { MOK }\end{array}$ $\begin{array}{llllllllll}.78 & 7 & 14 & 0 & 87 & .07 & \text { A } & .6 & 1.9 & \text { POL }\end{array}$

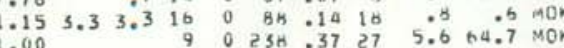



$26 \quad 211024.73 \quad 19 \quad 19.60 \quad 155 \quad 9.19$

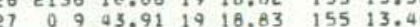

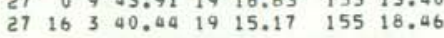

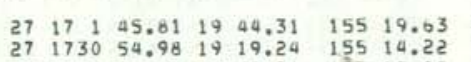
27 $\begin{array}{llllll}1850 & 31.97 & 19 & 19.91 & 155 & 12.00\end{array}$

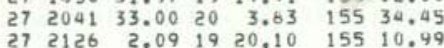

$\begin{array}{llllllll}27 & 22 & 4 & 53.75 & 19 & 26.52 \quad 155 \quad 36.38\end{array}$

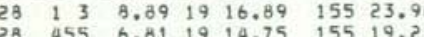
$\begin{array}{lllllll}28 & 455 & 6.81 & 19 & 14.75 & 155 & 19.21\end{array}$

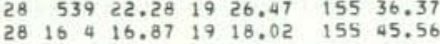

$\begin{array}{lllllll}28 & 1616 & 25.33 & 19 & 20.25 & 155 & 9.17\end{array}$

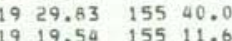

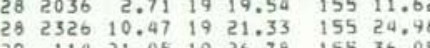

$29 \quad 148 \quad 7.451924 .56 \quad 155 \quad 16.05$

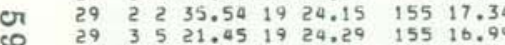

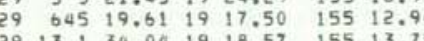
$29 \begin{array}{lllllll}1853 & 35.00 & 19 & 25.32 & 155 & 23.71\end{array}$ $\begin{array}{lllllll}29 & 1923 & 9.97 & 19 & 23.85 & 155 & 28.9\end{array}$

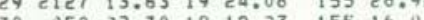
30 151 17.78 19 18.09 155 16.02

$\begin{array}{llllllll}30 & 152 & 36.18 & 19 & 18.33 & 155 & 16.93\end{array}$

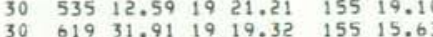

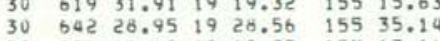
$\begin{array}{llll}30 & 828 \quad 44.10 \quad 19 \quad 19.33 \quad 155 & 13.16\end{array}$

$\begin{array}{lllllll}30 & 1121 & 1.99 & 19 & 32.99 & 155 & 36.73 \\ 30 & 1311 & 23.61 & 19 & 27.91 & 155 & 35.50\end{array}$ $\begin{array}{lllllll}30 & 1311 & 23.61 & 19 & 27.99 & 155 & 35.43 \\ 30 & 1410 & 28.34 & 19 & 23.45 & 155 & 26.46\end{array}$

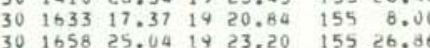

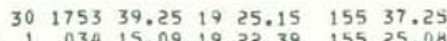

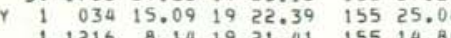
11623 46.32 19 25.41 $155 \quad 14.8$ $1 \quad 1727 \quad 41.591918 .48 \quad 15516.17$

$11922 \quad 16.42 \quad 19 \quad 15.64 \quad 15522.09$
12109

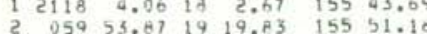

$\begin{array}{llllllllllll}9.41 & 1.82 & 2.020 & 0 & 86 & .08 & 7 & .7 & .9 & 9 & 9 & 0\end{array}$

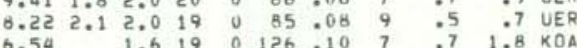

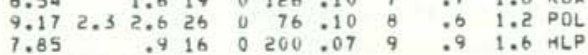
$\begin{array}{lllllllllll}25.91 & 2.5 & 1.4 & 27 & 0 & 103 & .10 & 28 & 1.0 & 3.6 & \text { KKU } \\ 7.89 & .9 & 9.6 & 18 & 0 & 91 & 10 & 7 & .7 & 1.0 & \text { UER }\end{array}$

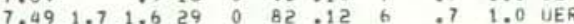

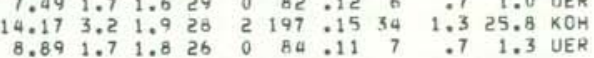
$\begin{array}{lllllllllllll}3.29 & 3.0 & 3.0 & 22 & 0 & 131 & .12 & 17 & .7 & 1.2 & \text { MOK }\end{array}$ $\begin{array}{lllllllllllll}5.10 & 1.2 & 1.7 & 22 & 0 & 117 & .13 & 10 & .7 & 1.0 & \text { SWR }\end{array}$

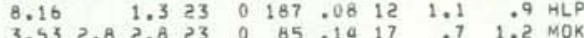

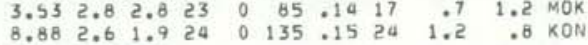
$\begin{array}{llllllllllllll}6.07 & 1.8 & 1.6 & 20 & 0 & 73 & .09 & 8 & .7 & .9 & \text { UER }\end{array}$

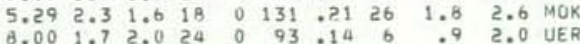

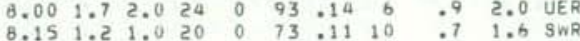

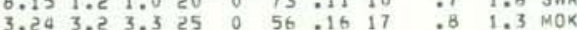
$\begin{array}{lllllllllll}15.05 & 3.4 & 3.7 & 33 & 0 & 64 & .10 & 2 & .6 & .9 & \text { DEP } \\ 14.46 & 2.5 & 2.5 & 32 & 0 & 34 & .09 & 2 & .5 & .7 & \text { DEP }\end{array}$

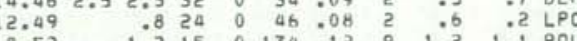

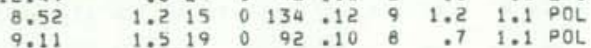
$\begin{array}{rrrrrrrrrrr}8.51 & 1.5 & 1.1 & 19 & 0 & 114 & .11 & 8 & .9 & 1.5 & \text { UKF } \\ 9.10 & 3.5 & 3.3 & 31 & 0 & 39 & .13 & 13 & .7 & 1.1 & \text { UKF }\end{array}$

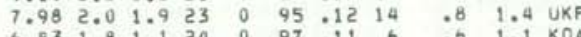
$\begin{array}{rrrrrrrrrrr}6.83 & 1.8 & 1.1 & 2 a & 0 & 97 & .11 & 6 & .6 & 1.1 & K O A \\ 9.74 & 2.0 & 2.3 & 27 & 0 & 122 & .13 & 6 & .8 & 1.0 & K 0 A\end{array}$ $\begin{array}{cccccccccccc}0.21 & 2.0 & 2.3 & 29 & 0 & 117 & .15 & 7 & .8 & 1.0 & \text { KOA } \\ 1.49 & & 1.3 & 15 & 0 & 84 & .05 & 6 & .3 & 99.0 & \text { SWR }\end{array}$

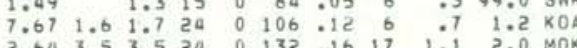

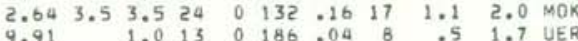

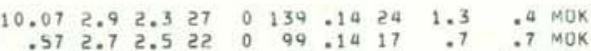

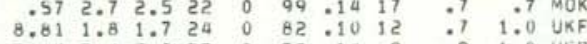

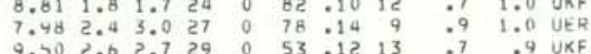
\begin{tabular}{llllllllll}
3.41 & 2.9 & 2.8 & 18 & 0 & 92 & .17 & 8 & .9 & 2.3 \\
\hline & MOK
\end{tabular}

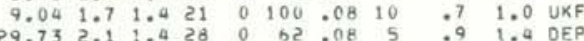

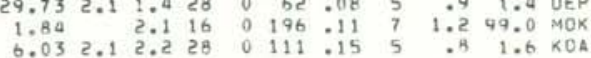
$\begin{array}{rrrrrrrrrrrr}0.80 & 2.4 & 2.7 & 29 & 0 & 144 & .13 & 9 & .8 & .9 & \text { LSW }\end{array}$ $4.553 .61 .619 \quad 0250.18323 .32 .7 \times 04$
HVO EARTHQUAKE SUMMARY LIST

PAGE 56

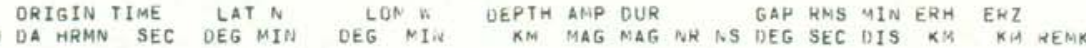

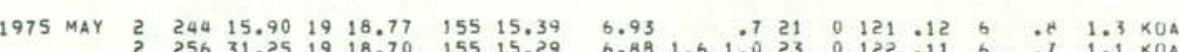

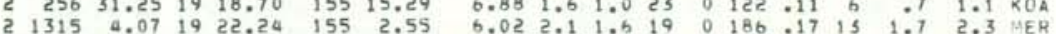

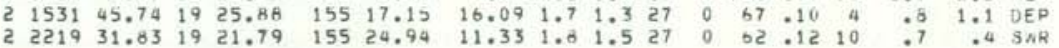

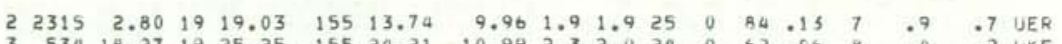

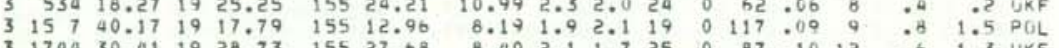

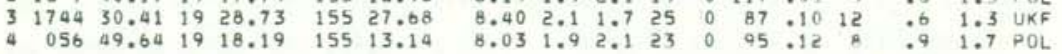

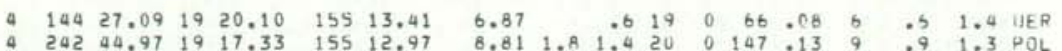

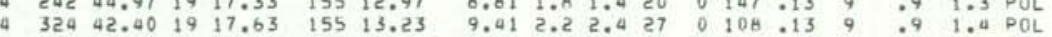

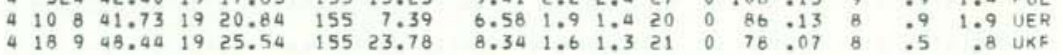

18340.6919 .19 .0315515 .39 4 2044 $59.75 \quad 19 \quad 31.77 \quad 155 \quad 15.39$ $42115 \quad 42.31 \quad 1920.15 \quad 15512.65$

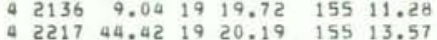

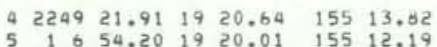

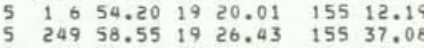
$553349.321931 .89 \quad 15541.67$

$\begin{array}{llllllll}5 & 7 & 7 & 21.07 & 19 & 25.44 & 155 & 36.36\end{array}$

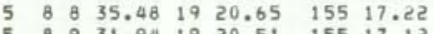
5
5 $93 \quad 42.791925 .80 \quad 15536.96$

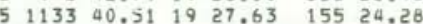

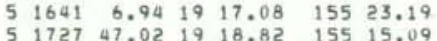

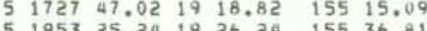
5 . $3128.0951926 .24 \quad 15556.01$ $5213930.591926 .01 \quad 15536.42$

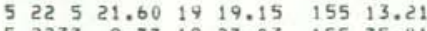

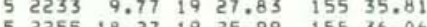

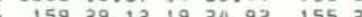
$622754.791920 .60 \quad 155 \quad 7.37$

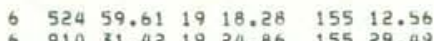

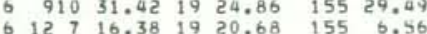

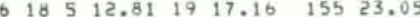

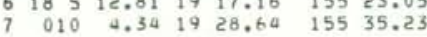

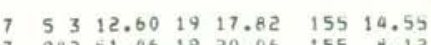
71300.15 ig 30.60 155 10.78 $\begin{array}{lllllllllllll}7.95 & 1.0 & 21 & 0 & 107 & .07 & 6 & .5 & .8 & \text { KOA }\end{array}$

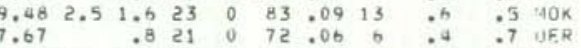

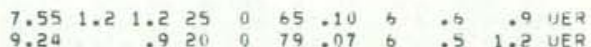

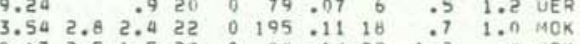

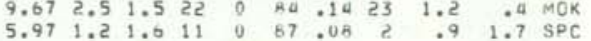

$\begin{array}{llllllrrrrr}3.34 & 2.8 & 2.8 & 20 & 0 & 95 & .13 & 16 & .8 & 1.6 & 10 K \\ 30.66 & 1.2 & 26 & 0 & 62 & .07 & 5 & : 0 & 1.2 & \text { DEP }\end{array}$

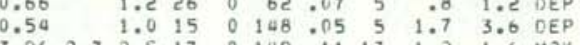

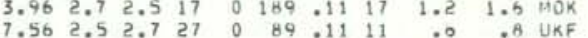
$\begin{array}{lllllllllll}5.93 & 1.8 & 2.0 & 25 & 0 & 120 & .13 & 8 & .8 & 2.3 & 5 \times R\end{array}$

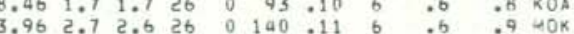
6.251 .61 .625 O 76.13 A .7 1.A UER $\begin{array}{llllllllllll}6.90 & 1.7 & 1.6 & 24 & 0 & 77 & .11 & 8 & .7 & 1.4 & \text { UER }\end{array}$

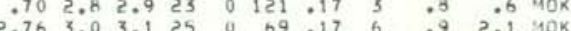
$\begin{array}{lllllllllllll}7.83 & 1.0 & 1.1 & 23 & 0 & 112 & .10 & 8 & .7 & 1.1 & \text { POL }\end{array}$

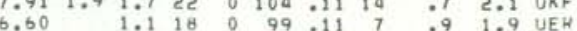

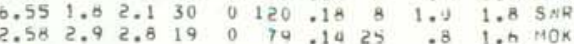
$\begin{array}{lllllllllll}8.34 & 1.0 & 21 & 0 & 134 & 10 & 7 & .0 & . A P O L\end{array}$

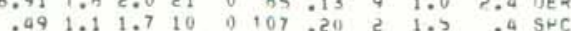

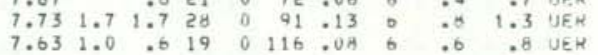
$8.611 .31 .25230061:-10010 \quad 0001.1$ UKF 
HVU EARTHOUAKE SUMRARY LIST

HAGE $\rightarrow 7$

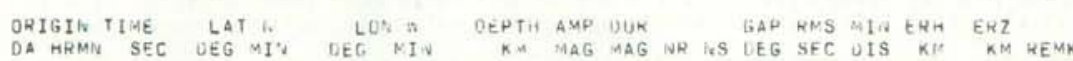
YEAR MON DA HRMM SEC UEG MI' DEG NIN KM MAG MAG INR FS DEG SEC UIS KI KM REMK

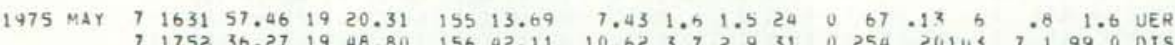

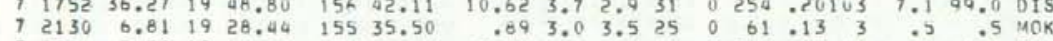

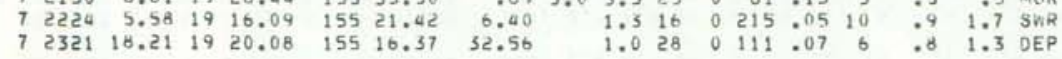

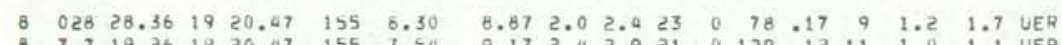

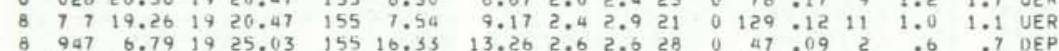

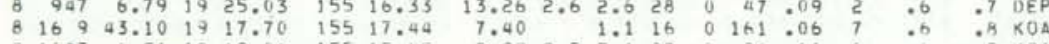

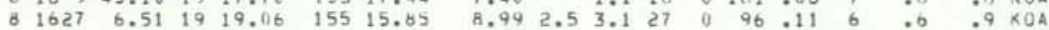

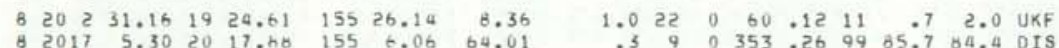

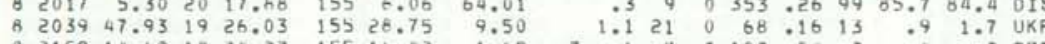

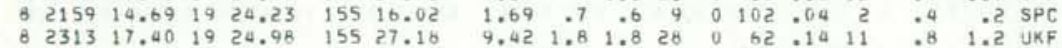

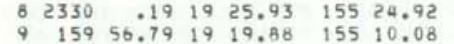

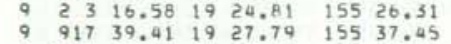
Q $1318,10.401927 .13$ 155 34.01

$\begin{array}{lllllll}9 & 1327 & 27.56 & 19 & 20.30 & 155 & 6.60 \\ 9 & 1551 & 2.36 & 14 & 23.74 & 155 & 25.45\end{array}$

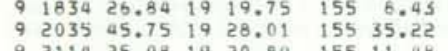

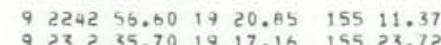

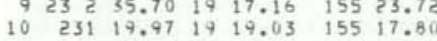
$10 \quad 349 \quad 43.73 \quad 19 \quad 19.06 \quad 155 \quad 13.80$ $55 \$ 3.8919 \quad 20.03 \quad 15511.84$ $\begin{array}{lllllll}10 & 621 & 16.37 & 19 & 18.78 & 155 & 15.39\end{array}$ $10 \quad 7338.56 \quad 1931.50 \quad 15539.01$ $10 \quad 135054.1319 \quad 17.81 \quad 15515.26$ $10 \quad 1921 \quad 17.04 \quad 19 \quad 19.32 \quad 155 \quad 13.80$ $\begin{array}{lllllll}10 & 1932 & 51.91 & 19 & 20.26 & 155 & 7.5\end{array}$ $101940 \quad 44.721920 .09 \quad 155 \quad 7.90$ $\begin{array}{rrrrrrr}10 & 2133 & 9.86 & 19 & 17.45 & 155 & 22.30 \\ 11 & 42 & 2.78 & 19 & 32.10 & 155 & 39.81\end{array}$

$\begin{array}{lllllll}11 & 418 & 29.48 & 19 & 18.95 & 155 & 12.95\end{array}$

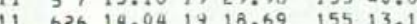

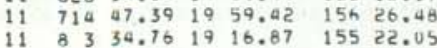

$\begin{array}{lllllll}11 & 916 & 10.03 & 19 & 12.72 & 155 & 00.53\end{array}$

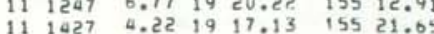

HVO EARTHQUAKE SUMMARY LIST

PATSE $5 B$

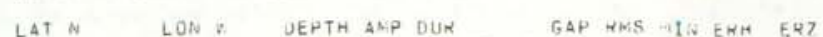

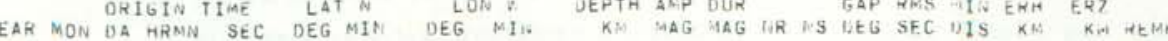

1975 MaY 11 1623 $\quad 0.04 \quad 19 \quad 17.36 \quad 135 \quad 12.31$ $11 \quad 1944 \quad 38.15 \quad 1929.45 \quad 15537.85$ $12030.531929 .25 \quad 15538.91$

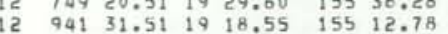

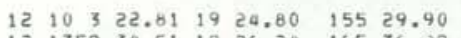

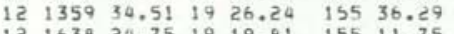

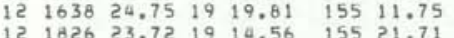
$13 \quad 751 \quad 7.84 \quad 19 \quad 19.52 \quad 155 \quad 15.93$

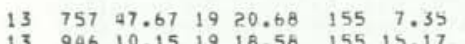

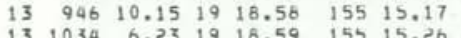

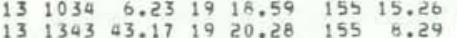
$13 \quad 1733 \quad 11.44 \quad 19 \quad 18.91 \quad 155 \quad 15.26$

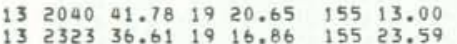
$14232336.611916 .86 \quad 155133.009$ $14 \quad 31651.81$ 19 $53.52 \quad 15535.89$

$\begin{array}{lllllll}14 & 428 & 3.93 & 19 & 17.53 & 155 & 14.23\end{array}$

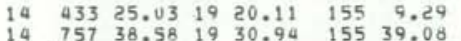

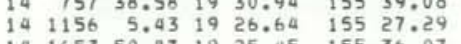
14165350.871925 .45 155 36.09 $\begin{array}{lllllll}14 & 2330 & 36.38 & 19 & 28.27 & 155 & 34.03 \\ 14 & 2343 & 52.61 & 19 & 18.78 & 155 & 15.04\end{array}$

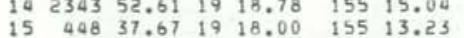
$15 \quad 73326.171917 .95 \quad 15513.65$ 151138.711920 .27 155 11.50 $\begin{array}{llllllll}15 & 11 & 8 & 38.71 & 19 & 20.27 & 155 & 11.50 \\ 15 & 1131 & 37.40 & 19 & 26.00 & 155 & 35.89\end{array}$

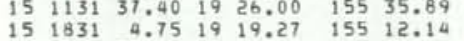
$1519737.891931 .02 \quad 15539.54$ $151916 \quad 34.8019 \quad 19.30 \quad 155 \quad 13.38$

$\begin{array}{lllllll}15 & 1929 & 41.41 & 19 & 20.24 & 155 & 8.07\end{array}$ 16 228 $15.141920 .21 \quad 15512.01$ $\begin{array}{lllllll}16 & 430 & 43.39 & 19 & 25.38 & 155 & 17.07 \\ 16 & 830 & 37.77 & 19 & 24.65 & 155 & 25.69\end{array}$

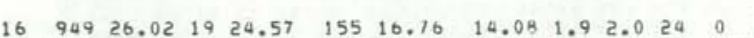

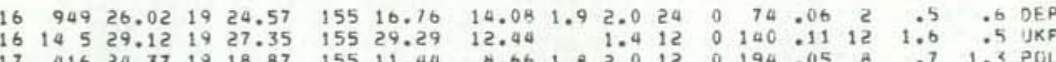

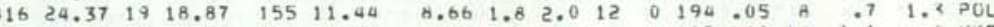

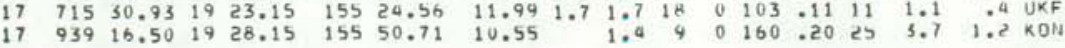
$\begin{array}{llllllllllllllllll}18 & 210 & 10.05 & 19 & 24.37 & 155 & 17.42 & 14.54 & 1.6 & 1.1 & 17 & 0 & \text { B } 1.05 & 2 & .6 & .6 & .0 & \text { DEP }\end{array}$

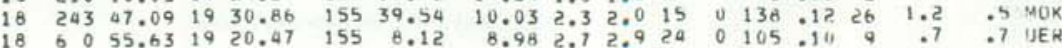




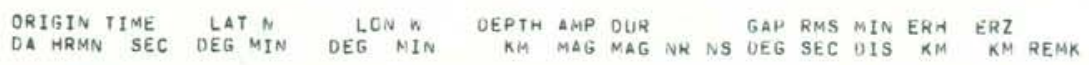

1975 MAY $18 \quad 610 \quad 11.81 \quad 19 \quad 19.88 \quad 155 \quad 8.36$

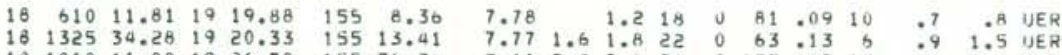

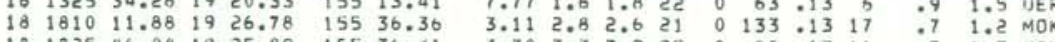

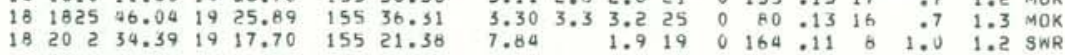

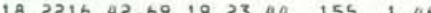

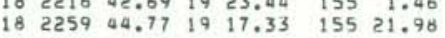

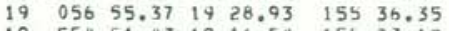

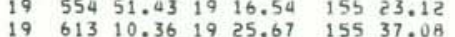

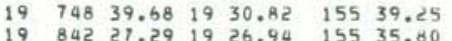

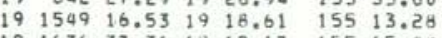
$\begin{array}{lllllll}19 & 1636 & 37.31 & 19 & 19.17 & 155 & 15.48 \\ 19 & 1743 & 32.09 & 19 & 17.77 & 155 & 13.37\end{array}$

$\begin{array}{lllllll}19 & 1837 & 13.06 & 19 & 18.44 & 155 & 13.40\end{array}$ $\begin{array}{lllllll}20 & 450 & 52.07 & 19 & 19.27 & 155 & 14.14 \\ 20 & 516 & 10.59 & 19 & 21.06 & 155 & 10.47\end{array}$ $201720.051921 .55 \quad 155 \quad 2.11$ $20 \quad 2027 \quad 20.78 \quad 19 \quad 18.05 \quad 155 \quad 14.23$

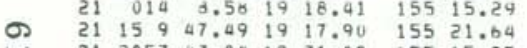
$\begin{array}{llllllll}21 & 2053 & 43.04 & 19 & 31.08 & 155 & 15.27 \\ 21 & 21 & 5 & 52.07 & 19 & 18.24 & 155 & 13\end{array}$ $21223258.18 \quad 20 \quad 18.72 \quad 155 \quad 36.78$ $\begin{array}{lllllll}22 & 446 & 5.44 & 19 & 22.69 & 155 & 27.83\end{array}$

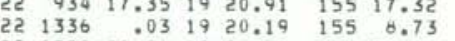

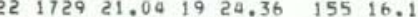
$23 \quad 413 \quad 13.51 \quad 19 \quad 18.34 \quad 155 \quad 23.51$

$23 \quad 522 \quad .45 \quad 19 \quad 31.51 \quad 155 \quad 39.07$ $\begin{array}{lllllll}23 & 643 & 47.15 & 19 & 20.08 & 155 & 11.97\end{array}$ $23 \quad 95431.16 \quad 18$ $23 \quad 105516.291924 .50 \quad 15517.40$

$\begin{array}{lllllll}23 & 1128 & 4.64 & 19 & 19.08 & 155 & 15.46\end{array}$ $2411525.03 \quad 1924.15 \quad 155 \quad 15.84$ $\begin{array}{rrrrrrr}24 & 1533 & 9.03 & 19 & 20.64 & 155 & 13.10 \\ 24 & 1956 & 31.69 & 19 & 16.04 & 155 & 24.02\end{array}$

$242123 \quad 39.94 \quad 19 \quad 16.01 \quad 155 \quad 23.97$ 2a $2158 \quad 36.58 \quad 19 \quad 18.87 \quad 155 \quad 15.16$

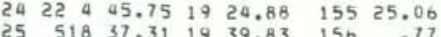

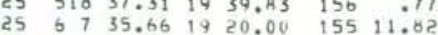

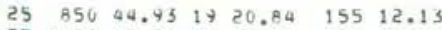

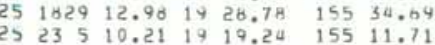

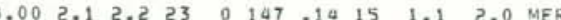

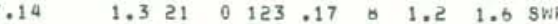

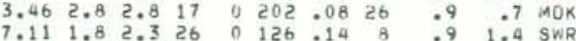

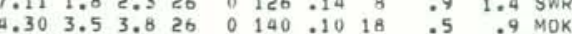

$\begin{array}{lllllllllll}0.74 & 2.7 & 2.7 & 18 & 0 & 139 & .13 & 26 & 1.2 & 1.8 & \text { MOK }\end{array}$

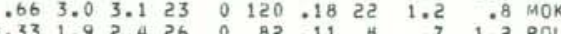

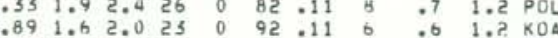
$0.451 .61 .626 \quad 0 \quad 92.1299 .801 .0001$ $\begin{array}{lllllllllll}8.25 & 1.7 & 1.3 & 20 & 0 & 81 & .09 & 8 & .7 & 1.2 & \text { POL }\end{array}$

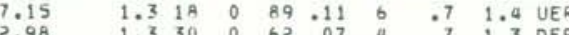

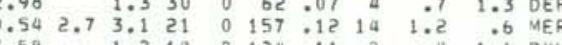

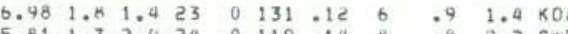

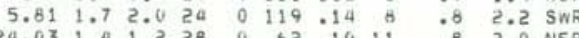

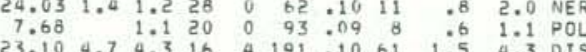

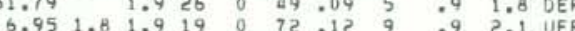

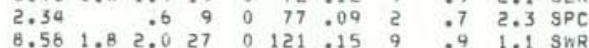
$\begin{array}{lllllllllll}9.34 & 2.5 & 2.1 & 24 & 0 & 83 & .15 & 26 & .9 & 1.5 & \text { MOK }\end{array}$

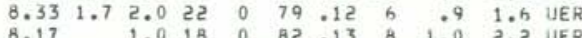

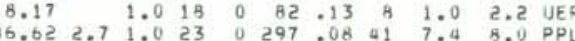

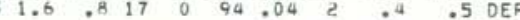

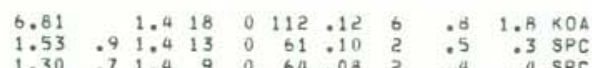
$\begin{array}{llllllll}1.30 & .7 & 1.4 & 9 & 0 & 64 & .08 & 5\end{array}$

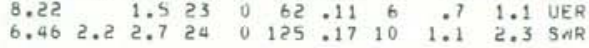
$\begin{array}{lllllllllll}6.26 & 1.9 & 2.0 & 24 & 0 & 125 & .15 & 14 & 1.1 & 2.1 & \text { SWR }\end{array}$

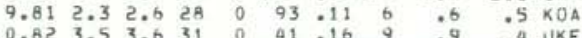

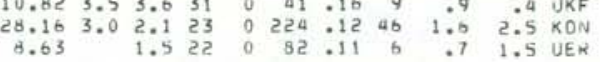
$\begin{array}{lllllllllll}9.09 & 1.7 & 1.4 & 24 & 0 & 67 & .11 & 7 & .7 & 1.1 & \text { UER }\end{array}$

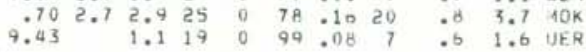

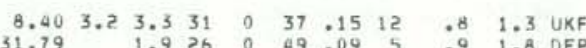

MVO EARTHQUAAE SUMRARY LIST

PAGE DO

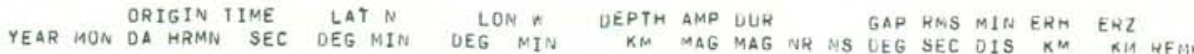

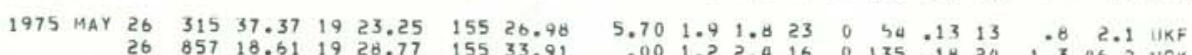

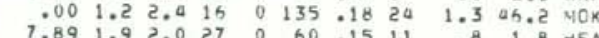

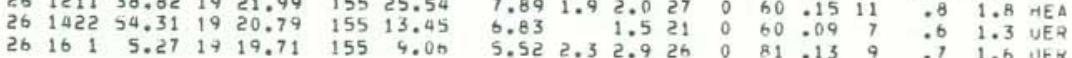

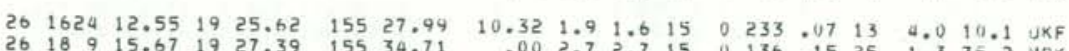

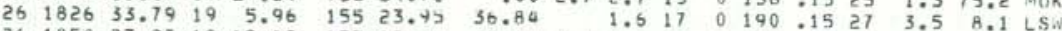

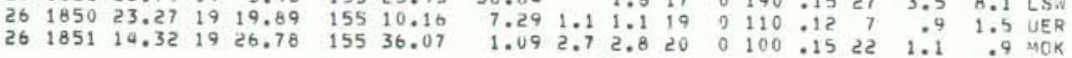

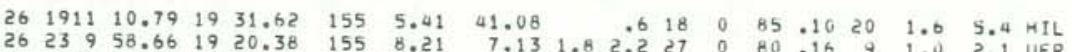

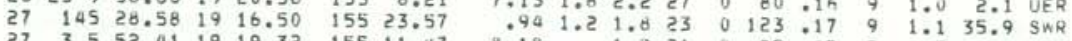
$\begin{array}{llllllllllllllll}27 & 35 & 52.41 & 19 & 19.32 & 155 & 11.47 & 8.18 & 1.2 & 21 & 0 & 99 & .07 & 7 & .5 & .8 \\ 27 & 335 & 30.37 & 19 & 20.50 & 155 & 7.74 & 6.90 & 1.1 & 24 & 0 & 86 & .13 & 9 & .9 & 1.7 \text { UER } \\ \text { UER }\end{array}$

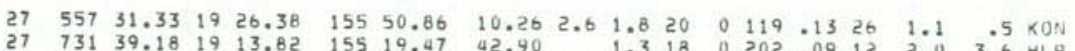

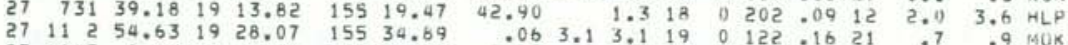

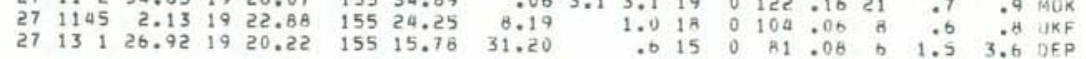

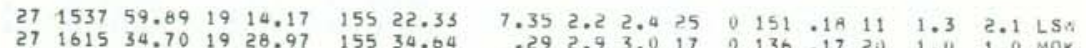

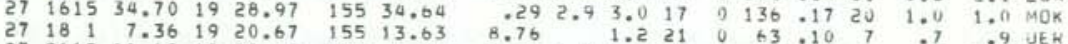

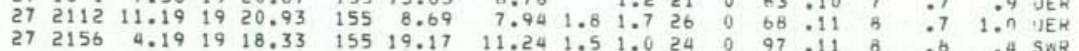

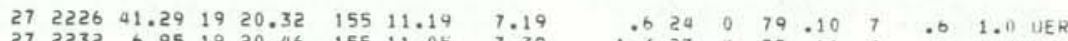

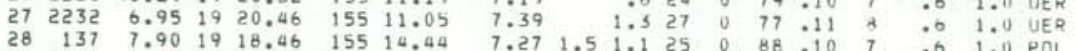

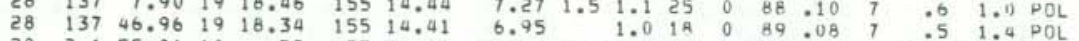

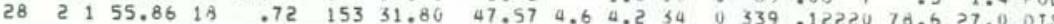

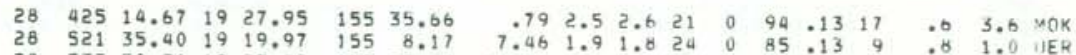

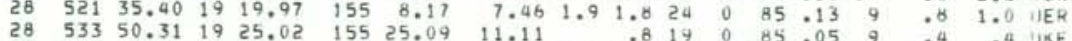

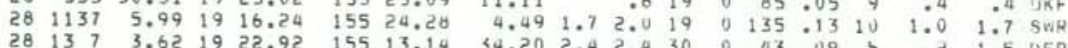

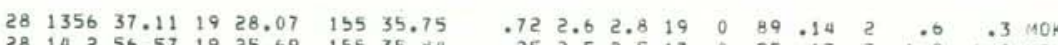

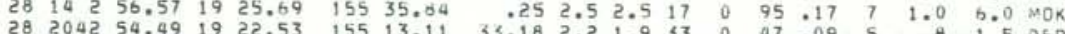

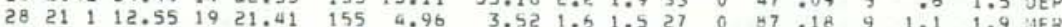

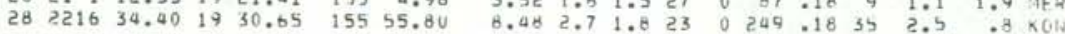

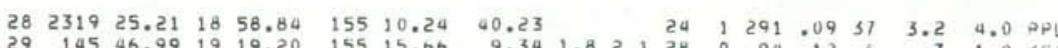

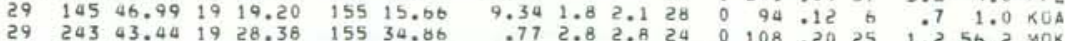

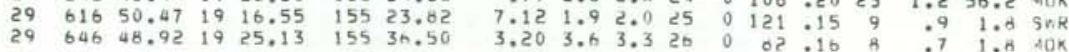

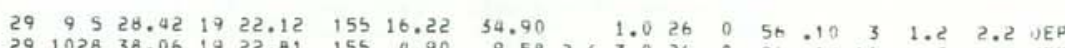

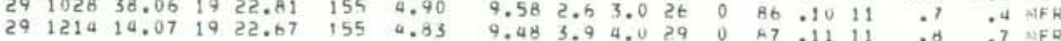




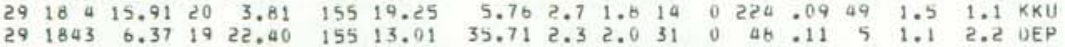

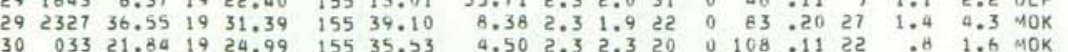

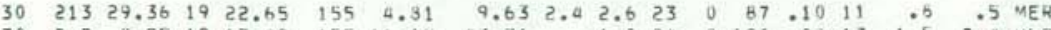

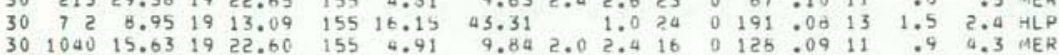

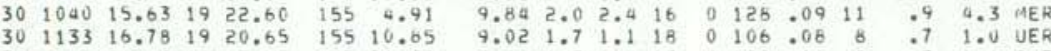

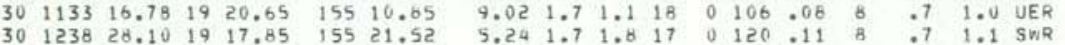

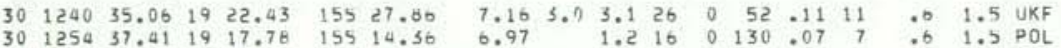

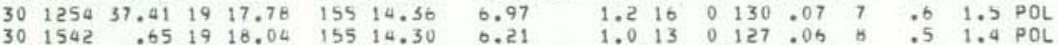

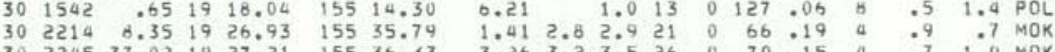
$\begin{array}{lllllllllllllllllll}31 & 0 & 3 & 6.23 & 19 & 19.04 & 155 & 15.26 & 8.47 & 1.6 & 2.0 & 23 & 0 & 111 & .08 & 6 & .5 & .7 & \text { KOA }\end{array}$

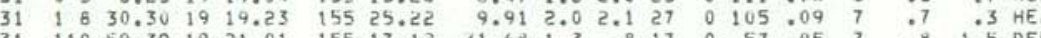

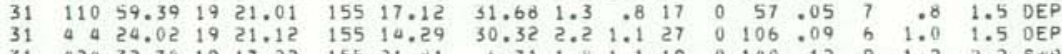

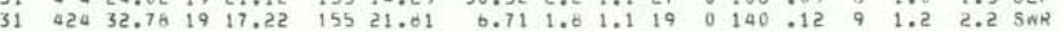

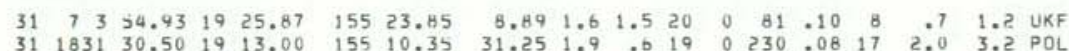

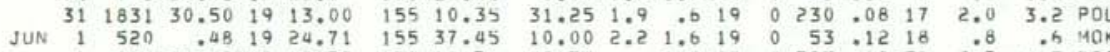

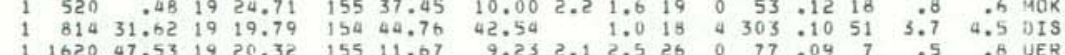

$\begin{array}{llllll}11725 & 57.66 \quad 19 & 24 . \mathrm{AB} & 155 & 25.90\end{array}$ $1253137.611925 .83 \quad 15528.69$ $\begin{array}{lllllll}2 & 510 & 14.47 & 19 & 22.76 & 155 & 27.87 \\ 2 & 812 & 50.15 & 19 & 18.25 & 155 & 15.70\end{array}$

$\begin{array}{llllll}2 & 958 & 36.99 & 19 & 27.39 \quad 155 \quad 35.99\end{array}$ $2124850.601919 .84 \quad 15511.32$

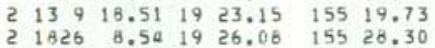
$\begin{array}{lllllll}2 & 1959 & 33.04 & 19 & 26.34 & 155 & 37.26\end{array}$

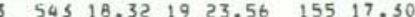

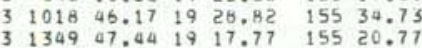
$3135923.051919 .48 \quad 15513.19$

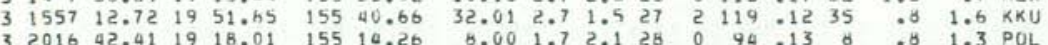

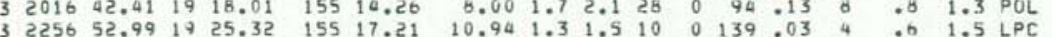

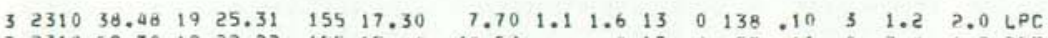

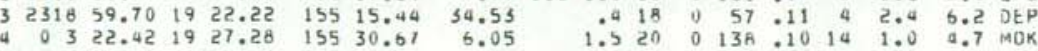

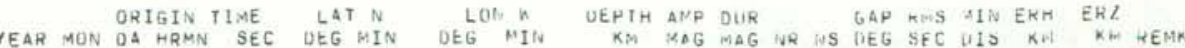

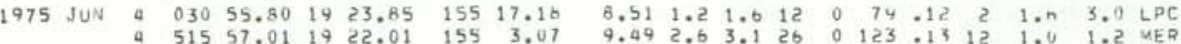

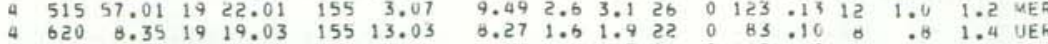

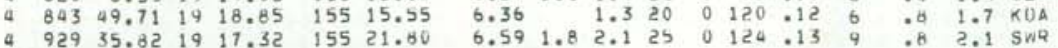

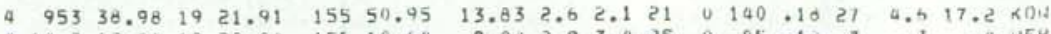

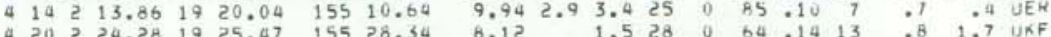

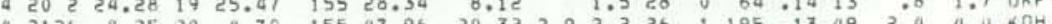

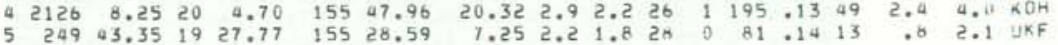

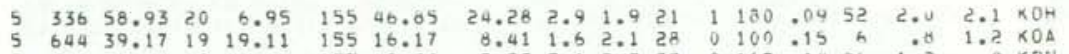

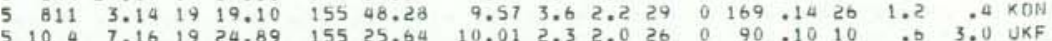

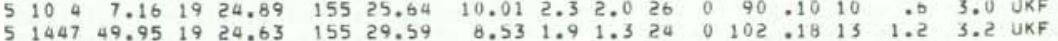

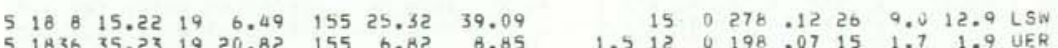

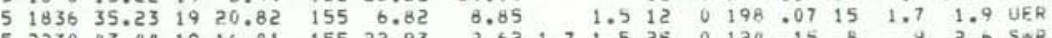

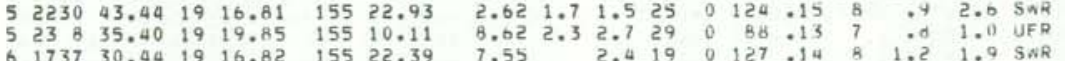

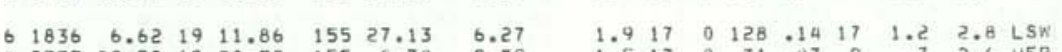

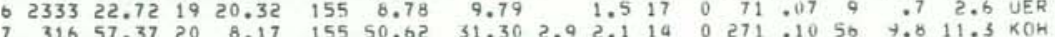

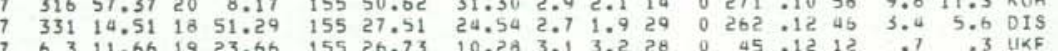

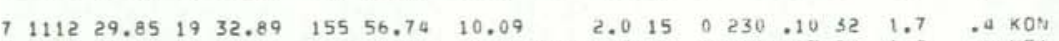

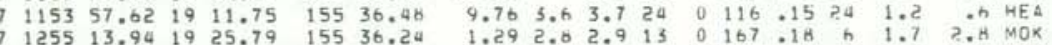

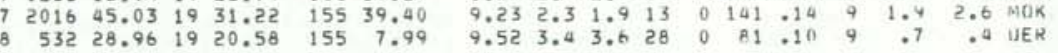

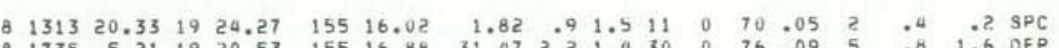

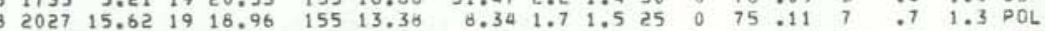

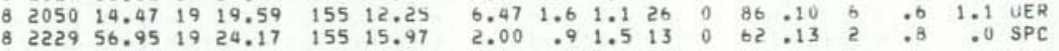

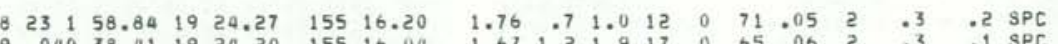
131
14.89
9

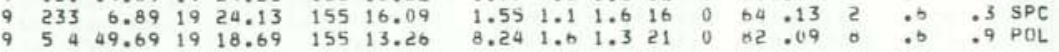

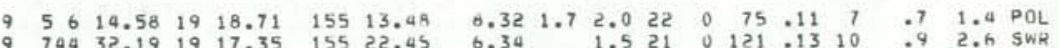

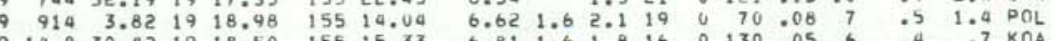

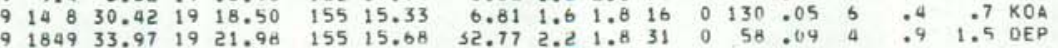

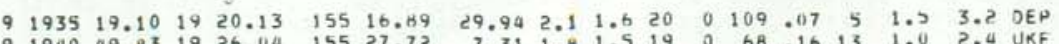

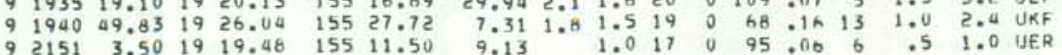


HVO EARTHQUARE SUMATANY LIST

PAGE 63

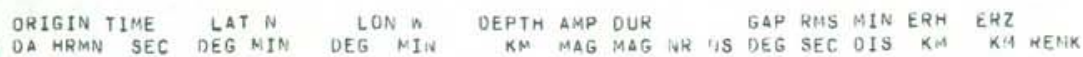

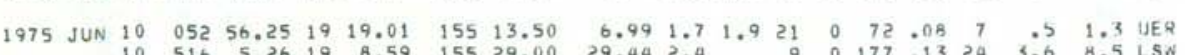

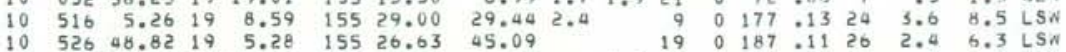

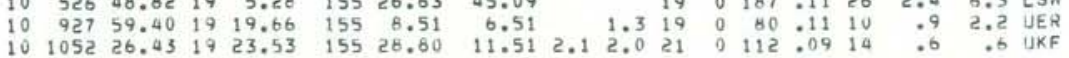
$\begin{array}{llllllllllllllllll}10 & 1218 & 39.54 & 19 & 19.12 & 155 & 15.92 & 8.88 & 2.4 & 3.1 & 29 & 0 & 96 & .09 & 6 & .5 & .8 & \text { KOA }\end{array}$

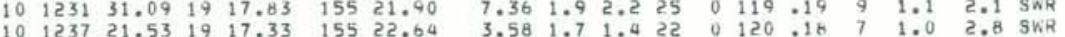

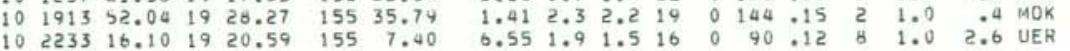
$\begin{array}{lllllllllllllllllll}10 & 2326 & 5.02 & 19 & 19.29 & 155 & 15.69 & 7.28 & 1.5 & 1.4 & 23 & 0 & 93 & .10 & 6 & .6 & 1.2 \text { KOA }\end{array}$

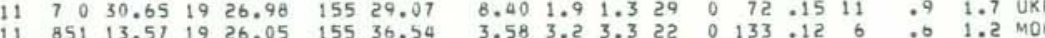

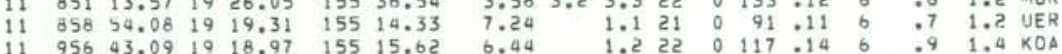

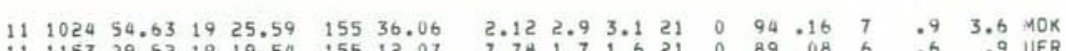

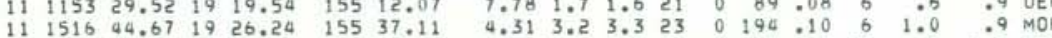

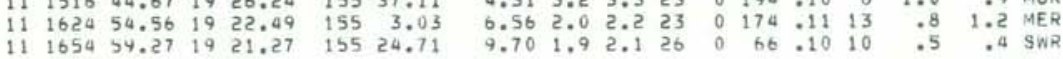

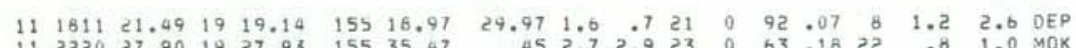

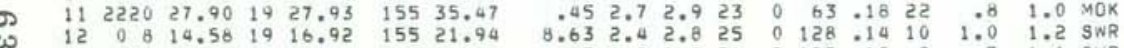

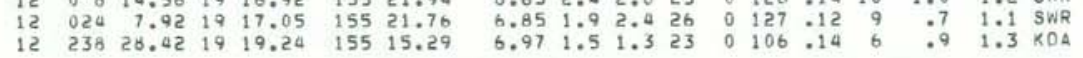

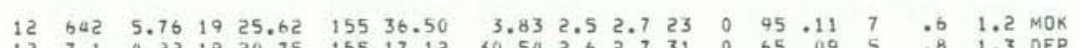

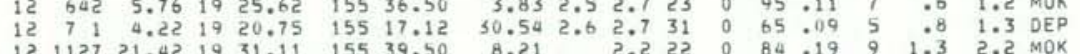

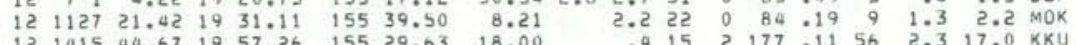

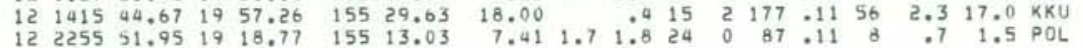

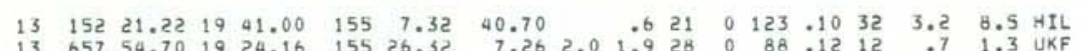

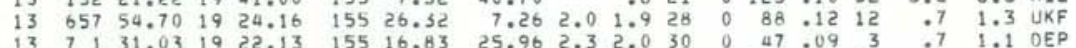

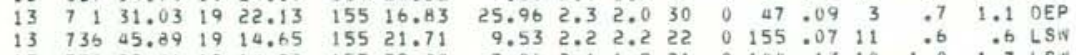

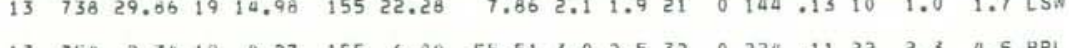

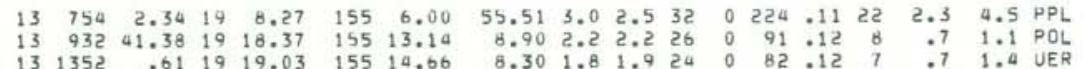

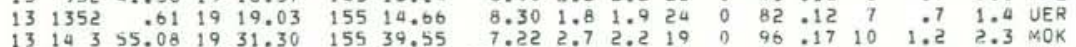

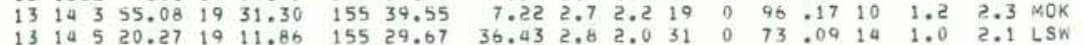

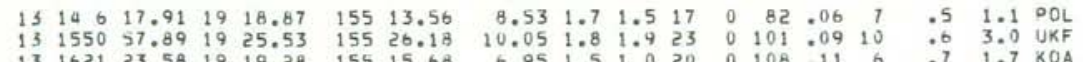

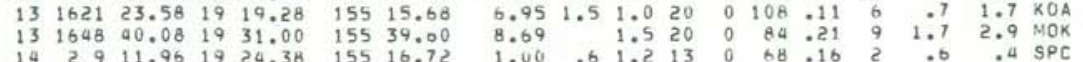

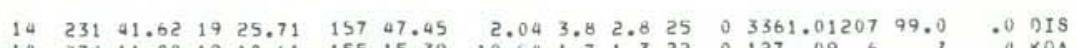

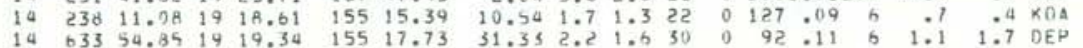

HVO EARTHOUAKE SUMMARY LIST

PAGE OQ ORIGIN THME LATN LUN D OEPTH AMP OUR GAP RMS MIN ERH ERZ KEMK

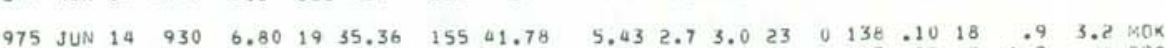
$14 \quad 936 \quad 35.631924 .36 \quad 15516.22$ $142021.241926 .66 \quad 15536.05$ $142247 \quad 6.821925 .90 \quad 15535.97$ $\begin{array}{lllllll}15 & 013 & 40.48 \quad 19 & 19.98 \quad 155 \quad 8.70\end{array}$ $15-3439.16$ 19 19.88 155 15.57

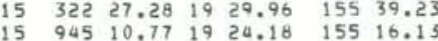

$\begin{array}{lllllll}15 & 1341 & 14.86 & 19 & 25.64 \quad 155 \quad 26.85\end{array}$

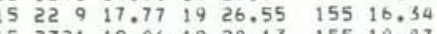
\begin{tabular}{l}
$19.06 \quad 1920.13 \quad 155 \quad 10.87$ \\
\hline
\end{tabular} $\begin{array}{llllrll}16 & 146 & 15.34 & 19 & 20.70 & 155 & 13.08 \\ 16 & 518 & 22.95 & 19 & 8.50 & 155 & 34.56\end{array}$

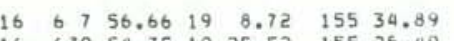
$16 \quad 73521.62 \quad 19 \quad 19.15 \quad 155 \quad 15.56$ $\begin{array}{rrrrrrr}16 & 939 & 10.15 & 19 & 17.58 & 155 & 21.39 \\ 16 & 1440 & 16.17 & 19 & 16.73 & 155 & 23.37\end{array}$

$\begin{array}{lllllll}16 & 1943 \quad 14.74 & 19 & 19.39 \quad 155 \quad 15.82\end{array}$

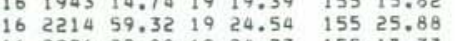
$\begin{array}{lllllll}16 & 2226 & 22.88 & 19 & 24.33 & 155 & 17.37\end{array}$

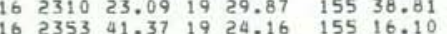

$\begin{array}{llllllll}17 & 022 & 24.37 & 19 & 24.39 & 155 & 16.87\end{array}$

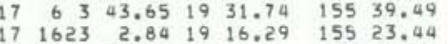
$17 \quad \begin{array}{llllll}1716 & 12.57 \quad 19 & 18.06 & 155 & 16.83\end{array}$

$\begin{array}{lllllll}17 & 1728 & 28.68 & 19 & 18.93 & 155 & 12.34\end{array}$ $\begin{array}{lllllll}17 & 1822 & 56.38 & 19 & 29.61 & 155 & 47.44\end{array}$

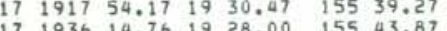
17193614.76 19 $28.00 \quad 15543.87$

$\begin{array}{llllllll}17 & 20 & 5 & 59.12 & 19 & 27.63 & 155 & 44.05\end{array}$ $17 \quad 2041 \quad 51.36 \quad 1927.48 \quad 155 \quad 44.27$ $17233955.751927 .76 \quad 15544.05$

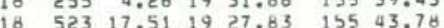
$\begin{array}{lllllll}18 & 1141 & 30.59 & 19 & 30.09 & 155 & 39.60 \\ 18 & 1337 & 47.00 & 19 & 17.09 & 155 & 22.35\end{array}$

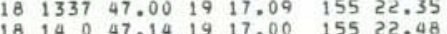

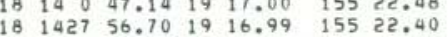
1816621.521918 .48 156 3.82 $\begin{array}{lllllll}18 & 2022 & 5.69 & 19 & 31.22 & 155 & 39.29\end{array}$

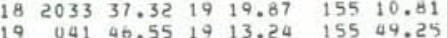

$\begin{array}{lllll}.08 & 5 & 1.2 & .4 & 5 P C\end{array}$

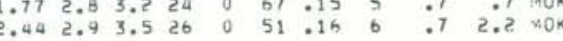
$\begin{array}{lllllllllll}9.06 & 2.4 & 3.5 & 28 & 0 & 73 & -11 & 9 & .7 & .9 & \text { UEH }\end{array}$

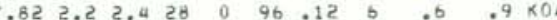

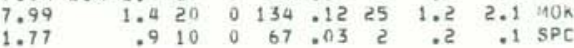
$\begin{array}{lllllllllll}8.14 & 1.9 & 2.1 & 16 & 0 & 165 & .07 & 11 & .7 & 1.8 & \text { UKF }\end{array}$

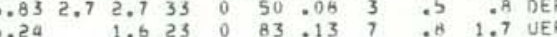

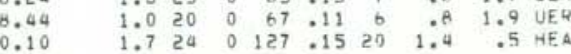
$\begin{array}{llllllllll}0.03 & 2.8 & 2.6 & 26 & 0 & 123 & .17 & 20 & 1.4 & .5\end{array}$ HEA $\begin{array}{lllllllllll}.50 & 1.7 & 1.4 & 20 & 0 & 99 & .10 & 9 & .8 & 1.4 & \text { UKF }\end{array}$ $\begin{array}{lllllllllll}.03 & 1.7 & 1.5 & 24 & 0 & 93 & .11 & 6 & .6 & 1.1 & \text { KOA }\end{array}$

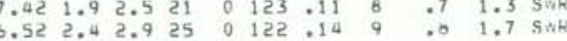

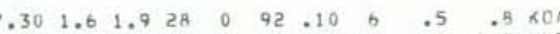

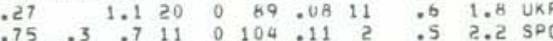

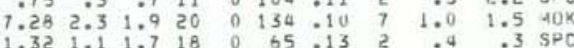

$\begin{array}{lllllllllll}3.66 & 1.6 & 2.4 & 15 & 0 & 59 & 19 & 2 & 1.2 & 2.0 & \mathrm{SPC}\end{array}$

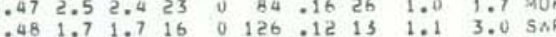

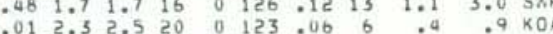

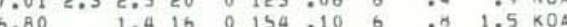
$\begin{array}{llllllllll}.60 & 1.4 & 19 & 0 & 99 & 09 & 7 & .7 & 1.7 & \text { POL }\end{array}$

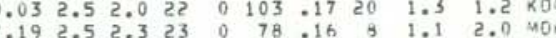

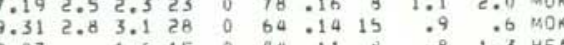
$\begin{array}{llllllllll}0.06 & 1.4 & 17 & 0 & 120 & .13 & 10 & 1.5 & .5 & \text { mok }\end{array}$

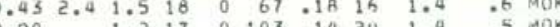
7.632 .31 .6 it 0 as $.1926 \quad 1.9$ 3.9 mox $\begin{array}{llllllllll}9.05 & 1.0 & 9 & 0 & 133 & .09 & 9 & 1.6 & 2.4 & \text { Mo }\end{array}$

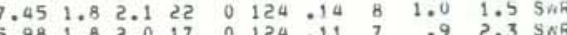

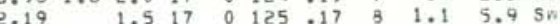

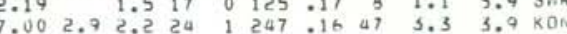
$\begin{array}{llllllllll}8.18 & 1.9 & 14 & 0 & 62 & .15 & 9 & 1.5 & 2.6 & \text { MO }\end{array}$

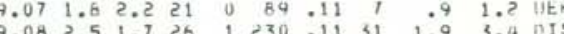

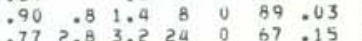




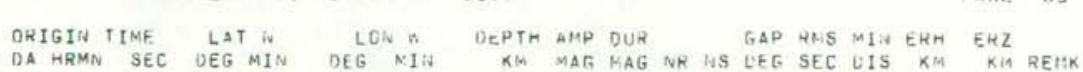
YEAH MON: DA HRMN SEC DEG MIN DEG NIIA KM MAG MAG NR HS LEE SEC UIS KM KIA RET:

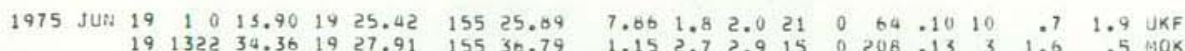

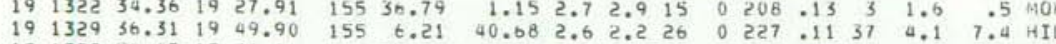

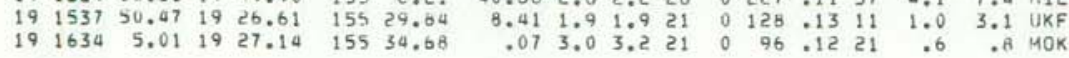

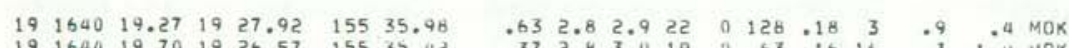

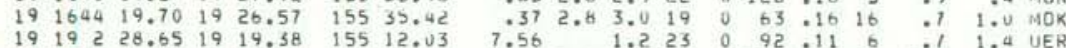

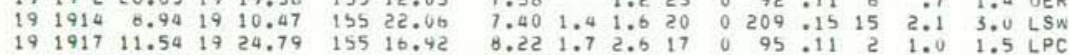

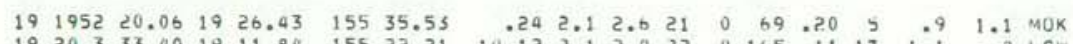

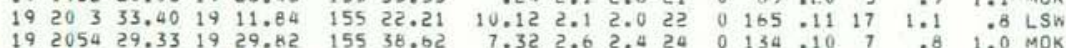

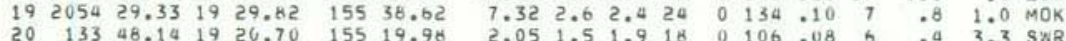

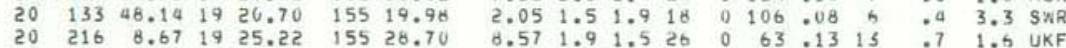

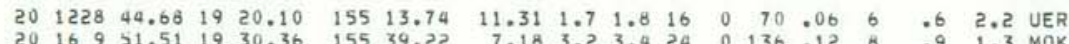

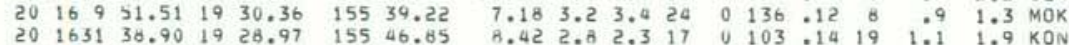

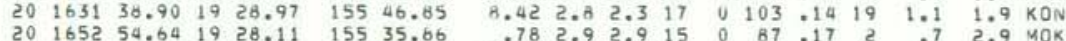

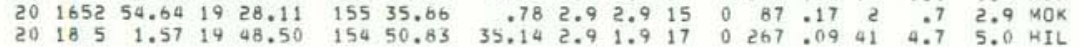

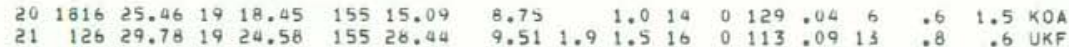

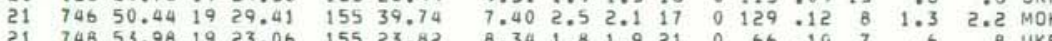

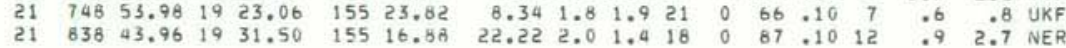

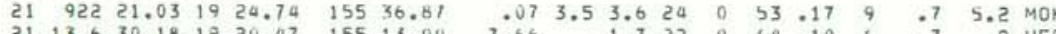

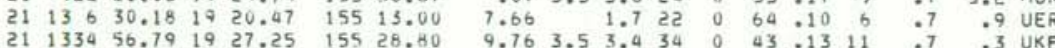

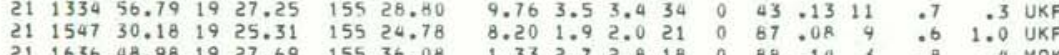
$21 \quad 1636 \quad 48.98 \quad 1927.69 \quad 15536,08$

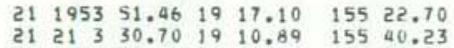
ट1 $211522.561928 .95 \quad 15535.59$ $212259 \quad 7.46 \quad 1918.55 \quad 15515.50$

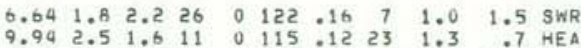

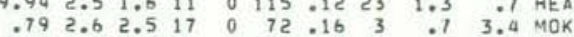

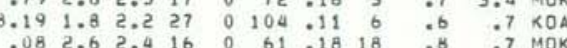
$\begin{array}{lllllllllllllllll}22 & 635 & 11.73 & 19 & 14.79 & 155 & 3.23 & 42.63 & 1.0 & 16 & 0 & 243 & 06 & 27 & 1.9 & 2.7 & 01 \mathrm{~S}\end{array}$

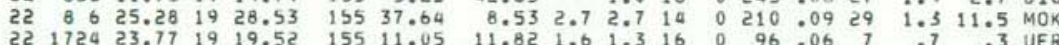

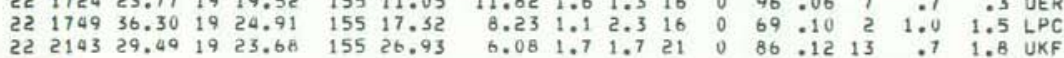

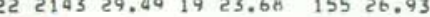

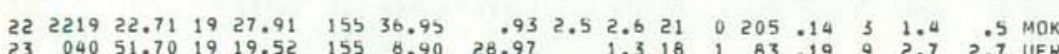

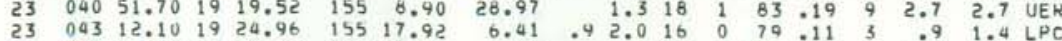

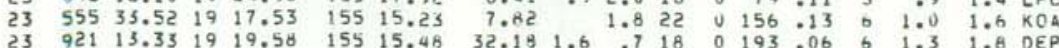
$\begin{array}{lllllllllllllllllll}23 & 10 & 51.33 & 19 & 27.34 & 155 & 28.62 & 7.22 & 2.3 & 2.5 & 25 & 0 & 87 & .15 & 11 & 1.0 & 2.2 & \text { UKF }\end{array}$

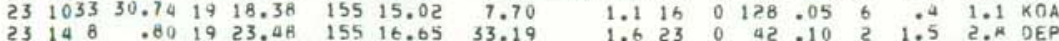

HVO EARTMQUARE SULAAGY LIST

Palit on

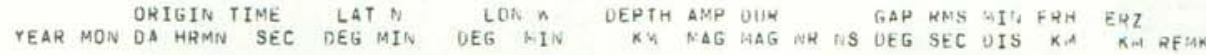

1975 JUN $23 \quad 1427 \quad 55.82 \quad 1924.97 \quad 155 \quad 25.06$

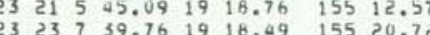

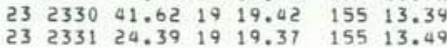

$24 \quad 0842.24 \quad 19 \quad 14.58 \quad 155 \quad 26.24$ $\begin{array}{llllll}354 & 23.90 & 19 & 27.26 & 155 & 35.90\end{array}$ $24 \quad 1831 \quad 45.76$ id $59.01 \quad 155 \quad 19.91$

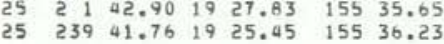

$\begin{array}{llllllll}25 & 414 & 5.41 & 19 & 20.21 & 155 & 12.81\end{array}$ $\begin{array}{lllllll}25 & 728 & 53.47 & 19 & 6.77 & 155 & 25.69\end{array}$ $\begin{array}{lllllll}25 & 729 & 42.24 & 19 & 7.57 & 155 & 26.12 \\ 25 & 735 & 52.46 & 19 & 25.22 & 155 & 36.63\end{array}$

$\begin{array}{lllllll}25 & 834 & 53.92 & 19 & 28.46 & 155 & 35.17\end{array}$

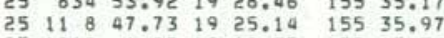
$\begin{array}{llllllll}25 & 1218 & 6.87 & 19 & 18.55 & 155 & 13.53 \\ 25 & 2142 & 41.94 & 19 & 31.32 & 155 & 30.5\end{array}$

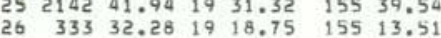

$\begin{array}{lllllll}26 & 610 & 12.49 & 19 & 26.49 & 155 & 30.30\end{array}$ $\begin{array}{lllllll}26 & 644 & 50.04 & 19 & 34.30 & 155 & 41.22\end{array}$ $\begin{array}{lllllll}26 & 1258 & 46.11 & 19 & 18.51 & 155 & 13.11 \\ 26 & 1511 & 57.72 & 19 & 25.69 & 155 & 37.67\end{array}$ $26 \quad 1732 \quad 55.43 \quad 19 \quad 13.01 \quad 15533.90$

26 $1735 \quad 15.7519 \quad 13.10 \quad 155 \quad 33.56$ $\begin{array}{lllllll}26 & 1850 & 56.44 & 19 & 25.79 & 155 & 15.61 \\ 26 & 20 & 13.95 & 19 & 36.71 & 155 & 26.44\end{array}$ $26 \quad 2154 \quad 50.76 \quad 19 \quad 17.96 \quad 155 \quad 15.53$ $\begin{array}{llllll}033 & 31.87 & 20 & 3.18 & 155 & 44.50\end{array}$

$\begin{array}{lllllll}27 & 133 & 20.82 & 19 & 28.64 & 155 & 38.17\end{array}$ $\begin{array}{rrrrrrrr}27 & 143 & 38.18 & 19 & 14.23 & 155 & 21.97 \\ 27 & 229 & 4.89 & 19 & 28.02 & 155 & 34.34\end{array}$ $27 \quad 855 \quad 33.43 \quad 19 \begin{array}{llll}19.65 & 155 & 15.41\end{array}$ $\begin{array}{lllll}110 & 19 & 25.81 & 155 \quad 35.61\end{array}$

$\begin{array}{lllllll}28 & 431 & 27.57 & 19 & 29.16 & 155 & 40.04\end{array}$ $28141651.991931 .02 \quad 15539.21$ $28 \quad 1739 \quad 57.8019 \quad 19.57 \quad 15515.45$ $\begin{array}{lllllll}26 & 2348 & 21.31 & 19 & 16.65 & 155 & 21.90\end{array}$ $2912941.0919 \quad 9.32 \quad 15542.31$ $39211959.12 \quad 1925.37 \quad 155 \quad 17.56$ $\begin{array}{llllll}2331 & 15.26 & 19 & 23.39 & 155 & 17.55\end{array}$ $\begin{array}{lllllll}30 & 116 & 40.21 & 19 & 25.34 & 155 & 17.60\end{array}$

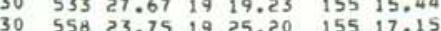

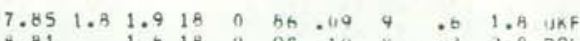

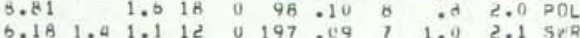
$\begin{array}{lllllllllll}8.17 & 2.1 & 2.4 & 23 & 0 & 70 & .10 & 7 & .6 & 1.3 & \text { UER } \\ 7.57 & 1.9 & 1.9 & 21 & 0 & 68 & .11 & 7 & .7 & 1.5 & \text { UER }\end{array}$ $\begin{array}{llllllllllll}12.59 & 2.1 & 10 & 0 & 173 & .13 & 20 & 3.3 & .7 & \text { LSA }\end{array}$

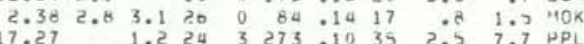

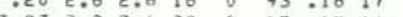
16 .8 1.5 10 $\begin{array}{llllllllll}7.51 & .5 & 17 & 0 & 69 & .12 & 0 & .9 & 2.1 & \text { UER }\end{array}$ $\begin{array}{lllll}43.80 & 2.0 & 1.5 & 23 \\ 39.0 & 0 & 0\end{array}$

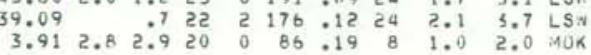
$\begin{array}{lllllllllll}1.20 & 2.9 & 3.0 & 15 & 0 & 126 & .13 & 3 & .8 & .4 & \text { MOK }\end{array}$

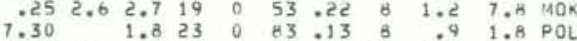

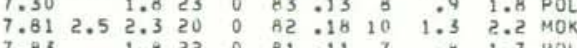
$3.3723 .93512330 .129013-5$ $\begin{array}{lllllll} & \end{array}$

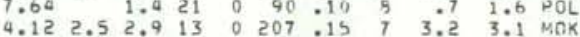

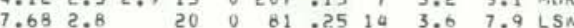
$\begin{array}{lllllllllll}38.44 & 2.8 & 12 & 0 & 185 & 15 & 51 & 0.2 & 13.8 & \text { LSN } \\ 11.95 & 2.2 & 2.5 & 28 & 0 & 51 & 13 & 3 & .9 & .4 & \text { LPC }\end{array}$

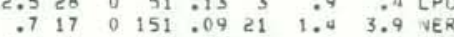
$\begin{array}{llllllllllll}7.58 & 1.1 & 15 & 0 & 146 & .07 & 5 & .0 & 1.6 & \times 04 \\ 15.87 & 2.7 & 2.2 & 29 & 2 & 141 & .14 & 42 & 1.4 & 4.2 & \text { KOH }\end{array}$ $\begin{array}{llllllllllll}6.91 & 2.6 & 2.5 & 25 & 0 & 115 & .12 & 22 & .4 & 1.1 & \text { NaK } \\ 9.52 & .6 & 1.5 & 22 & 0 & 157 & 09 & 11 & .0 & .6 & \text { LSW }\end{array}$

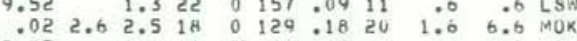

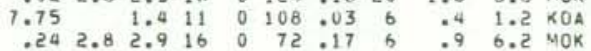
$\begin{array}{llllllllll}6.89 & 2.0 & 13 & 0 & 127 & .12 & 25 & 1.4 & 2.7 & \text { MOK }\end{array}$

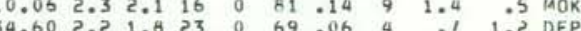

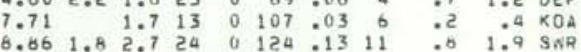
6.47 . 1.10190137 .28 21 2.0 2.2 HEA

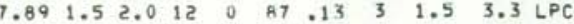
$\begin{array}{lllllllllll}8.19 & 1.4 & 2.0 & 16 & 0 & 91 & .12 & 3 & 1.2 & 1.7 & \text { LPC }\end{array}$

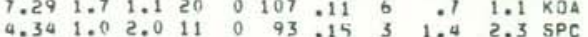
$\begin{array}{lllllllllll}8.18 & 1.7 & 14 & 0 & 250 & 05 & 10 & 1.7 & 1.9 & \text { SNR } \\ 8.11 & 2.7 & 3.1 & 19 & 0 & 137 & 0.28 & 21 & 3.0 & 2.2 & \text { HEA }\end{array}$

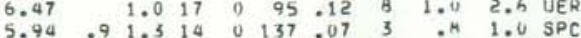



.921 द $304.11001 .4 \quad 1.1$ DEP $1851 \quad .331919 .54 \quad 15512.52 \quad 7.42 \quad 2.0220083 .13 \quad 7 \quad .8$ 1.8 UER

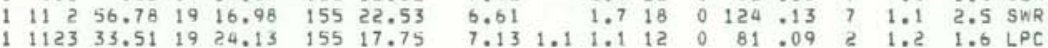

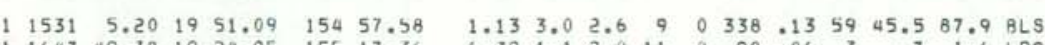

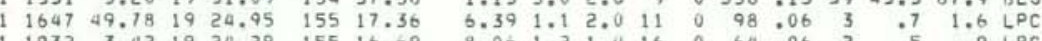

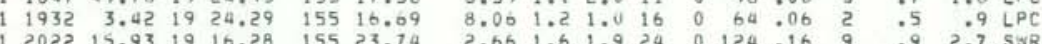

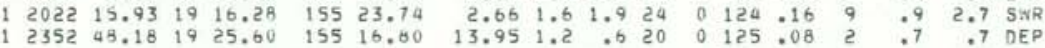

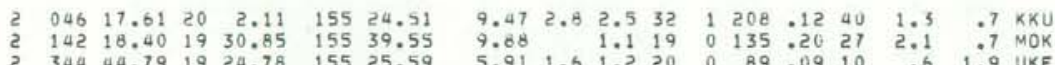

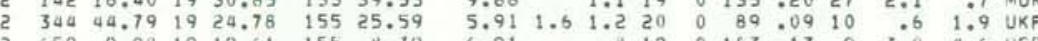

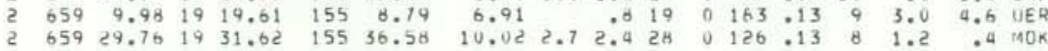
$\begin{array}{llllllllllllllllllll}2 & 7 & 4 & 25.97 & 19 & 19.00 & 154 & 59.77 & 2.06 & 1.7 & 1.0 & 14 & 0 & 271 & .17 & 28 & 9.3 & 3.3 & \text { DIS } \\ 2 & 725 & 50.44 & 19 & 26.50 & 155 & 23.79 & 11.48 & 1.7 & 1.3 & 17 & 0 & 80 & .06 & 9 & .6 & 2.1 & \text { UKF }\end{array}$

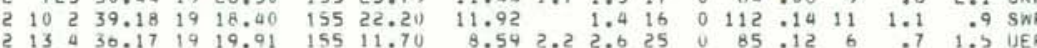

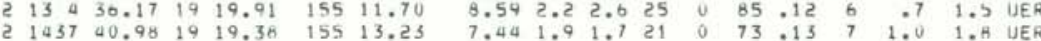

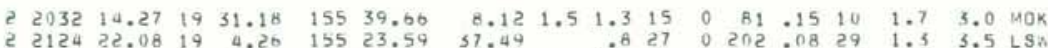

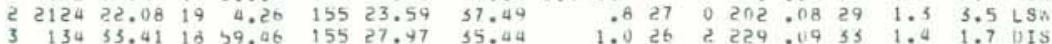
ORIGIN TIME LAT N LON N DEPTH AMP DUR GAP RMS MIVERA ERZ YEAQ MON OQATH 1975 JUL $3 \begin{array}{llllllllllllllllll}3 & 152 & 40.05 & 18 & 59.84 & 155 & 28.19 & 37.23 & 3.2 & 2.7 & 32 & 0 & 215 & 11 & 31 & 1.7 & 3.6 & 01 \mathrm{IS} \\ 3 & 2 & 26.55 & 19 & 23.30 & 155 & 48.95 & 10.12 & 1.5 & 20 & 0 & 112 & -13 & 25 & 1.2 & .5 \times 0 \mathrm{~N}\end{array}$

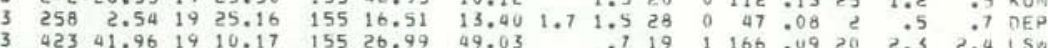

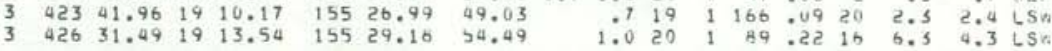

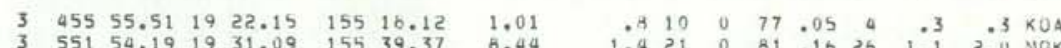

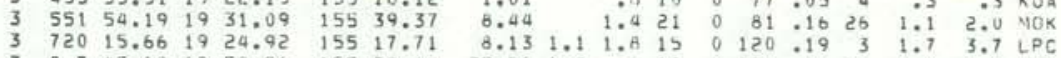

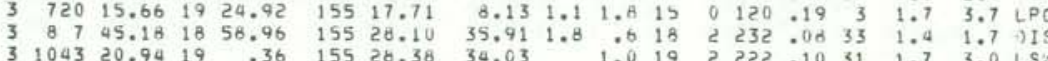

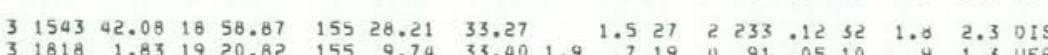

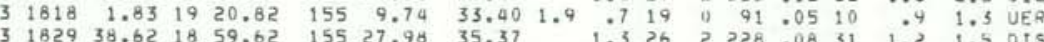

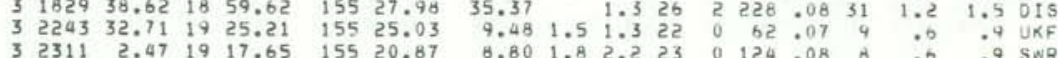

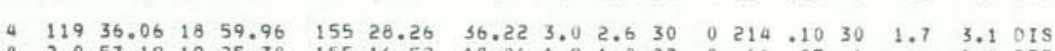

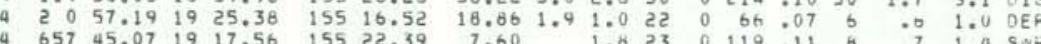

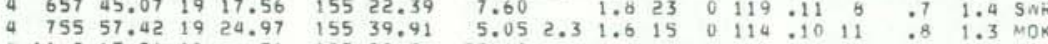

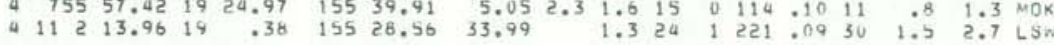

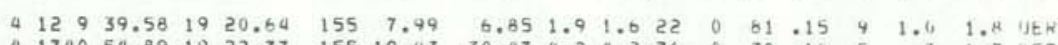

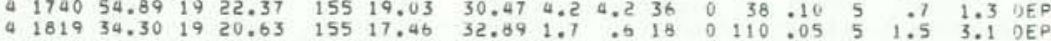

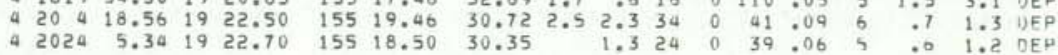

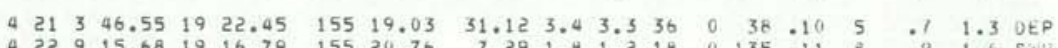

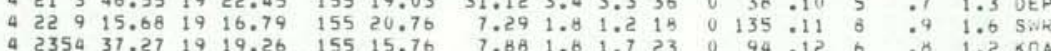

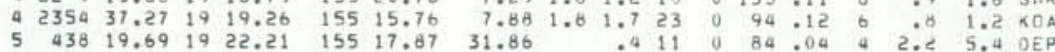

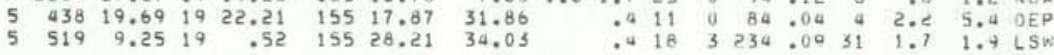

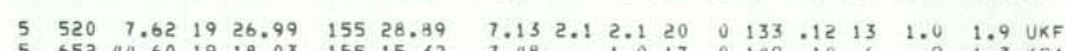

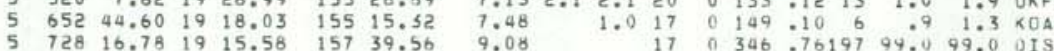

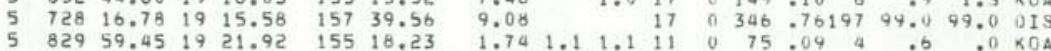

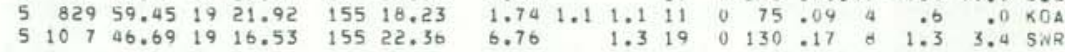

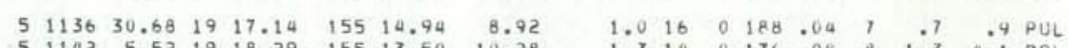

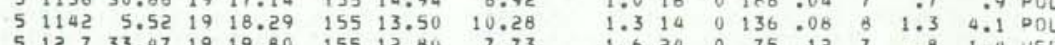

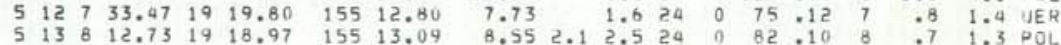

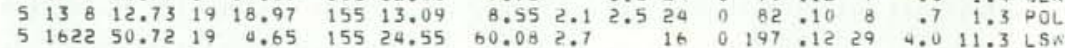

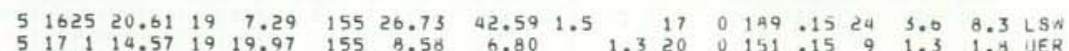

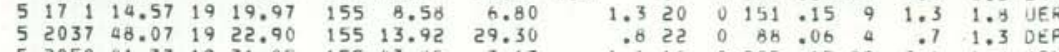

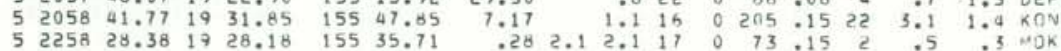

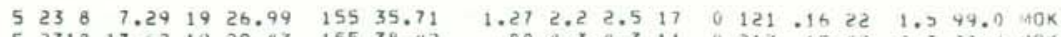

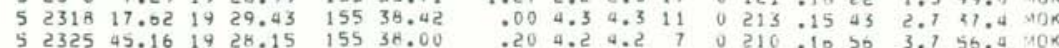




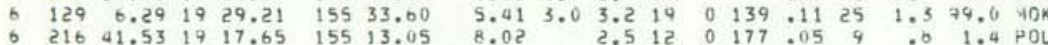

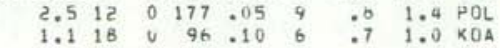

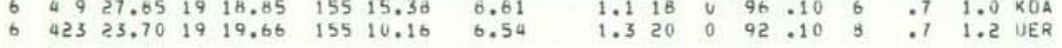

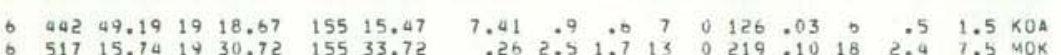

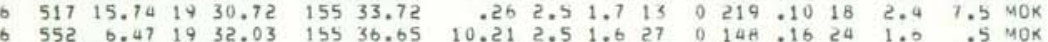

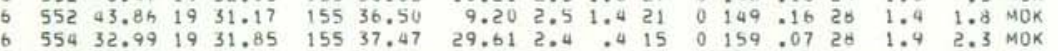
$\begin{array}{llllllllllllllllllll}6 & 6 & 6 & 21.61 & 19 & 32.63 & 155 & 35.43 & 22.84 & 2.6 & 1.4 & 19 & 0 & 168 & .16 & 27 & 2.8 & 4.3 & 40 \mathrm{~K}\end{array}$

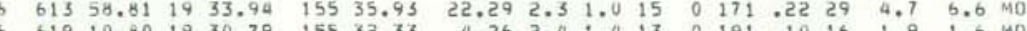

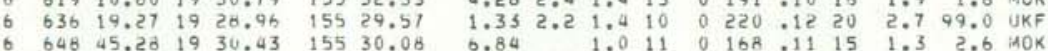

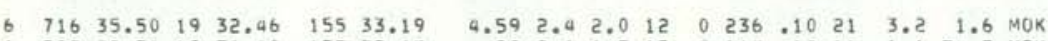

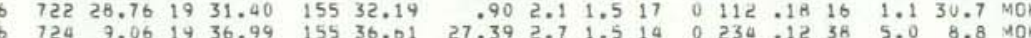

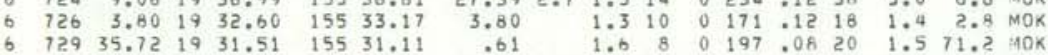

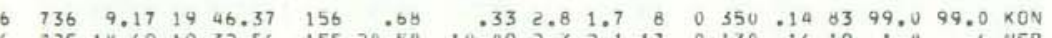

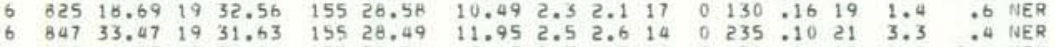

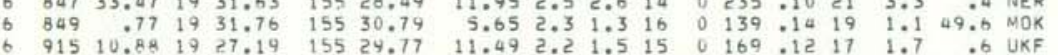

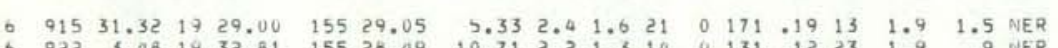

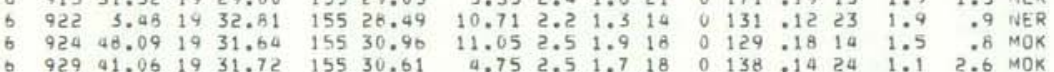

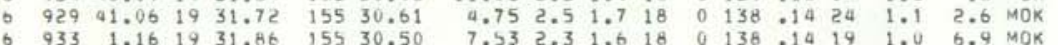

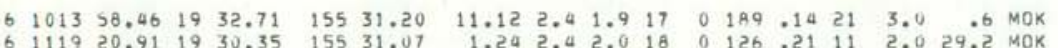

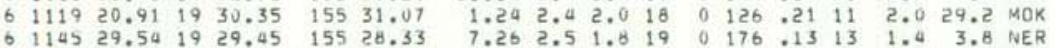

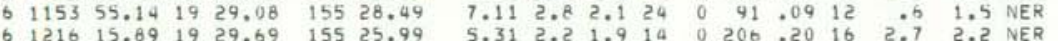

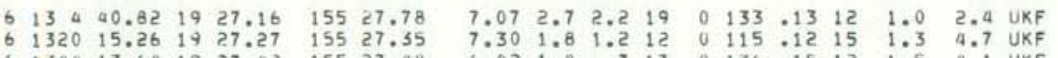

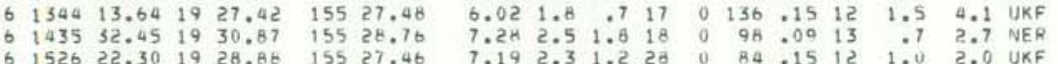
$\begin{array}{llllllllllllllllll}6 & 1528 & 26.64 & 19 & 31.04 & 155 & 25.82 & 2.34 & 2.6 & 2.0 & 17 & 0 & 98 & .14 & 16 & 1.1 & 5.2 & \text { NER } \\ 6 & 1538 & 53.93 & 19 & 29.38 & 155 & 28.67 & 5.00 & & .9 & 12 & 0 & 186 & 0.11 & 13 & 2.0 & 1.2 & \text { NER }\end{array}$

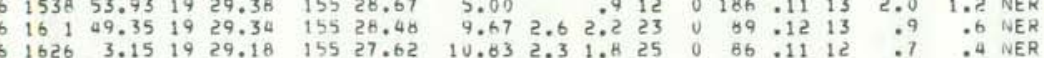

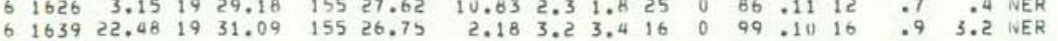

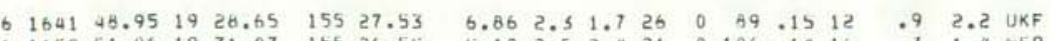

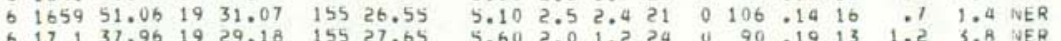

MVO EAKIMOUAKE SUMHARY LIST

PAGF 711 ORIGIN TIME LATN LON N OEPTH AMP DUR G GAP KES AIN ERM ERZ REM

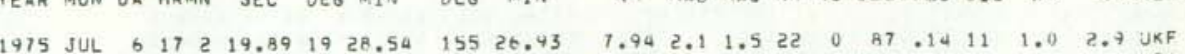

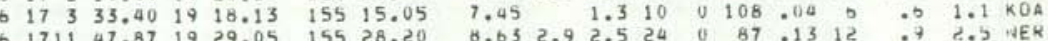

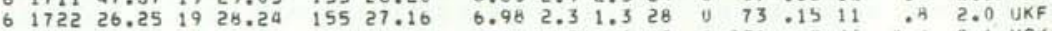
$6172327.881931 .11 \quad 15531.58$ 6
$1735 \quad 9.16 \quad 19 \quad 30.80 \quad 155 \quad 29.58$ 6 181033.921928 .51 155 26.01

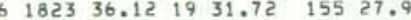
$6 \quad 195753.211927 .11 \quad 15528.31$

$62018 \quad 46.05 \quad 19 \quad 32.72 \quad 15528.71$ $62047 \quad 41.64 \quad 1931.77 \quad 155 \quad 29.93$ $62051 \quad 14.96 \quad 1931.61 \quad 155 \quad 30.35$

$6 \quad 2056 \quad 43.89 \quad 19 \quad 31.83 \quad 155 \quad 29.94$

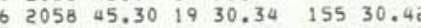
$\begin{array}{llllllll}6 & 21 & 1 & 41.22 & 19 & 30.93 & 155 & 30.07\end{array}$

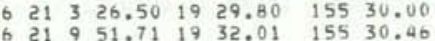

$\begin{array}{llllllll}6 & 2113 & 10.54 & 19 & 31.50 & 155 & 30.15\end{array}$ $6 \quad 2117 \quad 6.671931 .5415530 .12$

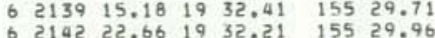

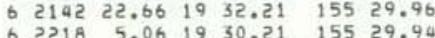

$62223 \quad 57.83 \quad 19 \quad 30.79 \quad 15527.06$ $6 \quad 2227 \quad 49.73 \quad 1931.13 \quad 15526.96$

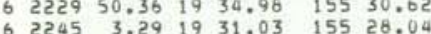
$62247 \quad 13.62 \quad 1929.25 \quad 15526.06$

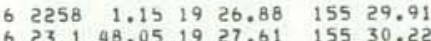

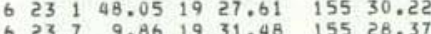

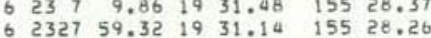

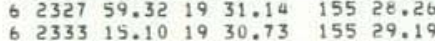

$62341 \quad 10.631930 .99 \quad 15530.46$

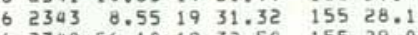

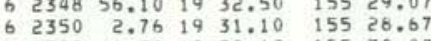
$62352 \quad 6.391932 .12 \quad 155 \quad 30.02$

\begin{tabular}{l}
$027 \quad 8.12 \quad 19 \quad 31.05 \quad 155 \quad 28.81$ \\
\hline
\end{tabular} $035 \quad 53.16 \quad 1929.96 \quad 15528.4$

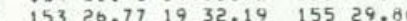
$2355.681931 .06 \quad 15528.20$
0

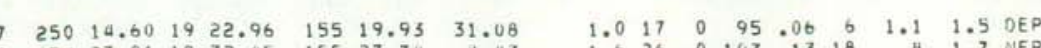

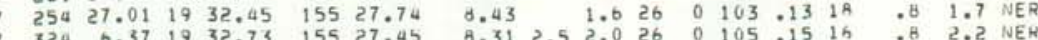
$\begin{array}{llllllllll}5.58 & .6 & 14 & 0 & 205 & .10 & 17 & 1.3 & 3.9 & \text { NER }\end{array}$

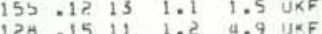

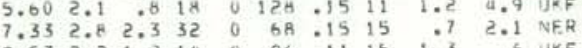

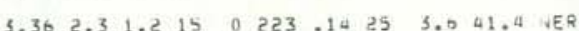

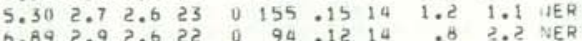

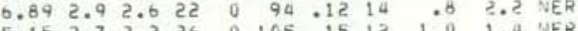

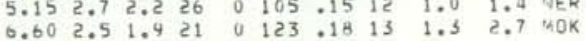

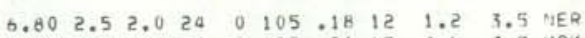

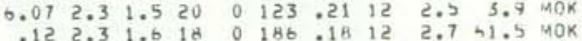

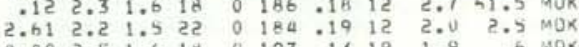
$\begin{array}{llllllllll}5.49 & 2.5 & 2.1 & 26 & 0 & 105 & .16 & 12 & 1.1 & 2.5 \\ 5.50 k\end{array}$

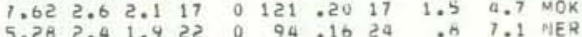

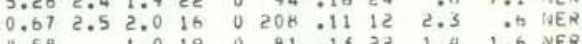

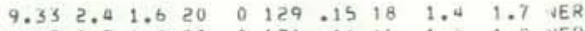

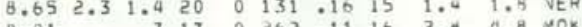

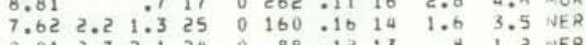
$\begin{array}{lllllllllll}8.93 & 1.9 & 1.3 & 13 & 0 & 172 & .11 & 11 & 1.4 & 3.1 & \text { UKF }\end{array}$

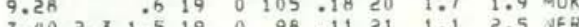

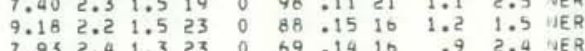
$\begin{array}{lllllllllll}7.22 & 2.0 & 1.0 & 18 & 0 & 226 & .09 & 18 & 1.6 & 2.7 & 40 \mathrm{~K}\end{array}$

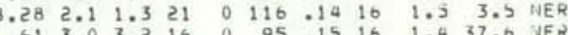

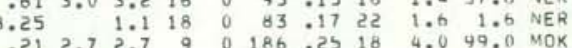

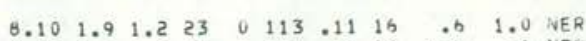

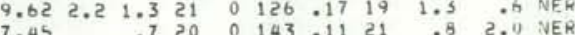


HVO EARTHQUAKE SUMMARY LIST

PAGE 71

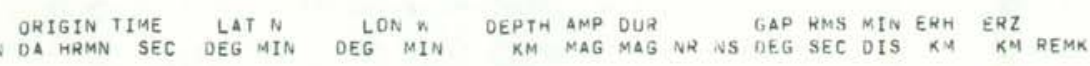
1975 JUL ? $34257.40 \quad 1931.96 \quad 15528.23$ 7 a $1153.531931 .97 \quad 15528.06$

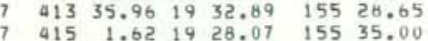

$\begin{array}{llllllll}7 & 420 & 27.07 & 19 & 32.51 & 155 & 27.04\end{array}$

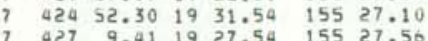
$\begin{array}{lllllll}7 & 427 & 9.41 & 19 & 27.54 & 155 & 27.56 \\ 7 & 434 & 4.11 & 19 & 32.07 & 155 & 28.18\end{array}$ \begin{tabular}{l}
$44322.32 \quad 19 \quad 32.29 \quad 155 \quad 27.95$ \\
\hline
\end{tabular}

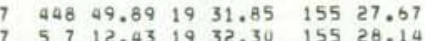

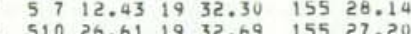

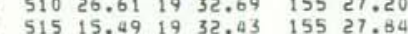

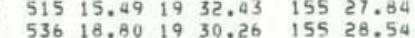

7
7 $53948.74 \quad 1932.28 \quad 155 \quad 27.78$ $\begin{array}{rrrrrrr}7 & 547 & 26.31 & 19 & 32.70 & 155 & 27.79 \\ 7 & 552 & 3.25 & 19 & 30.78 & 155 & 29.89\end{array}$

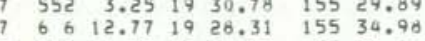

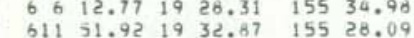

$\begin{array}{lllllll}7 & 010 & 30.84 & 19 & 30.66 & 155 & 23.84 \\ 7 & 626 & 21.89 & 19 & 30.29 & 155 & 26.14\end{array}$ 7 626 $21.8919 \quad 30.29$ 155 26.14

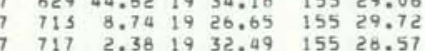

$\begin{array}{lllllll}7 & 723 & 5.25 & 19 & 31.23 & 155 & 26.40\end{array}$ 7.48
75.27
757 $\begin{array}{lllllll}7 & 811 & 27.64 & 19 & 32.06 & 155 & 28.12 \\ 7 & 817 & 24.23 & 19 & 32.55 & 155 & 27.08\end{array}$

$\begin{array}{lllllll}7 & 828 & 22.09 & 19 & 32.17 & 155 & 27.65\end{array}$ $\begin{array}{lllllll}916 & 40.61 & 19 & 32.24 & 155 & 27.65 \\ 918 & 40.75 & 19 & 32.19 & 155 & 27.94\end{array}$ $\begin{array}{llllll}922 & 51.77 & 19 & 31.54 & 155 & 25.19 \\ 931 & 29.02 & 19 & 31.77 & 155 & 26.83\end{array}$

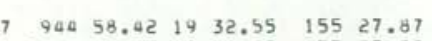

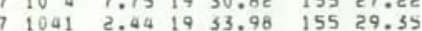

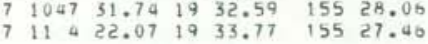
$\begin{array}{lllllll}7 & 1226 & 52.06 & 19 & 34.06 & 155 & 27.70\end{array}$

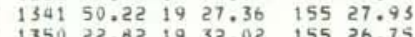

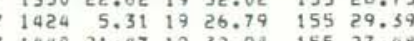

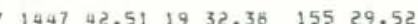
7164322.181931 .90 155 27.18

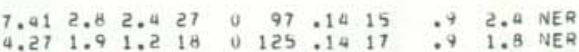

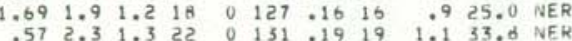
$.452 .82 .821 \quad 0 \quad 66.18 \quad 17 \quad .843 .3$ MOK

$\begin{array}{lllllllllll}5.06 & 2.0 & 1.0 & 25 & 0 & 95 & 17 & 18 & .9 & 3.7 & \text { NER } \\ 4.93 & 1.7 & 1.9 & 18 & 0 & 189 & 17 & 19 & 2.2 & 1.4 & \text { NER }\end{array}$

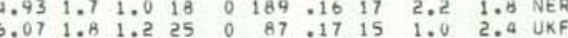
$\begin{array}{llllllllllll}5.16 & 2.1 & 1.7 & 20 & 0 & 114 & .13 & 15 & .9 & 1.7 & \text { NER } \\ .22 & 1.9 & 1.3 & 16 & 0 & 180 & 16 & 16 & 15 & 2.5 & 73.1 & \text { NER }\end{array}$ $\begin{array}{lllllllllll}5.73 & 2.2 & 1.5 & 16 & 0 & 192 & .10 & 21 & 1.3 & 4.9 & \text { NER } \\ 6.43 & 2.7 & 2.5 & 28 & 0 & 98 & .13 & 16 & .8 & 2.6 & \text { NER }\end{array}$

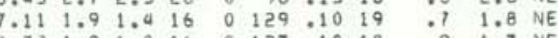

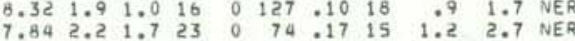
$\begin{array}{rrrrrrrrrrr}6.27 & 3.6 & 3.4 & 30 & 0 & 69 & .15 & 18 & .8 & 2.3 & \text { NER } \\ 7.07 & & .7 & 9 & 0 & 191 & 0.11 & 21 & 2.7 & 4.6 & \text { NER }\end{array}$

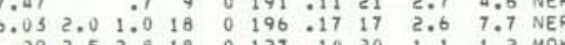

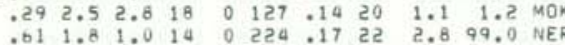
$6.58 \begin{array}{lllllllll}6.2 & 2.4 & 20 & 0 & 98 & .13 & 15 & 1.2 & 1.4 \\ 6 & \text { NER }\end{array}$

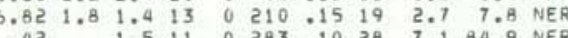

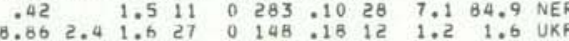

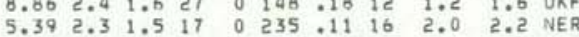

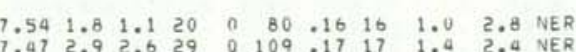

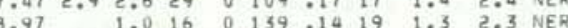

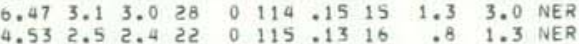
$\begin{array}{llllllllll}0.94 & 3.6 & 3.3 & 17 & 0 & 159 & .17 & 21 & 2.1 & 3.4 \\ 0.4 & \text { NER }\end{array}$

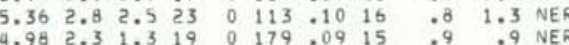

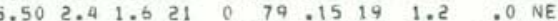

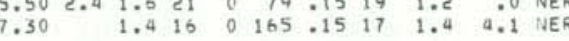
$\begin{array}{llllllllll}0.31 & 2.4 & 2.9 & 23 & 0 & 116 & .12 & 16 & 1.2 & 7.2 \\ 0 & \text { NER }\end{array}$

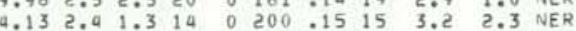
$\begin{array}{lllllllllll}5.26 & 2.1 & 2.0 & 19 & 0 & 183 & 13 & 15 & 2.1 & 1.3 & \text { NER } \\ 3.02 & 2.4 & 2.1 & 24 & 0 & 102 & 13 & 17 & 1.4 & 2.9 & \text { NER }\end{array}$ $\begin{array}{lllllllllll}3.02 & 2.5 & 1.7 & 12 & 0 & 307 & 09 & 21 & 11.8 & 4.3 & \text { NER }\end{array}$

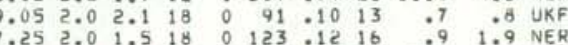

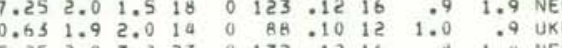

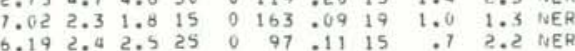
$\begin{array}{llllllllllll}3.75 & 4.1 & 4.6 & 30 & 0 & 119 & 20 & 13 & 1.4 & 2.3 & \text { NER }\end{array}$
HVO EARTHGUAKE SUMMARY LIST

PAGE 72

ORIGIN TIME LATN LON W DEPTH AMP DUR GAP RMS MIN ERH ERZ

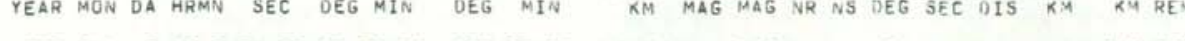

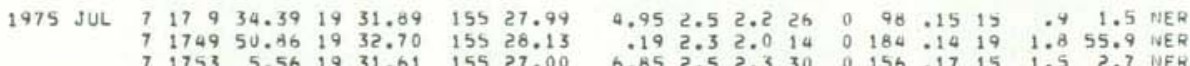
$1753 \quad 5.56 \quad 19 \quad 31.61$

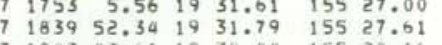
$1843 \quad 43.61 \quad 19 \quad 30.44 \quad 15528.16$ $\begin{array}{lllllll}7 & 1847 & 7.84 & 19 & 19.75 & 155 & 12.51 \\ 7 & 1848 & 51.58 & 19 & 32.62 & 155 & 26.92\end{array}$

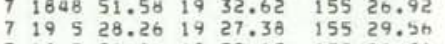
719758.241932 .1515528 .31 $71937 \quad 36.32 \quad 19 \quad 32.64 \quad 155 \quad 28.47$

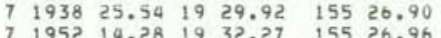

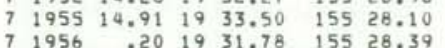

$72014 \quad 8.77 \quad 19 \quad 32.35 \quad 155 \quad 28.27$

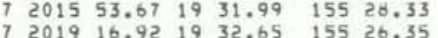

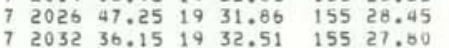
$\begin{array}{lllllll}7 & 2036 & 48.09 & 19 & 31.99 & 155 & 28.15\end{array}$

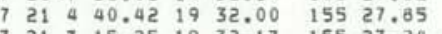

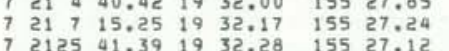

$\begin{array}{lllllll}7 & 2151 & 47.13 & 19 & 31.99 & 155 & 27.93\end{array}$

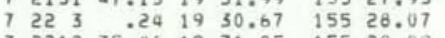
$7221235.46 \quad 1931.85 \quad 15528.04$ $\begin{array}{lllllll}7 & 2219 & 45.15 & 19 & 27.53 & 155 & 29.21 \\ 7 & 2233 & 41.05 & 19 & 32.12 & 155 & 27.63\end{array}$

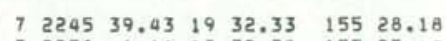

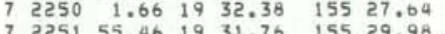
$\begin{array}{lllllll}7 & 2251 & 55.46 & 19 & 31.76 & 155 & 29.98 \\ 7 & 23 & 34.38 & 39 & 32.27 & 155 & 27.72\end{array}$

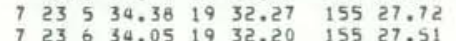

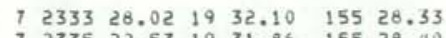
$\begin{array}{lllllll}7 & 2335 & 22.53 & 19 & 31.86 & 155 & 28.40\end{array}$

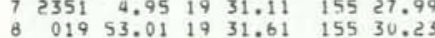

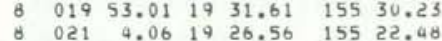

$\begin{array}{lllllll}8 & 028 & 5.12 & 19 & 32.31 & 155 & 27.59\end{array}$ $03818.201931 .49 \quad 155 \quad 29.41$
0.049

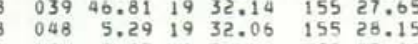
$\begin{array}{lllll}052 & 49.53 \quad 19 & 32.16 & 155 & 27.90\end{array}$ $\begin{array}{llllllll}8 & 1 & 7 & 6.86 & 19 & 31.35 & 155 & 28.65 \\ 8 & 1 & 9 & 7.71 & 19 & 31.82 & 155 & 28\end{array}$

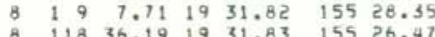

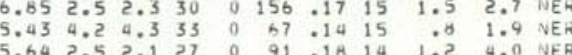

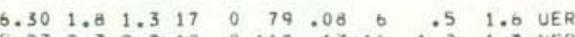
.642 .1
7.5 $\begin{array}{llllllllll}6.19 & 3.2 & 3.0 & 27 & 0 & 101 & .12 & 15 & 1.0 & 3.1 \\ \text { WIE }\end{array}$

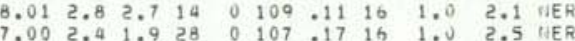
$\begin{array}{lllllllllll}7.41 & 2.1 & 1.4 & 18 & 0 & 244 & 010 & 16 & 2.0 & 1.5 & \text { IEER } \\ 8.11 & 2.0 & 1.6 & 23 & 0 & 109 & 14 & 16 & 1.0 & 2.1 & \text { NER }\end{array}$ $\begin{array}{llllllllllll}4.75 & 2.7 & 2.5 & 21 & 0 & 101 & .12 & 17 & .7 & 1.5 & \text { NER }\end{array}$

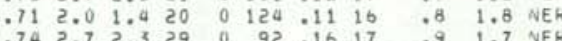
$.7 \quad 1,3$ N.EH $4.923 .53 .631 \quad 0 \quad 78.15$ is .8 1.4 NE $\begin{array}{lllllllllll}5.66 & 2.6 & 3.0 & 27 & 0 & 97 & .15 & 15 & .8 & 3.1 & \text { NEER }\end{array}$ $\begin{array}{lllllllllll}.19 & 2.6 & 2.7 & 28 & 0 & 96 & .16 & 15 & 1.1 & 1.5 & \text { NER }\end{array}$

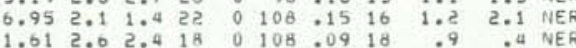

$\begin{array}{lllllllllll}5.29 & 2.6 & 2.6 & 17 & 0 & 131 & .14 & 14 & 1.4 & 2.6 & \text { NER }\end{array}$

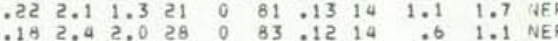
9.492 .31 .7 16 $0 \quad 109: 12$ 12 1.3 1.0 UK $\begin{array}{lllllllllllll}5.73 & 2.7 & 2.6 & 33 & 0 & 76 & 15 & 14 & 0 & 7 & 2.1 & \text { NER }\end{array}$

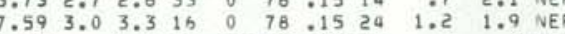

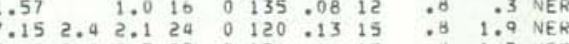
$.093 .02,732$

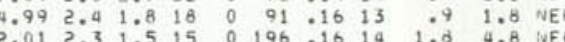

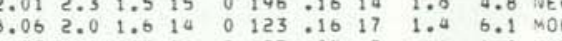

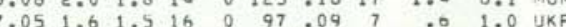

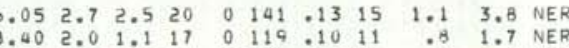

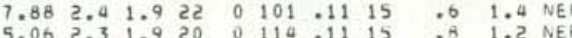
.873 .03 .029 o 100.14 is 75 1.2 NE $\begin{array}{rrrrrrrrrrr}7.65 & 1.9 & 1.1 & 22 & 0 & 112 & .12 & 13 & .9 & 2.2 & \text { NER } \\ 7.60 & 3.6 & 3.5 & 25 & 0 & 76 & .17 & 14 & 1.1 & 2.5 & 14 E R\end{array}$

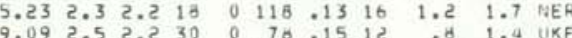

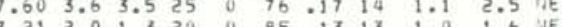



$6.153 .421 .7230112 .1214 \quad 1.0130 .8$ NER

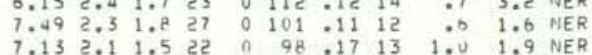

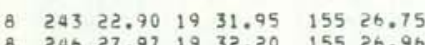
$\begin{array}{lllllll}8 & 246 & 27.97 & 19 & 32.20 & 155 & 26.96\end{array}$

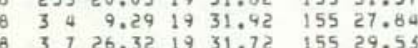

$\begin{array}{lllll}319 & .45 \quad 19 \quad 31.60 & 155 & 30.00\end{array}$ 323
33329.01
330 $\begin{array}{lllllll}8 & 336 & 49.47 & 19 & 31.63 & 155 & 27.61 \\ 8 & 353 & 59.63 & 19 & 31.10 & 155 & 29.67\end{array}$

8 $35858.711931 .01 \quad 155 \quad 29.85$

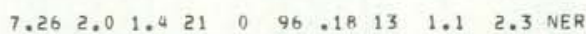

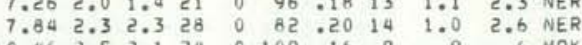

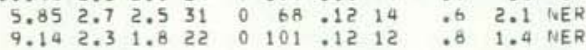
$\begin{array}{llllllllllllll}8.34 & 2.4 & 1.6 & 23 & 0 & 104 & .13 & 11 & .7 & 3.1 & \text { MOK }\end{array}$

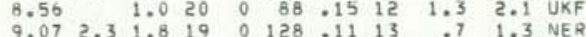

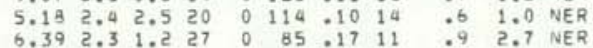
$\begin{array}{lllllllll}8.53 & 1.1 & 17 & 0 & 123 & -11 & 12 & 1.0 & 2.5 \\ 0.5 E R\end{array}$ $\begin{array}{lll}.16 & 14 & .8 \\ 2.2 & \text { NER }\end{array}$

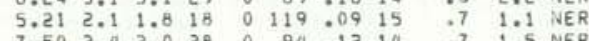

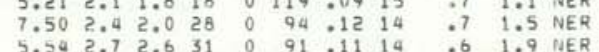
$416 \quad 0.621932 .08$ 43536.54 19 $31.29 \quad 15531.10$

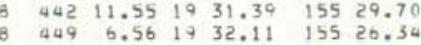
$\begin{array}{llllllllll}7.76 & 2.1 & 1.420 & 0 & 115.11 & 12 & .0 & 2.4 \text { NER }\end{array}$

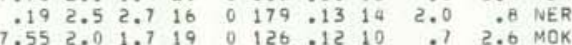

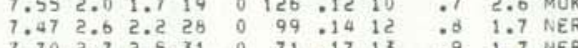
$\begin{array}{lllllllllllll}5.81 & 2.3 & 2.3 & 14 & 0 & 100 & .12 & 14 & 9 & 5.3 & \text { NER }\end{array}$

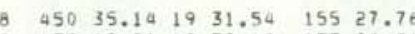

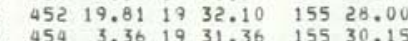

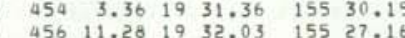

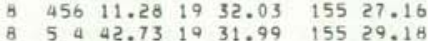

\begin{tabular}{l}
8 \\
\hline
\end{tabular}$\quad$ A $14.36 \quad 19 \quad 32.27 \quad 155 \quad 26.54$

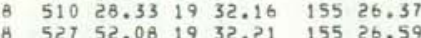
$\begin{array}{lrrrrrr}8 & 527 & 52.00 & 19 & 32.21 & 155 & 26.59 \\ 8 & 531 & 1.77 & 19 & 30.91 & 155 & 29.29\end{array}$ \begin{tabular}{l}
$542 \quad 6.221932 .47 \quad 15526.10$ \\
\hline
\end{tabular}

$\begin{array}{lllrllll}8 & 6 & 1 & 34.47 & 19 & 31.33 & 155 & 29.61 \\ 8 & 6 & 3 & 8.24 & 19 & 32.38 & 155 & 27.83\end{array}$ $61114.041932 .56 \quad 15528.07$

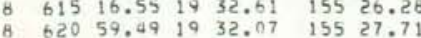

$\begin{array}{lllllll}8 & 626 & 17.96 & 19 & 31.96 & 155 & 29.54 \\ 8 & 627 & 31.21 & 19 & 30.94 & 155 & 29.69 \\ 8 & 032 & 89 & 19 & 31.94 & 155 & 28.64\end{array}$

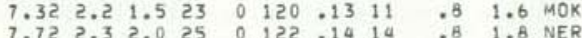

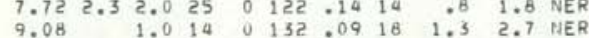
$\begin{array}{lllllllllll}7.42 & 2.3 & 1.7 & 20 & 0 & 127 & .16 & 13 & 1.0 & 2.0 & \text { NER }\end{array}$

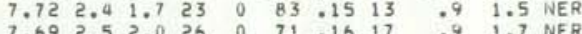

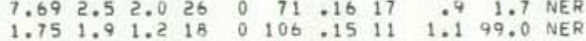

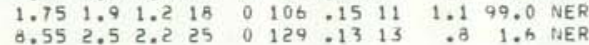

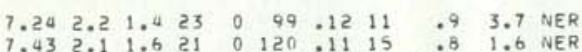

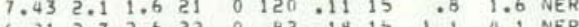

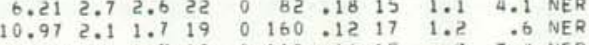

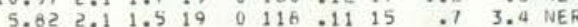
$\begin{array}{lllllllllll}7.29 & 2.1 & 1.5 & 18 & 0 & 224 & .12 & 12 & 1.7 & 2.0 & \text { NER } \\ 5.18 & 2.0 & 1.5 & 17 & 0 & 111 & .10 & 11 & .7 & 1.3 & \text { MER }\end{array}$

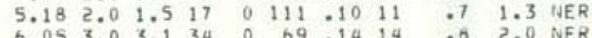

THU EAKTHOUAKE SUMMARY LIST Q $\triangle$ GE 7 ORIGIN TIME LATN LON A DEPTH AMP DUR GAP RHS MIN EKM ERL RTE

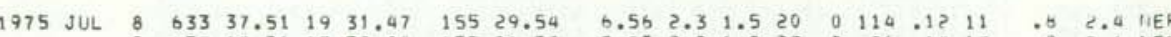

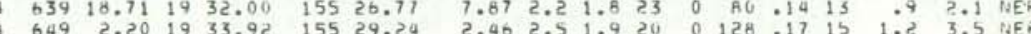

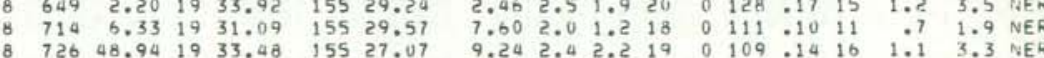
$8 \quad 726 \quad 48.94 \quad 1933.48 \quad 155 \quad 27.07$ $\begin{array}{lllllll}8 & 728 & 52.98 & 19 & 31.39 & 155 & 27.93\end{array}$ $730 \quad 53.81 \quad 14$
8

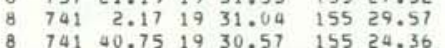
8 $745 \quad 48.44 \quad 1931.83 \quad 155 \quad 29.22$ $856 \quad 3.051931 .65$ 155 26.70 $\begin{array}{llllllll}8 & 8 & 1 & 36.08 & 19 & 31.60 & 155 & 27.65 \\ 8 & 8 & 4 & 40.20 & 19 & 18.79 & 155 & 15.29\end{array}$

$\begin{array}{llllllll}8 & 811 & 7.28 & 19 & 33.37 & 155 & 29.81\end{array}$ $\begin{array}{llllll}824 & 43.01 & 19 & 32.62 & 155 & 27.50\end{array}$ $\begin{array}{lllllll}841 & 4.78 & 19 & 32.52 & 155 & 27.62\end{array}$ $\begin{array}{lllllll}8 & 845 & 7.99 & 19 & 31.56 & 155 & 27.06 \\ 8 & 849 & 56.37 & 19 & 31.39 & 155 & 29.73\end{array}$ $\begin{array}{lllllll}8 & 856 & 4.17 & 19 & 32.57 & 155 & 29.60\end{array}$ $8943.71 \quad 1931.72 \quad 15529.88$

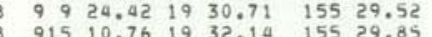
81510.76
825 $52.451932 .32 \quad 15526.36$

$8 \quad 932 \quad 46.4919 \quad 32.02 \quad 15526.53$

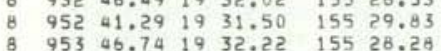

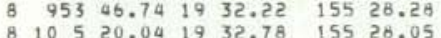

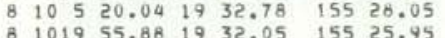

8 $1023 \quad 45.73 \quad 19 \quad 32.08 \quad 15526.23$ $\begin{array}{lllllll}8 & 1037 & 14.81 & 19 & 31.57 & 155 & 29.83 \\ 8 & 1055 & 53.00 & 19 & 30.80 & 155 & 30.97\end{array}$ $\begin{array}{lllllll}8 & 1055 & 52.00 & 19 & 30.80 & 155 & 30.97 \\ 8 & 1056 & 35.18 & 19 & 31.52 & 155 & 28.41\end{array}$ (1) $952.02 \quad 1931.93 \quad 15526.63$

$\begin{array}{llrllll}8 & 1112 & 3.89 & 19 & 32.03 & 155 & 28.43 \\ 8 & 1118 & 58.82 & 19 & 30.91 & 155 & 29.79\end{array}$ $\begin{array}{lllllll}8 & 1118 & 58.82 & 19 & 30.91 & 155 & 29.79 \\ 8 & 1120 & 40.19 & 19 & 32.03 & 155 & 27.56\end{array}$ $\begin{array}{lllllll}8 & 1120 & 40.19 & 19 & 32.03 & 155 & 27.56 \\ 8 & 1124 & 54.19 & 19 & 31.10 & 155 & 29.42\end{array}$

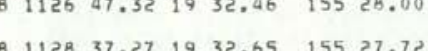
$\begin{array}{lllllll}8 & 1128 & 37.27 & 19 & 32.65 & 155 & 27.72 \\ 8 & 1138 & 19.72 & 19 & 31.29 & 155 & 29.85\end{array}$

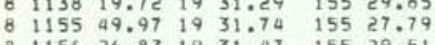

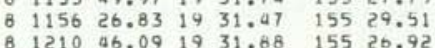
$\begin{array}{lllllll}8 & 1212 & 41.73 \quad 19 & 25.63 & 155 & 27.95\end{array}$ $81226 \quad 21.95 \quad 1932.21 \quad 15526.52$ $\begin{array}{llllllllllll}5.01 & 2.4 & 2.1 & 18 & 0 & 102 & .10 & 14 & .7 & 1.4 & \text { NER }\end{array}$ 9.101 .91 .616 o 87.14 14 $1.4 \% 3$ NEA

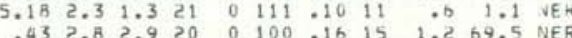

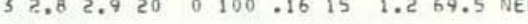
$\begin{array}{lllllllllllll}1.96 & 2.7 & 2.3 & 15 & 0 & 113 & .08 & 12 & 1.0 & .3 & 3 & \text { WEH }\end{array}$

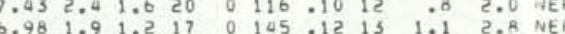

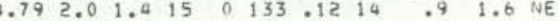
.

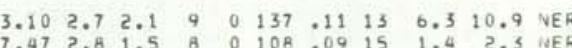

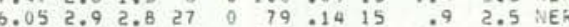

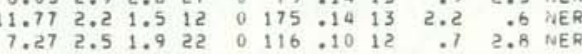
$\begin{array}{lllllllllll}1.48 & 1.5 & 17 & 0 & 245 & .11 & 18 & 2.7 & .9 & \text { A AER }\end{array}$

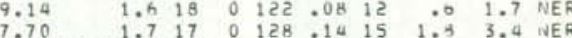

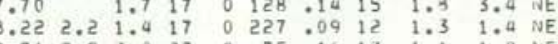

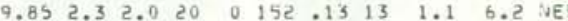

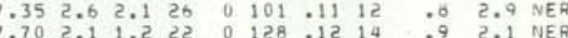

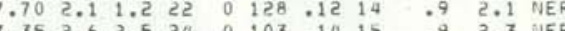

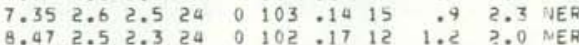
$\begin{array}{llllllllllll}9.37 & 2.3 & 2.0 & 23 & 0 & 90.14 & 13 & .9 & 1.3 & \text { NER } \\ 7.73 & 2.1 & 1.6 & 19 & 0 & 119 & 0.08 & 12 & : 8 & 2.8 & \text { NER }\end{array}$

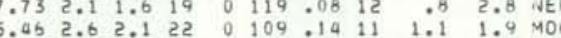

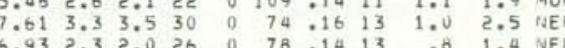
$\begin{array}{llllllllll}7.13 & 2.8 & 2.5 & 26 & 0 & 86 & .13 & 14 & .9 & 2.1 \\ 5.33 & 2.5 & 19 & 96\end{array}$

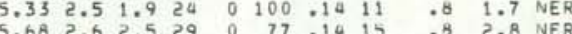
$6.062 .111 .423 \quad 00100: 13$ i1 9.9 4.2 NER $\begin{array}{llllllllll}7.74 & 2.3 & 2.0 & 25 & 0 & 142 & .14 & 15 & 1.0 & 2.4 \\ 7\end{array}$ $\begin{array}{lllllllllll}7.29 & 2.1 & 1.4 & 14 & 0 & 117 & .09 & 11 & 1.35 & 5.6 & \text { NER }\end{array}$

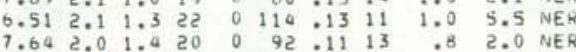
$\begin{array}{lllllllllll}7.89 & 2.1 & 1.6 & 20 & 0 & 158 & .16 & 13 & 1.3 & 2.0 & \text { UKF }\end{array}$

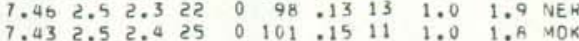


HVO EARTHOUAKE SUMMARY LIST

PAGE 75

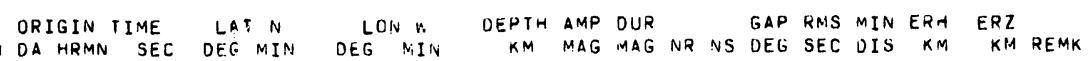
YEAR MON DA HRMN SEC DEG MIN DEG MIIN KM MAG MAG NR NS DEG SEC DIS KM KM REMK

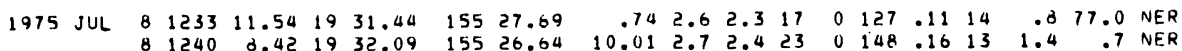

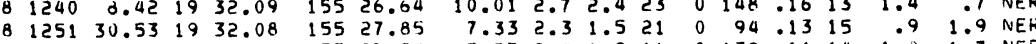

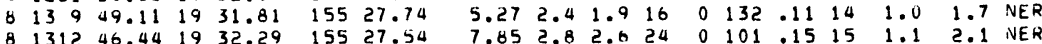
$\begin{array}{lllllll}8 & 1335 & 31.09 & 19 & 32.34 & 155 & 26.54 \\ 8 & 1336 & 41.60 & 19 & 32.38 & 155 & 27.49\end{array}$ $\begin{array}{llllllll}8 & 1333 & 28.17 & 19 & 32.21 & 155 & 26.36 \\ 8 & 1354 & 29.82 & 19 & 32.46 & 155 & 26.35\end{array}$

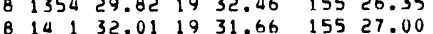
$\begin{array}{lllllll}8 \quad 1410 & 33.90 \quad 19 & 31.05 & 155 & 29.64\end{array}$ $\begin{array}{lllllll}8 & 1417 & 11.37 & 19 & 32.35 & 155 & 26.31 \\ 8 & 1423 & 24.18 & 19 & 20.74 & 155 & 13.40\end{array}$

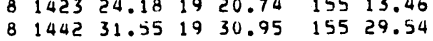
144231.551930 .9515529 .58
$145653.171931 .47 \quad 15526.68$

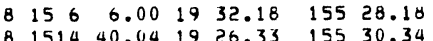
$\begin{array}{lllllll}8 & 1514 & 40.04 & 19 & 26.33 & 155 & 30.34 \\ 8 & 1518 & 2.13 & 19 & 30.93 & 155 & 29.43\end{array}$ $\begin{array}{lllllll}6 & 1518 & 2.13 & 19 & 30.93 & 155 & 29.43 \\ 8 & 1536 & 11.97 & 19 & 31.19 & 155 & 29.12\end{array}$ $8154653.911932 .35 \quad 15526.11$ $\begin{array}{lllllllll}8 & 16 & 94.64 & 19 & 31.60 & 155 & 27.01 \\ 8 & 1623 & .10 & 19 & 22.16 & 155 & 18.72\end{array}$ $\begin{array}{lllllll}8 & 1634 & 14.39 & 19 & 31.23 & 155 & 30.04 \\ 8 & 1634 & 19 & 31.03 & 155 & 29.55 \\ 8 & 175 & 19.38 & 19 & 33.00 & 155 & 26.88\end{array}$

$8171536.921932 .51 \quad 15528.42$ $173812.931931 .57 \quad 15526.84$

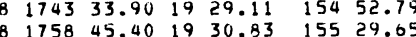
\begin{tabular}{l}
$8187 \quad 8.62 \quad 1924.97 \quad 155 \quad 28.42$ \\
\hline
\end{tabular}

$\begin{array}{lllllll}8 & 1811 & 29.79 & 19 & 32.18 & 155 & 26.69\end{array}$ $\begin{array}{lllllll}8 & 1827 & 32.67 & 19 & 25.18 & 155 & 28.24\end{array}$ $\begin{array}{lllllll}8 & 1848 & 1.30 & 19 & 35.94 & 155 & 28.23 \\ 8 & 1853 & 17.92 & 19 & 31.54 & 155 & 26.90\end{array}$ $\begin{array}{llllll}190 & 6.09 & 19 & 31.96 & 155 & 25.45\end{array}$

$\begin{array}{lllllll}8 & 1910 \quad 11.92 \quad 19 & 32.22 & 155 & 26.30\end{array}$ $\begin{array}{rrrrrrr}8 & 1911 & 11.83 & 19 & 31.60 & 155 & 28.36 \\ 8 & 1915 & 6.93 & 19 & 31.18 & 155 & 29.87\end{array}$

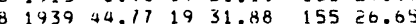
$81943 \quad 36.331931 .70 \quad 155 \quad 28.01$ $\begin{array}{llllllll}8 & 1950 & 0.07 & 19 & 31.59 & 155 & 28.14\end{array}$ 19599.731931 .98015527 .79

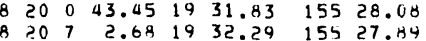

$2044 \quad 14.20 \quad 1932.36 \quad 155 \quad 21.09$ $\begin{array}{lllllllll}8 & 21 & 9 & 21.38 & 19 & 31.81 & 155 & 28.17\end{array}$

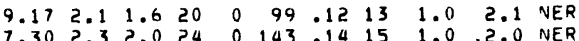

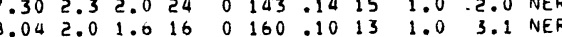

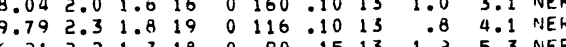
$\begin{array}{llllllllll}7.22 & 2.6 & 1.9 & 27 & 0 & 97.14 & 12 & .8 & 2.4 & \text { NER }\end{array}$

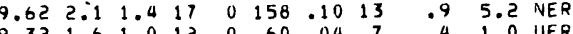
$6.122 .92 .927 \quad 0096.1311 \quad .8$ 2.6 NER

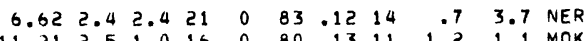

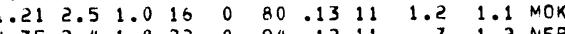

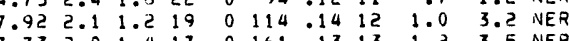

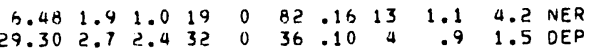

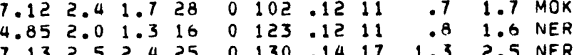
$\begin{array}{llllllllll}7.29 & 2.4 & 1.9 & 24 & 0 & 94 & .16 & 14 & 1.1 & 4.9 \\ 0\end{array}$

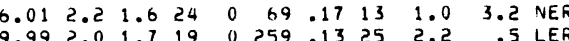

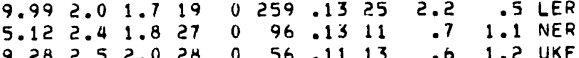

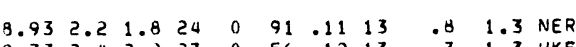

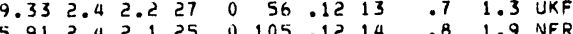

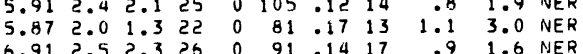
$\begin{array}{lllllllllll}7.93 & 2.0 & 1.6 & 22 & 0 & 152 & .14 & 13 & 1.0 & 1.9 & \text { NER }\end{array}$

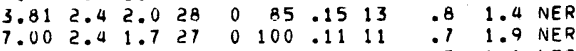

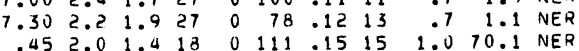
$\begin{array}{llllllllllll}.46 & 2.0 & 1.5 & 18 & 0 & 106 & .15 & 14 & 1.0 & 64.2 & \text { NER }\end{array}$ $\begin{array}{lllllllllll}8.38 & 2.3 & 1.7 & 26 & 0 & 71 & .12 & 12 & .7 & 1.9 & \text { UKF } \\ 5.08 & 2.3 & 1.6 & 20 & 0 & 132 & .10 & 14 & .6 & 1.1 & \text { NER }\end{array}$

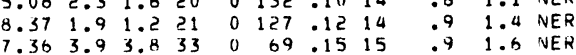
$\begin{array}{lllllllllll}7.12 & 2.1 & 1.2 & 20 & 0 & 149 & .10 & 14 & .7 & 1.4 & 1.4 \\ 0.15 R\end{array}$

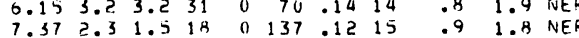

hVO EARTHQUAKE SUMMARY LIST

PAGE 76

ORIGIN TIME LAT N LON W DEPTH AMP DUR GAP RNS IIN ERH ERZ YEAR MON DA HRMN SEC DEG MIN DEG MIN OEMM MAG MAG NR HS DEG SEC OIS KM KM REMK

$\begin{array}{llllllll}1975 \text { JUL } & 8 & 2125 & 57.37 & 19 & 31.09 & 155 & 29.80 \\ 8 & 2130 & 46.11 & 19 & 32.35 & 155 & 27.74\end{array}$ $822939.991931 .71 \quad 15526.53$ $8223218.721930 .79 \quad 15520.72$ $82257 \quad 35.47 \quad 1932.19 \quad 155 \quad 26.30$ $\begin{array}{llllll}23 & 31.24 & 19 & 31.64 & 155 & 26.60\end{array}$

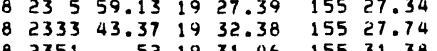
$9016 \quad 40.27 \quad 19 \quad 31.26 \quad 155 \quad 29.76$

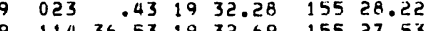

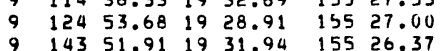
$\begin{array}{llllllll}9 & 0 & .65 & 19 & 27.77 & 155 & 28.92\end{array}$

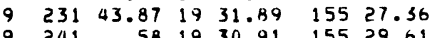

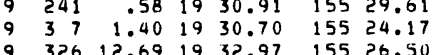

$\begin{array}{lllllll}330 & 2.03 \quad 19 & 32.70 \quad 155 \quad 27.65\end{array}$ $9342 \quad 28.44 \quad 1932.01 \quad 155 \quad 26.11$ $9 \quad 34927.551932 .18 \quad 15528.06$

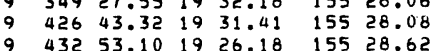

$944738.81 \quad 1930.94 \quad 155 \quad 29.99$ $95442.701932 .57 \quad 15527.95$ $955748.58 \quad 1932.46 \quad 15528.17$

$960043.27 \quad 1931.71 \quad 15528.31$ $9616 \quad 19.48 \quad 19 \quad 31.05 \quad 15528.78$ $963434.381932 .31 \quad 155 \quad 25.95$

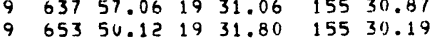

$974 \quad 34.04 \quad 19 \quad 31.80 \quad 155 \quad 27.80$ $939 \quad 28.40 \quad 19 \quad 31.97 \quad 15527.64$
9 $9754 \quad 6.23 \quad 1931.71 \quad 15526.60$ $\begin{array}{rrrrrrr}9 & 758 & 18.43 & 19 & 49.90 & 155 & 22.49 \\ 9 & 89 & 11.85 & 19 & 31.01 & 155 & 29.76\end{array}$

$9840 \quad 3.50 \quad 19 \quad 31.88 \quad 155 \quad 28.17$

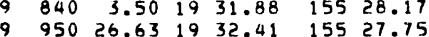
$9101044.98 \quad 1931.68 \quad 15528.28$

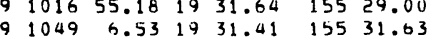

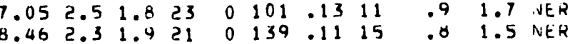

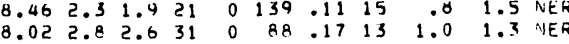

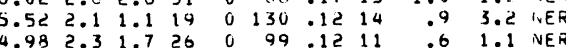
$\begin{array}{lllllllllll}8.23 & 2.4 & 2.1 & 25 & 0 & 94 & 0.12 & 13 & \text {. B } & 1.3 & 3 \text { NER }\end{array}$

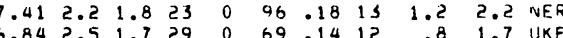
$5.43 \quad 3.033 .1290078 .16$ is 9.9019 NER 5.392 .31 .7 20 $00114.13 \quad 17 \quad 1.1$ 2.5 110 $\begin{array}{llllllllllll}7.54 & 2.6 & 2.0 & 31 & 0 & 69 & .13 & 11 & .8 & 1.5 \text { H.EK }\end{array}$

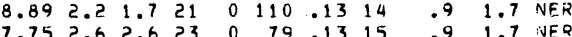
$8.261 .81 .024009 .1412 \quad 801.3$ UKF $8.492 .92 .730 \quad 0 \quad 97 \quad .1513 \quad 1.0 \quad 1.5$ NER $\begin{array}{llllllllllll}9.13 & 2.4 & 1.5 & 30 & 0 & 76 & .16 & 11 & .8 & 1.1 & \text { UKF }\end{array}$

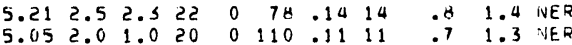

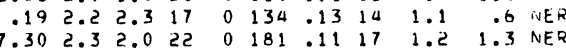
$\begin{array}{lllllllllll}6.58 & 2.5 & 2.4 & 26 & 0 & 79 & .14 & 15 & .9 & 2.4 & \text { NER }\end{array}$

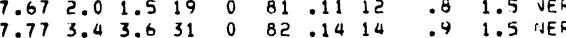

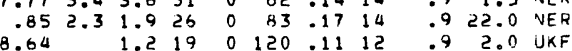
$\begin{array}{lllllllllll}7.25 & 2.0 & 1.5 & 17 & 0 & 115 & .12 & 11 & 1.0 & 2.9 & \text { NER }\end{array}$ $\begin{array}{lllllllllll}6.23 & 1.9 & 1.6 & 19 & 0 & 91 & .15 & 13 & 1.1 & 3.1 & \text { NER }\end{array}$ $\begin{array}{llllllll}2.7 & 2.6 & 29 & 0 & 100 & .17\end{array}$

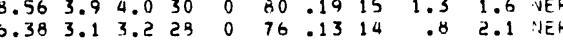
$\begin{array}{llllllllll}.44 & 2.2 & 1.4 & 15 & 0 & 109 & .15 & 13 & 1.0 & 52.5 \\ 7.5 & \text { NER }\end{array}$

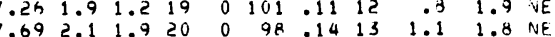

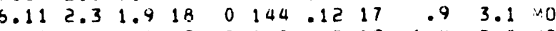
$\begin{array}{llllllllll}7.74 & 3.0 & 2.9 & 29 & 0 & 79 & .14 & 14 & .9 & 1.6\end{array}$

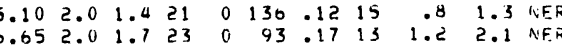
$\begin{array}{llllllllll}8.04 & .8 & 19 & 0 & 158 & .07 & 37 & 1.8 & 3.7 & \mathrm{kK}\end{array}$ $0.434 .544333 \quad 0 \quad 68.1514$

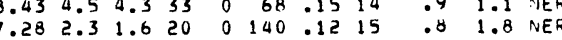

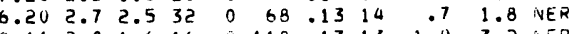

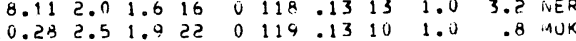

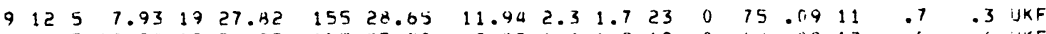

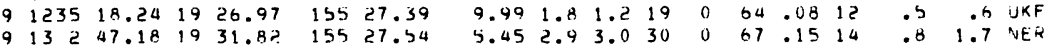


hVU EARThouare Summaky List

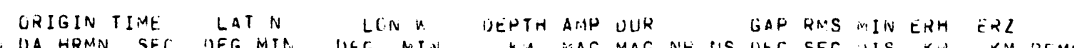

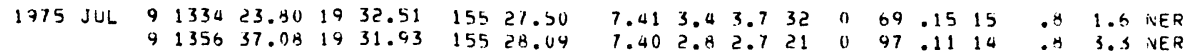

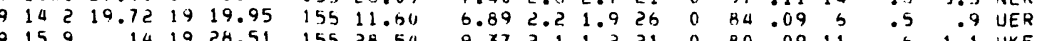

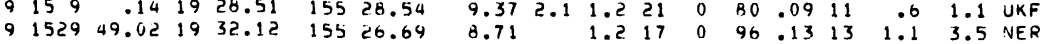

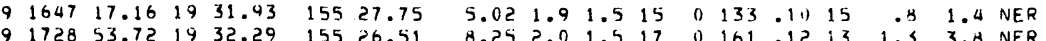

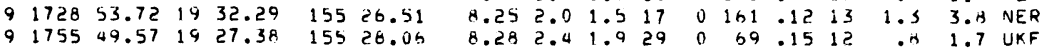

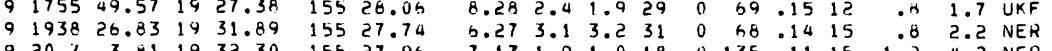

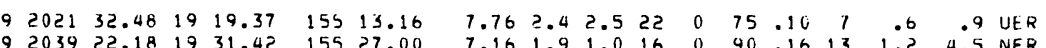

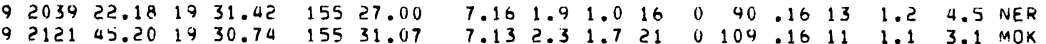

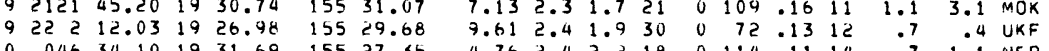
$\begin{array}{lllllll}10 & 153 & 2.36 & 19 & 32.77 & 155 & 25.97 \\ 10 & 253 & 32.04 & 19 & 30.81 & 155 & 31.92\end{array}$ $10025332.041930 .81 \quad 15531.12$ $\begin{array}{rrrrrrrr}10 & 3 & 1 & 24.26 & 19 & 31.13 & 155 & 29.85 \\ 10 & 4 & 9 & 0.20 & 19 & 31.39 & 155 & 27.71\end{array}$ $10 \quad 735 \quad 27.94 \quad 1932.15$ 155 27.42

$\begin{array}{rrrrrrr}10 & 816 & 37.48 & 19 & 31.64 & 155 & 27.85 \\ 10 & 25 & 49.25 & 19 & 32.65 & 155 & 27.94\end{array}$ $101216 \quad 53.87 \quad 19 \quad 30.75 \quad 15529.73$ $\begin{array}{lllllll}10 & 1432 & 38.10 & 19 & 31.60 & 155 & 28.47 \\ 10 & 1439 & 42.44 & 19 & 26.59 & 155 & 29.90\end{array}$

$10 \quad 1547 \quad 18.8219 \quad 27.66 \quad 155 \quad 26.94$

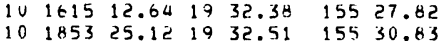

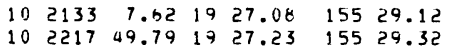

$\begin{array}{llllllll}10 & 23 & 0 & 10.86 & 19 & 26.95 & 155 & 29.29\end{array}$

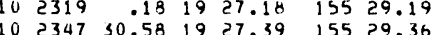

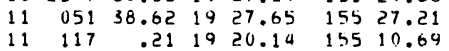

$\begin{array}{llllllllllllllllll}11 & 120 & 13.68 & 19 & 31.55 & 155 & 33.15 & 10.95 & 1.6 & 1.3 & 16 & 0 & 196 & .09 & 19 & 1.2 & .4 \text { MOK } \\ 11 & 318 & 18.00 & 19 & 32.33 & 155 & 27.93 & 7.919 & 2.6 & 2.4 & 25 & 0 & 136 & 14 & 15 & 1.0 & 2.4 & \text { NER }\end{array}$

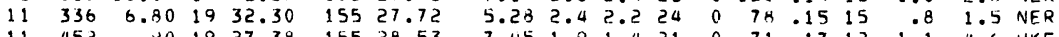

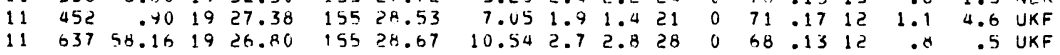

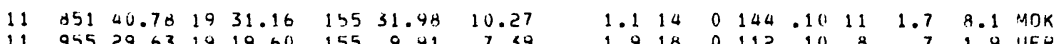

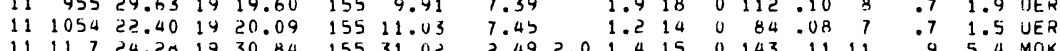

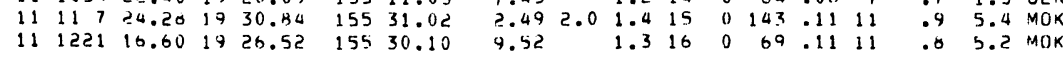

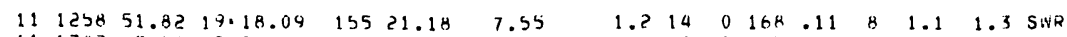

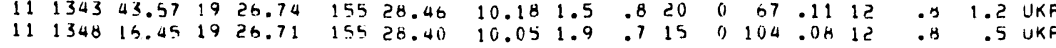

hUU Earthguakf. SUMimaky List

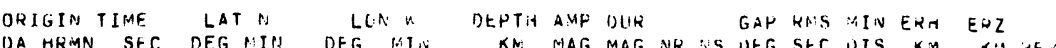
YEAQ जON OA HEYN

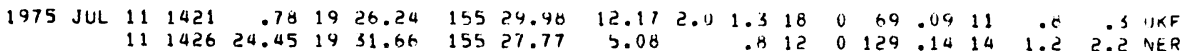

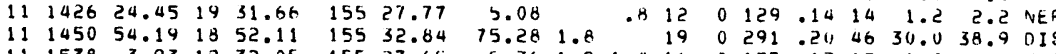

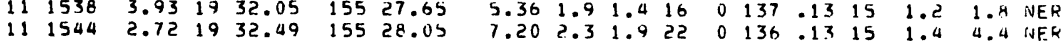

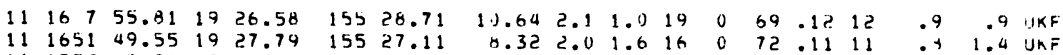

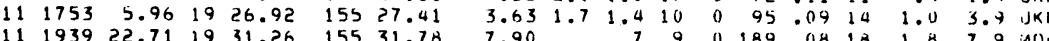

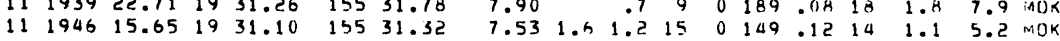

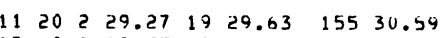
$1200019.331925 .32 \quad 15524.19$

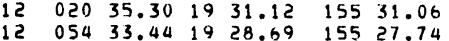
$12 \quad 331 \quad .65 \quad 19 \quad 18.50 \quad 155 \quad 13.14$ $\begin{array}{lllllll}12 & 350 & 15.02 & 19 & 30.01 & 155 & 26.61 \\ 12 & 415 & 53.07 & 19 & 32.03 & 155 & 27.46\end{array}$ $12 \quad 41553.07 \quad 1932.03 \quad 155 \quad 27.46$

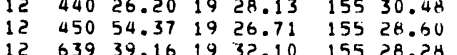
$\begin{array}{lllllll}12 & 1236 & 51.65 & 19 & 31.97 & 155 & 27.39\end{array}$ $12155217.671936 .70 \quad 15526.90$ $12 \quad 171012.24 \quad 1925.08 \quad 15516.34$ $1218414.26 \quad 1924.61 \quad 15516.35$

$\begin{array}{lllllll}13 & 016 & 4.32 & 19 & 24.84 & 155 & 28.86\end{array}$

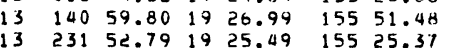
$\begin{array}{lllllll}13 & 525 & 31.12 & 19 & 50.89 & 155 & 49.97 \\ 13 & 611 & 13.03 & 19 & 20.23 & 155 & 12.15\end{array}$

$\begin{array}{lllllll}13 & 648 & 59.24 & 19 & 20.16 & 155 & 10.35\end{array}$ $\begin{array}{rrrrrrr}13 & 649 & 40.91 & 19 & 20.08 & 155 & 9.92 \\ 13 & 1655 & 22.79 & 19 & 28.92 & 155 & 28.53\end{array}$ $\begin{array}{llllllll}13 & 173 & 21.71 & 19 & 22.32 & 155 & 26.90 \\ 13 & 1720 & 36.00 & 19 & 25.47 & 155 & 17.14\end{array}$ $\begin{array}{llllllll}13 & 1743 & 6.19 & 19 & 26.65 & 155 & 24.75\end{array}$ $14 \quad 032 \quad 49.17 \quad 1919.01 \quad 15515.61$ $\begin{array}{llllllll}14 & 1 & 6 & 54.72 & 19 & 20.45 & 155 & 8.96\end{array}$ $14 \quad 551 \quad 59.25 \quad 1926.55 \quad 155 \quad 22.41$ $\begin{array}{rrrrrrr}14 & 932 & 38.37 & 19 & 17.41 & 155 & 12.97 \\ 14 & 1448 & 24.30 & 19 & 25.01 & 155 & 23.90\end{array}$ $\begin{array}{lllllll}14 & 1828 & 29.20 & 19 & 25.59 & 155 & 30.26 \\ 14 & 2011 & 35.42 & 19 & 31.52 & 155 & 24.70\end{array}$ $14201135.421931 .52 \quad 15524.70$ $15 \quad 345 \quad 23.65 \quad 19 \quad 18.91 \quad 15515.17$ $\begin{array}{rrrrrrr}15 & 420 & 13.68 & 19 & 17.99 & 155 & 13.05 \\ 15 & 1030 & 56.49 & 19 & 32.69 & 155 & 77.09\end{array}$

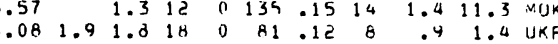

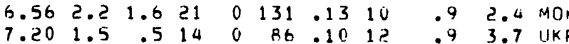

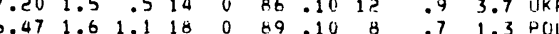

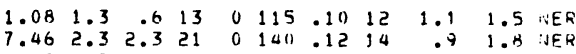

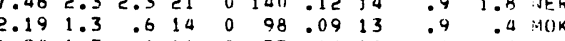

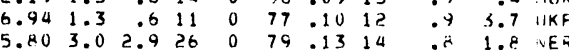
$\begin{array}{lllllllllll}7.53 & 2.4 & 2.2 & 23 & 0 & 140 & .15 & 14 & 1.1 & 1.9 & \text { INER }\end{array}$

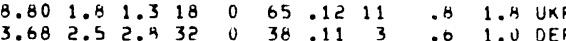

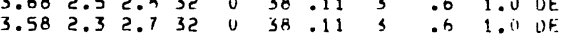
\begin{tabular}{llllllllll}
6.43 & 1.7 & 1.7 & 28 & 0 & 43 & .10 & 2 & .6 & 1.1 \\
\hline
\end{tabular}

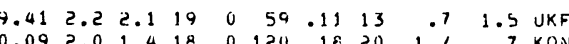

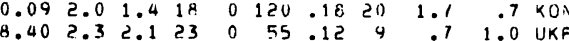

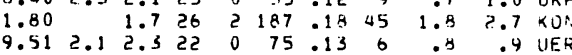
\begin{tabular}{lllllllllll}
7.68 & 2.1 & 2.2 & 21 & 0 & 82 & .12 & 1 & .8 & 1.0 & 1.0 \\
\hline
\end{tabular}

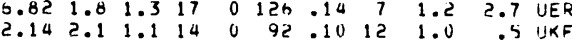

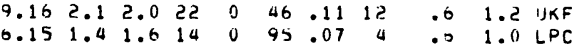
$\begin{array}{llllllrlllll}8.47 & 1.6 & 1.3 & 15 & 0 & 53 & .08 & 9 & .7 & 1.2 & \text { JKF }\end{array}$ 6.931 .91 .318 O 116.11 6 .

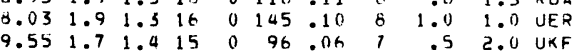
$\begin{array}{rllllllllllll}7.97 & 1.6 & 1.8 & 21 & 0 & 140 & .11 & 9 & .9 & 1.3 & P O L\end{array}$

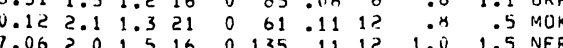

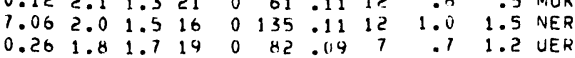

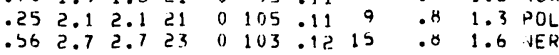


HVO EARTHQUAKE SUMMARY LIST

PAGE 79 ORIGIN TIME LATN LON W DEPTH AMP DUR GAP RMS MIN ERH ERZ REMK YEAR MON DA HRMN SEC OEG MIN TEE MIN

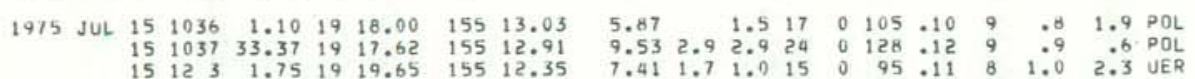

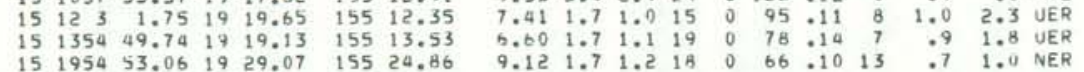

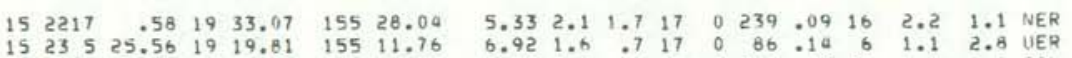

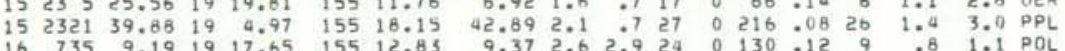

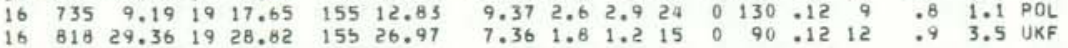

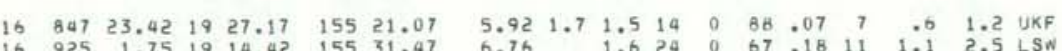

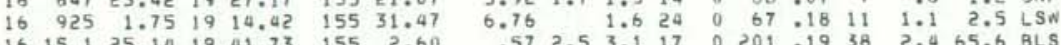

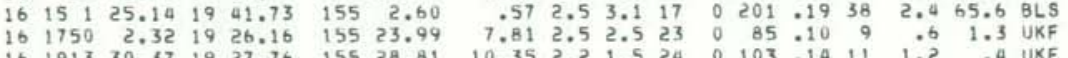

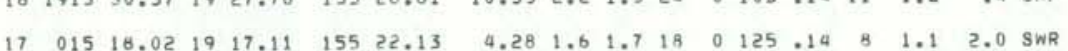

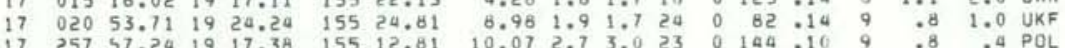

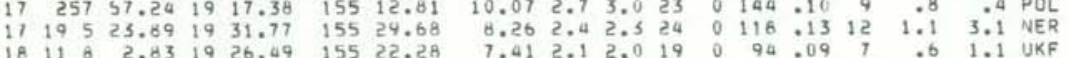

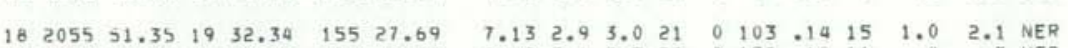

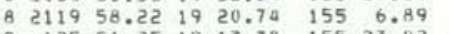

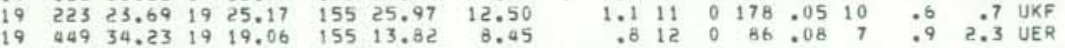
$\begin{array}{lllllllllllllllllll}19 & 6 & 0 & 54.27 & 19 & 17.87 & 155 & 20.11 & 8.72 & 2.2 & 2.5 & 18 & 0 & 124 & .09 & 7 & .7 & 1.1 & \text { SWR }\end{array}$

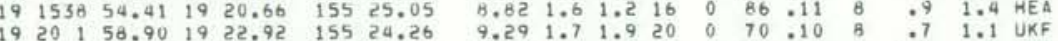

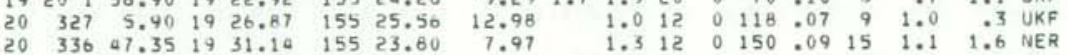

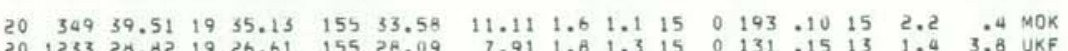

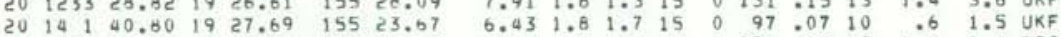

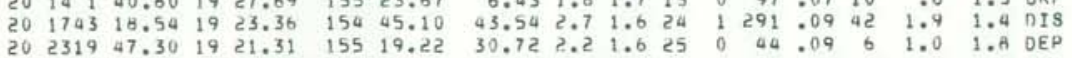

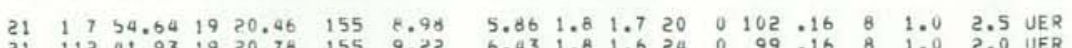

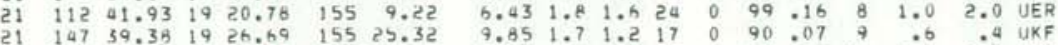

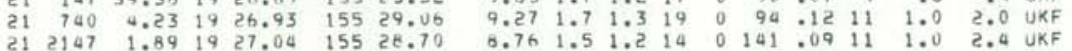
$\begin{array}{lllllllllllllllll}21 & 2314 & 43.29 & 19 & 31.24 & 155 & 23.54 & 7.46 & 2.4 & 2.1 & 24 & 0 & 101 & .13 & 15 & .8 & 1.0 \text { NER }\end{array}$

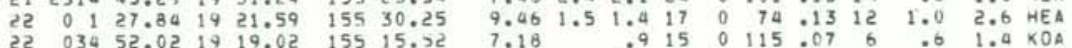

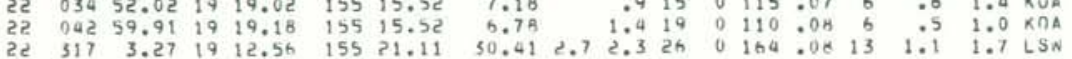

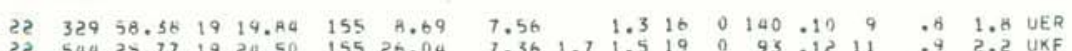

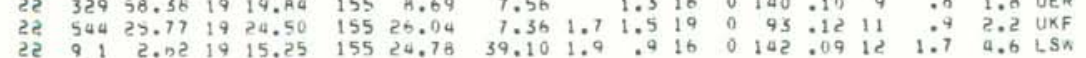

HVO EARTHEUARE SUMHARY LIST

PALE BU

ORIGIN TIME LAT N LON W DEPTH AMP DUR GAP RMS MIN ERN EHZ YEAR MON DA HRMN SEC DEG MIN DEG MIIN KM MAG MAG WR NS OAEG SEC UIS KM KA REAK

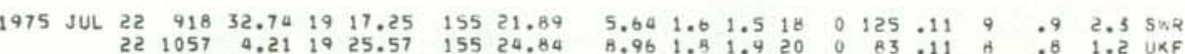

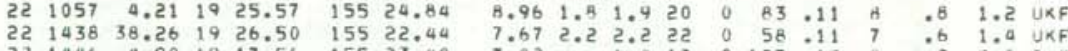

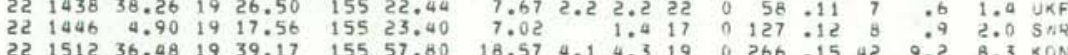
$\begin{array}{llllllllllllllllll}22 & 1933 & 20.32 & 19 & 26.57 & 155 & 22.61 & 8.11 & 1.5 & 1.2 & 14 & 0 & 98 & .06 & 7 & .5 & 1.4 \text { UKF }\end{array}$ 22 2159 49.57 19 27.88 15526.76 (15.26

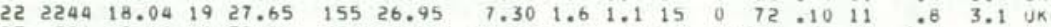
$23 \quad 32150.691859 .76$ 155 $25.78 \quad 34.97 \quad 1.8 \quad .7$ 18 $0244.0931 \quad 3.0 \quad 4.4015$

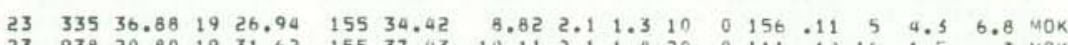

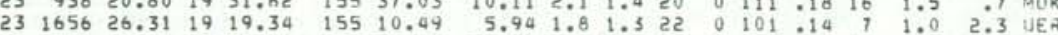

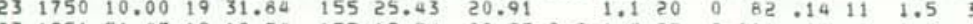

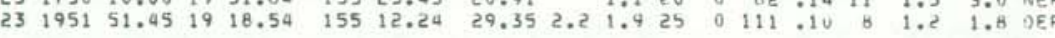

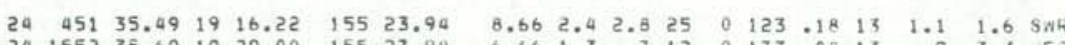

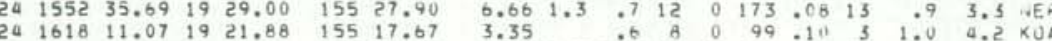

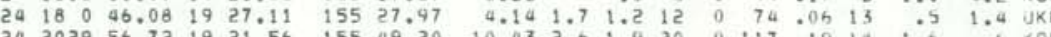

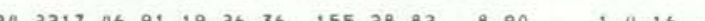
$\begin{array}{llllllllll}8.90 & 1.0 & 16 & 0 & 65 & .10 & 12 & .7 & 2.3 & \text { UKF }\end{array}$

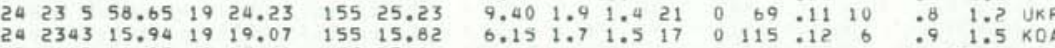

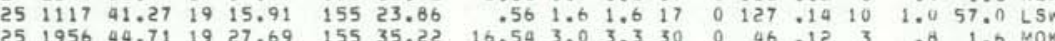
$\begin{array}{llllllllllllllllll}26 & 918 & 38.96 & 19 & 18.86 & 155 & 12.49 & 8.88 & 1.9 & 1.7 & 15 & 0 & 97 & .09 & 8 & .8 & 1.9 & \text { POL }\end{array}$

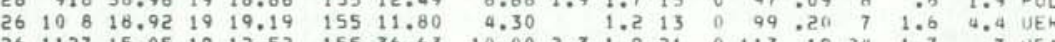

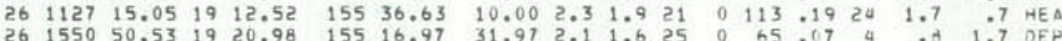

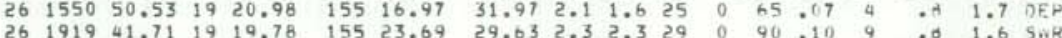

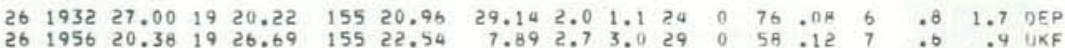

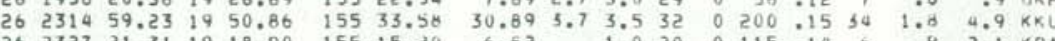

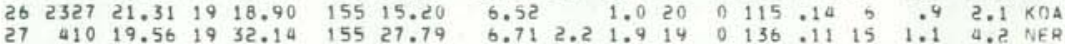

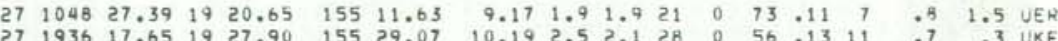

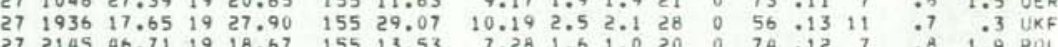

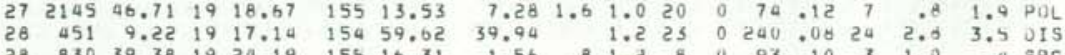

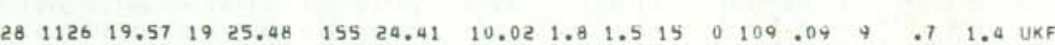

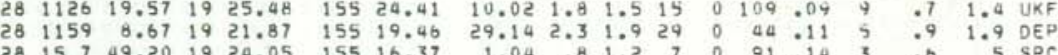

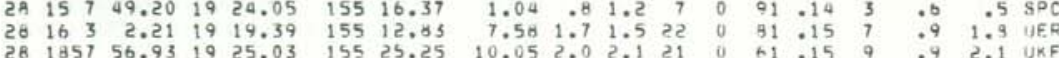
$\begin{array}{llllllllllllllllll}28 & 1913 & 17.79 & 19 & 24.30 & 155 & 16.29 & 1.75 & .6 & 1.0 & 8 & 0 & 94.08 & 5 & .8 & .3 & 50 C\end{array}$

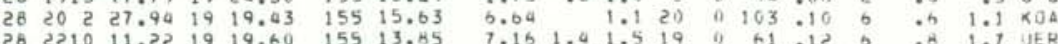




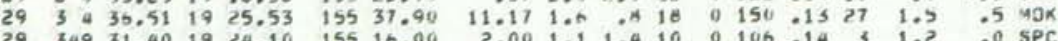

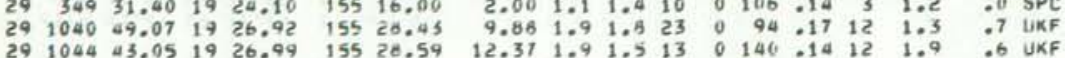

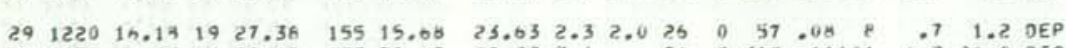

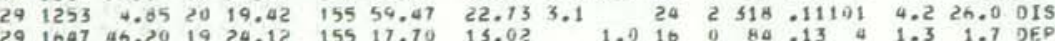

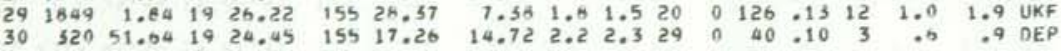

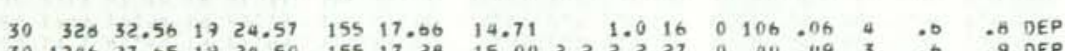

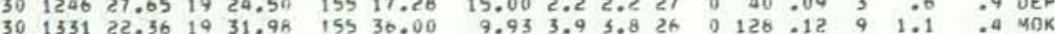

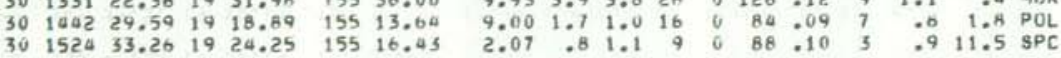

$\begin{array}{lllllllllllllllll}31 & 144 & 29.33 & 19 & 26.97 & 155 & 56.49 & 12.42 & 3.2 & 2.8 & 21 & 0 & 230 & .16 & 38 & 8.5 & 2.9 \\ 3 & \text { KON }\end{array}$

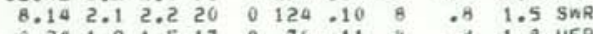

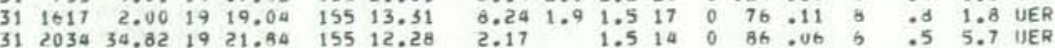

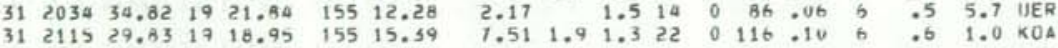

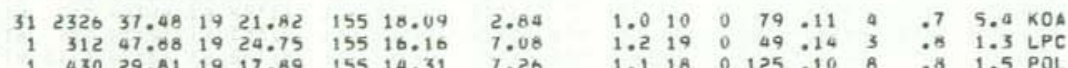

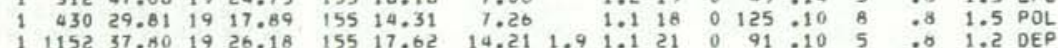
$\begin{array}{rrrrrrrrrrr}14.21 & 1.9 & 1.1 & 21 & 0 & 91 & .10 & 5 & 90 & 1.2 & \text { OEP } \\ 7.25 & 1.3 & 1.9 & 17 & 0 & 58 & .13 & 6 & : 9 & 2.0 & \text { UEK }\end{array}$

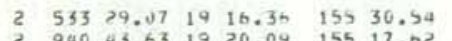
$\begin{array}{llllll}8 & 43.53 & 19 & 20.04 & 155 & 17.02 \\ \text { द } 1841 & 31.02 & 19 & 16.52 & 155 & 22.05\end{array}$ ट $20934.71926 .39 \quad 15522.7$

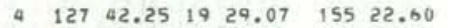

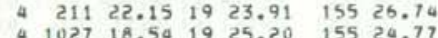

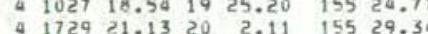

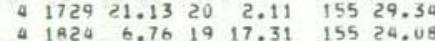

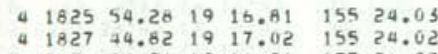
$\begin{array}{lllllll}4 & 2134 & 24.86 & 19 & 16.96 & 155 & 24.02 \\ 4 & 22 & 37.02 & 19 & 17.21 & 155 & 24.20\end{array}$

$\begin{array}{lllllll}5 & 736 & 28.76 & 17 & 16.95 & 155 & 23.42\end{array}$ 5 T36 737.731915 .99 155 23.07 5 750 47.301916 .83 155 23.25 $5 \quad 753 \quad 19.7919 \quad 16.48 \quad 15523.35$

$\begin{array}{llllllll}5 & \text { 8 } & 0 & 47.03 & 19 & 15.01 & 155 & 22.85\end{array}$ $\begin{array}{llllllll}5 & 8 & 2 & 41.43 & 17 & 16.81 & 155 & 23.20 \\ 5 & 910 & 47.32 & 19 & 17.10 & 155 & 23.42\end{array}$ $\begin{array}{llllllllll}3.62 & 2.5 & 2.4 & 22 & 0 & 70 & .17 & 15 & .9 & 2.2 \\ 2.5 E A\end{array}$

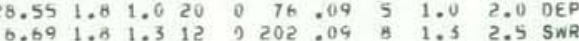
$\begin{array}{lllllllllll}7.91 & 2.4 & 2.6 & 23 & 0 & 67 & .11 & 7 & .7 & 1.2 & \text { UKF }\end{array}$ $\begin{array}{lllllllllll}9.25 & 2.0 & 1.6 & 17 & 0 & 127 & .07 & 12 & .6 & .9 & \text { NER }\end{array}$

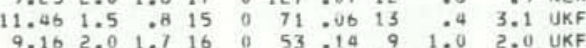

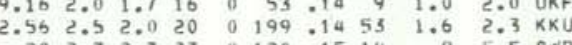

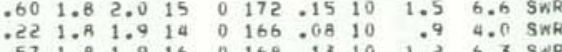

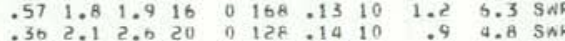
8.171 .92 .021 O OH.14 6 :9 1.7 UER $\begin{array}{lllllllllll}7.35 & 2.2 & 1.6 & 21 & 0 & 131 & .16 & 9 & 1.2 & 1.9 & \text { SWR }\end{array}$

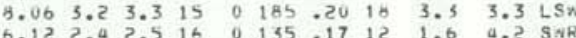

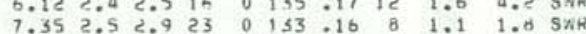

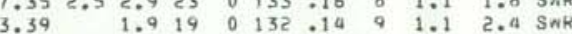
$\begin{array}{lllllllllll}10.92 & 1.9 & 1.6 & 16 & 0 & 167 & .12 & 9 & 1.9 & .6 & \text { LSW }\end{array}$

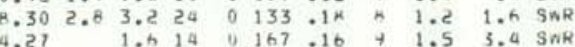

page as

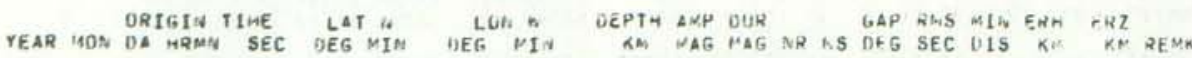

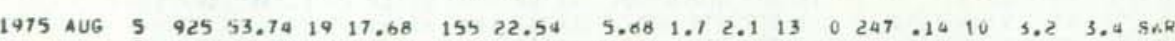

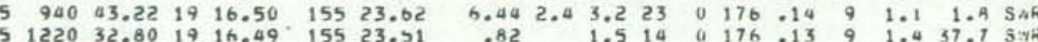

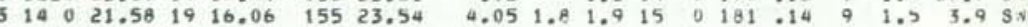
5144051.641916 .13 155 23.23

$\begin{array}{lllllll}5 & 15 & 1 & 57.17 & 19 & 16.36 \quad 155 & 23.21\end{array}$

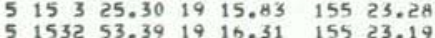

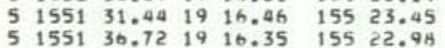

$51653 \quad 51.0414 \quad 16.34 \quad 155 \quad 23.42$
5

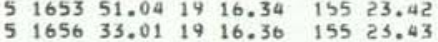
5172916.21
5 $19 \quad 15.95 \quad 15523.74$ $\begin{array}{lllllll}5 & 1733 & 24.12 & 19 & 15.96 & 155 & 23.52 \\ 5 & 1749 & 37.59 & 19 & 16.36 & 153 & 23.82\end{array}$ $\begin{array}{lllllll}5 & 1918 & 33.06 & 19 & 16.40 & 155 & 23.52\end{array}$ 5 2054 $33.07 \quad 1920.33 \quad 155 \quad 9.86$

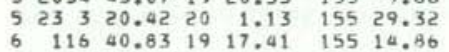

$\begin{array}{lllllll}6 & 214 & 34.67 & 19 & 31.61 & 155 & 27.89\end{array}$

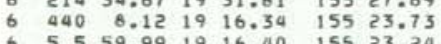

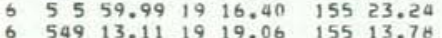
$6 \quad 54913.11 \quad 1919.06 \quad 155 \quad 13.78$

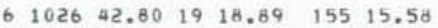

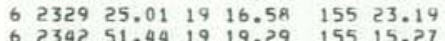

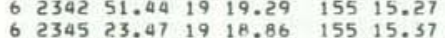
6234523.471918 .8615515 .37

$\begin{array}{llllllll}7 & 4 & 7 & 6.90 & 19 & 16.97 & 155 & 23.06\end{array}$ $\begin{array}{lllllll}7 & 428 & 26.38 & 19 & 13.16 & 155 & 35.77 \\ 7 & 737 & 51.08 & 19 & 231.71 & 155 & 3.49\end{array}$

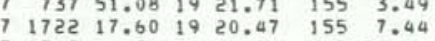
4.95 1.8 18 0 129.18 9 1.4 1.A SAN

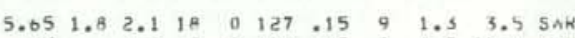

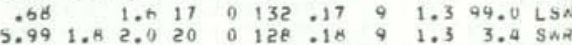

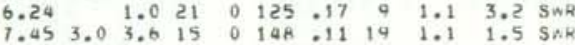

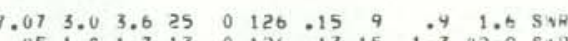

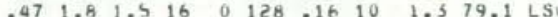

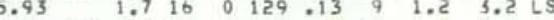

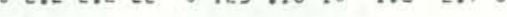
$\begin{array}{llllllllllll}3.63 & 1.6 & 1.7 & \text { ट1 } & 0 & 125 & .16 & 9 & 1.0 & 2.3 & \mathrm{~s} \times \mathrm{m}\end{array}$

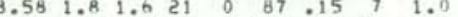
$\begin{array}{lllllllllll}4.06 & 2.4 & 1.7 & 21 & 0 & 194 & .16 & 52 & 1.9 & 3.1 & \mathrm{kK}\end{array}$

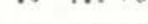
$\begin{array}{lllllllllll}4.37 & 2.3 & 2.1 & 20 & 0 & 107 & .13 & 10 & .7 & 1.4\end{array}$

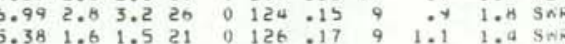

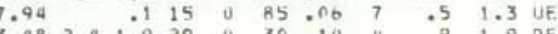

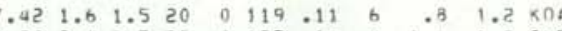

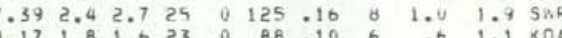

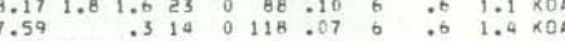

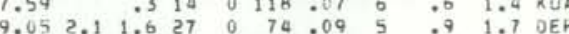
\begin{tabular}{lllllllllll}
5.07 & 1.4 & 1.3 & 20 & 0 & 122 & .15 & 8 & 1.0 & 1.4 & SNR \\
\hline 0.018 & 2.4 & 2.0 & 22 & 0 & 114 & .21 & 25 & 1.6 & .4 & HEA
\end{tabular}

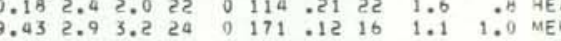
5.260 .51 .516 O 175.11 11 1.51 .0 UER

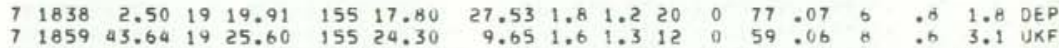

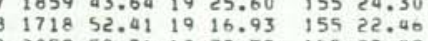

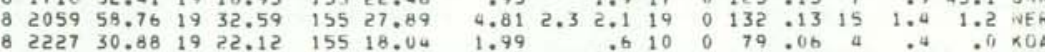

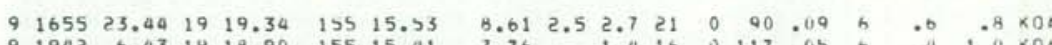

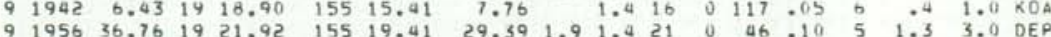

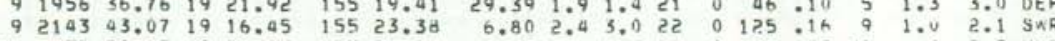

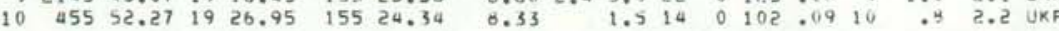

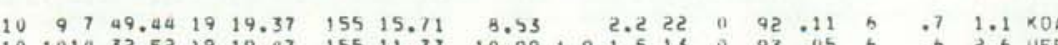

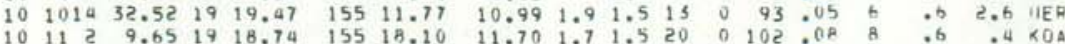


HVO EARTHQUAKE SUMMARY LIST

HAGE 83 ORIGIN TIME LAT N LON W OEPTH AMP DUR GAP KMS MIN ERH ERZ REMK

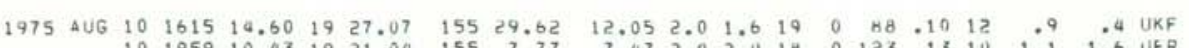

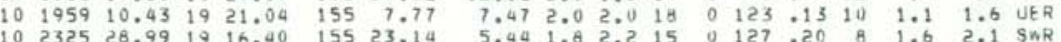

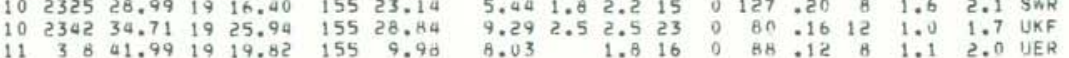
$\begin{array}{llllllllllllllllll}11 & 440 & 2.62 & 19 & 23.03 & 155 & 16.96 & 1.71 & .8 & 1.2 & 8 & 0 & 122 & .10 & 3 & 1.1 & .4 & \text { SPC }\end{array}$

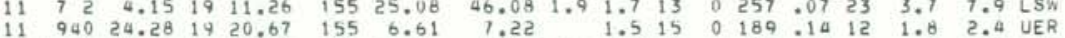

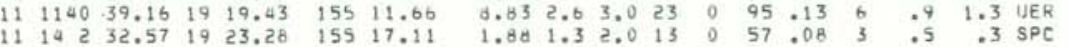
$\begin{array}{lllllllllllllllll}11 & 1530 & 45.86 & 19 & 24.23 & 155 & 17.15 & 14.61 & 1.3 & 20 & 0 & 43 & .06 & 3 & .4 & .7 & \text { OEP }\end{array}$

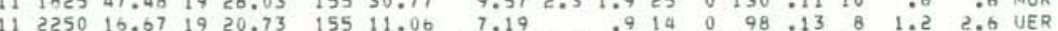

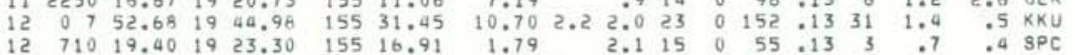

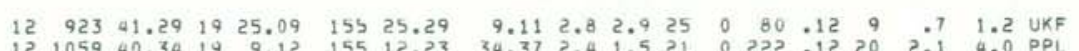

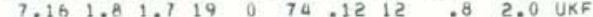

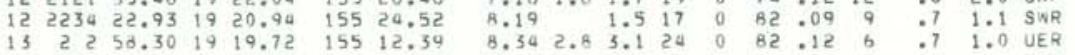

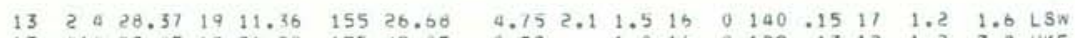

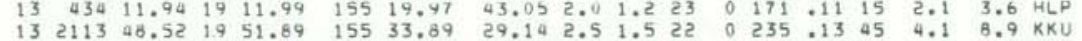

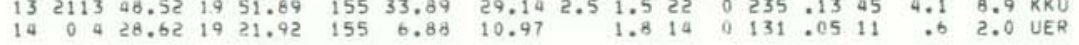

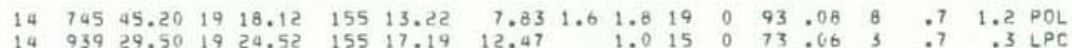

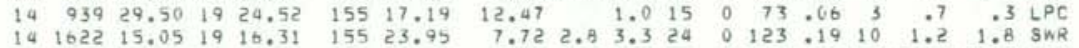

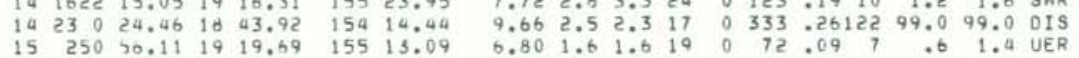

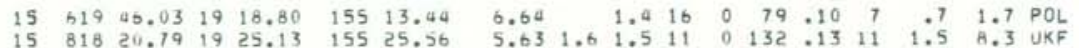

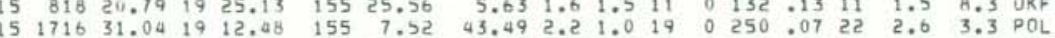

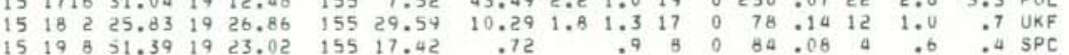

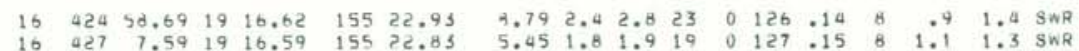

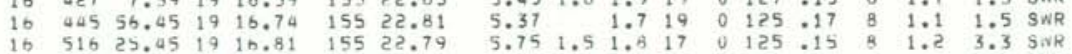

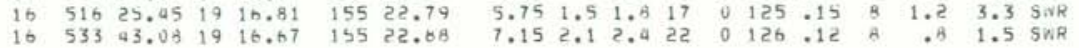
$\begin{array}{lllllllllllllllllll}16 & 720 & 23.09 & 19 & 19.92 & 155 & 11.30 & 7.76 & 1.8 & 1.9 & 20 & 0 & 86 & .15 & 7 & 1.0 & 2.0 & \text { UER } \\ 17 & 2 & 1 & 47.25 & 19 & 27.19 & 155 & 23.27 & 3.81 & 1.6 & 1.7 & 12 & 0 & 138 & .06 & 12 & .5 & 1.3 & \text { UKF }\end{array}$

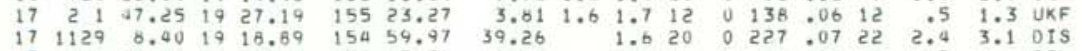

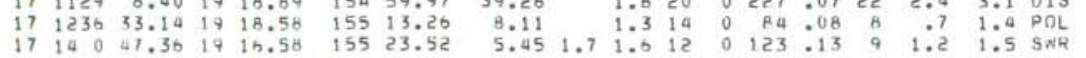

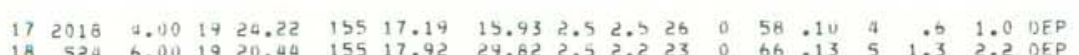

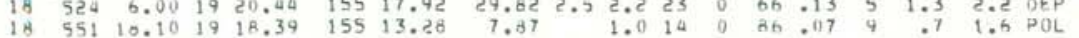

HVO EARTHQUARE SURMRY LIST

PAFt HA

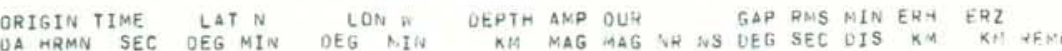

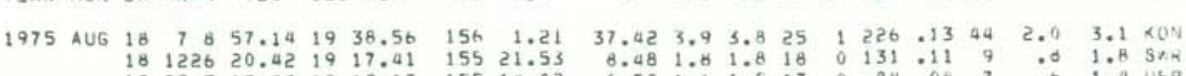

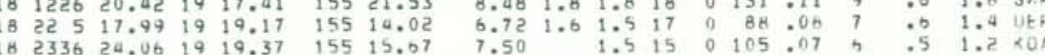
$19048 \quad 15.6419 \quad 19.62 \quad 15510.03$

$19 \quad 412 \quad 5.47 \quad 19 \quad 19.88 \quad 155 \quad 12.25$ 19. $95954.751918 .94 \quad 15515.1$

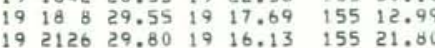

$20 \quad 050 \quad 1.23 \quad 19 \quad 19.60 \quad 155 \quad 11.60$ $\begin{array}{llllllll}20 & 3 & 3 & 3.29 & 19 & 16.61 & 155 & 22.9\end{array}$

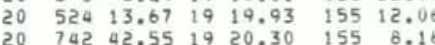

$20 \quad 1746 \quad 18.301920 .63 \quad 155 \quad 12.82$ $201925 \quad 45.941928 .10 \quad 15528.04$ $21 \quad 13937.9017 \quad 16.81 \quad 155 \quad 22.72$

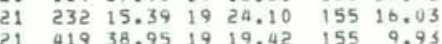

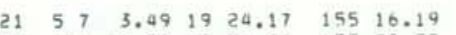
$22 \quad 03621.79 \quad 1956.30 \quad 15530.52$

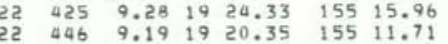
25 $448 \quad 18.501920 .20 \quad 15511.93$

$22 \quad 518 \quad 6.841920 .19 \quad 155 \quad 12.14$ $\begin{array}{lllllll}22 & 644 & 46.21 & 19 & 18.08 & 155 & 32.40 \\ 22 & 851 & 40 & 01 & 19 & 24 & \end{array}$

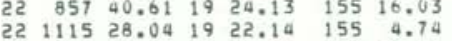
$221115 \quad 28.041922 .14 \quad 155 \quad 4.74$

$23 \quad 932 \quad 13.991921 .92 \quad 155 \quad 3.18$ $23192430.691947 .45 \quad 15532.98$

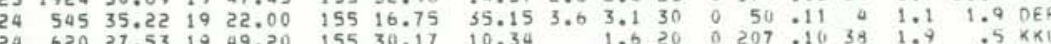

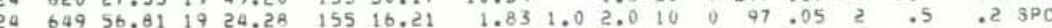

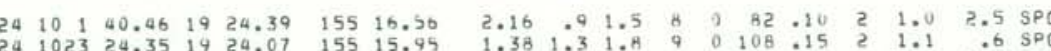

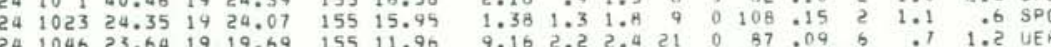

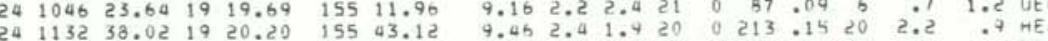
$24145712.661953 .60 \quad 15515.19 \quad 9.532 .72 .22000266 .10459 .5$ की PPL $\begin{array}{llllllllllllllllll}24 & 1641 & 15.74 & 19 & 52.39 & 155 & 30.63 & 17.00 & 2.4 & 1.4 & 23 & 0 & 129 & .13 & 40 & 1.3 & 8.6 & \times \times 1\end{array}$

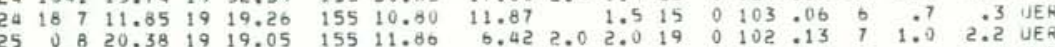

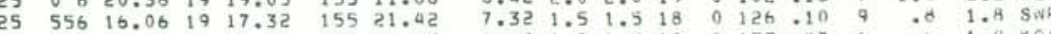

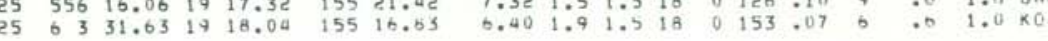

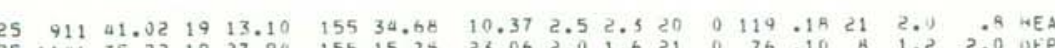

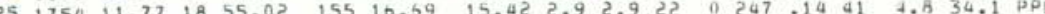




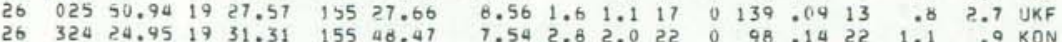

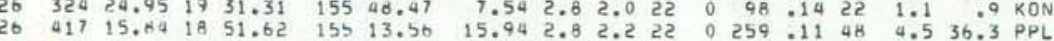
$26 \quad 55050.52 \quad 1926.09$ 155 27.75 $9.451 .71 .316 \quad 0 \quad 122.1313 \quad 1.2 \quad 3.2$ UKF $\begin{array}{lllllllllllllllll}26 & 943 & 57.56 & 16 & 45.49 & 155 & 14.69 & 16.28 & 3.0 & 13 & 0 & 308 & .11 & 64 & 14.7 & 82.0 & \text { PPL }\end{array}$

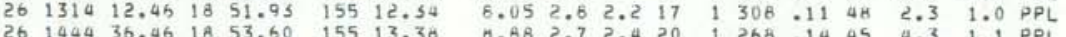

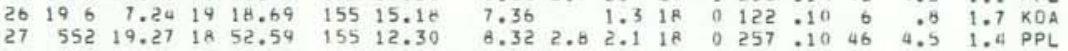

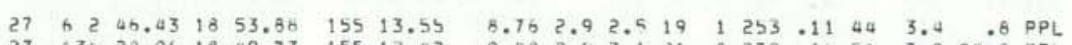

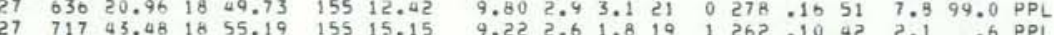

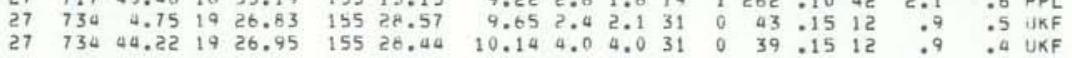
$\begin{array}{llllllllllllllllll}27 & 1324 & 31.36 & 18 & 52.32 & 155 & 13.72 & 15.09 & 3.0 & 3.2 & 21 & 1 & 288 & .10 & 47 & 2.9 & 44.7 & P P L\end{array}$

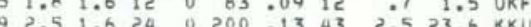
?7 234909.86 19 $51.85 \quad 15530.55 \quad 14.29$ 2..5 1.6

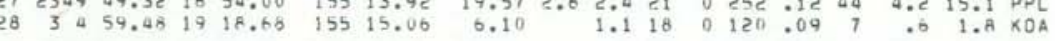
$\begin{array}{lllllllllllllllll}28 & 636 & 50.56 & 19 & 17.28 & 155 & 22.44 & 6.32 & 1.7 & 1.7 & 18 & 0 & 122 & .12 & 8 & .9 & 2.3\end{array}$ SWR

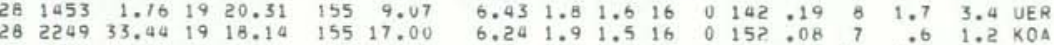

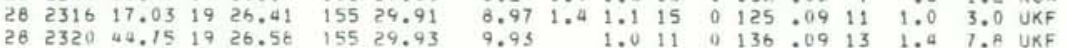

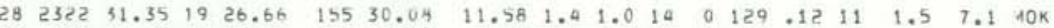
$\begin{array}{llllllllllllllllll}29 & 151 & 27.76 & 19 & 18.48 & 155 & 15.75 & 9.31 & 2.4 & 2.7 & 25 & 0 & 106 & .11 & 5 & .7 & \text {. A } & \times 0 A\end{array}$

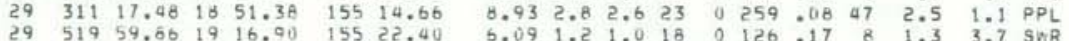

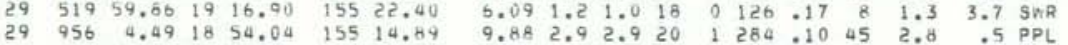

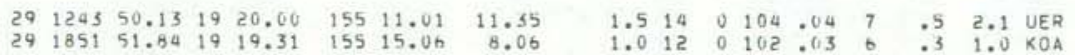

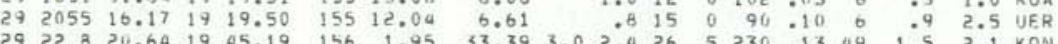

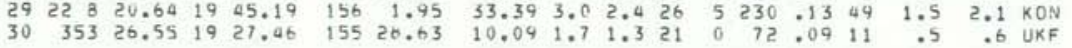
$\begin{array}{llllllllllllllllll}30 & 355 & 42.55 & 19 & 27.55 & 155 & 26.4 & 10.17 & 1.6 & 1.3 & 17 & 0 & 72 & .06 & 14 & .5 & .9 & \text { UKF }\end{array}$

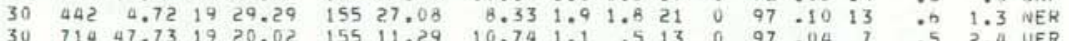

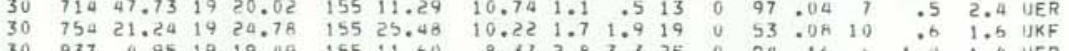
$\begin{array}{lllllllllllllllll}30 & 1325 & 35.96 & 19 & 16.55 & 155 & 26.05 & 8.00 & 1.6 & 1.9 & 14 & 0 & 106 & .12 & 11 & .9 & 1.4 \\ 3 E A A & \end{array}$

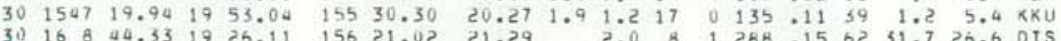

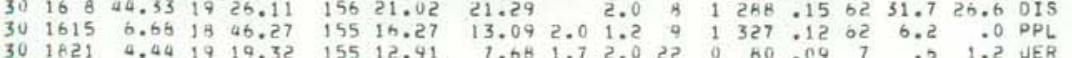

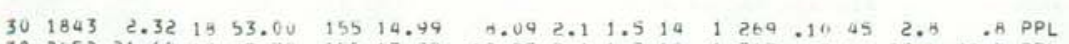

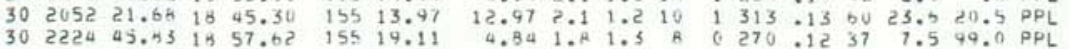

Tho eantwouane suniranr List

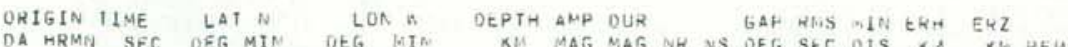

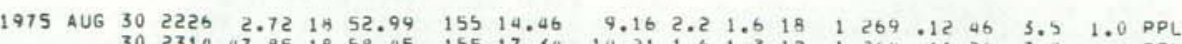

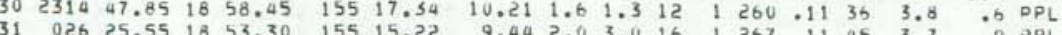

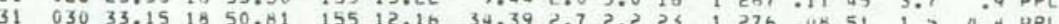

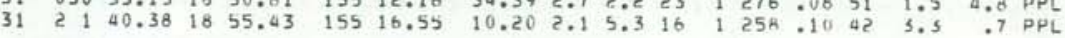
$\begin{array}{llllllllllllllllll}31 & 746 & 16.26 & 19 & 21.18 & 155 & 5.42 & 11.50 & 2.0 & 2.2 & 16 & 0 & 151 & .07 & 13 & .4 & .5 \text { MFER }\end{array}$

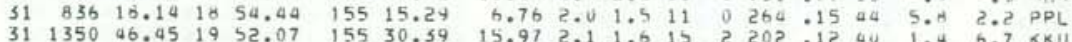

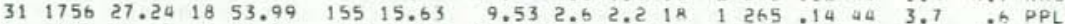

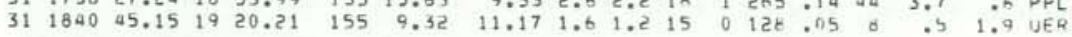

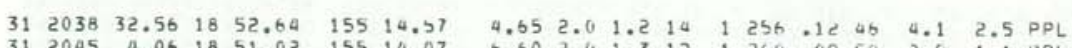

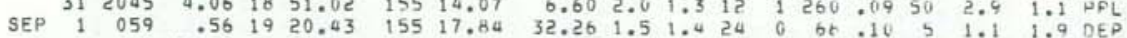

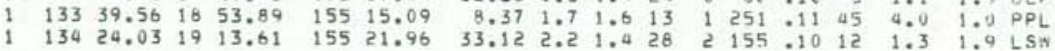
$\begin{array}{llllllllllllllllll}1 & 143 & 53.76 & 18 & 53.90 & 155 & 15.96 & 9.57 & 1.9 & 1.4 & 12 & 0 & 251 & 11 & 45 & 4.9 & .6 & P P L\end{array}$

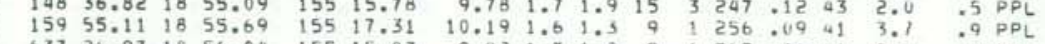

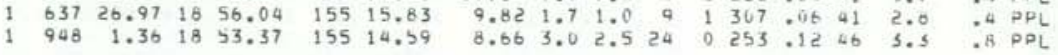

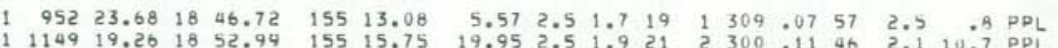

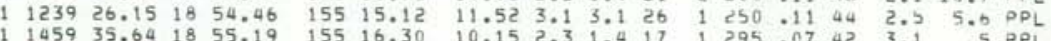

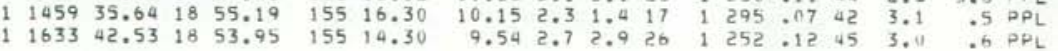

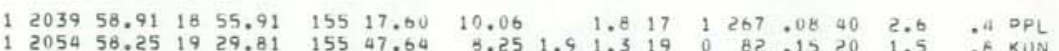

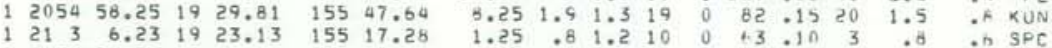

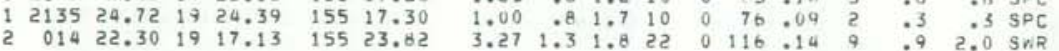

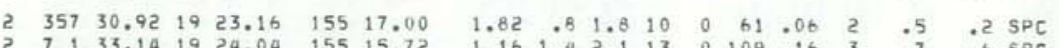

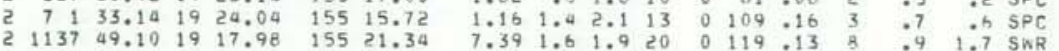

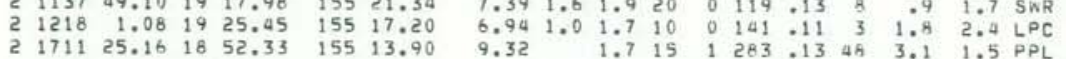
$\begin{array}{llllllllllllllllll}2 & 1721 & 4.17 & 18 & 51.04 & 155 & 14.08 & 9.68 & 2.6 & 2.1 & 25 & 1 & 274 & .10 & \text { AB } & 1.5 & \text {.4 PPL }\end{array}$

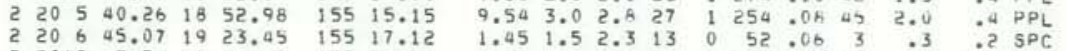

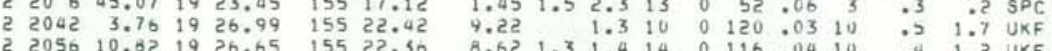

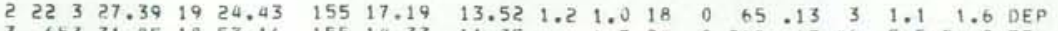

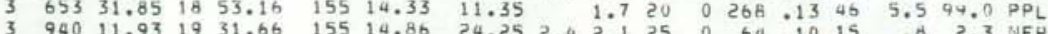

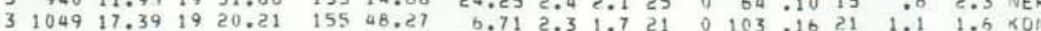

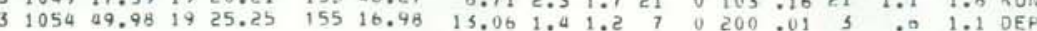
$\begin{array}{llllllllllllllllll}3 & 1923 & 57.60 & 19 & 24.39 & 155 & 17.84 & 6.03 & 1.0 & 1.9 & 11 & 0 & 102 & .11 & 3 & 1.2 & \text { 2.2 LPC }\end{array}$

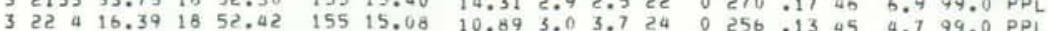


HVO EARTHRUAKE SUMATARY LIST

PAGF HT

ORIGIN TIME LATN LON W UEPTH AMP DUR W GAP KMS MIN ERH ERZ

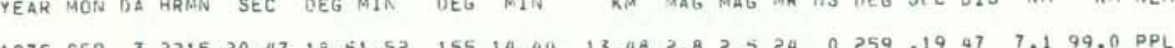
$\begin{array}{lllllllllllllllllll}1975 \text { SEP } & 3 & 2215 & 20.47 & 18 & 51.52 & 155 & 14.40 & 13.48 & 2.8 & 2.5 & 24 & 0 & 259 & .19 & 47 & 7.1 & 99.0 & P P L \\ & 3 & 2228 & 45.63 & 18 & 51.87 & 155 & 15.34 & 20.28 & 2.7 & 2.5 & 23 & 0 & 257 & .15 & 47 & 5.5 & 17.01 & P P L\end{array}$

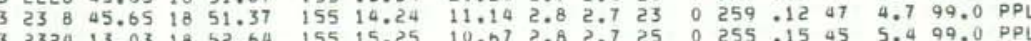

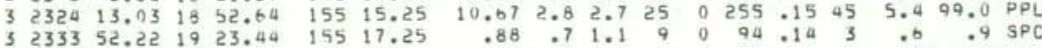

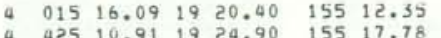
4 425 $10.911924 .90 \quad 155 \quad 17.78$ - 6.630 .181926 .47 155 23.34 464436,01 18 53.21 155 14.83

$\begin{array}{lllllll}4 & 655 & 49.08 & 18 & 46.77 & 155 & 13.42\end{array}$ $4945.53 \quad 18 \quad 51.05 \quad 155 \quad 13.2$ 473834.631918 .260155 21.35

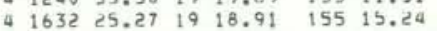

$\begin{array}{lllllll}4 & 2022 & 27.11 & 19 & 26.52 & 155 & 27.51\end{array}$

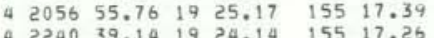

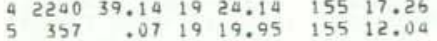
$\begin{array}{lllllll}5 & 1629 & 50.56 & 19 & 15.36 & 155 & 13.49\end{array}$

$\begin{array}{lllllll}5 & 1641 & 19.23 & 18 & 53.54 & 155 & 16.14\end{array}$

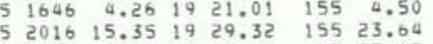

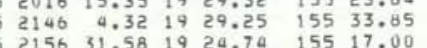

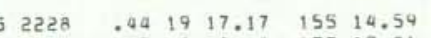
$\begin{array}{llllll}2259 & 33.63 & 19 & 19.41 & 155 & 13.81\end{array}$ $\begin{array}{llllll}041 & 25.95 & 19 & 34.20 & 155 & 10.79\end{array}$

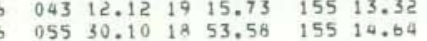

$\begin{array}{llllllll}6 & 3 & 6 & 32.94 & 19 & 17.04 & 155 & 13.43\end{array}$ $\begin{array}{lllllll}547 & 35.75 & 18 & 53.56 & 155 & 15.48\end{array}$

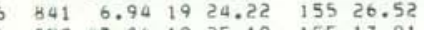
$\begin{array}{rrrrrrr}6 & 852 & 47.00 & 19 & 25.19 & 155 & 17.91 \\ 6 & 1345 & 8.06 & 19 & 19.18 & 155 & 13.31\end{array}$

$\begin{array}{lllllll}6 & 1428 & 26.88 & 19 & 36.69 & 155 & 11.23\end{array}$ $\begin{array}{llllllll}6 & 22 & 1 & 23.01 & 19 & 16.65 & 155 & 13.30\end{array}$ $\begin{array}{lllllll}62329 & 9.48 & 18 & 54.39 & 155 & 15.01\end{array}$ $\begin{array}{lllllll}058 & 58.77 & 19 & 13.23 & 155 & 21.50\end{array}$ $\begin{array}{lllllll}\text { द } 2 & 58.90 & 19 & 13.53 & 155 & 21.43\end{array}$ $\begin{array}{llllll}248 & 12.49 & 19 & 14.57 & 155 & 2.39 \\ 248 & 44.31 & 19 & 24.06 & 155 & 1.69\end{array}$ $\begin{array}{llllll}248 & 48.31 & 19 & 24.06 & 155 & 1.69 \\ 422 & 50.23 & 18 & 55.14 & 155 & 16.01\end{array}$

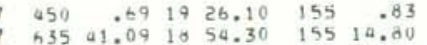
797 5.50 19 45.74 155 33.22

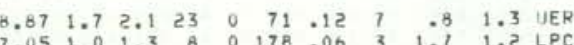

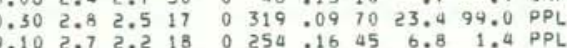
$\begin{array}{llllllllll}5.84 & 2.9 & 2.3 & 20 & 0 & 296 & .08 & 57 & 9.8 & 3.4 \\ \text { PPL }\end{array}$

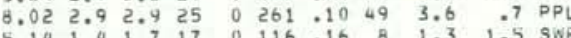

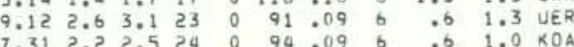
$\begin{array}{lllllllllll}7.90 & 1.8 & 1.0 & 19 & 0 & 64 & .13 & 14 & .8 & 2.9 & \text { UKF }\end{array}$

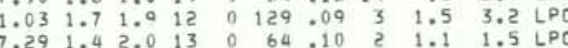

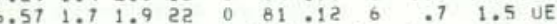
$6.43 \quad 1.91 .4 \quad 14$ o 242.1111 2.1 2.1 pol

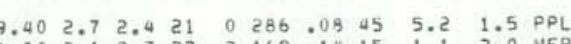
$8.69 \quad 1.7 \quad 1.614 \quad 0 \quad 83.1014$.9 2.1 NEP

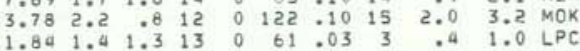

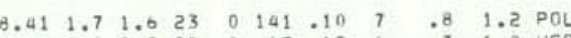
$\begin{array}{rrrrrrrrrrr}.46 & 2.1 & 2.2 & 28 & 0 & 62 & .12 & 6 & .7 & 1.0 & \text { UER } \\ 30.25 & 1.5 & .7 & 17 & 0 & 187 & 0.07 & 17 & 1.5 & 3.2 & \text { NER }\end{array}$ \begin{tabular}{llllllllll}
8.72 & 1.9 & 1.7 & 21 & 0 & 195 & .14 & 10 & 1.3 & 1.9 \\
\hline
\end{tabular} $8.41 \quad 1.819 \quad 0 \quad 152.11 \quad 9 \quad 99 \quad 1.4$ POL $\begin{array}{lllllllllllll}5.98 & 1.7 & 1.5 & 16 & 0 & 57 & .10 & 12 & .6 & 2.0 & \text { UKF }\end{array}$

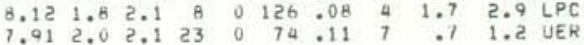

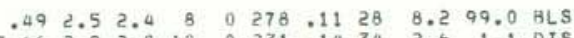

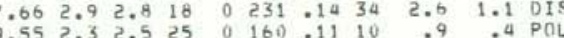

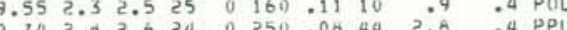

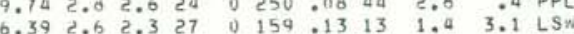

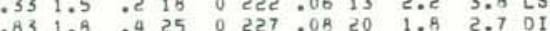

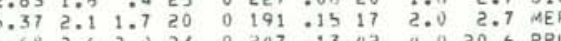
$\begin{array}{lllllllll}6.11 & 1.5 & 21 & 0 & 149 & .10 & 11 & 2.8 & 5.5 \\ 6 & \text { LEM }\end{array}$

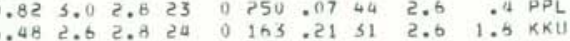

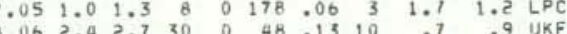
$\begin{array}{llllllllllll}20 & 2.0 & 1.2 & 24 & 0 & 15 B & .10 & 13 & 1.3 & 2.3 & \text { LSW }\end{array}$
HVO EARTHOUACE SUMNGRY LIST

PAGE BA

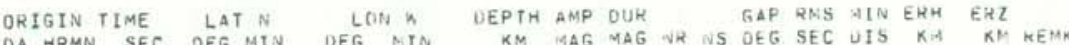

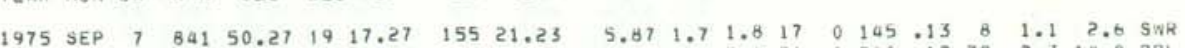

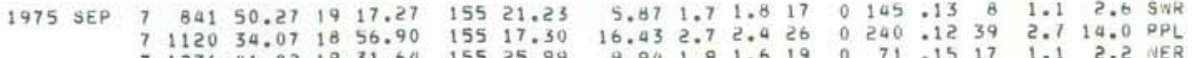

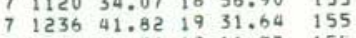

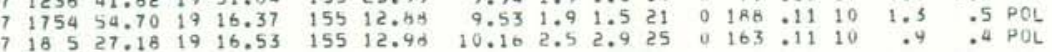

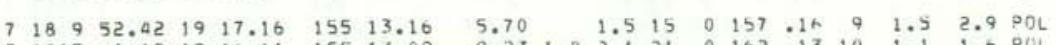
$\begin{array}{llllllllllll}7 & & & \end{array}$

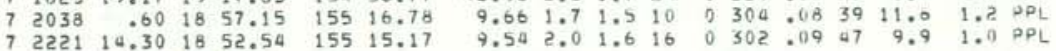

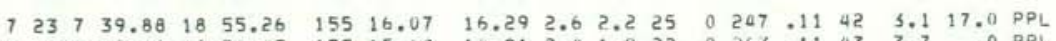

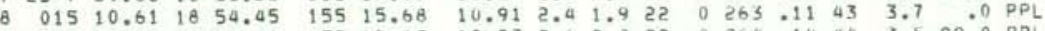

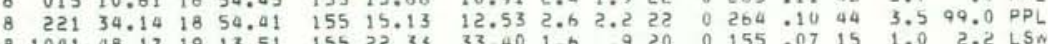

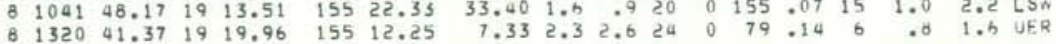

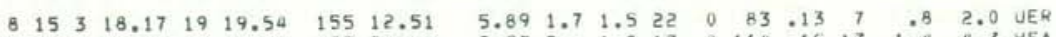

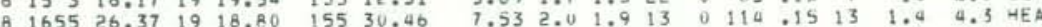

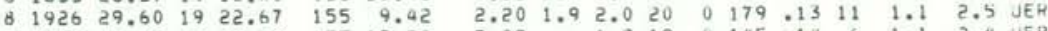

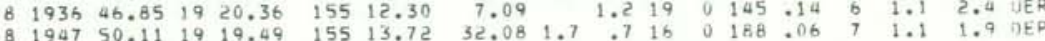

\begin{tabular}{l}
$8036 \quad 13.75 \quad 19 \quad 18.14 \quad 155 \quad 15.33$ \\
\hline
\end{tabular}

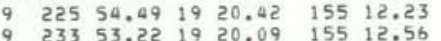
$93165.421924 .53 \quad 15516.79$

$9 \quad 317 \quad 38.64 \quad 1923.96 \quad 155 \quad 16.74$

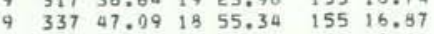

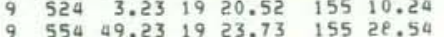
$915719.831926,70 \quad 155 \quad 29.41$ $\begin{array}{lllllll}1814 & 18.87 & 19 & 13.15 & 155 & 22.04 \\ 9 & 1820 & 24.07 & 19 & 28.86 & 155 & 26.76\end{array}$

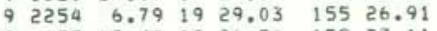
10015347.591926 .5415523 .11

$10 \quad 713 \quad 00.351912 .40 \quad 155 \quad 20.09$ $\begin{array}{lllllll}10 & 723 & 41.28 & 19 & 20.48 & 155 & 7.12 \\ 10 & 834 & 13.49 & 19 & 19.79 & 155 & 8.56\end{array}$ $10 \quad 846 \quad 25.91 \quad 1928.05 \quad 15527.41$ 10,246 45.80 19 19.09 15515.80

$\begin{array}{lllllll}10 & 1512 & 2.52 & 19 & 18.36 & 155 & 13.11\end{array}$

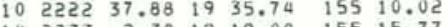
$102233 \quad 2.301919 .00 \quad 15515.77$ $\begin{array}{lllllll}11 & 358 & 22.14 & 19 & 22.47 & 155 & 15.11\end{array}$

$11 \quad 443 \quad 55.34 \quad 18 \quad 19.94 \quad 155 \quad 45.08$ $11 \quad 45012.891919 .02 \quad 155 \quad 13.58$

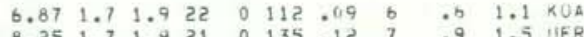

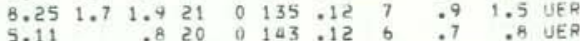
$\begin{array}{llllllllllll}7.86 & 1.3 & 1.7 & 11 & 0 & 78 & 06 & 2 & .9 & 1.6 & \text { LPC }\end{array}$

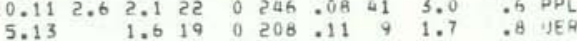

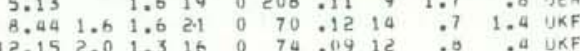
$\begin{array}{lllllllllllll}35.58 & 2.5 & 2.3 & 28 & 0 & 157 & .11 & 12 & 1.3 & 2.4 & \text { LSW }\end{array}$

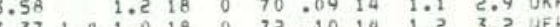
8.52 1.5 1.6 18 0 82 .06 11 94 . 4 JKF

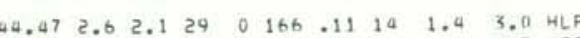

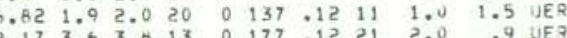

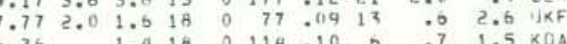
$\begin{array}{llllllllllll}6.58 & 1.7 & 1.8 & 21 & 0 & 93 & .11 & 8 & .7 & 1.6 & \text { POL }\end{array}$

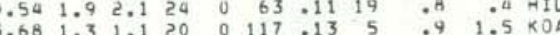

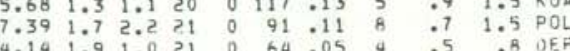
$11.783 .12 .429 \quad 0322.1510329 .399 .6015$

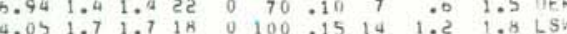
$7.961 .41 .313 \quad 0 \quad 76: 05$ a $\quad: 0 \quad \therefore L P C$ 


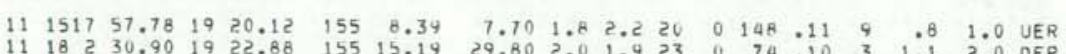

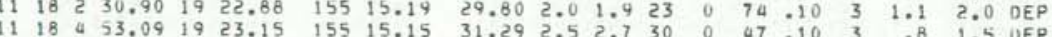

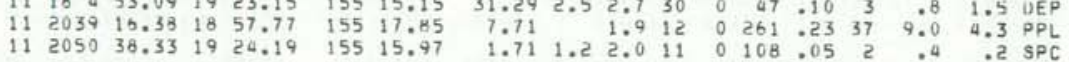

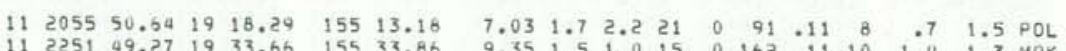

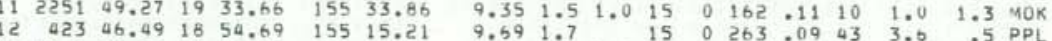

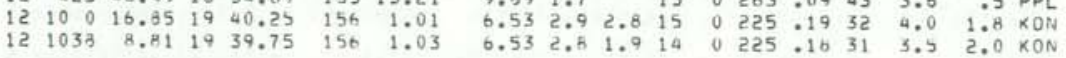

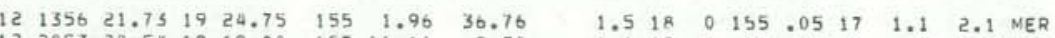

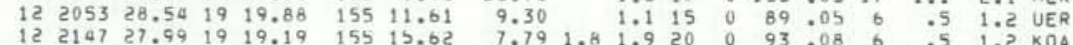

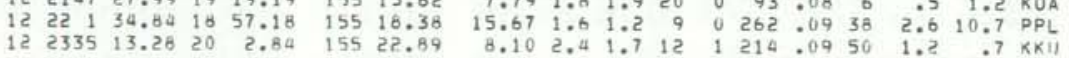
$\begin{array}{lllllllllllllllll}13 & 042 & 17.80 & 19 & 19.83 & 155 & 8.72 & 7.53 & 1.8 & 1.8 & 17 & 0 & 105 & .10 & 9 & .8 & 1.8 \\ 13 & 11 & 0.8 & 15 & 19.8\end{array}$

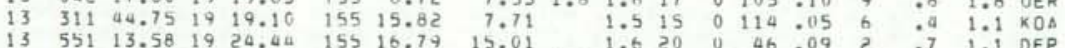

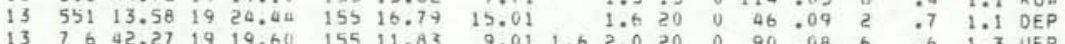

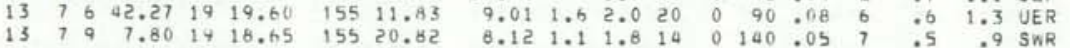

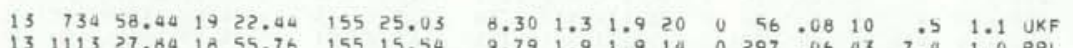

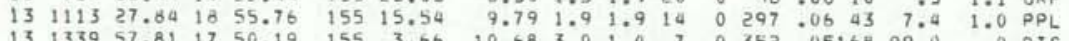

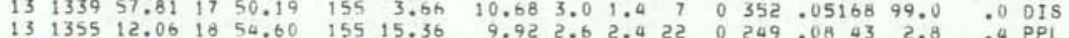

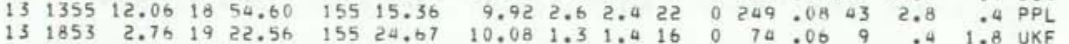

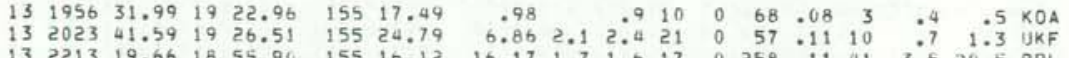

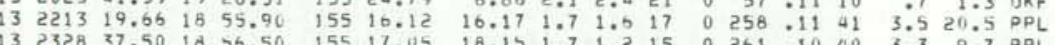

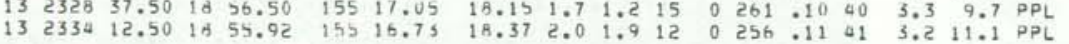

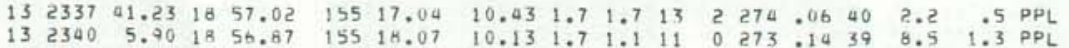

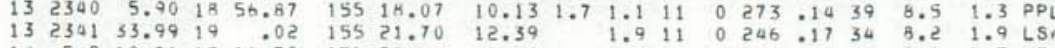

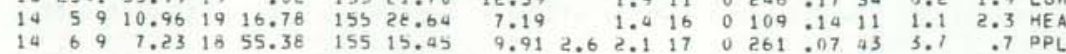

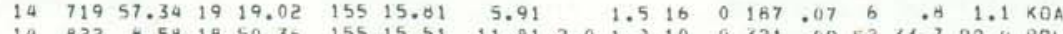

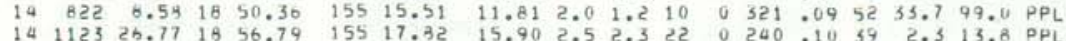

HVD EARTHGUANE SUM:AGY LIST

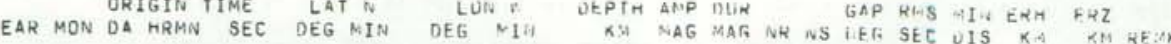

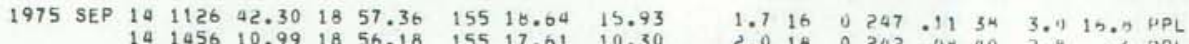

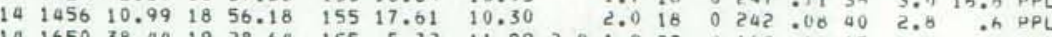

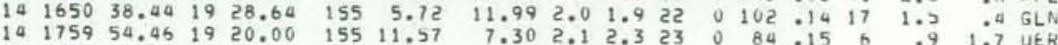

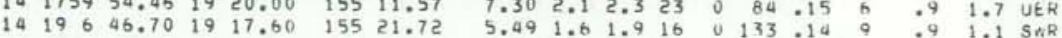

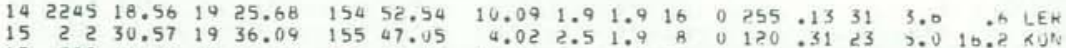

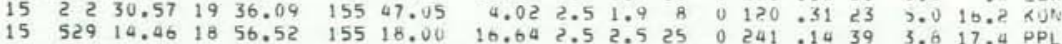

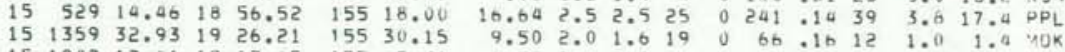

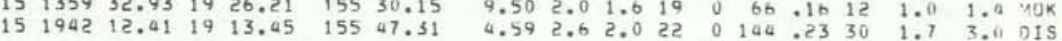

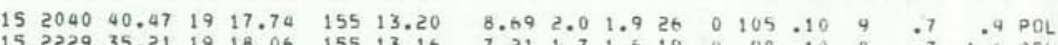

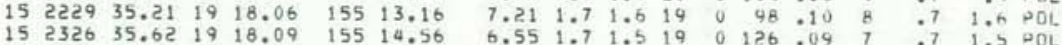

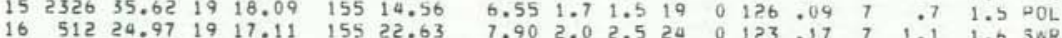

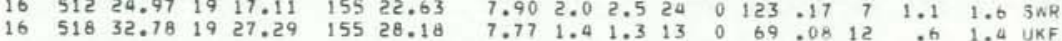

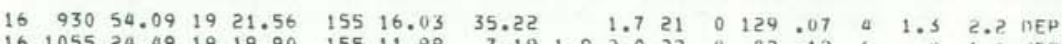

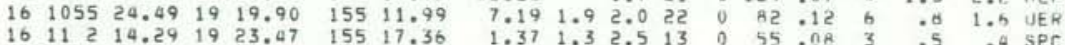

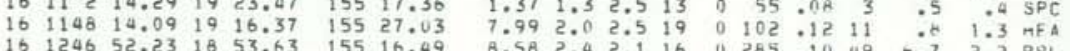
$\begin{array}{lllllllllllllllll}16 & 198 & 39.91 & 19 & 10.92 & 155 & 36.73 & 8.85 & 2.6 & 2.3 & 18 & 0 & 95 & .19 & 20 & 1.5 & 2.4\end{array}$ HEA

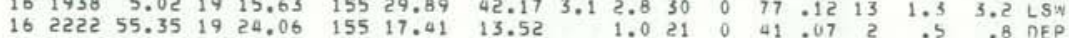

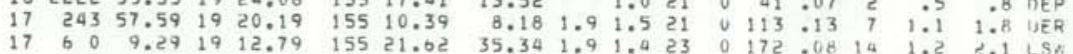

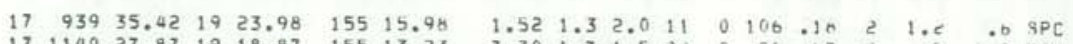

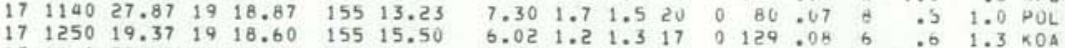

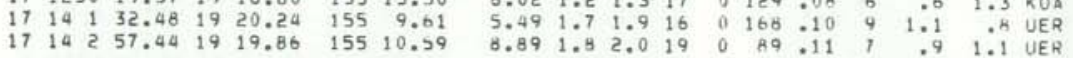
$\begin{array}{lllllllllllllllll}17 & 1414 & 3.53 & 19 & 24.08 & 155 & 16.02 & 1.56 & 1.3 & 2.2 & 11 & 0 & 105 & .11 & 2 & .7 & .45 P C\end{array}$

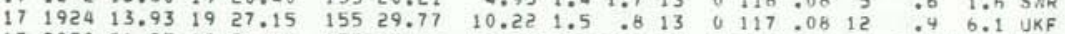

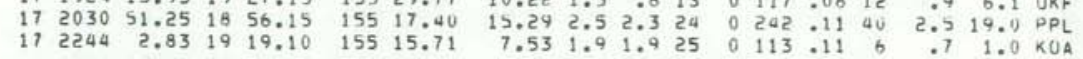

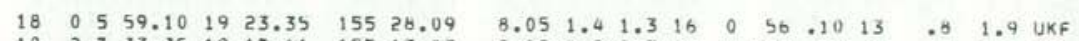

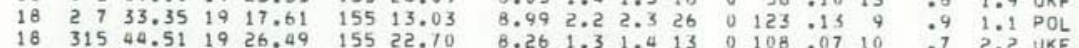

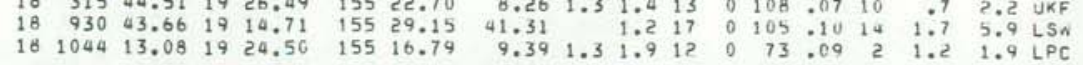

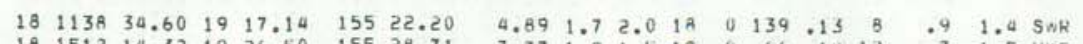

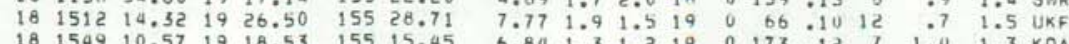

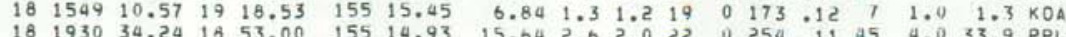

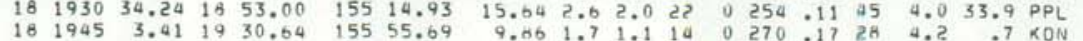

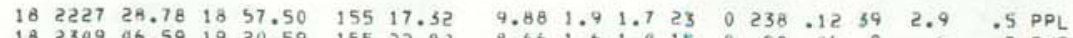

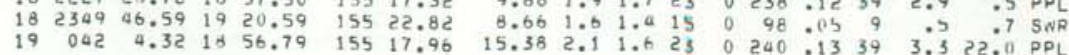




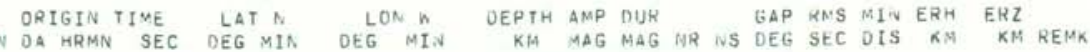

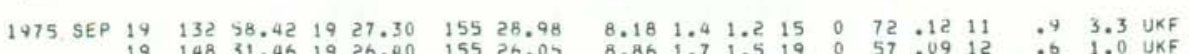

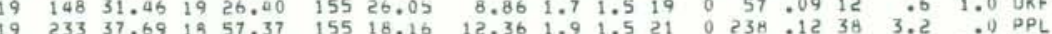

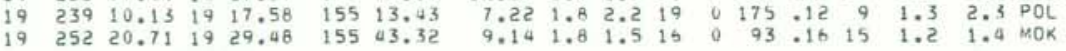
\begin{tabular}{llllllllllllllll}
19 & 326 & 15.43 & 19 & 17.15 & 155 & 13.30 & 6.56 & 1.4 & 16 & 0 & 230 & .08 & 11 & 1.4 & 2.5 \\
\hline
\end{tabular}

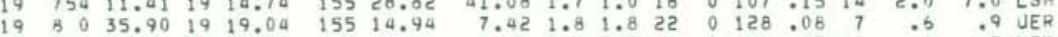

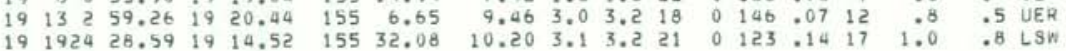

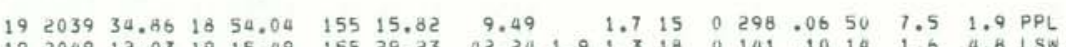

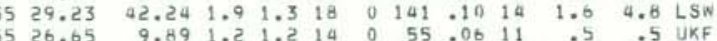

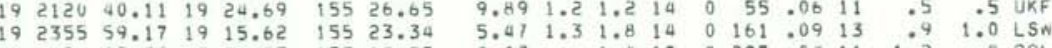

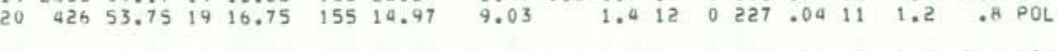
$\begin{array}{lllllllllllllllll}20 & 656 & 7.71 & 19 & 15.25 & 155 & 29.53 & 41.59 & 2.0 & 1.3 & 19 & 0 & 95 & .09 & 13 & 1.2 & 3.8 \text { LSW }\end{array}$

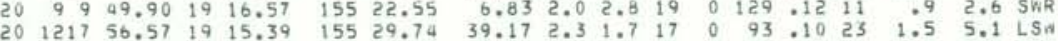

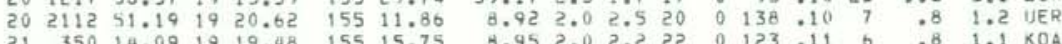

(155 59.140

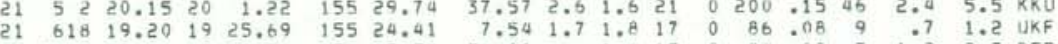

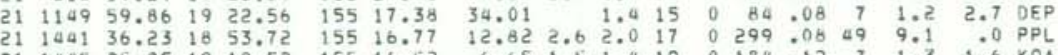

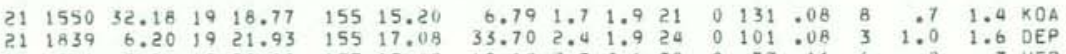

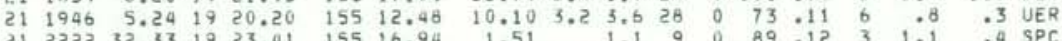

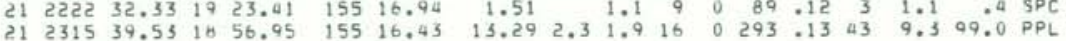
$\begin{array}{lrllllllllllllllll}22 & 026 & 58.72 & 19 & 15.28 & 155 & 29.70 & 43.15 & 2.6 & 2.0 & 27 & 0 & 93 & .13 & 13 & 1.4 & 3.7 & \text { LSW } \\ 32 & 134 & 38.31 & 19 & 22.18 & 155 & 17.34 & 33.14 & 1.8 & 1.0 & 17 & 0 & 132 & .07 & 7 & 1.0 & 1.5 & 0.5\end{array}$

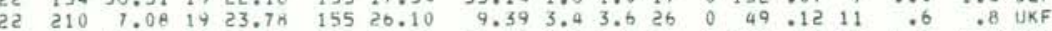

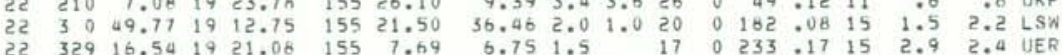

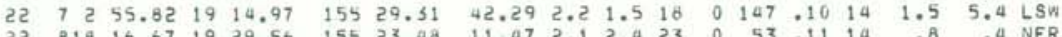

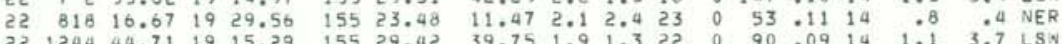

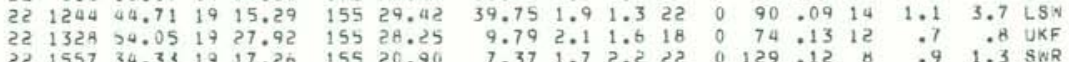
$\begin{array}{lllllllllllllllllll}22 & 17 & 7 & 25.85 & 19 & 23.84 & 155 & 17.19 & 1.43 & .9 & 1.5 & 10 & 0 & 55 & .09 & 2 & .7 & .4 & \mathrm{SPC}\end{array}$

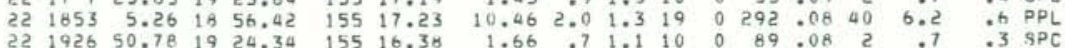

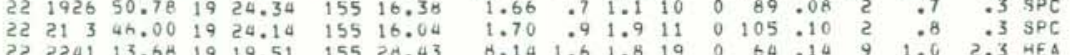

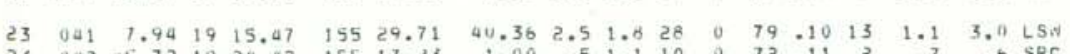

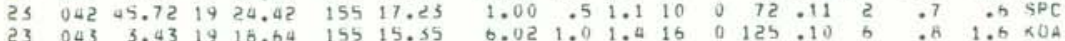

ORIGIN TIAE LATA LON DE DEPTH AMP DUH GAP KMS MTIN EHM ERZ YEAR MON DA HRMN SEC DEG MIN UEG MIN KM MAG MAG WR HS OEG SFC UIS KA KM HENK

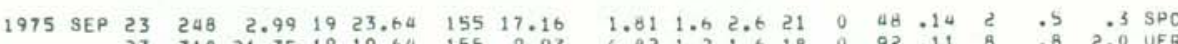

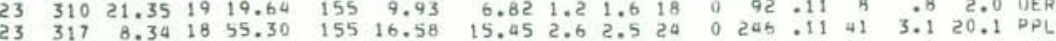

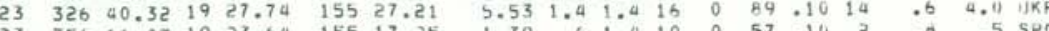

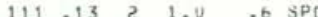

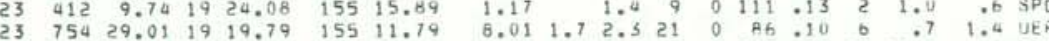

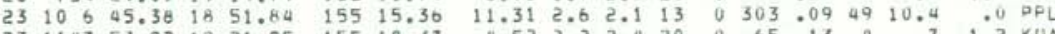

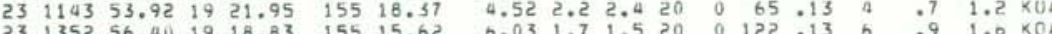

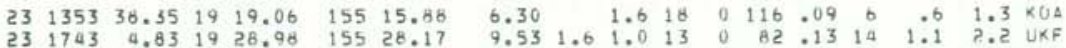

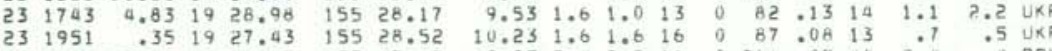

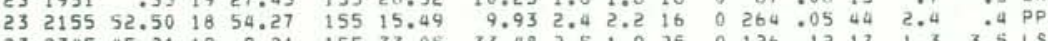

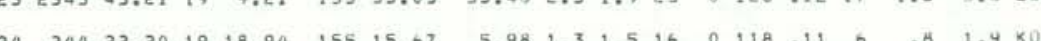
$\begin{array}{lrrrrrrrrrrrrrrrrr}24 & 244 & 22.20 & 19 & 18.94 & 155 & 15.67 & 5.98 & 1.3 & 1.5 & 16 & 0 & 118 & .11 & 6 & .8 & 1.4 & \text { KOA } \\ 24 & 3 & 5 & 57.17 & 19 & 20.46 & 155 & 8.73 & 5.11 & 2.1 & 1.5 & 21 & 0 & 71 & .15 & 10 & .3 & 1.3 \\ \text { ULER }\end{array}$

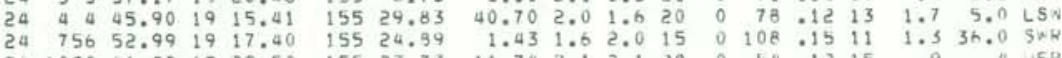

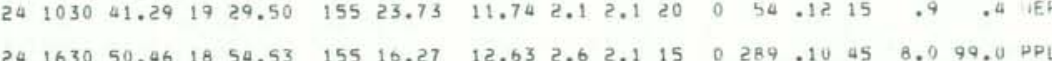

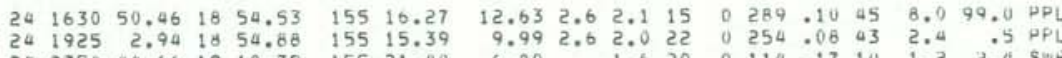

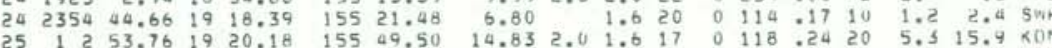

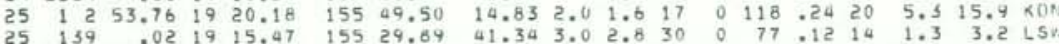

$\begin{array}{llllllllllllllllll}25 & 158 & 22.53 & 19 & 15.51 & 155 & 29.73 & 39.54 & 1.5 & .6 & 18 & 0 & 125 & .09 & 14 & 1.3 & 4.4 & \text { LS }\end{array}$

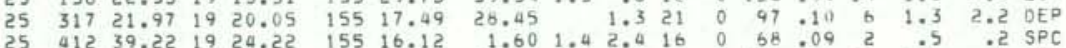

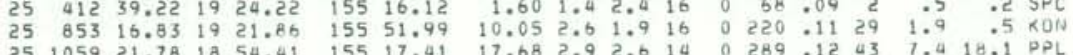
$\begin{array}{llllllllllllllllll}25 & 1129 & 41.18 & 19 & 24.32 & 155 & 16.89 & 15.48 & 2.2 & 2.3 & 29 & 0 & 46 & 09 & 2 & .6 & .8 & 0\end{array}$

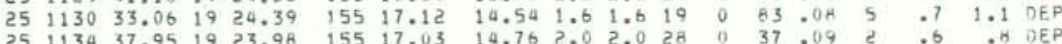

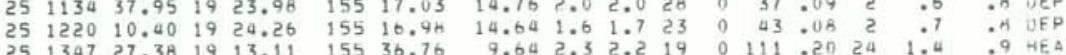

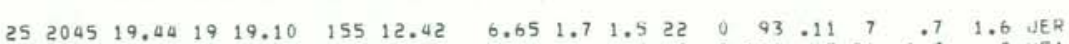

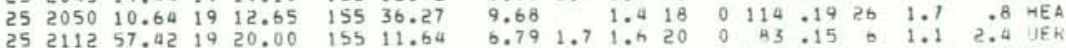

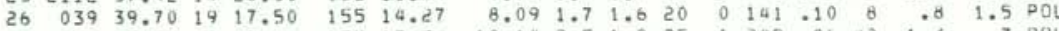

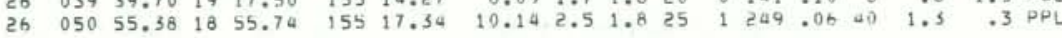
$\begin{array}{llllllllllllllllllll}26 & 3 & 1 & 13.92 & 19 & 10.76 & 155 & 31.42 & 3.25 & 1.7 & 1.2 & 16 & 0 & 104 & .13 & 22 & .9 & 2.3 & \text { LSW } & 0.4\end{array}$

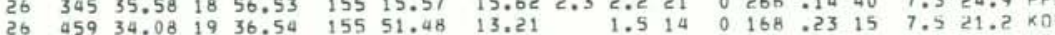

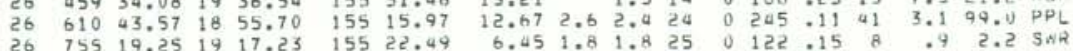

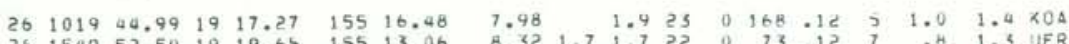

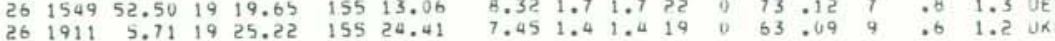




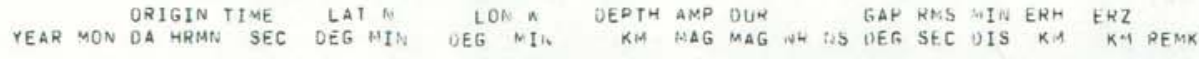

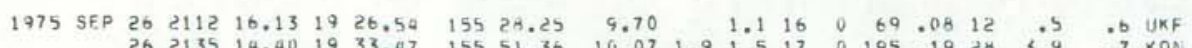

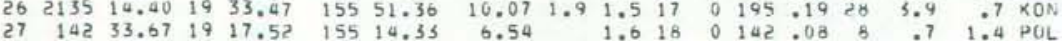

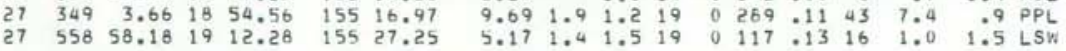

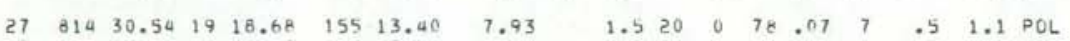

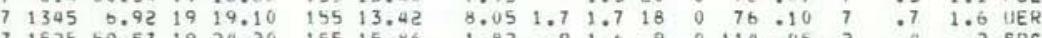

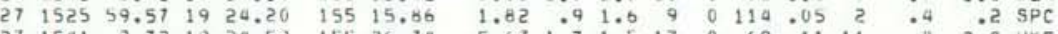

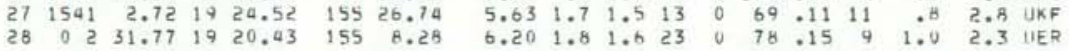

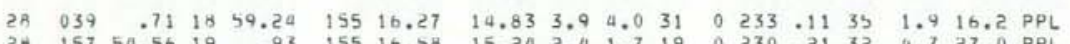

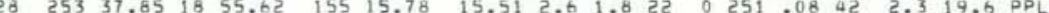

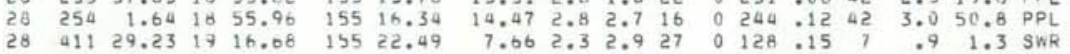
$\begin{array}{llllllllllllllll}28 & 416 & 59.65 & 19 & 13.02 & 155 & 29.99 & 39.86 & 1.6 & 31 & 0 & 72 & .12 & 13 & 1.3 & 3.1 \text { LSW }\end{array}$

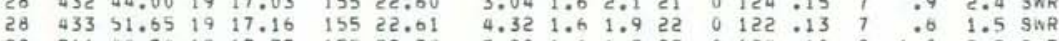

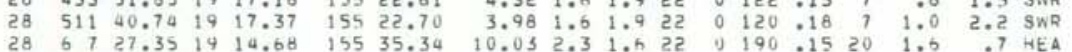

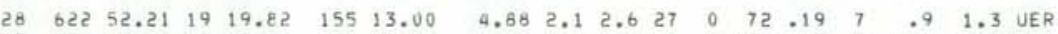

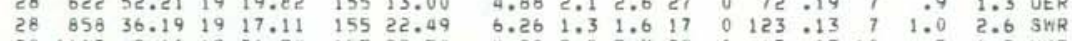

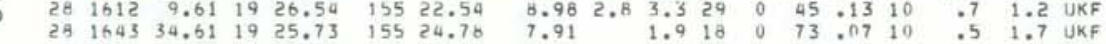

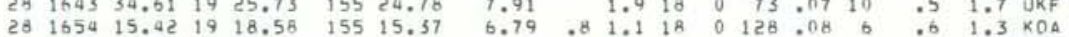

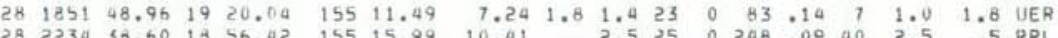

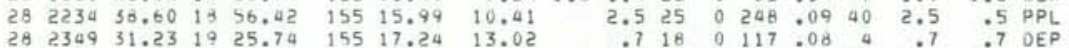

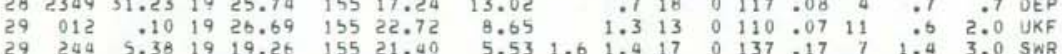
$\begin{array}{rrrrrrrrrrrrrrrrrr}29 & 432 & 33.35 & 19 & 13.76 & 155 & 22.03 & 33.27 & 1.8 & 1.2 & 26 & 2 & 164 & : 10 & 13 & 1.4 & 2.0 & \text { LSW } \\ 29 & 437 & 45.58 & 19 & 19.60 & 155 & 10.04 & 7.33 & 1.8 & 1.7 & 23 & 0 & 93 & : 12 & 8 & .8 & 1.5 & \text { UER }\end{array}$

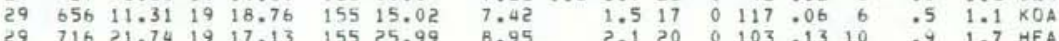

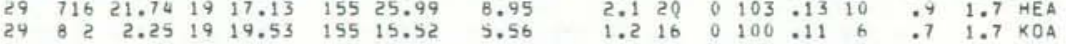

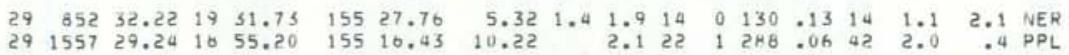

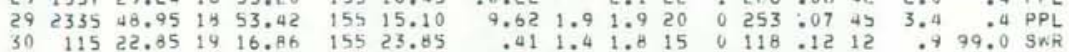

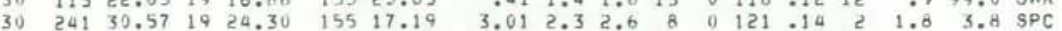

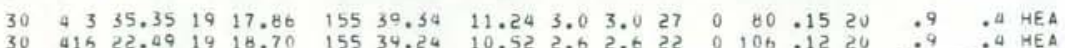

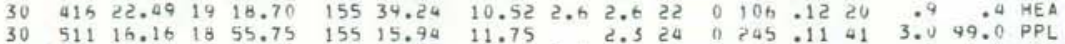

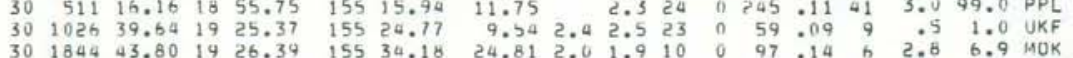

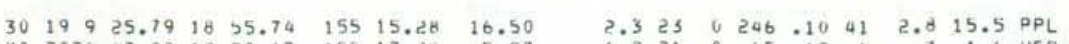

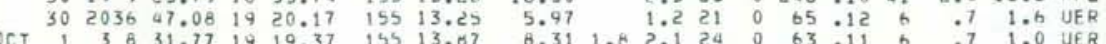

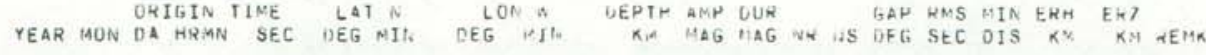

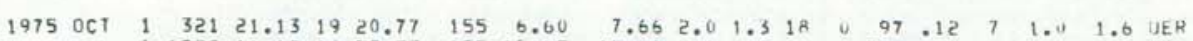

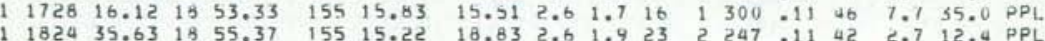

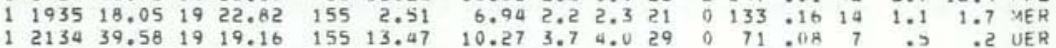

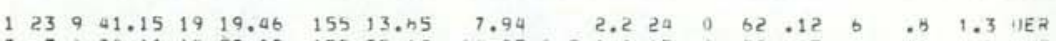

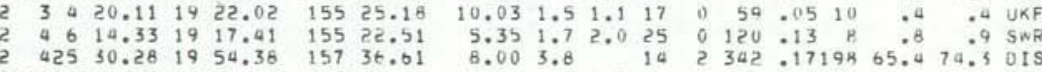

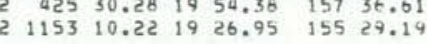
द $1340 \quad 40.96 \quad 1920.19 \quad 155 \quad 12.47$ द 16910.9618 171211.241924 .19
25516.07 2330 25.10 $19+10.05 \quad 155+33.75$ $\begin{array}{llllll}3 & 0 & 0 & 27.91 & 19\end{array}$ $\begin{array}{lllllll}3 & 033 & 42.69 & 18 & 56.91 & 155 & 16.81\end{array}$ 3 3 $048 \quad 18.98 \quad 18 \quad 59.41 \quad 155 \quad 15.84$

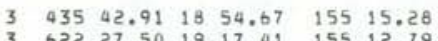
$\begin{array}{lllllll}3 & 622 & 27.50 & 19 & 17.41 & 155 & 12.79\end{array}$ $\begin{array}{lrlllll}3 & 714 & 6.86 & 18 & 57.60 & 155 & 12.60 \\ 3 & 1457 & 36.16 & 19 & 22.84 & 155 & 3.12\end{array}$

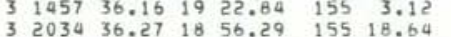

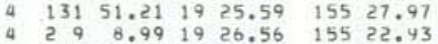

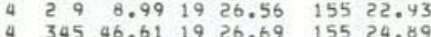
345
-56.611926 .69
0 $4,1318 \quad 46.57 \quad 1924.75 \quad 155 \quad 17.31$

$\begin{array}{lllllll}4 & 1616 & 12.04 & 19 & 38.82 & 156 & 4.73\end{array}$ $417514.611924 .39 \quad 15517.12$ 492858.61
4 $1924.22 \quad 15516.61$

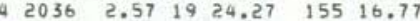

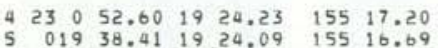
$5 \quad 01938.411924 .09 \quad 15516.69$ $55345 \quad 55.061919 .08 \quad 15512.62$ $5 \quad 514 \quad 5.28 \quad 1924.09 \quad 15516,50$ $\begin{array}{lllllllllll}2.17 & 1.1 & 1.3 & 9 & 0 & 88 & .08 & 5 & .8 & 1.7 & \text { SPC }\end{array}$

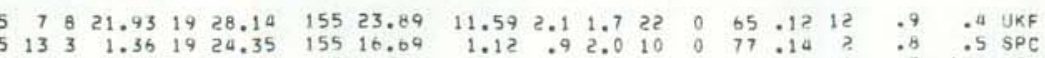

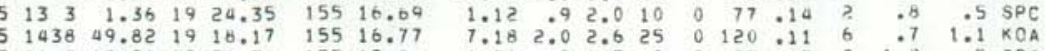

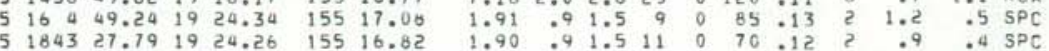

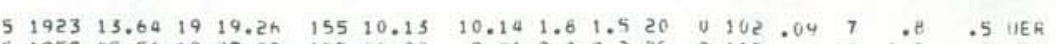

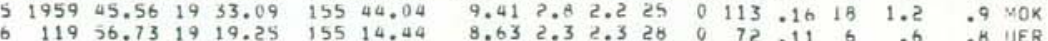


HVO EARTHQUAKE SUMTARY LIST

PAGE 95

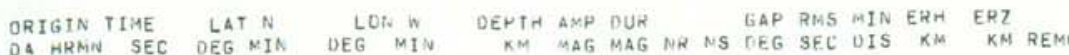

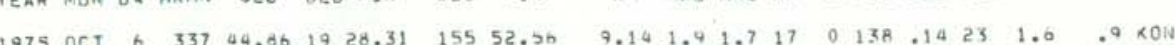

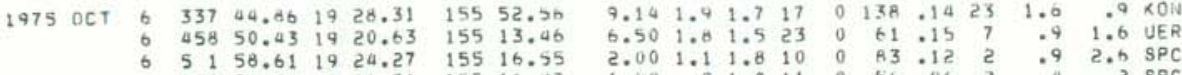

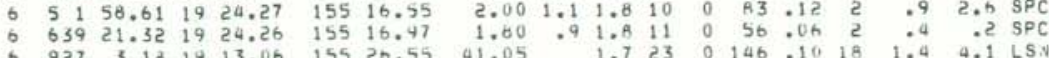

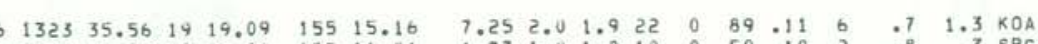

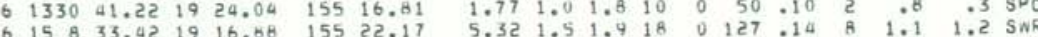

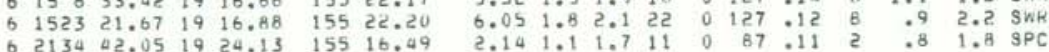

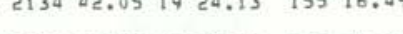

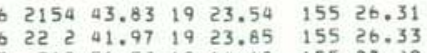

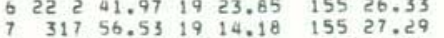
$\begin{array}{lllllll}7 & 321 & 54.70 & 19 & 6.50 & 155 & 24.36 \\ 7 & 437 & 37.68 & 19 & 24.27 & 155 & 16.78\end{array}$

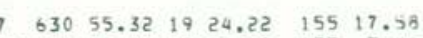

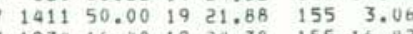
$8 \quad 13146.621924 .23 \quad 15516.84$

$\begin{array}{llll}1159 & 52.24 \quad 19 \quad 19.18 \quad 155 \quad 15.56\end{array}$

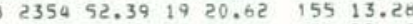
$\begin{array}{lrlllll}9 & 254 & 56.97 & 19 & 34.21 & 156 & 29.00 \\ 9 & 648 & 18.92 & 19 & 19.96 & 155 & 12.62\end{array}$

$9822 \quad 1.19 \quad 1922.18 \quad 15518.00$

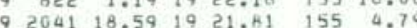
7. $2113 \quad 10.67 \quad 19 \quad 17.78 \quad 15514.34$ 9. 231421.661951 .09 155 45.10

$\begin{array}{lllllll}10 & 125 & 6.50 & 19 & 6.27 & 155 & 24.42\end{array}$ $10 \quad 234 \quad 42.25 \quad 1924.26 \quad 155 \quad 16.78$

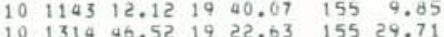

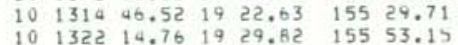

$\begin{array}{lllllll}10 & 1952 & 29.35 & 19 & 29.76 & 155 & 53.12\end{array}$

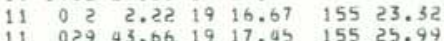
$11 \quad 029 \quad 43.66 \quad 19 \quad 17.45 \quad 155 \quad 25.94$ 1143048.991919 .29015515 .97

$\begin{array}{lllllll}11 & 1338 & 55.12 & 19 & 26.92 & 155 & 28.21 \\ 11 & 1540 & 36.77 & 19 & 19.04 & 155 & 13.49\end{array}$ $\begin{array}{lllllll}11 & 1540 & 36.77 & 19 & 19.04 & 155 & 13.49 \\ 11 & 1621 & 20.56 & 19 & 19.26 & 155 & 9.82\end{array}$ $11162536.491918 .19 \quad 15529.40$ $12030 \quad 53.98 \quad 1854.23 \quad 155 \quad 16.03$ 12 Bटl 20.40 is $35.96 \quad 155$ 19.01
HVO EARTHGUAKE SUHRARY LIST

Pagt o

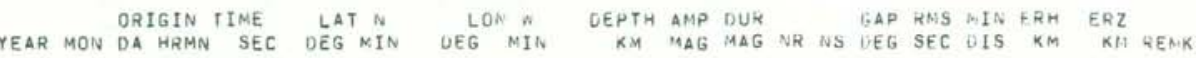

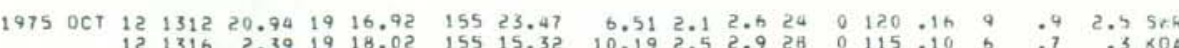

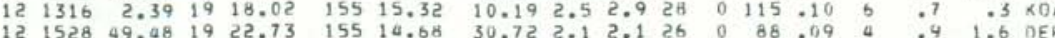

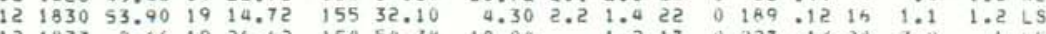

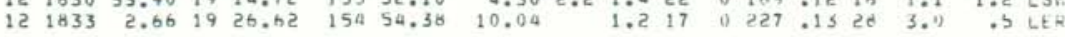
$\begin{array}{llllllllllllllllll}12 & 1957 & 9.23 & 18 & 55.55 & 155 & 17.30 & 11.72 & 2.7 & 2.9 & 25 & 0 & 245 & .12 & 40 & 5.5 & 99.0 & \text { PPL }\end{array}$

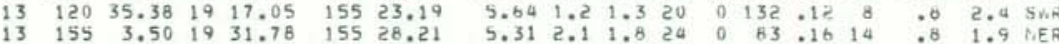

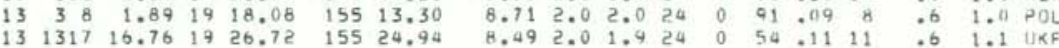
$\begin{array}{lllllllllllllllll}13 & 1346 & 31.99 & 19 & 24.38 & 155 & 17.15 & 13.22 & 1.6 & 1.6 & 24 & 0 & 59.06 & 2 & .5 & .6 & 0 E P\end{array}$

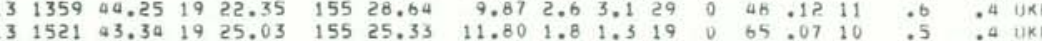

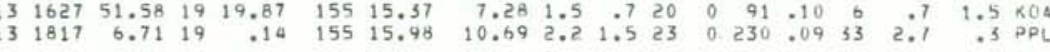

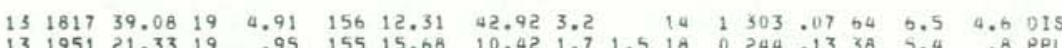

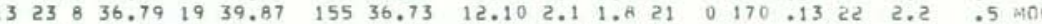

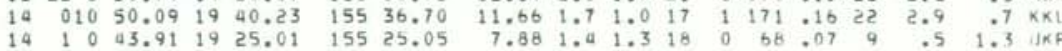

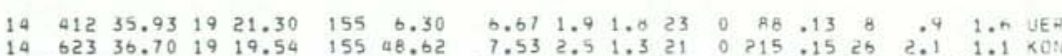

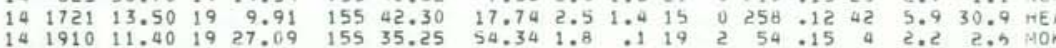

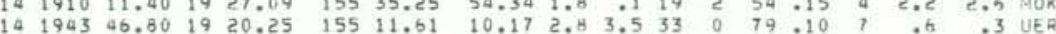

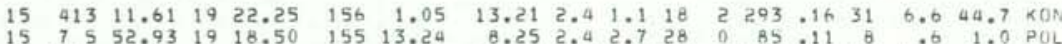

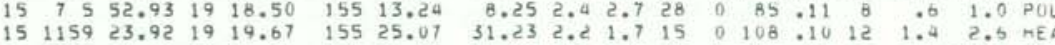

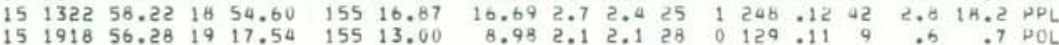

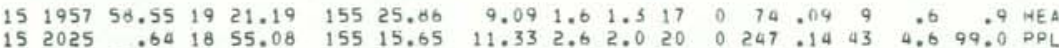

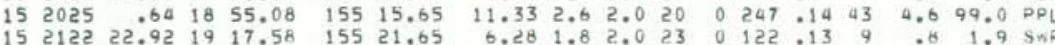

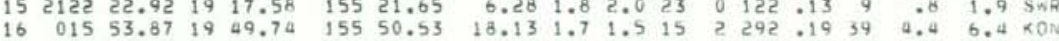

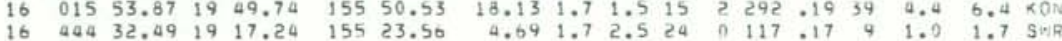

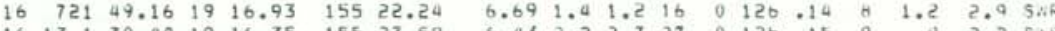

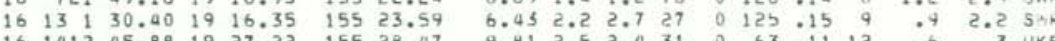

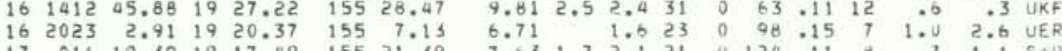

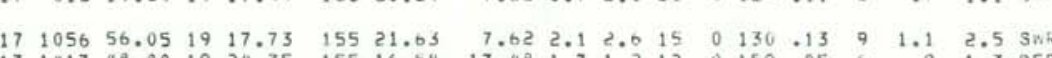

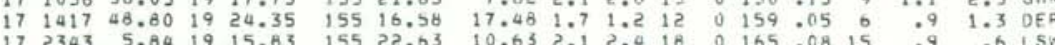

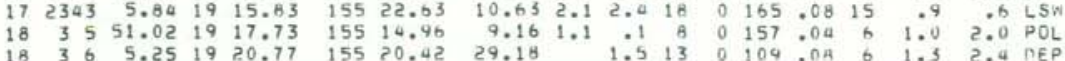

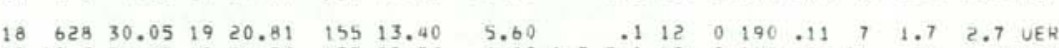

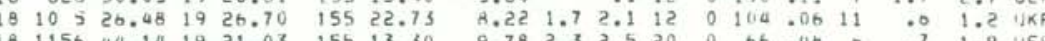




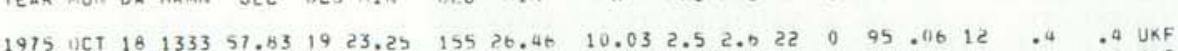

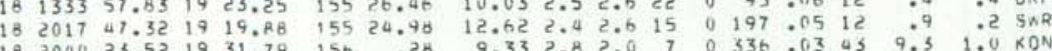

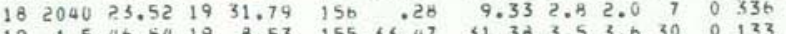

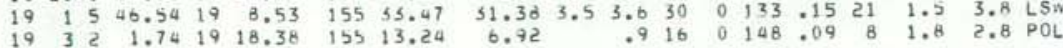

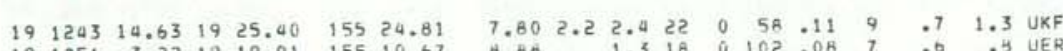

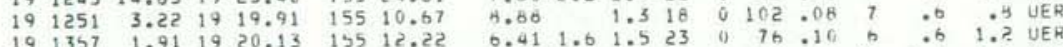

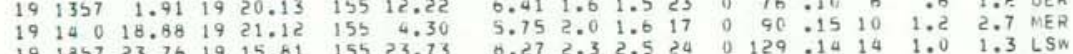

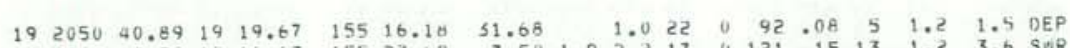

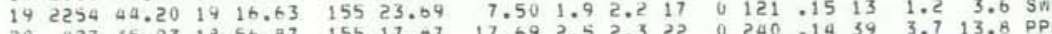

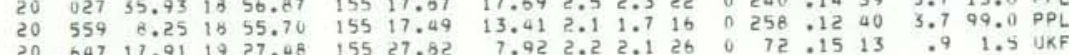

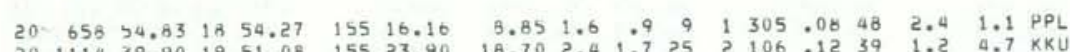

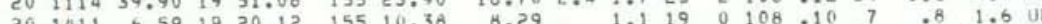

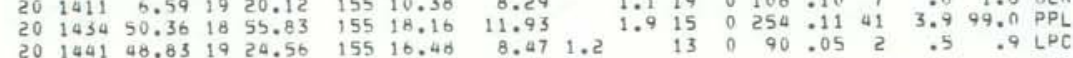

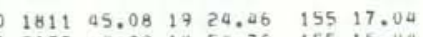

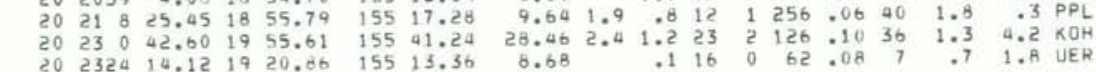

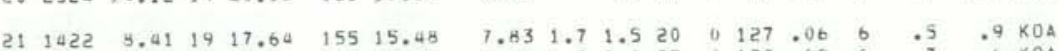

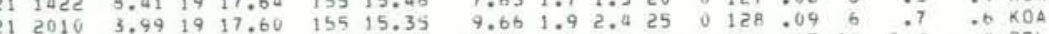

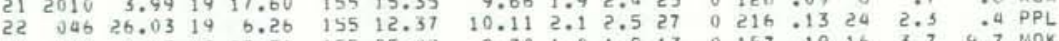

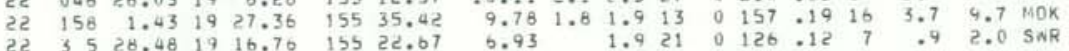

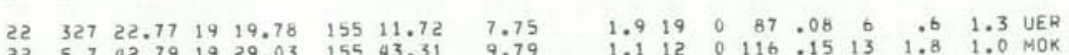

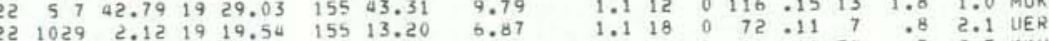

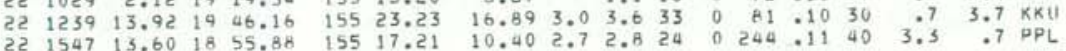

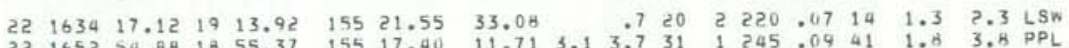

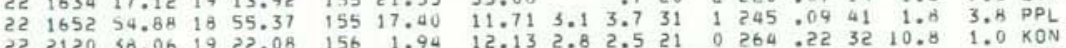

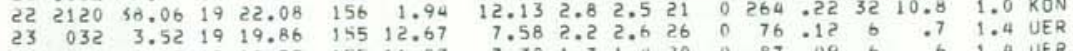

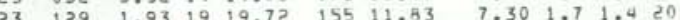

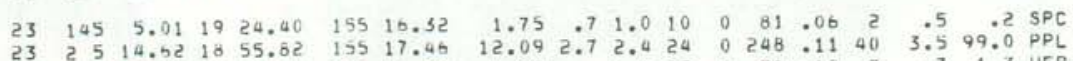

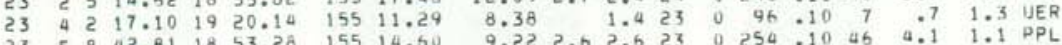

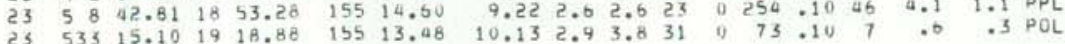

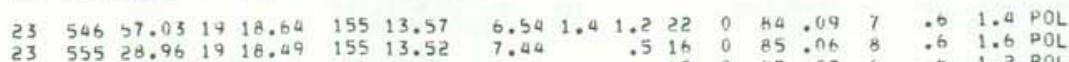

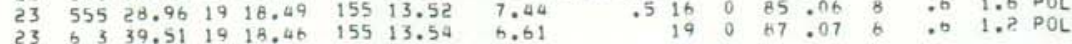

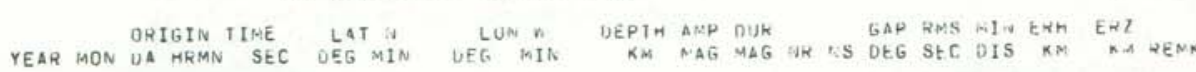

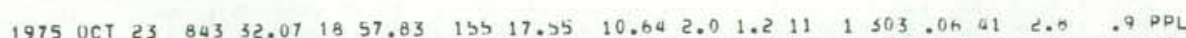
$3580.9619 \quad 18.75 \quad 155$

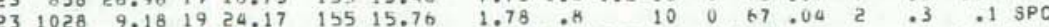

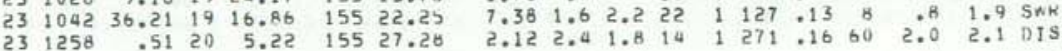
$\begin{array}{llllllllllllllllll}23 & 1735 & 50.61 & 19 & 17.77 & 155 & 14.26 & 6.70 & 2.1 & 2.9 & 27 & 0 & 98 & 0.10 & 8 & .6 & .6 & 00\end{array}$

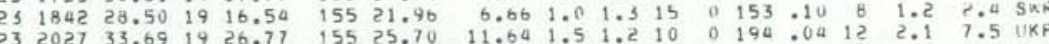

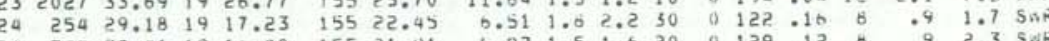
$2434832.411916 .88 \quad 15521.41$ $24 \quad 429 \quad 44.54 \quad 19 \quad 18.93 \quad 155 \quad 12.50$ \begin{tabular}{lllllllllllllllll}
24 & 632 & 30.90 & 18 & 55.59 & 155 & 17.71 & 15.24 & 2.7 & 2.4 & 24 & 1 & 249 & 11 & 40 & 2.5 & 24.2 \\
\hline
\end{tabular}

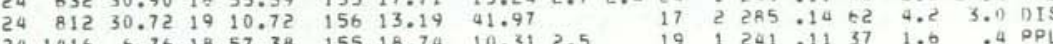
$50.15,19.20 .40015513 .00$ 15515.39 25 is $3 \quad 5.98$ is 57.81 is 18.02 $25161510.312011 .19 \quad 154,45.20$ - 25. $25 \quad 1632 \quad 11.15 \quad 19 \quad 18.99 \quad 155 \quad 13.51$

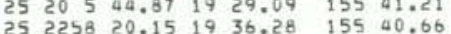
$25225922.35 \quad 19 \quad 36.43 \quad 155 \quad 00.52$

$\begin{array}{llllllll}26 & 0 & 0 & 41.74 & 19 & 24.36 & 155 & 17.36\end{array}$ $26 \quad 115511.931910 .69 \quad 15532.67$

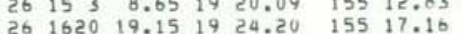

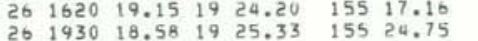

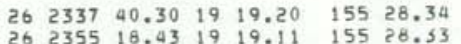

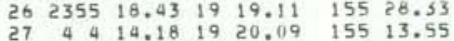
$27 \quad 630 \quad 44.9119 \quad 16.43 \quad 15522.67$ $27 \quad 631 \quad 25.37 \quad 19 \quad 16.55 \quad 155233.10$

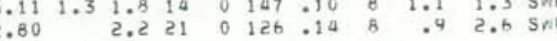
\begin{tabular}{lllllllllllllllll}
27 & 828 & 37.18 & 18 & 56.50 & 155 & 17.78 & 10.20 & 2.5 & 1.6 & 11 & 1 & 307 & .12 & 44 & 9.4 & 2.3 \\
\hline$P P L$
\end{tabular}

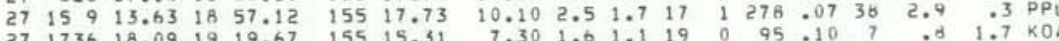

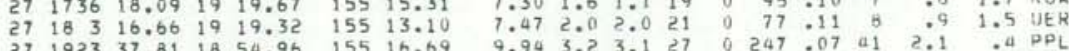
$\begin{array}{lllllllllllllllllll}27 & 1935 & 52.87 & 19 & 18.92 & 155 & 13.37 & 10.20 & 3.7 & 3.9 & 29 & 0 & 76 & .11 & 7 & .8 & .3 & 00 L\end{array}$

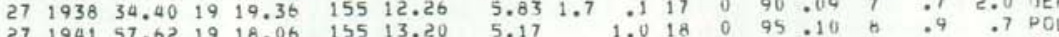

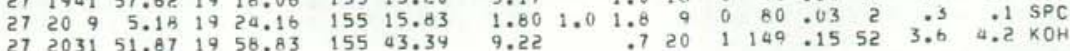
\begin{tabular}{lllllllllllllllll}
28 & 350 & 5.50 & 19 & 26.19 & 155 & 28.45 & 8.46 & 1.4 & .7 & 15 & 0 & 120 & .10 & 12 & .8 & 2.4 \\
\hline
\end{tabular}

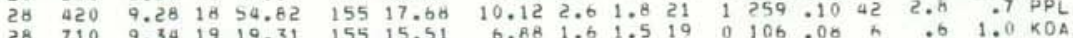


ORIGIN TIME LATN LON W DEPTH AMP DUR N GAP RMS MIN ERH ERT
OA HRMN SEC DEG MIN UEG MIN KM MAG MAG INR WS DEG SEC DIS KH KM REMK

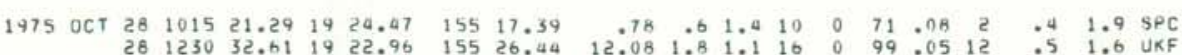

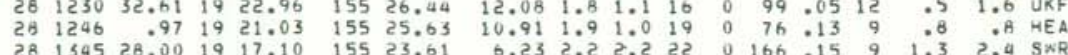

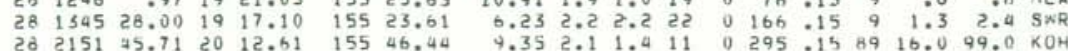

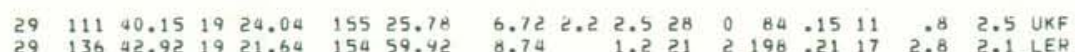

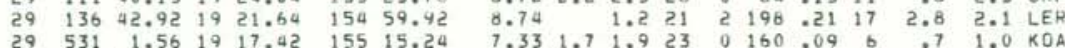

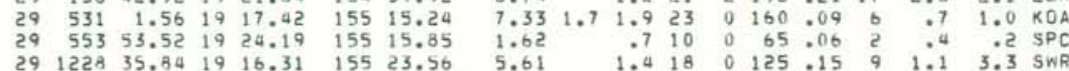
$\begin{array}{lllllllllllllllllllll}29 & 18 & 1 & 1.84 & 19 & 27.11 & 155 & 27.61 & 8.37 & 2.0 & 1.7 & 17 & 0 & 66 & .11 & 13 & .8 & 2.5 & \text { UKF } \\ 29 & 19 & 9 & 51.44 & 19 & 14.41 & 155 & 16.49 & 27.38 & 1.8 & 1.0 & 23 & 1 & 232 & .07 & 15 & 1.3 & 1.2 & \text { HLP }\end{array}$

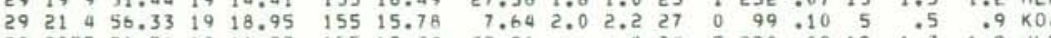

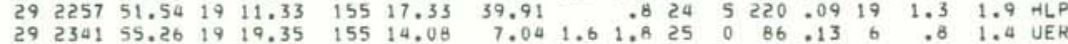

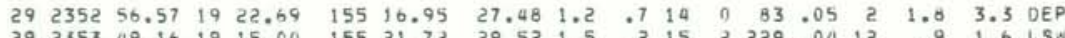

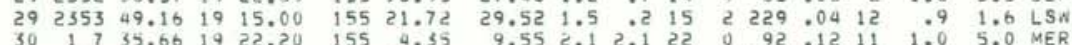

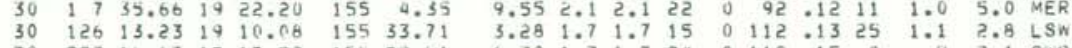

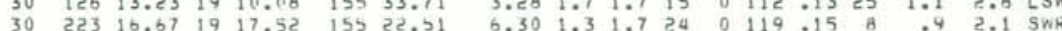

$\begin{array}{lllllllllllllllllll}30 & 4 & 3 & 47.58 & 19 & 16.99 & 155 & 21.09 & 24.78 & 1.3 & .5 & 17 & 0 & 222 & .05 & 9 & 1.2 & 2.0 & \text { SWR }\end{array}$

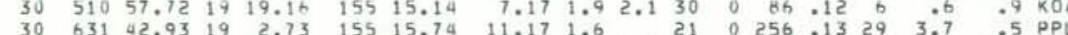

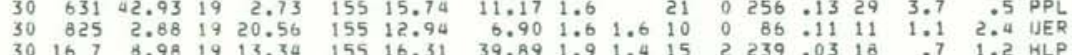

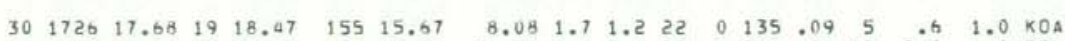

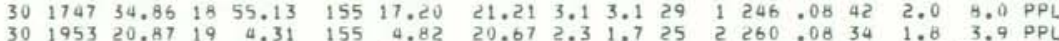

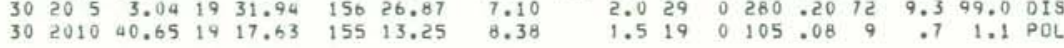
$\begin{array}{llllllllllllllllll}30 & 2123 & 48.33 & 19 & 22.35 & 155 & 29.75 & 8.67 & 1.8 & 2.0 & 26 & 0 & 43 & .12 & 12 & .6 & 1.8 & \text { UKF }\end{array}$

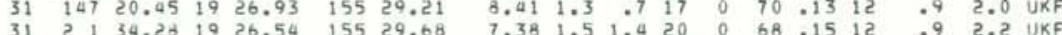

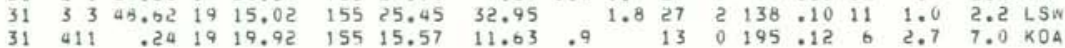

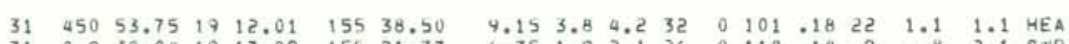

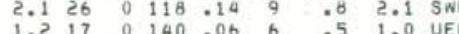

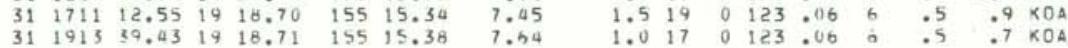

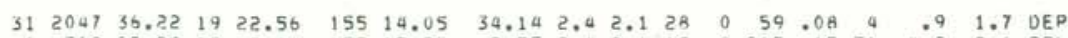

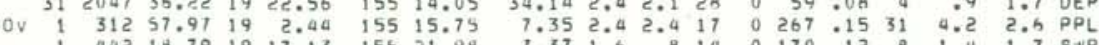

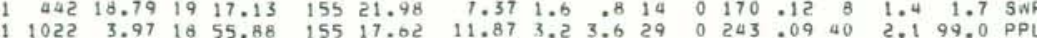

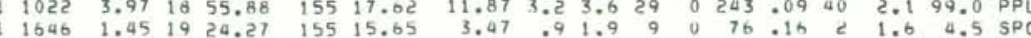

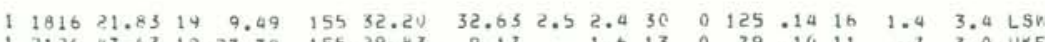

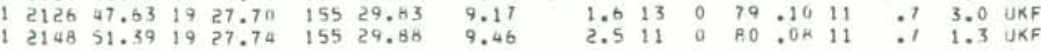

HVO EARTHQUAKE SUMHAARY LIST

PAGE 100

RRIGIN TIME LAT N LON W DEPTH AMP OUJR GAP KNS MIN ERH EKZ YEAR MON DA HRMN SEC DEG MIN DEG MIL DE KM AMP OAK UR US GEG SEC UIS KM KM KEMA

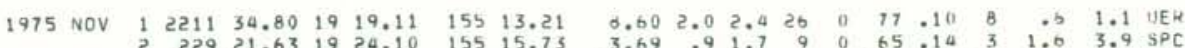

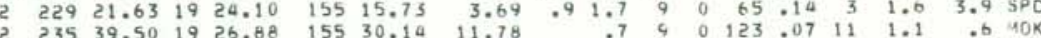

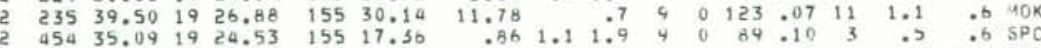

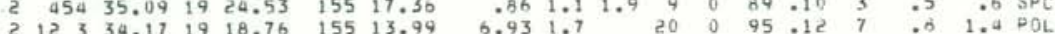

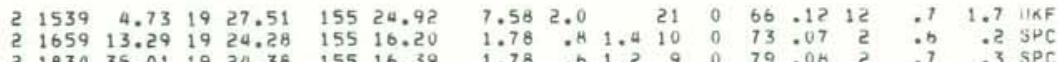

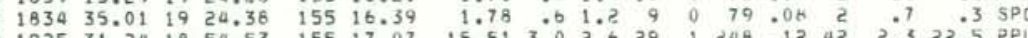

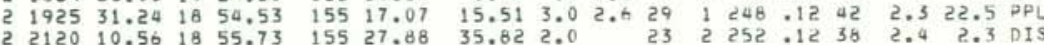
$\begin{array}{lllllllllllllllllll}3 & 313 & 52.57 & 19 & 27.44 & 155 & 27.99 & 9.80 & 1.9 & 1.7 & 24 & 0 & 50 & 10 & 12 & .6 & .4 & \text { UKF }\end{array}$

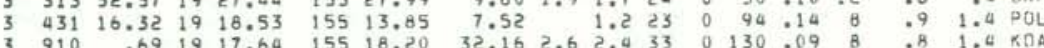

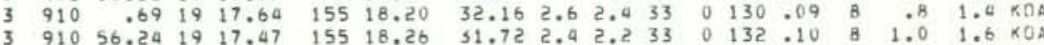

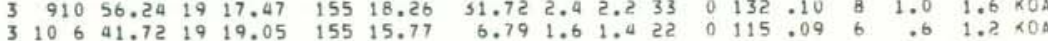
$\begin{array}{llllllllllllllllll}3 & 1226 & 59.29 & 19 & 20.53 & 155 & 12.44 & 8.47 & 1.6 & 1.7 & 25 & 0 & 69 & 11 & 7 & .6 & 1.2 & \\ 3 & \text { UER }\end{array}$

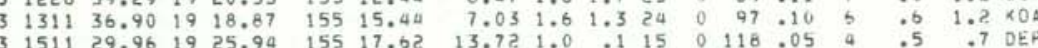

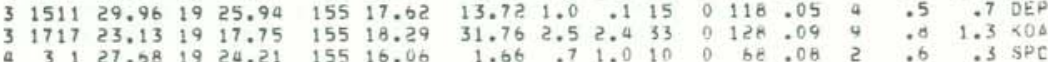
$4 \quad 31253.62 \quad 1920.56 \quad 155 \quad 7.10 \quad 6.82 \quad 1.2240095 \quad .127 ? \quad .8 \quad 1.4$ UER

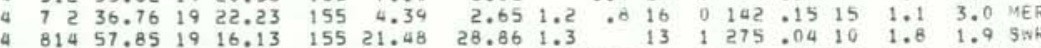

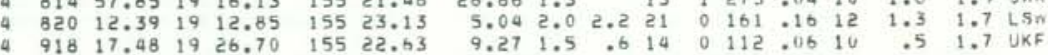

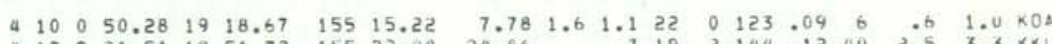

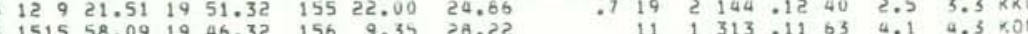

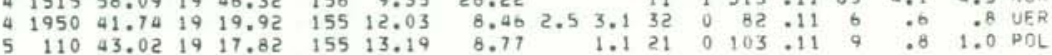
$\begin{array}{lllllllllllllllll}5 & 251 & 58.34 & 19 & 17.94 & 155 & 16.24 & 31.28 & .4 & 19 & 0 & 158 & .06 & 9 & .9 & 1.8 & 004\end{array}$

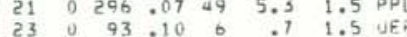

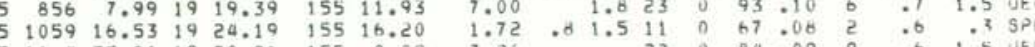
$511435.011920 .21 \quad 1558.09 \quad 7.26$

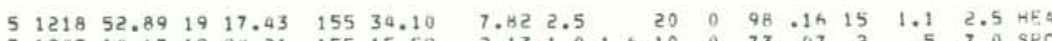

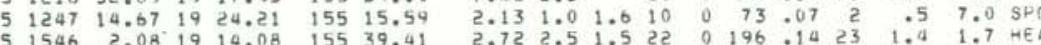

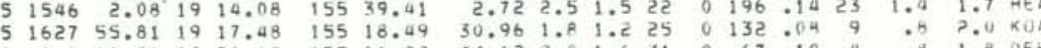

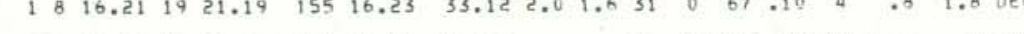

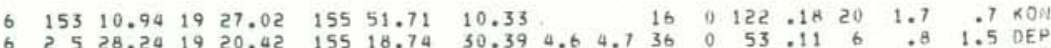

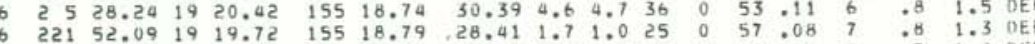

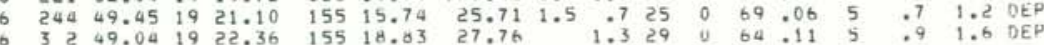
$\begin{array}{llllllllllllllllll}6 & 313 & 44.17 & 19 & 24.19 & 155 & 16.15 & 1.71 & 1.1 & 1.5 & 12 & 0 & 47 & .08 & 2 & .6 & .2 & 5 P C\end{array}$

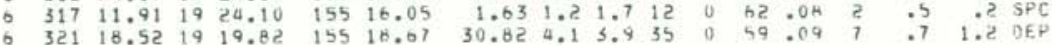




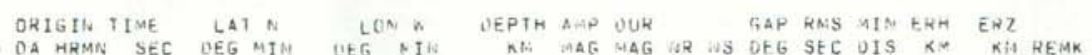

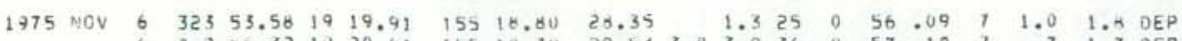

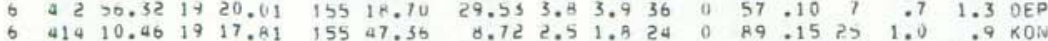

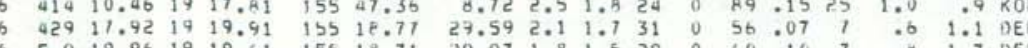

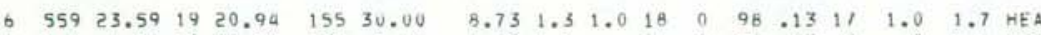

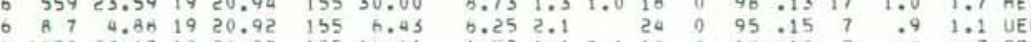

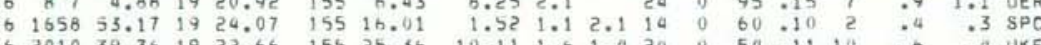

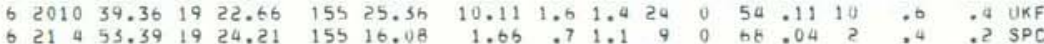

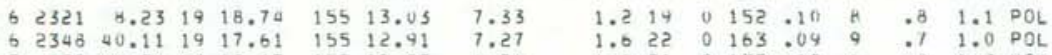

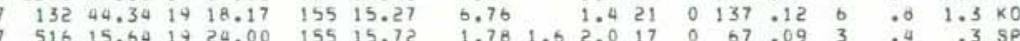

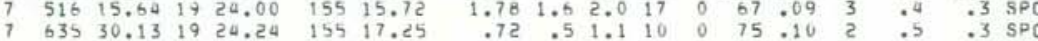

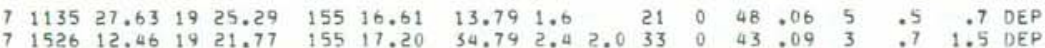

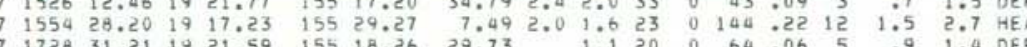

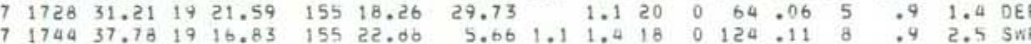

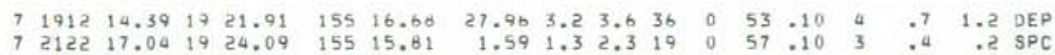

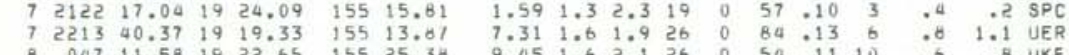

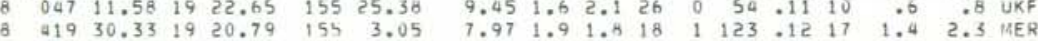

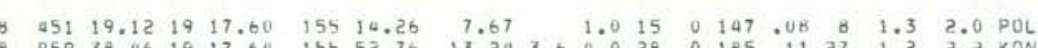

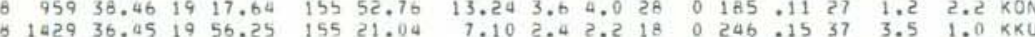

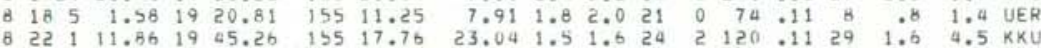

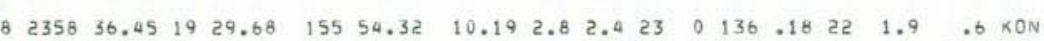
9
9

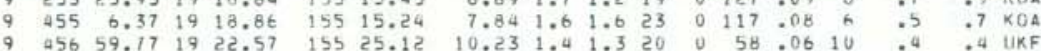

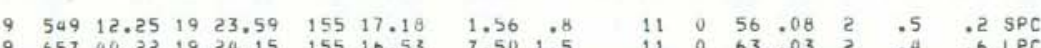

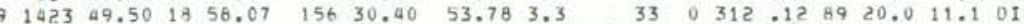

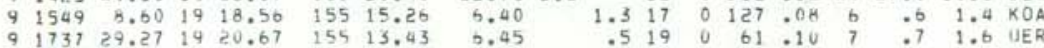

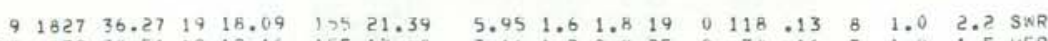

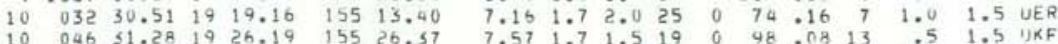

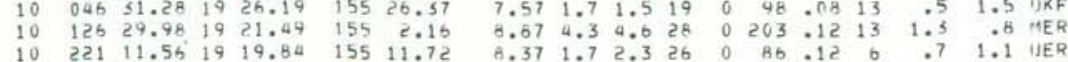

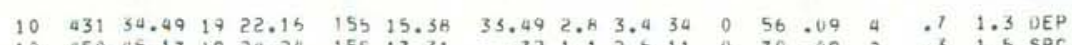

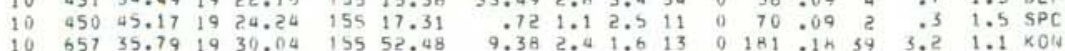

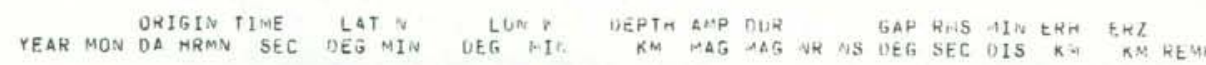

$\begin{array}{rrrrrrrrrrrrrrrrrrr}1975 \text { NOV } & 10 & 9 & 2 & 27.64 & 19 & 21.37 & 155 & 1.34 & 7.56 & 2.6 & 3.1 & 22 & 0 & 180 & .11 & 15 & .9 & 1.06 \text { MER } \\ 10 & 1622 & 5.02 & 19 & 17.78 & 155 & 22.12 & 5.91 & 2.0 & 2.4 & 21 & 0 & 110 & 10 & 0 & 1.1 & 2.7 & \text { SNR }\end{array}$

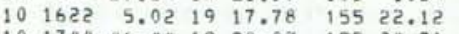

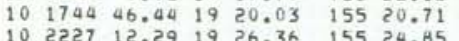
$\begin{array}{rrrrrrr}10 & 2227 & 12.29 & 19 & 26.36 & 155 & 24.85 \\ 11 & 020 & 59.95 & 19 & 19.14 & 155 & 13.84\end{array}$

$11 \quad 559 \quad 11.90 \quad 19 \quad 24.17 \quad 155 \quad 15.87$

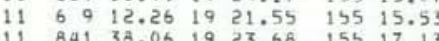
$11 \quad 841 \quad 33.06 \quad 1923.68 \quad 155 \quad 17.13$ $\begin{array}{llllllll}11 & 10 & 5 & 32.00 & 19 & 12.55 & 155 & 21.23 \\ 11 & 10 & 8 & 55.49 & 18 & 56.93 & 155 & 17.52\end{array}$

$\begin{array}{lllllll}11 & 1038 & 53.57 & 19 & 18.35 & 155 & 13.75\end{array}$

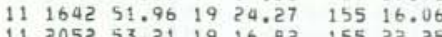
$\begin{array}{lllllll}11 & 2052 & 53.21 & 19 & 16.82 & 155 & 22.25 \\ 11 & 2313 & 36.83 & 18 & 55.50 & 155 & 16.45\end{array}$

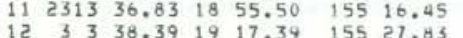

$\begin{array}{lllllll}12 & 543 & 41.63 & 19 & 24.2 \mathrm{~A} & 155 & 16.34 \\ 12 & 838 & 36.53 & 19 & 16.55 & 155 & 22.67\end{array}$ $\begin{array}{lllllll}12 & 838 & 36.53 & 19 & 16.55 & 155 & 22.67\end{array}$

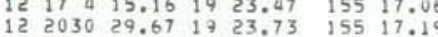
$12205148.91 \quad 1923.62 \quad 155 \quad 16.91$

$\begin{array}{lllllll}12 & 2348 & 59.03 & 19 & 23.15 & 155 & 17.05 \\ 13 & 153 & 25.04 & 19 & 23.20 & 155 & 17.09\end{array}$

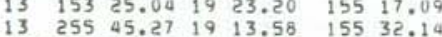
$\begin{array}{lllllll}13 & 255 & 45.27 & 19 & 13.58 & 155 & 32.14 \\ 13 & 1157 & 34.58 & 19 & 23.74 & 155 & 17.14\end{array}$ $\begin{array}{lllll}3 & 1521 & 38.87 & 19 & 19.44 \quad 155 \quad 9.70\end{array}$ $\begin{array}{rrrrrrr}13 & 1521 & 38.87 & 19 & 19.44 & 155 & 9.70 \\ 13 & 1533 & 0.15 & 19 & 19.56 & 155 & 9.60\end{array}$ $\begin{array}{rrrrrrr}13 & 1533 & 0.15 & 19 & 19.56 & 155 & 9.60 \\ 13 & 1536 & 13.30 & 19 & 23.00 & 155 & 16.97\end{array}$

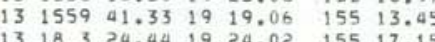

$\begin{array}{lllllll}13 & 1844 & 9.26 & 19 & 24.04 & 155 & 17.17\end{array}$ $\begin{array}{rrrrrrr}13 & 1844 & 9.26 & 19 & 24.04 & 155 & 17.17 \\ 13 & 232 & 4.91 & 19 & 21.09 & 155 & 3.36 \\ 14 & 041 & 36.55 & 19 & 23.87 & 155 & 17.26\end{array}$

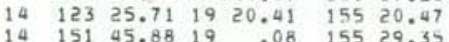

$14 \quad 2 \quad 3 \quad 21.69 \quad 1920.69 \quad 15520.60$

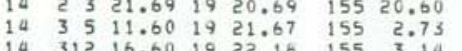
$14312 \quad 16.60 \quad 1922.16 \quad 155 \quad 3.14$ $\begin{array}{lllllll}14 & 358 & 10.23 & 19 & 23.12 & 155 & 17.08 \\ 14 & 5 & 93.57 & 19 & 24.13 & 155 & 17.53\end{array}$ $\begin{array}{lllllll}14 & 758 & 45.86 & 18 & 56.75 & 155 & 33.98\end{array}$

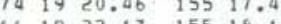

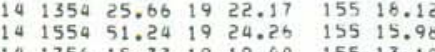

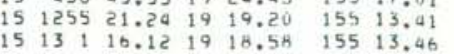

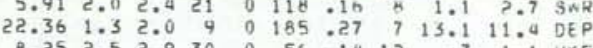
$\begin{array}{lllllllllllll}8.25 & 2.5 & 2.9 & 30 & 0 & 56 & .14 & 12 & 71 & 7 & 1.1 & \text { UKF } \\ 6.78 & 1.6 & 1.4 & 16 & 0 & 85 & .05 & 7 & .4 & 1.2 & \text { IJER }\end{array}$ $\begin{array}{lllllllllll}3.58 & 1.9 & 2.9 & 25 & 0 & 55 & .12 & 2 & .5 & 1.0 & 3+C\end{array}$

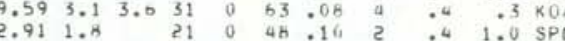

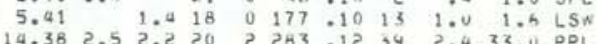

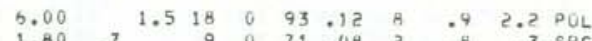
$\begin{array}{llllllllllll}1.80 & .7 & 9 & 0 & 71 & .08 & 2 & .0 & .3 & 5 P C\end{array}$

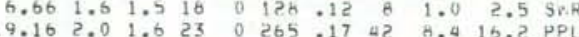

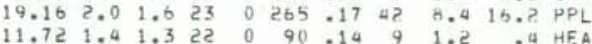

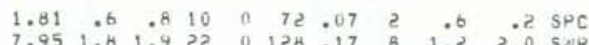

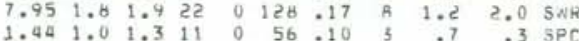

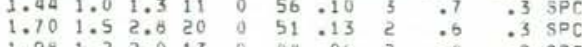
$\begin{array}{lllllllllll}1.50 & .8 & 1.8 & 11 & 0 & 62 & -16 & 2 & .7 & .3 & \mathrm{SPC} \\ 1.46 & .7 & 1.1 & 11 & 0 & 60 & .07 & 3 & : 5 & : 5 & \mathrm{SPC}\end{array}$

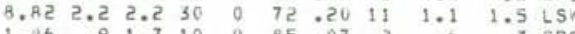

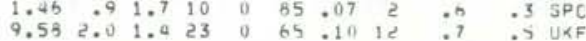

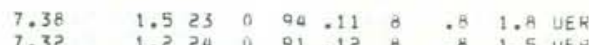

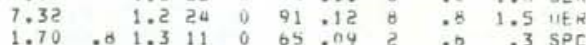

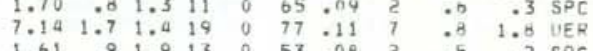
$.61 \quad .91 .913$ $\begin{array}{llllllllllll}1.65 & 1.0 & 2.6 & 14 & 0 & 53 & .07 & 2 & .4 & .2 & \text { SPC }\end{array}$

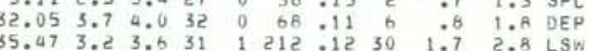
$\begin{array}{llllllllll}8.95 & 1.3 & 23 & 0 & 88 & 07 & 6 & .7 & 1.4 & \text { DEP }\end{array}$

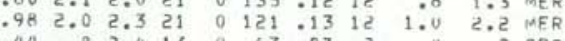

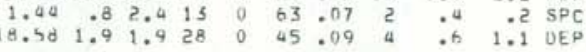
$\begin{array}{lllllllllll}30.03 & 3.0 & 2.9 & 14 & 0 & 239 & .09 & 38 & 3.3 & 5.1 & 01 \mathrm{~s}\end{array}$

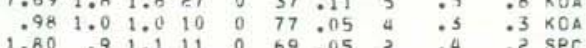

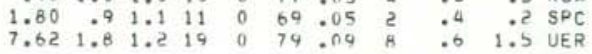
$\begin{array}{lllllllllll}1.00 & .3 & 1.3 & 11 & 0 & 58 & .13 & 2 & .7 & .4 & \mathrm{SPC}\end{array}$

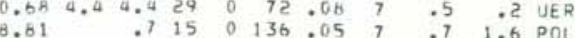


HVO EARTHDUAKE SUMMAKY LIST

HAGE 103 ORIGIN TIME LAT LON LON OEPTH AMP DUR GAP RMS MTN ERH ERZ REMK

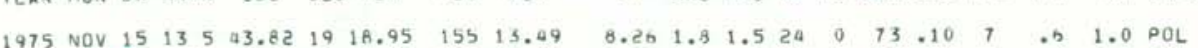

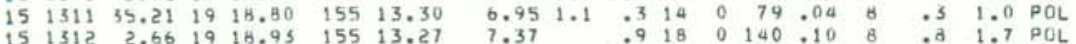

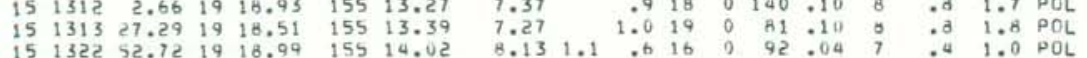
$\begin{array}{llllllllllllllllll}15 & 1328 & 33.97 & 19 & 18.81 & 155 & 13.34 & 7.17 & .8 & 17 & 0 & 78 & .10 & 8 & .0 & 2.0 & \text { POL }\end{array}$

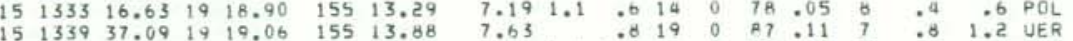

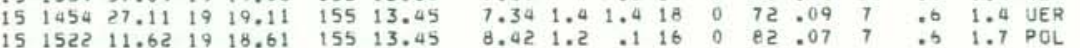
$\begin{array}{llllllllllllllllll}15 & 1751 & 3.23 & 18 & 59.40 & 155 & 29.50 & 37.67 & 2.6 & 2.0 & 33 & 3 & 216 & .12 & 32 & 1.8 & 2.7 & 01 \mathrm{~S}\end{array}$

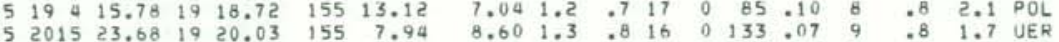

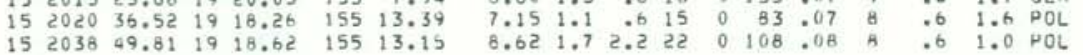

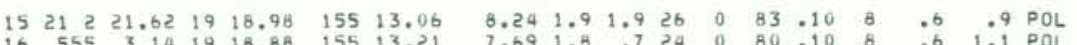

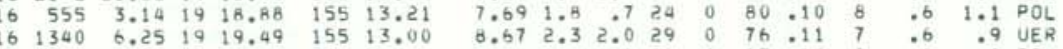

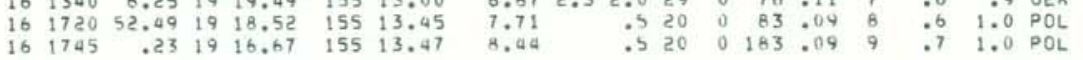

1604313.36 t9 $26.06 \quad 15523.58$

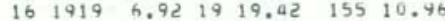

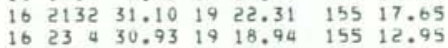
$162315 \quad 7.4419 \quad 18.90 \quad 15515.10$ $\begin{array}{llllllll}17 & 1 & 2 & 19.54 & 19 & 14.49 & 155 & 29.51 \\ 17 & 1 & 8 & 39.81 & 19 & 19.23 & 155 & 12.41\end{array}$

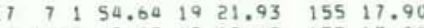
$\begin{array}{lllllll}17 & 1027 & 42.87 & 19 & 18.69 & 155 & 13.29 \\ 17 & 127 & 31.08 & 19 & 19.11 & 155 & 13.52\end{array}$

$\begin{array}{rrrrrrr}17 & 1242 & 15.74 & 19 & 23.19 & 155 & 16.94 \\ 17 & 1559 & 6.48 & 19 & 13.32 & 156 & 10.17\end{array}$ $\begin{array}{lllllll}17 & 1559 & 6.48 & 19 & 13.32 & 1556 & 16.94\end{array}$ $1771453 \quad 30.21$ 19 25.20 155 25.15 $17 \quad 1810 \quad 30.86 \quad 19 \quad 19.42 \quad 155 \quad 13.26$

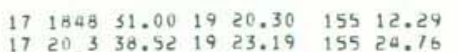

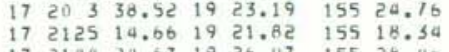

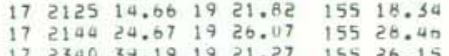

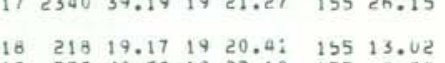

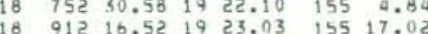

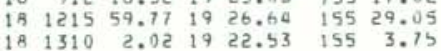

$\begin{array}{llllll}18 & 1336 \quad 37.00 \quad 19 & 19.10 \quad 155 \quad 13.32\end{array}$

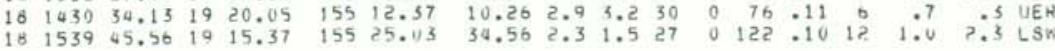

HVO EARTHQUAKE SUMAAREY LIST

PAGE 104 ORIGIN TIME LATN LON $W$ DEPTA ANP OUR GAP KMS ATI EHW ERL DEPTA AWP OHA NH WS DEG SEC DIS KM KM REMK

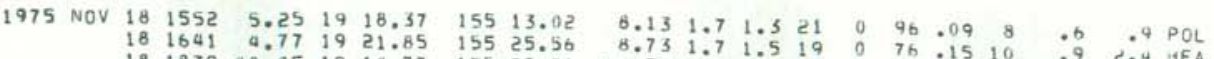

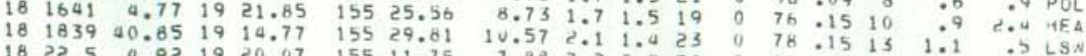

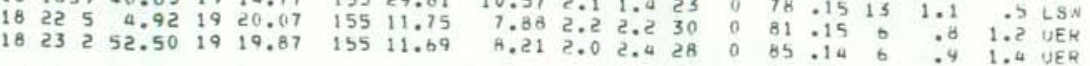

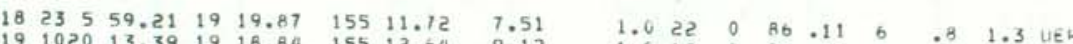

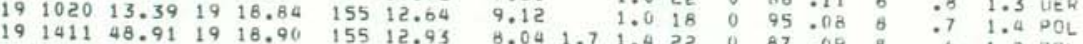

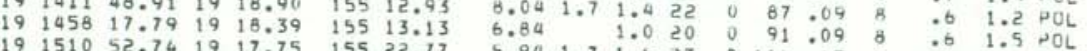

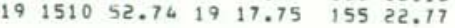
$19 \quad 193540.19 \quad 1923.05 \quad 155 \quad 17.18$ $\begin{array}{lllllllll}20 & 650 & 13.87 & 19 & 28.40 & 155 & 32.72\end{array}$

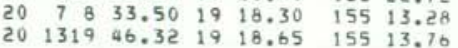

$\begin{array}{lllllll}20 & 1321 & 24.95 & 19 & 19.66 & 155 & 13.78\end{array}$ 201341 a4.22 $19 \begin{array}{llll}33.74 & 155 & 41.78 \\ 40 & 0.00 & 0\end{array}$

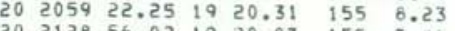

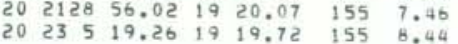

$\begin{array}{lllllll}21 & 044 & 49.49 & 19 & 26.91 & 155 & 22.07\end{array}$ $2124523.98 \quad 19$

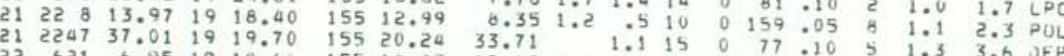

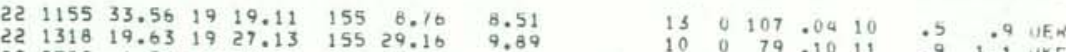

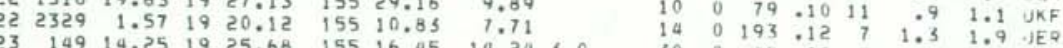

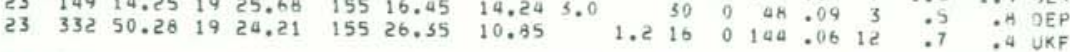

$\begin{array}{lllllllllllllllllll}23 & 19 & 7 & .48 & 19 & 21.50 & 155 & 18.69 & 2.09 & 1.2 & 1.4 & 16 & 0 & 74 & .07 & 5 & .4 & 27.8 & 004\end{array}$

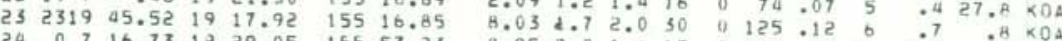

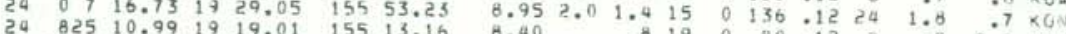

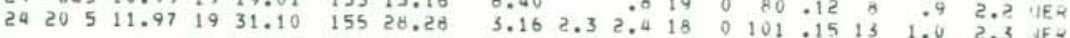

$\begin{array}{lllllll}25 & 012 & 16.01 & 19 & 18.45 & 155 & 13.55\end{array}$ 25 . 32452.471918 .35 155 13.64 $25 \quad 63213.87$ 19 19.04155 . 35 $25 \quad 754 \quad 49.6919 \quad 18.71 \quad 155 \quad 15.60$

$25 \quad 9230.651926 .27 \quad 15529.11$ 25225230.48 19 $25.97 \quad 15528.33$

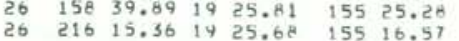

$\begin{array}{lllll}26 & 217 & 28.69 & 19 & 28.41 \quad 155 \quad 45.45\end{array}$ $26 \quad 23550.24$ 19 $30.76 \quad 155 \quad 51.74$ $\begin{array}{llllllllllll}6.92 & 1.3 & 24 & 0 & 84 & .10 & 8 & .7 & 1.1 & \text { POL }\end{array}$

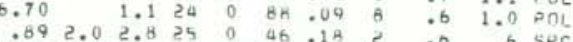

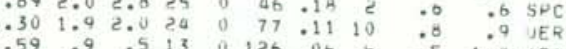

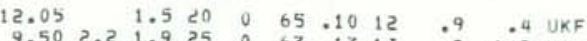

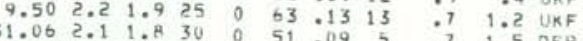

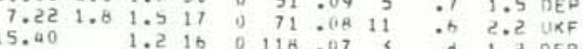

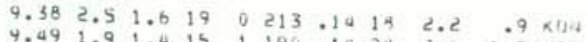

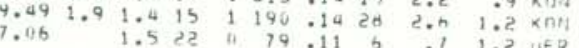




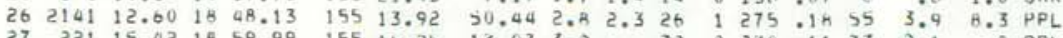

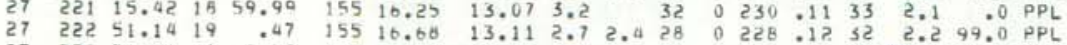

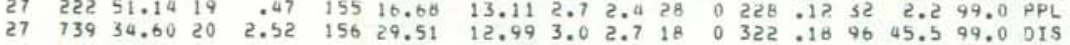
$\begin{array}{llllllllllllllllllll}27 & 12 & 4 & 59.00 & 19 & 19.46 & 155 & 15.23 & 6.80 & .8 & 1.1 & 18 & 0 & 99 & .11 & 6 & .8 & 1.8 & \text { KOA } \\ 27 & 1615 & 40.88 & 19 & 34.90 & 155 & 58.24 & 9.11 & 3.6 & 3.2 & 30 & 0 & 247 & 016 & 23 & 2.6 & .8 & .8 & \text { KON }\end{array}$

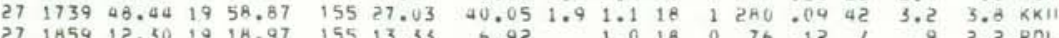

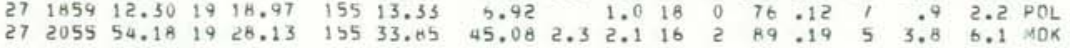
$\begin{array}{lllllllllllllllll}28 & 150 & 9.19 & 19 & 22.41 & 155 & 9.44 & 5.09 & 1.7 & 1.5 & 23 & 0 & 78 & .16 & 10 & 1.0 & 1.2 \text { UEK }\end{array}$

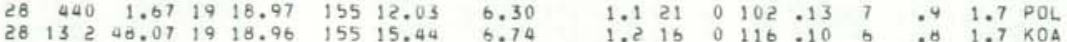

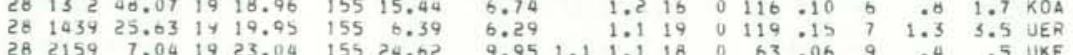

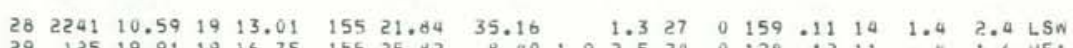

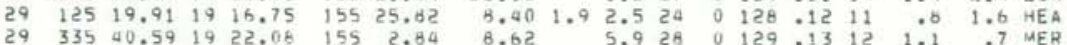

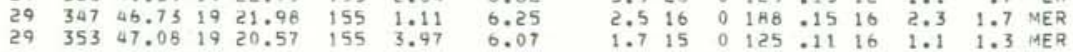

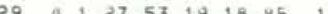

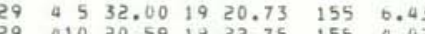

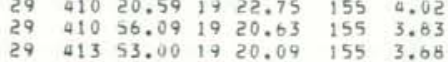
द9 $415 \quad 20.80 \quad 19 \quad 19.49 \quad 155 \quad 13.70$

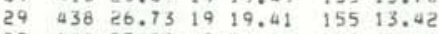

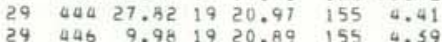
$\begin{array}{lllllll}24 & 446 & 9.98 & 19 & 20.89 & 155 & 4.59 \\ 24 & 447 & 40.06 & 19 & 20.90 & 155 & .94\end{array}$

$\begin{array}{lllllll}29 & 834 & 56.13 & 19 & 22.75 & 155 & 5.21\end{array}$ $\begin{array}{lllllll}29 & 837 & 30.40 & 19 & 23.77 & 155 & 0.02 \\ 29 & 842 & 12.78 & 19 & 2004 & 155 & 4.70\end{array}$ $\begin{array}{lllllll}29 & 842 & 12.78 & 19 & 20.41 & 155 & 4.70 \\ 29 & 843 & 59.97 & 19 & 8.63 & 155 & 15.37\end{array}$ $29 \quad 855 \quad 59.891919 .26 \quad 15520.93$

$\begin{array}{lllllll}29 & 858 & 24.23 & 19 & 21.13 & 155 & 15.54 \\ 29 & 859 & 34.94 & 19 & 19.36 & 155 & 7.37\end{array}$ $29,9250.041923 .41 \quad 15516.07$ $29 \quad 91320.151917 .84 \quad 155,15.79$

$\begin{array}{lrrrrrr}29 & 918 & 5.71 & 19 & 24.15 & 155 & 3.73 \\ 29 & 921 & 40.59 & 19 & 19.41 & 155 & 20.97\end{array}$

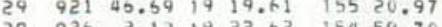

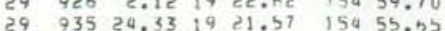
$29 \quad 938 \quad 25.73 \quad 19 \quad 16,41 \quad 156 \quad 16.28$

$29 \begin{array}{lllllll}238 & 58.61 & 19 & 19.54 & 155 & 20.90\end{array}$ $\begin{array}{lllllll}29 & 951 & 10.14 & 19 & 20.45 & 155 & 4.09 \\ 29 & 954 & 25.12 & 19 & 23.84 & 155 & 13.27\end{array}$

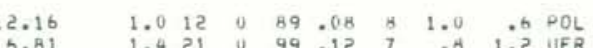

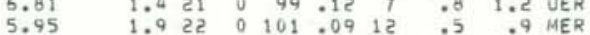
$\begin{array}{lllllllll}6.21 & 1.9 & 14 & 0 & 100 & -15 & 11 & 1.3 & 2.1 \\ 7.91 & 3.1 & 20 & 0 & 131 & .12 & 11 & 1.2 & \text { MER } \\ 0.8 & \text { MER }\end{array}$ $\begin{array}{llllllllll}6.42 & 1.3 & 17 & 0 & 77 & .10 & 7 & .7 & 1.7 & \text { UER }\end{array}$

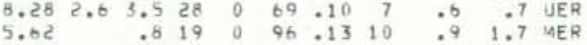

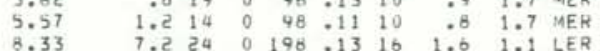

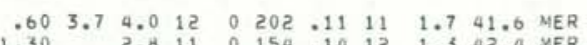
$\begin{array}{lllllllll}1.30 & 2.0 & 11 & 0 & 154 & .19 & 12 & 1.3 & 42.4 \text { MER }\end{array}$

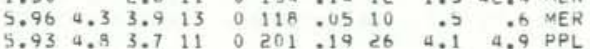

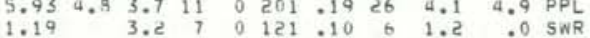
$\begin{array}{rrrrrrrrrr}4.78 & 2.5 & 12 & 0 & 85 & .09 & 5 & 1.1 & 2.7 & \text { KOA } \\ 6.56 & 2.6 & 19 & 0 & 113 & .13 & 8 & .8 & 1.2 & \text { UER } \\ 6.43 & 2.5 & 10 & 0 & 100 & .47 & 5 & .8 & 1.6 & \text { SRE }\end{array}$

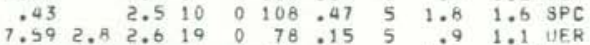
$\begin{array}{rrrrrrrrrrr}7.59 & 2.8 & 2.6 & 19 & 0 & 78 & .15 & 5 & .9 & 1.1 & \text { UER } \\ 4.97 & 5.6 & 4.0 & 7 & 0 & 179 & .11 & 8 & 2.1 & 3.2 & \text { KOA }\end{array}$ $\begin{array}{rrrrrrrrrrr}2.52 & 2.9 & 3.4 & 13 & 0 & 170 & .12 & 11 & 1.2 & 3.0 & \text { MER } \\ 4.65 & 3.3 & 11 & 0 & 113 & .15 & 8 & 1.7 & 2.7 & \text { SNR }\end{array}$

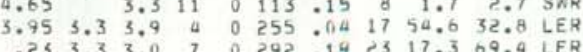

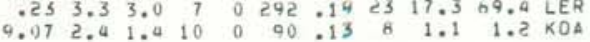
$\begin{array}{rrrrrrrrrrr}.34 & 3.0 & 2.6 & 10 & 0 & 113 & .16 & 6 & 1.5 & \text { A.6 } & \text { SWR } \\ 9.86 & 3.8 & 2.9 & 19 & 0 & 160 & .10 & 10 & 1.1 & .04 & \text { UER }\end{array}$

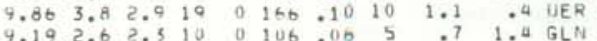

HVO EARTHOUARE SUMAARY LIST

Maliti 100

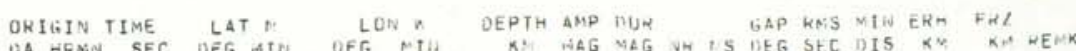

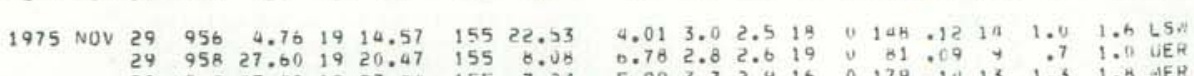
$29100045.48 \quad 1923.44 \quad 155 \quad 3.23$

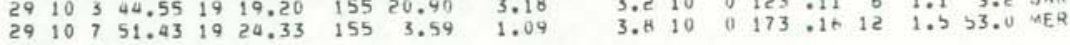

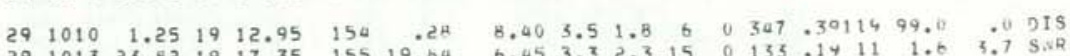

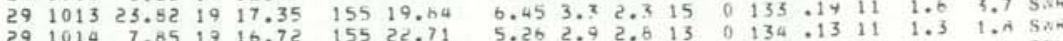

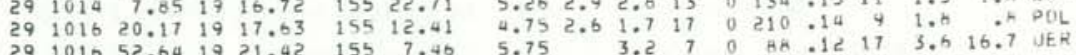
$\begin{array}{lllllll}29 & 1018 & 7.91 & 19 & 21.58 & 155 & 15.14 \\ 29 & 1019 & 5.18 & 19 & 21.49 & 155 & 15.56\end{array}$

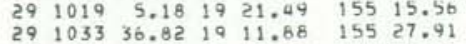
29103519.551913 .99 154 55.06 $29 \quad \begin{array}{llllll}1039 & 17.91 & 19 & 19.59 & 155 & 21.64\end{array}$ 29104529.03 19 19.3935 154 59.03

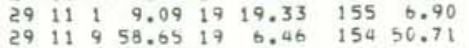
$\begin{array}{lllllll}29 & 1111 & 49.30 & 19 & 20.39 & 155 & 7.04\end{array}$ $291116 \quad 58.38$ 19 21.29 155 6.84 $\begin{array}{lllllll}29 & 1121 & 58.52 & 19 & 18.83 & 155 & 17.51 \\ 29 & 1124 & 28.21 & 19 & 20.48 & 155 & 5.65\end{array}$ $291124 \quad 28.21 \quad 1920.48 \quad 155 \quad 5.65$

$\begin{array}{llllllll}29 & 1128 & 22.51 & 19 & 16.84 & 155 & 12.14\end{array}$

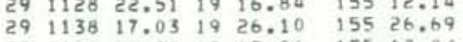
$291144 \quad 16.811917 .96 \quad 15512.05$ $29114625.261920 .47 \quad 15511.14$

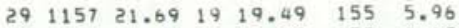

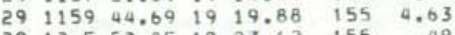
2912552.851923 .62 15s 0.49 $291210 \quad 6.491919 .69 \quad 15520.07$

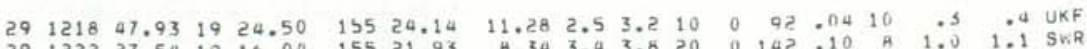

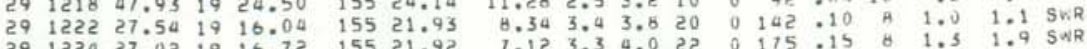

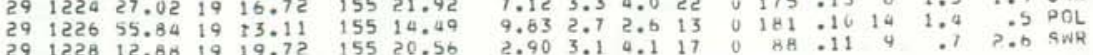

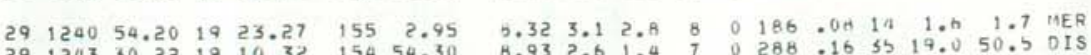

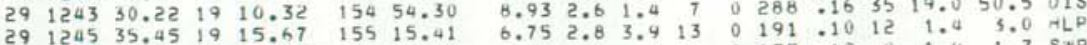

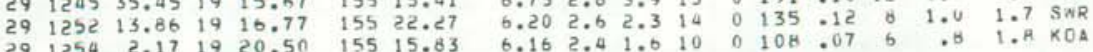

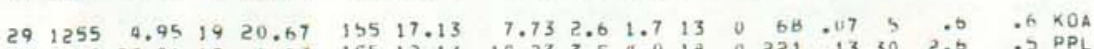

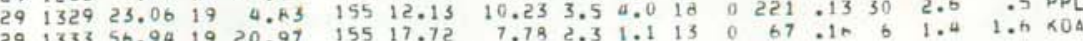


HVU EARTHQUAKE SUMAARY LIST

PAFE 107

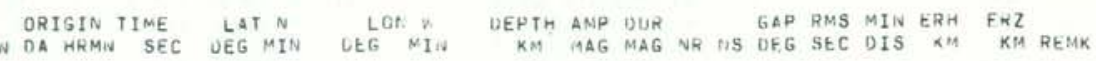
1975 NOV $291344 \quad 36.48 \quad 19 \quad 18.68 \quad 155 \quad 9.38$

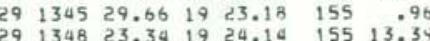

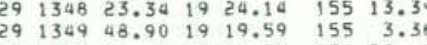
$291412 \quad 58.68 \quad 1924.26 \quad 15458.45$ $29 \begin{array}{llllll}1414 & 33.34 & 19 & 19.96 \quad 155 & 15.70\end{array}$ $291442 \quad 45.091923 .19 \quad 155 \quad 1.33$ 29 1452 22.12 $1918.42 \quad 15511.34$ $\begin{array}{llllll}29 & 1459 \quad 37.40 & 19 & 21.83 & 155 & 18.59\end{array}$

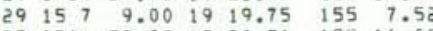

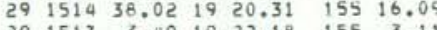

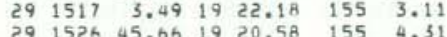

$\begin{array}{lllllll}29 & 1528 & 54.02 & 19 & 17.39 & 155 & 22.17\end{array}$ $\begin{array}{lllllll}29 & 1535 & 37.96 & 19 & 21.53 & 155 & 19.34\end{array}$ 29153954.081920 .10015516 .80 $29 \quad 1550 \quad 21.57 \quad 19 \quad 15.02 \quad 155 \quad 14.92$

$\begin{array}{lllllll}29 & 1552 & 33.67 & 19 & 19.61 & 155 & 21.28 \\ 29 & 1557 & 2.32 & 19 & 22.44 & 155 & 5.44\end{array}$

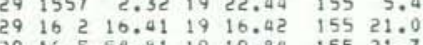

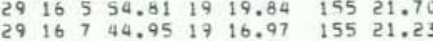
$\begin{array}{lllllll}29 & 1611 & 49.96 & 19 & 23.14 & 155 \quad .52\end{array}$ $29 \quad 1617 \quad 23.18 \quad 19 \quad 19.69 \quad 155 \quad 18.04$ $391623 \quad 8.08 \quad 1921.71 \quad 155 \quad 18.95$

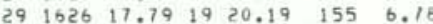

$\begin{array}{lllllll}29 & 1627 & 7.08 & 19 & 22.49 & 155 & 1.10\end{array}$ $\begin{array}{lllllll}29 & 1629 & 29.26 & 19 & 24.59 & 155 & 24.00\end{array}$

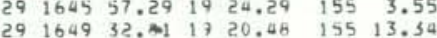
$\begin{array}{llllllll}29 & 1650 & 52.01 & 19 & 19.85 & 155 & 12.17\end{array}$ $\begin{array}{lllllll}29 & 1657 & 35.31 & 19 & 19.76 & 155 & 11.75\end{array}$

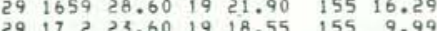

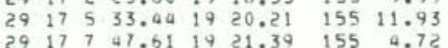
29 $1713 \quad 52.05 \quad 19 \quad 19.94 \quad 155 \quad 17.60$

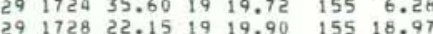

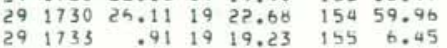
$\begin{array}{lllllll}29 & 1739 & 25.38 & 19 & 20.01 & 155 & 0.13\end{array}$ 29 175234.001922 .01 155 18.57 UEPTH ANO OUA YEAR MON DA HRMN SEC DEG MIH DEG MIN KM KM MAG MAG NH NS DFG SEC OIS KM KM KEMK 1975 NOV $29 \quad 1755 \quad 7.88 \quad 19 \quad 24.02 \quad 155 \quad 13.02$

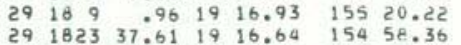

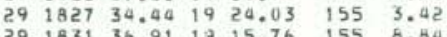

$29 \quad 1837 \quad 50.70 \quad 1923.22 \quad 154 \quad 59.25$

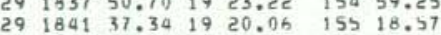

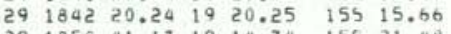
29185041.171914 .79 155 21.48

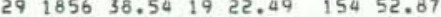

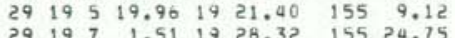

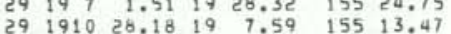
$29.913 \quad 37.03 \quad 1919.45 \quad 15519.82$

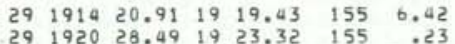

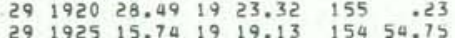
$291928 \quad 56.051919 .20 \quad 15522.00$ $29193531.48 \quad 1913.37 \quad 155 \quad 23.07$

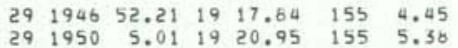
$591951 \quad .32 \quad 19 \quad 19.82 \quad 15519.60$

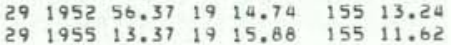
$\begin{array}{lllllll}29 & 1959 & 1.54 & 19 & 19.14 & 155 & 21.00\end{array}$

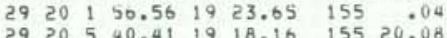
$29201011.931920 .20 \quad 15520.03$ $\begin{array}{lllllll}29 & 2015 \quad .25 \quad 19 & 20.27 \quad 155 & 21.21\end{array}$

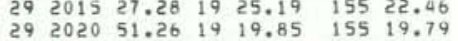

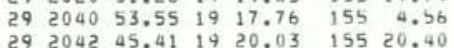
$292043 \quad 50.65 \quad 19 \quad 15.62 \quad 155 \quad 15.32$

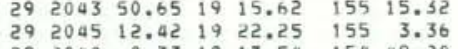

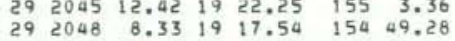

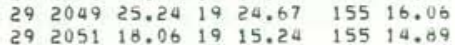
$29 \quad 2057 \quad 9.62 \quad 19 \quad 18.98 \quad 155 \quad 11.55$

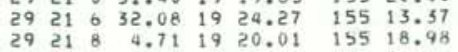
$29219 \quad 3.5419 \quad 16.65 \quad 15522.01$

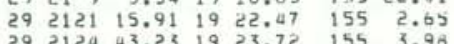

$9.22 \quad 2.6 \quad 13 \quad 0 \quad 173.04$ 6 1.251 .7 GLN

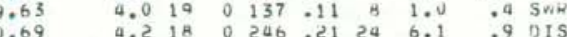

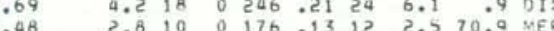

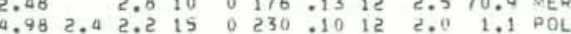
$\begin{array}{lllllllllll}5.83 & 3.7 & 3.1 & 22 & 0 & 182 & 14 & 15 & 1.4 & 1.9 & \text { LER } \\ 7.57 & & 1.5 & 10 & 0 & 90 & .44 & 0 & .4 & 1.1 & \text { KU }\end{array}$

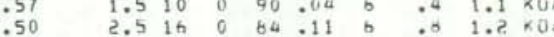
9.922 .73 .1 18 0192.15 11 1.4 . 9 LS $\begin{array}{lllllllllll}7.19 & 3.8 & 3.7 & 16 & 0 & 247 & 15 & 30 & 5.3 & 2.0 & \text { LEM }\end{array}$

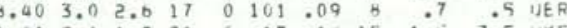

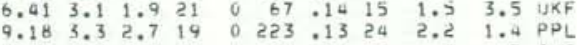

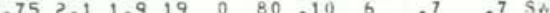
$\begin{array}{lllllllllll}8.04 & 2.8 & 2.5 & 9 & 0 & 133 & .05 & 8 & .8 & .9 & \text { UER } \\ 7.01 & 2.8 & 2.9 & 21 & 0 & 168 & .19 & 16 & 1.7 & 1.8 & \text { LEK }\end{array}$ $5.922 .4 \quad 1.57900278 .232625 .2$ 12.2 DIS

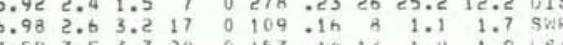

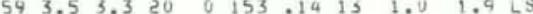

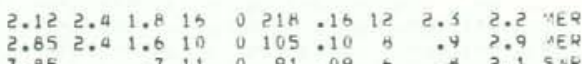

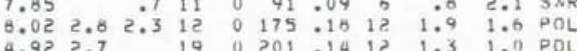

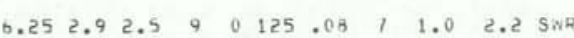

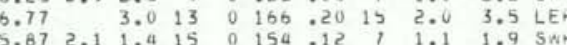
$\begin{array}{llllllllllllll}9.57 & 2.5 & 2.1 & 20 & 0 & 72 & .10 & 6 & .0 & .4 & \text { SWF } \\ 9.26 & 2.6 & 1.8 & 13 & 0 & 263 & .43 & 26 & 23.1 & 13.4 & \text { OIS }\end{array}$ $\begin{array}{llllllllllllll}1.41 & 2.2 & 1.0 & 9 & 0 & 100 & .27 & 7 & 2.5 & 99.0 & \text { SWR }\end{array}$

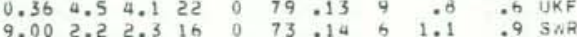

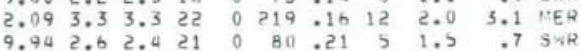
$\begin{array}{lllllllllll}5.65 & 2.6 & 1.0 & 20 & 0 & 158 & .16 & 13 & 1.4 & 1.0 & \text { HLP }\end{array}$

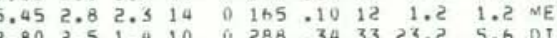

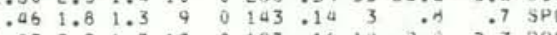
$\begin{array}{lllllllllll}0.22 & 2.2 & 1.7 & 17 & 0 & 197 & .16 & 10 & 2.0 & 2.3 & \text { POL }\end{array}$ $\begin{array}{lllllllllll}7.98 & 2.5 & 1.0 & 18 & 0 & 107 & .13 & 1 & 1.0 & 1.4 & \text { POL }\end{array}$

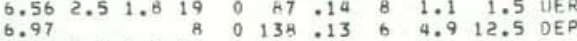

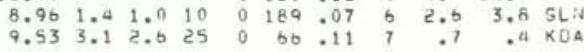

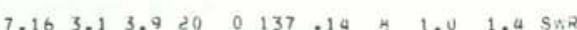

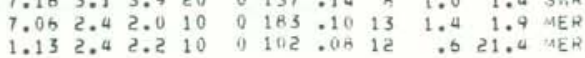


aVO EARTHQUAKE, SUMMARY LIST

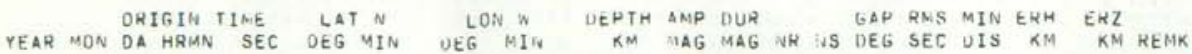
1475 Nov $292132 \quad 4.501920 .22 \quad 15520.24$

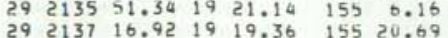

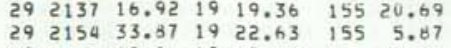

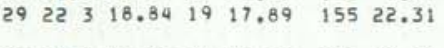
$\begin{array}{llllllll}29 & 22 & 8 & 32.12 & 19 & 19.59 & 155 & 20.73 \\ 29 & 25 & 9 & 12.48 & 19 & 22.17 & 155 & 5.14\end{array}$ 29 $2210 \quad 34.57 \quad 19 \quad 19.02 \quad 155 \quad 1.22$

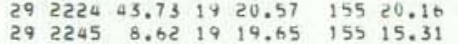

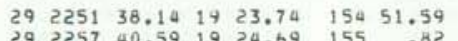
$\begin{array}{lllllll}29 & 2557 & 40.59 & 19 & 24.69 & 155 & .82 \\ 29 & 23 & 11.29 & 19 & 19.38 & 155 & 20.35\end{array}$

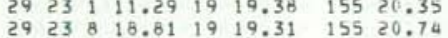

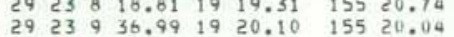

$\begin{array}{lllllll}29 & 2311 & 33.52 & 19 & 20.93 & 155 & 17.56\end{array}$

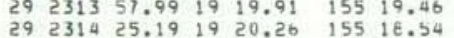

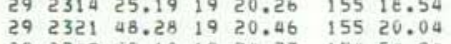

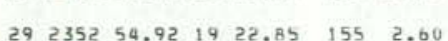
$\infty \quad 292357 \quad 3.05 \quad 1922.74 \quad 155 \quad .14$

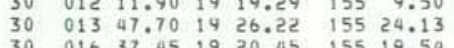
$\begin{array}{lllllll}30 & 029 & 2.86 & 14 & 24.81 & 155 & 23.55\end{array}$ $\begin{array}{lllllll}30 & 030 & 13.70 & 19 & 26.97 & 154 & 57.47\end{array}$ $30 \quad 044 \quad 28,40 \quad 19 \quad 20,83 \quad 155 \quad 19,48$ $\begin{array}{lllllll}30 & 048 & 37.40 & 19 & 20.20 & 155 & 17.16\end{array}$ $\begin{array}{llllllll}30 & 111 & 35.13 & 19 & 16.31 & 155 & 22.22\end{array}$ $\begin{array}{lllllll}30 & 112 & 14.51 & 19 & 20.55 & 155 & 7.69 \\ 30 & 115 & 41.55 & 17 & 20.70 & 155 & 18.56\end{array}$

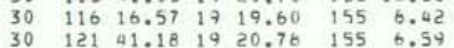

$\begin{array}{lllllll}30 & 159 & 43.68 & 19 & 21.81 & 155 & 6.65\end{array}$

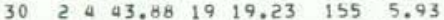
$\begin{array}{llllllll}30 & 2 & 6 & 31.74 & 19 & 14.10 & 155 & 23.47 \\ 30 & 2 & 9 & 13.70 & 19 & 15.78 & 155 & 9.23\end{array}$ $\begin{array}{lllllll}30 & 217 & 35.96 & 19 & 19.37 & 155 & 20.83\end{array}$

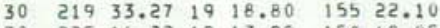

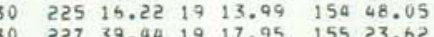
$\begin{array}{lllllll}30 & 227 & 39.44 & 19 & 17.95 & 155 & 23.62 \\ 30 & 228 & 6.77 & 19 & 20.57 & 155 & 18.65\end{array}$

$\begin{array}{lllllll}30 & 232 & 3.57 & 19 & 21.56 \quad 155 & 18.29\end{array}$

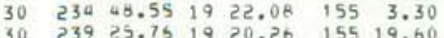

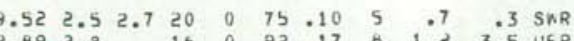

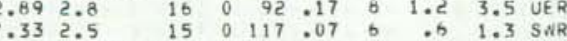
6.823 .03 .224 O 120.1410 . 60 1.3 MER $\begin{array}{lllllllllll}7.86 & 2.1 & 1.5 & 16 & 0 & 111 & .17 & 6 & 1.2 & 1.5 & \text { SWR }\end{array}$

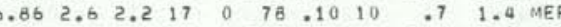

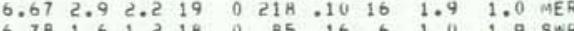

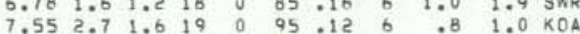
$\begin{array}{llllllllllll}35.07 & 3.0 & 2.5 & 7 & 0 & 308 & .30 & 31 & 72.1 & 67.8 & \text { LER } \\ .62 & 2.2 & 1.4 & 17 & 0 & 140 & .22 & 17 & 1.9 & 41.8 & \text { LER }\end{array}$

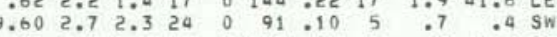

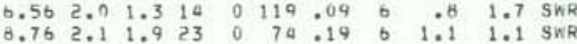
$\begin{array}{rrrrrrrrrrr}8.33 & 2.4 & 1.4 & 16 & 0 & 67 & .09 & 5 & .8 & .8 & \text { KOA } \\ 7.17 & & 1.0 & 12 & 0 & 117 & .05 & 7 & .5 & 1.2 & \text { SWR }\end{array}$

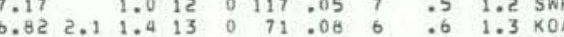

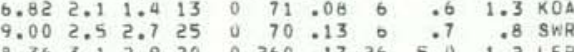
$\begin{array}{llllllllllll}3.21 & 2.3 & 1.7 & 16 & 0 & 183 & .09 & 14 & .9 & 1.2 & \text { MEH }\end{array}$

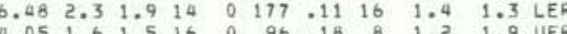

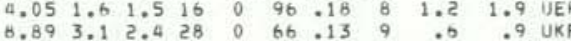

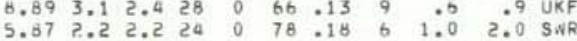
$\begin{array}{lllllllllll}8.47 & 2.4 & 1.9 & 19 & 0 & 90 & .10 & 9 & .6 & .9 & \text { UKF }\end{array}$

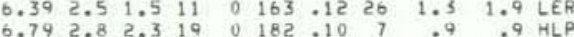

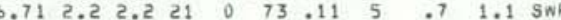

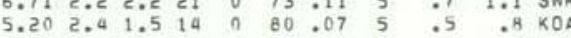
$\begin{array}{lllllllllll}7.01 & 2.6 & 2.2 & 20 & 0 & 13 B & .15 & 8 & 1.1 & 2.7 & \text { SWR }\end{array}$

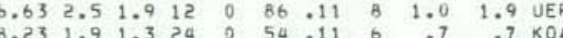

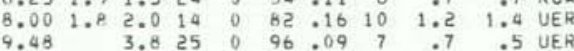
$\begin{array}{llllllllll}7.80 & 2.8 & 2.6 & 23 & 0 & 100 & .17 & 9 & 1.1 & 1.3 \\ 0.3 & \text { UER }\end{array}$

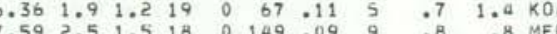

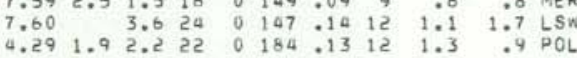
$\begin{array}{llllllllllll}9.81 & 2.4 & 2.4 & 25 & 0 & 97 & .10 & 6 & .6 & .3 & \text { SWR }\end{array}$

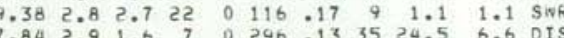

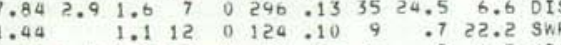
$.081 .91 .3170073 .130909 .5 \times 04$ $\begin{array}{lllllllllll}5.89 & 1.6 & .8 & 14 & 0 & 64 & .12 & 4 & .9 & 1.9 & \times 04\end{array}$

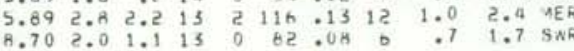

hVO EARTHQUARE SUMNAKY LIS

Patig 110

ORIGIN TIME LATN LUN TEAR MON DA NQMN

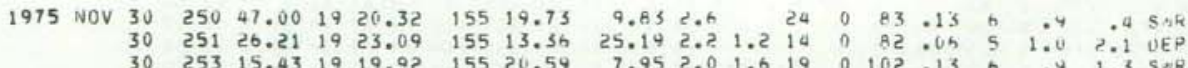

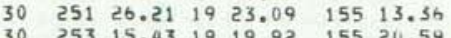
$3025315.431919 .92 \quad 15520.54$ $30.256 \quad 1.791920 .36$ 155 18.96 $\begin{array}{lllllll}30 & 257 & 36.18 & 19 & 24.05 & 155 & .24\end{array}$

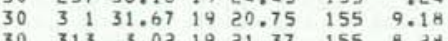
(30

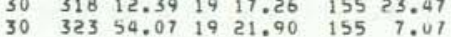
$30 \quad 325 \quad 45.641914 .21 \quad 15514.02$ $30328827.071925 .67 \quad 15454.53$ 30 349 35.07 19 14.73 155 23.13 $30 \quad 358 \quad 58.92 \quad 19 \quad 9.41 \quad 155 \quad 15,40$ $\begin{array}{llllllll}30 & 4 & 1 & 58.73 & 19 & 21.98 & 155 & .75 \\ 30 & 4 & 6 & 30.22 & 19 & 22.07 & 155 & 3.41\end{array}$

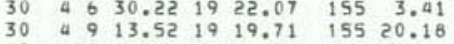

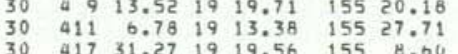

$\begin{array}{lllllll}30 & 427 & 19.33 & 19 & 18.28 & 155 & 11.57\end{array}$ 3004458.14 19 20.93 155 11.24

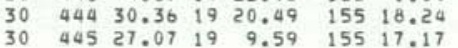

$\begin{array}{lllllll}30 & 448 & 48.12 & 19 & 22.93 & 155 & 1.82\end{array}$ $\begin{array}{lllllll}30 & 453 & 43.36 & 19 & 21.73 & 155 & 3.82\end{array}$

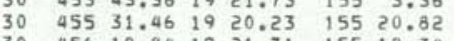
$\begin{array}{llrllll}30 & 456 & 10.84 & 19 & 21.71 & 155 & 18.34 \\ 30 & 459 & 7.65 & 19 & 24.54 & 155 & 13.33\end{array}$ $\begin{array}{llllllll}30 & 5 & 4 & 39.52 & 19 & 20.27 \quad 155 & 19.55\end{array}$

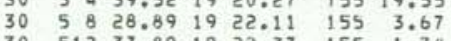
$\begin{array}{lllllll}30 & 512 & 37.89 & 19 & 22.37 & 155 & 1.74\end{array}$ $\begin{array}{lllllll}30 & 516 & 33.35 & 19 & 23.77 & 154 & 59.30 \\ 30 & 521 & 21.38 & 19 & 20.29 & 155 & 20.55\end{array}$ $\begin{array}{lllllll}30 & 542 & 34.72 & 19 & 16.92 & 155 & 6.73\end{array}$

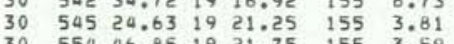

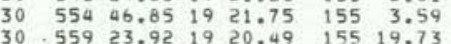

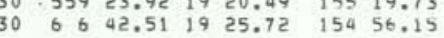
$\begin{array}{lllllll}30 & 610 & 12.25 & 19 & 19.14 & 155 & 20.80\end{array}$ $\begin{array}{lllllll}30 & 623 & 13.40 & 19 & 20.17 & 155 & 18.95\end{array}$ $\begin{array}{lllllll}30 & 632 & 49.59 & 19 & 23.78 & 154 & 59.54 \\ 30 & 657 & 43.92 & 19 & 20.41 & 155 & 18.65\end{array}$

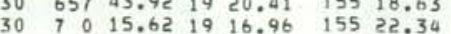

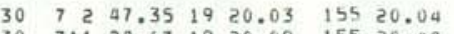
$\begin{array}{lllllll}30 & 711 & 28.67 & 19 & 20.09 & 155 & 20.48 \\ 30 & 715 & 50.98 & 19 & 23.93 & 154 & 58.41\end{array}$

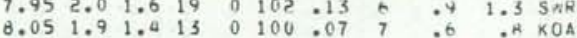

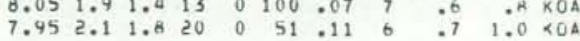

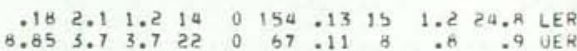

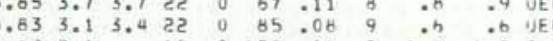

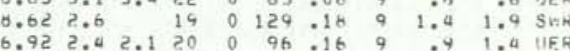

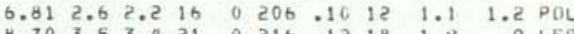

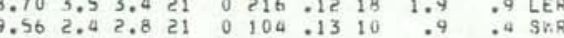

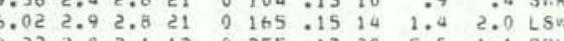

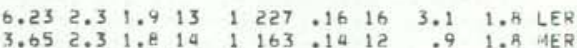

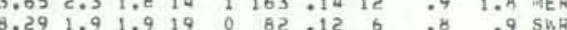

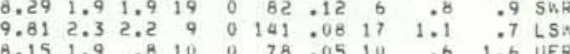

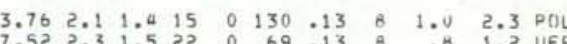

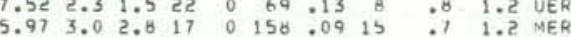

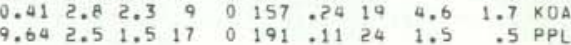
$\begin{array}{lllllllllll}3.23 & 1.9 & 1.4 & 14 & 2 & 146 & .14 & 14 & 1.0 & 1.7 & \text { MEF }\end{array}$

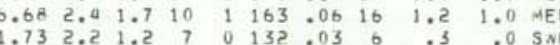
$\begin{array}{lllllll} & 0\end{array}$

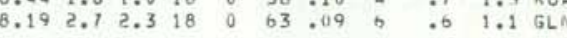
$\begin{array}{rrrrrrrrrrr}7.21 & 1.9 & 1.5 & 20 & 0 & 63 & .12 & 6 & .7 & 1.0 & \text { SWR } \\ 5.94 & 3.9 & 3.4 & 20 & 0 & 107 & 12 & 12 & .7 & 1.3 & \text { MER }\end{array}$ \begin{tabular}{llllllllll}
1.22 & 2.2 & 1.5 & 12 & 1 & 204 & -15 & 15 & 5.4 & 1.3 \\
\hline
\end{tabular}

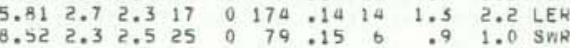
$\begin{array}{lllllllllllll}3.24 & 1.9 & 2.0 & 15 & 0 & 207 & .30 & 10 & 6.1 & 7.6 & \text { POL }\end{array}$

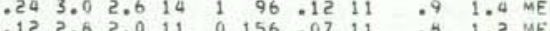

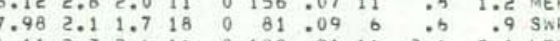

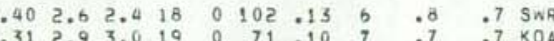

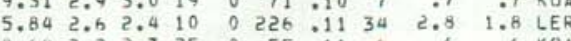

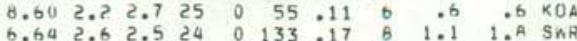

$8.913 .03 .114 \quad 0 \quad 75.12$ 1s 1.0 1.2 Sk.R

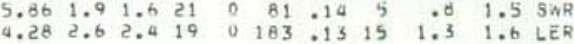


HVO EARTHGUAKE SUMR.ARY LIST

PAGE 111

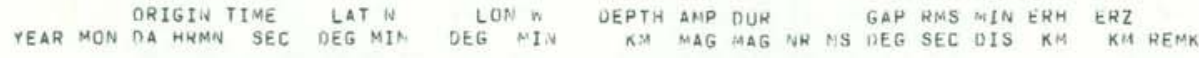
1975 NOV $30 \quad 724 \quad 20.29 \quad 19 \quad 14.08 \quad 155 \quad 20.15$

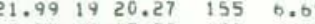

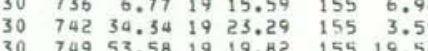

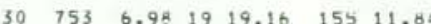

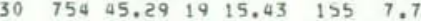
$\begin{array}{llllllll}30 & 8 & 0 & 14.61 & 19 & 21.72 & 155 & 15.12\end{array}$

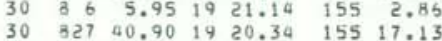

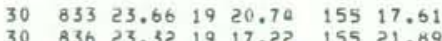
$\begin{array}{llllllll}30 & 836 & 23.32 & 19 & 17.22 & 155 & 21.89\end{array}$

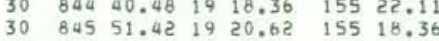
$23.31+155 \quad 3.08$

$\begin{array}{lllllll}30 & 854 & 6.47 & 19 & 24.26 & 155 & 13.49\end{array}$

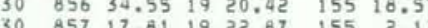

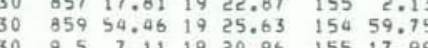

$\begin{array}{llllllll}30 & 9 & 7 & 3.32 & 19 & 22.64 & 155 & .6 \\ 30 & 9 & 8 & 3.83 & 19 & 19.76 & 155 & 5.16\end{array}$

$\begin{array}{lllllll}30 & 912 & 11.47 & 19 & 19.05 & 155 & 18.42 \\ 30 & 914 & 38.72 & 19 & 20.60 & 155 & 18.6\end{array}$

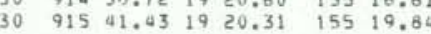

$\begin{array}{lllllll}30 & 926 & 57.12 & 19 & 19.43 & 155 & 20.73\end{array}$

$\begin{array}{lllllll}30 & 933 & 30.77 & 19 & 20.71 & 154 & 58.19\end{array}$

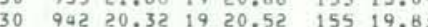

$\begin{array}{lllllll}30 & 946 & 10.30 & 19 & 17.90 & 155 & 23.64\end{array}$

$\begin{array}{lllllll}30 & 946 & 52.77 & 19 & 16.85 & 155 & 21.01\end{array}$

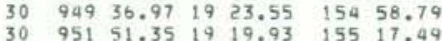

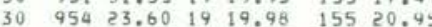

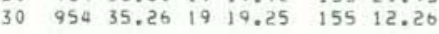

$\begin{array}{lllllll}30 & 957 & 20.21 & 19 & 18.98 & 155 & 14.56\end{array}$ 30 954 $40.121921 .10 \quad 15519.10$

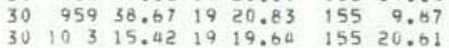
$\begin{array}{llllllll}30 & 10 & 4 & 2.49 & 19 & 19.32 & 155 & 20.49\end{array}$

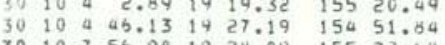
$3010756.98 \quad 1928.09 \quad 15522.68$

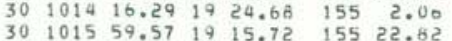

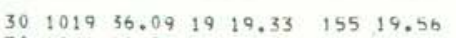
$\begin{array}{lllllll}30 & 1021 & 46.24 & 19 & 25.17 & 155 & 1.75 \\ 30 & 1028 & 51.03 & 19 & 20.46 & 155 & 17.03\end{array}$

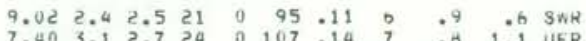
$\begin{array}{lllllllllll}11.08 & 2.4 & 1.6 & 13 & 0 & 231 & 13 & 13 & 2.5 & .7 & P O L\end{array}$ $\begin{array}{rrrrrrrrrr}7.69 & 2.6 & 1.9 & 13 & 0 & 165 & -10 & 13 & 1.0 & 1.2 \\ 8.08 & 2.3 & 2.0 & 23 & 0 & 67 & .16 & 7 & .9 & .8 \\ 8 & .8 & \text { SWR }\end{array}$ $\begin{array}{lllllllllll}6.78 & 2.8 & 2.6 & 26 & 0 & 100 & .14 & 7 & .7 & 1.0 & \text { UER }\end{array}$

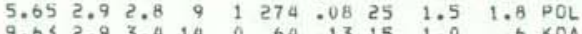

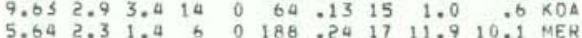

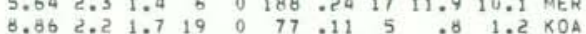
$\begin{array}{lllllllllll}7.66 & 1.6 & 1.4 & 14 & 0 & 65 & 09 & 5 & .7 & 1.7 & \text { KOA }\end{array}$ $\begin{array}{llllllllllll}9.88 & 1.9 & 1.6 & 12 & 0 & 90 & .07 & 5 & .9 & .5 & \text { GLN }\end{array}$

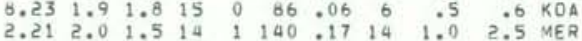

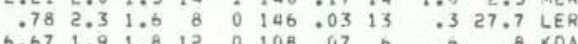

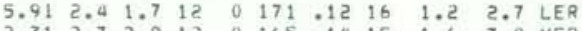
9.072 .92 .9120 165 .14 15 $1.6 \quad 7.0$ MER

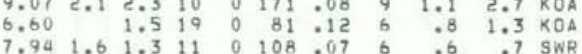
$\begin{array}{llllllllllll}7.94 & 1.7 & 1.4 & 13 & 0 & 115 & .08 & 6 & .0 & .8 & \text { SWR } \\ 3.96 & 2.8 & 2.8 & 20 & 0 & 221 & .016 & 18 & 3.0 & 1.7 & \text { LER }\end{array}$

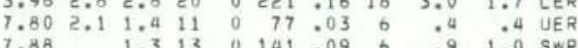
$\begin{array}{llllllllllll}7.88 & 1.7 & 1.3 & 13 & 0 & 141 & .09 & 6 & .9 & 1.0 & \text { SWR } \\ 2.75 & 1.9 & 11 & 0 & 147 & .09 & 9 & .8 & 3.2 & \text { SWR }\end{array}$ $\begin{array}{lllllllllll}9.13 & 2.5 & 1.8 & 13 & 0 & 161 & .08 & 9 & .9 & .9 & \text { SWR }\end{array}$

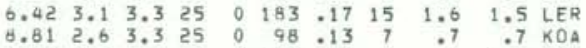
$\begin{array}{lllllllllll}9.82 & 1.9 & .9 & 11 & 0 & 126 & .07 & 7 & 1.0 & 3.4 & \text { POL }\end{array}$

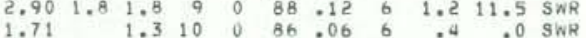

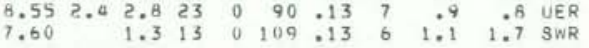
$\begin{array}{llllllllllllllllll}6.44 & 1.5 & 9 & 2 & 161 & .14 & 6 & 2.4 & 3.0 & \text { SWR }\end{array}$ $4.852 .01 .526 \quad 0400.06$ 12 $1.4 \quad 3.9$ UKF

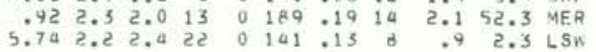
$\begin{array}{llllllllll}6.40 & 1.3 & 13 & 0 & 99 & .13 & 7 & 1.1 & 2.5 & \text { SWR }\end{array}$

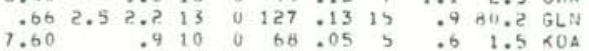

HVO EARTHQUAKE SUMMARY LIST

PAGE 112

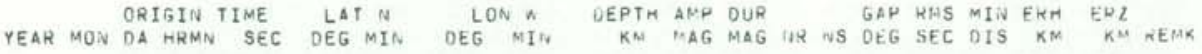

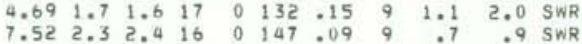

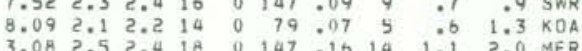

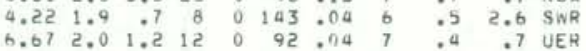

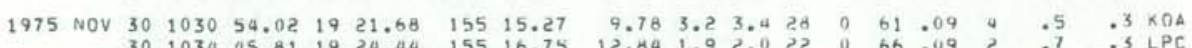

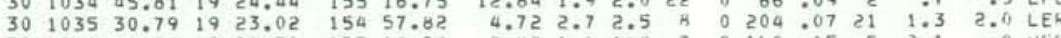

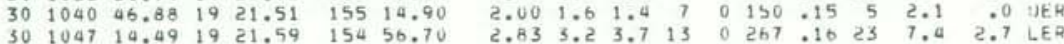

$30-1050236.3419-31.75 \quad 155 \quad 5.80$

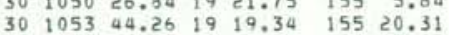
$30105450.001922 .36 \quad 15514.63$ $3011{ }^{2} \quad 3.341920 .32 \quad 155 \quad 7.60$

$\begin{array}{llllllll}30 & 11 & 6 & 13.80 & 19 & 18.72 & 155 & 14.07\end{array}$

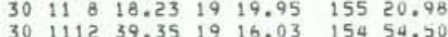
$\begin{array}{lllllll}30 & 1112 & 39.35 & 19 & 16.03 & 154 & 54.50 \\ 30 & 1118 & 54.90 & 19 & 21.53 & 155 & 19.07\end{array}$

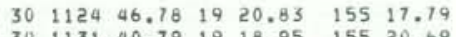

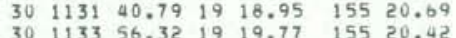

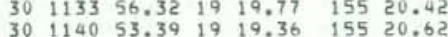

$\begin{array}{lllllll}30 & 1145 & 44.98 & 19 & 20.14 & 155 & 20.76 \\ 30 & 1146 & 33.27 & 19 & 18.97 & 155 & 11.22\end{array}$

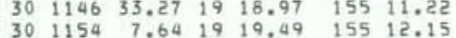
$\begin{array}{rrrrrrr}30 & 1154 & 7.64 & 19 & 19.49 & 155 & 12.15 \\ 30 & 1157 & 19.71 & 19 & 20.21 & 155 & 20.36\end{array}$

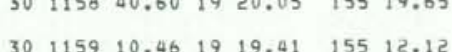
$\begin{array}{llllllll}30 & 1159 & 10.46 & 19 & 19.41 & 155 & 12.12 \\ 30 & 12 & 42.28 & 32.20 & 19 & 20.93 & 155 & 17.32\end{array}$

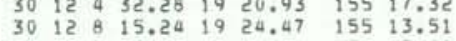

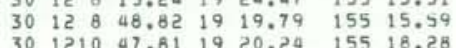
$\begin{array}{llllll}30 & 1213 & 19.57 & 19 & 19.05 \quad 155 \quad 20.26\end{array}$ $30122013.221919 .94 \quad 15519.38$ $\begin{array}{lllllll}30 & 1229 & 55.81 & 19 & 14.35 & 155 & 19.07 \\ 30 & 1234 & 39.79 & 19 & 20.39 & 155 & 18.93\end{array}$

$\begin{array}{lllllll}30 & 1244 & 39.15 & 19 & 18.56 & 155 & 12.03\end{array}$

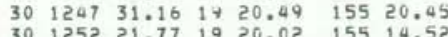

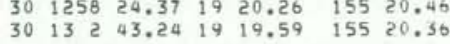
$\begin{array}{llll}30 & 13 & 56.951920 .08 \quad 15519.42\end{array}$

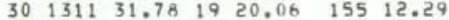

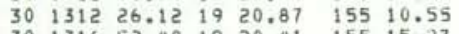

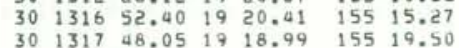

$\begin{array}{llllll}30 & 1322 & 43.94 & 19 & 20.06 \quad 155 & 20.93\end{array}$ $30133821.651918 .69 \quad 15522.59$ $\begin{array}{lllllllllll}6.55 & 2.0 & 1.6 & 16 & 0 & 110 & .12 & 9 & .8 & 1.8 & A E R\end{array}$

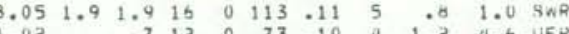

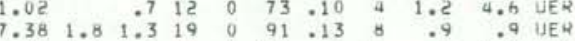

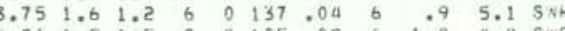

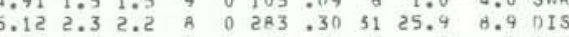

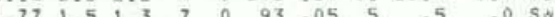

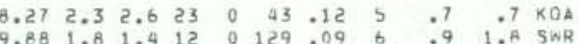

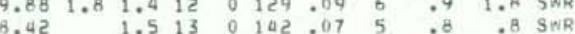
$\begin{array}{lllllllllll}5.13 & 1.9 & 1.7 & 13 & 0 & 134 & .09 & 6 & .8 & 1.5 & 5 n k \\ 7.19 & 2.1 & 2.3 & 21 & 0 & 110 & .15 & 6 & 1.1 & 1.5 & \text { PDo }\end{array}$

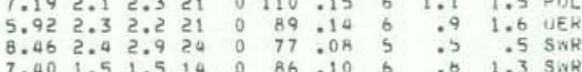

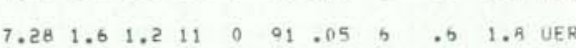

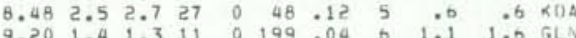

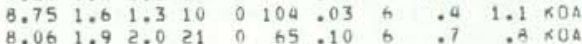
$8.41 \quad 1.92 .32100115 .16 \quad 9 \quad 1.1 \quad 1.0$ SWR

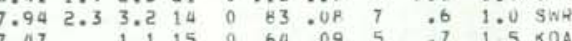

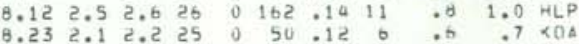
$\begin{array}{lllllllllll}8.14 & 2.6 & 3.0 & 25 & 0 & 114 & .13 & \text { B } & .4 & 1.2 & \text { POL }\end{array}$

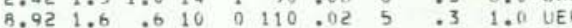

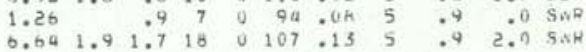
$\begin{array}{llllllllllll}8.09 & 1.5 & 1.6 & 16 & 0 & 70 & -11 & 0 & .7 & .8 & \text { SNR }\end{array}$ $10.71 \quad 1.91 .514 \quad 0 \quad 116.09$ \& 1.3 .5 UER

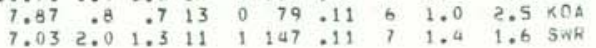
$\begin{array}{llllllllllll}5.09 & 1.5 & 1.5 & 11 & 0 & 102 & .07 & 6 & .0 & 1.0 & \operatorname{SnR} R\end{array}$

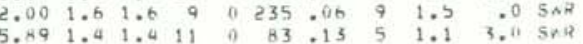
$\begin{array}{llllllll}30 & 117 & 15.71 & 19 & 20.06 & 155 & 20.71 \\ 30 & 11 & 15 & 18.23 & 19 & 19.95 & 155 & 20.93\end{array}$

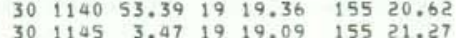

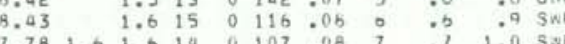


HVO EARThQUARE SLMMARY LIST

PAGE 113

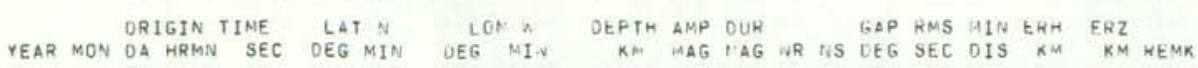
1975 NOV $30 \quad 1346 \quad 40.791921 .41 \quad 155 \quad 16.94$ $\begin{array}{lllllll}30 & 1348 & 4.33 & 19 & 20.25 & 155 & 19.9 \\ 30 & 1352 & 12.49 & 19 & 22.18 & 155 & 1.20\end{array}$ $\begin{array}{lllllll}30 & 1353 & 13.36 & 19 & 19.75 & 155 & 20.46 \\ 30 & 1354 & 20.76 & 19 & 20.46 & 155 & 20.33\end{array}$

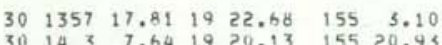

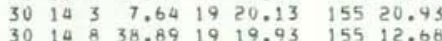

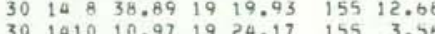

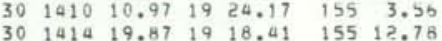

$\begin{array}{lllllll}30 & 1215 & 17.76 & 19 & 16.43 & 155 & 12.84\end{array}$ $\begin{array}{lllllll}30 & 1415 & 58.77 & 19 & 20.50 & 155 & 20.33 \\ 30 & 1417 & 39.66 & 19 & 25.76 & 155 & 23\end{array}$

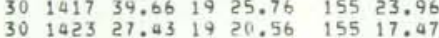
$30 \quad 1424 \quad 40.69 \quad 19 \quad 21.10 \quad 155 \quad 6.39$ $\begin{array}{rrrrrrr}30 & 1428 & 25.19 & 19 & 20.07 & 155 & 18.75 \\ 30 & 1440 & 2.76 & 19 & 20.52 & 155 & 20.65\end{array}$ $\begin{array}{lllllll}30 & 1441 & 36.21 & 19 & 18.43 & 155 & 23.5\end{array}$

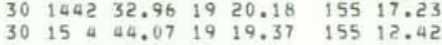

$\begin{array}{llllllll}30 & 15 & 5 & 22.96 & 19 & 20.38 & 155 & 12.14\end{array}$

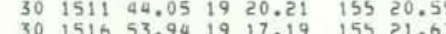

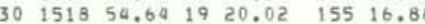

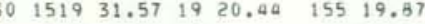
$\begin{array}{lllllll}30 & 1524 & 41.33 & 19 & 21.17 & 155 & 17.60\end{array}$ $30152724.241920 .05 \quad 15521.09$ $\begin{array}{lllllll}30 & 1550 & 53.03 & 19 & 19.85 & 155 & 20.00 \\ 30 & 1553 & 40.29 & 19 & 20.11 & 155 & 13.31\end{array}$ $\begin{array}{lllllll}30 & 1556 & 58.82 & 19 & 20.80 & 155 \quad 5.02\end{array}$ 30 1551 50.3619211 .74 155 12.90

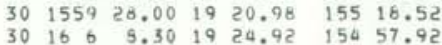
$\begin{array}{lllllll}30 & 16 \quad 7 & 40.13 \quad 19 & 19.92 \quad 155 & 19.07\end{array}$ 15521.60 $30 \quad 162111.581925 .82$ 155 20.58

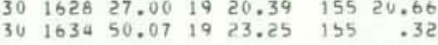

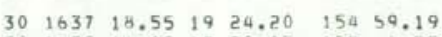

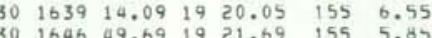

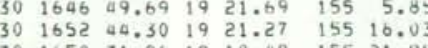

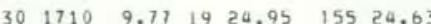

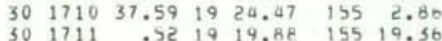
$\begin{array}{llllllllll}1.32 & 1.6 & 1.6 & 10 & 0 & 79.05 & 5 & .4 & 99.0 & 004\end{array}$

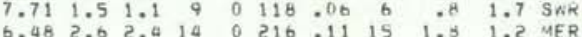

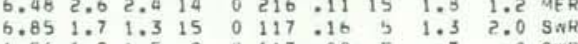
$3.352 .62 .521 \quad 1 \quad 173.1313 \quad 9 \quad 1.6$ MER

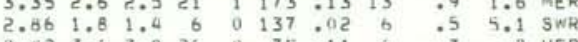

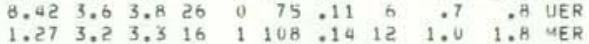

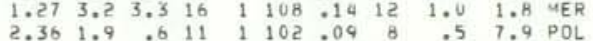

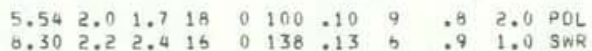

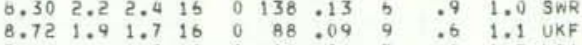

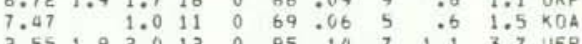
$\begin{array}{rrrrrrrrrrr}7.94 & 1.5 & 1.6 & 14 & 0 & 95 & .06 & 7 & .5 & .9 & \times 04 \\ .57 & 1.7 & 1.0 & 10 & 0 & 120 & .06 & 6 & .6 & 3.6 & 9 \times R\end{array}$ $\begin{array}{lllllllllll}4.96 & 2.0 & 1.9 & 11 & 0 & 123 & .13 & 9 & 1.3 & 2.0 & \text { SWR } \\ 6.90 & & 1.0 & 9 & 0 & 153 & 0.03 & 5 & .5 & 9.1 & \text { SWR }\end{array}$

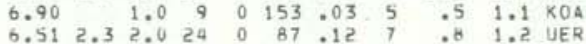
$\begin{array}{llllllllllll}2.16 & 1.8 & .7 & 12 & 2 & 75 & .14 & 7 & 1.1 & 63.3 & \text { UER }\end{array}$

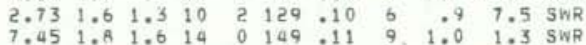

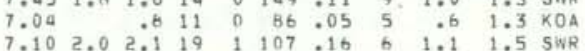
$\begin{array}{lllllllllll}8.76 & 2.8 & 5.2 & 21 & 0 & 94 & .11 & 4 & 1.1 & .6 & \text { KOA }\end{array}$

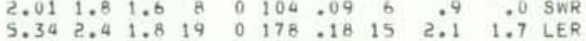
$\begin{array}{rrrrrrrrrrr}8.25 & 1.9 & 1.6 & 16 & 0 & 77 & .11 & 6 & .8 & .9 & \text { SWR } \\ 11.17 & 1.9 & 1.1 & 9 & 0 & 188 & .04 & 7 & 1.0 & 4.0 & \text { UER }\end{array}$ $\begin{array}{lllllllllll}3.74 & 2.0 & 1.6 & 16 & 0 & 102 & .20 & 7 & 1.3 & 2.7 & \text { MER }\end{array}$

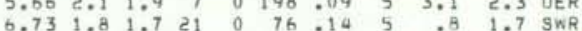
$\begin{array}{lllllllllll}6.34 & 1.3 & 13 & 0 & 69 & .09 & 5 & .8 & 1.9 & \text { KOA } \\ 5.97 & 2.6 & 1.9 & 17 & 0 & 176 & .13 & 14 & 1.5 & 2.1 & \text { LER }\end{array}$ $\begin{array}{llllllllllllll}7.93 & 1.3 & 13 & 0 & \text { R4 } & .0 R & 7 & .8 & 1.1 & \text { SWR }\end{array}$

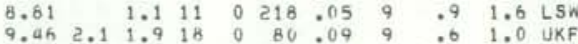

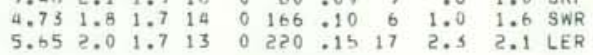
$\begin{array}{llllllllll}6.94 & 2.9 & 3.1 & 22 & 0 & 170 & .16 & 14 & 1.7 & 1.4 \\ 5 & \text { LER }\end{array}$

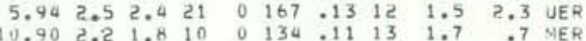

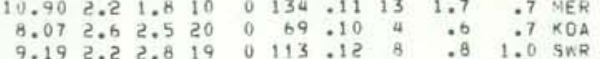
$\begin{array}{llllllllllllllllll}7.15 & 2.0 & 1.7 & 17 & 0 & 77 & .09 & 9 & .5 & 2.0 & \text { UKF }\end{array}$

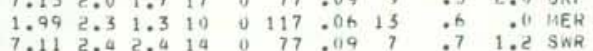

HVO EARTHOLARE SUMMAKY LIST

Paif 110

ORIGIN TIME LAT HA LON DE DETH ANO DUR

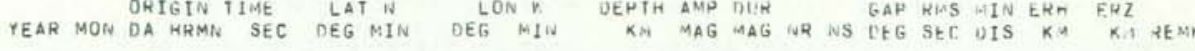

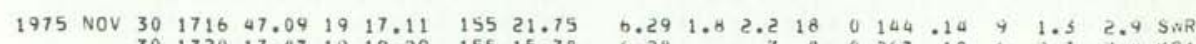

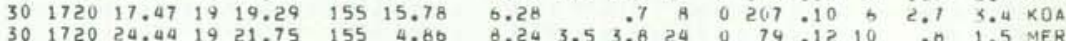

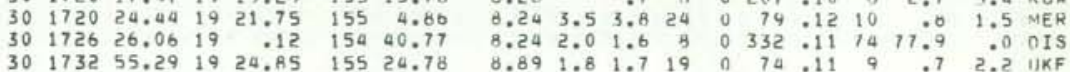
$30 \quad 1732 \quad 55.29 \quad 1924.85$ is 20.7 $\begin{array}{lllllll}30 & 1733 & 33.74 & 19 & 28.28 & 155 & 22.04\end{array}$

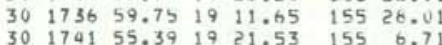

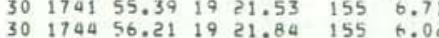

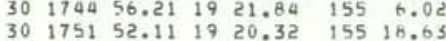
$\begin{array}{llllllll}30 & 1758 & 49.76 & 19 & 16.93 & 155 & 20.35 \\ 30 & 1810 & 8.13 & 19 & 19.40 & 155 & 19.69\end{array}$

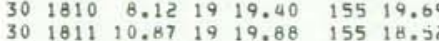

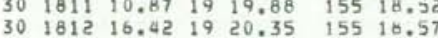
$\begin{array}{lllllll}30 & 1813 & 47.19 & 19 & 15.31 & 155 & 16.12\end{array}$ $\begin{array}{lrrrrrr}30 & 1810 & 8.12 & 19 & 19.40 & 155 & 19.69 \\ 30 & 1811 & 10.87 & 19 & 19.88 & 155 & 10.52\end{array}$ $\begin{array}{lllllll}30 & 1811 & 10.87 & 19 & 19.80 & 155 & 16.52\end{array}$ $301813 \quad 47.191915 .31 \quad 15516.12$

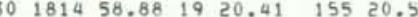
$\begin{array}{lllllll}30 & 1819 & 1.48 & 19 & 20.41 & 155 & 12.01\end{array}$ 30182011.221921 .26 155 19.46

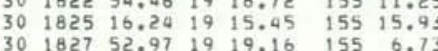

$\begin{array}{lllllll}30 & 1829 & 14.73 \quad 19 & 22.19 & 155 & 6.01\end{array}$

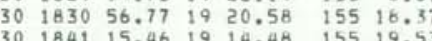
$\begin{array}{lllllll}30 & 1851 & 43.05 & 19 & 22.37 & 154 & 59.77 \\ 30 & 1854 & 51.46 & 19 & 18.07 & 155 & 19.47\end{array}$

$\begin{array}{llllllll}30 & 19 & 6 & 43.40 & 19 & 19.67 & 155 & 20.83\end{array}$

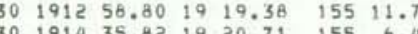

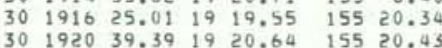

$\begin{array}{lllllll}30 & 1921 & 9.45 & 19 & 22.01 & 155 & 15.25\end{array}$

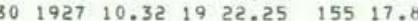
$\begin{array}{lllllll}30 & 1928 & 5.10 & 19 & 22.64 & 155 & 1.63 \\ 30 & 1933 & 50.27 & 19 & 19.38 & 155 & 13.40\end{array}$

$\begin{array}{lllllll}30 & 1938 & 46.50 & 19 & 17.31 & 155 & 21.09\end{array}$ 30
1939 $9.48 \quad 1919.78 \quad 15514.42$ $\begin{array}{lllllll}30 & 1941 & 46.26 & 19 & 24.09 & 154 & 59.20\end{array}$ $\begin{array}{lllllll}30 & 1943 & 50.34 & 19 & 18.95 & 155 & 22.15 \\ 30 & 1945 & 39.67 & 19 & 14.91 & 155 & 9.49\end{array}$

$\begin{array}{lllllll}30 & 1947 \quad 36.80 & 19 & 23.90 & 155 & 13.55\end{array}$

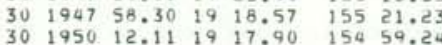

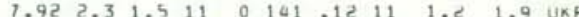

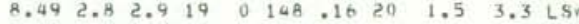

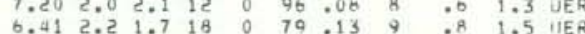

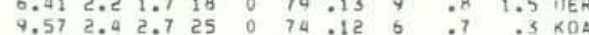
$6.47 \quad 2.3 \quad 1.813 \quad 1 \quad 166.08$ \& $\quad .7$ 1.5 SNR

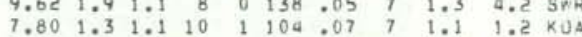

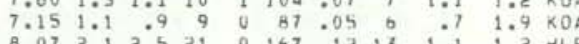
$\begin{array}{lllllllllll}9.62 & 1.9 & 1.1 & 8 & 0 & 138 & .05 & 7 & 1.3 & 4.2 & \text { SWH }\end{array}$ $\begin{array}{lllllllllll}7.80 & 1.3 & 1.1 & 10 & 1 & 104 & .07 & 7 & 1.1 & 1.2 & \times 04\end{array}$ $\begin{array}{lllllllllll}7.07 & 2.1 & 2.5 & 21 & 0 & 167 & 0.12 & 13 & 1.0 & 1.9 & \text { KLP }\end{array}$ $\begin{array}{lllllllllll}7.89 & 2.5 & 2.2 & \text { iा } & 0 & 138 & .07 & 8 & .7 & 1.0 & \text { UEA }\end{array}$

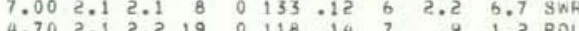

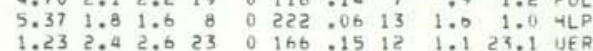
$\begin{array}{lllllllllll}0.12 & 2.4 & 2.3 & 17 & 0 & 108 & .10 & 9 & 1.1 & 1.2 & \text { IJER }\end{array}$

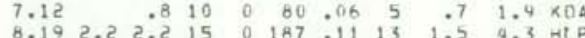
$\begin{array}{lllllllllll}6.95 & 2.2 & 1.6 & 13 & 0 & 188 & .17 & 16 & 2.2 & 6.5 & \text { LER } \\ 6.68 & 1.0 & 13 & 0 & 172 & .09 & 7 & .8 & 1.8 & \text { SWR }\end{array}$ $\begin{array}{lllllllllll}8.27 & 2.1 & 2.3 & 21 & 0 & 131 & .15 & \text { a } & 1.0 & 1.5 & \text { SWR }\end{array}$

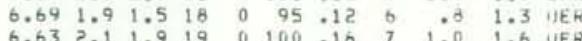

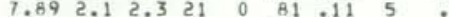

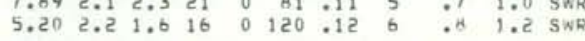

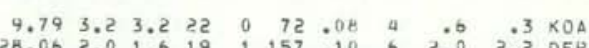

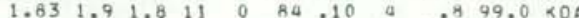

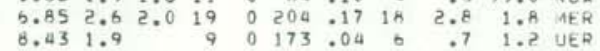
$\begin{array}{llllllllllllllll}7.14 & 1.7 & 1.4 & 13 & 0 & 127 & .14 & \text { \& } & 1.3 & 2.0 & \text { SWR }\end{array}$

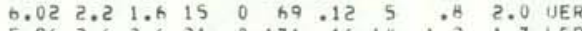
$\begin{array}{lllllllllll}5.96 & 2.6 & 2.6 & 21 & 0 & 171 & .16 & 14 & 1.2 & 1.7 & \text { LER }\end{array}$ $\begin{array}{llllllllllll}9.10 & 2.6 & 2.7 & 27 & 0 & 103 & .15 & 9 & .8 & .9 & \text { SWR } \\ 9.13 & 2.3 & 2.4 & 24 & 0 & 199 & .13 & 15 & 1.4 & .8 & \text { POL }\end{array}$

$\begin{array}{llllllllllll}0.19 & 1.8 & .9 & 10 & 0 & 161 & .05 & \text { y } & 1.5 & 1.9 & \text { GLW }\end{array}$ 


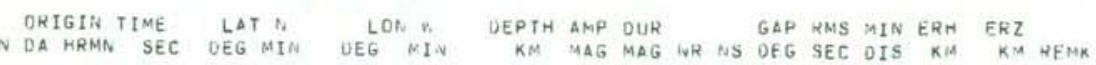

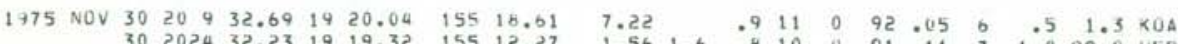

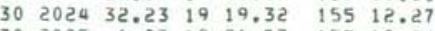
$\begin{array}{lllllll}30 & 2025 & 6.23 & 19 & 21.73 & 155 & 12.00\end{array}$ 3020253.231920 .11015519 .13

$\begin{array}{lllllll}30 & 2031 & 18.62 & 17 & 57.29 & 154 & 58.91 \\ 30 & 2037 & 34.94 & 19 & 20.21 & 155 & 19.23\end{array}$

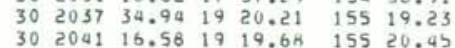
$\begin{array}{lllllll}30 & 2041 & 16.56 & 19 & 19.64 & 155 & 20.45 \\ 30 & 2043 & 16.77 & 19 & 20.20 & 155 & 19.72\end{array}$ $302045 \quad 46.101920 .66 \quad 15518.14$

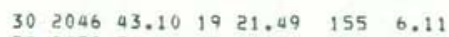
$\begin{array}{llllllr}30 & 2050 & 56.46 & 19 & 20.07 & 155 & 19.38 \\ 30 & 2057 & 18.34 & 19 & 22.59 & 155 & 2.97\end{array}$

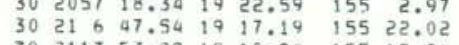
$\begin{array}{llllll}30 & 2113 \quad 53.29 & 19 & 18.94 & 155 & 19.26\end{array}$ $\begin{array}{lllllll}30 & 2117 & 5.19 & 19 & 18.25 & 155 & 5.85 \\ 30 & 2118 & 40.29 & 19 & 18.02 & 155 & 21.67\end{array}$ 30 2118 $40.291918 .02 \quad 15521.67$

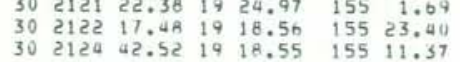

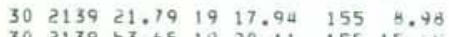

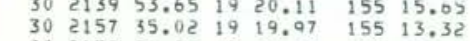

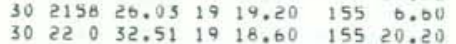

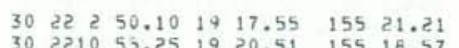
$30221145.8619218 .08 \quad 15513.72$ $\begin{array}{lllllll}30 & 2218 & 49.98 & 19 & 24.45 & 155 & 12.58 \\ 30 & 2222 & 50.38 & 19 & 18.25 & 155 & 23.48\end{array}$ $\begin{array}{lllllll}30 & 2224 & 19.43 & 19 & 20.58 & 155 & 13.47\end{array}$

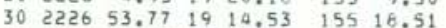
$\begin{array}{rrrrrrr}30 & 2237 & 6.33 & 19 & 21.09 & 155 & 17.66 \\ 30 & 2240 & 42.06 & 19 & 19.81 & 155 & 3.53\end{array}$ $\begin{array}{lllllll}30 & 2249 & 52.41 & 19 & 16.25 & 155 & 21.71\end{array}$

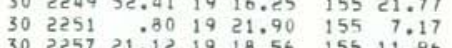

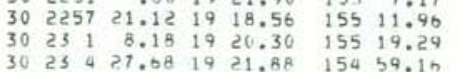

$\begin{array}{llllllllll}30 & 2310 & 37.91 & 19 & 17.79 \quad 155 & 21.75\end{array}$

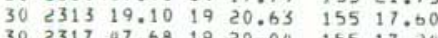
$\begin{array}{lllllll}30 & 2317 & 47.68 & 19 & 20.04 & 155 & 17.24 \\ 30 & 2322 & 11.78 & 19 & 20.05 & 155 & 18.5\end{array}$ $\begin{array}{lllllll}30 & 2322 & 11.78 & 19 & 20.65 & 155 & 18.50 \\ 30 & 2328 & 57.70 & 19 & 21.05 & 155 & 4.28\end{array}$

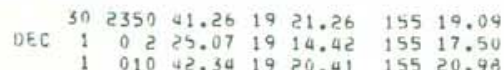

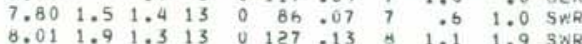

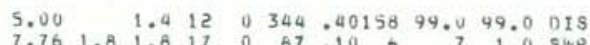

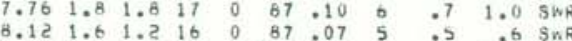

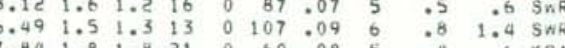
$\begin{array}{lllllllllll}6.39 & 2.3 & 1.7 & 15 & 0 & 106 & .15 & \text { B } & 1.2 & 2.7 & \text { UER }\end{array}$

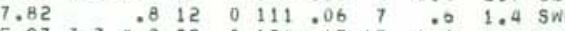

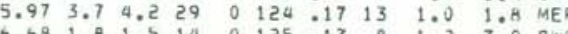
$\begin{array}{lllllllllll}6.68 & 1.8 & 1.5 & 14 & 0 & 125 & .13 & 8 & 1.2 & 3.0 & \text { SNR } \\ 9.55 & 1.7 & 1.7 & 13 & 0 & 92 & .09 & 7 & 1.0 & .5 & \text { SWR }\end{array}$ $\begin{array}{llllllllll}2.12 & 2.3 & 2.4 & 21 & 0 & 186 & .16 & 10 & 1.5 & 2.7 \\ 0.0 & .3 & .3\end{array}$

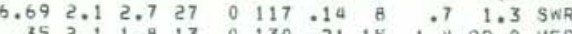

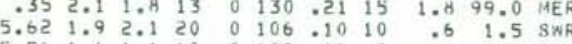
$\begin{array}{rrrrrrrrrrr}4.95 & 1.6 & .9 & 13 & 1 & 187 & .11 & 8 & 2.2 & 2.1 & P O L \\ 2.93 & 1.4 & 1.7 & 19 & 0 & 81 & .13 & 0 & .6 & 1.7 & K O A\end{array}$

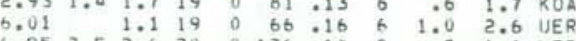

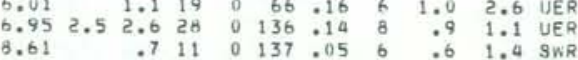

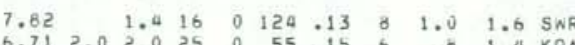

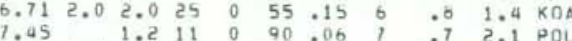
$\begin{array}{lllllllllll}.12 & 1.7 & 1.7 & 17 & 0 & 88 & .21 & 7 & 1.7 & 5.4 & 64\end{array}$

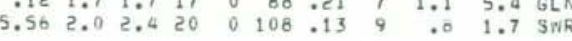
$\begin{array}{llllllllll}7.46 & .9 & 13 & 0 & 62 & .07 & 7 & .8 & .9 & \text { UER }\end{array}$

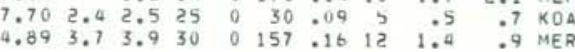
$\begin{array}{llllllllll}5.92 & 1.3 & 17 & 0 & 136 & .12 & 9 & 1.0 & 2.1 & \text { SNR }\end{array}$

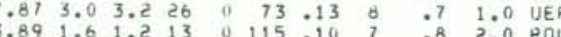

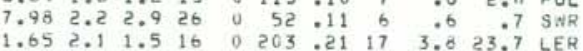
$\begin{array}{llllllllllllll}6.66 & 1.7 & 1.7 & 20 & 0 & 120 & .13 & \text { g } & .9 & 5.2 & \text { SNP }\end{array}$ $\begin{array}{lllllllllll}0.78 & 1.7 & 1.3 & 19 & 0 & 68 & .14 & 5 & .9 & 1.5 & \mathrm{k0}\end{array}$ $6.581 .51 .117 \quad 0 \quad 82.12, \quad 991.2$ KOA $\begin{array}{rrrrrrrrrrr}7.47 & .9 & .9 & 11 & 0 & 153 & -13 & 6 & 1.7 & 3.2 & \text { KOA } \\ 5.94 & 2.4 & 2.1 & 21 & 0 & 91 & .15 & 10 & 1.0 & 1.7 & \text { MER }\end{array}$ $\begin{array}{lllllllllllllll}1.46 & 1.0 & 11 & 0 & 83 & .08 & 0 & .6 & 99.0 & \text { SWR }\end{array}$

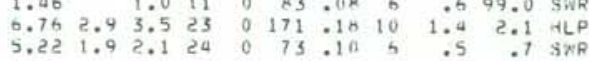

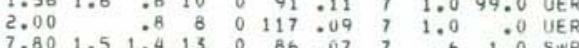
$\begin{array}{llllllllll}.07 & 1.5 & 15 & 0 & 76 & .07 & 8 & .6 & 1.0 & \text { UER }\end{array}$

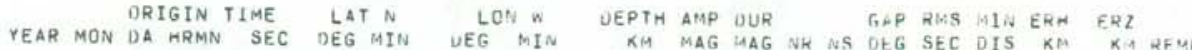

$\begin{array}{llllll}011 & 34.46 & 19 & 19.87 & 155 & 20.10 \\ 013 & 15.88 & 19 & 19.98 & 155 & 21.12\end{array}$

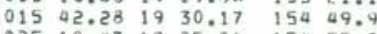
$\begin{array}{llllll}025 & 10.47 & 19 & 25.56 & 154 & 55.2 \\ 025 & 55.30 & 19 & 21.36 & 155 & 19.1\end{array}$

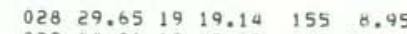

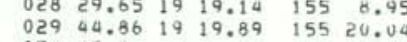

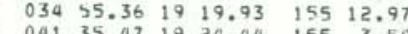

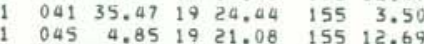

$\begin{array}{lllll}046 \quad 15.57 \quad 19 & 16.87 \quad 155 \quad 21.88\end{array}$ $05128.031922 .42 \quad 155 \quad 3.69$

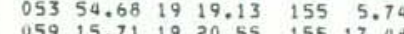

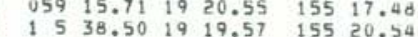

$\begin{array}{lll}17 & 15.9919 & 17.84 \quad 155 \quad 19.76\end{array}$ $1748.74 \quad 1920.79 \quad 155 \quad 19.64$ $1832.701920 .18 \quad 15520.60$

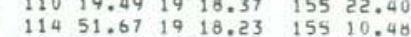

$1 \quad 120 \quad 4.501917 .47 \quad 15521.75$

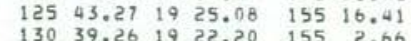

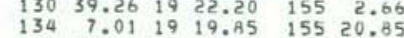
$\begin{array}{lllll}136 & 36.16 \quad 19 & 20.24 & 155 \quad 17.16\end{array}$

$\begin{array}{llllll}137 & 36.59 & 17 & 19.29 & 155 & 11.73 \\ 139 & 23.19 & 19 & 19.95 & 155 & 7.85\end{array}$

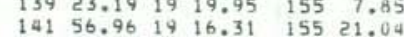

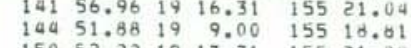

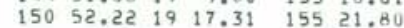

$\begin{array}{lllllll}151 & 52.38 & 19 & 24.23 & 155 & 13.41 \\ 153 & 57.25 & 19 & 18.67 & 155 & 13.44\end{array}$ $15357.251918 .67 \quad 15513.44$ $15943.231921 .11 \quad 15514.07$

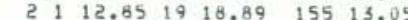
$\begin{array}{lllllll}2 & 1 & 53.95 & 19 & 20.67 & 155 & 8.60\end{array}$

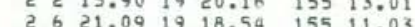

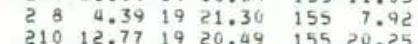
$\begin{array}{lllll}219 & 31.10 & 19 & 21.65 \quad 155 \quad 16.41\end{array}$ $22547.901920 .44 \quad 15519.08$

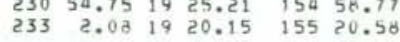
$\begin{array}{ll}235 & 58.35 \quad 19 \quad 18.90 \quad 155 \\ 21.25\end{array}$

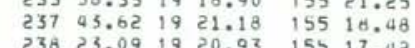
$\begin{array}{rrrrrrrrrrr}8.38 & 1.6 & .9 & 16 & 0 & 97 & -10 & 6 & .1 & 1.0 & \text { SAR } \\ 4.23 & 1.5 & 1.2 & 11 & 0 & 100 & 05 & 7 & 0 & 1.0 & 0\end{array}$

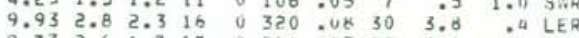

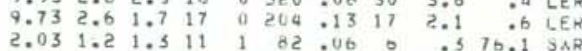
$6.81 \quad 1.112 \quad 0 \quad 92.06 \quad 9 \quad .6 \quad 1.5$ UEH

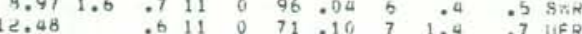
$\begin{array}{rrrrrrrrrr}.25 & 3.1 & 3.9 & 18 & 0 & 108 & .22 & 12 & 1.5 & 98.0 \\ 8.12 & 1.9 & 1.9 & 26 & 0 & 61 & .14 & 6 & .7 & .9\end{array}$ $\begin{array}{llllllllllll}7.75 & 2.5 & 3.3 & 29 & 0 & 129 & .14 & 8 & .8 & 1.0 & \text { SNR }\end{array}$ \begin{tabular}{llllllllll}
5.63 & 2.3 & 2.3 & 18 & 0 & 158 & .12 & 12 & .0 & 1.0 \\
\hline
\end{tabular} $\begin{array}{llllllll}1.50 & .9 & 11 & 0 & 156 & .13 & 9\end{array}$ $\begin{array}{lllllllllll}7.32 & .5 & 10 & 0 & 94 & .04 & 5 & .5 & 1.1 & K 0 A \\ 8.46 & 1.8 & 2.1 & 23 & 0 & 83 & .14 & 6 & .8 & 1.0 & \text { S } \\ 7.4 R\end{array}$ $\begin{array}{llllllllll}7.15 & 1.3 & \geq 0 & 0 & 125 & .14 & 8 & .9 & 1.4 & 5 \times R\end{array}$ $\begin{array}{llllllllll}2.45 & .9 & 7 & 1 & 99 & .06 & 6 & .5 & 12.2 & 504\end{array}$

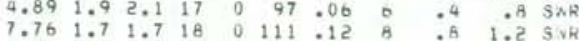
7.57 1.6 1.2 12 00158.13 1. $\begin{array}{lllllllll}7.66 & 1.819 & 0 & 123 & 16 & \text { y } & 1.5 & 1.4 & 5 \times 1\end{array}$

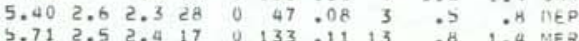

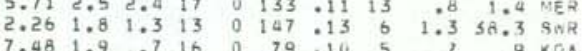

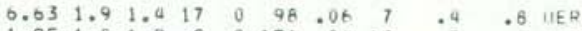

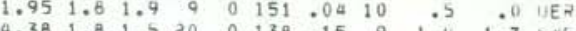

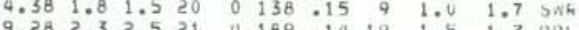

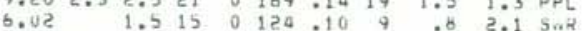

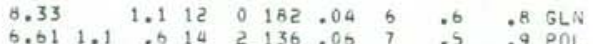

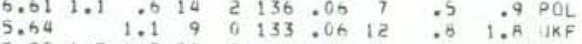

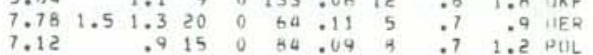
$\begin{array}{lllllllllll}6.84 & 1.5 & 1.1 & 17 & 0 & 72 & .15 & 9 & 1.1 & 1.9 & \text { UER }\end{array}$

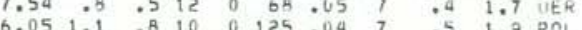

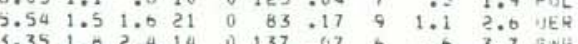

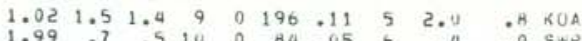

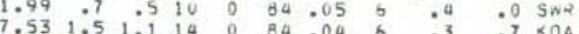

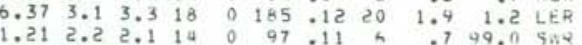
$\begin{array}{lllllllllll}5.78 & 1.6 & 1.6 & 24 & 0 & 103 & .10 & 7 & .4 & 5.5 & 5 N\end{array}$

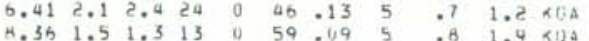




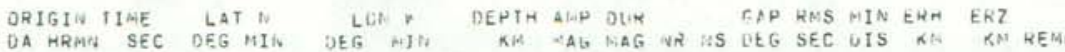

1975 DEC $1238 \quad 46.021914 .94 \quad 15521.20$

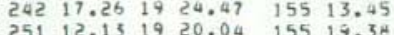
$\begin{array}{llllll}252 & 12.77 & 19 & 24.20 & 154 & 59.18\end{array}$ $25458.13 \quad 1919.41 \quad 155 \quad 12.34$

$\begin{array}{llllll}32 & 32.26 \quad 19 & 21.00 \quad 155 & 17.03\end{array}$

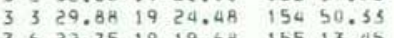
$19.68 \quad 155 \quad 13.45$

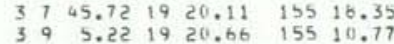
$\begin{array}{lllll}323 & 52.27 \quad 19 & 17.99 \quad 155 & 21.20\end{array}$ $\begin{array}{llllll}325 & 55.53 & 19 & 17.33 & 155 & 21.24\end{array}$

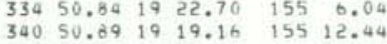
$\begin{array}{llllll}346 & 34.84 & 19 & 18.19 & 155 & 21.17\end{array}$

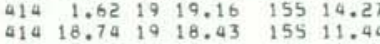
10.06 ins 19.09

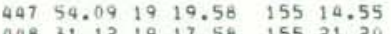

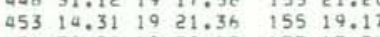
$\begin{array}{lllll}4565 & 50.98 & 1920.89 & 155 & 13.73\end{array}$

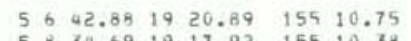

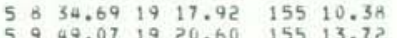

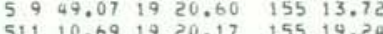

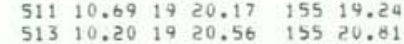

$\begin{array}{llllll}516 & 03.07 & 19 & 20.62 & 155 & 20.01 \\ 517 & 19.81 & 19 & 16.82 & 155 & 11.58\end{array}$ 5टa $97.101916 .94-15521.85$ $533 \quad 12.18 \quad 19 \quad 14.32 \quad 15516.42$ $\begin{array}{rrrrrr}534 & 55.44 & 19 & 23.89 & 154 & 56.83 \\ 540 & 1.58 & 19 & 17.56 & 155 & 21.19\end{array}$ $54549.241920 .54 \quad 15516.35$ 546
54859.78

$\begin{array}{llllll}556 & 37.17 & 19 & 20.67 & 155 & 17.52\end{array}$

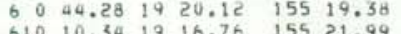

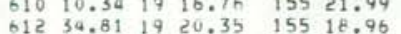
$\begin{array}{llll}613 & 42.15 \quad 19 & 19.04 & 155\end{array} 1.12$

$\begin{array}{llllll}618 & 58.58 & 19 & 12.69 & 155 & 23.72\end{array}$

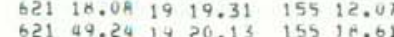

$\begin{array}{lllllllllllll}0.86 & 1.9 & 1.8 & 14 & 0 & 101 & .13 & 7 & 1.0 & 2.5 & \text { SWE }\end{array}$

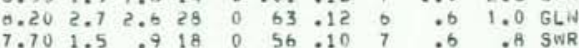
5.211 .61 .212 o $88.03, .3$.6 UER $\begin{array}{lllllllllll}7.20 & 1.7 & 1.8 & 15 & 0 & 64 & .08 & 5 & .5 & 1.3 & \text { KOA }\end{array}$

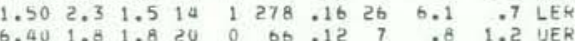
$\begin{array}{rrrrrrrrrrr}7.12 & .8 & 1.3 & 11 & 0 & 84 & .05 & 0 & .5 & 1.2 & \text { KOA } \\ 7.61 & 2.0 & 2.3 & 15 & 0 & 109 & .05 & 8 & \text {.5 } & 1.0 & \text { UER }\end{array}$ $\begin{array}{llllllllllllllllll}7.50 & 1.2 & 13 & 0 & 119 & .08 & 8 & .7 & . \text {. A SWR }\end{array}$

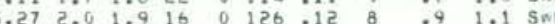
$\begin{array}{llllllllll}0.93 & 9 & 13 & 0 & 92 & .11 & 7 & 1.0 & 1.9 & 9 \text { UER }\end{array}$ $\begin{array}{lllllllllllllll}7.68 & 1.9 & 1.5 & 16 & 0 & 117 & .11 & 7 & .8 & 1.3 & \text { SWR } \\ .15 & 2.4 & 3.5 & 12 & 0 & 150 & .19 & 12 & 2.0 & 53.9 & \text { LEF }\end{array}$

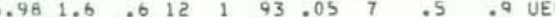

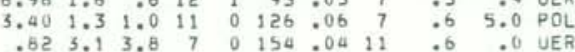
$\begin{array}{lllllllllll}7.45 & 2.2 & 1.5 & 25 & 0 & 71 & .09 & 6 & .5 & .8 & \text { UER }\end{array}$ $\begin{array}{rrrrrrrrrr}.65 & 1.3 & 10 & 0 & 82 & .06 & 6 & .5 & 1.7 & \text { SWR } \\ 5.61 & .7 & 10 & 0 & 104 & .04 & 7 & .4 & 1.3 & \text { UER }\end{array}$

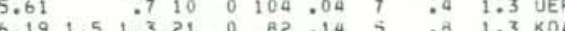
$\begin{array}{llllllllllll}7.58 & 1.7 & 1.5 & 16 & 0 & 107 & .06 & 0 & .6 & .7 & \text { UER }\end{array}$

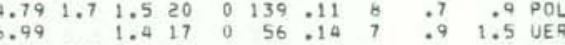

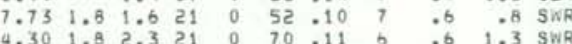
$\begin{array}{lllllllllll}3.58 & 1.7 & 1.2 & 18 & 3 & 116 & .14 & 6 & .8 & 3.4 & \text { SWh }\end{array}$

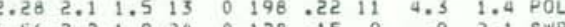

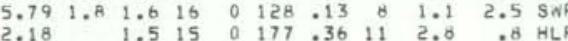
$\begin{array}{lllllllllll}6.62 & 2.7 & 2.3 & 24 & 0 & 178 & 15 & 15 & 1.4 & 1.1 & \text { LER }\end{array}$

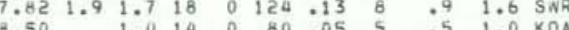

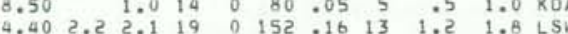
5.791 .8 .914 o 101.11 \& 1.2 3.8 4ER $\begin{array}{lllllllllllll}8.54 & 2.1 & 2.1 & 25 & 0 & 36 & .12 & 9 & .6 & .6 & \times 0 A\end{array}$

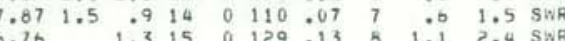

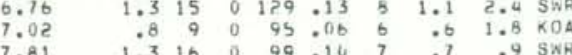
$\begin{array}{llllllllll}36 & 2.2 & 2.5 & 18 & 0 & 162 & .16 & 13 & 1.4 & 1.7 \quad \text { LS }\end{array}$ $\begin{array}{lllllllllll}5.9 \mathrm{~B} & 2.4 & 1.9 & 14 & 0 & 170 & 16 & 14 & 1.7 & 2.9 & \text { LER }\end{array}$ $\begin{array}{llllll}.112 .62 .7 & 23 & 71 & .16 & 10 & 0\end{array}$

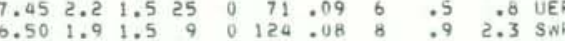

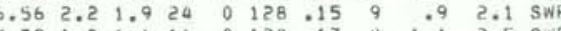
$\begin{array}{rrrrrrrrrrr}5.36 & 2.2 & 2.5 & 18 & 0 & 162 & .16 & 13 & 1.4 & 1.7 & \text { LS } \\ 3.41 & .7 & 11 & 0 & 90.0 \% & 0 & .7 & 5.7 & \text { UEE }\end{array}$
HVO EARTHGUAKE SUMMAKY LISI

PA6t 118

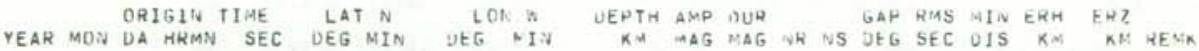

1975 DEC 1 626 $4.53 \quad 19 \quad 20.08 \quad 155 \quad 10.29$

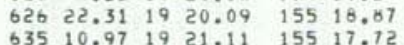
$63726.621924 .71 \quad 1553.21$

$648 \quad 12.71 \quad 1920.19 \quad 15520.26$

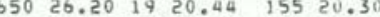

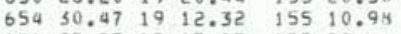

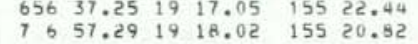
$\begin{array}{llllll}79 & 35.60 & 19 & 8.79 \quad 155 & 16.35\end{array}$

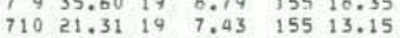
$\begin{array}{llllll}714 & 42.20 & 19 & 19.07 & 155 & 21.07\end{array}$

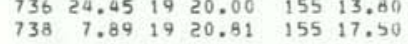
$\begin{array}{llllll}738 & 51.74 & 19 & 21.39 & 155 & 19.16\end{array}$ $\begin{array}{llllll}740 & 8.59 & 19 & 21.04 & 155 & 19.47\end{array}$

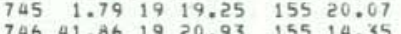

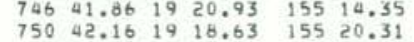

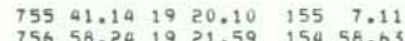
$\begin{array}{llllll}756 & 58.24 & 19 & 21.59 & 154 & 58.63\end{array}$ $\begin{array}{rrrrrr}759 & 15.72 & 19 & 17.42 & 155 & 21.41 \\ 86 & 8.19 & 19 & 24.39 & 155 & 2.92\end{array}$

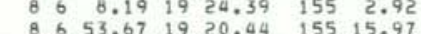

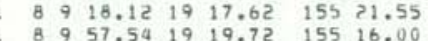

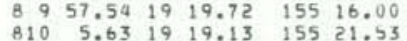
$\begin{array}{lllll}813 & 5.63 & 19 & 19.13 & 155 \\ 816 & 19 & 17.16 & 155 & 22.07\end{array}$

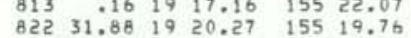

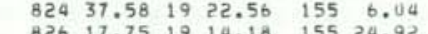

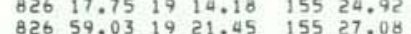

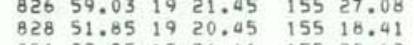
$\begin{array}{llll}22.95 \quad 19 & 26.41 \quad 155 \quad 28.62\end{array}$

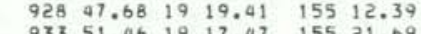
93331.461917 .47 155 21.09 103955.20
103548.35
19 $1127 \quad 10.91 \quad 19 \quad 9.25 \quad 15520.08$

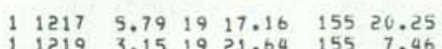
$1219 \quad 3.151921 .64$ 155 7.46 $\begin{array}{llllll}1247 & 29.56 & 19 & 17.90 & 155 & 21.07\end{array}$ \begin{tabular}{l}
$124930.84 \quad 1920.05 \quad 155 \quad 16.13$ \\
\hline
\end{tabular} $\begin{array}{lllllll}1 & 1352 & 58.41 & 19 & 21.70 & 155 & 15.30\end{array}$ $153925.30 \quad 19 \quad 17.79 \quad 15524.90$

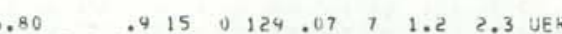

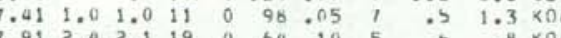
6.991 .91 .620 O $80: 16 \quad 7 \quad 1.1198$ UER $\begin{array}{lllllllllll}4.88 & 1.5 & 1.4 & 14 & 0 & 69 & .11 & 5 & .0 & 1.5 & \text { SWR }\end{array}$

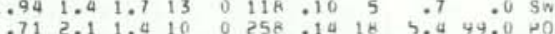

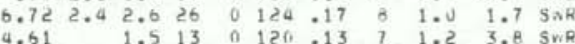
$\begin{array}{lllllllllllllll}4.38 & 2.7 & 2.6 & 52 & 0 & 191 & .12 & 17 & 1.3 & .4 & \mathrm{PPL}\end{array}$

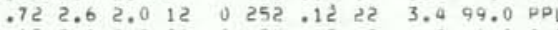

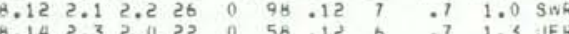

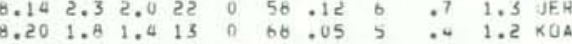
$\begin{array}{llllllllllll}1.51 & 1.5 & 1.7 & 13 & 0 & 62 & .09 & 6 & .5 & .0 & \text { SWR }\end{array}$

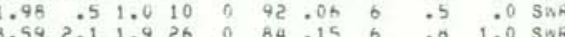

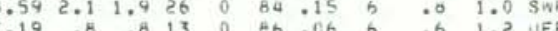

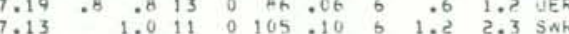

$\begin{array}{llllllllllllll}7.43 & 2.0 & 1.7 & 16 & 0 & 103 & .11 & 7 & 1.0 & 1.5 & \text { UER }\end{array}$

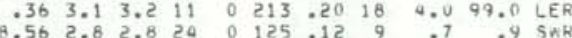

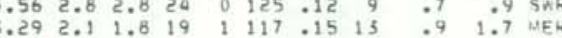
$5.952 .62 .5240114 .150_{0} 01.3 \times 00$ $\begin{array}{llllllllll}7.54 & 1.5 & 16 & 0 & 125 & .12 & \text { y } & 1.0 & 5.4 & \text { Sn }\end{array}$

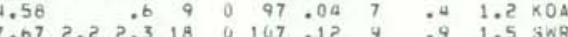
$6.582 .52 .629 \quad 0125.15$ \& $.8 \quad 1.6$ SWR $\begin{array}{lllllllllll}.21 & 3.2 & 3.2 & 22 & 0 & 116 & .11 & 10 & .7 & 1.0 & \text { UER } \\ .17 & 1.5 & 1.2 & 11 & 0 & 136 & .08 & 11 & .8 & 1.7 & \text { LS }\end{array}$

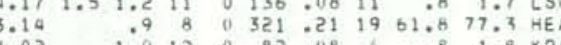

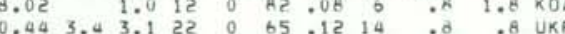

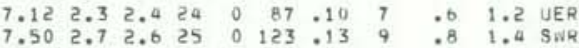

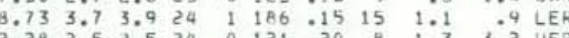

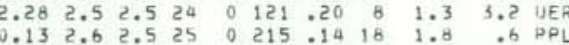

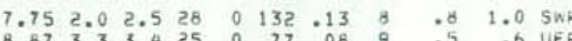
8.673 .33 .425 o 77.08 a 52.06 Uea

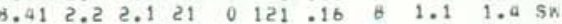

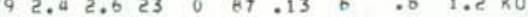
$\begin{array}{lllllllllll}8.11 & 1.9 & 2.1 & 23 & 0 & 61 & .13 & 4 & .7 & 1.1 & \text { KOA }\end{array}$

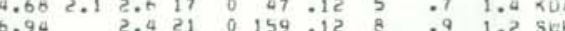


$\begin{array}{llllllllll}8.21 & 3.0 & 3.1 & 19 & 0 & 171 & .16 & 14 & 1.9 & 1.4 \\ 0.05 R\end{array}$

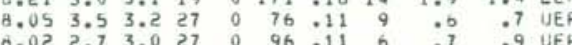

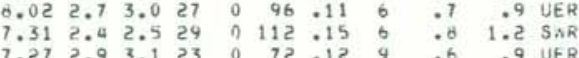

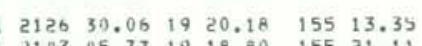

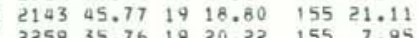

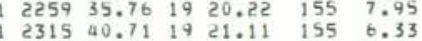
1233534.611924 .0515524 .26

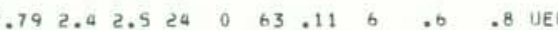
9.978 .

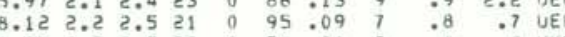
$\begin{array}{lllllll}1 & 2337 & 28.83 & 19 & 20.14 & 155 & 19.10\end{array}$ 0
5 $04025.191922 .40 \quad 155 \quad 6.71$

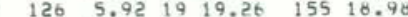
$129 \quad 50.30 \quad 19 \quad 20.14 \quad 155 \quad 6.69$

$\begin{array}{lllllll}140 & 49.39 & 19 & 19.66 & 155 & 6.91\end{array}$ $\begin{array}{llllll}322 & 37.03 & 19 & 18.57 & 155 & 23.91 \\ 336 & 55.90 & 14 & 15.32 & 155 & 12.35\end{array}$

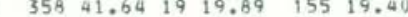

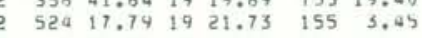

$\begin{array}{lllllll}2 & 545 & 55.77 & 19 & 27.21 & 154 & 52.32\end{array}$ $\begin{array}{lllllll}2 & 724 & 5.86 & 19 & 16.67 & 155 & 20.82\end{array}$ $\begin{array}{rrrrrr}753 & .50 & 19 & 16.40 & 155 & 11.64 \\ 756 & 53.03 & 19 & 20.33 & 155 & 9.12\end{array}$ $\begin{array}{llllll}1011 & 19.33 & 19 & 22.50 & 155 & 1.13\end{array}$

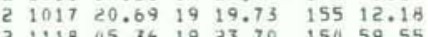
$\begin{array}{lllllll}1118 & 45.36 & 19 & 23.70 & 154 & 59.55\end{array}$

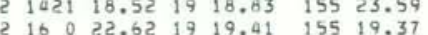

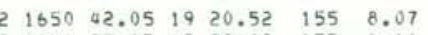

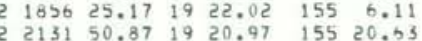

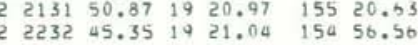

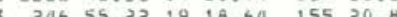

$\begin{array}{lllllll}3 & 343 & 57.01 & 19 & 19.97 & 155 & 11.88 \\ 3 & 430 & 20.82 & 19 & 20.43 & 155 & 12.70\end{array}$

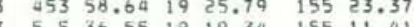

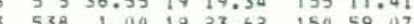
$\begin{array}{lllllll}3 & 731 & 45.30 & 19 & 17.21 & 155 & 21.74 \\ 3 & 749 & 25.08 & 19 & 24.18 & 155 & 24.41\end{array}$

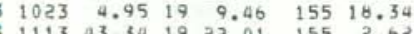
$3111923.04 \quad 1922.06 \quad 15456.97$ $\begin{array}{lllllll}3 & 1147 & 57.93 & 19 & 17.33 & 155 & 21.8\end{array}$

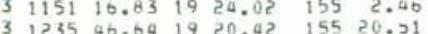

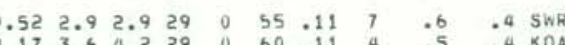

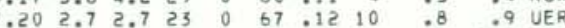

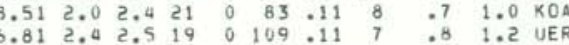

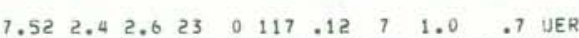

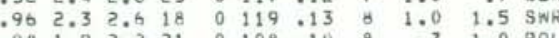
$\begin{array}{lllllllll}9.04 & 1.9 & 2.2 & 21 & 0 & 108 & .10 & 8 & 0\end{array}$

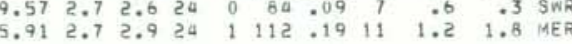
$9.95 \quad 3.1 \quad 3.125 \quad 0 \quad 265 \quad .16253 .1 \quad .5$ LER

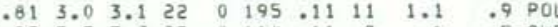

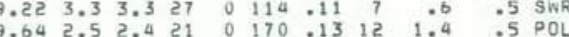
.

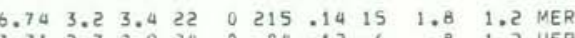

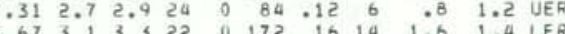

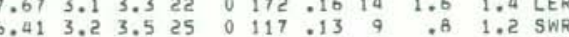

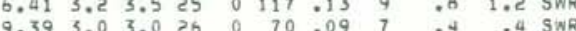

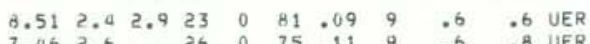

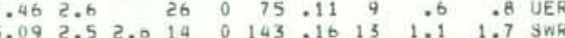

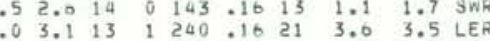
3.

$\begin{array}{rrrrrrrrrrr}45 & 2.9 & 3.1 & 16 & 0 & 169 & -12 & 10 & 1.3 & 1.0 & \text { UER } \\ 7.51 & 2.8 & 3.0 & 18 & 0 & 191 & -15 & 8 & 1.8 & 1.3 & \text { UER }\end{array}$ 9.843 .53 .4 ट1 0 47 013 10 10.8 .5 UKF

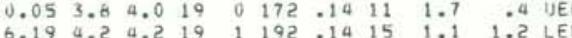
$6.612 .3 \quad 2.6 \quad 18 \quad 0 \quad 157 \quad .15 \quad 18 \quad 1.4 \quad 1.9$ SNR

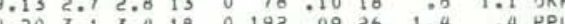
$0.475 .020 .919 \quad 0 \quad 176: 1913$ 1.8 2.5 UER

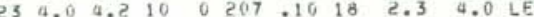
$\begin{array}{lllllllllll}.58 & 1.7 & 2.4 & 19 & 0 & 168 & 0.15 & 9 & 1.4 & 2.4 & \text { Sw. }\end{array}$

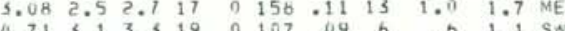

HVO EARTHGUUAKE SUMMARY LIST

PAGE 120 DRIGIN TIME LATN LON K OEPTH AMP OUN GAP KHA AIN ERH EHZ

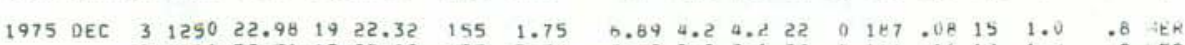

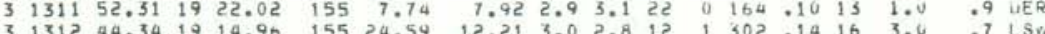

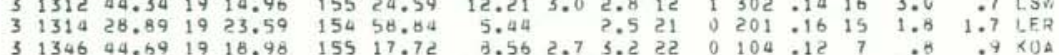

$\begin{array}{lllllll}3 & 1529 & 1.47 & 19 & 19.73 \quad 155 \quad 17.46\end{array}$ $3 \quad 1748 \quad 26.591923 .74 \quad 15458.20$

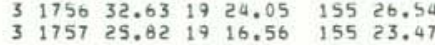

$\begin{array}{lllllll}31851 & 9.58 & 19 & 21.90 \quad 155 & 15.04\end{array}$

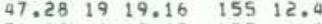

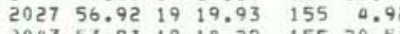

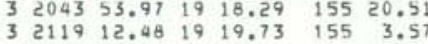

$\begin{array}{lllllll}3 & 2244 & 55.49 & 19 & 19.77 \quad 155 & 12.72\end{array}$ $\begin{array}{llllll}32311 & 16.00 & 19 & 20.42 & 155 & 20.57\end{array}$ $4 \quad 02955.04 \quad 1920.51 \quad 15520.44$

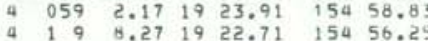

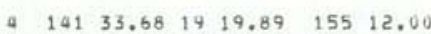

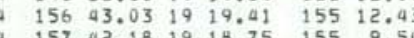

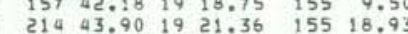
$43053.321921 .58 \quad 15457.66$

$\begin{array}{llllll}450 & 4.91 & 19 & 22.72 & 155 & 6.44\end{array}$

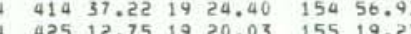

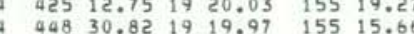

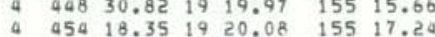

$\begin{array}{lllllll}4 & 537 & 55.42 & 19 & 22.00 & 155 & 6.97\end{array}$ \begin{tabular}{l}
$548 \quad 50.091920 .42 \quad 15520.45$ \\
\hline
\end{tabular}

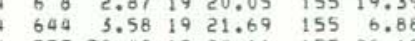

\begin{tabular}{l}
$747 \quad 12.59 \quad 19 \quad 17.29 \quad 155 \quad 22.0$ \\
\hline 59
\end{tabular}

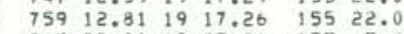

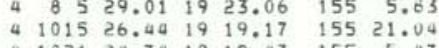
$\begin{array}{ll}1039 & 0.47 \quad 19 \quad 19.77 \quad 155 \quad 5.39\end{array}$ $\begin{array}{rrrrrrr}4 & 1029 & 8.47 & 19 & 19.77 & 155 & 5.59 \\ 4 & 1035 & 11.25 & 19 & 23.63 & 154 & 56.99\end{array}$ $4104010.051924 .87 \quad 15456.89$

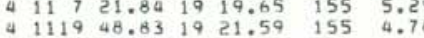
4 $1139 \quad 10.05 \quad 1921.01 \quad 154 \quad 57.79$ 4 1238 51.09 19 19.03 155 13.00 6.173 .03 .12300193 .17 15 199 1. H LER

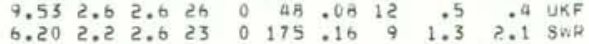
$\begin{array}{lllllllllll}1.93 & 1.8 & 2.3 & 20 & 0 & 58 & .11 & 4 & .7 & .9 & \text { KOA }\end{array}$

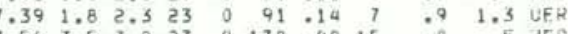
$\begin{array}{lllllllllll}7.56 & 3.5 & 3.8 & 23 & 0 & 178 & 0.08 & 15 & .6 & .5 & \text { IER }\end{array}$

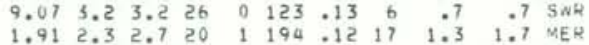

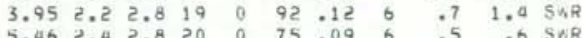

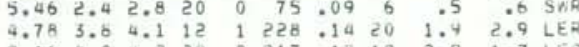

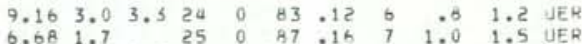

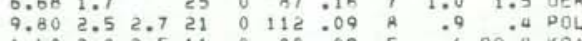

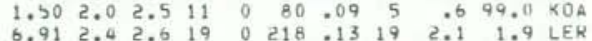
$\begin{array}{llllllllllllll}7.17 & 2.9 & 3.1 & 25 & 0 & 166 & .13 & 14 & 1.2 & .9 & \text { LER } \\ 7.55 & 2.8 & 3.0 & 26 & 1 & 199 & 22 & 16 & 3.0 & 2.0 \text { LER }\end{array}$

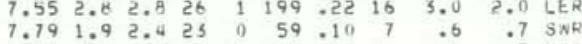

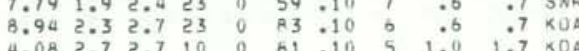
$\begin{array}{rrrrrrrrrrrr}6.44 & 2.2 & 2.7 & 22 & 0 & 130 & .11 & 11 & .8 & 1.5 & \text { UER } \\ 4.38 & 1.9 & 2.5 & 15 & 0 & 91 & -10 & 6 & .6 & 1.0 & \text { SWR }\end{array}$

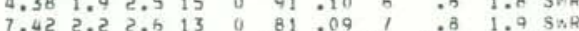

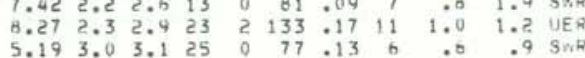
$\begin{array}{llllllllllll}6.82 & 1.8 & 2.4 & 25 & 0 & 131 & .15 & 8 & 1.0 & 1.6 & 3 \times .8\end{array}$

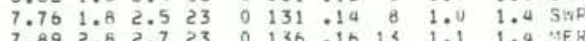
8.55
5.62 $\begin{array}{llllllllll}5.38 & 3.1 & 19 & 0 & 178 & 11 & 15 & 1.1 & 1.0 & \text { AE }\end{array}$ $\begin{array}{lllllllllll}.90 & 2.5 & 3.0 & 20 & 1 & 183 & .12 & 14 & 1.1 & 1.3 & \text { MER }\end{array}$

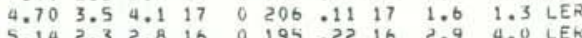

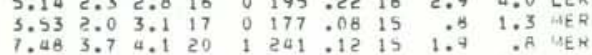

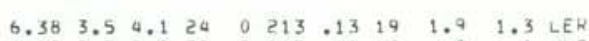

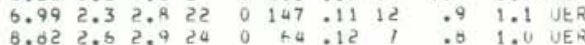

$\begin{array}{rllllllllllll}7.69 & 1.9 & 2.3 & 15 & 0 & 86 & .67 & 6 & .6 & 1.2 & K 0 A\end{array}$ $\begin{array}{lllllllllll}6.07 & 1.6 & 2.3 & 21 & 0 & 76 & .16 & 7 & 1.0 & 2.2 & \text { JER }\end{array}$ 


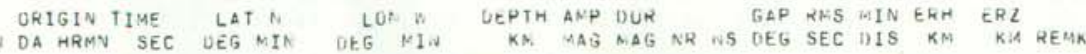

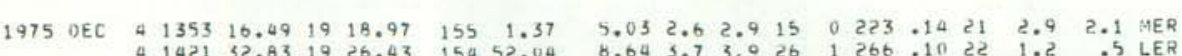

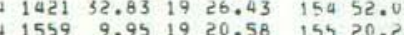

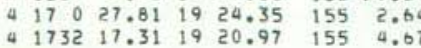

$41754 \quad 42.35 \quad 19 \quad 17.52 \quad 155 \quad 22.10$

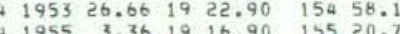
$420949.571921 .46 \quad 155$ 8. $421431.531922 .60 \quad 1550.17$

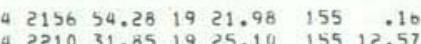
$4232011.07 \quad 1920.26 \quad 155 \quad 18.6$

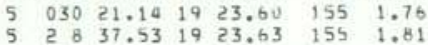

$\begin{array}{lllllll}5 & 214 & 12.65 & 19 & 20.64 & 155 & 7.17\end{array}$ 5 256 $21.021923 .58 \quad 155 \quad 16.96$ 526
716 9.841919 .68 155 7.87

$\begin{array}{lllllll}5 & 1099 & 8.54 & 14 & 22.01 \quad 155 \quad 15.14\end{array}$

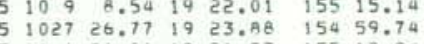

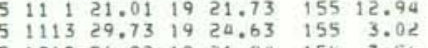

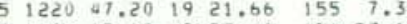
$\begin{array}{llllll}5 & 1226 & 17.42 & 19 & 23.41 & 154 \quad 53.19\end{array}$

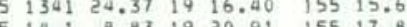
5 1421 $15.8319 \quad 17.92 \quad 15521.0$

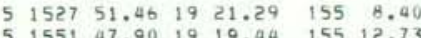

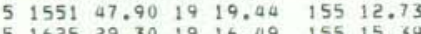
$5 \quad 161155.16 \quad 19 \quad 17.36 \quad 155 \quad 21.30$ 5 18ट2 22.04 $19 \quad 17.16 \quad 15521.67$ $\begin{array}{lllllll}5 & 1851 & 59.56 & 19 & 17.32 & 155 & 21.42\end{array}$ $519832.06 \quad 1916.45 \quad 15521.91$ $5202540.821920 .47 \quad 15451.77$

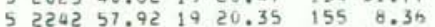
$\begin{array}{lllllll}5 & 2316 & 45.53 & 19 & 20.80 & 155 & 17.97\end{array}$

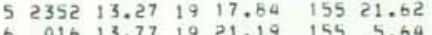
$6 \quad 032 \quad 16.851721 .51$ 155 8.78 $6 \quad 03 A 54.291921 .56 \quad 15 b \quad 8.51$

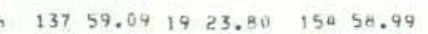
$\begin{array}{lllllll}6 & 218 & 13.71 & 19 & 20.62 & 155 & 7.32 \\ 6 & 229 & 18.92 & 19 & 19.92 & 155 & 12.95\end{array}$ $\begin{array}{llllllllllll}.33 & 2.0 & 2.7 & 13 & 0 & 113 & 0.10 & 6 & .6 & .0 & \text { SWR }\end{array}$

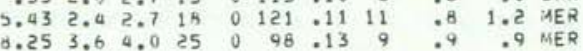
$\begin{array}{lllllllllll}7.39 & 2.6 & 3.2 & 26 & 0 & 121 & .17 & 8 & 1.1 & 1.5 & \text { SNR }\end{array}$ $\begin{array}{lllllllllll}.42 & 2.6 & 2.8 & 11 & 1 & 199 & .17 & 14 & 2.2 & 4.3 & \text { LER }\end{array}$ $\begin{array}{rlllllllllll}.47 & 1.8 & 2.6 & 24 & 0 & 133 & .15 & 8 & .9 & 1.7 & \text { SWR } & 0\end{array}$

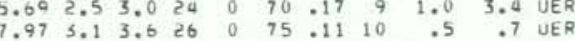
$\begin{array}{llllllllll}5.74 & 3.13 .127 & 0 & 189 & .12 & 16 & 1.2 & .9 \text { LER }\end{array}$

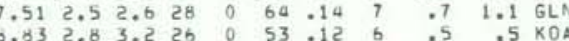

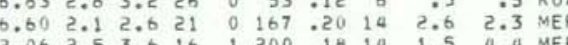

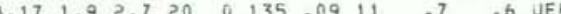

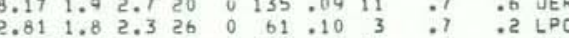
$\begin{array}{lllllllllll}8.23 & 2.6 & 3.1 & 25 & 0 & 185 & .13 & 13 & .9 & .9 & \text { POL } \\ 3.30 & 2.8 & 2.8 & 22 & 1 & 182 & .09 & 12 & 1.0 & .6 & \text { UER }\end{array}$

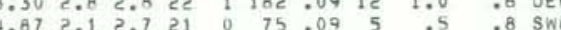
$\begin{array}{lllllllllllll}9.56 & 5.3 & 3.7 & 27 & 0 & 57 & .11 & 4 & .5 & .5 & K 0 A\end{array}$

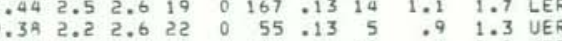

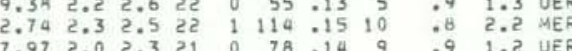
$\begin{array}{lllllllllll}7.71 & 3.1 & 3.3 & 25 & 0 & 75 & .07 & 8 & .4 & .6 & \text { UER }\end{array}$

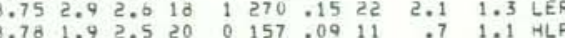

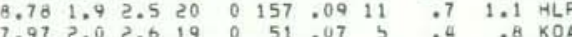

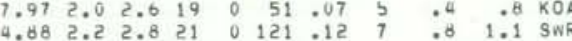
$\begin{array}{lllllllllll}8.53 & 2.1 & 2.5 & 20 & 0 & 85 & .10 & 9 & .7 & .6 & \text { UER }\end{array}$

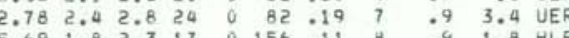

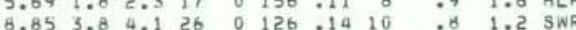

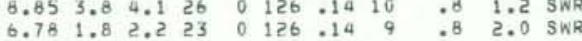
$\begin{array}{lllllllllll}5.81 & 2.0 & 2.7 & 23 & 0 & 126 & .11 & 9 & .7 & 1.8 & \text { SWR }\end{array}$

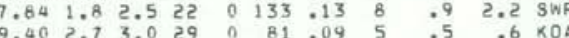

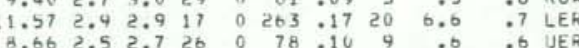
$\begin{array}{llllllllll}1.79 & 1.9 & 2.3 & 25 & 0 & 53 & .10 & 5 & .1 & .3 \\ \text { KOA }\end{array}$

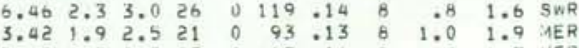

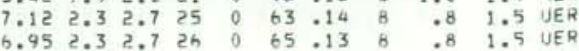

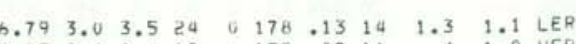

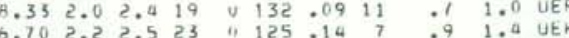

HVU EARTHGUAKE. SUINAGY LIST

PAGE 152

ORIGIN TIME LAT" LQN

1975 DEC $6 \quad 237 \quad 16.061918 .46 \quad 155 \quad 20.74$ 655
6 $250.211920 .41 \quad 155 \quad 7.84$ $\begin{array}{llll}6 & 353 & 25.27 & 19 \\ 6 & 16.88 & 154 & 55.10\end{array}$

$\begin{array}{llll}012 & 2.60 \quad 19 & 20.26 \quad 155 \quad 20.35\end{array}$

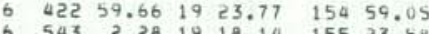
6432.281918 .14
6 15523.50

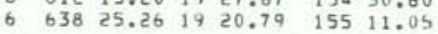

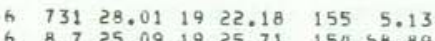

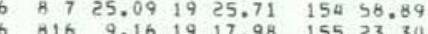
$84124.041921 .45 \quad 155 \quad 6.31$

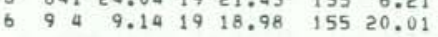

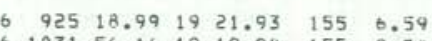

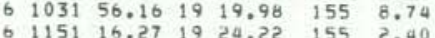

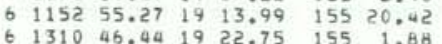
$\begin{array}{lllllll}6 & 1331 & 3.51 & 19 & 20.01 & 155 & 4.90\end{array}$ $\begin{array}{lllllll}6 & 1452 & 13.97 & 19 & 19.87 & 155 & 16.42\end{array}$ $\begin{array}{lllllll}6 & 150 & 55.46 & 19 & 20.29 & 155 & 20.40 \\ 6 & 1534 & 7.87 & 19 & 23.38 & 155 & 25.21\end{array}$ $\begin{array}{lllllll}6 & 1553 \quad 0.34 & 19 & 22.00 & 155 \quad 6.96\end{array}$

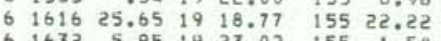
$\begin{array}{lllllll}6 & 1632 & 5.95 & 19 & 23.02 & 155 & 1.54\end{array}$ $\begin{array}{llrllll}6 & 1815 & 21.68 & 19 & 20.31 & 155 & 6.44 \\ 6 & 1817 & 3.67 & 19 & 17.89 & 155 & 21.61\end{array}$

$\begin{array}{llllllll}6 & 19 & 0 & 56.97 & 19 & 21.01 & 155 & 0.93\end{array}$ $\begin{array}{lllllll}6 & 1910 & 8.98 & 19 & 20.21 & 155 & 7.33\end{array}$

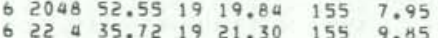

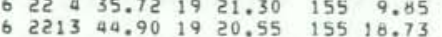

$\begin{array}{lllllll}6 & 2244 & 3.59 & 19 & 21.93 & 155 & 6.72\end{array}$

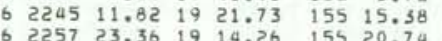

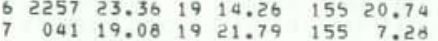

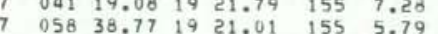

$\begin{array}{lllllll}7 & 145 & 18.58 & 19 & 28.06 & 155 & 23.00\end{array}$ $\begin{array}{llllll}238 & 42.05 & 1922.07 & 155 & 1.09 \\ 347 & 20.88 & 19 & 21.56 & 155 & 15.45\end{array}$

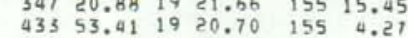

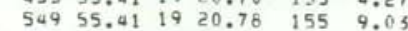

$\begin{array}{llllll}6 & 756.07 & 19 & 16.98 & 155 & 22.21 \\ 631 & 31.51 & 19 & 29.86 & 154 & 49.86\end{array}$ $\begin{array}{llllll}631 & 31.51 & 19 & 29.86 & 154 & 49.86 \\ 645 & 36.45 & 19 & 19.85 & 155 & 21.94\end{array}$

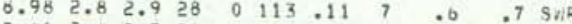

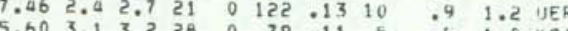

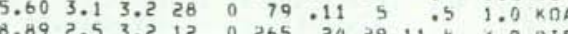

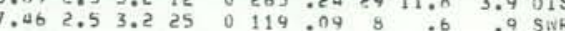
$\begin{array}{llllllllllll}4.57 & 2.1 & 2.6 & 15 & 0 & 93 & .06 & 5 & .5 & 1.5 & 3 n d\end{array}$

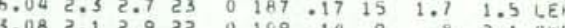

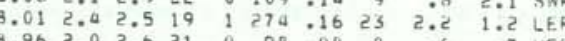

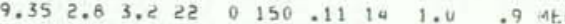

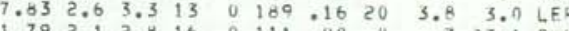

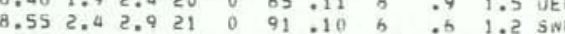

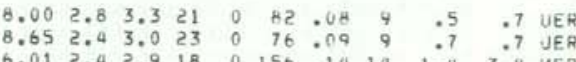

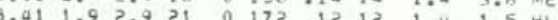
$7.433 .1 \quad 3.624 \quad 0 \quad 167.94 \quad 14 \quad 2.0 \quad 1.5$ Ne

$\begin{array}{llllllllllllll}7.10 & 1.8 & 2.3 & 18 & 0 & 102 & .08 & 9 & . b & 1.3 & 3 & \text { UER }\end{array}$

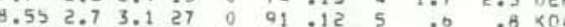
$\begin{array}{lllllllllll}4.26 & 2.0 & 2.6 & 15 & 0 & 93 & .08 & 5 & 0 & 1.4 & \text { SNH } \\ 9.22 & 2.2 & 2.7 & 25 & 0 & 56 & .11 & 10 & 0 & 9 & \text { UKF }\end{array}$

$\begin{array}{llllllllllll}7.35 & 2.3 & 3.1 & 25 & 0 & 73 & .11 & 9 & .7 & 1.2 & \text { IER }\end{array}$

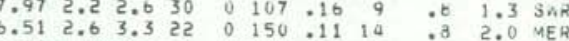
$\begin{array}{lllllllllll}1.07 & 1.9 & 2.5 & 21 & 0 & 109 & .08 & 7 & .5 & .9 & .9 \\ 8.24 & 2.5 & 3.1 & 27 & 0 & 119 & .13 & 0 & .7 & 1.2 & \text { SW }\end{array}$ $\begin{array}{lllllllllll}.66 & 2.3 & 2.6 & 23 & 0 & 73 & .11 & 8 & .6 & 1.0 & \text { UER }\end{array}$

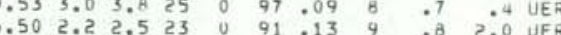

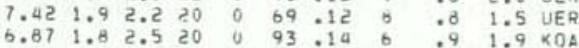

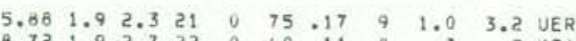

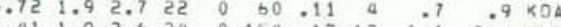
$\begin{array}{lllllllllll}.41 & 1.9 & 2.6 & 24 & 0 & 154 & .17 & 12 & 1.1 & 2.4 & \mathrm{ML}\end{array}$

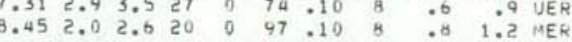
$\begin{array}{lllllllll}5.62 & 2.4 & 2.7 & 25 & 0 & 172 & .11 & 15 & .8 \\ 9.97 & 3.3 & 3.8 & 29 & 1.4 & \text { MER }\end{array}$

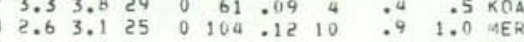

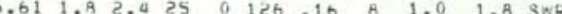

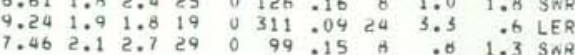

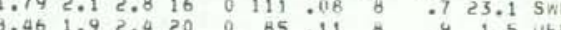

$\begin{array}{lllllllllll}.00 & 2.7 & 5.0 & 59 & 0 & 92 & .16 & 13 & .0 & 1.6 & \text { UKF }\end{array}$ 


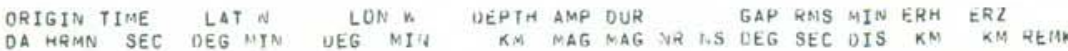

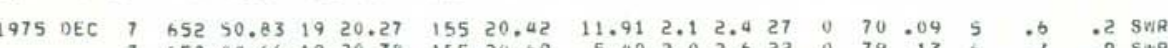

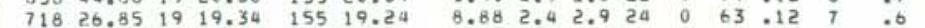

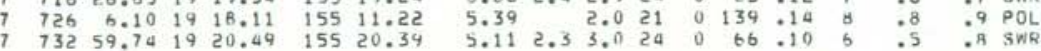

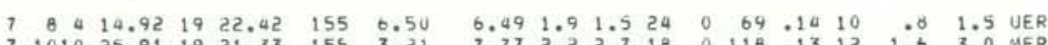

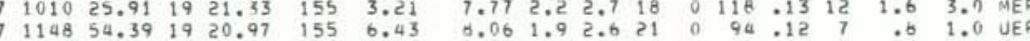

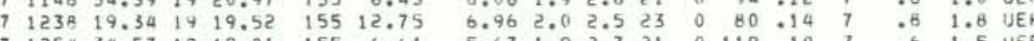
155 6.6

$\begin{array}{lllllll}7 & 139 & 22.46 \quad 19 & 16.47 \quad 155 & 12.11\end{array}$

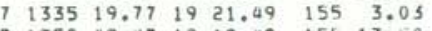

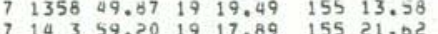

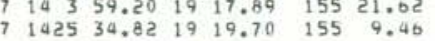
$\begin{array}{lllllll}7 & 1550 & 5.14 & 19 & 22.09 & 155 & 1.00\end{array}$

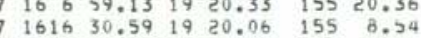

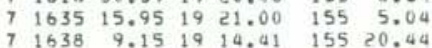

$\begin{array}{llllllll}7 & 17 & 9 & 2.07 & 19 & 21.33 & 155 & 15.59\end{array}$ $\begin{array}{llllll}17 & 92.73 & 19 & 18.16 & 155 & 20.17 \\ 1731 & 27.96 & 19 & 20.57 & 155 & 12.82\end{array}$

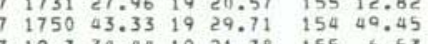

$\begin{array}{lllllll}7 & 1956 & 43.81 & 19 & 24.61 & 155 & 2.62 \\ 7 & 2040 & 50.30 & 19 & 21.77 & 155 & 3.24\end{array}$ $\begin{array}{lllllll}7 & 2040 & 50.34 & 19 & 21.77 & 155 & 3.28 \\ 7 & 2144 & 45.08 & 19 & 19.79 & 155 & 12.07\end{array}$

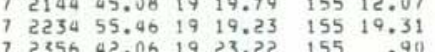

$\begin{array}{lllllll}8 & 027 & 43.54 & 19 & 19.04 & 155 & 19.90\end{array}$

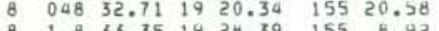

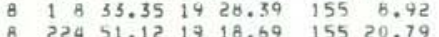
$\begin{array}{lllll}357 & 44.67 \quad 19 & 26.60 & 154 & 53.04\end{array}$

$\begin{array}{lllllll}\text { b } & 435 & 37.29 & 19 & 22.21 & 155 & 6.43 \\ \text { g } & 440 & 15.08 & 19 & 18.61 & 155 & 8.07\end{array}$ of $44015.08 \quad 19 \quad 18.61 \quad 155 \quad 8.07$

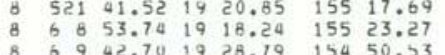

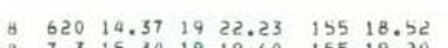
748 5.

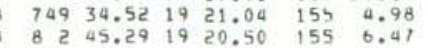

$8 \quad 829 \quad 9.15 \quad 19 \quad 22.50 \quad 155 \quad 6.03$
MVO EARTHGUAKE SUMMAKY LIST

PAGE 124 ORIGIN TIME LAT N LOH K DEPTH AMP DUR GAP RIMS MIN ERH EHZ YEAR MON DA HRMN SEC DEG MIN CEGG MIN

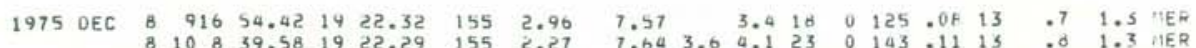

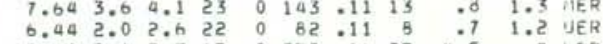

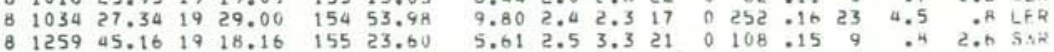

$8 \begin{array}{llll}1334 & 21.43 \quad 19 & 19.53 \quad 155 \quad 18.7\end{array}$ $81526 \quad 43.69 \quad 1919.95 \quad 155 \quad 6.90$

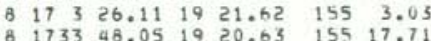
8. 175048.621918 .6915521 .91

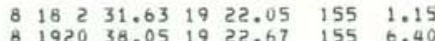

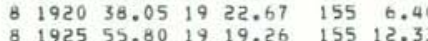

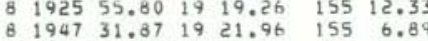
$403634.921919 .96 \quad 15519.57$

$82129 \quad .791919 .31 \quad 155 \quad 13.82$

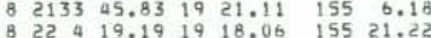

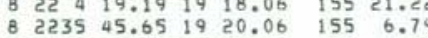
B. $2239 \quad 11.25 \quad 1723.16 \quad 155 \quad 4.50$

$9021 \quad 17.31 \quad 1924.21 \quad 15513.57$

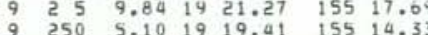
$9 \begin{aligned} & 254 \\ & 9 \\ & 4\end{aligned}$ \begin{tabular}{l}
$512 \quad 10.16 \quad 19 \quad 17.25 \quad 15521.9$ \\
\hline
\end{tabular}

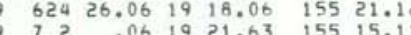
$72754.041919 .44 \quad 15512.39$ $744 \quad 35.73 \quad 19 \quad 19.42 \quad 155 \quad 12.49$ $\begin{array}{llllll}932 & 40.96 \quad 19 & 18.28 & 155 & 20.74\end{array}$ $\begin{array}{lllllll}10 & 1 & 21.81 & 19 & 21.69 & 155 & 16.4\end{array}$ $1018 \quad 8.341921 .40 \quad 155 \quad 7.25$ $104411.521921 .88 \quad 155 \quad 3.02$

$9131952.94 \quad 1920.91 \quad 155 \quad 6.46$

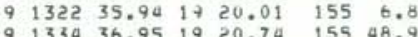

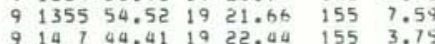

$9 \begin{aligned} & 911 \\ & 96.18 \quad 19 \quad 19.36 \quad 155 \quad 11.03\end{aligned}$

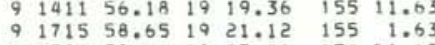
$9172639.16 \quad 1927.81 \quad 15451.65$

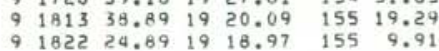

$9201020.29 \quad 19 \quad 19.68 \quad 15519.34$

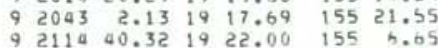

$\begin{array}{lllllllllll}9.05 & 2.3 & 3.1 & 25 & 0 & 58 & 11 & 7 & .6 & .7 & \text { KUA } \\ 4.84 & 3.4 & 4.0 & 21 & 0 & 145 & 10 & 12 & 99 & 1.0 & \text { UER }\end{array}$

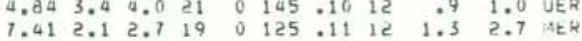

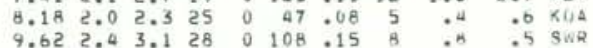

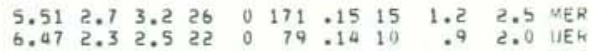

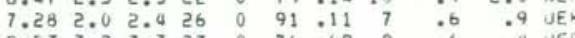

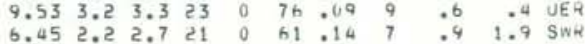

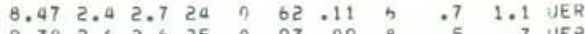

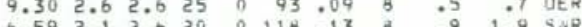

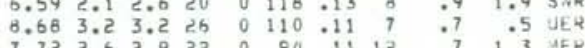

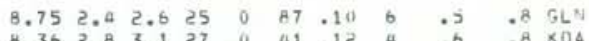

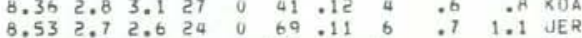

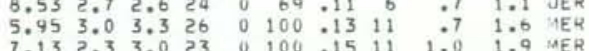
$\begin{array}{llllllllllll}7.25 & 2.7 & 3.2 & 24 & 0 & 124 & .14 & 8 & .6 & 1.9 & 5 \times 2\end{array}$

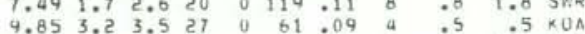

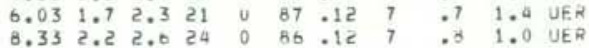
$\begin{array}{llllllllllll}8.96 & 3.6 & 3.9 & 25 & 0 & 117 & .09 & 7 & .5 & .8 & \text { SAR }\end{array}$

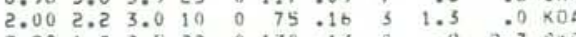

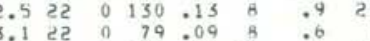

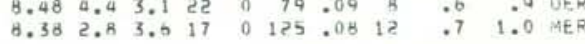

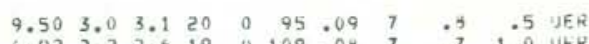
$10.853 .0 \geq .729 \quad 0 \quad 112.1919 \quad 1.4 \quad .5 \mathrm{kON}$ $\begin{array}{rrrrrrrrrrr}9.16 & 4.0 & 4.1 & 25 & 0 & 77 & .11 & \text { b } & .7 & .5 & .5 \\ 6.17 & 2.4 & 2.9 & 16 & 0 & 100 & .04 & 11 & .7 & 1.1 & \text { UER }\end{array}$ \begin{tabular}{llllllllll}
8.65 & 2.6 & 2.9 & 25 & 0 & 97 & .15 & 7 & .9 & 1.4 \\
\hline
\end{tabular} $\begin{array}{llllllllllll}7.07 & 2.5 & 3.0 & 16 & 0 & 180 & .11 & 13 & 1.2 & 1.1 & 1 & 1.2 E\end{array}$

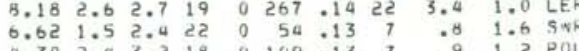

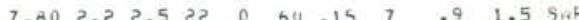

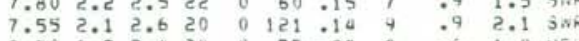


DRIGIV TIME LAT TO LON F DEPTH AMP DUR GAP RMS MIN ERH ERZ YEAR MON DA HRMM SEC DEG HIN DEG MIN KL MAG MAG NR NS WEE SEC DIS KM KH REMA

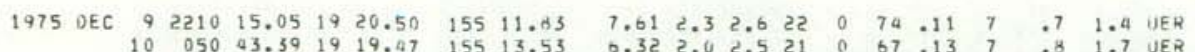

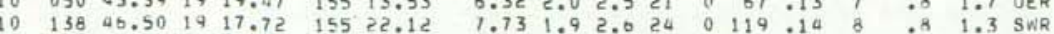

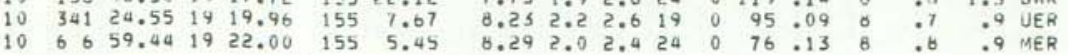

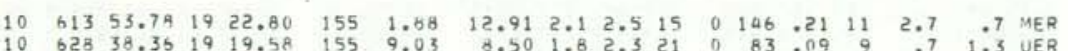
$\begin{array}{lllllllllllllllll}10 & 637 & 42.52 & 19 & 19.24 & 155 & 20.10 & 8.44 & 2.4 & 2.7 & 27 & 0 & 85 & .15 & 6 & 0 & 1.2\end{array}$

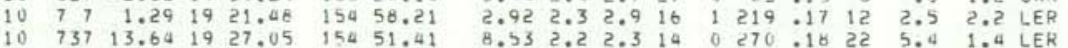

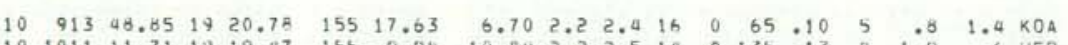

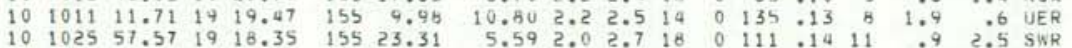

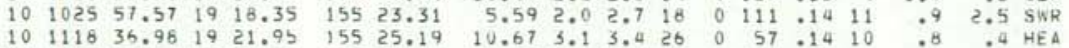

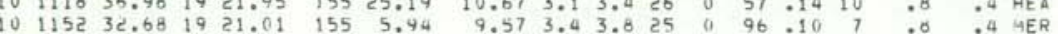
$\begin{array}{llllllllllllllllll}10 & 12 & 3 & 11.91 & 19 & 23.08 & 155 & .57 & 7.11 & 2.5 & 2.8 & 22 & 0 & 166 & .15 & 9 & 1.5 & 1.0 \\ 10 & 1224 & 52.78 & 19 & 19.70 & 155 & 11.99 & 7.89 & 2.2 & 2.6 & 23 & 0 & 46 & 15 & 6 & 1.0 & 1.7 & \text { LER }\end{array}$ $101351 \quad 47.571923 .79$ 155 $4.07 \quad 6.622 .03 .112200101 .1011$.6 1.2 MERR

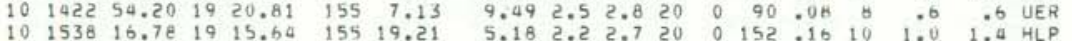

$10 \quad 1543 \quad 16.8819 \quad 20.06 \quad 15512.03$

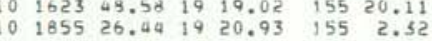
$1002116 \quad 32.3919 \quad 27.55 \quad 15451.54$ $\begin{array}{llllll}10 & 2343 & 8.24 & 14 & 20.58 \quad 150 & 59.57\end{array}$ $102350 \quad 51.641920 .16 \quad 155 \quad 8.03$ 11 040 19.101919 .4015519 .25 $11 \quad 045 \quad 50.46 \quad 1923.29 \quad 154 \quad 59.13$

$\begin{array}{lllllll}11 & 156 & 58.20 & 19 & 20.71 & 155 & 16.47\end{array}$ $11 \quad 246 \quad 53.68 \quad 1924.28 \quad 155 \quad 13.36$ $11 \quad 316,34.24 \quad 1929,05 \quad 154,46.94$ $11 \quad 350 \quad 11.31 \quad 1921.05 \quad 155 \quad 4.48$

$\begin{array}{lllllll}11 & 541 & 19.06 & 19 & 21.90 & 153 & 7.13\end{array}$

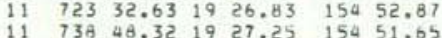

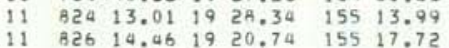

$\begin{array}{lllllll}11 & 627 & 4.44 & 19 & 20.97 & 155 & 16.31\end{array}$

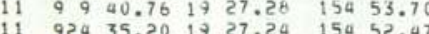

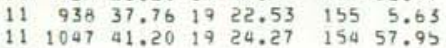

$\begin{array}{lllllll}11 & 1117 & 27.57 & 19 & 25.47 & 154 & 57.38\end{array}$

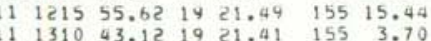
$\begin{array}{lllllllllll}9.65 & 4.0 & 4.0 & 26 & 0 & 79 & .11 & 6 & .7 & .4 & \text { UER } \\ 8.51 & 2.4 & 2.9 & 26 & 0 & 91 & .15 & 6 & .9 & 1.1 & \text { SWR }\end{array}$ $\begin{array}{lllllllllll}8.47 & 2.4 & 2.7 & 16 & 0 & 162 & 08 & 12 & .8 & 1.1 & \text { MER } \\ 8.18 & 2.3 & 2.5 & 18 & 0 & 268 & 0.16 & 22 & 4.4 & 1.1 & \text { MER }\end{array}$ $6.832 .62 .92100133,060$

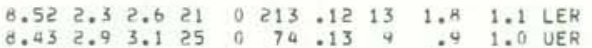

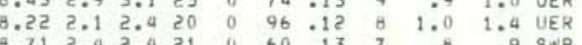
$5.402 .52 .624 \quad 1183$.11 $901.1 \quad 1.1$ LER $\begin{array}{lllllllllll}6.92 & 1.4 & 2.2 & 17 & 0 & 74 & .09 & 5 & .6 & 1.1 & \mathrm{KOA} \\ 8.98 & 2.7 & 2.7 & 26 & 0 & 87 & .12 & 6 & .5 & .8 & 6 L N\end{array}$

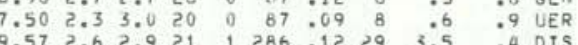

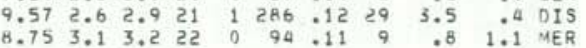
$\begin{array}{llllllllllll}8.87 & 3.2 & 3.5 & 26 & 0 & 73 & .09 & 8 & .6 & . & . \text { A UER }\end{array}$

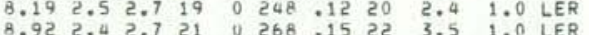

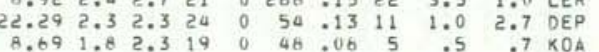
$\begin{array}{lllllllllllll}7.73 & 1.4 & 2.1 & 17 & 0 & 55 & .05 & 5 & .4 & .8 & \text { KOA }\end{array}$

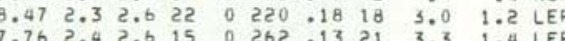

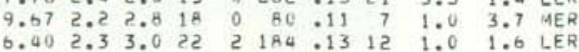
$\begin{array}{llllllllll}4.44 & 2.4 & 3.0 & 15 & 0 & 173 & .12 & 12 & 1.2 & .9 \\ 0 \text { LER }\end{array}$

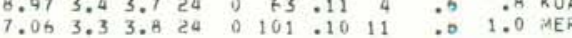

HVO EARTHLUAKL SUARARY LIST

P4GF 126 YEAR MON DRIGIN TIME LAT N TH LON SEC DEG MIN OEG NIN KEPTH ANP DUR GAP HMS AIU ERH ERZ 1975 DEC $11 \quad 131451.641920 .76 \quad 155 \quad 3.45$

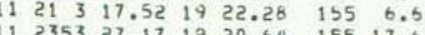
$\begin{array}{llllllll}12 & 0 & 6 & 11.45 & 19 & 21.35 & 155 & 13.06\end{array}$ $12 \quad 041 \quad 4.7919 \quad 18.88 \quad 15521.26$

$\begin{array}{lllllll}12 & 447 & 34.10 & 19 & 21.07 \quad 155 \quad 17.41\end{array}$

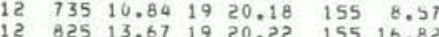

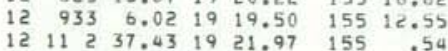
$12 \quad 1129 \quad 59.17 \quad 1920.22 \quad 155 \quad 4.40$

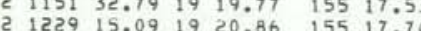
$\begin{array}{lllllll}12 & 1327 & 49.70 & 19 & 20.35 & 155 & 19.07 \\ 12 & 1328 & 52.21 & 19 & 18.98 & 155 & 21.00\end{array}$ $12142123.44 \quad 19 \quad 17.40 \quad 15522.26$ 12142455.33 i9 20.93 155 17.59

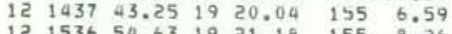
$\begin{array}{lllllll}12 & 1536 & 54.63 & 19 & 21.18 & 155 & 8.26 \\ 12 & 1556 & 58.86 & 19 & 21.24 & 155 & 8.73\end{array}$ $\begin{array}{lllllll}12 & 1651 & 7.72 & 19 & 22.48 & 155 & 2.40\end{array}$ $121753 \quad 32.331919 .11 \quad 155 \quad 13.45$

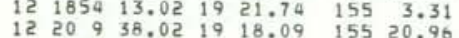
$12204318.611922 .09 \quad 155 \quad 6.45$ $\begin{array}{lllllll}13 & 042 & 3.56 & 19 & 19.50 & 155 & 18.64\end{array}$ $\begin{array}{lllllll}13 & 053 & 36.33 & 19 & 22.66 & 155 & 3.18\end{array}$ $\begin{array}{lllllll}13 & 145 & 26.73 & 19 & 19.38 & 155 & 19.22 \\ 13 & 231 & 48.78 & 19 & 19.42 & 155 & 12.15\end{array}$

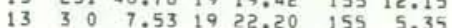
$\begin{array}{lllllll}13 & 412 & 52.30 & 19 & 20.88 & 155 & 8.85\end{array}$

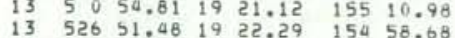
$\begin{array}{lllllll}13 & 526 & 51.48 & 19 & 22.29 & 154 & 58.68 \\ 13 & 77 & 58.52 & 19 & 29.93 & 155 & 47.82\end{array}$ $\begin{array}{lllllll}13 & 724 & 12.43 & 19 & 22.40 & 155 & 0.01\end{array}$

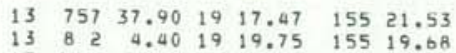

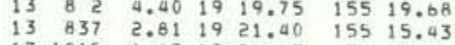
$131015 \quad 6.671924 .65 \quad 15522.24$

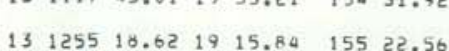
$\begin{array}{llllllll}13 & 1255 & 18.62 & 19 & 15.84 & 155 & 22.56 \\ 13 & 1319 & 33.34 & 19 & 19.94 & 155 & 16.97\end{array}$

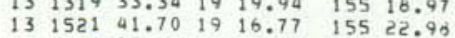
$\begin{array}{rrrrrrr}13 & 1551 & 25.29 & 19 & 22.31 & 155 & 5.47 \\ 13 & 1737 & 1.16 & 19 & 19.85 & 155 & 19.30\end{array}$

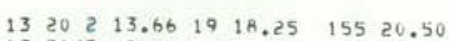

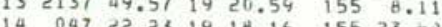

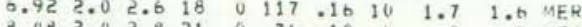

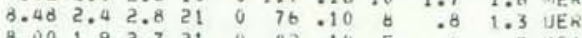

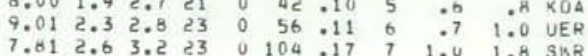

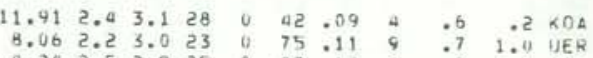

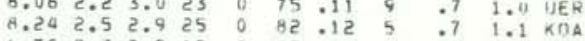

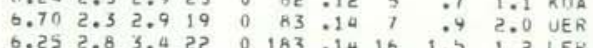

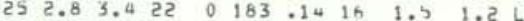
$\begin{array}{llllllllllll}7.16 & 2.4 & 2.7 & 21 & 0 & 125 & .10 & 8 & .6 & .9 & \text { MER }\end{array}$

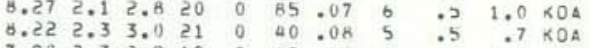
$\begin{array}{rrrrrrrrrrr}7.98 & 2.3 & 2.9 & 19 & 0 & 48 & 08 & 6 & .5 & 1.1 & \text { SAR } \\ 8.48 & 1.8 & 2.7 & 20 & 0 & 100 & .11 & 7 & .7 & 1.1 & \text { SNR }\end{array}$

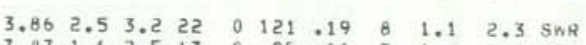

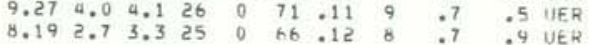
$\begin{array}{lllllllllll}6.98 & 3.2 & 3.6 & 23 & 0 & 138 & .09 & 12 & .6 & 1.1 & \text { MER }\end{array}$ $6.522 .12 .521 \quad 0 \quad 116.09 \quad 11 \quad 0 \quad 09458$

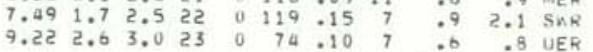
$\begin{array}{lllllllllll}9.65 & 2.6 & 3.2 & 27 & 0 & 63 & .12 & 7 & .7 & .5 & \times 04\end{array}$

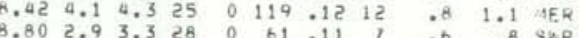

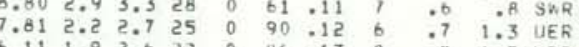
$\begin{array}{lllllllllllll}5.48 & 2.7 & 3.4 & 28 & 0 & 67 & .13 & 8 & .6 & .7 & \text { UER }\end{array}$

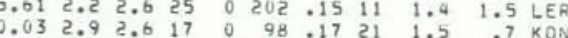

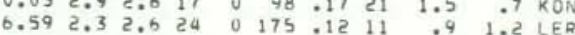

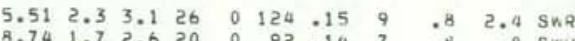

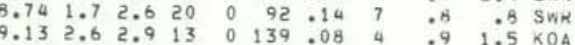

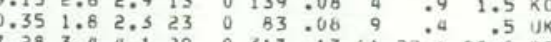

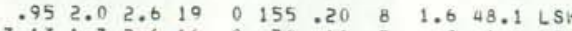

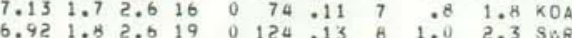

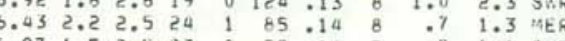
$\begin{array}{rrrrrrrrrrr}8.87 & 2.6 & 3.1 & 28 & 0 & 116 & .16 & 0 & .9 & 1.2 & \text { Suk } \\ 8.61 & 2.2 & 2.7 & 20 & 0 & 79 & .13 & 9 & 1.0 & 1.3 & \text { UER }\end{array}$

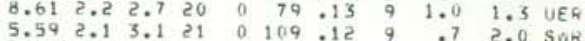

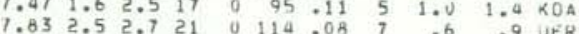

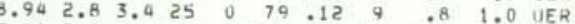


ORIGIN TIME LATN LON W DEPTH AMP DUR N GAP RMS MIN ERH ERZ
OA MRMN SEC DEG MIN DEG IN KM MAG MAG NR NS DEG SEC OIS KM KM REMK

1975 OEC $14 \quad 14 \quad 33.84 \quad 19 \quad 18.27 \quad 15523.34$ $18.59 \quad 155 \quad 23.37$ $14.430 \quad .62 \quad 1916.90 \quad 155$. 11.39 $14 \quad 432 \quad 28.72 \quad 1916.92 \quad 15521.39$ $\begin{array}{lllllll}14 & 540 & 43.06 & 19 & 20.16 & 155 & 6.48\end{array}$ 14 $718 \quad 9.53 \quad 1920.66 \quad 155 \quad 17.75$ $14 \quad 95951.041922 .35 \quad 155 \quad 6.56$ $\begin{array}{rrrrrrr}14 & 1048 & 49.26 & 19 & 20.64 & 155 & 6.53 \\ 14 & 1147 & 7.38 & 19 & 21.87 & 155 & 15.13\end{array}$

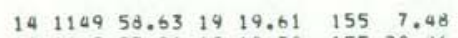

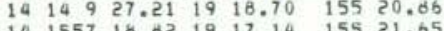
$14 \quad 1557 \quad 18.6219 \quad 17.14 \quad 15521.65$

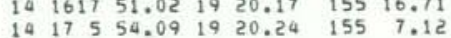

$\begin{array}{lllllll}14 & 2051 & 58.79 & 19 & 21.02 \quad 155 \quad 6.80\end{array}$

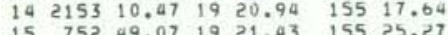
$\begin{array}{lllllll}15 & 752 & 49.07 & 19 & 21.43 & 155 & 25.27\end{array}$ $15924 \quad 11.251919 .23 \quad 15515.51$

$\begin{array}{lllllll}15 & 940 & 24.37 & 14 & 19.27 & 155 & 10.30\end{array}$ $15132356.661921 .49 \quad 15515.25$

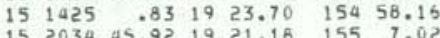

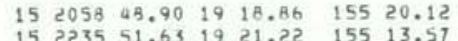
$15223551.631921 .22 \quad 15513.57$

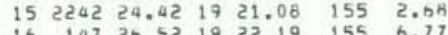
16 16 $239 \quad 30.55 \quad 1919.47 \quad 155 \quad 12.06$

$\begin{array}{lllllll}16 & 240 & 46.77 & 19 & 19.55 & 155 & 12.90\end{array}$ 16 $244 \quad 10.87 \quad 19 \quad 24.22 \quad 155 \quad 13.09$ $16 \quad 24038.581910 .46$ 155 20.50 16 $35112.161919 .95 \quad 15519.57$

$\begin{array}{lllllll}16 & 433 & 44.21 & 19 & 19.63 & 155 & 6.75\end{array}$ $16 \quad 436 \quad 45.97191917 .19 \quad 15520.02$

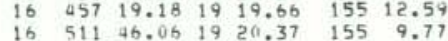

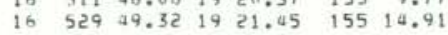

$\begin{array}{lllllll}16 & 543 & 17.18 & 14 & 24.97 & 155 & 12.88\end{array}$

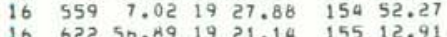

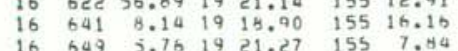

in $\quad 655 \quad 15.93 \quad 19 \quad 20.70 \quad 155 \quad 4.04$

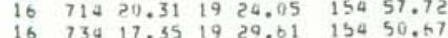

$\begin{array}{lllllllllll}.70 & 2.2 & 2.9 & 19 & 0 & 109 & .12 & 8 & .7 & 38.5 & \text { SWR }\end{array}$

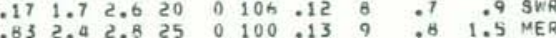

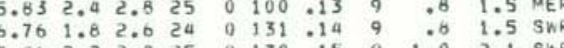
$\begin{array}{lllllllllll}0.62 & 2.1 & 2.5 & 23 & 0 & 112 & .10 & 7 & .8 & .6 & \text { UER }\end{array}$

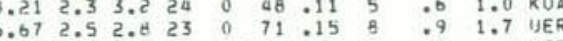

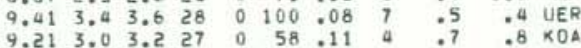
$\begin{array}{lllllllllll}7.92 & 2.4 & 3.0 & 17 & 0 & 105 & .10 & 8 & .8 & 2.1 & \text { UER }\end{array}$

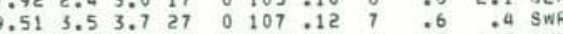

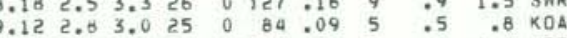

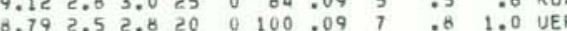
$\begin{array}{lllllllllll}9.10 & 2.1 & 2.9 & 22 & 0 & 90 & .07 & 7 & .5 & .6 & \text { UER } \\ 1.30 & 2.3 & 2.9 & 23 & 0 & 40 & 010 & 5 & .6 & 1.0 & \text { KOA }\end{array}$ 9.4112 .252 .925 0 $68.1010 \quad .6 \quad .7 \mathrm{HEA}$ $\begin{array}{lllllllllll}6.81 & 1.8 & 2.3 & 21 & 0 & 123 & .14 & 8 & 1.0 & 2.4 & \text { SNR } \\ 8.24 & 2.5 & 2.8 & 23 & 0 & 69 & .10 & 7 & .7 & 1.3 & \text { UER }\end{array}$

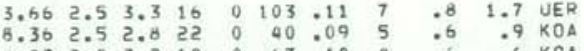

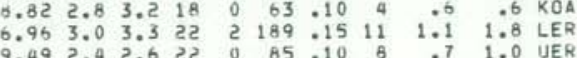
$\begin{array}{lllllllllll}8.16 & 2.2 & 2.6 & 24 & 0 & 96 & .14 & 6 & .8 & 1.1 & \text { SWR }\end{array}$ $\begin{array}{rrrrrrrrrrr}8.34 & 2.7 & 3.0 & 24 & 0 & 53 & 14 & 7 & .8 & 1.4 & \text { UER } \\ 8.94 & 2.6 & 3.1 & 17 & 0 & 143 & .07 & 12 & .7 & 1.3 & \text { MER }\end{array}$ $9.22 \quad 2.83 .321 \quad 0 \quad 76.08$ 9 $52.5 \quad .6$ UER $\begin{array}{lllllllllll}6.00 & 1.6 & 1.8 & 22 & 0 & 77 & .11 & 7 & .7 & 1.6 & \text { UER }\end{array}$

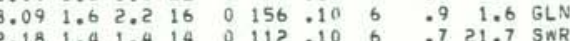

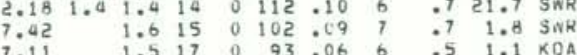
$\begin{array}{lllllllllllll}6.68 & 2.2 & 20 & 0 & 121 & .10 & 7 & .8 & 1.5 & \text { JER }\end{array}$ $\begin{array}{rrrrrrrrrrr}.91 & 1.5 & 1.5 & 18 & 0 & 133 & .08 & 8 & .7 & 1.4 & \text { SWR } \\ 6.14 & & .4 & 12 & 0 & 80 & .05 & 7 & : 5 & 1.5 & \text { UER }\end{array}$

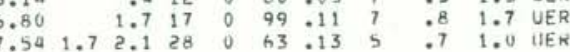
$\begin{array}{lllllllllllllll}7.29 & 3.1 & 3.1 & 29 & 0 & 91 & .13 & 7 & .7 & 1.1 & \mathrm{GLN}\end{array}$

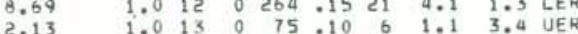

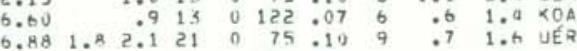
$\begin{array}{lllllllll}2.71 & 1.5 & 16 & 0 & 101 & -15 & 9 & 1.0 & 3.0 \\ 0 & \text { MER }\end{array}$

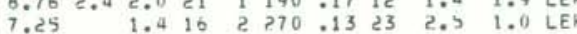

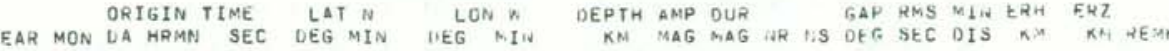
1975 DEC 16 $735 \quad 35.43 \quad 19 \quad 17.84 \quad 15521.35$

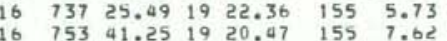

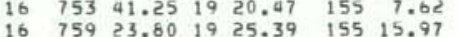

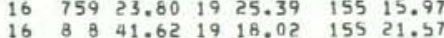

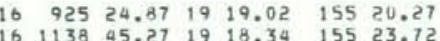
$\begin{array}{lllllll}16 & 1138 & 45.27 & 19 & 18.34 & 155 & 23.72\end{array}$ $16 \quad 1355 \quad 11.34 \quad 1922.34 \quad 155 \quad 5.00$ $16 \quad 1355 \quad 11.341922 .39 \quad 155 \quad 5.80$

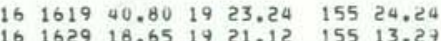
$16162918.6519210 .12 \quad 15513.27$ $161827 \quad 22.18 \quad 1920.73 \quad 15517.91$

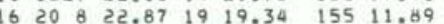
$\begin{array}{rrrrrrr}16 & 2310 & 30.85 & 19 & 21.49 & 155 & 8.15 \\ 16 & 2327 & 24.76 & 19 & 19.42 & 155 & 18.98\end{array}$

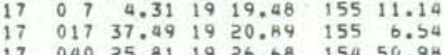

$\begin{array}{lllllll}17 & 048 & 42.66 & 19 & 20.63 & 155 & 17.92\end{array}$ $17 \quad 439 \quad 57.851927 .23 \quad 15451.62$ $\begin{array}{lllllll}17 & 736 & 40.00 & 19 & 23.28 & 155 & 4.00\end{array}$

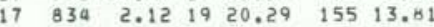

$17 \quad 954 \quad 30.16 \quad 1921.75 \quad 155 \quad 15.36$
177 $\begin{array}{lllllll}17 & 1116 & 31.03 & 19 & 12.39 & 155 & 27.18 \\ 17 & 1142 & 27.66 & 19 & 20.94 & 155 & 4.72\end{array}$

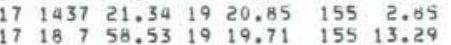

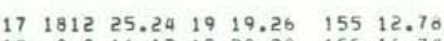
$\begin{array}{lllll}18 & 0 & 8 & 16.12 & 19 \\ 18 & 357 & 14.90 .20 & 155 & 16.74\end{array}$

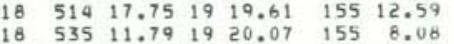
$\begin{array}{lllllll}18 & 547 & 45.21 & 19 & 17.19 & 155 & 22.28\end{array}$ $1917.25 \quad 15522.33$ $49.53 \quad 1920.41 \quad 155 \quad 17.69$ 18 $121 \quad 2.481928 .58$ 154 51.92 $\begin{array}{lllllll}18 & 811 & 21.25 & 19 & 19.46 & 155 & 14.09\end{array}$

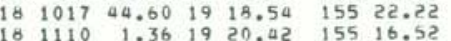
$\begin{array}{rrrrrrr}18 & 1110 & 1.36 & 19 & 20.42 & 155 & 16.52 \\ 18 & 1314 & 17.93 & 19 & 19.31 & 155 & 19.17\end{array}$ $\begin{array}{lllllll}18 & 1014 & 41.48 & 19 & 20.74 & 155 & 4.80\end{array}$ $\begin{array}{lllllll}18 & 1425 & 39.15 & 19 & 19.24 & 155 & 12.34\end{array}$

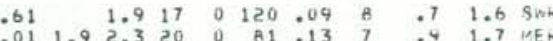

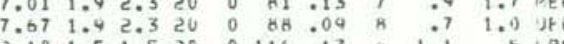

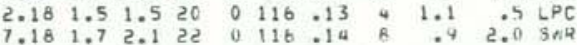

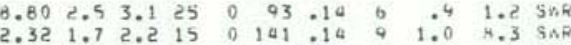
.841 .82 .317 O 98.06 1 .6 .9 Je

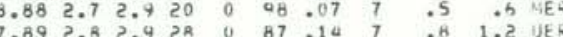

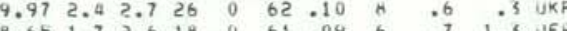

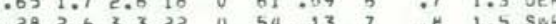

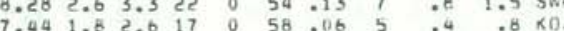

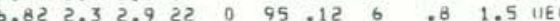

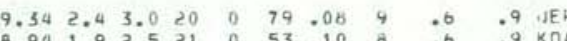

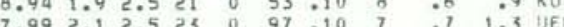

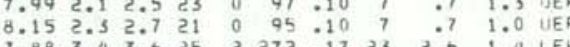
\begin{tabular}{l}
$.88 \quad 3.43 .625 \quad 2272.17$ \\
\hline
\end{tabular} $\begin{array}{lllllllllll}.21 & 1.4 & 2.3 & 18 & 0 & 57 & .13 & 5 & .8 & 1.7 & \times 0\end{array}$

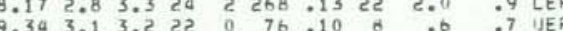

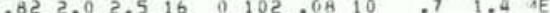
$6.801 .62 .2 \quad 23$ o 82.13 o .4 1.5 ue $\begin{array}{lllllllllll}9.41 & 3.83 .9 & 28 & 0 & 60 & .09 & 4 & .5 & .6 & 604\end{array}$

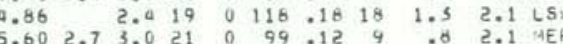

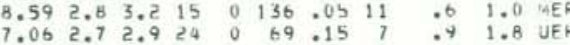

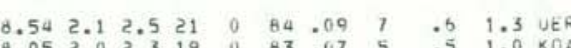
$9.242 .125 .520 \quad 0 \quad 96.07 \quad 7 \quad 5 \quad 1.0$ UEE

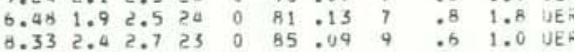
$\begin{array}{lllllllllll}5.82 & 2.2 & 2.5 & 21 & 0 & 123 & .14 & 8 & .9 & 2.6 & \text { SnR }\end{array}$

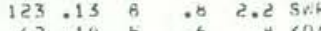

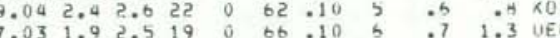

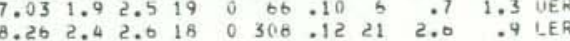
$\begin{array}{llllllllll}7.70 & 1.6 & 2.5 & 19 & 0 & 66 & .09 & 6 & .6 & 1.2 \\ 7 E R\end{array}$

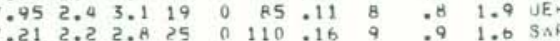

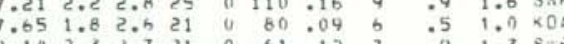

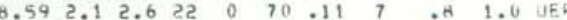

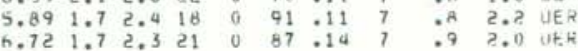


HVO EARTHGUARE SUMAARY LIST

PAISE 129

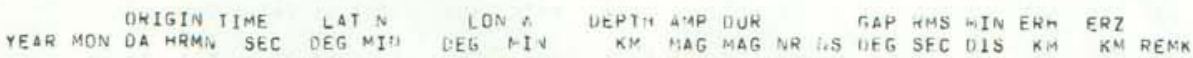

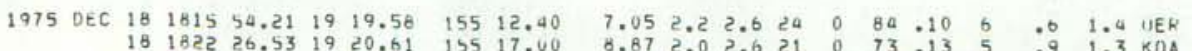

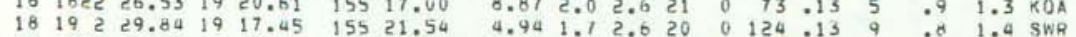

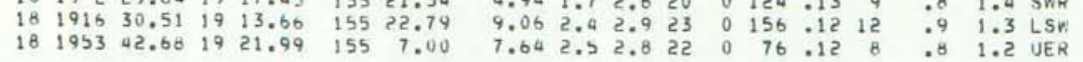

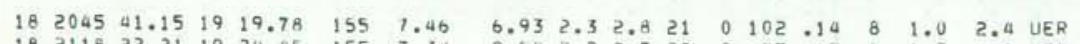

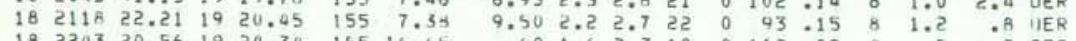

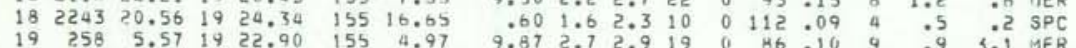

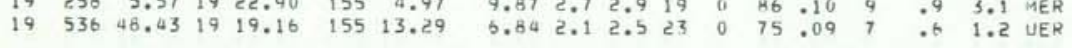

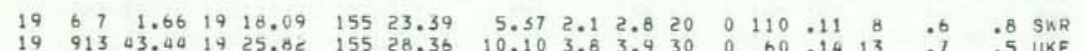

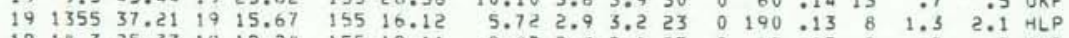

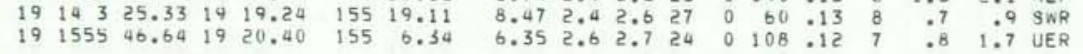

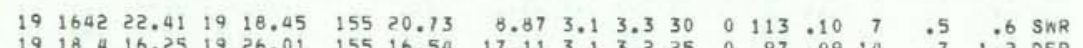

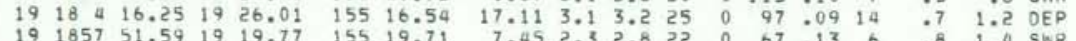

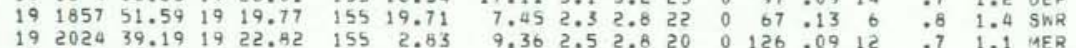

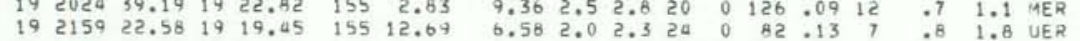

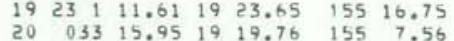
$\begin{array}{lllllll}20 & 412 & 38.16 & 19 & 20.79 & 155 & 6.50\end{array}$

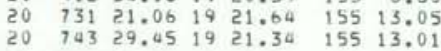

$\begin{array}{llllllll}20 & 8 & 5 & 24.23 & 19 & 19.94 & 155 & 12.90 \\ 20 & 800 & 32.03 & 19 & 22.59 & 155 & 5.50\end{array}$ $\begin{array}{rrrrrrr}20 & 840 & 32.03 & 19 & 22.59 & 155 & 5.50 \\ 20 & 1355 & 48.09 & 19 & 18.40 & 155 & 23.54\end{array}$ $\begin{array}{lllllll}20 & 1355 & 48.09 & 19 & 18.40 & 155 & 23.54 \\ 20 & 1414 & 57.25 & 19 & 21.56 & 155 & 2.77\end{array}$ $201936 \quad 43.42 \quad 19 \quad 17.03 \quad 15521.50$ $\begin{array}{rrrrrrr}20 & 2330 & 44.52 & 19 & 22.83 & 155 & 1.71 \\ 21 & 028 & 21.74 & 19 & 16.76 & 155 & 21.96\end{array}$ $\begin{array}{lllllll}21 & 028 & 21.74 & 19 & 16.76 & 155 & 21.96 \\ 21 & 036 & .07 & 19 & 24.08 & 155 & 24.08\end{array}$ 21 $122 \quad 35.96 \quad 1924.00 \quad 15524.08$ 21 $642 \quad 40,11 \quad 1920,20 \quad 155 \quad 11.06$ $\begin{array}{rrrrrrr}21 & 821 & 4.13 & 19 & 22.12 & 155 & 15.46 \\ 21 & 1126 & 16.54 & 19 & 19.08 & 155 & 21.86\end{array}$ 21 11220 (16.52 19 19.06 155 21.86

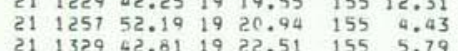
$\begin{array}{lllllll}21 & 1340 & 21.74 & 19 & 21.52 & 155 & 5.86\end{array}$ 211013 13.19 1921.78 155 2.60 $\begin{array}{lllllll}21 & 1453 & 55.49 & 19 & 21.74 & 155 & 17.83 \\ 21 & 1525 & 48.77 & 19 & 20.26 & 155 & 16.61\end{array}$ $21 \quad 1612 \quad 37.08 \quad 19 \quad 19.60 \quad 155 \quad 18.06$

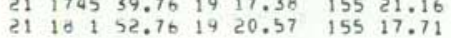
$\begin{array}{lllllllllllllll}9.63 & 2.7 & 3.0 & 30 & 0 & 61 & .11 & 7 & .6 & .4 & \times 0\end{array}$

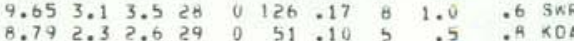

HVO EARTHQUAKE SLHMAKY LIST

HALE 136 ORIGIN TIME LATM LON $M$ DERTH ARAP DUR GAP NMS MIN ERH EW YEAR MON DA HRMN SEC DEG MIN DEG MIN KM TAG MAG NR NS TEEG SFC. DIS KM KM: PEMK

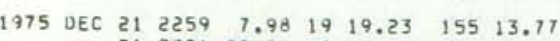
$2264938.91 \quad 1919.23 \quad 15512.24$

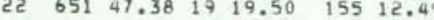
$22 \quad 916 \quad 36.46 \quad 19 \quad 27.69 \quad 15451.6$ र2 $1312 \quad 8.2919$ 18. 136 155

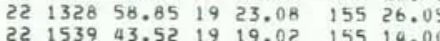

$22 \quad 1540 \quad 25.12 \quad 1921.66 \quad 155 \quad 1.90$

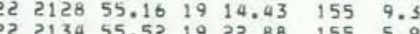
$\begin{array}{lrrrrrr}22 & 2227 & 35.48 & 19 & 27.85 & 154 & 52.30 \\ 23 & 058 & .80 & 19 & 20.95 & 155 & 13.36\end{array}$

$\begin{array}{lllllllll}23 & 1 & 4 & 30.75 & 19 & 15.47 & 155 & 9.67\end{array}$

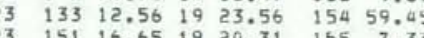
$23 \quad 350 \quad 55.801920 .51 \quad 155 \quad 7.35$

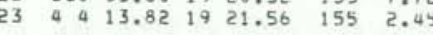

$\begin{array}{lllllll}23 & 619 & 33.31 & 19 & 23.50 & 155 & 4.39\end{array}$

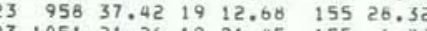

$23 \quad 14 \quad 4 \quad 53.56 \quad 19 \quad 21.81 \quad 155 \quad 1.35$ 23 $162416.001925 .19 \quad 15524.16$

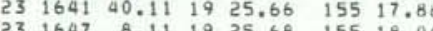

$\begin{array}{lllllll}23 & 1727 & 42.26 & 19 & 26.14 & 154 & 54.26\end{array}$ $23 \quad 1841 \quad 47.11 \quad 1921.89 \quad 155 \quad 6.89$

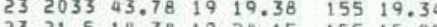
$2332127 \quad 03.2519 \quad 17.56 \quad 155$ 20.95

$24 \quad 044 \quad 34.90 \quad 19 \quad 20.24 \quad 155 \quad 16.60$

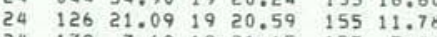
. $24 \quad 43139.36 \quad 19,16.55 \quad 155 \quad 12.50$

$24 \quad 616 \quad 51.04 \quad 19 \quad 19.04 \quad 155 \quad 20.16$

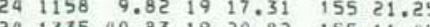

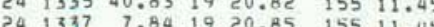
$24 \quad 1451 \quad 53.66 \quad 19 \quad 20.97 \quad 155 \quad 17.57$ $24 \quad 1643 \quad 45.791926 .10 \quad 154 \quad 56.23$

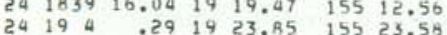

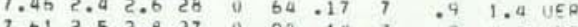

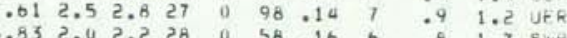

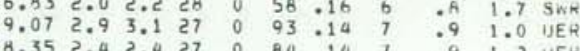

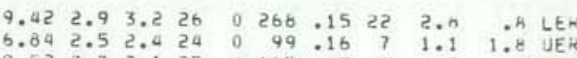

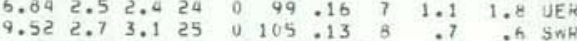
9.272 .52 .525 0 50.14 511 :0 1.5 UKF

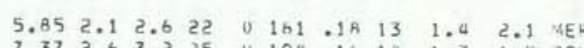

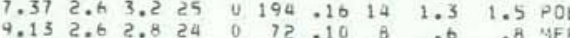
$8.742 .83 .02400264 .1 \%$ ट1 5.251 .0 LER $\begin{array}{lllllllllll}9.58 & 2.3 & 2.7 & 24 & 0 & 185 & -12 & 14 & 1.2 & .9 & \text { POL }\end{array}$

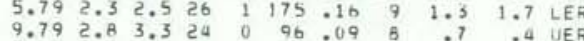

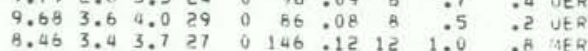
$\begin{array}{lllllllllll}8.75 & 3.1 & 3.4 & 25 & 0 & 96 & .10 & 10 & .6 & .7 & \text { YEW }\end{array}$

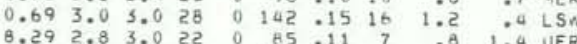

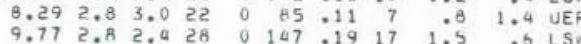
$10.493 .43 .428 \quad 0 \quad 143.1616 \quad 1.5$.5 LSW $\begin{array}{lllllllllll}1.48 & 2.8 & 3.0 & 25 & 0 & 171 & .12 & 12 & 1.1 & 1.0 & \text { MER }\end{array}$

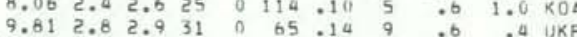
$\begin{array}{llllllllll}8.54 & 2.7 & 2.7 & 27 & 0 & 216 & .09 & 18 & 1.4 & .5 \\ 8 & \text { LER }\end{array}$

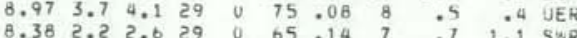

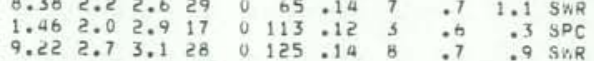
$\begin{array}{lllllllllll}9.44 & 3.13 .5 & 3.5 & 0 & 83 & .10 & 0 & .5 & .6 & \text { KOA }\end{array}$

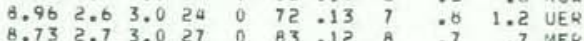

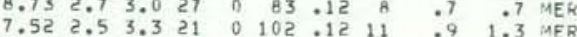

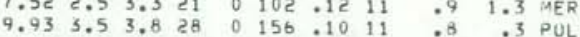

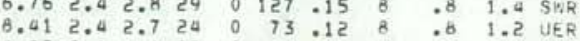

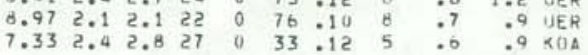
$\begin{array}{rrrrrrrrrrr}7.84 & 3.1 & 3.3 & 28 & 0 & 182 & .15 & 14 & 1.5 & .9 & \text { LER } \\ 4.17 & 2.7 & 2.9 & 29 & 0 & 44 & .15 & 7 & .9 & 1.3 & \text { UER }\end{array}$ $22 \quad 14359.491920 .46 \quad 15511.47$

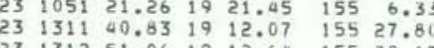

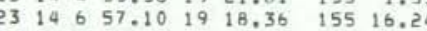

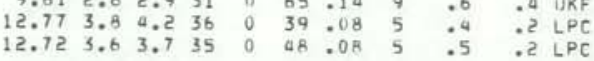
$\begin{array}{lllllllllllllll}9.01 & 2.5 & 2.6 & 31 & 0 & 90 & .13 & 6 & .7 & .7 & \text { SWR }\end{array}$ 
HVO EARTHQUAKE SUMMARY LIST

HAGE 131 ORIGIN TIME LATN LONN JEPTH AMP DUR GAP RMS MIN ERH ERZ KEMK YEAR MON DA 1975 UEC $242016 \quad 51.801924 .72 \quad 15456.82$ ट4 $2043 \quad 14.26 \quad 1720.64 \quad 155 \quad 6.79$

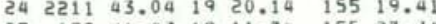
$2515848.091925 .56 \quad 15516.99$

$\begin{array}{llllllll}25 & 818 & 17.78 & 19 & 26.16 & 155 & 27.93\end{array}$

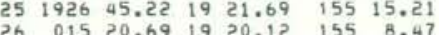
$26 \quad 058 \quad 33.62 \quad 1920.99 \quad 15513.12$ 23118.89
19 $20.18 \quad 155 \quad 16.95$

$\begin{array}{lllllll}26 & 432 & 20.35 & 19 & 27.10 & 155 & \mathbf{4 9} .38 \\ 26 & 524 & 31.11 & 19 & 22.31 & 155 & 5.22\end{array}$

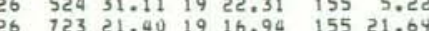
$\begin{array}{lllllll}26 & 927 & 8.36 & 19 & 20.53 & 155^{\circ} & 6.54\end{array}$

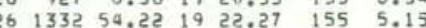

$26 \quad 1852 \quad 19.801924 .46 \quad 155 \quad 13.12$

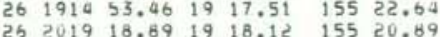
टक 201918.891918 .12155 20.09 26 $2053 \quad 57.191921 .90 \quad 15515.39$

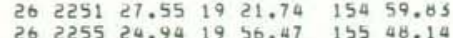

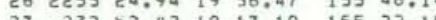
$27 \quad 71635.301920 .20155$ 11 15

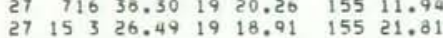
$\begin{array}{lllllll}27 & 1550 & 30.61 & 19 & 19.93 & 155 & 6.58\end{array}$ ट7 1559 41.51 19 25.89 155 16.22

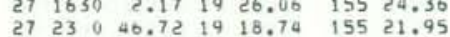

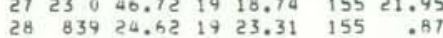
$\begin{array}{llllllll}28 & 9 & 6 & 39.29 & 19 & 19.57 & 155 & 12.64\end{array}$

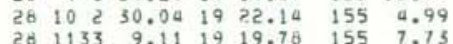

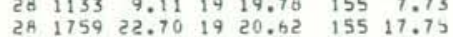

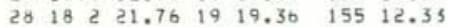
28 $1823 \quad 54.60 \quad 19 \quad 13.87 \quad 155 \quad 20.19$

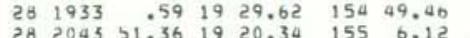
$\begin{array}{lllllll}29 & 228 & 57.69 & 19 & 21.10 & 155 & 6.16 \\ 29 & 338 & 54.65 & 19 & 20.33 & 155 & 4.72\end{array}$

$\begin{array}{lllllll}29 & 832 & 33.96 & 19 & 19.97 \quad 155 & 12.33\end{array}$ $29 \quad 926 \quad 29.71 \quad 1921.35 \quad 155 \quad 6.33$ $\begin{array}{lllllll}29 & 939 & 40.60 & 19 & 19.31 & 155 & 11.47 \\ 29 & 956 & 17.75 & 19 & 17.16 & 155 & 23.57\end{array}$

$29 \quad 1016 \quad 35.41 \quad 17 \quad 18.71 \quad 155 \quad 19.01$

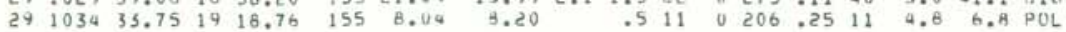
$\begin{array}{rrrrrrrrrrr}9.53 & 2.4 & 2.3 & 21 & 0 & 192 & .14 & 13 & 1.7 & 1.4 & \text { LER } \\ 3.97 & 2.4 & 2.7 & 21 & 0 & 97 & .10 & 7 & .7 & 1.4 & \text { UER }\end{array}$

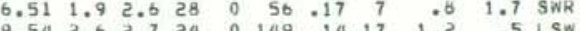

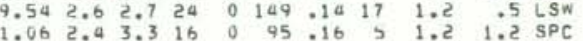
$\begin{array}{llllllllllll}8.28 & 2.4 & 2.4 & 26 & 0 & 61 & .14 & 13 & .8 & 1.8 & \text { UKF } \\ 9.61 & 3.2 & 3.4 & 29 & 0 & 60 & 110 & 4 & .6 & .4 & \text { KO }\end{array}$

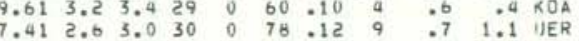
$8.972 .42 .726 \quad 0 \quad 58.12$ 6 $50.7 \quad 1.1$ UER $\begin{array}{lllllllllllll}0.89 & 5.1 & 3.1 & 31 & 0 & 102 & -14 & 21 & .9 & .9 & \text { KON }\end{array}$

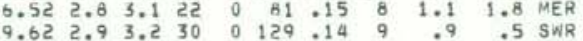

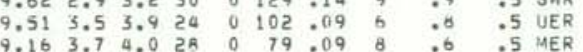
$\begin{array}{rrrrrrrrrrr}8.72 & 2.4 & 2.6 & 22 & 0 & 88 & .10 & 6 & .7 & 1.1 & \text { GLN } \\ 7.19 & 2.4 & 2.9 & 26 & 0 & 119 & .17 & 10 & .9 & 1.9 & \text { SNR }\end{array}$ $7.732 .32 .511 \quad 00124.16$ 14 1.5 3.9 SNR

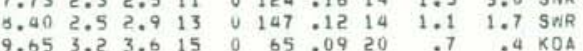
$\begin{array}{llllllllll}5.86 & 2.9 & 3.1 & 13 & 0 & 243 & .09 & 16 & 2.5 & 1.3 \\ .0 E R\end{array}$

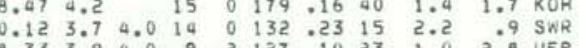

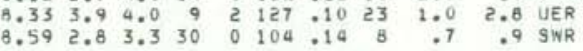
$\begin{array}{llllllllllll}8.06 & 3.1 & 3.1 & 24 & 0 & 116 & .11 & 7 & .7 & 1.0 & \text { UER } \\ 1.00 & 1.9 & 2.2 & 11 & 0 & 174 & .14 & 5 & .9 & .7 & \text { SPC }\end{array}$ 8.963 .13 .032 0 63.14 9 $.7 \quad 1.1$ UKF

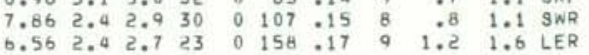
$\begin{array}{lllllllllll}6.91 & 2.5 & 2.8 & 26 & 0 & 81 & .15 & 7 & .9 & 1.6 & 1.6 \\ 0 E R\end{array}$

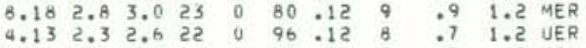

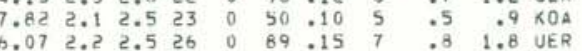
$\begin{array}{llllllllllll}9.18 & 2.5 & 2.7 & 30 & 0 & 96 & .17 & 6 & .9 & .7 & \text { SWR }\end{array}$

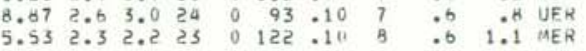

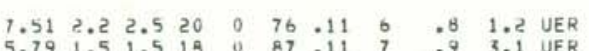
$7.13 \quad 1,311 \quad 0 \quad 89.10 \quad 5 \quad 1.0 \quad 2,0 \quad K O A$ $\begin{array}{rrrrrrrrrr}8.08 & .7 & 12 & 0 & 99 & .05 & 7 & .5 & 1.4 & \text { UER } \\ .42 & 2.0 & 22 & 0 & 117 & .18 & 9 & 1.0 & 45.1 & \text { SMR }\end{array}$
HVO EARTHIUUARE SUMMARY LIST

PAGE 132 ORIGIN TIME LATN LONN DEPTH AMP UUR GAP RMS MIN ERH ELZ

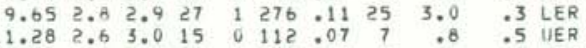

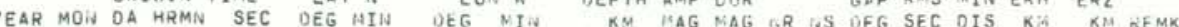

1975 DEC $29105258.911920 .77 \quad 15517.92$

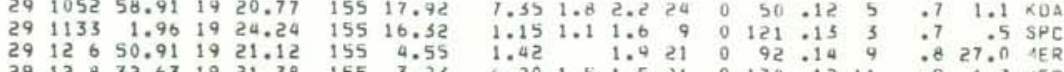

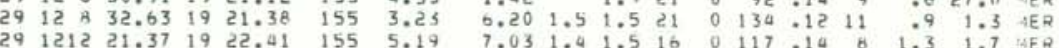

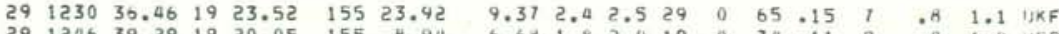

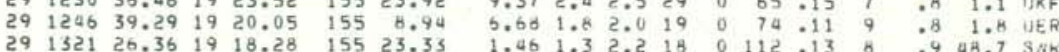

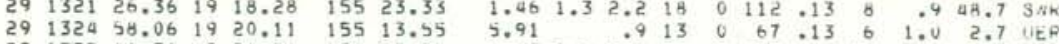

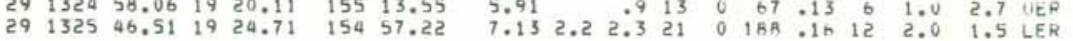

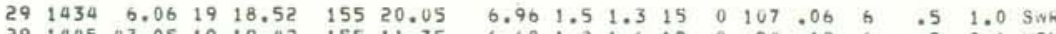

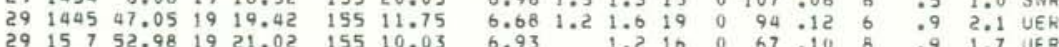

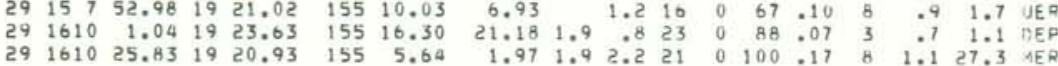

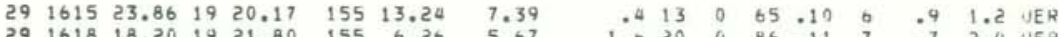

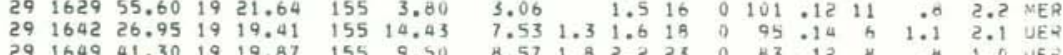
$291649 \quad 41.30 \quad 19 \quad 19.87 \quad 155 \quad 9.50$

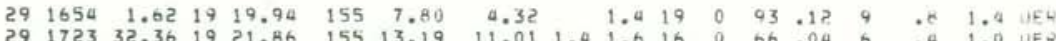

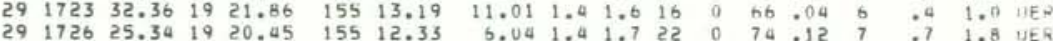

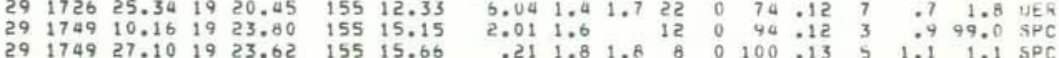

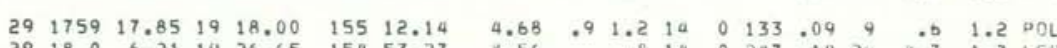

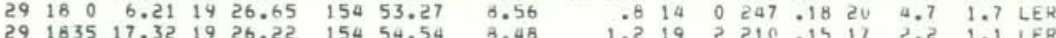

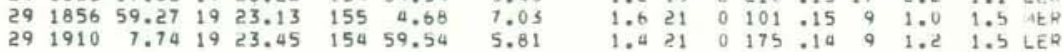

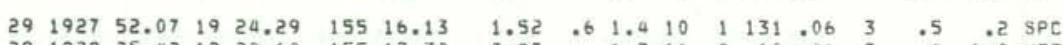

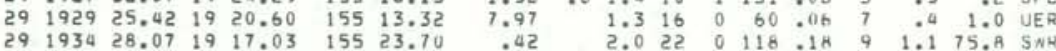

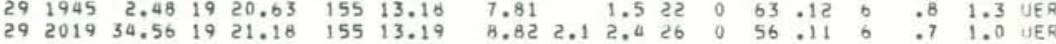

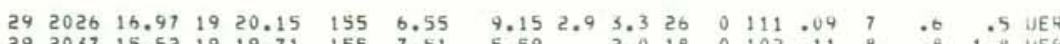

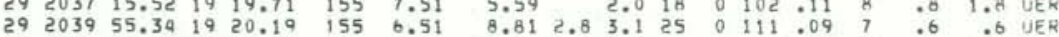

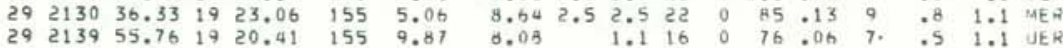

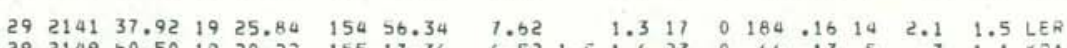

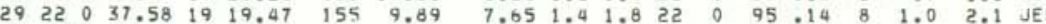
333.03 .08 19

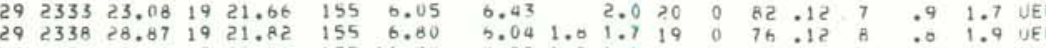

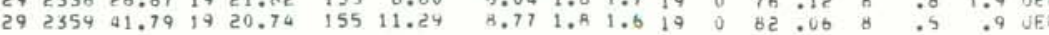

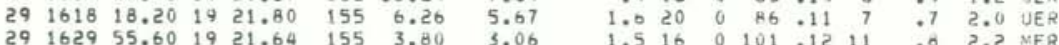

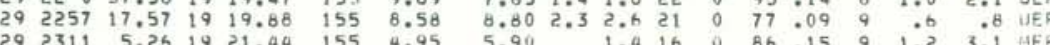


ORIGIN TIME LATH LON W UEPTH MMP DUK GAP KNS MIN ERH ERZ YEAR MON OA

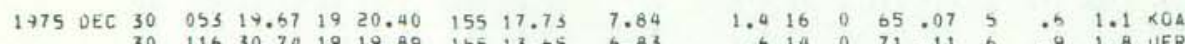

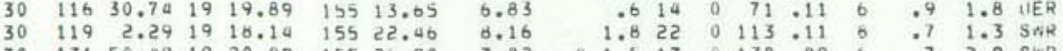

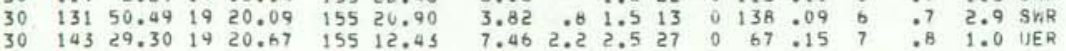

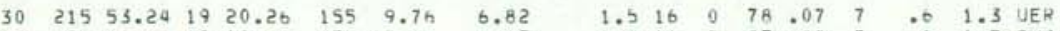

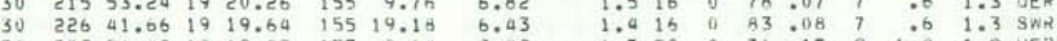

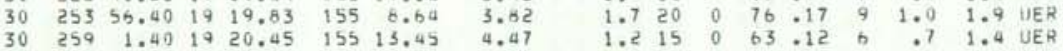

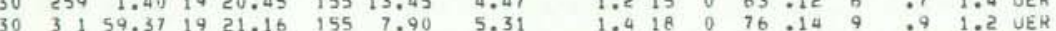

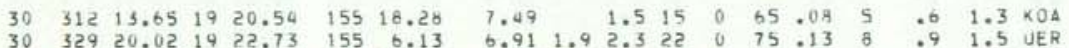
$\begin{array}{lllllllll}1.6 & 19 & 0 & 73 & .10 & 7 & .7 & 1.7 & \text { UER }\end{array}$

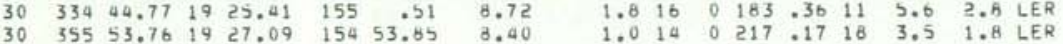

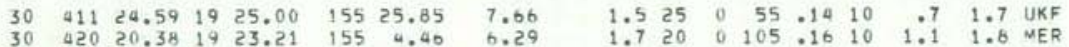

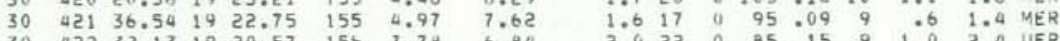

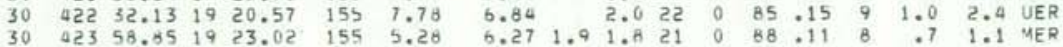

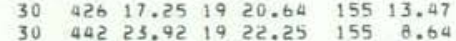

$30044818.941919 .60 \quad 15511.82$

$\begin{array}{lllllll}30 & 450 & 10.58 & 19 & 19.49 & 155 & 8.00 \\ 30 & 451 & 3.00 & 19 & 17.73 & 155 & 21.63\end{array}$

$\begin{array}{lllllll}30 & 512 & 8.28 & 19 & 17.90 & 155 & 22.25\end{array}$ $30 \quad 51235.261917 .85 \quad 155$ 22. 25

30 532 53.921927 .32 154 52.65

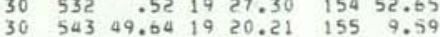

$\begin{array}{llllllll}30 & 553 & 19.77 & 19 & 20.73 & 155 & 11.05\end{array}$

$\begin{array}{llllllll}30 & 6 & 1 & 5.76 & 19 & 22.56 & 155 & 25.02 \\ 30 & 636 & 38.34 & 19 & 20.05 & 155 & 8.02 & 10\end{array}$

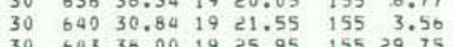

$\begin{array}{lllllll}30 & 645 & .33 & 19 & 21.23 & 155 & 9.55\end{array}$

$\begin{array}{rrrrrrr}30 & 645 & .53 & 19 & 21.23 & 155 & 9.53 \\ 30 & 653 & 50.41 & 19 & 21.27 & 155 & 14.28\end{array}$

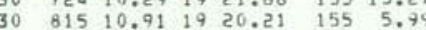

$30 \quad 818 \quad 13.601922 .04 \quad 15526.94$

$\begin{array}{lllllll}30 & 821 & 24.14 & 19 & 23.67 & 154 & 56.74\end{array}$

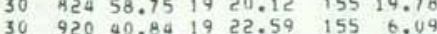

$\begin{array}{rrrrrrr}30 & 922 & 19.90 & 19 & 21.59 & 155 & 6.11 \\ 30 & 1032 & 16.74 & 19 & 19.99 & 155 & 19.81\end{array}$

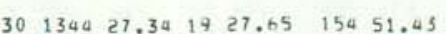
$\begin{array}{lllllll}30 & 1613 & 34.37 & 19 & 23.21 & 155 & 16.41\end{array}$

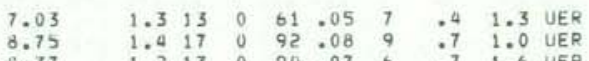

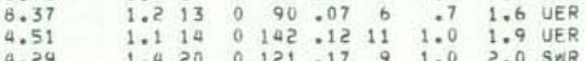

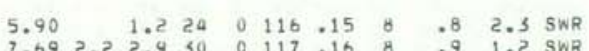

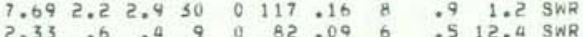

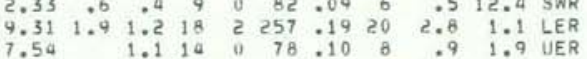
$\begin{array}{llllllllll}7.75 & 1.5 & 19 & 0 & 80 & .11 & 8 & .8 & 1.4 & 1.4 \\ \text { UER }\end{array}$

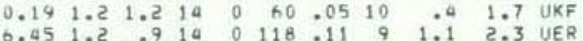

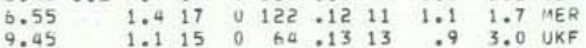
$\begin{array}{llllllllll}7.50 & 1.1 & 15 & 0 & 77 & .11 & 8 & .9 & 1.6 & \text { UER }\end{array}$

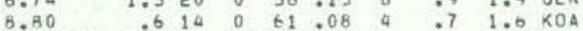

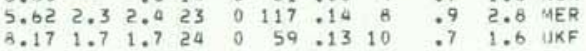
$\begin{array}{lllllllllll}0.54 & 2.8 & 2.7 & 24 & 0 & 182 & .15 & 10 & 1.1 & 1.2 & \text { LER }\end{array}$ $9.213 .65 .125 \quad 0074.11 \quad 7 \quad: 6 \quad .7$ UER $\begin{array}{lllllllllll}4.59 & 2.5 & 2.4 & 17 & 2 & 83 & .22 & 7 & 1.0 & 2.9 & \text { UER } \\ 7.42 & 1.9 & 2.5 & 27 & 0 & 65 & .15 & 6 & .8 & 1.3 & \text { SWR }\end{array}$

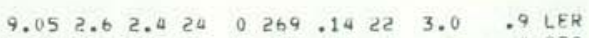

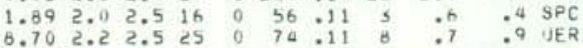

YEAR MON ORIGIN TIME LATN LONB DEPTH ANP NUR GN GAP KEIS AIN ERH ERZ

1975 DEC $30 \quad 17938.82 \quad 19 \quad 19.69 \quad 155 \quad 4.56$ $301752 \quad 7.321928 .11$ 154 51.00

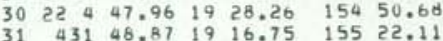
31 4 57.85 19 $21.49 \quad 155 \quad 8.52$

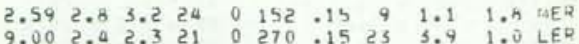

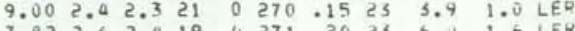

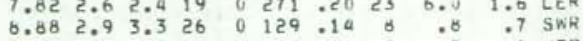

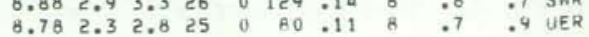

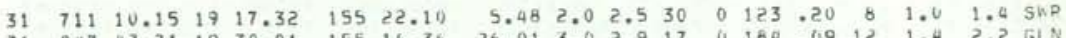

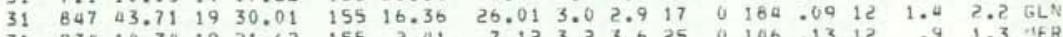

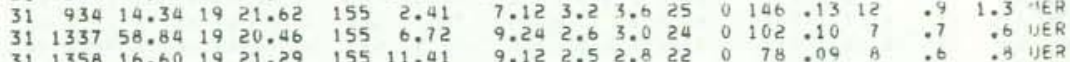
$31358,16.601921 .29 \quad 15511.41$

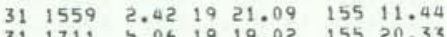

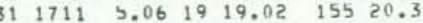

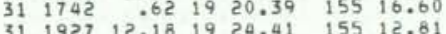

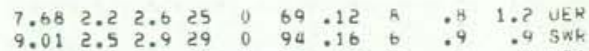
$9.612 .93 .129 \quad 0 \quad 80.119$ b :4 40 KOA 
ORIGIN TIME

LAT N YEAR MON DA HRIMN SEC DEG MIN

\begin{tabular}{|c|c|c|c|c|c|}
\hline 1975 JAN & 1 & 029 & 5.59 & 19 & 14.28 \\
\hline & 1 & 12 & 7.25 & 19 & 10.55 \\
\hline & 1 & 241 & 10.86 & 19 & 12.83 \\
\hline & 1 & 35 & 45.82 & 19 & 13.26 \\
\hline & 1 & 911 & 35.42 & 19 & 14.4 \\
\hline
\end{tabular}

$1946 \quad 45.66 \quad 19 \quad 14.40$

$\begin{array}{lllll}1 & 1027 & 5.19 & 19 & 12.50\end{array}$

$11033 \quad 47.74 \quad 1919.12$

$11046 \quad 49.39 \quad 19 \quad 16.61$

$11128 \quad 54.56 \quad 1910.95$

$1134429.79 \quad 1920.06$

$11410 \quad 58.84 \quad 19 \quad 19.78$

$11535 \quad 18.87 \quad 19 \quad 17.72$

$121355.79 \quad 1911.98$

$1211752.68 \quad 19 \quad 17.43$

$\begin{array}{llllll}12137 & 20.36 \quad 1926.00\end{array}$

$1232658.07 \quad 1914.01$

$12356 \quad 50.00 \quad 19 \quad 17.70$

2. $239 \quad 9.72 \quad 1912.06$

$\begin{array}{lllll}2 & 327 & 43.12 & 19 & 13.04\end{array}$

2 33953.431911 .25

? 4823.051916 .63

\begin{tabular}{l}
$2.825 \quad 29.20 \quad 1920.43$ \\
\hline
\end{tabular}

द $1148 \quad 16.63 \quad 19 \quad 19.95$

$\begin{array}{lllll}\text { 2 } & 1533 & 19.51 & 19 & 19.76\end{array}$

2 $1548 \quad 36.811911 .20$

314550.391910 .22

$3333 \quad 39.83 \quad 1913.14$

$3 \quad 732 \quad 49.30 \quad 1912.26$

381142.431914 .63

$381446.08 \quad 1913.01$

$\begin{array}{llllll}3 & 9 & 9 & 35.27 & 19 & 16.90\end{array}$

$\begin{array}{lllll}3 & 1027 & 9.55 & 19 & 14.45\end{array}$

$\begin{array}{llllll}3 & 1117 & 28.94 & 19 & 15.39\end{array}$

$\begin{array}{lllll}3 & 1153 & 10.10 & 19 & 19.28\end{array}$

$\begin{array}{lllll}3 & 1247 & 8.91 & 19 & 14.56\end{array}$

$\begin{array}{lllll}3 & 1630 & 9.44 & 19 & 17.45\end{array}$

3203552.381920 .27

$\begin{array}{lllll}4 & 213 & 28.10 & 19 & 14.45\end{array}$

$\begin{array}{lllll}4 & 358 & 15.22 & 17 & 52.96\end{array}$

$\begin{array}{lllll}415 & 26 & 26.61 & 19 & 14.47\end{array}$

$41532 \quad 5.221914 .89$

$41610 \quad 31.55 \quad 19 \quad 13.31$

$4 \quad 1613 \quad 18.24 \quad 19 \quad 13.92$

$41622 \quad 57.97 \quad 19 \quad 17.18$

$41624 \quad 53.85 \quad 19 \quad 15.48$

$4 \quad 1650 \quad 36.82 \quad 19 \quad 13.44$

$\begin{array}{lllll}4 & 1928 & 3.14 & 19 & 13.57\end{array}$

LON W DEPTH AMP DUR $K M \quad M A G$ MAG

GAP RMS MIN ERH ERZ OEG MIIN

15526.11

15520.87

15521.30

15522.40

15522.64

15524.31

15522.26

15513.90

15523.97

15521.80

15521.93

15521.33

15514.04

15520.90

15524.44

15527.66

15523.28

15516.07

15523.90

15523.19

15522.45

15524.58

15520.23

15519.68

15521.26

15522.19

$155 \quad 23.08$

15524.42

15521.72

15523.43

15523.00

15522.49

15522.68

15522.09

15521.29

15522.33

15543.06

1557.65

15523.27

15541.40

$155 \quad 22.28$

15522.39

15523.10

15521.94

15522.89

15520.90

15522.21

15520.94
$5.16 \quad 3.5$

$7.66 \quad 4.1$

5.954 .6

$9.30 \mathrm{s.5}$

9.493 .5

$5.814 .3 \quad 4.122$

$2.513 .6 \quad 3.6 \quad 28$ $11.30 \quad 3.4 \quad 3.8 \quad 20$

$7.924 .4 \quad 4.325$

$7.74 \quad 4.13 .927$

$2.96 \quad 3.0 \quad 3.6 \quad 15$

$3.32 \quad 3.1 \quad 3.6 \quad 19$

$\begin{array}{lllll}10.29 & 3.7 & 3.6 \quad 25\end{array}$

9.423 .43 .526

1.783 .13 .923

$9.63 \quad 3.6 \quad 3.7 \quad 32$

6.164 .24 .225

$9.76 \quad 3.7 \quad 3.3 \quad 28$

$10.813 .6 \quad 3.423$

6.155 .04 .724

$9.68 \quad 3.6 \quad 3.5 \quad 18$

8.443 .43 .526

$\begin{array}{llll}3.22 & 2.9 & 3.7 & 27\end{array}$

$\begin{array}{lllll}2.60 & 2.8 & 3.5 & 20\end{array}$

$1.02 \quad 2.6 \quad 3.6 \quad 26$

$\begin{array}{llll}8.21 & 3.5 & 3.7 & 23\end{array}$

$9.86 \quad 3.9 \quad .130$

$9.46 \quad 3.4 \quad 3.7 \quad 27$

9.624 .94 .726

$8.89 \quad 3.4 \quad 3.8 \quad 26$

$9.13 \quad 3.5 \quad 3.5 \quad 25$

$10.32 \quad 3.3 \quad 3.6 \quad 27$

$\begin{array}{lllll}5.80 & 3.5 & 3.9 & 28\end{array}$

$10.12 \quad 3.8 \quad 3.926$

$\begin{array}{llll}3.36 & 3.1 & 3.5 & 24\end{array}$

$8.30 \quad 3.5 \quad 3.6 \quad 28$ $\begin{array}{llll}15.50 & 3.7 & 2.8 \quad 5\end{array}$ $8.98 \quad 4.4 \quad 4.226$ $\begin{array}{llll}4.89 & 3.7 & 3.8 & 27\end{array}$ $\begin{array}{llll}71.81 & 3.7 & 15\end{array}$

$\begin{array}{llll}10.26 & 3.7 \quad 3.8 \quad 26\end{array}$ $9.70 \quad 5.0 \quad 4.828$ $5.39 \quad 3.4 \quad 3.5 \quad 22$ $7.26 \quad 3.5 \quad 3.5 \quad 26$ $8.693 .0 \quad 3.5 \quad 26$

$\begin{array}{lllll}4.93 & 3.2 & 3.6 \quad 27\end{array}$ $\begin{array}{lllll}5.71 & 3.0 & 3.7 \quad 18\end{array}$ 9.253 .93 .926 99

KM REMK

$1.6 \mathrm{LSW}$

2. 8 HLP

3. 1 LSW

2. 1 LSW

1. $7 \mathrm{LSW}$

0151.1811

0140.1511

$\begin{array}{llll}0 & 161 & .17 \quad 12\end{array}$

$\begin{array}{llll}0 & 127 & .19 & 7\end{array}$

$0 \quad 133.1515$

$0 \quad 171.16 \quad 14$

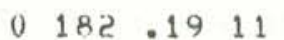

$\begin{array}{llll}0 & 151.118\end{array}$

$0 \quad 172.08 \quad 9$

$\begin{array}{llll}0 & 168 & .17 \quad 16\end{array}$

0110.1610

$044.16 \quad 13$

$0 \quad 153.1211$

1) $125.10 \quad 5$

$0 \quad 172.1214$

$0 \quad 158.1912$

$\begin{array}{llll}0 & 194.08 \quad 17\end{array}$

$0132.14 \quad 12$

66.125

$0 \quad 63.136$

$0 \quad 86.147$

$\begin{array}{llll}0 & 188 & .11 & 14\end{array}$

$\begin{array}{llll}0 & 171 & .19 & 16\end{array}$

$\begin{array}{lllll}0 & 151 & .15 & 13\end{array}$

0 164.1712

0144.1610

0163.1312

$\begin{array}{llll}0 & 125 & .17 & 7\end{array}$

$\begin{array}{llll}0 & 151 & .15 & 11\end{array}$

0144.12

05.117
0.117

$0152.1411 \quad 1.0 \quad 1.6 \mathrm{LSW}$ 0355.234897 .897 .8 HEA $\begin{array}{llllll}0 & 91 & .10 & 8 & .7 & .8\end{array}$ UER

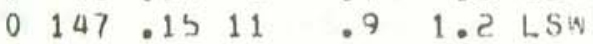
0336.4016484 .984 .4 DIS

$0 \quad 153.11 \quad 11$

0144.1610

0162.1112

$\begin{array}{llll}0 & 153 & 17 & 12\end{array}$

$0121.16 \quad 8$

$0146.17 \quad 10$

$0 \quad 173.10 \quad 19$

$\begin{array}{llll}0 & 141 & 13 & 13\end{array}$
1.

1. 1

. $y$

1.5

1.1

.9
1.4

1. 1
L LSW

.7 LSW

1.1 LSW

2.6 LSW

1. $5 \mathrm{SWR}$

1. 4 HLP 3.0 LSW

1.? L.Sw 
ORIGIN TIME LAT N LON W YEAR MON DA HRMN SEC DEG MIN DEG MIN

1975 JAN $5 \quad 048 \quad 39.62 \quad 19 \quad 13.59$ $\begin{array}{llllll}6 & 1747 & 2.90 & 19 & 16.61\end{array}$ 855250.231915 .32 $8 \quad 813 \quad 31.33 \quad 19 \quad 14.62$ $\begin{array}{llll}17 & 1399 & 9.13 \quad 19 & 17.92\end{array}$

FEB $\quad 7 \quad 646 \quad 52.37 \quad 19 \quad 14.85$ $20 \quad 1440 \quad 44.87 \quad 1925.57$ $21111940.47 \quad 1921.51$

MAR $10 \quad 014 \quad 12.67 \quad 1925.53$ $\begin{array}{lllllll}26 & 10 & 6 & 7.73 & 19 & 27.23\end{array}$

$\begin{array}{llllll}29 & 1456 & 26.36 & 19 & 17.15\end{array}$ $A P R \quad 4 \quad 13955.86 \quad 19 \quad 19.24$ $\begin{array}{lllll}4 & 1335 & 17.35 & 19 & 19.22\end{array}$ $\begin{array}{lllllll}5 & 8 & 3 & 3.20 & 19 & 16.70\end{array}$ $510249.91 \quad 1916.63$

\begin{tabular}{l}
$51156 \quad 58.28 \quad 19 \quad 17.02$ \\
\hline
\end{tabular}

$6 \quad 1215 \quad 55.40 \quad 1918.69$

$\begin{array}{lllll}7 & 424 & 56.27 & 19 & 24.85\end{array}$

$10 \quad 1418 \quad 40.24 \quad 1920.45$

$17 \quad 2330 \quad 14.73 \quad 19 \quad 18.31$

$\begin{array}{llllll}18 & 322 & 28.98 & 19 & 18.56\end{array}$

$\begin{array}{llllll}29 & 148 & 7.45 & 19 & 24.56\end{array}$

$\begin{array}{llll}30 & 642 \quad 28.95 \quad 19 & 28.56\end{array}$

$\begin{array}{lllll}\text { MAY } 12118 & 4.06 & 18 & 2.67\end{array}$

$\begin{array}{lllll}7 & 1752 & 36.27 & 19 & 48.80\end{array}$

$\begin{array}{llllll}7 & 2130 & 6.81 & 19 & 28.44\end{array}$ 10193251.911920 .26 $\begin{array}{lllll}19 & 613 & 10.36 & 19 & 25.67\end{array}$ 21 2232 $58.18 \quad 20 \quad 18.72$ $\begin{array}{llllll}24 & 22 & 4 & 45.75 & 19 & 24.88\end{array}$

$\begin{array}{lllllll}28 & 2 & 1 & 55.86 & 18 & .72\end{array}$ $\begin{array}{llllll}29 & 646 & 48.92 & 19 & 25.13\end{array}$ $\begin{array}{lllllll}29 & 1214 & 14.07 & 19 & 22.67\end{array}$ $\begin{array}{lllll}30 & 2245 \quad 37.02 \quad 19 & 27.21\end{array}$

JUN $5 \quad 811 \quad 3.14 \quad 19 \quad 19.10$

$7115357.62 \quad 19 \quad 11.75$

$\begin{array}{lllll}8 & 532 & 28.96 & 19 & 20.58\end{array}$ $\begin{array}{llllll}14 & 231 & 41.62 & 19 & 25.71\end{array}$ $\begin{array}{llllll}14 & 2247 & 6.82 & 19 & 25.90\end{array}$ $1501340.48 \quad 1919.98$

$\begin{array}{llllll}21922 & 21.03 & 19 & 24.74\end{array}$ $\begin{array}{llllll}21 & 1334 & 56.79 & 19 & 27.25\end{array}$ $\begin{array}{lllll}25 & 239 & 41.76 \quad 19 & 25.45\end{array}$ JUL $41740 \quad 54.89 \quad 19 \quad 22.37$ $5 \quad 2318 \quad 17.621929 .43$

$52325 \quad 45.16 \quad 1928.15$

$\begin{array}{llllll}7 & 539 & 48.74 & 19 & 32.28\end{array}$

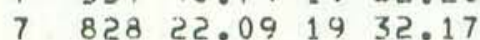

15523.28

15524.05

15520.55

15520.38

15523.61

$155 \quad 32.72$ $155 \quad 35.98$ 1556.48 15528.29 $155 \quad 35.47$

15522.57 15513.69 15513.64 15522.87 15523.03

1552.2 .64 $155 \quad 13.31$

$155 \quad 36.13$

1558.32

15513.26

$155 \quad 13.35$ 15516.65 15535.14 15543.69 $156 \quad 42.11$

$155 \quad 35.50$ 1557.58 15537.08 15536.78 $155 \quad 25.06$

15331.80 15536.50

1554.83

15536.33

15548.28

15536.48 1557.99 15747.45 $155 \quad 35.97$ $155 \quad 8.78$

$155 \quad 36.87$ 15528.80 15536.25 15519.03 15538.42

15538.00 15527.78 15527.65
DEPTH AMP DUR

6.024 .14 .124 $8.68 \quad 4.5 \quad 4.3 \quad 27$ $7.73 \quad 4.14 .0 \quad 26$ $7.88 \quad 3.5 \quad 3.9 \quad 24$ $5.44 \quad 3.0 \quad 3.7 \quad 23$

$9.26 \quad 3.6 \quad 3.9 \quad 30$ $3.49 \quad 3.5 \quad 3.8 \quad 26$

9.293 .43 .623

9.614 .03 .831 .804 .44 .225 $4.7 \quad 17$

$10.75 \quad 3.9 \quad 3.9 \quad 24$ $\begin{array}{lllll}10.38 & 3.5 & 3.7 & 27\end{array}$

$\begin{array}{llll}7.49 & 3.0 & 3.6 & 27\end{array}$

$\begin{array}{llll}7.74 & 3.5 & 3.5 & 28\end{array}$

$7.08 \quad 3.8 \quad 4.0 \quad 26$ $\begin{array}{llll}10.34 & 3.8 & 3.9 & 27\end{array}$

$4.70 \quad 3.9 \quad 3.9 \quad 22$ $9.613 .3 \quad 3.5 \quad 24$ $\begin{array}{llll}10.05 & 3.5 & 3.7 & 31\end{array}$

$\begin{array}{llllll}10.21 & 3.7 & 3.8 & 30\end{array}$ $15.05 \quad 3.4 \quad 3.7 \quad 33$ $2.643 .5 \quad 3.524$ $\begin{array}{lllll}59.22 & 3.0 & 4.0 & 29\end{array}$ $\begin{array}{lllll}10.62 & 3.7 & 2.9 & 31\end{array}$

$.893 .03 .5 \quad 25$ 9.494 .04 .129 $4.30 \quad 3.5 \quad 3.8 \quad 26$ $23.10 \quad 4.7 \quad 4.3 \quad 16$ $10.82 \quad 3.5 \quad 3.6 \quad 31$

$47.57 \quad 4.6 \quad 4.2 \quad 34$ $3.20 \quad 3.6 \quad 3.3 \quad 26$ $9.48 \quad 3.9 \quad 4.0 \quad 29$ $3.26 \quad 3.2 \quad 3.526$ $9.57 \quad 3.6 \quad 2.2 \quad 29$

$9.76 \quad 3.6 \quad 3.7 \quad 24$ $9.52 \quad 3.4 \quad 3.6 \quad 28$ $2.043 .8 \quad 2.8 \quad 25$ $2.44 \quad 2.93 .5 \quad 26$ $9.062 .8 \quad 3.5 \quad 28$

$.073 .53 .6 \quad 24$ $9.76 \quad 3.5 \quad 3.4 \quad 34$ $3.253 .2 \quad 3.628$ 30.474 .24 .236 $.004 .3 \quad 4.311$

.204 .24 .27 $6.27 \quad 3.6 \quad 3.4 \quad 30$ $6.94 \quad 3.6 \quad 3.3 \quad 17$ 100
GAP RMS MIN ERH ERZ

KM REMK

$\begin{array}{lllllll}0 & 155 & .12 & 11 & 1.0 & 2.4 & \text { LSW } \\ 0 & 119 & .18 & 10 & 1.1 & 1.7 & \text { SWR }\end{array}$

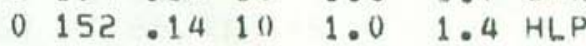

$\begin{array}{llllll}0 & 157 & .13 & 11 & .9 & 1.3\end{array}$ HLP

$\begin{array}{lllll}0110.1311 .8 & 1.0 & \text { SWR }\end{array}$

$\begin{array}{lllllll}0 & 67 & .18 & 13 & 1.0 & 1.3 \quad \mathrm{LSW}\end{array}$

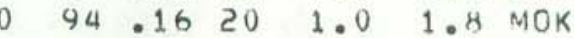

$\begin{array}{lllllll}0 & 139 & .09 & 12 & .7 & .8 & \text { UER }\end{array}$

\begin{tabular}{llllll}
0 & 60 & .14 & 13 & .7 & .4 \\
\hline
\end{tabular}

$\begin{array}{llll}0 & 83 & 17 & 4\end{array}$

$.91 .4 \mathrm{MOK}$

1.12 .7 SWR

.8 .4 UER

.6 .3 UER

.81 .4 SWR

1.11 .8 SWR

1.02 .1 SWR

$.6 \quad .3 \mathrm{POL}$

2. 1.9 MOK

.1 .5 UER

$.6 .3 \mathrm{POL}$

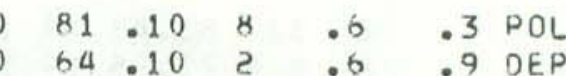
$\begin{array}{lllllllllll}0 & 132 & 16 & 17 & 1.1 & 2.0 & \text { MOK }\end{array}$ $\begin{array}{llllll}0 & 327 & .21136 & 55.4 & 23.5 & \text { DIS }\end{array}$ 0254.201037 .199 .0 DIS

$\begin{array}{rrrrrrr}0 & 61 & .13 & 3 & .5 & .5 & \text { MOK } \\ 0 & 92 & .10 & 8 & .7 & .7 & \text { UER } \\ 0 & 140 & .10 & 18 & .5 & .9 & \text { MOK } \\ 4 & 191 & .10 & 61 & 1.5 & 4.3 & \text { DIS } \\ 0 & 41 & .16 & 9 & .9 & .4 & \text { UKF }\end{array}$

0339.1222078 .627 .0 DIS $\begin{array}{llllll}0 & 82.16 & 8 & .7 & 1.8 & \text { MOK }\end{array}$ $\begin{array}{lllllll}0 & 87 & .11 & 11 & .8 & .7 & \text { MER }\end{array}$ $\begin{array}{llllll}0 & 70.15 & 4 & .1 & 1.0 & \text { MOK }\end{array}$ $\begin{array}{llllll}0 & 169 & .14 & 26 & 1.2 & .4 \mathrm{kON}\end{array}$

$\begin{array}{llllll}0 & 116 & .15 & 24 & 1.2 & .6\end{array}$ HEA $\begin{array}{llllll}0 & 81 & .10 & 9 & .7 & .4\end{array}$ UER $03361.0120799 .0 \quad$.0 DIS $\begin{array}{llllll}0 & 51.16 & 6 & .7 & \text { ?.2 } & \text { MOK }\end{array}$ $\begin{array}{llll}73.119 & .7 & .7\end{array}$

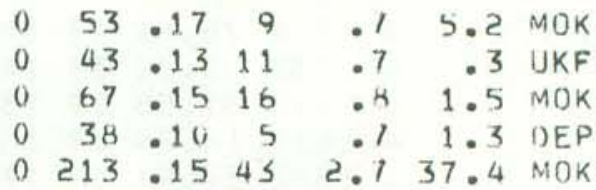

$2.737 .4 \mathrm{MOK}$

$3.756 .4 \mathrm{MOK}$

.8 2.3 NER

2. 1 3.4 NER 
ORIG IN TIME LAT iv YEAR MON DA HRMN SEC DEG MIN

1475 JUL $71447 \quad 42.51 \quad 19 \quad 32.38$

$\begin{array}{llllll}7 & 1839 & 52.34 & 19 & 31.79\end{array}$

$72032 \quad 36.15 \quad 19 \quad 32.51$

$\begin{array}{lllllll}8 & 1 & 9 & 7.71 & 19 & 31.82\end{array}$

$8 \quad 1056 \quad 35.18 \quad 19 \quad 31.52$

$\begin{array}{llllll}8 & 20 & 7 & 2.68 & 19 & 32.29\end{array}$

$\begin{array}{llll}9 & 349 & 27.55 \quad 19 & 32.18\end{array}$

$\begin{array}{lllll}9 & 547 & 42.70 & 19 & 32.57\end{array}$

$9840 \quad 3.50 \quad 19 \quad 31.88$

$9 \quad 1334 \quad 23.80 \quad 19 \quad 32.51$

$\begin{array}{llllll}18 & 2119 & 58.22 & 19 & 20.74\end{array}$

$\begin{array}{lllll}22 & 1512 & 36.48 & 19 & 39.17\end{array}$

$\begin{array}{lllll}26 & 2314 & 59.23 \quad 19 & 50.86\end{array}$

$\begin{array}{lllll}30 & 1331 & 22.36 & 19 & 31.98\end{array}$

AUG 5155136.721416 .35

5165351.041916 .34

$\begin{array}{llllll}18 & 7 & 8 & 57.14 & 19 & 38.56\end{array}$

2454535.221922 .00

$\begin{array}{lllll}27 & 734 & 44.22 & 19 & 26.95\end{array}$

$312140.38 \quad 1855.43$

SEP $322 \quad 416.3918 \quad 52.42$ $\begin{array}{llllll}10 & 834 & 13.49 & 19 & 19.79\end{array}$

$\begin{array}{llllll}21 & 1946 & 5.24 & 19 & 20.20\end{array}$

$\begin{array}{lllll}22 & 210 \quad 7.08 \quad 19 & 23.78\end{array}$

ट० $039 \quad .7118 \quad 59.24$

OCT $12134 \quad 39.58 \quad 19 \quad 19.16$ द $425 \quad 30.28 \quad 1954.38$

ट $1417 \quad 49.9418 \quad 26.72$

$818538.0119 \quad 16.70$

14194346.801920 .25

$19 \quad 1546.54 \quad 198.53$ $22123913.9219 \quad 46.16$ 2ट $165254.88 \quad 1855.37$ 2353315.101918 .88 $26 \quad 2337 \quad 40.30 \quad 19 \quad 19.20$

$\begin{array}{lllll}27 & 1935 & 52.87 & 19 & 18.92\end{array}$ $31450 \quad 53.75 \quad 19 \quad 12.01$ NOV $11022 \quad 3.97 \quad 18 \quad 55.38$ 62523.241920 .42

$6 \quad 32118.521919 .82$

$\begin{array}{lll}64256.32 & 1920.01\end{array}$

$\begin{array}{lllll}7 & 1912 & 14.39 & 19 & 21.91\end{array}$

$895938.46 \quad 1917.64$

$\begin{array}{lllll}10 & 126 & 29.98 & 19 & 21.49\end{array}$

$\begin{array}{llllll}11 & 6 & 9 & 12.26 & 19 & 21.56\end{array}$

$\begin{array}{lllll}13232 & 4.91 & 19 & 21.49\end{array}$

$14 \quad 123 \quad 25.71 \quad 1920.41$

$14 \quad 15145.8819 \quad .08$
LON W DEPTH AMP DUR DEG MIN

15529.52

15527.61

15527.80

15528.35

15528.41

15527.59

15528.06

15527.95

15528.17

15527.50

$155 \quad 6.49$

15557.00

15533.58

15536.00

15522.98

15523.42 $156 \quad 1.21$

$155 \quad 16.75$

15528.44

15516.55

15515.08

1558.56

15512.48

15526.10

$155 \quad 16.27$

$155 \quad 13.47$

15736.61

$156 \quad 43.43$

15525.91

15511.61

$155 \quad 33.47$

$155 \quad 23.23$

$155 \quad 17.40$

$155 \quad 13.48$

15528.34

$\begin{array}{ll}155 & 13.37\end{array}$

15538.50

15517.62

15518.74

15518.67

15518.10

15516.68

15552.76

15 ל 2.16

15515.35

1553.56

$155 \quad 20.47$

15529.35
$2.75 \quad 4.7 \quad 4.6 \quad 30$

5.434 .24 .333

4.92 $3.5 \quad 3.6 \quad 31$

$\begin{array}{llll}7.60 & 3.6 & 3.5 & 25\end{array}$

$7.613 .3 \quad 3.5 \quad 30$

$7.36 \quad 3.9 \quad 3.8 \quad 33$

$\begin{array}{lllll}7.77 & 3.4 & 3.6 & 31\end{array}$

$8.56 \quad 3.9 \quad 4.0 \quad 30$

8.434 .54 .335

$7.413 .43 .7 \quad 32$

$9.40 \quad 3.4 \quad 3.5 \quad 22$

$18.57 \quad 4.1 \quad 4.3 \quad 19$

$\begin{array}{llll}30.89 & 3.7 & 3.532\end{array}$

$9.9 .5 \quad 3.9 \quad 3.8 \quad 26$

$7.45 \quad 3.0 \quad 3.6 \quad 15$

$\begin{array}{llll}7.07 & 3.0 & 3.6 \quad 25\end{array}$ $\begin{array}{llll}37.42 & 3.9 & 3.8 & 25\end{array}$ $35.15 \quad 3.6 \quad 3.130$ $10.14 \quad 4.0 \quad 4.0 \quad 31$

10.202 .15 .316

$10.89 \quad 3.0 \quad 3.7 \quad 24$

$9.17 \quad 3.6 \quad 3.8 \quad 13$

$10.10 \quad 3.2 \quad 3.6 \quad 28$

$9.393 .43 .6 \quad 26$

14.833 .94 .031

$10.27 \quad 3.7 \quad 4.0 \quad 29$

$8.003 .8 \quad 14$

$73.243 .6 \quad 20$

$8.413 .4 \quad 4.0 \quad 29$

$10.17 \quad 2.8 \quad 3.5 \quad 33$

$31.38 \quad 3.5 \quad 3.6 \quad 30$

$16.89 \quad 3.0 \quad 3.6 \quad 33$

$\begin{array}{lllll}11.71 & 3.1 & 3.7 & 31\end{array}$

$10.152 .9 \quad 3.8 \quad 31$

$9.97 \quad 3.3 \quad 3.925$

$10.20 \quad 3.7 \quad 3.9 \quad 29$

$9.15 \quad 3.8 \quad 4.232$

11.875 .23 .629

$30.394 .6 \quad 4.7 \quad 36$

30.824 .13 .935

$29.53 \quad 3.8 \quad 3.9 \quad 36$ $27.96 \quad 3.2 \quad 3.6 \quad 36$ $13.243 .6 \quad 4.0 \quad 28$ $8.87 \quad 4.3 \quad 4.6 \quad 28$ 9.593 .13 .631

प्र.83 $3.7 \quad 4.231$ $32.05 \quad 3.7 \quad 4.0 \quad 32$ $35.47 \quad 3.2 \quad 3.6 \quad 31$ 101

GAP KMS MIN ERH ERZ

DEG SEC DIS KM KM REMK

$\begin{array}{rrrl}0 & 119 & .20 & 15 \\ 0 & 67 & .14 & 15 \\ 0 & 78 & .15 & 15 \\ 0 & 76 & .17 & 14 \\ 0 & 74 & .16 & 15 \\ 0 & 69 & .15 & 15 \\ 0 & 82 & .14 & 14 \\ 0 & 80 & .19 & 15 \\ 0 & 68 & .15 & 14 \\ 0 & 69 & .15 & 15\end{array}$

1.42 .3 NER

. 8 1.9 NER

.8 1.4 NER

1.12 .5 NER

1.0 2.5 NER

.9 1.6 INER

.9 1.5 NER

1.3 1.6 NER

-9 1.1 NER

.81 .6 NER

0139.1011

0266.1542

$0200 \quad .15 \quad 34$

0128.129

0148.1119

.8

.7 UEK

$9.28 .3 \mathrm{KON}$

$1.4 \quad 4.9 \mathrm{KKU}$

$1.1 .4 \mathrm{MOK}$

1.1 1.5 SWR

0126.159

$.9 \quad 1.6$ SWR

2. 0 3.1 KOiv

1.11 .9 DEP

.9 .4 UKF

$3.3 .7 \mathrm{PPL}$

4.799.0 PPL

2. 0.9 UER

. 8 .3 UER

.6 .8 UKF

1.916 .2 PPL $\begin{array}{rrrrrrr}0 & 71 & .08 & 7 & .3 & .2 & \text { UER } \\ 2 & 342 & .17198 & 65.4 & 74.3 & \text { DIS }\end{array}$ $\begin{array}{llll}034 & .12143 \quad 84.7 & 41.3 & \text { DIS }\end{array}$ $0119.20101 .1 \quad 1.6$ SWR

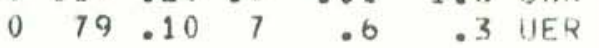
$0133.1521 \quad 1.5 \quad 3.4 \quad \mathrm{LSW}$ $\begin{array}{lllllll}0 & 81 & .10 & 50 & .7 & 3.7 & \mathrm{KKli}\end{array}$ $1245.0941 \quad 1.8$
$0.8 \mathrm{PPL}$

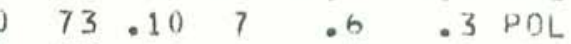
$\begin{array}{lllll}0.11 & 9 & .7 & .3 & \text { HEA }\end{array}$ $\begin{array}{rrrr}0 & 76 & .11 & 7 \\ 0 & 101 & .18 & 22\end{array}$ 0243.0940 $0 \quad 53.116$

$.6 \quad .3$ POL 1.1 1.1 HEA 2. $199.0 \mathrm{PPL}$ .81 .5 DEP

.7 1.? UEP

.71 .3 DEP

$\begin{array}{lll}.7 & 1.5 & 1.5 \mathrm{EP}\end{array}$

1.5 5. 5 KON

$\begin{array}{rrrrrrr}0 & 185 & .11 & 27 & 1.2 & 2.2 & K O N \\ 0 & 203 & .12 & 13 & 1.3 & .8 & M E R \\ 0 & 63 & .08 & 4 & .4 & .3 & K O A\end{array}$

0 161.12 11

1.0

- 6 NIER

0 कह 1116

.4

1.8 DEP

1.7 ?. $R$ LS N 
ORIGIIN TIME LAT IN YEAR MON DA HRMN SEC DEG MIN

1975 NOV 15125521.241919 .20

$\begin{array}{lllll}18 & 1310 & 2.02 & 19 & 22.53\end{array}$

$\begin{array}{lllll}27 & 1615 & 40.88 & 19 & 34.90\end{array}$

$2933540.59 \quad 1922.08$

$\begin{array}{lllll}29 & 438 & 26.73 & 19 & 19.41\end{array}$

$29 \quad 447 \quad 40.06 \quad 1920.90$

$\begin{array}{llllll}29 & 834 & 56.13 & 19 & 22.75\end{array}$

$29842 \quad 12.78 \quad 1920.41$

$\begin{array}{lllll}29 & 843 & 59.97 & 19 & 8.63\end{array}$

$2991320.15 \quad 19 \quad 17.84$

$\begin{array}{lllll}29 & 926 & 2.12 & 19 & 22.62\end{array}$ $\begin{array}{llllll}29 & 951 & 10.14 & 19 & 20.85\end{array}$ $\begin{array}{llllll}29 & 10 & 0 & 45.48 & 19 & 23.44\end{array}$ $\begin{array}{lllllll}29 & 10 & 7 & 51.43 & 19 & 24.33\end{array}$ $291010 \quad 1.25 \quad 1912.95$

$\begin{array}{lllll}29 & 1019 & 5.18 & 19 & 21.49\end{array}$ $29 \quad 1045 \quad 1.0319 \quad 19.33$ $\begin{array}{llllll}29 & 11 & 1 & 9.09 & 19 & 19.33\end{array}$ $\begin{array}{llllll}29 & 1151 & 38.86 & 19 & 20.38\end{array}$ $\begin{array}{lllll}29 & 125 & 52.8519 & 23.62\end{array}$

29 1222 27.54 19 16.04 29 122427.021916 .72 $\begin{array}{llllll}29 & 1228 & 12.88 & 19 & 19.72\end{array}$ $\begin{array}{llllll}29 & 1245 & 35.45 & 19 & 15.67\end{array}$ $29132923.06 \quad 19 \quad 4.83$

$29 \quad 141258.681924 .26$ $29 \quad 1414 \quad 33.34 \quad 19 \quad 19.96$ 29144245.091923 .19 $\begin{array}{llllll}29 & 1526 & 45.66 & 19 & 20.58\end{array}$ $\begin{array}{lllll}29 & 1627 & 7.08 & 19 & 22.49\end{array}$

$29 \quad 1629 \quad 29.26 \quad 1924.59$ 29164557.291924 .29 $29 \quad 1724 \quad 35.60 \quad 19 \quad 19.72$ $291733 \quad .911919 .23$ $\begin{array}{lllll}29 & 1739 & 22.38 & 19 & 24.01\end{array}$

$29 \quad 1745 \quad 18.17 \quad 19 \quad 23.83$ $\begin{array}{lllllll}29 & 189 & .96 & 19 & 16.93\end{array}$ $29 \quad 1823 \quad 37.61 \quad 1916.64$ $29183750.70 \quad 1923.22$ $29185256.32 \quad 1925.97$

29 $1856 \quad 38.54 \quad 19 \quad 22.49$ $29 \quad 1935 \quad 31.48 \quad 19 \quad 13.37$ $\begin{array}{lllll}29 & 2015 & 27.28 & 19 & 25.19\end{array}$ $\begin{array}{llllll}29 & 219 & 3.54 & 19 & 16.65\end{array}$ $\begin{array}{llllll}30 & 121 & 41.18 & 19 & 20.78\end{array}$

$3026 \quad 31.74 \quad 19 \quad 14.10$ $\begin{array}{llllll}30 & 3 & 1 & 31.67 & 19 & 20.75\end{array}$ $\begin{array}{lllll}30 & 328 & 27.07 & 19 & 25.67\end{array}$
DEG MIN

$155 \quad 13.41$

$155 \quad 3.75$

$155 \quad 58.29$

$155 \quad 2.84$

$155 \quad 13.42$

$155 \quad .94$

$155 \quad 5.21$

$155 \quad 4.70$

$155 \quad 15.37$

$155 \quad 15.75$

15459.70

1559.09

1553.23

1553.59

154.28

15515.56

15520.71

$155 \quad 6.90$

$154 \quad 58.73$

155.49

15521.93

15521.92

15520.56

15515.41

15512.13

15458.45

15515.70

$155 \quad 1.33$

1554.31

1551.16

15524.00

1553.55

1556.28

$155 \quad 6.45$

155.15

$154 \quad 59.03$ 15520.22 15458.30 15459.25 $155 \quad 6.61$

$154 \quad 52.87$ $155 \quad 23.07$ 15522.46 15522.01

$155 \quad 6.59$

$155 \quad 23.97$ 1559.18 15454.53
DEPTH AMP DUR GAP RMS MIN ERH ERZ KM MAG MAG NR NS DEG SEC DIS KM KM REMK

$\begin{array}{rrrrrrrrrrr}10.68 & 4.4 & 4.4 & 29 & 0 & 72 & .08 & 7 & .5 & .2 & \text { UER } \\ 8.66 & 3.1 & 3.6 & 30 & 0 & 106 & .14 & 12 & 1.0 & .7 & M E R \\ 9.11 & 3.6 & 3.2 & 30 & 0 & 247 & .16 & 23 & 2.6 & .8 & K O N \\ 8.62 & & 5.9 & 28 & 0 & 129 & .13 & 12 & 1.1 & .7 & \text { MER } \\ 8.28 & 2.6 & 3.5 & 28 & 0 & 69 & 10 & 7 & .6 & .7 & \text { UER }\end{array}$

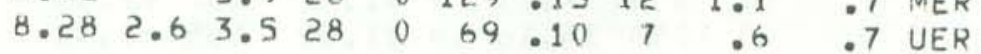

$8.33 \quad 7.224 \quad 0 \quad 198 \quad .13 \quad 16 \quad 1.6 \quad 1.1$ LER $\begin{array}{lllllllllll}.60 & 3.7 & 4.0 & 12 & 0 & 202 & .11 & 11 & 1.7 & 41.6 & 1\end{array}$ $\begin{array}{llllllllll}5.96 & 4.3 & 3.9 & 13 & 0 & 118 & .05 & 10 & .5 & .6\end{array} \mathrm{NER}$ $\begin{array}{llllllllllll}5.93 & 4.8 & 3.7 & 11 & 0 & 201 & .19 & 26 & 4.1 & 4.9 & \text { PPL }\end{array}$ $\begin{array}{lllllllllll}4.97 & 3.6 & 4.0 & 7 & 0 & 179 & .11 & 8 & 2.1 & 3.2 & \text { KOA }\end{array}$

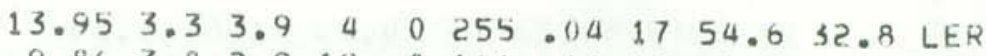
$9.86 \quad 3.82 .919 \quad 0 \quad 166.10 \quad 10 \quad 1.1 \quad .4$ UER $\begin{array}{llllllllll}5.99 & 3.7 & 2.9 & 16 & 0 & 179 & .14 & 13 & 1.3 & 1.8\end{array}$ $1.09 \quad 3.810 \quad 0 \quad 173.16 \quad 12 \quad 1.5 \quad 53.0$ MER $\begin{array}{llllllll}8.40 & 3.5 & 1.8 & 6 & 0 & 347 & .39119 & 99.0 \quad \text {.1 DIS }\end{array}$

$\begin{array}{rrrrrrrrrr}9.57 & 3.7 & 4.0 & 20 & 0 & 70 & .11 & 4 & .8 & .4 \\ 1.40 & 3.3 & 3 & 8 & 10 & 0 & 118\end{array}$

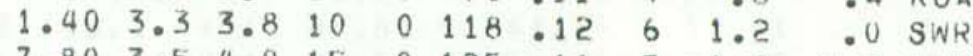
$\begin{array}{lllllllllll}7.80 & 3.5 & 4.0 & 15 & 0 & 125 & .11 & 7 & 1.2 & 2.8 & 1.2\end{array}$ \begin{tabular}{llllllllll}
1.41 & 3.0 & 3.8 & 6 & 0 & 277 & .17 & 20 & 20.8 & 98.6 \\
\hline
\end{tabular}

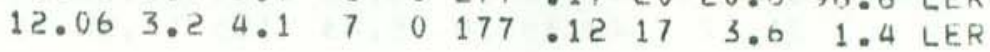

$8.343 .4 \quad 3.820 \quad 0 \quad 142 \quad .10 \quad 8 \quad 1.0 \quad 1.1$ SWk

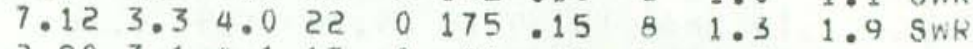
$\begin{array}{lllllllllll}2.90 & 3.1 & 4.1 & 17 & 0 & 88 & .11 & 9 & .7 & 2.6 & \text { SWR }\end{array}$ $\begin{array}{llllllllllll}6.75 & 2.8 & 3.9 & 13 & 0 & 191 & .10 & 12 & 1.4 & 3.0 & \text { HL.P }\end{array}$ $10.23 \quad 3.54 .018 \quad 0221.1350 \quad 2.6 \quad .5$ PPL

$\begin{array}{lllllllllll}7.09 & 3.1 & 3.5 & 16 & 0 & 178 & .20 & 20 & 3.0 & 2.1 & 17 E R\end{array}$ $\begin{array}{lllllllllll}8.92 & 3.4 & 3.6 & 15 & 0 & 118 & .15 & 9 & 1.4 & 1.2 & \text { KOA }\end{array}$

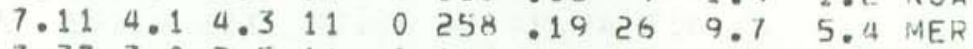
$\begin{array}{lllllllllll}7.37 & 3.0 & 3.5 & 16 & 0 & 110 & .10 & 10 & .9 & 1.0 & \text { MER }\end{array}$

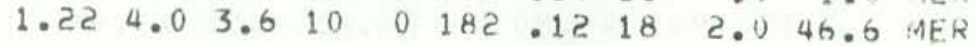

$\begin{array}{llllllllll}9.25 & 3.5 & 2.8 & 21 & 0 & 80 & .15 & 8\end{array}$ $\begin{array}{lllllllll}.33 & 3.0 & 3.9 & 15 & 0 & 108 & .12 & 12\end{array}$ $\begin{array}{lll}7.66 & 3.8 & 11\end{array}$ $8.08 \quad 3.5 \quad 3.3 \quad 16$ 3.713 .43 .818

$0 \quad 127 \cdot 128$ $\begin{array}{llll}0 & 138 & .14 \quad 8\end{array}$

$\begin{array}{lllll}5.76 & 3.9 & 4.0 & 18\end{array}$ 9.634 .019 $10.69 \quad 4.218$

$\begin{array}{lllll}5.83 & 3.7 & 3.1 & 22\end{array}$ 0160.1115

.91 .0 UKF .973 .6 MER $1.7 \quad 1.6$ UER 1.2 1.0 UER . 81.1 LER .203 .94 .04

$\begin{array}{llll}0 & 177.11 & 14\end{array}$

$1.31 .1 \mathrm{LER}$

$1.0 .4 \mathrm{SWR}$

$6.1 \quad .9$ DIS

1.4 1.9 LER

$7.699 .0 \mathrm{GLN}$

$\begin{array}{lllll}7.19 & 3.8 & 3.7 & 16\end{array}$

$\begin{array}{llll}3.59 & 3.5 & 3.3 & 20\end{array}$

$10.36 \quad 4.5 \quad 4.122$

$7.16 \quad 3.1 \quad 3.920$
.98 .45

9.48

3.825

$7.60 \quad 3.6 \quad 24$

$8.85 \quad 3.7 \quad 3.7 \quad 2$ ?

$8.70 \quad 3.5 \quad 3.4 \quad 21$ 102
5.S 2.0 LER

1.1) $1.9 \mathrm{LSW}$

. 5 U UKF

1. () $1.4 \mathrm{SWK}$

.7 .5 UER

$1.11 .7 \mathrm{LSW}$ 1.9. $9 \mathrm{LEK}$ .8 .9 UER 
ORIGIN TIME LAT N YEAR MON DA HRMN SEC DEG MIN

1975 NOV $30 \quad 1047 \quad 14.491921 .59$

$\begin{array}{lllllll}30 & 14 & 8 & 38.89 & 19 & 19.93\end{array}$

$\begin{array}{llllll}30 & 1720 & 24.44 & 19 & 21.75\end{array}$

$\begin{array}{lllll}30 & 2057 & 18.34 & 19 & 22.59\end{array}$

$\begin{array}{lllll}30 & 2240 & 42.06 \quad 19 & 19.81\end{array}$

\section{DEC}

LON W
DEG MIN

15456.70

15512.68

1554.86

$\begin{array}{ll}155 & 2.97\end{array}$

1553.53

15517.50

1553.50

1557.49

15458.71

$155 \quad 6.87$

$110955.20 \quad 1923.43$

118334.411922 .04

2 $015 \quad 48.111921 .73$

द $1421 \quad 18.52 \quad 1918.83$

$345358.64 \quad 1925.79$

$\begin{array}{llllll}3 & 5 & 5 & 36.55 & 19 & 19.34\end{array}$

$3538 \quad 1.04 \quad 1923.62$

$31119 \quad 23.041921 .66$

$\begin{array}{llllll}3 & 1250 & 22.98 & 19 & 22.32\end{array}$

$32027 \quad 56.921919 .93$

$\begin{array}{lllll}059 & 2.17 & 19 & 23.91\end{array}$

$\begin{array}{llll}4 & 19 & 8.27 \quad 19 & 22.71\end{array}$

$4103511.25 \quad 19 \quad 23.63$

$41119 \quad 48.83 \quad 1921.59$

4113910.051921 .01

4142132.831926 .43

4173217.311920 .97

$421431.33 \quad 1922.66$

$\begin{array}{llllll}5 & 2 & 8 & 37.53 & 19 & 23.63\end{array}$

$\begin{array}{llllll}5 & 109 & 8.54 & 19 & 22.01\end{array}$

$5 \quad 181155.16 \quad 1917.36$

6
137 $59.09 \quad 1923.80$

$\begin{array}{lllll}6 \quad 1310 \quad 46.44 \quad 19 & 22.75\end{array}$

$\begin{array}{lllll}6 & 1910 & 8.98 & 19 & 20.21\end{array}$

$041 \quad 19.08 \quad 1921.79$

34720.861921 .66

$\begin{array}{llll}7 & 13922.46 \quad 19 & 16.47\end{array}$

$\begin{array}{llllll}8 & 620 & 14.37 & 19 & 22.23\end{array}$

89253.101921 .30

$\begin{array}{llllll}8 & 10 & 8 & 39.58 & 19 & 22.29\end{array}$

$\begin{array}{lllll}8 & 1526 & 43.69 & 19 & 19.95\end{array}$

$972.06 \quad 1921.63$

$9 \quad 932 \quad 40.96 \quad 19 \quad 18.28$

$9 \begin{array}{lllll}9 & 1018 & 0.34 & 19 & 21.40\end{array}$

9104411.521921 .88

$9135554.52 \quad 1921.66$

10115232.681921 .01

$10 \quad 1543 \quad 16.88 \quad 1920.06$ $\begin{array}{lllll}11 & 541 & 19.06 & 19 & 21.90\end{array}$ 11121555.621921 .49

15515.30

15523.59

15523.37

15511.41

15459.07

$154 \quad 58.97$

$155 \quad 1.75$

1554.92

15456.25

15456.49

1554.78

15457.79

$154 \quad 52.04$

$155 \quad 6.17$

$155 \quad 15.14$

15521.30

15458.99

1551.88

1557.33

1557.28

15515.45

15512.11

15518.52

15515.55

1552.27

1556.96

15515.11

15520.79

1557.25

1553.02

1557.59

$155 \quad 5.94$

$155 \quad 12.03$

1557.13

$155 \quad 15.44$

DEPTH AMP DUR

GAP RMS MIN ERH ERZ

KM MAG MAG NR NS DEG SEC OIS KM KM REMK

$\begin{array}{llllllll}2.83 & 3.2 & 3.7 & 13 & 0 & 267 & .16 & 23\end{array}$

$8.42 \quad 3.6 \quad 3.8 \quad 26$

$8.24 \quad 3.5 \quad 3.8 \quad 24$

$5.97 \quad 3.7 \quad 4.229$

$\begin{array}{llll}4.89 & 3.7 & 3.9 & 30\end{array}$

$6.762 .9 \quad 3.523$

.253 .13 .918

$\begin{array}{llll}.82 & 3.1 & 3.8 \quad 7\end{array}$

$8.73 \quad 3.73 .924$

$8.05 \quad 3.5 \quad 3.227$

15458.83

$155 \quad 4.67$

$155 \quad 1.81$
9.173 .64 .229

$6.413 .2 \quad 3.525$

$9.84 \quad 3.5 \quad 3.4 \quad 21$

$10.05 \quad 3.8 \quad 4.0 \quad 19$

6.194 .24 .219

5.234 .04 .210

6.894 .24 .222

$7.56 \quad 5.53 .023$

$4.78 \quad 3.8 \quad 4.1 \quad 12$

9.164 .04 .220

$4.70 \quad 3.5 \quad 4.1 \quad 17$

$7.48 \quad 3.7 \quad 4.120$

$6.38 \quad 3.5 \quad 4.1 \quad 24$

8.643 .73 .926

$8.25 \quad 3.6 \quad 4.0 \quad 25$

$\begin{array}{lllll}7.97 & 3.1 & 3.6 & 26\end{array}$

$2.06 \quad 2.5 \quad 3.6 \quad 16$

$\begin{array}{llll}9.56 & 3.3 & 3.7 & 27\end{array}$

$8.85 \quad 3.8 \quad 4.126$

$6.79 \quad 3.0 \quad 3.5 \quad 24$

$7.43 \quad 3.1 \quad 3.6 \quad 24$

$9.53 \quad 3.0 \quad 3.8 \quad 25$

$\begin{array}{llll}7.31 & 2.9 & 3.5 & 27\end{array}$

$9.97 \quad 3.3 \quad 3.8 \quad 29$

$6.22 \quad 3.3 \quad 3.7 \quad 25$

$\begin{array}{llll}12.11 & 2.9 & 3.5 & 29\end{array}$

$\begin{array}{lllll}13.56 & 3.4 & 5.7 & 29\end{array}$

$7.64 \quad 3.6 \quad 4.123$

$4.84 \quad 3.4 \quad 4.021$

$9.853 .2 \quad 3.5 \quad 27$

$\begin{array}{lllll}8.96 & 3.6 & 3.9 & 25\end{array}$

$8.48 \quad 4.43 .122$

$8.38 \quad 2.8 \quad 3.6 \quad 17$

$9.16 \quad 4.0 \quad 4.125$

$9.57 \quad 3.4 \quad 3.825$

9.854 .04 .026

8.813 .23 .526

$8.973 .4 \quad 3.724$

103 $\begin{array}{rrr}7.4 & 2.7 & \text { LER } \\ .1 & .8 & \text { UER } \\ .8 & 1.5 & \text { MER } \\ 1.0 & 1.8 & \text { MER } \\ 1.4 & .9 & \text { MER }\end{array}$

1.42 .1 HLP

1.5 98.0 MER

.6 .0 UER

1.1 .9 LER

.0 .7 UER

$.5 .4 \mathrm{KOA}$

.81 .2 Sivk

. .5 UKF

1.1 .4 UER

1.1 1.2. LER

2.S 4.0 LER

1.0 .8 MER

.0 .5 MER

1.92 .9 LER

2.91 .3 LER

1.6 1.3 LER

1.9 . 8 MER

1.9 1.3 LER

1.2 .5 LER

9 MER

.7 UER

4. 4 MER

$.5 \mathrm{KOA}$

$\begin{array}{ll}.8 & 1.2 \\ 1.3 & 1.1\end{array}$

2.0 1.5 MER

.7 .4 HER

.6 .9 UER

$.5 \mathrm{KOA}$

$1.12 .1 \mathrm{PUL}$

.2 KOA

.7 DEP

1.3 MER

1. 0 UIER

$.5 \mathrm{KOA}$

.8 SWK

.9 UER

1.0 MER

.5 IIER

.4 MER

- 4 UER

. 4 UER

- 8 KOA 
ORIGIN TIME

LAT $N$ YEAR MON DA HRMN SEC DEG MIN
LON W DEPIH AMP DUR DEG NIIN

$155 \quad 3.70$

1556.26

1552.40

$155 \quad 3.18$

$154 \quad 31.92$

$\begin{array}{lllll}13 & 053 & 36.33 & 19 & 22.6\end{array}$

13111743.011953 .21

$14 \quad 1048 \quad 49.26 \quad 19 \quad 20.64$

$\begin{array}{lllll}1449 & 27.211918 .70\end{array}$

$\begin{array}{lllll}17 & 040 & 25.81 & 19 & 26.68\end{array}$

$\begin{array}{lllll}17 & 954 & 30.16 \quad 19 & 21.75\end{array}$

$19913 \quad 43.44 \quad 1925.82$

$20135548.09: 918.40$

20 $1936 \quad 43.42 \quad 19 \quad 17.03$

$\begin{array}{llllll}20 & 2330 & 44.52 & 19 & 22.83\end{array}$

$\begin{array}{lllll}21 & 1745 & 39.76 & 19 & 17.38\end{array}$

$\begin{array}{llllll}23 & 350 & 55.80 & 19 & 20.52\end{array}$

$2344 \quad 13.82 \quad 1921.56$

23164140.111925 .66

$\begin{array}{llllll}23 & 1647 & 8.11 & 19 & 25.68\end{array}$

$23 \quad 184147.111921 .89$

$\begin{array}{llllll}24 & 044 & 34.90 & 19 & 20.24\end{array}$

$\begin{array}{llllll}24 & 431 & 39.36 & 19 & 16.55\end{array}$

$\begin{array}{llllll}26 & 927 & 8.36 & 19 & 20.53\end{array}$

$\begin{array}{llllll}26 & 1332 & 54.22 & 19 & 22.27\end{array}$

$\begin{array}{llllll}26 & 2053 & 57.19 & 19 & 21.90\end{array}$

$\begin{array}{lllll}26 & 2255 & 24.94 & 19 & 56.47\end{array}$

$27 \quad 232 \quad 52.42 \quad 19 \quad 17.19$

$\begin{array}{llllll}27 & 716 & 38.30 & 19 & 20.26\end{array}$

$30920 \quad 40.841922 .59$

$31934 \quad 14.341921 .62$
$155 \quad 6.53$

15520.86

15450.98

$155 \quad 15.36$

15528.36

15523.59

15521.58

$155 \quad 1.71$

15521.16

$155 \quad 7.72$

$155 \quad 2.45$

15517.86

15518.04

$155 \quad 6.89$

15516.60

15512.50

1556.54

$155 \quad 5.15$

15515.29

15546.14

15522.15

15511.94

1556.09

1552.41
KM MAG MAG

GAP RMS MIN ERH

EKZ

0101.1011

$9.27 \quad 4.0 \quad 4.1 \quad 26$

6.983 .23 .623

8.424 .14 .325

$\begin{array}{llll}7.28 & 3.4 & 4.129\end{array}$

$9.413 .4 \quad 3.6 \quad 28$

$9.513 .5 \quad 3.7 \quad 27$

$7.88 \quad 3.4 \quad 3.6 \quad 25$

$9.413 .8 \quad 3.928$

$10.103 .8 \quad 3.930$

$0 \begin{array}{lll}0 & 7 \\ 0 & 1 & 13\end{array}$

0 1 119

119.1212

.6 1.0 MER

.7 . .5 UER

.6 1.1 MER

$.8 \quad 1.1 \mathrm{MER}$

$5.66 \quad 2.9 \quad 3.5 \quad 24$

8.902 .93 .629

$6.623 .43 .8 \quad 29$

$9.65 \quad 3.1 \quad 3.5 \quad 28$

$9.683 .6 \quad 4.0 \quad 29$

0107

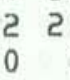

$8.46 \quad 3.4 \quad 3.7 \quad 27$

$0 \quad 60.1413$

$12.77 \quad 3.8 \quad 4.236$

$\begin{array}{llll}0 & 109 \quad .18 \quad 9\end{array}$

$\begin{array}{llll}0 & 128 & .17 & 9\end{array}$

3149.1511

0126.17

$0 \quad 86.08$

$\begin{array}{lllll}12.72 & 3.6 & 3.7 & 35\end{array}$

$8.973 .7 \quad 4.129$

9.443 .13 .529

0146.1212

$0 \quad 39.085$

$\begin{array}{llll}0 & 48.08 & .08\end{array}$

$075.08 \quad 8$

$9.93 \quad 3.5 \quad 3.8 \quad 28$

$9.51 \quad 3.5 \quad 3.9 \quad 24$

$9.16 \quad 3.7 \quad 4.0 \quad 28$

$9.65 \quad 3.2 \quad 3.6 \quad 15$

8.474 .2

15

$0 \quad 156.10 \quad 11$

0102.096

$\begin{array}{rrrr}0 & 79 & .09 & 8 \\ 0 & 65 & .09 & 20\end{array}$

$0 \quad 179.16 \quad 40$

$10.12 \quad 3.7 \quad 4.0 \quad 14 \quad 0 \quad 132 \quad .2315$

$8.33 \quad 3.94 .0 \quad 9 \quad 2 \quad 127 \quad .1023$

9.213 .63 .125

$\begin{array}{llll}7.12 & 3.2 & 3.6 & 25\end{array}$ $\begin{array}{rrr}.5 & .4 & \text { UER } \\ .6 & .4 & \text { SWR } \\ 2.6 & 1.4 & \text { LER } \\ .5 & .6 & \text { KOA } \\ .7 & .5 & \text { UKF }\end{array}$

.93 .6 SWR

1.0 1.6 SWR

. $\$$ 1.2 MER

1.0 .6 SWR

.S .2 UER

1.0 . A MEK

.4 .? LPC

.5 .2 LPC

.5 . 4 UER

.5 . 5 KOA

.8 .3 POL

.8 . 5 UER

.6 . .5 MEK

$.7 .4 \mathrm{KUA}$

1. $41.7 \mathrm{KOH}$

2.5.9 SWR

1.0 2.8 UER

. 6 . 7 UER

.91 .3 IMER 
Table 7. Felt earthquakes of magnitude $\geq 3.0$

Jan 1-5 earthquake swarm following volcanic activity at Kilauea. Many hundreds of earthquakes felt in the Ka'u and Puna districts of Hawaii. Records obscured, only well-recorded, large events are listed.

\begin{tabular}{|c|c|c|c|c|c|c|}
\hline \multicolumn{2}{|c|}{ Date } & \multicolumn{3}{|c|}{ Time } & \multirow[t]{2}{*}{ Magnitude } & \multirow[t]{2}{*}{ Felt report } \\
\hline & & $\underline{\mathrm{H}}$ & $\mathrm{M}$ & S & & \\
\hline Jan & 1 & 01 & 02 & 06 & 4.1 & Glenwood, Pahala, Volcano, Hilo \\
\hline & 1 & 02 & 41 & 10 & 4.6 & $\begin{array}{l}\text { Captain Cook, Hilo, Pahala, Volcano } \\
\text { Kamuela }\end{array}$ \\
\hline & 1 & 03 & 05 & 45 & 3.6 & Hilo, Pahala \\
\hline & 1 & 05 & 18 & 12 & 3.5 & Hilo, Pahala \\
\hline & 1 & 09 & 11 & 35 & 3.6 & Hilo, Pahala \\
\hline & 1 & 09 & 46 & 45 & $4 \cdot 3$ & Hilo, Volcano, Pahala \\
\hline & 1 & 10 & 27 & 04 & 3.6 & Hilo, Pahala \\
\hline & 1 & 10 & 46 & 48 & 4.3 & Hilo, Pahala, Puna \\
\hline & 1 & 11 & 28 & 54 & 4.1 & Hilo, Pahala \\
\hline & 1 & 15 & 35 & 18 & 3.7 & Hilo, Pahala \\
\hline & 2 & 03 & 27 & 42 & 4.9 & Volcano, Kamuela, Hilo, Pahala \\
\hline & 2 & 03 & 49 & 07 & 3.0 & South Kona, Hilo, Pahala \\
\hline & 3 & 01 & 45 & 50 & 3.9 & Hilo, Pahala, Volcano \\
\hline & 3 & 07 & 32 & 49 & 4.9 & $\begin{array}{c}\text { Pahala, Glenwood, Kamuela, Volcano, } \\
\text { Kealakekua, Captain Cook, Hilo }\end{array}$ \\
\hline & 3 & 08 & 14 & 45 & 3.5 & Pahala, Volcano \\
\hline & 3 & 11 & 17 & 28 & 3.7 & Volcano, Pahala \\
\hline & 3 & 20 & 35 & 52 & 4.4 & $\begin{array}{l}\text { Keaau, Kealakekua, Kamuela, Hilo, } \\
\text { Volcano, Pepeekeo, Glenwood, } \\
\text { Pahala, Pahoa }\end{array}$ \\
\hline & 4 & 02 & 13 & 27 & 3.8 & Hilo, Pahala, Volcano \\
\hline & 4 & 15 & 32 & 04 & 4.9 & $\begin{array}{l}\text { Volcano, Kamuela, Kealakekua, } \\
\text { Hilo (Island-wide) }\end{array}$ \\
\hline & 4 & 19 & 28 & 03 & 3.8 & Pahala, Volcano \\
\hline & 5 & 00 & 48 & 39 & 4.0 & Pahala, Volcano, Hilo \\
\hline & 6 & 17 & 47 & 02 & 4.4 & Pahala, Volcano \\
\hline & 8 & 05 & 52 & 49 & 4.1 & South Kona, Volcano, Pahala \\
\hline & 8 & 08 & 13 & 31 & 3.5 & South Kona, Pahala \\
\hline $\mathrm{Feb}$ & 7 & 04 & 04 & 43 & 3.3 & Volcano, Hawaii National Park \\
\hline & 7 & 06 & 46 & 52 & 3.6 & South Kona \\
\hline & 19 & 15 & 42 & 48 & 3.2 & Hilo \\
\hline & 21 & 11 & 19 & 40 & 3.5 & Hilo, Pahoa \\
\hline Mar & 10 & 00 & 14 & 12 & 4.0 & $\begin{array}{l}\text { Hilo, Captain Cook, Volcano, } \\
\text { Mt. View, Pahala }\end{array}$ \\
\hline & 16 & 20 & 57 & 58 & 3.2 & Volcano \\
\hline & 26 & 10 & 06 & 03 & 4.3 & $\begin{array}{l}\text { Mauna Loa Observatory, Hawaiian } \\
\text { Volcano Observatory, South Kona, } \\
\text { Pahala }\end{array}$ \\
\hline
\end{tabular}




\begin{tabular}{|c|c|c|c|c|c|c|}
\hline \multicolumn{2}{|c|}{ Date } & \multicolumn{3}{|c|}{ Time } & \multirow[t]{2}{*}{ Magnitude } & \multirow[t]{2}{*}{ Felt report } \\
\hline & & $\underline{\mathrm{H}}$ & M & $\underline{S}$ & & \\
\hline \multirow[t]{13}{*}{ Apr } & 4 & 13 & 09 & 55 & 3.9 & Hilo, Pahoa \\
\hline & 4 & 13 & 35 & 17 & 3.5 & Kahuku, Hilo, Pahoa \\
\hline & 5 & 10 & 24 & 09 & 3.5 & Kahuku \\
\hline & 5 & 11 & 56 & 57 & 3.8 & Kahuku \\
\hline & 6 & 12 & 15 & 55 & 3.8 & $\begin{array}{l}\text { Hilo, Hawaii National Park, } \\
\text { Pohakuloa, Volcano }\end{array}$ \\
\hline & 10 & 14 & 18 & 40 & 3.4 & Volcano \\
\hline & 11 & 06 & 22 & 24 & 3.3 & Volcano \\
\hline & 17 & 23 & 30 & 14 & 3.6 & Volcano, Mt. View, Hilo \\
\hline & 18 & 03 & 22 & 28 & 3.7 & Volcano, Glenwood, Hilo \\
\hline & 24 & 14 & 46 & 55 & 3.1 & Kapapala \\
\hline & 29 & 01 & 14 & 20 & 3.3 & Kona \\
\hline & 29 & 01 & 48 & 07 & 3.5 & Glenwood, Keaau, Volcano \\
\hline & 29 & 19 & 23 & 09 & 3.4 & Kapapala \\
\hline \multirow[t]{5}{*}{ May } & 10 & 19 & 32 & 51 & 3.9 & Hilo, Volcano, Kurtistown \\
\hline & 21 & 22 & 32 & 58 & 4.7 & Island-wide of Hawaii, Maui, Oahu \\
\hline & 22 & 04 & 46 & 53 & 3.2 & Kapapala \\
\hline & 27 & 16 & 15 & 34 & 3.0 & Kapapala \\
\hline & 29 & 12 & 14 & 13 & 3.9 & Volcano, Hilo, Pahoa, Kurtistown \\
\hline \multirow[t]{2}{*}{ Jun } & 7 & 11 & 53 & 57 & 3.7 & Ocean View Estates \\
\hline & 21 & 13 & 34 & 56 & 3.6 & Volcano \\
\hline
\end{tabular}

Jul 5 earthquake swarm following volcanic activity of Mauna Loa. Many quakes were felt but only the well recorded, large events are listed.

\begin{tabular}{|c|c|c|c|c|c|c|}
\hline \multicolumn{2}{|c|}{ Date } & \multirow[b]{2}{*}{$\underline{\mathrm{H}}$} & \multicolumn{2}{|c|}{ Time } & \multirow[t]{2}{*}{ Magnitude } & \multirow[t]{2}{*}{ Felt report } \\
\hline & & & M & $\underline{S}$ & & \\
\hline JuI & 4 & 17 & 40 & 55 & 4.1 & Island-wide \\
\hline & 5 & 23 & 18 & 18 & $4 \cdot 3$ & Hilo, Volcano, Pahala, Kona \\
\hline & 5 & 23 & 25 & 47 & 4.2 & Hilo, Volcano, Pahala, Kona \\
\hline & 7 & 05 & 39 & 48 & 3.6 & Volcano \\
\hline & 7 & 14 & 47 & 42 & 4.7 & Southern parts of the island \\
\hline & 7 & 18 & 39 & 52 & 4.2 & Hilo, Volcano, Pahala, Kona \\
\hline & 8 & 01 & 09 & 07 & 3.6 & Pahala \\
\hline & 8 & 20 & 07 & 02 & 3.9 & Hilo, Volcano, Pahala, Kona \\
\hline & 9 & 05 & 47 & 43 & 3.9 & Hilo, Volcano, Pahala, Kona \\
\hline & 9 & 08 & 40 & 03 & 3.4 & Island-wide \\
\hline & 18 & 21 & 19 & 58 & 3.4 & Hilo \\
\hline & 22 & 15 & 12 & 33 & 4.4 & Kona \\
\hline & 26 & 23 & 14 & 59 & 3.7 & Kona \\
\hline & 30 & 13 & 31 & 22 & 3.9 & Waimea, Pahala, South Kona \\
\hline Aug & 5 & 07 & 36 & 39 & 3.3 & Kapapala \\
\hline & 18 & 07 & 08 & 56 & 3.9 & Kona \\
\hline & 24 & 05 & 45 & 35 & 3.6 & Volcano \\
\hline & 27 & 07 & 34 & 44 & 4.1 & $\begin{array}{l}\text { Volcano, South Kona, Keaau, } \\
\text { Pahoa, Hualalai }\end{array}$ \\
\hline Sep & 10 & 08 & 34 & 11 & 3.5 & Volcano, Hilo \\
\hline & 21 & 19 & 46 & 05 & 3.2 & Kurtistown, Hilo, Volcano \\
\hline
\end{tabular}


In addition to the seismic network, a network of spirit-level tilt stations (dry), borehole tiltmeters, and water-tube (wet) tilt stations is maintained. The network is located on the summits and flanks of Kilauea and Mauna Loa Volcanoes. In December 1975 the tilt network consisted of:

54 spirit level tilt stations (dry)

8 borehole tiltmeters

10 water-tube tilt stations (wet)

1 continuous recording Ideal-Arrowsmith tiltmeter

Dry and wet tilt stations are generally occupied at irregular intervals. Critical stations are measured more frequently than the entire network. Digital borehole tiltmeters data are telemetered by VHF radio and recorded at the observatory. An Ideal-Arrowsmith mercury-pool capacitor-type tiltmeter with a $1 \mathrm{~m}$ base is located at the Uwekahuna vault, and the analog signal is recorded at the observatory. 
Tilting of the ground around the surmit of Kilauea is monitored daily by a short-base water-tube tiltmeter in Uwekahuna Vault, and at irregular intervals it is measured on a regional scale by means of a network of field tilt-bases and a portable water-tube tiltmeter. The attitude of the ground surface at each tilt-base is reported in terms of north-south and east-west tilt coordinates. Both coordinates at each station were arbitrarily set equal to 500 when measurements at that station were begun. Increasing tilt coordinates correspond to northward and eastward tilting of the earth's surface; that is, to a relative subsidence toward the north and east. A oneunit change in coordinate corresponds to a tilting of 1 microradian ( $1 \mathrm{~mm}$ per $\mathrm{km}$ ) in the direction indicated.

Location of and essential data on each tiltmeter station are listed in table.

Table 8.--Tilt Coordinates at Uwekahuna

\begin{tabular}{|c|c|c|}
\hline Date (1975) & $\mathrm{N}-\mathrm{S}$ & $E-W$ \\
\hline $\begin{array}{r}\operatorname{Jan} 5 \\
12 \\
19 \\
26\end{array}$ & $\begin{array}{l}716 \\
709 \\
706 \\
706\end{array}$ & $\begin{array}{l}341 \\
336 \\
337 \\
336\end{array}$ \\
\hline $\begin{array}{ll}\text { Feb } 2 \\
9 \\
\\
16 \\
23\end{array}$ & $\begin{array}{l}707 \\
707 \\
708 \\
710\end{array}$ & $\begin{array}{l}330 \\
330 \\
328 \\
326\end{array}$ \\
\hline $\begin{array}{r}\text { Mar } 2 \\
9 \\
16 \\
23 \\
30\end{array}$ & $\begin{array}{l}711 \\
712 \\
715 \\
716 \\
717\end{array}$ & $\begin{array}{l}324 \\
323 \\
321 \\
319 \\
315\end{array}$ \\
\hline $\begin{array}{rr}\text { Apr } & 6 \\
13 \\
20 \\
27\end{array}$ & $\begin{array}{l}718 \\
720 \\
721 \\
723\end{array}$ & $\begin{array}{l}314 \\
313 \\
313 \\
310\end{array}$ \\
\hline $\begin{array}{r}4 \\
\text { May } \\
11 \\
18 \\
25\end{array}$ & $\begin{array}{l}725 \\
727 \\
728 \\
728\end{array}$ & $\begin{array}{l}309 \\
307 \\
311 \\
312\end{array}$ \\
\hline 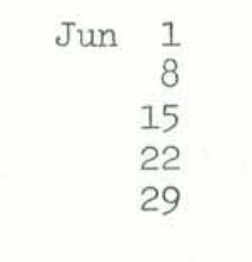 & $\begin{array}{l}728 \\
731 \\
731 \\
732 \\
734 \\
\mathbf{1 0 8}\end{array}$ & $\begin{array}{l}312 \\
310 \\
305 \\
306 \\
303\end{array}$ \\
\hline
\end{tabular}


Table 8.--Tilt Coordinates at Uwekahuna (Continued)

\begin{tabular}{|c|c|c|}
\hline Date (1975) & $\mathrm{N}-\mathrm{S}$ & $\mathrm{E}-\mathrm{W}$ \\
\hline $\begin{array}{r}\text { Jul } 6 \\
13 \\
20 \\
27\end{array}$ & $\begin{array}{l}735 \\
737 \\
737 \\
739\end{array}$ & $\begin{array}{l}302 \\
301 \\
302 \\
299\end{array}$ \\
\hline Aug $\begin{array}{r}3 \\
10 \\
17 \\
24 \\
31\end{array}$ & $\begin{array}{l}739 \\
742 \\
745 \\
747 \\
751\end{array}$ & $\begin{array}{l}298 \\
296 \\
294 \\
291 \\
285\end{array}$ \\
\hline $\begin{array}{r}\quad 7 \\
14 \\
21 \\
28\end{array}$ & $\begin{array}{l}752 \\
755 \\
756 \\
759\end{array}$ & $\begin{array}{l}286 \\
277 \\
277 \\
276\end{array}$ \\
\hline Oct $\begin{array}{r}5 \\
12 \\
19 \\
26\end{array}$ & $\begin{array}{l}759 \\
760 \\
759 \\
760\end{array}$ & $\begin{array}{l}277 \\
275 \\
272 \\
268\end{array}$ \\
\hline Nov $\begin{array}{r}2 \\
9 \\
16 \\
23 \\
30\end{array}$ & $\begin{array}{l}760 \\
762 \\
763 \\
766 \\
679\end{array}$ & $\begin{array}{l}268 \\
264 \\
262 \\
259 \\
342\end{array}$ \\
\hline $\begin{array}{rr}7 \\
14 \\
21 \\
28\end{array}$ & $\begin{array}{l}601 \\
588 \\
582 \\
576\end{array}$ & $\begin{array}{l}406 \\
409 \\
405 \\
406\end{array}$ \\
\hline
\end{tabular}


Table 9.--U.S. Geological Survey water-tube tiltmeter stations in Hawaii

\begin{tabular}{|c|c|c|c|c|c|c|}
\hline \multirow{2}{*}{ Station } & \multirow{2}{*}{ Symbol } & \multicolumn{2}{|c|}{ Location } & \multirow{2}{*}{$\begin{array}{c}\text { Frequency } \\
\text { of } \\
\text { reading }\end{array}$} & \multirow{2}{*}{$\begin{array}{c}\text { Base } \\
\text { length } \\
M\end{array}$} & \multirow{2}{*}{ Description } \\
\hline & & $\begin{array}{ll}\text { Lat. N. } \\
\text { Deg. } \\
\text { M1n. }\end{array}$ & $\begin{array}{l}\text { Long. W. } \\
\text { Deg. Min. }\end{array}$ & & & \\
\hline Tree Molds & $\mathrm{TM}$ & $19-26.3$ & $155-17.3$ & & 50.79 & NS. and EW. \\
\hline Sand Sp1t & ss & $19-24.1$ & $155-16.8$ & & 25.40 & $\begin{array}{l}\text { Equilateral } \\
\text { triangle. }\end{array}$ \\
\hline Keamoku & Kea & $19-25.1$ & $155-19.0$ & & 47.55 & do \\
\hline Ahua & & & & & & \\
\hline Kamokukolau & Kam & $19-22.7$ & $155-16.6$ & & 50.79 & do \\
\hline K1puka Nene & $\mathrm{KN}$ & $19-19.4$ & $155-16.7$ & & 47.73 & do \\
\hline Hilina Pall & HP & $19-18.2$ & $155-18.6$ & & 47.73 & do \\
\hline Kapapala & & & & & & \\
\hline Ranch & Kap & $19-20.5$ & $155-23.8$ & & 50.79 & do \\
\hline Mehana & M & $19-26.2$ & $155-14.3$ & & 25.00 & do \\
\hline Uwekahuna & $\mathrm{U}$ & $19-25.5$ & $155-17.4$ & & 50.79 & do \\
\hline $\begin{array}{l}\text { Uwekahuna } \\
\text { Vault }\end{array}$ & & $19-25.4$ & $155-17.6$ & Daily & 3.48 & NS. and EW. \\
\hline
\end{tabular}


Table 10--Tilt coordinates and changes at bases around Kilauea caldera. (See fig 10)

\begin{tabular}{|c|c|c|c|c|c|c|}
\hline Tilt base & $\begin{array}{c}\text { Date } \\
\text { (1975) }\end{array}$ & $\begin{array}{l}\text { Tilt } \\
\mathrm{N}-\mathrm{S}\end{array}$ & $\begin{array}{c}\text { Coordinates } \\
\qquad E-W\end{array}$ & $\begin{array}{l}\text { Rate ( } \\
\text { and di } \\
\text { tiltin } \\
\text { last } r\end{array}$ & $\begin{array}{l}6 \mathrm{rad} / \mathrm{mo}) \\
\text { tion of } \\
\text { ince } \\
\text { inf }\end{array}$ & $\begin{array}{c}\text { Date of last } \\
\text { reading } \\
(1975)\end{array}$ \\
\hline Uwekahuna（U) & $14 \mathrm{Apr}$ & 742.7 & 268.0 & 14.23 & $\mathrm{~N} 42.0^{\circ} \mathrm{W}$ & $14 \mathrm{Jan}$ \\
\hline Tree Molds (TM) & $14 \mathrm{Apr}$ & 565.5 & 485.8 & 5.11 & $\mathrm{~N} 17.8^{\circ} \mathrm{W}$ & $14 \mathrm{Jan}$ \\
\hline Sand Spit (SS) & $15 \mathrm{Apr}$ & 947.1 & 743.5 & 9.25 & $\mathrm{~N} 25.2^{\circ} \mathrm{W}$ & $15 \mathrm{Jan}$ \\
\hline Keamoku (Kea). & $16 \mathrm{Apr}$ & 751.3 & 271.3 & 9.10 & $\mathrm{~N} 53.3^{\circ} \mathrm{W}$ & $16 \mathrm{Jan}$ \\
\hline Ahua Kamokukolau (Kam). & $15 \mathrm{Apr}$ & 465.9 & 603.2 & 14.49 & $\mathrm{~S} 10.9^{\circ} \mathrm{E}$ & $15 \mathrm{Jan}$ \\
\hline Kipuka Nene (KN) & $17 \mathrm{Apr}$ & 256.7 & 517.9 & 1.09 & $\mathrm{~S} 42.5^{\circ} \mathrm{W}$ & $17 \mathrm{Jan}$ \\
\hline Hilina Pali (HP) & 17 Apr & 397.1 & 526.1 & 0.46 & $\mathrm{~S} 35.0^{\circ} \mathrm{W}$ & $28 \mathrm{Jan}$ \\
\hline Kapapala Ranch (Kap). & 16 Apr & 573.5 & 454.0 & 2.92 & $\mathrm{~S} 43.2^{\circ} \mathrm{E}$ & $16 \mathrm{Jan}$ \\
\hline Mehana (M) & $14 \mathrm{Apr}$ & 620.3 & 592.0 & 1.41 & $N 81.9^{\circ} \mathrm{E}$ & $14 \mathrm{Jan}$ \\
\hline
\end{tabular}




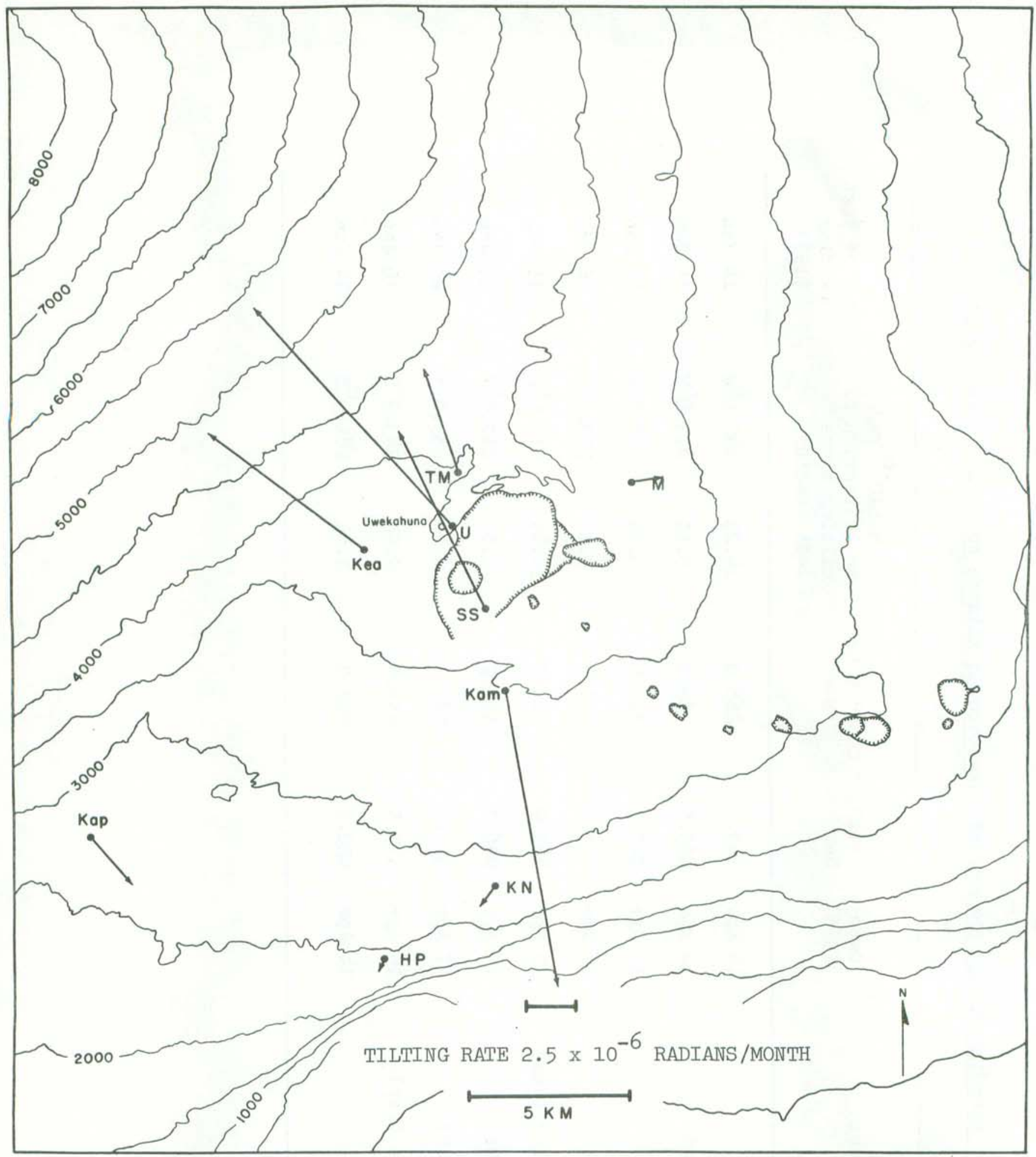

Figure 10 January to April 1975 tilting of the ground around Kilauea Caldera. The vector depicting tilt at a given tilt base points in the direction of maximum relative subsidence, and its length is proportional to the rate of tilting during the measurement interval. Closed circles represent field tilt bases; open circles, short-base watertube tiltmeters. See Table 9 for explanation of abbreviations. 
Table 11. Tilt coordinates and changes at bases around Kilauea caldera. (See fig.11)

\begin{tabular}{|c|c|c|c|c|c|c|}
\hline \multirow[b]{2}{*}{ Uwekahuna (U) } & $\begin{array}{l}\text { Date } \\
(1975)\end{array}$ & \multicolumn{2}{|c|}{$\begin{array}{l}\text { Tilt Coordinates } \\
\mathrm{N}-\mathrm{S} \\
\text { E-W }\end{array}$} & \multicolumn{2}{|c|}{$\begin{array}{l}\text { Rate }\left(10^{-6} \mathrm{rad} / \mathrm{mo}\right) \\
\text { and direction of } \\
\text { tilting since } \\
\text { last reading }\end{array}$} & \multirow{2}{*}{$\frac{\begin{array}{c}\text { Date of la } \\
\text { reading } \\
(1975)\end{array}}{14 \mathrm{Apr}}$} \\
\hline & $15 \mathrm{Sep}$ & 788.2 & 233.0 & 11.17 & $\mathrm{~N} 37.5^{\circ} \mathrm{W}$ & \\
\hline Tree Molds (TM) & $17 \mathrm{Sep}$ & 592.3 & 478.1 & 5.34 & $\mathrm{~N} 15.9^{\circ} \mathrm{W}$ & $14 \mathrm{Apr}$ \\
\hline Sand Spit (SS) & $19 \mathrm{Sep}$ & 1018.3 & 683.0 & 17.86 & $\mathrm{~N} 40.3^{\circ} \mathrm{W}$ & $15 \mathrm{Apr}$ \\
\hline Mehana (M) & $17 \mathrm{Sep}$ & 627.1 & 603.7 & 2.59 & $\mathrm{~N} 60.2^{\circ} \mathrm{E}$ & $14 \mathrm{Apr}$ \\
\hline Keamoku (Kea) & $15 \mathrm{Sep}$ & 781.6 & 225.3 & 10.88 & $\mathrm{~N} 56.6^{\circ} \mathrm{W}$ & $16 \mathrm{Apr}$ \\
\hline Ahua Kamokukolau (Kam) & $16 \mathrm{Sep}$ & 352.7 & 605.2 & 21.09 & $51.0^{\circ} \mathrm{E}$ & $15 \mathrm{Apr}$ \\
\hline Kipuka Iene (KN) & $22 \mathrm{Sep}$ & 252.2 & 517.0 & 0.88 & $\mathrm{~S} 11.6^{\circ} \mathrm{W}$ & $17 \mathrm{Apr}$ \\
\hline Hilina Pali (HP) & $18 \mathrm{Sep}$ & 398.1 & 518.1 & 1.60 & $\mathrm{~N} 82.9^{\circ} \mathrm{W}$ & $17 \mathrm{Apr}$ \\
\hline Kapapala Ranch (Kap) & $19 \mathrm{Sep}$ & 570.4 & 455.6 & 0.67 & $\mathrm{~S} 27.5^{\circ} \mathrm{W}$ & $16 \mathrm{Apr}$ \\
\hline
\end{tabular}




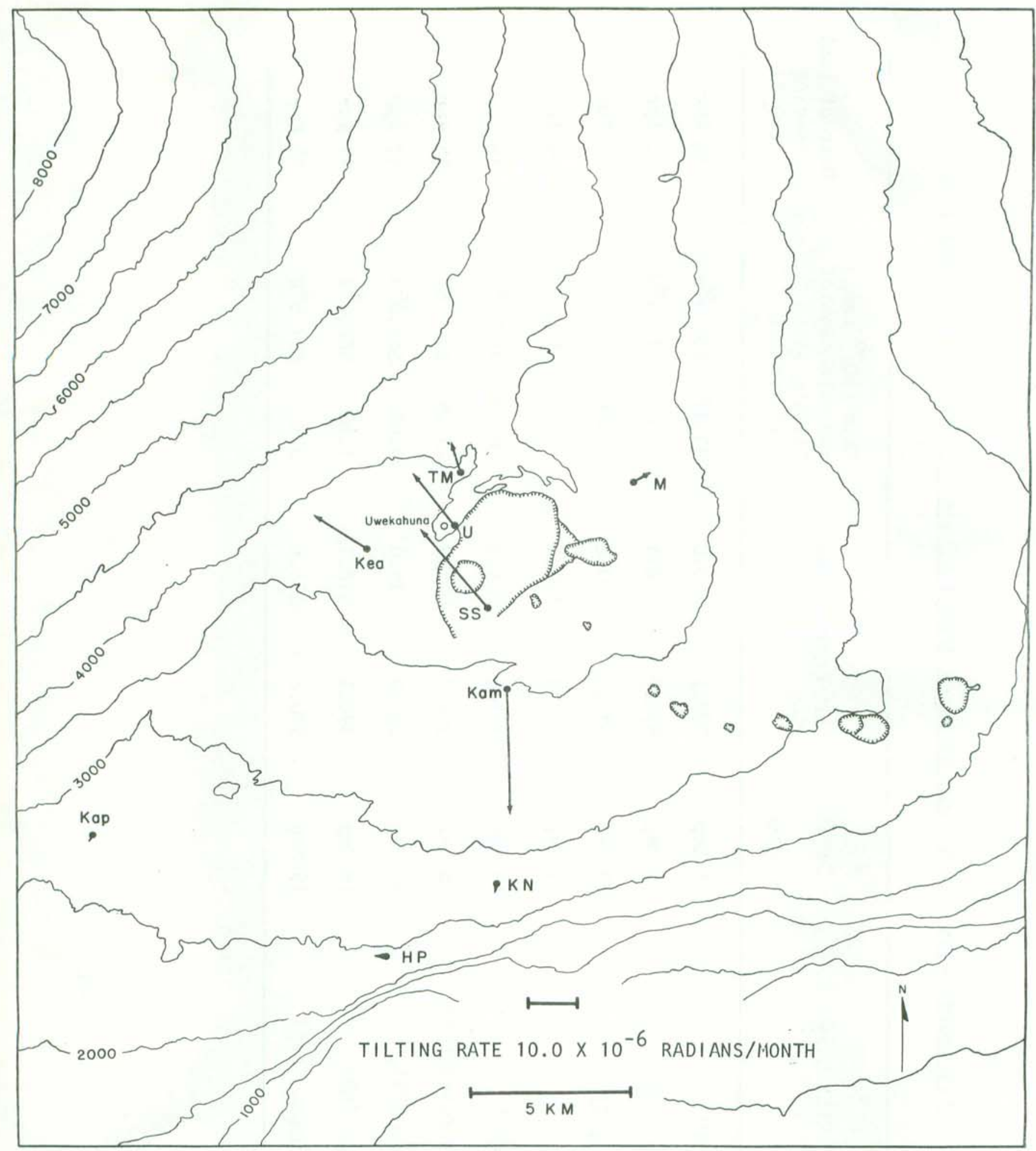

Figure 11 April to September 1975 tilting of the ground around Kilauea Caldera. 
Table 12. Tilt coordinates and changes at bases around Kilauea caldera. (See fig.12)

\begin{tabular}{|c|c|c|c|c|c|c|}
\hline \multirow{2}{*}{$\begin{array}{r}\text { Tilt base } \\
\text { Uwekahuna (U) }\end{array}$} & $\begin{array}{l}\text { Date } \\
\text { (1975) }\end{array}$ & \multicolumn{2}{|c|}{$\begin{array}{cc}\text { Tilt Coordinates } \\
\mathrm{N}-\mathrm{S} & \mathrm{E}-\mathrm{W}\end{array}$} & \multicolumn{2}{|c|}{$\begin{array}{l}\text { Rate }\left(10^{-6} \mathrm{rad} / \mathrm{mo}\right) \\
\text { and direction of } \\
\text { tilting since } \\
\text { last reading }\end{array}$} & $\begin{array}{l}\text { Date of las } \\
\text { reading } \\
(1975)\end{array}$ \\
\hline & $19 \mathrm{Dec}$ & 591.1 & 369.1 & 75.69 & $\mathrm{~S} 34.6^{\circ} \mathrm{E}$ & 15 Sep \\
\hline Tree Molds (TM) & $16 \mathrm{Dec}$ & 489.7 & 518.7 & 36.41 & $\mathrm{~S} 21.6^{\circ} \mathrm{E}$ & $17 \mathrm{Sep}$ \\
\hline Sand Spit (SS) & $18 \mathrm{Dec}$ & 841.7 & 820.5 & 73.87 & $\mathrm{~S} 37.9^{\circ} \mathrm{E}$ & 19 Sep \\
\hline Mehana (M) & $16 \mathrm{Dec}$ & 562.0 & 562.0 & 25.52 & $\mathrm{~S} 32.6^{\circ} \mathrm{W}$ & 17 Sep \\
\hline Keamoku (Kea) & $17 \mathrm{Dec}$ & 600.9 & 480.2 & 100.80 & $\mathrm{~S} 54.7^{\circ} \mathrm{E}$ & 15 Sep \\
\hline Ahua Kamokukolau (Kam) & $18 \mathrm{Dec}$ & 656.2 & 560.5 & 98.96 & $\mathrm{~N} 8.4^{\circ} \mathrm{W}$ & $16 \mathrm{Sep}$ \\
\hline Kipuka Nene (KN) & $15 \mathrm{Dec}$ & 141.2 & 589.7 & $47 \cdot 40$ & $\mathrm{~S} 33.2^{\circ} \mathrm{E}$ & $22 \mathrm{Sep}$ \\
\hline Hilina Pali (HP) & $15 \mathrm{Dec}$ & 285.9 & 565.0 & 41.46 & $\mathrm{~S} 22.7^{\circ} \mathrm{E}$ & $18 \mathrm{Sep}$ \\
\hline Kapapala Ranch (Kap) & $17 \mathrm{Dec}$ & 518.3 & 506.0 & .22 .42 & $\mathrm{~S} 44.1^{\circ} \mathrm{E}$ & $19 \mathrm{Sep}$ \\
\hline
\end{tabular}




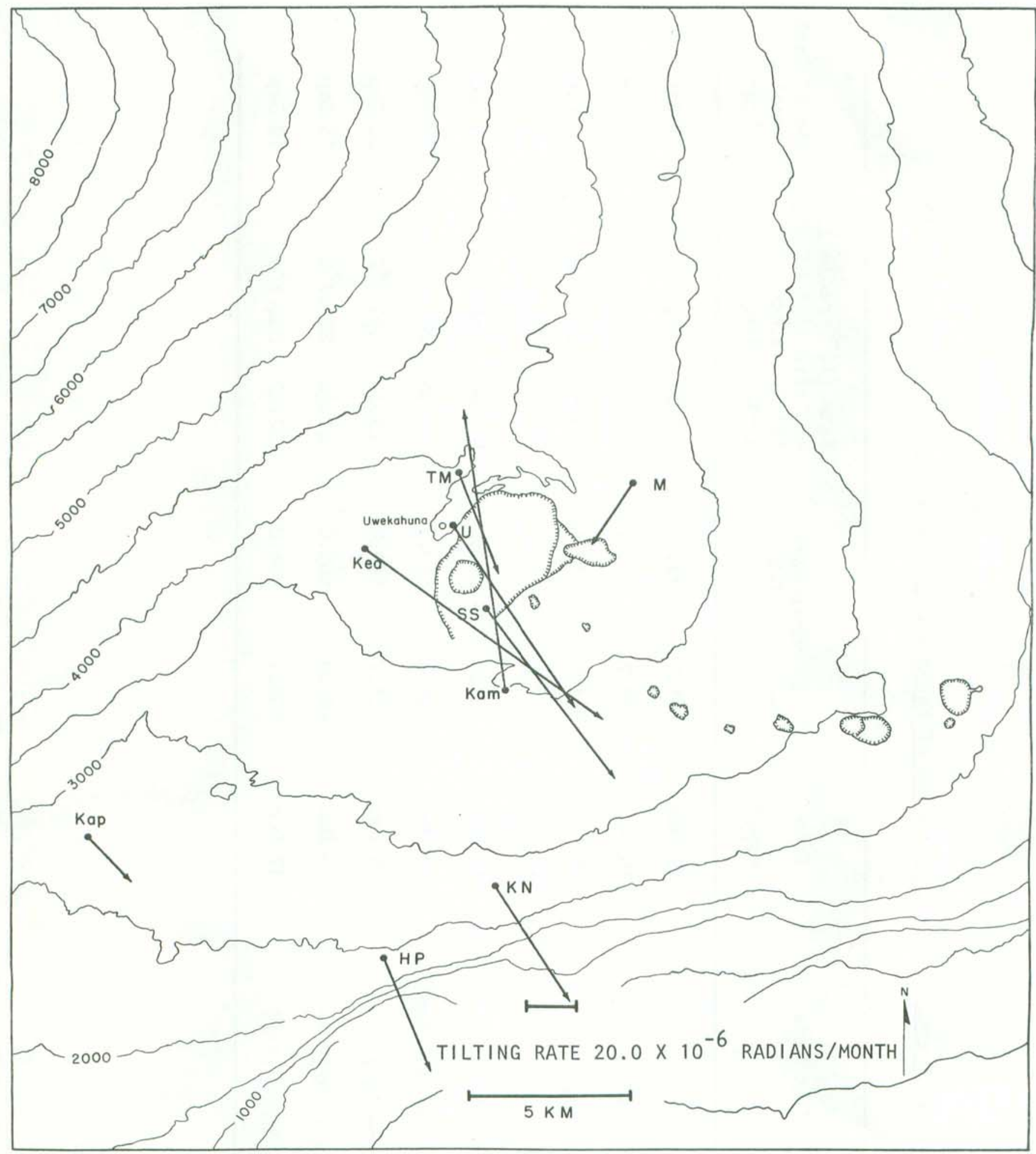

Figure 12. September to December 1975 tilting of the ground around Kilauea Caldera. 
Crosson, R. S., 1976, Velocity structure below the Island of Hawaii from earthquake modelling, (abstract), EOS Trans. Am. Geoph. Union, 57, 961.

Koyanagi, R. Y., Stevenson, P., Endo, E. T., and Okamura, A. T., Hawaiian Volcano Observatory Summary 74, January to December 1974.

Lockwood, J. P., Koyanagi, R. Y., Tilling, R. I., Holcomb, R. T., and Peterson, D. W., 1976, Mauna Loa threatening: Geotimes, v. 21, no. 6, p. 12-15.

Tilling, R. I., Koyanagi, R. Y., Lipman, P. W., Lockwood, J. P., Moore, J. G., and Swanson, D. A., 1976, Earthquakes and related catastrophic events, Island of Hawaii, November 29, 1975 -- a preliminary report: U.S. Geol. Survey Circ. 740,33 p. 